\title{
Mass Customization Services
}

Edwards, Kasper; Blecker, Thorsten; Hvam, Lars; Salvador, Fabrizio; Friedrich, Gerhard

Publication date:

2008

Document Version

Publisher's PDF, also known as Version of record

Link back to DTU Orbit

Citation (APA):

Edwards, K. (Ed.), Blecker, T. (Ed.), Hvam, L. (Ed.), Salvador, F. (Ed.), \& Friedrich, G. (2008). Mass Customization Services. (1 ed.) DTU Management Engineering.

\section{General rights}

Copyright and moral rights for the publications made accessible in the public portal are retained by the authors and/or other copyright owners and it is a condition of accessing publications that users recognise and abide by the legal requirements associated with these rights.

- Users may download and print one copy of any publication from the public portal for the purpose of private study or research.

- You may not further distribute the material or use it for any profit-making activity or commercial gain

- You may freely distribute the URL identifying the publication in the public portal

If you believe that this document breaches copyright please contact us providing details, and we will remove access to the work immediately and investigate your claim. 
Mass Customization Services 


\section{Kolofon:}

Ass. Prof. Dr. Kasper Edwards Technical University of Denmark

Department of Management Engineering

Building 423

2800 Lyngby, Denmark

edwards@ipl.dtu.dk

Prof. Dr. Thorsten Blecker

Hamburg University of Technology (TUHH)

Institute of Business Logistics and General Management (5-11)

Schwarzenbergstr. 95

21073 Hamburg, Germany

blecker@ ieee.org

Prof. Dr. Fabrizio Salvador

Instituto de Empresa Business School

Academic Area Operations \& Technology

Maria de Molina, 11

28006 Madrid, Spain

fabrizio.salvador@ie.edu

Associate Prof. Dr. Lars Hvam

Technical University of Denmark

Department of Management Engineering

Building 425

2800 Lyngby, Denmark

lhv@ipl.dtu.dk

Prof. Dr. Gerhard Friedrich

University of Klagenfurt

Computer Science and Manufacturing

Universitaetsstr. 65 - 67

9020 Klagenfurt, Austria

gerhard.friedrich@uni-klu.ac.at

ISBN: 978-87-90855-12-3 DTU Management Engineering

Layout: J-Grafik, Copenhagen, Denmark

Print: Vesterkopi, Copenhagen, Denmark

(C) Kasper Edwards and Technical University of Denmark.2008 


\section{Mass Customization Services}

Edwards, $K$.

Blecker, Th.

Salvador, F.

Hvam, $L$.

Friedrich, $G$.

(Eds.): 
9 Supply-side Collaboration with Modular Supply:

Cases from Chinese Automotive Industry.

By Yong Lin, Yongjiang Shi, Shihua Ma

25 Customer Co-creation in the Dutch Financial Services.

By Marcel Weber

41 Different configuration problems at different stages in the service life cycle.

By Jose Angel Lakunza, Juan Carlos Astiazaran, Maria Elejoste

$55 \quad$ Configuring an open pipeline fulfilment system

- a simulation study in an automotive context.

By Philip G. Brabazon, Andy Woodcock, Bart L MacCarthy

70 Replanning in an open pipeline fulfilment system - a simulation study in an automotive context.

By Andrew Woodcock, Philip G. Brabazon, Bart L MacCarthy

86 An approach for the Real Time Intelligent Production Control to fulfill Mass Customization requirements.

By Sarfraz-ul-Haque, Minhas, Ralf, Kretzschmann, Veronica, Vargas, Ulrich, Berger

102 Driving Mass Customisation in Supply Networks.

A Machine Tool Sector Case Study.

By Eduardo Saiz, Eduardo Castellano, Juan Manuel Besga, Jone Uribetxeberria, Iñaki Zugasti

118 A cost management viewpoint to mass customizable services: a review and considerations.

By Author: Matti Sievänen

130 Products vs. services: The point of view of the customer. By Jose Angel Lakunza, Juan Carlos Astiazarán

140 Dimensions for classifying service mass customizers. By Mikko Heiskala, M.Sc. (Tech), Project Manager

158 Cognition of retrieved product information in configuration projects.

By Anders Haug 
171 Time Postponement: Modified Models to Improve Deli-very Lead Time.

By Soroosh Saghiri

182 Web-based VR for Pre-Sales Service Customization. By Boris Tudjarov, Angel Bachvarov, Ilia Boyadjiev

199 A software tool for design and documentation of configurator knowledge bases.

By Anders Haug, Torben Lisbjerg and Lars Hvam

219 Configuration of product-centric services. By Philipp Ackermann

229 Coupling interactively Product and Project Configuration: a Proposal using Constraints Programming. By Elise VAREILLES, Michel ALDANONDO, Meriem DJEFEL, Paul GABORIT

245 Issues in the implementation process of tailored logistics services for mass customization. By Frank Straube, Stefan Alexander Doch and Kathrin Klaar

259 Configuration and Product Modularization for the flexible Network: A Contribution to Mass Customization. By Wendelin Gross, Sven Kuhn

277 Development of Product Configurators - Levels of Customisation.

By Kaj A. Jørgensen

290 Developing appropriate metrics to measure supplier load in Mass Customization and high variety environments.

By Lorena Scarpulla, Bart MacCarthy, Philip Brabazon

307 Fuzzy Cognitive Map based Health ServiceCustomisation.

By Dimitrios K. Kardaras, Bill Karakostas, Eleutherios A. Papathanassiou, Stavroula G. Barbounaki, Aikaterini P. Kikilia

321 Developing a Frame of Reference for understanding configuration systems. By Klaes Ladeby Jensen and Kasper Edwards 



\section{Preface}

The International Mass Customization Meeting (IMCM) and the International Conference on Economic, Technical and Organizational Aspects of Product Configuration Systems (PETO) has been collaborating since 2006. For the third time the two conferences collaborate to offer a platform for advancing mass customization research. 22 papers have been accepted for presentation at the conference, this time in Copenhagen at the Technical University of Denmark

Topics of the IMCM'08 \& PETO'08 and this book are: Mass customization in service, mass customizing financial services, mass customization in supply networks, implementation issues in logistics, product life cycle and mass customization. The research field of mass customization is more than 15 years old but as the topics illustrate, quite a diverse field. This is expected to continue as long as all fields continue to evolve in their own direction. From a research point of view this provides an opportunity as practitioners from diverse fields have an interest in meeting and sharing ideas. The IMCM'08 \& PETO'08 is one such opportunity.

We would like to offer our special thanks to the authors who by submitting their work made this conference possible and thereby continue to support this conference as a platform for mass customization research.

Kasper Edwards, Thorsten Blecker, Lars Hvam, Fabrizio Salvador and Gerhard Friedrich.

\section{Conference Chairs}





\title{
Supply-side Collaboration with Modular Supply: Cases from Chinese Automotive Industry
}

\author{
Yong Lin, Yongjiang Shi, Shihua Ma
}

\begin{abstract}
The rules and practices of supply-side collaboration changed with the automaker moving to modular assembly, and modular supply becomes dominate of the emerging automotive industry. The major purpose of this paper is to identify how modular supply affects the supply-side collaboration, and how to organize the upstream supply network effectively to supply these two type modules. Based on the case studies in Chinese automotive industry and literature reviews, we conclude that there are two type modules, fully integrated module and partial integrated module, and there are two types module supplier correspondingly. This paper observes the supply-side collaboration not only on the function level to identify the function deployment among design, production, inbound logistics and information, but also on the role level to analysis the relationship among automaker, module supplier and 3PL provider. And we summarized the findings into a $3 \mathrm{C}$ framework followed the 3C framework approach, which includes the context and capability requirements for supply-side collaboration when providing the two different type modules, and the configuration regarding the upstream supply network structure, the supply side collaboration process structure, and the supported information architecture.
\end{abstract}

\section{Keywords}

Supply-side collaboration; Modular supply; Modularity; Supply chain; Automotive

\section{Introduction}

Modularity has becomes one of the most prevalent means to support product variety (Baldwin and Clark, 1997; Duray, Ward et al., 2000; Pil and Holweg, 2004) and to achieve mass ustomization (Duray, Ward et al., 2000; Mikkola, 2007). Recently, more and more literature and industrial practitioners put their focus on modularity (Fixson, 2007; Salvador, 2007), not only on modular product design (Sako, 2003; Kreng and Lee, 2004; Mikkola, 2006; Mikkola, 2007; Tseng, Chang et al., 2007), but also on modular production (Sturgeon, 2002; Brusoni and Prencipe, 2006; Kotabe, Parente et al., 2007).

Baldwin and Clark (1997) describe modularity as: "building a complex product or process from smaller subsystems that can be designed independently yet functions together as a whole". Since 1990s, modularity was prevalent applied in com- 
puter industry, and the most famous successful case was DELL Company, they use modularity as a strategy for mass customization to achieve product variety at lower cost (Pine II, 1993). The automotive industry is also trying to apply modularity to achieve competitive advantages from mass customization. Recent studies on the automotive industry from different countries (including Brazil (Kotabe, Parente et al., 2007), French (Doran, Hill et al., 2007), U.K. (Doran, 2004) and U.S. (Ro, Liker et al., 2007)) proved that the automotive industry is moving to modularity and modular supply will leads to risk sharing, cost reduction, speed to market increasing and flexibility increasing.

Volkswagen and Mercedes-Benz (Daimler Chrysler since 2000) are the first automakers introduced modularity into the automotive industry in 1996 (plant in Resende, Brazil) and 1997 (plant in Hambach, France) (Roberto, Mauro et al., 1997; Ramalho and Santana, 2002; Takeishi and Fujimoto, 2003). In their plants, some individual components once delivered to the final assembly line one by one are first sub-assembled on a separate line, and then delivered to the assembly line and installed into the vehicle body as a module. General Motors opened the Blue Macaw plant in Gravatai of Brazil in 2000, is followed the logic of modularity introduced by Volkswagen and Mercedes-Benz (Reichhart and Holweg, 2007).

One of the most famous case of modularity is the 'Smart' car project cooperatively launched the joint venture of Mercedes-Benz and SMH (Swatch, the Swiss watchmakers) (Takeishi and Fujimoto, 2003). Even a typical car manufacturer have to deal with around 200 300 suppliers, the Smart car has only 25 module suppliers (called as 'system partners') who provide modules including complete dashboard systems, body structure, breaking control systems and seating modules (Doran, Hill et al., 2007).

Most recently, Audi starts a program called "The Audi modular longitudinal platform technology" on the A5/S5 coupes to switch to a modular architecture that will allow most of its product lines to share major components (Kurylko, 2007), through the modular approach, Audi has developed modules for costlier components, including heating and ventilation and key components of the body, driver train and suspension. And Volkswagen AG (VW), a pioneer in module sharing, then will learn some new module sharing tricks from sister company Audi AG (Stein, 2007). VW will cut its development times by a year and expand its portfolio significantly through using Audi's modular-architecture strategy. While the largest modular assembly in the Hyundai plant in Montgomery is the cockpit, which is supplied from a Hyundai-owned module supplier Hyundai Mobis, and the right cockpit can be delivered in the right place at the right time and at the right quality level (Wortham, 2007).

As automaker moving to modular assembly, the rules and practices of supplyside collaboration changed. And with the complex product structure and supplier network, it is not very easy to apply modularity and collaborate with the upstream 
supply chain. Actually, organizations are increasingly compelled to deal with more and more complexities in the global supply chain, both in the number of suppliers and in their varying characteristics (Erevelles and Stevenson, 2006). For example, General Motors has approximately 30,000 suppliers, with approximately 9000 members in its supply chain (Gould, 2001). It is hard to collaborate with their huge number ofsuppliers and to exploit the enormous competitive potential advantages of applying modularity in the supply chain management. Furthermore, there is an obvious trend towards stronger collaboration with suppliers for improving the performance of the buying company (Avittathur and Swamidass, 2007).

However, literature on the affects of modularity on supply-side collaboration and the methods to improve the performance of supply-side collaboration in the modular supply environment is quite limited. This motives us to explanatory the context of modular supply and investigates the framework of supply-side collaboration when moving to modular supply in the automotive industry.

The paper is organized as follows. In the next section the methodology will be described. Case study methodologies are adapted in our research, and we observed the function level and role level in each case followed the $3 \mathrm{C}$ framework approach. Then in section 3, we analysis two cases of seat and air-conditioner supply to identify the context feature, capability feature and configuration feature of supply-side collaboration with modular supply. The results therefore lead to managerial insights and discussions in section 4 and summarized into a $3 \mathrm{C}$ framework for the future modularity application in the automotive industry. Finally, section 5 concludes our findings and future research directions are provided.

\section{Methodology}

The research aims to obtain a broader perspective on the development of modular supply and the practices in Chinese automotive industry. Hence case study methodologies were adopted in our research, which given the explanatory nature of the research questions being posed (Yin, 1994). Cases were chosen and administered in accordance with replication logic (Eisenhardt, 1989), and the case company were mainly from the Chinese automotive industry. Data were collected via plant visits, semi-structured interviews during 2005-2007 with senior engineers, senior managers, R\&D managers and logistics operations managers.

Our research focus on the supply-side collaboration among the different roles, then followed the 3C framework first proposed by (Zhang, Shi et al., 2007) as a generic approach to the study of network organizations, this research studies the supply-side collaboration from the perspectives of the context, capabilities and configuration. The main purpose of this research is to explanatory the environment features, key success features and organizational features for the supply-side collaboration within the supply side network when moving to modular supply. The research framework of this paper is described in Figure.1. 
Based on the interviews conducted with the automotive manufacturer and component suppliers from 2005 to 2007, it was observed that 3PL provider plays a very important role in modular supply. Some 3PL providers take responsibility for the sub-assembly of the modules (such as plugging in electronic cluster) before it is delivered to the assembly line for final assembly of the vehicle, and the sub-assembly and delivery are synchronized with the final assembly which is followed by the information sharing.

Consequently, design, production, inbound logistics and information (see Figure.1) are the major function activities we analysed in our study, and they are also the fields where we are trying to identify the capability features required by supply-side collaboration when applying modular supply in automotive industry. As a result, we mainly consider the related roles including module supplier (or component supplier), manufacture (automaker) and 3PL provider (see Figure.1) in our research when specify the configurations to support achieving the capabilities the supply-side collaboration needed. The concept of supply-side collaboration therefore refers to not only the collaborations between the manufacturer and the module suppliers, but also the collaborations among the module suppliers, the manufacturer and the 3PL provider.

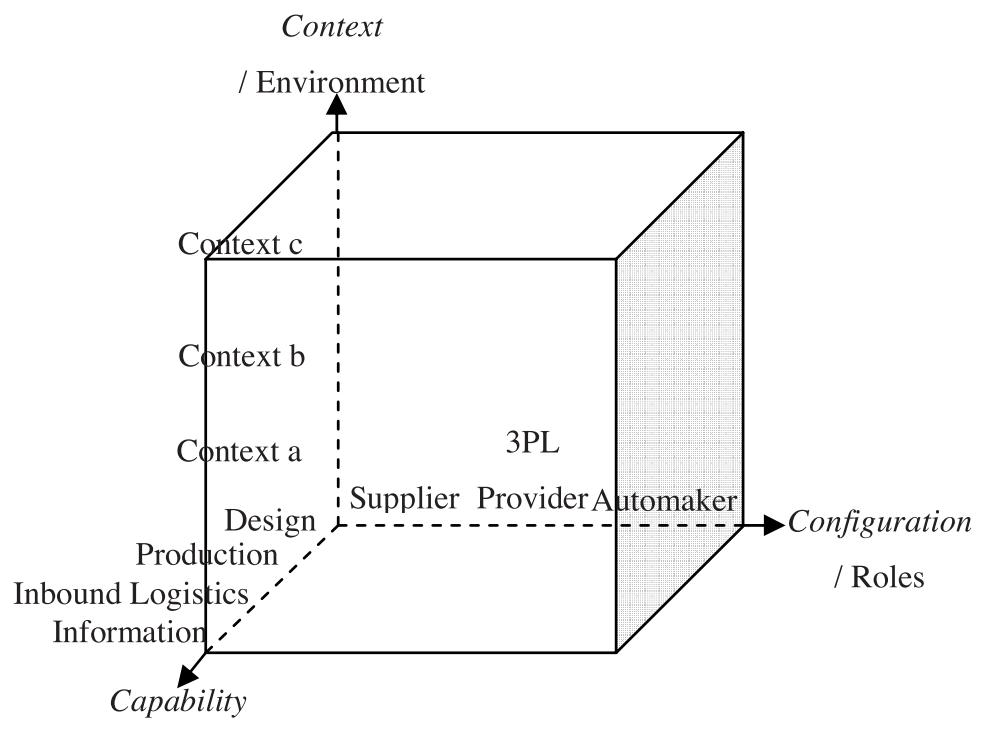

/ Function

Figure.1 Research framework for supply-side collaboration

Different from the research of Doran and Roome (2003) which argues that the second-tier supplier plays important role within the modular landscape, our research only put focus on the first tier suppliers. 


\section{Findings}

The findings starts with a case summary of each case, and then the analysis on the context, capability and configuration are presented. All the findings are not just from these two cases, the same features derived from other cases are summarized into these two cases.

\subsection{Case A: Seat Module}

\section{1) Case summary}

The supplier we investigated in this case is a joint venture (JV) of a Chinese seat supplier and an U.S. automotive supplier who advanced in module integration technology and is one of the largest component suppliers in the world. While the automaker is one of the 2007 top ten best sale companies in china, and it is also a JV.

The module the JV provided to the automaker is a complete integrated seat module which doesn't need any more assembly process before it delivered to the final assembly line. And the module is delivered directly to the assembly line by the supplier with just in time (JIT) logic. As the seniormanager from the module supplier said that they are providing completed solution to the automaker, not only the design and production, but also the logistics service.

The JV also sets up several plants in other cities as the major supplier of seat module to some other automakers.

\section{2) Capability to successful supply-side collaboration}

The concept of the module is originated from the automaker, but the design and engineering are all outsourced to the module supplier. In fact, the JV has advanced technologies in design and engineering in their own specified fields, especially in module integration, which is immigrated from the U.S. parent company. With the developed and comprehensive knowledge of modularity and advanced R\&D capability, it ensures that the supplier can provide a total solution (including design, production and inbound logistics) to the automaker, and actually the $\mathrm{JV}$ becomes the only supplier providing the seat module to the automaker for the three series vehicles.

Furthermore, the JV not only has high capability in module integration, but also in upstream supplier integration. The JV only holds some high value-added assembly activities, while the low value-added activities are outsourced to his upstream supplier. As a result, the module supplier should integrate the second-tier supplier to make the module for the automaker efficiently and effectively.

Obviously, the automaker is a great integrator of vehicle with a high assembly capability, while the module supplier is a great integrator of module or system with a high R\&D, production and supplier management capabilities.

The module supplier is located close to the automaker's assembly plant $(1 \mathrm{~km}$ away), hence it is easy to operate on a JIT basis with the help of Kanban information 
system. On the other hand, it is possible for the supplier to deliver the seat module directly to the assembly line by themselves at right time and at right place with right number, that also means they don't use 3PL provider in this scenario.

3) Configurations for supply-side collaboration

The supply network structure for this case is described in Figure.2.

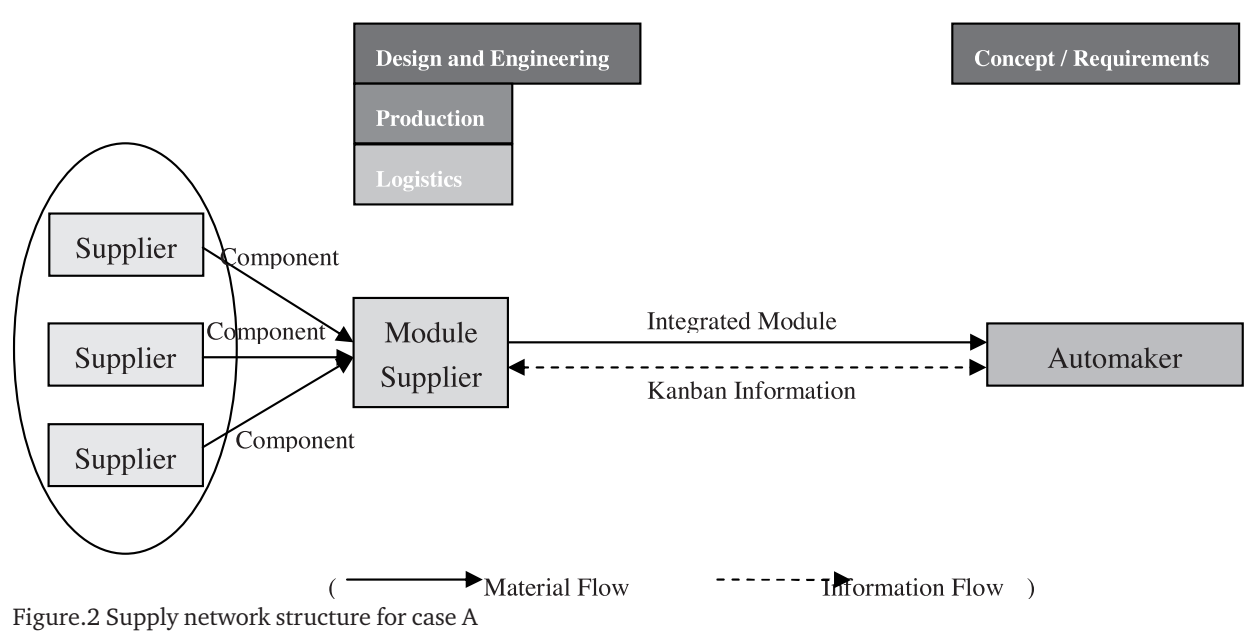

The major roles in this supply chain are the automaker and the module supplier. But the module supplier has a complex upstream supply network to manage. Hence, the collaboration between supplier and automaker is relatively simple because there is only with two roles. But the supply chain management upwards the module supplier is more complex.

Figure. 3 illustrated the process and information structure in this case. The automaker share their production plan and related information with the module supplier one month ahead. Hence the module supplier can organize his upstream supplier and his own plant to make the module for the automaker with the shared production plan information. And then the supplier deliveries the integrated module directly to the automaker with a JIT logic followed by the Kanban information, which is an order with time, place and number for diary deliver. This dairy detailed delivery order information are transferred through Kanban system to the module supplier two hours ahead, and then the module supplier will organize to make the integrated module and deliver it directly to the assembly line on time with the right number. 


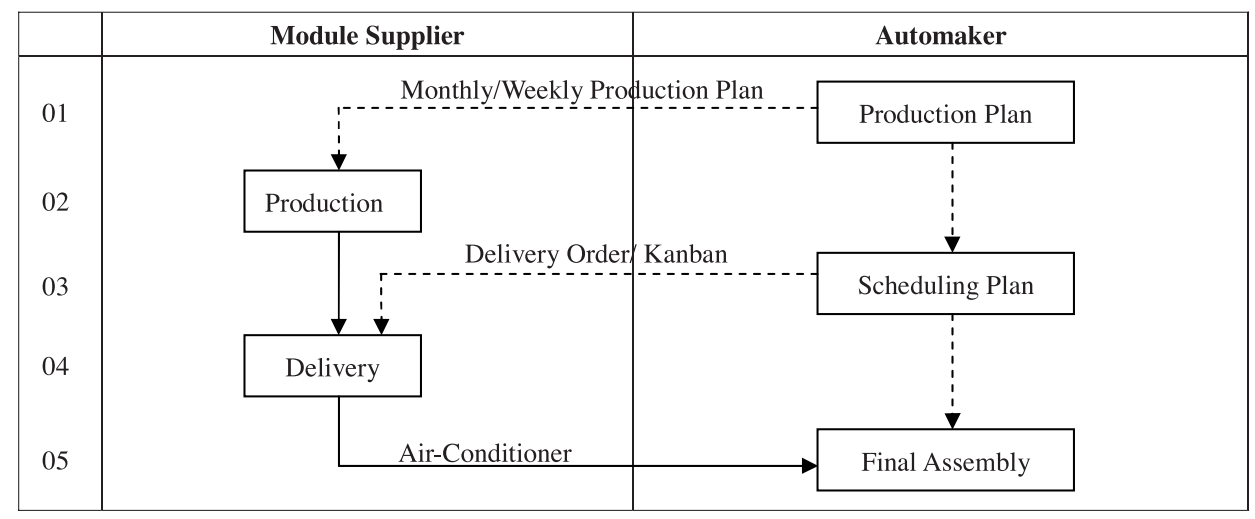

Figure.3 Process structure for fully integrated module

Before the JV formed, the automaker had to hold 240 units inventory to meet the daily production demand, while the module supplier had to store two-day inventory to satisfy the requirement from the automaker. That is due to the module supplier is lack of enough R\&D capabilities to design integrated seat module, and also lack of sufficient production capability to modular assembly. This leads to a big financial burden to both the manufacturer and the supplier.

After the establishment of the JV, the modular design was improved with the involvement of the US company's advanced R\&D capability. Around 1998, the JV was asked to using Kanban information system followed by the implement of JIT supply logic in the automaker. With the highly integrated seat module and extended production capability, the JV reduced their inventory level to two-hour standard.

For the two roles are all great integrator in their own field now, the information sharing plays a very important role in the success of supply-side collaboration. To some extent, they achieved synchronized production with real-time information sharing. The information architecture mainly includes ERP/MRP information system and Kanban information system just sharing information between the module supplier and the automaker.

\subsection{Case B: Air-conditioner}

\section{1) Case summary}

The air-conditioning supplier is also a JV founded in 1994. The parent company is an independent France industrial group, which is fully focused on the design, production and sale of components, systems and modules for cars and trucks, both on the original equipment market and the aftermarket. And it ranks among the world's top automotive suppliers and supplies all major vehicle manufacturers, especially, the annual investment in R\&D represents $6.6 \%$ of the Group's total operating revenues (Figures at December 31, 2006 from the company website), which leads to a high capabilities of module design and assembly. 
The $\mathrm{JV}$ provides air-conditioner to the same automaker in case A for their six series vehicles. The module is not delivered to the automaker directly as the case A, but first delivered to a 3PL provider who providing warehouse and inbound logistics services to the automaker. And the module is processed with some sub-assembly (such as plug-in electronic clusters) by the 3PL provider before feeding the assembly line followed by the automaker's delivery order.

The 3PL provider is a new company issued from a French logistics company who having a long experience of automotive logistics (who owns the third integrated logistics network in Europe)and a Chinese logistics company who owning a wide network established throughout the country. The establishment of the JV is to make full use of the advantages from both the French company's management technology and the Chinese company's national network.

\section{2) Capability to successful supply-side collaboration}

The supplier offers air-conditioner with state-of-the-art technology which can save energy, increase driving comfort and contribute to better environmental protection. The parent group of the JV has $5 \mathrm{R} \& \mathrm{D}$ centres in 7 countries to develop their advanced technology on compressor, not focus on the whole module integration. In fact, such compressor technology is their core competency.

As a result of focusing on the compressor technology development, the JV outsources some low-value components to other component suppliers. These components will be delivered to the 3PL provider monthly. Hence, the module provided by the air-conditioner supplier is not a complete integrated module, and it will be transferred into a complete integrated module after finishing the final sub-assembly activities performed by the 3PL provider. But the JV still has high capability of supplier integration to organize the upstream supplier to make the air-conditioner module.

The 3PL provider will finish the final sub-assemblies after received the delivery order from the automaker two hours ahead. Consequently, the 3PL provider should hold some production equipment and human force to provide such capability.

And the 3PL provider should also take responsible for collecting all the low-value components to ensure the final sub-assembly activities. Here the 3PL provider plays an important role on collaboration among the automaker, module supplier and component suppliers.

\section{3) Configurations for supply-side collaboration}

Figure.4 illustrates the basic supply network structure for case B. The 3PL provider here is not only performs the logistics functions, but also sometimes performs the production functions (sub-assembly) to transfer the air-conditioner into a complete integrated module like case A before delivering it to the assembly line. The module supplier, automaker and 3PL provider are the three key roles in this supply network 
structure. There are still some component suppliers providing low-value components for the sub-assembly performed by the 3PL provider. For the more complex supply network than Case A, close collaboration is needed to ensure the efficient operations of inbound logistics.

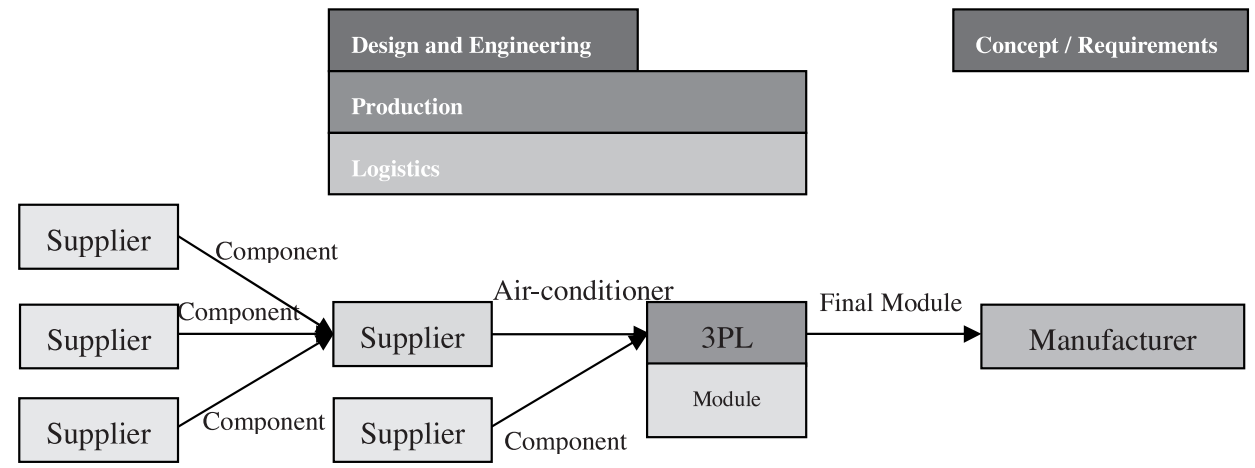

Figure.4 Supply network structure for Case B

The process and information structure is described in Figure.5.

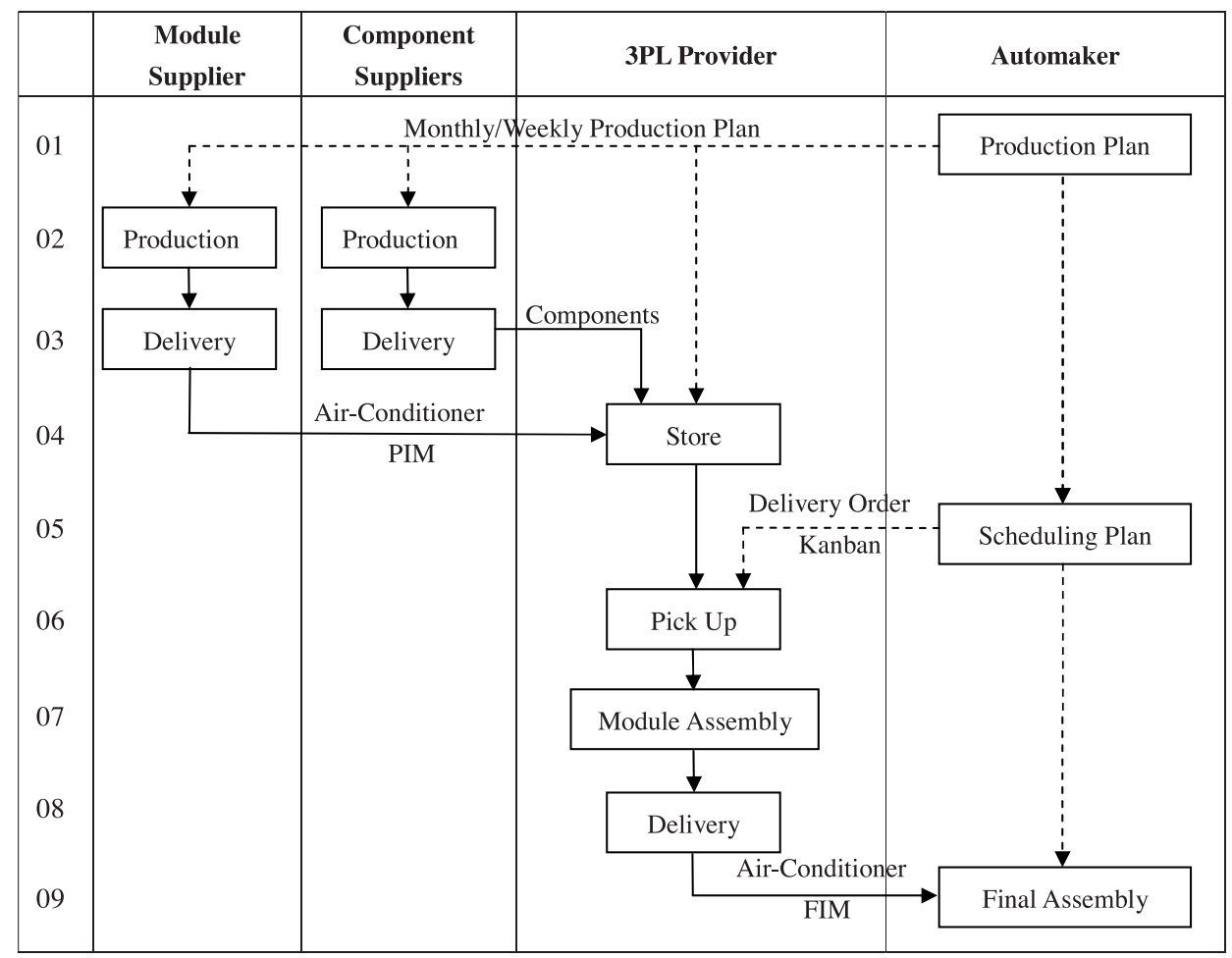

Figure.5 Process structure for partial integrated module 
In this scenario, the air-conditioner module was first delivered to the 3PL provider. Normally, there holds two-day inventory in stock in 3PL provider's warehouse. When the 3PL provider received the delivery order from the automaker through Kanban information system, the air-conditioner will be picked up from the shelf and finished the sub-assembly with the components provided by the low-value component suppliers, and then it will be delivered as final completed module ready to assembly to the assembly line, with the right number to the right places at the right time.

Even those low value components for the final sub-assembly maybe not with high value and high importance to the air-conditioner, it is really needed to be managed efficiently to ensure the JIT delivery to the assembly line. Sometimes those components' suppliers received delivery order from the 3PL provider directly. Definitely, there is a 'big contract' ahead between the automaker and the suppliers cover the supply for regular periods (a quarter, half year or one year, depends on the automaker's decision priorities).

The information architecture mainly includes not only the automaker sharing production information with the PIM supplier, component suppliers and 3PL provider, but also the 3PL provider sharing delivery information with both the PIM and component supplier.

ERP/MRP and Kanban system are still the main information sharing enablers.

\section{Discussions}

The major purpose of this research is to identify the affections of modular supply on supply-side collaboration and to explore how to improve the performance of supply-side collaboration with different module supply.

The findings show that there are normally two types of module according to their integration level before being feed to the final assembly line, which is the fully integrated module (FIM) and the partial integrated module (PIM). The FIM is the module that complete integrated without any more sub-assembly before feeding to the final assembly line, while the PIM is the module that not complete integrated and needs some sub-assembly activities before feeding to the assembly line. Through all our case studies on Chinese automotive industry, the suppliers we investigated who adapting modular supply can be classified into two categories supplying FIM and PIM.

These two types of module are the typical modules in the reality. For the seat module, its modularity has become common practice across the automotive industry (Ro, Liker et al., 2007). The capabilities of R\&D especially the module integration becomes more and more mature technology in the automotive industry. Not like the cockpit, seat module almost accepted by all the automaker with developed modularity logic. But only a few suppliers can supply fully integrated cockpit, while lots of supplier still can't, they only supply partial integrated cockpit modules to the automakers. That's also the reason why we choose the seat module as the fully integrated module case in our research. For the air-conditioner, its module integra- 
tion technology is also mature across their industry. And because of the suppliers usually focus on the core technology development (such as the compressor in the air-conditioner) as their methods to gain more competitive advantage, most of the suppliers are just involved in the logic of modularity, but they are not devoted into the modular logic.

Followed this classification, the operational characteristics of supply side collaboration within these two different modules supplying are summarized in Table.1 from the perspectives of context, capability and configuration.

The context identifies the environmental features of providing these two different modules. The capabilities specify the key capability requirements for the successful supply-side collaboration within these two modules supply, followed the functional view point of design, production, inbound logistics and information management. The configuration defines the supply network structure, process structure and information architecture to support and ensure to get those capabilities needed for the supply-side collaboration when providing FIM or PIM.

The findings suggest that modular supply is a complex system, the degree of involvement into modularity is largely depends on the module suppliers' corporate strategy on technology innovation. Whether the supplier is devoted into modularity or just get involved into the modular logic is depends on the supplier's corporate strategy of being a comprehensive modular integrator or an advanced core-technology seeker. If the strategy priority is on core-technology development to gain special competitive advantages, the supplier will left the low-value components to the other component suppliers. And it leads to a complex collaboration among the automaker, the module supplier and the component supplier. Then the solution provided by the PIM supplier is not a total completed solution, there are still some sub-assembly activities should be finished by third party, such as the 3PL provider. Also the degree of modular involvement reflects the automaker's trade-off decision between modularity and outsourcing. The modularization progress of the automaker and the module supplier are inter-dependent.

The results demonstrate that the Chinese automaker is followed the international trends of transferring design and production responsibility to supplier (Fine, 1998; Camuffo, 2000), that means the design outsourcing. While such outsourcing is different from the original idea of the Volkswagen and Mercedes-Benz's modularity case, but it is similar with the cases of European and U.S. automakers (Corrêa, 2001). And it also demonstrate that the Chinese component suppliers, not only the FIM supplier but also the PIM supplier, are in the wave of M\&As Operations (Camuffo, 2000), and these two suppliers in our cases are all joint venture of a Chinese company and a world-leader component supplier who have a advanced capability on modular integration in their specific fields.

Along with the design and production outsourcing, the research findings suggest that the module supplier should take more responsibility to manage the up- 
stream supply network effectively and efficiently. To the automaker, the complexity of supply chain management is decreased with the responsibility transformation of supply chain management from itself to the module supplier. At the same time, it increases the demand on supply chain management skills development for the module supplier.

Table.1 3C framework for supply side collaboration with FIM and PIM supply

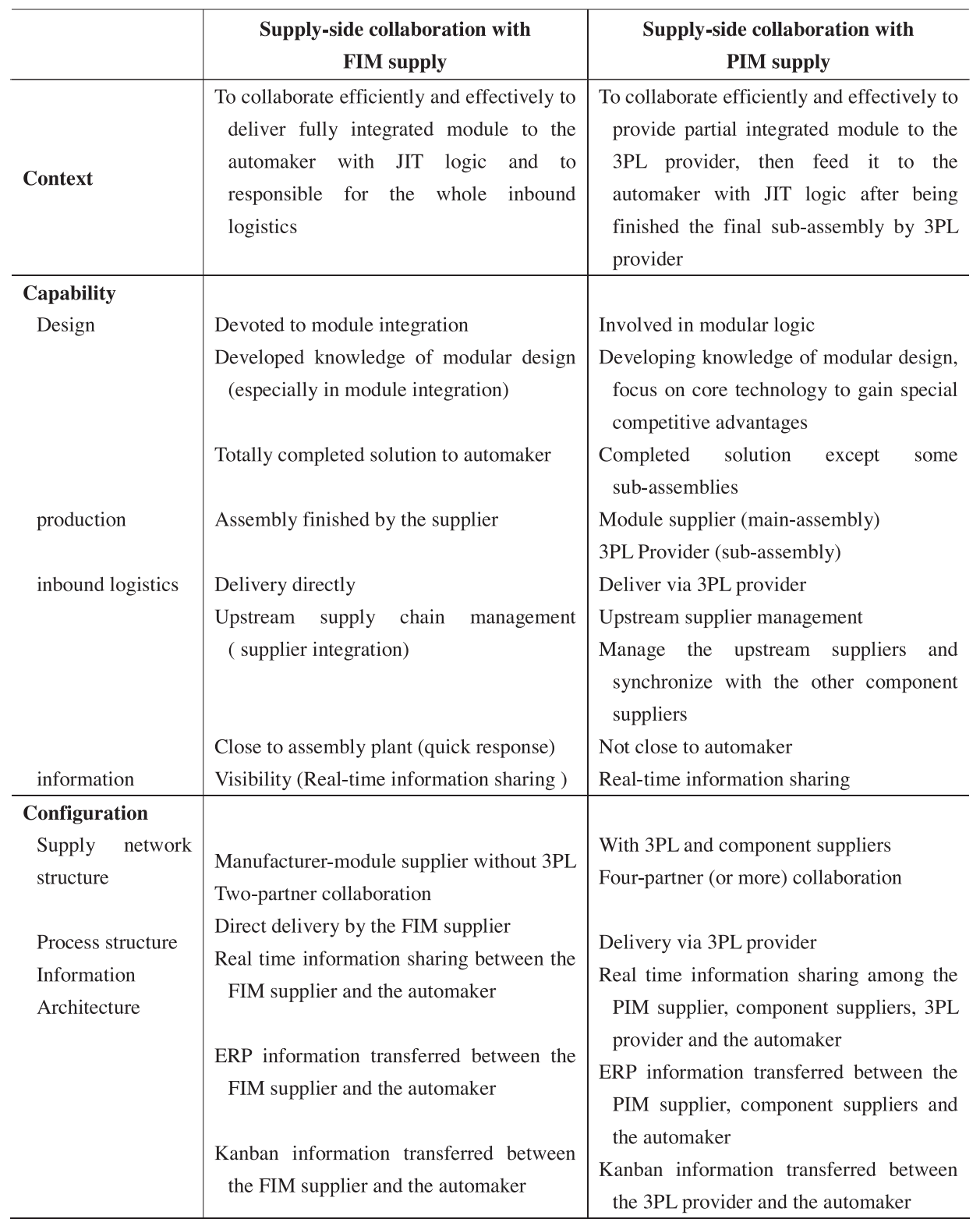


The important role of 3PL provider plays in PIM supply is showed from the findings. It can provide not only the logistics services but also the sub-assembly activities. More interesting is that with the help of 3PL provider it makes postponement possible, which is the same as the function of the supplier park (Reichhart and Holweg, 2007). And the findings also suggest that the close proximity between the module suppliers and the automaker will be helpful to modular supply. That's the reason why the supplier park (Reichhart and Holweg, 2007) are popular introduced to achieve JIT supply in automotive industry. Actually, close to assembly plant is a success factor to achieve postponement and JIT logic.

The results show the complexities of the supply chain roles, supply network structures, process structure, information architectures, process of production and inbound logistics decreased when moving to more integrated module supply, which is also noted by (Baldwin and Clark, 1997; Camuffo, 2000; Doran, Hill et al., 2007).

\section{Conclusions}

Through literature reviews and case studies, this explanatory research concludes two type modules, fully integrated model and partial integrated module. And then findings are summarized into a $3 \mathrm{C}$ framework from the perspectives of context, capability and configuration. The $3 \mathrm{C}$ framework suggests a way to improve the performance of supply-side collaboration when moving to modular supply. Also it shows the practitioners the fields of how to improve and enhance the modular logic in the automotive industry.

The findings suggest that the module suppliers should devoted to improve the capability not only on module integration (product design) and process integration (production process design), but also on upstream supplier integration, that is to say, they should develop their skills on supply chain management which traditionally regarded as the responsibility of the automaker the focal company in the supply chain. And the research results also imply that not only the automaker but also the module should match their modularity strategy with their corporate strategy. It will directly affect the performance of supply-side collaboration.

And the findings also suggest that the 3PL provider should also hold production capabilities of sub-assembly for the requirements of the modular supply context. Hence the supply-side collaboration between the 3PL provider and the automaker focus not only on synchronized delivery with JIT logic, but also on synchronized production between the sub-assembly in 3PL's warehouse or DC and the final-assembly in the automaker's assembly line. All these need well-organized information system to achieve real-time information sharing, not only on the planning level, but on the operational level.

Since the research is conducted in Chinese automotive industry, especially the seat and air-conditioner module, the findings and implications are not universally applicable across the sector. Regarding the future research on this problem, more 
in-depth case studies and comparisons across a wider range of countries would improve the validity and reliability of the findings. The type of the module would be extended in the future researches, and it will be more detailed and specified. Also the strategies and tools for the supply-side collaboration with modular supply will be more specified in the future.

\section{Acknowledgements}

Project 70502015 supported by National Science Foundation of China. Project 2006AA04Z153 supported by National High Technology Research and Development Program of China.

Corresponding author: Dr Yong Lin now is a visiting scholar in the Institute for Manufacturing, Department of Engineering, University of Cambridge, Mill Lane Cambridge, CB2 1RX. Tel: +44 (0) 7867 514476; E-mail: yl366@cam.ac.uk; yonglin1212@mail.hust.edu.cn.Dr.

Yong Lin

School of Management, Huazhong University of Science \& Technology.

Wuhan, Hubei, P. R. China. 430074

yonglin1212@mail.hust.edu.cn

Yongjiang Shi

Institute for Manufacturing, Department of Engineering,

University of Cambridge,

Mill Lane, Cambridge U.K.,

CB2 1RX

ys@eng.cam.ac.uk

Shihua Ma

School of Management,

Huazhong University of Science \& Technology.

Wuhan, Hubei, P. R. China. 430074

shihuama@mail.hust.edu.cn

\section{References}

Avittathur, B. and P. Swamidass (2007): Matching plant flexibility and supplier flexibility: Lessons from small suppliers of U.S. manufacturing plants in India. Journal of Operations Management 25(3): 717-735.

Baldwin, C. Y. and K. B. Clark (1997): Managing an age of modularity. Harvard Business Review 75(5): 84-93. 
Brusoni, S. and A. Prencipe (2006): Making Design Rules: A Multidomain Perspective. Organization Science 17(2): 179-189.

Camuffo, A. (2000): Rolling Out a "World Car": Globalization, Outsourcing and Modularity In the Auto Industry. IMVP working paper.

Corrêa, H. L. (2001): The VW Resende (Brazil) plant modular consortium SCM model after 5 years of operation. Proceedings of the International Conference of the Operations Management Society. Orlando, Fla.

Doran, D. (2004): Rethinking the supply chain: an automotive perspective. International Journal of Supply Chain Management 9(1): 102 - 109.

Doran, D., A. Hill, et al. (2007): Supply chain modularisation: Cases from the French automobile industry. International Journal of Production Economics 106(1): 2-11. Doran, D. and R. Roome (2003): An evaluation of value-transfer within a modular supply chain. Proceedings of the Institution of Mechanical Engineers Part D: Journal of Automobile Engineering 217: 521-527.

Duray, R., P. T. Ward, et al. (2000): Approaches to mass customization: configurations and empirical validation. Journal of Operations Management 18(6): 605-625.

Eisenhardt, K. M. (1989): Building Theories from Case Study Research. Academy of Management Review 14(4): 532-550.

Erevelles, S. and T. H. Stevenson (2006): Enhancing the business-to-business supply chain: Insights from partitioning the supply-side. Industrial Marketing Management 35(4): 481-492.

Fine, C. H. (1998): Clockspeed: Winning Industry Control in the Age of Temporary Advantage, Perseus Books, U.S.

Fixson, S. K. (2007): Modularity and Commonality Research: Past Developments and Future Opportunities. Concurrent Engineering 15(2): 85-111.

Gould, L. S. (2001): Keeping Track With Symbology. Automotive Manufacturing \& Production 113(3): 64.

Kotabe, M., R. Parente, et al. (2007): Antecedents and outcomes of modular production in the Brazilian automobile industry: a grounded theory approach. Journal of International Business Studies 38(1): 84-106.

Kreng, V. B. and T.-P. Lee (2004): Modular product design with grouping genetic algorithm--a case study. Computers \& Industrial Engineering 46(3): 443-460.

Kurylko, D. T. (2007): Audi adopts modular architecture to share parts. Automotive News 81(6255): 46-46.

Mikkola, J. H. (2006): Capturing the Degree of Modularity Embedded in Product Architectures. Journal of Product Innovation Management 23(2): 128-146.

Mikkola, J. H. (2007): Management of Product Architecture Modularity for Mass Customization: Modeling and Theoretical Considerations. IEEE Transactions on Engineering Management 54(1): 57-69.

Pil, F. K. and M. Holweg (2004): Linking Product Variety to Order-Fulfillment Strategies. Interfaces 34(5): 394-403. 
Pine II, B. J. (1993): Mass Customization: The New Frontier in Business Competition, Harvard Business School Press.

Ramalho, J. R. and M. A. Santana (2002): VW's modular system and workers' organization in Resende, Brazil. International Journal of Urban and Regional Research 26(4): 756-766.

Reichhart, A. and M. Holweg (2007). Do we still need supplier parks? Automotive Logistics: 52-58.

Ro, Y. K., J. K. Liker, et al. (2007): Modularity as a Strategy for Supply Chain Coordination: The Case of U.S. Auto. IEEE Transactions on Engineering Management 54(1): 172-189.

Roberto, M., Z. Mauro, et al. (1997): The modular consortium in a new VW truck plant in Brazil: new forms of assembler and supplier relationship. Integrated Manufacturing Systems 8: 292-298.

Sako, M. (2003): Modularity and Outsourcing: The Nature of Co-evolution of Product Architecture and Organization Architecture in the Global Automotive Industry. The Business of Systems Integration. A. Prencipe, A. Davies and M. Hobday, Oxford University Press: 392.

Salvador, F. (2007): Toward a Product System Modularity Construct: Literature Review and Reconceptualization. IEEE Transactions on Engineering Management 54(2): 219-240.

Stein, J. (2007): VW will adopt Audi's modules of development. Automotive News 81(6245): 3-3.

Sturgeon, T. J. (2002): Modular production networks: a new American model of industrial organization. Industrial and Corporate Change 11: 451-496.

Takeishi, A. and T. Fujimoto (2003): Modularization in the Car Industry. The Business of Systems Integration. A. Prencipe, A. Davies and M. Hobday, Oxford Scholarship Online. 1: 254-279.

Tseng, H.-E., C.-C. Chang, et al. (2007): Modular design to support green life-cycle engineering. Expert Systems with Applications In Press, Corrected Proof.

Wortham, A. (2007): Exec says suppliers must deliver more. Automotive News 81(6256): 42-42. Yin, R. K. (1994): Case Study Research: Design and Methods (Applied Social Research Methods) Sage Publications, Inc.

Zhang, Y., Y. Shi, et al. (2007): Global engineering networks: The integrating framework and key patterns. Proceedings of the I MECH E Part B Journal of Engineering Manufacture 221: 1269-1283.v 


\title{
Customer Co-creation in the Dutch Financial Services
}

\author{
Author: Marcel Weber
}

\begin{abstract}
The Dutch Financial (FS) Services industry is known for its variety and diversity in financial products and services, that have been created and developed by firms themselves. Customer involvement in the development of new products is something very unusual, because of its risk and because consumers consider financial matters as complex and not exciting at all. However, Dutch FS firms are slowly becoming aware of the potential to create new products and services with the participation of their customers, leading to products and services that are really wanted and needed by consumers. As a consultant and as a part of his Ph.D. programme, the author of this paper intends to develop a 'protocol' on how to involve customers in the innovation process, based on best practices (in all kinds of industries) and literature on the subject. So far, a preliminary protocol, consisting of guidelines on recruiting participants, and methods and procedures for involving the participants, has been developed. This preliminary protocol has been tested in several cases, some in the financial services. Two cases of firms that have taken this first step to involve their customers in this co-creation will be discussed.
\end{abstract}

\section{Keywords}

Customer Co-creation, Open Innovation, Customer Involvement, Financial Services, Netherlands.

\section{Introduction}

In order to create products and services that customers really want, firms can involve customers in the value creation process, either by mass customization, by participation of the customer in the new product development or innovation process, or both. In comparison with sectors with high-technology and several consumer products, the Dutch financial services (FS) industry (banks, insurance companies, intermediaries, financial brokers) has ignored this possibility to involve its customers for a long time.

This paper deals with the reasons for this low interest for customer involvement by Dutch Financial Services (FS) industry. It identifies the causes and discusses the open innovation possibility for FS with their customers, based on insights developed in other industries and innovation theory. It illustrates that the Dutch FS is becoming aware of this customer potential with the introduction of its first simple mass customization products and involvement of the customer in the innovation 
process. To substantiate this observation, two cases where customers have been involved in the innovation process, will be presented and discussed. One case is about a mortgage bank, where consumers participated in the development and configuration of new mortgage products and services. The other one is about an insurance company that renewed it's marketing with the aid of consumers.

The paper concludes with an advise towards the Dutch Financial Services firms.

\section{Customer orientation in Dutch FS innovations}

The Dutch Financial Services (FS) industry is known for its large variety and diversity in products and services. If one is looking for a way to finance a new house, people have diverse possibilities of achieving this, for instance through a mortgage, plain lending, leasing, investing in stocks, taking a life insurance, or by taking a combination of, or combinations within these products, e.g. a mortgage based on investing and savings. This may indicate that the Dutch FS is highly innovative. But if one looks more closely to these products, one will discover that they are all based on or are clones of the traditional financial products, developed for $\mathrm{P} \& \mathrm{C}$ insurance, loans and savings. Over the years these basic products have evolved into diverse and complex financial products through modularisation and the, from time to time, creation of new combinations. In this way a potential base for mass customization of financial services has been created. The distinction between al the products can be found in name, the interest rate, the currency of the product, or its purpose, but they are basically the same. And the mass customization opportunity that lies within the modularization of the products has been hardly utilized until now, because of reasons to be discussed further on.

Brand distinction has largely disappeared; consumers don't really distinguish any differences between all these financial products and become confused or mislead by product names or advisers' information. The result is that Dutch consumers have an average of four different financial service providers with whom they possess many similar products, like two bank accounts (one for daily payments and one for savings), several property insurances covering the same occurrence multiple times, and such (GfK, 2005). Consumers don't feel any need to switch from provider, because they feel they are all the same.

Over the past decade the Dutch FS have been more concerned with growing through mergers and acquisitions, and cost cutting programs, like the closing of bank branches, standardizing products and services, and striving for mass production, than in serving their customers with the tailored products and services. Compared to other industries like the chemical, and electro technical industries, innovativeness in the Dutch FS is relatively low and declining each year. Only 15\% of the FS innovations are developed with or by external partners (Langenberg \& Plant, 2000; van der Veen, 2007), of which a large part because of governmental legislation on consumer protection and financial solidity for financial institutions. 
Each year Dutch corporations spend about 8 billion Euros - of which about 300 million Euros by the FS industry - on innovations. About $80 \%$ of those expenditures are spent on new products and services that the marketplace has little use for. Certainly no one consciously pursues failure but lacking refined tools to improve performance, much is wasted. In that environment the cost of getting it wrong weighs heavily on financial product and brand managers and marketers. The Dutch FS industry is on the outlook for new innovation initiatives as it is obvious that the few new products and services that are developed in the FS industry are not what consumers really are waiting for.

\section{Low customer focus and involvement by the Dutch FS: an exploration of the motives}

\subsection{Low customer interest, high customer loyalty}

Research shows that the success of innovations is highly dependent on the market- and customer orientation of the firm that innovates (Ahmed, 1998; Cooper, 1980; Deshpande, Farley, \& Webster, 1993; Kelly \& Storey, 2000; Storey \& Easingwood, 1996). FS firms should therefore need to focus more on customer- and market needs to create effective innovations. One of the ways to achieve this is to focus on the perception that customers have on financial issues in life and their expectations form the industry.

The poor results of product development and introduction have common roots in the way organizations view their customers, according to Zuboff and Maxmin (Zuboff \& Maxmin, 2002). During the same period that FS have been stalling in the development of customer-centered innovations, society has also been changing with a noticeable trend towards greater consumer empowerment, resulting in consumers making their own decisions on products to purchase in financial services, like insurance policies or pension plans. Zuboff and Maxmin claim that the corporate business model of mass production and mass consumption (abetted by mass media marketing) are a poor fit for a population of consumers that is richer, better educated, and more time starved than any generation in history. Today's customers are taught from an early age to think of themselves and their needs as unique and they expect tailored solutions from vendors, not mass market products (Zuboff \& Maxmin, 2002). Nevertheless, consumers don't seem to switch easily from providers because of a low involvement and high switching costs. This viewpoint is supported by academic research that has shown that the majority of individuals do not have an inherent interest or derive much pleasure from financial services (Howcroft, Hamilton, \& Hewer, 2007). There also seems to be a great loyalty with customers to financial services because of inertia, rather than any strong feelings of brand loyalty, caused by a lack of differentiation in the market (Colgate \& Hedge, 2001; Dick \& Basu, 1994; Knights, Sturdy, \& Morgan, 1994). Because of this FS firms have little incentive to encourage customer involvement (Howcroft, Hamilton, \& Hewer, 2007), thereby reinforcing consumers' indifference. 
Research indicates that a growing part of consumers reveal a desire to have more information about products and emphasize the need for increased involvement with financial service providers via greater deliberation and talking (Howcroft, Hamilton, \& Hewer, 2007). In the wider service literature it has been shown that increased customer involvement either during the search or at the point of purchase can reduce levels of perceived risk (Bowen \& Larsson, 1989; Hocutt, 1998) and increase levels of customers confidence when making product choices (Bateson \& Hui, 1987; Parker \& Ward, 2000). This is underlined by the Green Paper COM(2007) 226 by the EC which strives to empower consumers to make the right decisions for their financial circumstances, based on several elements, including financial literacy; clear, appropriate and timely information provision; high-quality advice; and a level playing field between products perceived as having similar characteristics. Empowered consumers may be more confident in seeking out the best deals to meet their needs, regardless of the location of the financial services provider (Commission of the European Communities, 2007). The ongoing development in information technology has also accelerated this phenomenon by making information on products, costs, risks, and such more accessible for consumers. Unless these issues are addressed, the majority of customers will continue to lack confidence when purchasing financial products (Howcroft, Hamilton, \& Hewer, 2007).

Other review studies have concluded that to develop successful new products, a firm must acquire an in-depth understanding of user needs, which requires user involvement in the development process (Brown \& Eisenhardt, 1995; Craig \& Hart, 1992; Montoya-Weiss \& Calantone, 1994). Similarly, research has shown that user or customer involvement is an important success factor for new services as well (de Brentani, 1995; Edgett, 1994). The idea of enabling customers to co-design and codevelop innovations has caught academic attention with the research of Eric von Hippel, a professor of the MIT. In the late 1970s he discovered that a large part of innovations by firms are accounted for by users and not by manufacturers solely (von Hippel, 1988, , 2005). In the last decade a large amount of academic research has been executed on this subject, showing that customer involvement in new product or service development is beneficial to the success of the innovation. Involvement of customers in an act of co-production or co-creation, can also lead to an increase in affective customer loyalty (Auh, Bell, McLeod, \& Shih, 2007).

\subsection{Fraud and attrition when involving customers}

Another reason for not involving customers in the deliverance, production or innovation is be that FS firms think this is too great a risk, as customers can freely access product or service data, information, and modules, enabling them to change features in a way that gives the best result and thereby decreasing profits for the firms, or even enabling them to commit fraud. There is research that argues that less direct contact between the customer and the service provider enables a greater 
chance for maximum efficiency (Chase, 1978). Involving customers in the service process also increases workload and the chance for conflicts for service employees because of the additional uncertainty (Hsieh, Yen, \& Chin, 2004).

There is a part of the academic world that warns for caution on the subject of relying too much on customers in innovations (Christensen \& Bower, 1996; Esselman, 2006; Gardiner \& Rothwell, 1985; J. Martin, 1995). Customers can be protective or closed concerning their inventions or innovations, or simply can't be trusted concerning their commitment to participate in the innovation process, often leading to an early withdrawal from the process (Gardiner \& Rothwell, 1985) or a low productivity because of a lack of knowledge what to do (C. R. Martin, Horne, \& Schultz, 1999). FS firms could therefore argue that it would be better not to allow customers to co-create, in order not to make extra, unnecessary costs or risks. As a result of this thinking, new products and services, new combinations of product and service modules are invented, developed, created, and diffused by the firms themselves. The only involvement of consumers in new product or service development consists of an occasional participation in market research to find out what consumer needs are, and, in some cases, the testing of new product and service concepts. Therefore, it is justified to observe that, until now, Dutch financial services have been very risk aversive and reluctant in involving their customers in the value creation process, whether by mass customization or by co-innovation.

Even though that there is no consensus on whether or not to involve users and consumers directly in innovations, a growing body of empirical work shows that customers are the first to develop many and perhaps most new industrial and consumer products and services (Lilien, Morrison, Searls, Sonnack, \& Hippel, 2002; Lundvall, Johnson, Andersen, \& Dalum, 2002; Lüthje, Herstatt, \& von Hippel, 2005; Prahalad \& Ramaswamy, 2003). If firms don't take consumers' demands and needs into account, this neglect could lead to consumers taking over the control of creation and production of goods and services at the expense of the firms. Further, the contribution of customers is growing steadily larger as a result of continuing advances in computer and communications capabilities. And, as we have argued before, consumers are more and more demanding a vast role in the creation of goods and services. The manufacturer-centric model, which resembles the Closed Innovation model described by Chesbrough (Chesbrough, 2003) is losing ground.

\section{Needed: a protocol for customer involvement}

\subsection{Research objectives}

As has been demonstrated in the previous sections, management theory has discovered the phenomenon of engaging customers in innovations, but it should also be observed that its research hasn't reached a sufficient level of completeness, comprehensiveness and applicability for firms to start engaging customers without the risk of failure or premature abandonment by the participants. On top of this, it has 
been discussed that there isn't even an academic consensus on the benefits of involving customers in NPD and NSD. It isn't that surprising that even if firms and organizations acknowledge the importance of customer involvement of some kind, only a small percentage of them really give meaning to this conviction by really involving customers in their innovation process.

However, we can observe a growing interest for the phenomenon, both in the academic field as among firms, making followers aware of the importance of engaging with the customer to innovate. Some of these firms may actually want to start one or more innovation projects with the involvement of their customers. They will be looking for a quick and workable guide on how to do this, not for a participation in the academic discussion. While previous research provides pieces for the procedures firms can follow, they do not provide a robust and generalizable framework for these firms. Firms need a comprehensive, robust protocol for engaging customers and users in innovations. A protocol which addresses the following questions: How can firms engage and involve customers in the innovation process in an effective way? What kind of customers, process, procedures and methods should be engaged and followed, what are the tools to be used? What pitfalls or disadvantages exist in engaging customers in this innovation process, and how can they be overcome and avoided? Such a protocol will aid firm managers in choosing the right participants and right methods.

Developing such a protocol is a prescriptive research in its kind, and will therefore be based on the insights of the design science of research in management (van Aken, 1994, , 2004, , 2007), with the following design objectives: (1) to develop a method on how to identify, select, and motivate potential customers to participate in customer-open innovations, and (2) to develop a way on how to engage and involve these customers in the innovation process in an effective way, i.e. the process, procedures and methods one can follow, the tools one can use to accomplish this, and how to avoid and overcome pitfalls or disadvantages that exist in engaging customers in this customer-open innovation process

These design objectives will be the author's research objectives for his $\mathrm{PhD}$ dissertation, thus contributing to theory in management science. Apart from this theoretical relevance this design will also contribute to (1) firm managers, who will receive a method to involve customers in an effective way also, so that total firm costs because of new product or service failure, or innovation failure most likely will be decreased, (2) society which will encounter less waste than when a firm innovates on its own, (3) customers, who will receive what they really need form firms, to get things done in their own environment, and (4) participants in the innovation process, who gain psychological and social benefits.

\subsection{Theory from practice}

Partly because of the omission that literature isn't comprehensive, the future design 
of the protocol will be based not only literature findings, but on author's experience in projects as well. As a consultant in a firm that specializes in advising and supporting its clients in programs for obtaining customer insights, shaping customer interactions, developing new business and retaining customers, the author has lately been more and more involved in several projects in which firms seek to involve their customers in order to better understand their needs and to develop services that are really needed by these customers. These projects can be divided roughly into five categories that correspond with a phase in the innovation process:

1. Collecting Customer Insights: using qualitative research like the Zaltman Metaphor Elicitation Technique (ZMET) (Zaltman, 2003) and the Outcome Based Research (Ulwick, 2005). These techniques reach further than the traditionally used Voice of the Customer (Griffin \& Hauser, 1993) techniques to capture and explicit needs and requirements from users, because they also capture frustrations, sacrifices, and emotional outcomes from users. This category of activities can be seen as a first phase in an innovation process.

2. Idea Generation with customers and users: using brainstorm techniques (Osborn, 1963), open discussions, Delphi techniques (Linstone \& Turoff, 2002) and nominal group techniques (Stewart \& Shamdasani, 2004) to collect, categorize and prioritize ideas for solution to the above mentioned Customer Insights.

3. Concept Development in which users and customers are involved in the design and development process of a firm with the use of online tools, as well as physical involvement.

4. Concept Testing by presenting product ideas, designs, solutions, and such to customers to capture their rational and emotional reaction, in order to find improvements for this design, idea or solution, before committing funds for the market launch (Moore, 1982). In this phase again qualitative methods are used, like the ZMET, focus groups, and such.

5. Customer and User Safaris, meaning as much as to visit and meet with users in places where they usually stay when using the product or service the firm supplies. During such a visit or meeting the firm uses techniques like behavioural observation, interviews and critical incident analysis (Fivars, 1980) in order to capture what users experience when really using the product or service in normal, day to day life. These visits explicit customer sacrifices better than in depth interviews in the firm's office, but they also reveal solutions users have invented for problems they encounter, but haven't reported to the firm, either because they think that it will not be appreciated or adopted by the firm, or because they think the idea is not worth mentioning to others. This activity is deployed when the product or service is already in use, but can also be applied for prototypes that have been made available or a certain amount of users. 
The aforementioned categories of activity have been executed in several projects in the past 3 to 4 years, either as single activity in an innovation project, either as a combination of activities(two or more of the categories) in a same innovation project, most of the time a comprehensive concept development project based on customer insights, the so called Customer Insights Based Concept Development. One of the interesting projects executed in this category was the Client-Co-Creation Lab, a project done for and with SME's subsidized by a Dutch provincial government and in cooperation with the research department of Philips, a large consumer electronics producer, that made its facilities available for this project. One of the facilities was an imitation house, the Philips HomeLab, where users can live and dwell as in their own home, but which is equipped with several observational technologies, like camera's, microphones, and other sensors to register user behaviour. Participating firms were able to test their product and services in a home environment, thus gathering more useful data on usage performance than with other ways of testing (HighTechCampusEindhoven, 2005).

\section{Dutch FS into co-creation}

\subsection{Awareness is growing}

Dutch FS firms are becoming aware of this potential role for their customers, and are willing to surpass their initial inhibitions or reluctance in involving or engaging with the customers. Evidence for this can be observed: banks are reopening the previously closed branches, insurance providers start to mass customize insurance service by providing online tools so consumers can tailor the insurance to their needs (for an example see figure 1), the two largest organizations for financial services intermediaries have announced to improve their image towards consumers bytaking their needs and demands seriously (NBVA, 2006).

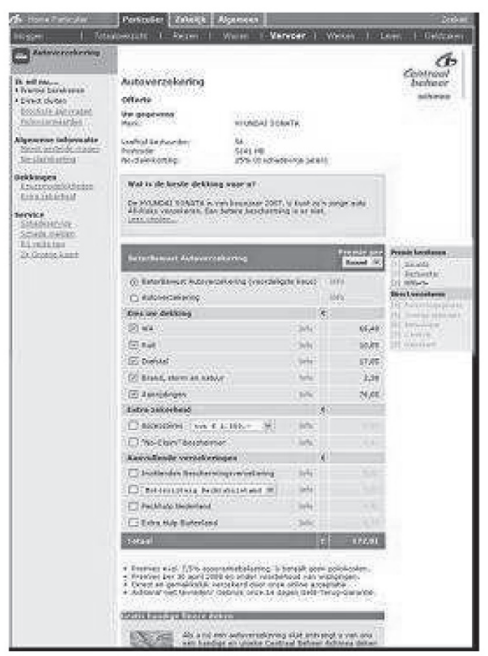

Figure 1: Example of a mass customized car insurance (www.centraalbeheer.nl) 
One example of the developments that indicates this is the growing demand for ZMET® researches. ZMET® stands for Zaltman Metaphor Elicitation Technique, and is a qualitative indepth consumer research method that exposes the unconscious thoughts and feelings of participants on certain subjects (Zaltman, 2003). The author's firm is licensed for this method and has now performed over 30 of these research projects of which about 70 percent for FS companies. Apart form this development we can also observe a growing demand for customer involvement in new product or service development by these FS companies. Two of these projects, executed in the Dutch Financial Services, will be presented from here on.

\subsection{Case study \# 1: new mortgage development}

The first case presented in this paper is about the consumer involvement in the ideation of some new mortgage products for starters. The FS firm that is concerned in this case is a small subsidiary bank of a large insurance company in the Netherlands. The bank is mainly specialized in mortgages, but also delivers other bank services like savings, loans, etc., all except for payment accounts. The bank is only a small player in the field of mortgages with less than 5 percent market share. It is, however, known as a trustworthy money-lender, but also as very particular and conservative about its customers and financial services.

The mortgage market in the Netherlands has been booming but is reaching its limits. Booming because the prospering Dutch society leads to everyone wanting his or her own home or place to live and having the money to fulfil this dream. Reaching limits because the demand for new houses is greater than what is built every year. The effect of this latter phenomenon is that the flow of house-owners to newer and more expensive houses is stagnating, thus leaving little space for starters in the house-owning market to acquire a first and cheap house. These starters are mainly young adults that also have this dream to own their own house and are yearning to fulfil it. Because of the stagnating construction of new houses prizes for existing houses are rising every year, making it, together with the stagnating exit of former house-owners from these existing houses, the more difficult for starters to acquire their own first house at reasonable and affordable costs. Apart from the obstacles of physically acquiring a house, this group also has difficulties in getting a proper mortgage to finance the acquisition.

The goal of the project was to develop some new mortgage products for this starters group, making it easier and more comfortable for them to get a mortgage. Whereas the developing time of new products and services could take more than a year in this bank, the ambition was set to aim for a time to market of 6 months at the most. Because the bank wished to launch at least 2 or 3 new marketable mortgage services it was planned for to involve consumers in the project. This would ensure that only those ideas for which consumers saw the relevance towards their needs would be developed further on. 
A project team, consisting of 3 bank employees - marketing, market intelligence and communication - and 3 consultants - of which the author was one -, was appointed to carry out the mission. The following steps and activities were executed. First the project team performed a desk research to identify the needs of starters, the context in which these needs existed, and the possible frustrations and problems these starters encountered in fulfilling their needs. This step resulted in the identification of three starter segments which make up about 75 to $80 \%$ of the total starter population, briefly described here.

One segment consists of youngsters, still living at home, being cared for by their parents, sometimes still in college or other education, sometimes already working, but in most cases with at least a part time job enabling them to earn some money. This money is an extra saving because they do not need to spend it for housekeeping, living, and such, and thus, so to speak, saving money for the day that they would leave the nest. Most of these youngsters have a fiancée in the same situation - still living with their parents - and thus doubling the amount that is being saved. When leaving home, either to marry or live together, the people from this segment will probably opt for an own house, because, as the team later learned, this is what they have been exposed to all the time - their parents being house-owners themselves. Later on, one other learning was that most parents of these youngsters donate their children some extra cash when leaving house, which is hardly taken into consideration when applying for a mortgage.

The second segment consists of mainly single young people, already on a well paid, but also demanding job, that want to leave the parental house as quickly as possible, and own their own place, to be, as we later learned, independent from family and to show off this independence status to friends.

The last segment identified, consists of mostly older starters, that have been living in a hired house for a few years first, in most cases with a partner and a kid or the wish to have children. For this reason - family expansion - they are looking for an own place, where they can have more children and live a family life like most other couples do. Often, both partners have a job and therefore a double income, making it easier to acquire a new house and a mortgage. The difficulty lies in the fact that having kids would probably mean that one of the partners has to quit the job or work less to give the children a proper education, making the sustaining redemption of the mortgage difficult.

The next step entailed a focus group session for each segment to further understand the needs, the motives for having an own house, the strategies that the consumers made to finance their ambitions. For each focus group session five to six participants, representing a member of the segment looking for or on the verge of buying a new house, were invited for an 2 to 3 hours discussion in an evening with the project team members. Participants received a monetary incentive for this. The session identified several - varying from six to ten per segment - consumer insights 
for the project objectives. But it also helped the participants to learn from each other; they were all peers in the same situation, but everyone had his or her own strategy or tactic to acquire a new house, which was in some occasions an eye-opener for other participants.

The project team also observed all participants closely on their coping strategies, their creativeness and inventive ways and means to fulfil their needs on the subject. From each segments one participant was identified as a 'lead user' (von Hippel, 2005), and was later on invited for a co-creation session with the project team. In this co-creation four more bank employees - marketers and product specialists - were invited, totalling a number of 13 participants. This co-creation session was held about two weeks after the focus group sessions, and was intended to generate some useful product and service ideas to be developed. The session took about six hours, with a lunch break, in a creative environment, where nobody could be interrupted for daily business. In this session the participants jointly created, based on the insights acquired through desk research and the focus group sessions, personas for each segment, brainstormed on ideas to handle these insights and persona needs, discussing these possibilities and translating them in service and product ideas. The session ended with a selection of the best and feasible ideas, on which the participating consumers could reflect concerning their own needs. The whole session was done in an interactive manner, with the aid of simple tools like mood boards, magazine pictures, markers and post its, and common techniques like brainstorming, open discussion, and voting. The composite picture in figure 2 gives an impression of the session and the tools used.

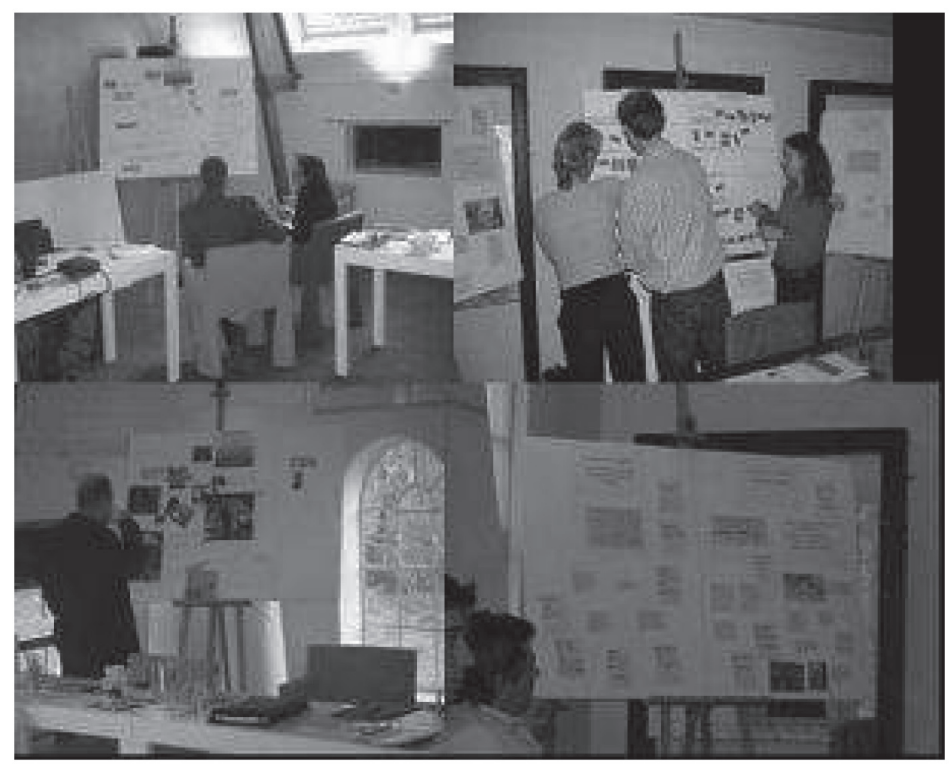

Figure 2: Impression of a co-creation session with consumers 
Based on this session the project team was able to work out some propositions for each segment. These propositions were presented to a decision board, consisting of senior management in the bank. Only a few alterations in terms of estimated revenues and communication style were made by this board, confirming that the project team was on a right track. Five were selected to be developed. The selected propositions were also presented to other product specialists, and to the people that are going to sell these new financial products, namely the financial intermediary advisors, to check the underlying assumptions in and the viability of these propositions. These intermediaries are independent advisors, with offices and operating in all regions of the country, advising consumers on all sorts of financial products of almost all insurance companies. These advisors can also be viewed as customers of the insurance companies. This check pointed out that these assumptions were correct and that the advisors foresee a success for these new products. Only 5 months after the project start two from the five propositions were ready for launch. The reason that the other three had not seen daylight yet was because of tax complications, international standardization legislation, and competitive motives.

\subsection{Case study \# 2: promises in insurance}

The second and last case presented in this paper is about customer involvement in the development of the brand communication for an insurance company. As has been argued before, consumers have a low interest in financial problems and solutions. The insurance company had the ambition of making the insurance process and product understandable, enjoyable and transparent for their customers.

First a ZMET® research was performed with 14 participants - consisting of consumers and small-business owners; half of them customers of the insurance company, half of them customers of other insurance companies. These participants were interviewed in-depth about their thoughts and feeling on transparency in insurance. The outcome of this research showed that people really wanted to understand the process, but were unable to do so because of several obstacles they perceived. Most of these obstacles were mere communication issues, like jargon by financial advisors, small print conditions in policies, and such, but in total making the whole insurance process a complete blur for consumers and business-owners.

Based on these outcomes, as well as the insights that the research gave on the needs, motives and desires people have when insuring something, a team of communication specialists developed about a dozen of so called promises the company was going to make to make the process more understandable and enjoyable for its customers. These promises were derived from the frustrations and obstacles that people had expressed in the ZMET® research. These promises were scrutinized carefully by a steering committee, resulting in the survival of eight promises for further development.

These eight promises were then tested in a total of four focus group sessions, 
consisting of consumers as well as small-business owners, both customers as noncustomers, for agreement and the formulation of the proof that participants wanted to see to such promises. For each promise the focus group discussed the way that the insurance company could show upfront that it was really going to live up its promise. An example was for instance the promise to keep claiming simple and not bureaucratic at all. This resulted in a proof that asked for trust in the claiming customer, instead of distrust, by not demanding bills or other proof of ownership when somebody issued a claim. Two promises were discarded, because participants judged them either too pushy or incredible. From the other six, one was reformulated in a way that participants valued more as a promise. For all promises about two to five proofs were identified.

This result was incorporated in the following steps of the brand communication development. The whole process took about 14 weeks, in the eyes of the insurance company a remarkable acceleration of the process that normally took a year or more.

\section{Conclusions}

It has been argued that innovation of FS companies in the Netherlands have been little customer oriented over the past decade. The reasons for doing this it this way are that 1) consumers do not seem to be highly involved with financial matters, and 2) firms have a certain fear for failure or fraud when involving customers in this process.

It has been also argued that low involvement of consumers in itself is a result of the way that FS communicate and develop their services. Evidence from other industries shows that customer involvement in new product development enhances the chance for success of the new product, so we may conclude that FS can benefit from involving customers in their innovation process. The two presented cases substantiate this conclusion and show us that the time-to-market as well as the quality of the innovation can be greatly improved by involving customers in this process.

Dutch FS firms are becoming aware of this and can benefit from these examples in other industries as well as the intended development of a protocol from the author.

\section{References}

Ahmed, P. K. (1998). Benchmarking innovation best practice. Benchmarking for Quality Management \& Technology, 5(1), 45-58

Auh, S., Bell, S. J., McLeod, C. S., \& Shih, E. (2007). Co-production and customer loyalty in financial services. Journal of Retailing, 83(3), 359-370.

Bateson, J. E. G., \& Hui, M. K. M. (1987). Perceived control as a crucial perceptual dimension of the service experience. In C. Suprenant (Ed.), Add Value to Your Service (pp. 187-193). Chicago, IL.: American Marketing Association.

Bowen, D. E., \& Larsson, R. (1989). Organisation and customer: managing design and coordination of services. Customer participation. Academy of Management Review, 2, 213-233. 
Brown, S. L., \& Eisenhardt, K. M. (1995). Product development: Past research, present findings, and future directions. Academy of Management. The Academy of Management Review, 20(2), 343.

Chase, R. B. (1978). Where Does the Customer Fit in a Service Operation? Harvard Business Review, 56(Nov-Dec), 137-142.

Chesbrough, H. W. (2003). Open Innovation. The New Imperative For Creating and Profiting from Technology. Boston, Massachusetts: Harvard Business School Publishing.

Christensen, C. M., \& Bower, J. L. (1996). Customer power, strategic investment, and the failure of leading firms. Strategic Management Journal, 17(3), 197-218.

Colgate, M., \& Hedge, R. (2001). An investigation into the switching process in retail banking services. Journal of Bank Marketing, 19(5), 201-212.

Commission of the European Communities. (2007). Green Paper on Retail Financial Services in the Single Market COM(2007) 226 Final. Retrieved. from.

Cooper, R. G. (1980). Project new prod: factors in new product success. European Journal of Marketing, 15(5/6), 277-292.

Craig, A., \& Hart, S. (1992). Where to Now in New Product Development Research? European Journal of Marketing, 26(11), 2-49.

de Brentani, U. (1995). New industrial service development: Scenarios for success and failure. Journal of Business Research, 32(2), 93-103.

Deshpande, R., Farley, J. U., \& Webster, F. E., Jr. (1993). Corporate culture, customer orientation, and innovativeness. Journal of Marketing, 57(1), 23.

Dick, A., \& Basu, K. (1994). Customer loyalty: towards an integrated conceptual framework. Journal of Marketing Science, 22(2), 99-113.

Edgett, S. (1994). The Traits of Successful New Service Development. Journal of Services Marketing, 8(3), 40 - 49

Esselman, T. (2006). Innovation in Science and Engineering. "Why Does it Matter?” (pp. 31). Dublin, Ireland: Ryan Academy for Entrepreneurship.

Fivars, G. (Ed.). (1980). Critical Incident Technique. Washington DC: American Institutes for Research.

Gardiner, P., \& Rothwell, R. (1985). Tough Customers: Good Designs. Design Studies, 6(1), 7-17.

GfK. (2005). TOF. Dongen, NL: GfK (not public).

Griffin, A., \& Hauser, J. (1993). The Voice of the Customer. Marketing Science, 12(1), 1-27.

HighTechCampusEindhoven. (2005, August 2005). Client Co Creation Lab provides access for Small and Medium-sized Enterprises. E-Magazine.

Hocutt, M. (1998). Relationship dissolution model: antecedents of relationship commitment and the likelihood of dissolving a relationship. International Journal of Services Industries Management, 9(2), 189-200.

Howcroft, B., Hamilton, R., \& Hewer, P. (2007). Customer involvement and inter- 
action in retail banking: an examination of risk and confidence in the purchase of financial products. Journal of Services Marketing, 21(7), 481-491.

Hsieh, A. T., Yen, C. H., \& Chin, K. C. (2004). Participative Customers as Partial Employees and Service Provider Workload. International Journal of Service Industry Management, 15(2), 187-199.

Kelly, D., \& Storey, C. (2000). New service development: initiation strategies. International Journal of Service Industry Management, 11(1).

Knights, D., Sturdy, A., \& Morgan, G. (1994). The consumer rules? An examination of the rhetoric and 'reality' of marketing in financial services. European Journal of Marketing, 28(3), 42-54.

Langenberg, H., \& Plant, R. (2000). Innoveren loont. Grote rol dienstensector bij innovaties [Electronic Version]. Index, Centraal Bureau voor de Statistiek, 12-13. (in Dutch).

Lilien, G. L., Morrison, P. D., Searls, K., Sonnack, M., \& Hippel, E. v. (2002). Performance assessment of the lead user idea-generation process for new product development. Management Science, 48(8), 1042-1059.

Linstone, H. A., \& Turoff, M. (Eds.). (2002). The Delphi Method: Techniques and Applications Newark, NJ New Jersey Institute of Technology.

Lundvall, B., Johnson, B., Andersen, E. S., \& Dalum, B. (2002). National systems of production, innovation and competence building. Research Policy, 31(2), 213-231.

Lüthje, C., Herstatt, C., \& von Hippel, E. (2005). User-innovators and "local" information: The case of Mountain Biking. Research Policy, 34, 951-965.

Martin, C. R., Horne, D. A., \& Schultz, A. M. (1999). The business-to-business customer in the service innovation process. European Journal of Innovation Management, 2(2).

Martin, J. (1995). IgnoreYour Customers. Fortune, 123-126.

Montoya-Weiss, M. M., \& Calantone, R. (1994). Determinants of new product performance: A review and meta-analysis. Journal of Product Innovation Management, 11(5), 397-417.

Moore, W. L. (1982). Concept Testing. Journal of Business Research, 10(3), 279-294. NBVA. (2006). "Financieel bekwaam is ook tijdig om deskundige hulp vragen" NBVA website: http://www.nbvanet.nl/nieuws.phtml?berichtid=1185 (in Dutch).

Osborn, A. F. (1963). Applied imagination: Principles and procedures of creative problem solving (Third Revised Edition ed.). New York, NY: Charles Scribner's Sons.

Parker, C., \& Ward, P. (2000). An analysis of role adoptions and scripts during customer-tocustomer encounters. European Journal of Marketing, 34(3/4), 341-358.

Prahalad, C. K., \& Ramaswamy, V. (2003). The Future of Competition: Co-Creating Unique Value with Customers Boston, Mass.: Harvard Business School Press.

Stewart, D. W., \& Shamdasani, P. N. (2004). Focus Groups: Theory and Practise. Thousand Oaks: Sage Publications.

Storey, C. D., \& Easingwood, C. J. (1996). Determinants of new product perform- 
ance: A study in the financial services sector. International Journal of Service Industry Management, 7(1).

Ulwick, A. W. (2005). What Customers Want. Using Outcome-Driven Innovation to Create Breakthrough Products and Services. New York: McGraw-Hill.

van Aken, J. E. (1994). Bedrijfskunde als Ontwerpwetenschap. Bedrijfskunde(66), 16-22.

van Aken, J. E. (2004). Management Research Based on the Paradigm of the Design Sciences: The Quest for Field-Tested and Grounded Technological Rules. Journal of Management Studies, 41(2), 219-246.

van Aken, J. E. (2007). The Research Design for design science research in management (pp. 6): Eindhoven University of Technology.

van der Veen, G. (2007). Het Nederlandse ondernemingsklimaat in cijfers 2007.

Heerlen/Voorburg NL: Centraal Bureau voor de Statistiek (in Dutch).

von Hippel, E. (1988). The Sources of Innovation. New York: Oxford University Press.

von Hippel, E. (2005). Democratizing Innovation. Cambridge, Massachusetts: MIT Press.

Zaltman, G. (2003). How Customers Think. Essential Insights into the Mind of the Market. Boston, Mass.: Harvard Business School Press.

Zuboff, S., \& Maxmin, J. (2002). The Support Economy. New York: Penguin Books.

\section{Correspondence}

Altuition BV, Rompertsebaan 60,

5231 GT 's-Hertogenbosch,

the Netherlands

email: mweber@altuition.nl

Eindhoven University of Technology,

Faculty of Technology Management, Department

Organisation Science and Marketing,

Postbox 513, 5600 MB Eindhoven. The Netherlands

\section{Biographical notes}

Marcel Weber holds a position as a senior consultant at Altuition BV, a consultancy specialized in advising and supporting its clients in programs for obtaining customer insights, shaping customer interactions, developing new business and retaining customers. He has an MBA and a MSc in Strategy and Organisation Science. At the moment he is an executive PhD student at Eindhoven University of Technology. He has over 25 years experience as an Air Force officer in aircraft maintenance and logistics, with a specialization in total quality management and customer focus. 


\title{
Different configuration problems at different stages in the service life cycle
}

\author{
Authors: Jose Angel Lakunza, Juan Carlos Astiazaran, Maria Elejoste
}

\begin{abstract}
Service is a concept difficult to define. It is a term that has been extensively used in different contexts. According with different authors a service can be seeing: as an activity or as an output of a process. As an output it represents what a customer receives. As an activity it represents how a customer receives the service.

By service customization we mean the definition, adaptation and/or the modification of a service according to the customer' requirements, when this customization process is carried out in a systematic way.

A company can respond to users needs diversity offering products than can be customized easily, and/or increasing the product variety offered to the market. The same idea could be a good strategy in the field of services. We should take into account that the increments in the service variety have also drawbacks. The increasing in the service offer variety or the service customization raises also the variety and complexity in the company transactions that involves cost increments for these transactions. Therefore, the challenge will be to extend the range of services offered to the market maintaining the efficiency (without cost rise), that is similar to the Mass Customization concept.
\end{abstract}

\section{Keywords}

Service customization, service configuration, service architecture.

\section{Introduction}

Nowadays, the important of service industry in the economy is growing (Grönroos, C. (2000); Fitzsimmons \& Fitzsimmons (2004)). More and more customers are demanding services that meet their needs. The differences between goods and services and services are fuzzy as it has been argued that products always contain a service dimension (Kotler et al. (1996)). Human needs are satisfied not only with physical goods but also services.

Different authors argue that research on service customization is sparse and needs to be developed in depth (Harvey et al. (1997), da Silveira et al. (2001)). In the other hand, due to the service nature, it should be adapted to the customer needs.

Our research goal has been focused on the service customization, we try to apply similar approaches to the service customization than those used for the cus- 
tomization of goods. The main research questions we try to answer are: how can we define a generic service model that represents a "Service Family"?; what are the different configuration moments in the service life cycle?

We carried out the research as follow. First we made a literature review on the service concept. Next, we developed a service classification. After that, we focused on the definition of a generic model for the services that might be configured taking into account the customer needs. And finally, we analyzed the applicability of configuration along the service life.

\section{Literature Background}

Service is a difficult term to define, because it is extensively used in different contexts. This difficulty has been widely mentioned in the literature by different authors (Johns (1999) "What is this thing called service?", Grönroos (2000), Fitzsimmons \& Fitzsimmons (2004)). Some of the service definitions find in the literature are the following:

Kotler (1994) defines service as "any act or performance that one party can offer to another that is essentially intangible and does not result in the ownership of anything".

Grönroos (2000) defines service as "a process consisting of a series of a more or less intangible activities that normally, but not necessarily always, take place in interactions between the customer and service employees and/or physical resources or goods and/or systems of the service provider, which are provided as solutions to customer problems".

Fitzsimmons \& Fitzsimmons (2004) defines service as "a time-perishable, intangible experience performed for customer acting in the role of coproducer".

According to Jiao et al. (2003) two main streams can be identified in the service definition literature: from the service provider's view as an activity (we call it service) and from the customer view as an output of a system (we call it service result).

Johns (1999) mentions these different approaches for the service. The provider sees the service offering in terms of process, related to service operation. On the other hand, the customer views it as a personal experience, present in service outcomes. As an output a service represents what a customer receives. As an activity it represents how a customer receives the service

\section{Service Concept}

\subsection{Transaction concept}

Any kind of commercial relationship between a provider and a customer can be considered as a "commercial transaction" as we can see in the figure 1. At any commercial transaction the customer gets something (a good and/or a service) from the provider in exchange for money.

As we can see in the figure, other things can be involved in this interchange between the provider and the customer: resources (materials or humans), supplies, etc. 


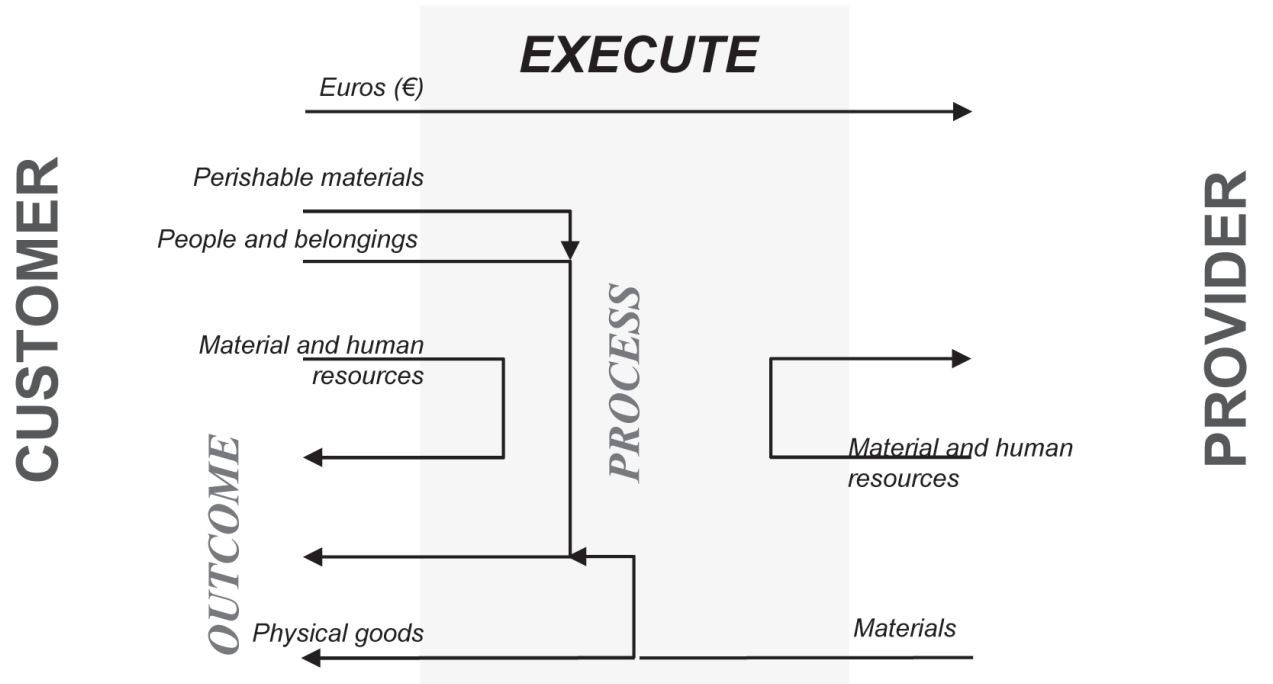

Figure 1: Transaction between customer and provider

From the customer point of view, this transaction might imply money to pay the provider, supplies to be used in the transaction, human and/or material resources to carry out the transaction.

After the transaction some changes can affect the customer: change in their physical possessions (is owner of new goods) or change in their intangible assets (welfare, state of mind, information, knowledge...).

From the provider point of view, to perform the transaction, he must to provide resources (human and/or material) to be used in the transaction, and materials in order to get the goods or to support the service.

As the result, the provider will receive money from the customer and will take back the resource used for the transaction.

The transaction itself is done by means of resources (people, devices...). These resources can execute two kinds of actions: obtaining goods from materials or transforming (physically or mentally) the customer.

\subsection{Service classification}

In accordance with the increasing importance of services as a primary component of the economies in developed countries, a number of service typologies have been developed, although there has been virtually no empirical validation of the proposed ideas.

One such classification, is the service process matrix, originally proposed by Schmenner, Roger (1986) and latterly adapted by Lovelock (1983). This classification defines two dimensions: 
- Degree of labour intensity: Defined as the "ratio of labour cost to capital cost". Services with high labour intensity are schools and retailers (mass services) which depends on the manual labour of educators and the retail managers-employees who perform the services

- Degree of interaction and customization: Ability of the service client to affect the value that is co-created. Most professional services, (e.g., doctors, lawyers, and IT services) require a high-degree of customization

The service classification we propose is an adaptation of the proposed by Lovelock. We define also two dimensions for the classification schema:

- What level of configuration is proposed?: This can be standard, catalogue, menu (combination), a framework.

- When does the customization moment happen?: represent different moments for the service configuration. These options can be no customization, contract customization or service order customization.

\begin{tabular}{|c|c|c|c|c|c|}
\hline & & \multicolumn{4}{|c|}{ Level of flexibility } \\
\hline & & Standard & Catalogue & Menu & Framework \\
\hline \multirow{3}{*}{ 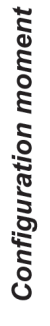 } & No customization & $\begin{array}{l}\text { Baggage } \\
\text { store }\end{array}$ & Underground & No valid & No valid \\
\hline & Contract customization & No valid & $\begin{array}{c}\text { Maintenance } \\
\text { contract }\end{array}$ & $\begin{array}{c}\text { Car } \\
\text { insurance }\end{array}$ & $\begin{array}{l}\text { Repair } \\
\text { service } \\
\text { contract }\end{array}$ \\
\hline & Service order customization & No valid & $\begin{array}{l}\text { Transport of } \\
\text { goods }\end{array}$ & $\begin{array}{c}\text { Car } \\
\text { accident }\end{array}$ & $\begin{array}{c}\text { Machine } \\
\text { breakdown } \\
\text { repair }\end{array}$ \\
\hline
\end{tabular}

Table1: Service classification

\section{Service Model}

\subsection{Process map}

In order to establish a good service architecture that allows us the configuration of services to the customers needs, we first focus on the map of process that can be executed along the service life cycle. 


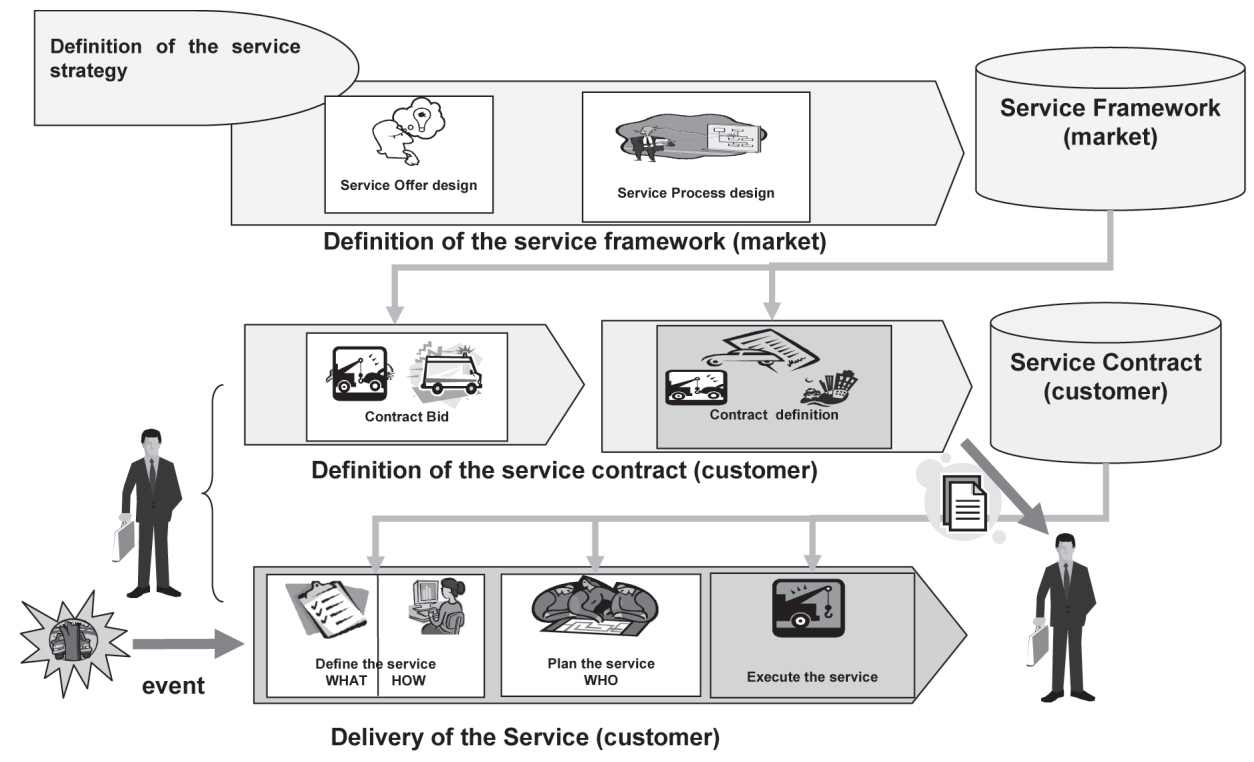

Figure 2: Process Map

As we can see in the figure 2, we have defined three different process levels depending in the time horizon for the process:

- Process level with large time horizon. The input for the process is the market requirements and the output is also the offer to the market. At this level we can distinguish the process for the service strategy definition and the process for the service framework definition for the market (for instance the definition of the city bus lines and timetables).

- Process level with a medium time horizon. In this case the input for the process is the customer needs and output for the process is the service contract for the customer. At this level we can see the process for the development of the contract bid and the process for the development of the contract service. Both processes are famed by the framework defined in the previous level (for instance the definition of the car insurance conditions for a customer).

- Process level with a short time horizon. In this case the input is an event for the customer and the output is the executed service for the customer. At this level we can see the following activities in the process for the delivery of the service: definition of the facts for the service and the activities needed to execute the service (WHAT \& HOW); definition of the resources to execute the service (WHO); and the execution of the service itself. These activities are framed by the framework 
(contract) defined in the previous level (for instance the response for a car accident from the insurance company).

\subsection{Service architecture}

According with Goldstein et al. (2002), the service should describe what are the customer needs, how this is going to be achieved, and with what kind of resources. The breakdown of a service into its components, allows the designer to check them against the customers' needs. This approach is necessary in order to customize services in a mass customization schema.

Meyer \& De Torre (2001) are using product platform concepts that have been used for physical products. Applying modularization techniques to the service is seen as one way to achieve service customization. As in the case of goods, modules can be combined in response to specific customer needs. Usually these service modules are seen as work tasks, parts of processes or groups of activities.

The figure 3 shows a high level conceptualization of service proposed by Heiskala (2005) as the result of the service literature analysis.

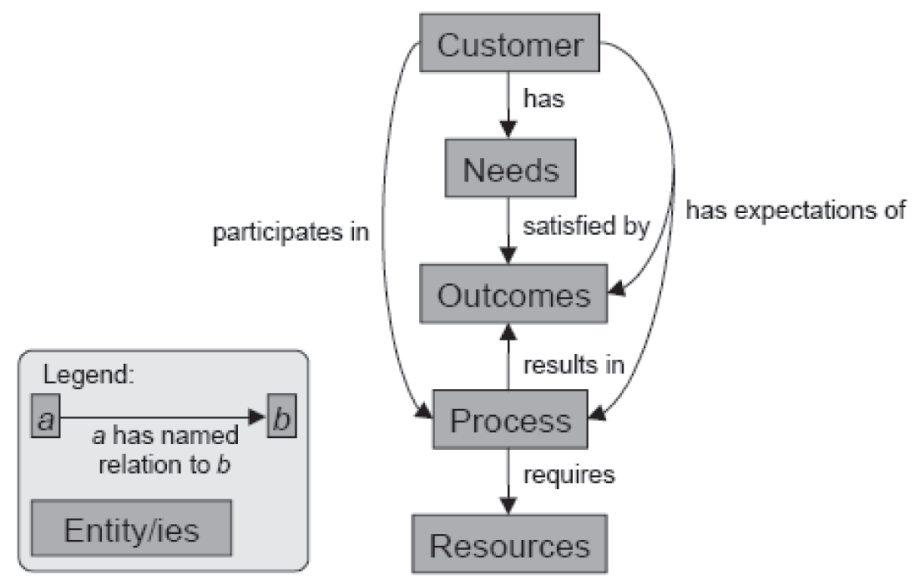

Figure 3: A high level conceptualization of services based on service literature

The aim of our work has been to apply the ideas of Product Family Architecture (PFA) developed by Jianxin, J. (1998) in the field of services, in order to define a service architecture that can be used by a service configurator. Our approach for the definition of this service architecture is based in the analysis of the different moments identified in the service life (see figure 4):

A. Definition of the service offer to be provided by the company.

B. Definition of the processes to be carried out inside this service offer. 
C. Sale of a service contract.

D. Specification of the service to be provided to the customer as response to an event.

E. Planning and scheduling of the service.

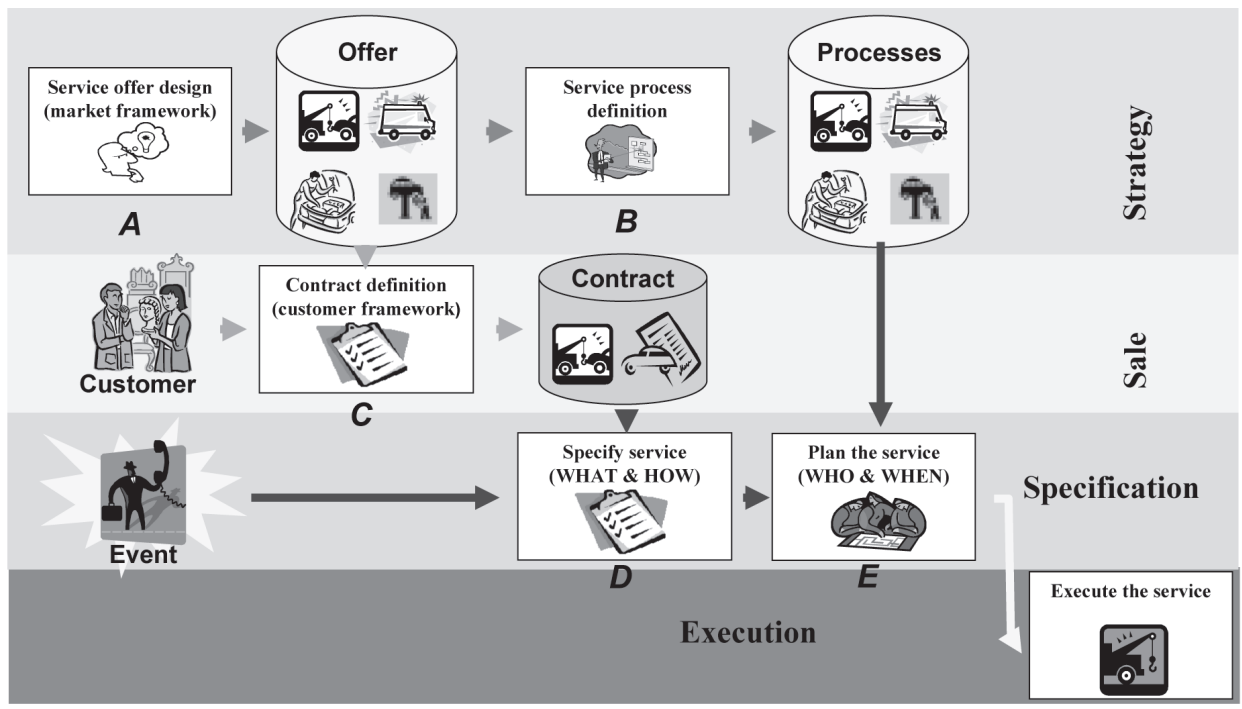

Figure 4: Different moments in the service life

To broach the service customization, a service family architecture is needed to characterize customer needs and subsequently to fulfill these needs by configuring and modifying wellestablished service modules and service components (termed as building blocks).

As the result of this work and as answer to the question how can we define a generic service model that represents a "Service Family"?, we propose the following service architecture.

The entities identified to define the service framework that the service company offers to the market are the following:

- Service Offering: framework offered by the company to the market (service family). This service offering is composed by service elements.

- Service Element: service module that can be offered by the company to a customer. A service process will be executed by a resource to give this service. A service element can be hierarchically structured by using service components and service bundles. 
- Service Component: elementary service used to make up the Service Element.

- Service Bundle: group of elementary services that make up the structure of a Service Element.

- Resource: element needed to execute the process associated to a service. This element can be an input for the process or an output of the process. Resources can be material or human.

- Service Process: Services are produced by processes. It represents the set of activities o be carried out to give a service to a customer.

- Relationship: the relationships between service elements can be of different type as proposed by Baida et al. (2003). These types are enhance, support, bundle, substitute, exclude and optional.

In the following figure 5 we represent graphically the architecture define for a service offering.

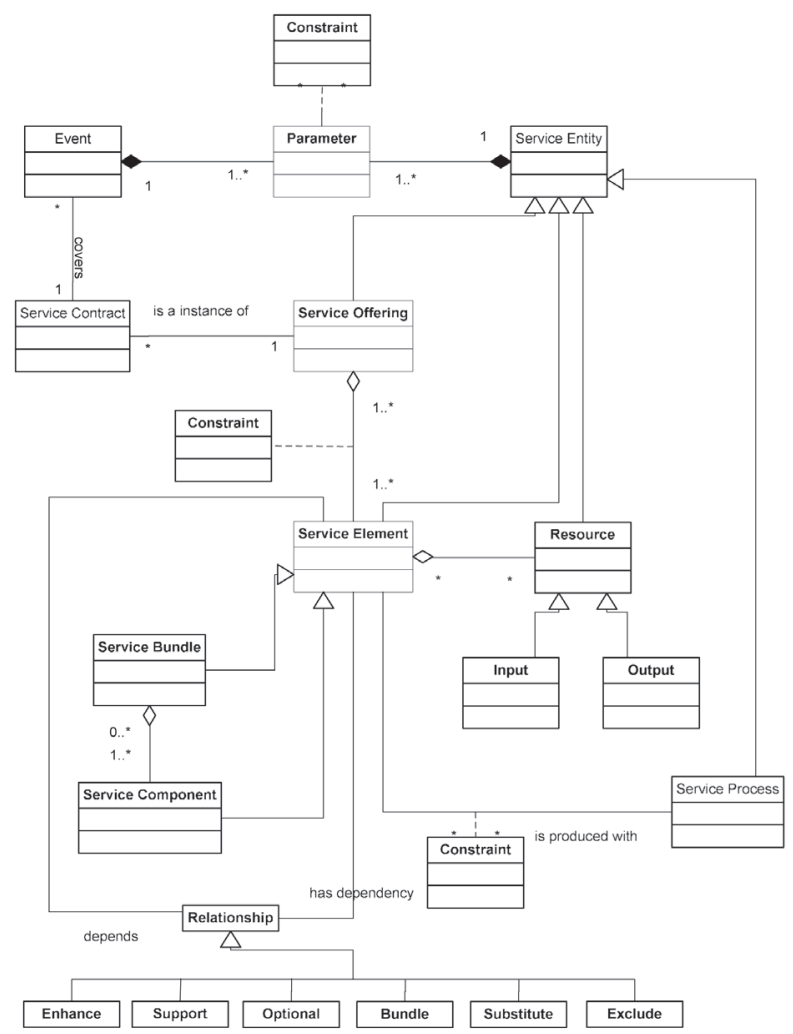

Figure 5: Service architecture 
Other entities that appear in the figure as part of the model defined for the service offering are:

- Parameters: all the entities of the model are characterized by parameters. Each parameter will be defined by its value domain. These parameters give to the model its generic characteristic allowing the model to be instantiated.

- Constraints: the components of the service offering can be constrained. Also the values that a parameter can take can be constrained.

- Service Contract: instance of the service offering that responds to the requirements o a customer. This service contract covers several service events that lead to a service.

- Event: context in which the customer needs the service from the provider.

\section{Service configuration}

In order to answer the possed question what are the different configuration moments in the service life cycle?, we have identified two different service contexts from the point of view of the service configuration: services without contract definition and services that need contract definition.

\subsection{Configuration of services without contract}

For services that don't need the definition of a service framework (contract) customized for the customer, the service framework is the same for any customer that wants the service. That means the company has defined a general framework for the service offer.

As we can see in the figure 6, in a first step, this general framework for the service has been defined by the company, when the company defined the service offer to the market (when a launching new service offers for the market). In a second stage, the company sells a service to a customer, according with the specifications required for the customer.

As we can see, the configuration of the service is carried out during the sale and specification definition of the service from the required needs of the customer. This service configuration takes into account the service offer defined previously by the company. In fact in this moment the process needed to answer to the customer requirement is configured (HOW) and also the type of resources needed to execute the process.

In this scenario of services, there is only a configuration possibility for the service: at the moment of specification definition and sale of the service. An example of this service could e the mail service. In this case the customer defines the specifications for the mail at the same moment that pays for the service. 


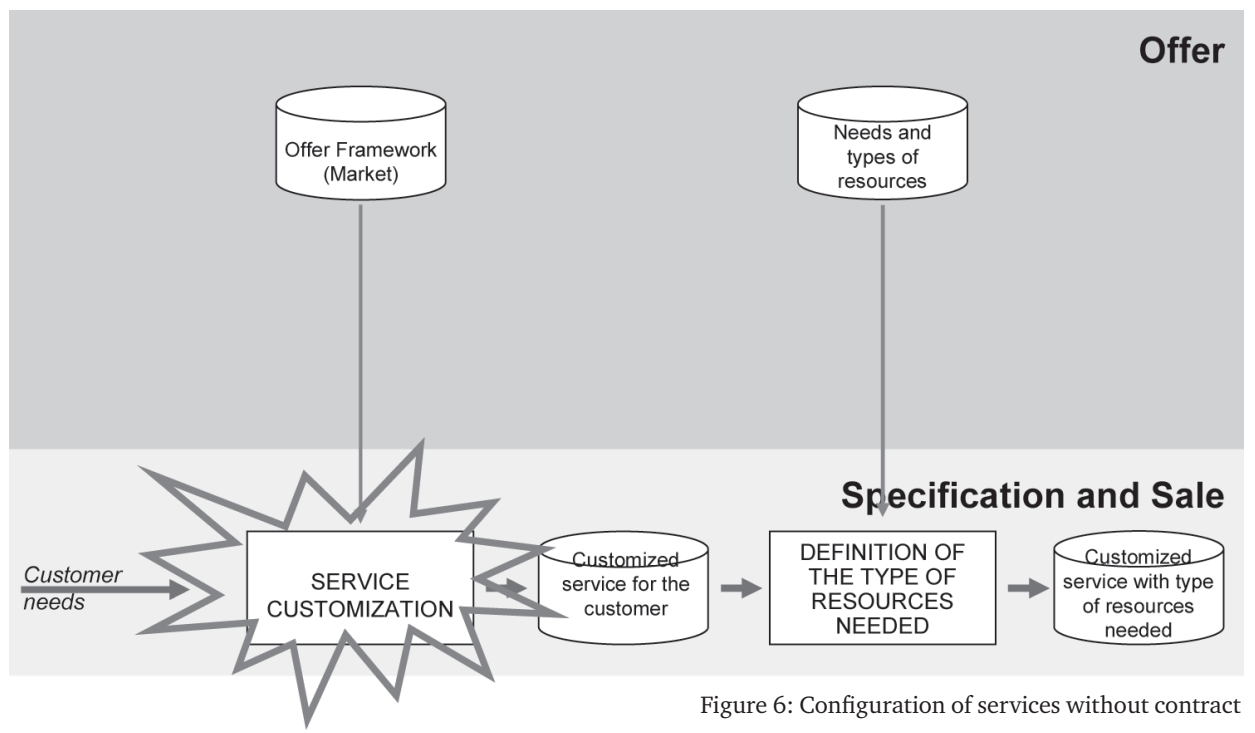

\subsection{Configuration of services with contract}

In the field of services, there are a lot of examples in which is necessary to define previously a contract for the customer establishing the characteristics of the service to be provided in the future to the customer. Some examples are insurance services, maintenance services, etc.

In these cases of services we can distinguish three different moments along the service life cycle:

1. Service offer definition for the market.

2. Service contract definition and sale for the customer

\section{Customer event turn up and service execution for the customer}

This is the case of car insurance. In a first moment, the company defines the overall service offer for the insurance of a car (1). Next, the customer in collaboration with the company, defines a specific contract for the insurance of the customer car that mentions all the options covered by the company (2). Once the contract has been established, as response to any kind of event suffered by the customer (and accident, a car breakdown, etc.), the company provides the customer with the adequate service (3).

As we can see in the figure 7 (next page), two different service configuration moments can be identified along the service life cycle. 


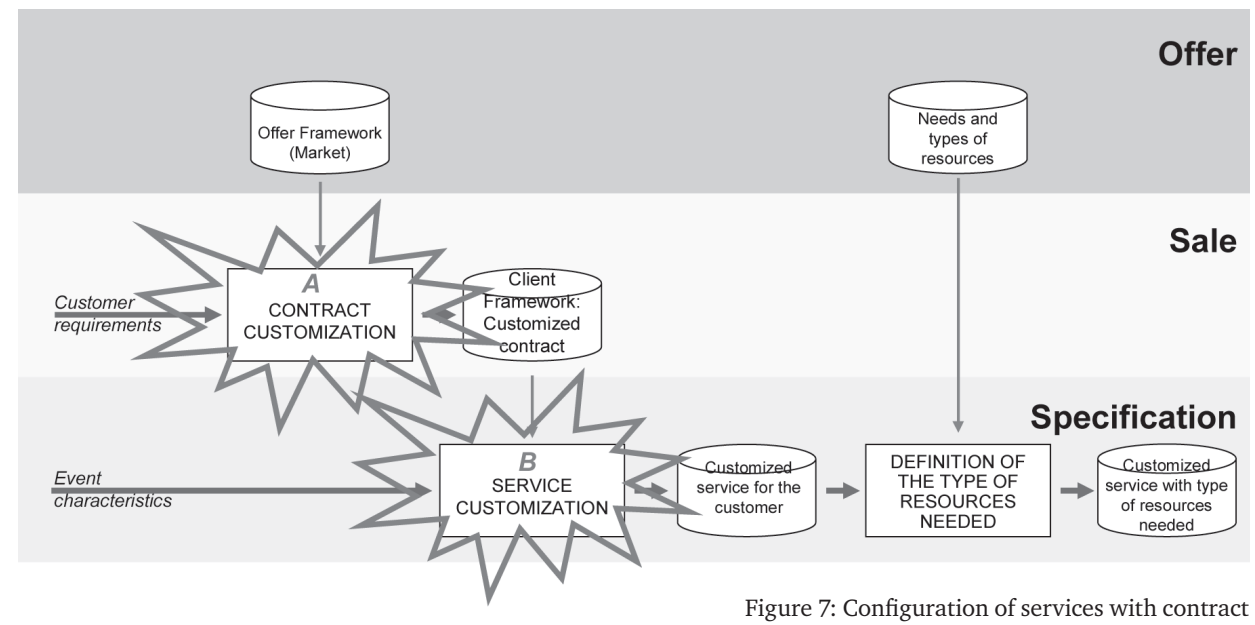

A. A first moment is the configuration of the service contract, taking into accout the customer requirement for the service contract and the overall service offer defined for the company. This kind of configuration is quite similar to the used for the configuration of goods. Defines WHAT is going to be provided by the company. For instance when a customer establish with the insurance company the contract for the car insurance.

B. In a second moment the company, as a response to a customer event, configures the service process to be execute (HOW), and the kind of resources to be used, taking into account the framework posed by the service contract. For instance when the customer suffers and accident with his car and the insurance company provides the adequate service.

\subsection{Service execution planning}

As we can see in the previous figure 7, in this scenario of services we could configure the service contract (WHAT the contract covers) and when an event happens HOW (the process for the service) is going to be delivered the service, taking into account the event characteristics and the service contract defined for the customer and the defined type of resources to be used for the execution of the process.

Alter this definition and in order to execute the defined service in response to an event, we must to define exactly which are the resources to be used in the service process (WHO) and when this resources are going to be used (the scheduling). In short to plan the service execution.

In the figure 8 we present a diagram for the development of the service plan. In this process we can distinguish two different activities: 


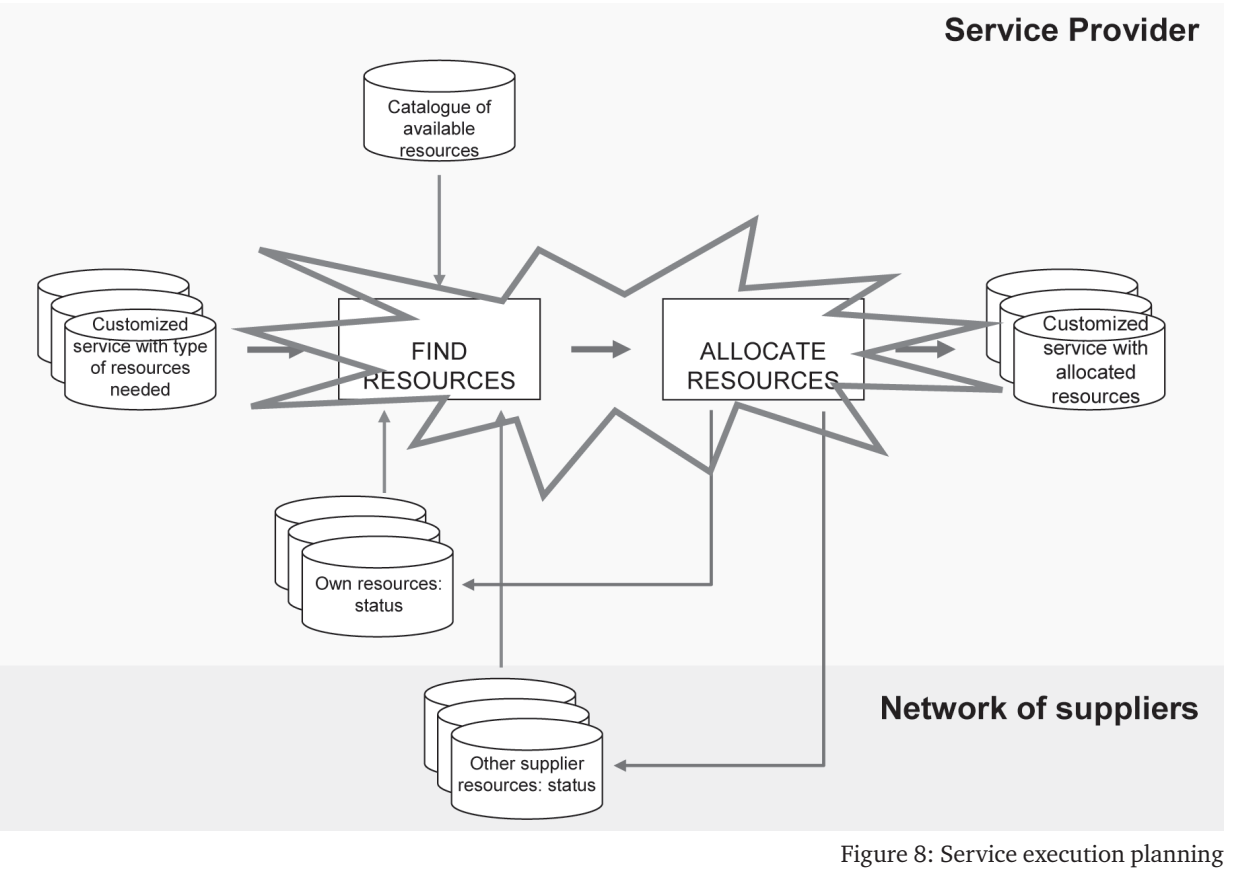

- To find resources in the supplier's network: form the information generated as result of the service configuration (HOW the service will be executed and the type of resources needed for the execution of the service) and taking into account the resources available in the network of suppliers, its finds the appropriate resources for the service execution.

- To allocate resources for the service process: once identified the resources, these will be allocated to the corresponding service process activity and the scheduling will be defined. As result of this we will have the customized service process with the allocated resources plus the execution scheduling for the service.

\section{Conclusions}

As result of our research goal, we have answered the main research questions posed: how can we define a generic service model that represents a "Service Family"?; what are the different configuration moments in the service life cycle?

Answering the first question we have defined a service architecture that allows the model of services that can be customized to the customer needs and that takes into account the two aspects posed by the literature: service as an outcome and service as a process.

To answer the second question we have identified the different moments for the service configuration in the two different scenarios of the service: service without contract and service with contract. 
As future work we are going to go deeply into the following:

- To adapt the configurator tool developed for the configuration of goods (physical products) to the configuration of service contracts.

- To develop new configurator tool for the configuration of the service processes to be executed by the service provider company, as answer to the event.

These developments will be tested, during the 2009, in quasi-real cases and later on in real cases of services.

\section{References}

Baida, Z., Gordijn, J., Akkermans, H. (2003): Service Ontology, Deliverable D6.1 of OBELIX IST project, 2003.

Da Silveira, G., Borenstein, D. \& Fogliato, F.S. (2001): Mass Customization: Literature review and research directions, International Journal of Production Econo-mics, 2001.

Fitzsimmons, J.A. \& Fitzsimmons, M.J. (2004): Service Management - Operations, Strategy, and Information Technology, Fourth Edition, International Edition, ISBN 0-07- 121457-7, McGraw-Hill, 2004

Goldstein, S.M., Johnston, R., Duffy, J.A., Rao, J. (2002): The service concept: the missing link in service design research, Journal of Operations Management, 2002.

Grönroos, C. (2000): Service Management and Marketing: A Customer Relationship Management Approach, Second Edition, ISBN 0-471-72034-8, John Wiley \& Sons, Ltd., 2000

Harvey, J., Lefebvre, .A. \& Lefebvre, E. (1997): Flexibility and technology in services: a conceptual model, International Journal of Operations \& Production Management, 1997.

Heiskala, M. (2005): A conceptual model for modeling configurable services from a customer perspective, Master's Thesis, Helsinki University of Technology, 2005.

Jiao, J. (1998), Design for Mass Customization by Developing Product Family Architecture, GMRG thesis, 1998.

Jiao, J., Ma, Q., Tseng, M.M. (2003): Towards high value-added products and services: mass customization and beyond, Technovation, Vol. 23 No. 10, 2003.

Johns, N. (1999): What is this thing called service, European Journal of Marketing, Vol. 33 No. 9, 1999.

Kotler, P (1994): Marketing management: analysis, planning implementation and control, London, Prentice Hall, 1994.

Kotler, P., Armstrong, G., Saunders, J. \& Wong, V. (1996): Principles of Marketing, Prentice Hall, 1996.

Lovelock, C. (1983): Classifying services to gain strategic marketing insights, Journal of Marketing, 1983. 
Meyer, M.H. \& DeTorre, A. (2001): PERSPECTIVE: Creating a platform-based approach for developing new services, The Journal of Product innovation Management, 2001.

Schmenner, Roger W. (1986): How Can Service Businesses Survive and Prosper?, Sloan Management Review, 1986. 


\title{
Configuring an open pipeline fulfilment system - a simulation study in an automotive context
}

\author{
Philip G. Brabazon, Andy Woodcock, Bart L MacCarthy
}

\begin{abstract}
Automotive producers are adopting multi-modal fulfillment models in which customers can be fulfilled by products from stock, by allocating as yet unmade products that are in the planning pipeline, or by building a product to order. This study explores how fulfillment is sensitive to several parameters of the system and how they interact with different methods for sequencing products into the production plan.
\end{abstract}

\section{Keywords}

Order fulfillment, product variety, pipeline, automotive

\section{Introduction}

In the automotive sector it has become common practice for the mass market producers to use several different mechanisms to fulfil customers in their major markets (Holweg and Pil, 2004). A suitable vehicle for a customer can be sought by searching: the stock of finished vehicles, among the vehicles planned for production, or by building a vehicle to order (BTO). The fundamental behaviour of this form of system has been studied by Brabazon \& MacCarthy (2006) and been shown to be different from a conventional system in which the pipeline is closed from view. Methods for sequencing products into the pipeline have been described and compared in Brabazon et al (2008). The study reported here extends the previous research by assessing how the configuration of the system impacts performance. The model captures customer compromise behaviour i.e. the customer being prepared to compromise on vehicle specification in their purchase decision in order to reduce lead time.

To study the system a discrete event simulation has been created which models the pipeline as a sequence of products. At each time step of the simulation these products increment one position along the pipeline and one leaving the downstream end. The exiting product goes into stock unless it has been sold already in which case it is removed from the system. Customer arrivals are synchronised with the incrementing pipeline, with one customer served in each time period. Thus, production and customer demand are assumed to be in balance with respect to volume. Every customer is allocated a product, either from stock or the pipeline or by requesting a buildto-order product. 
The study investigates the following configurations of the system:

- Amount of finished stock

- Open and closed pipeline

- Length of pipeline

- Batch feeding of the pipeline. The smallest batch size is one (i.e. a product is fed into the pipeline at every time step) and the largest batch size is half the length of the pipeline.

\section{Description of the model and pipeline feed methods}

A schematic of the order fulfilment system is shown in Figure 1. In this system there are three mechanisms for fulfilling a customer with a product - from finished stock, from allocating a product in the planning pipeline to the customer, or requesting a build-to-order (BTO) product

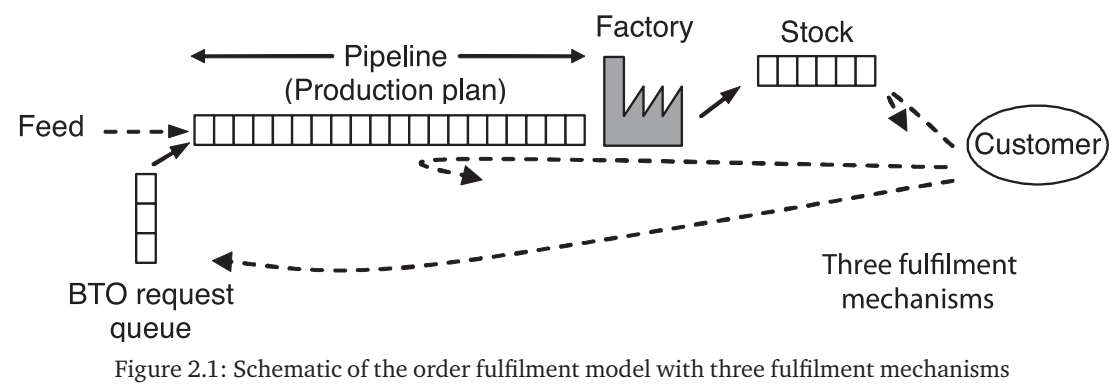

The challenge for a producer is to configure the system in a way that give the best fulfilment to customers. The amount of product variety being offered by producers is large, with some passenger vehicles coming in thousands if not millions of feature and option combinations.

In this study, the relative demand for each variant follows an 80/20 distribution, i.e. $20 \%$ of the variants account for $80 \%$ of demand as illustrated in the right plot in Figure 3. This is modelled in the simulation using a Beta distribution with the shape parameters set to 1 and 7.5 .
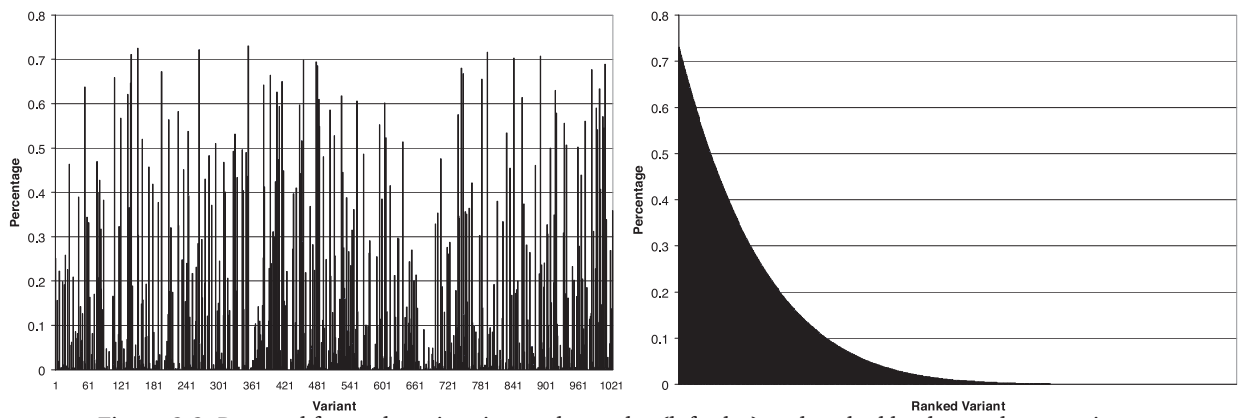

Figure 2.2: Demand for each variant in number order (left plot) and ranked by demand proportion to show the shape of the $80 / 20$ distribution (product range 1024) 
Several variety levels from 64 to 16384 are simulated. Figure 2.2 shows the relative demand for each variant when there are 1024 variants and it is important to note that demand per variant is randomised to avoid a correlation between variant specification and variant demand. This is to emulate the real world situation in which the most commonly requested variants from a product range differ greatly.

The producer has control over the configuration of the system and the question is how fulfilment performance is affected by the parameter. The parameters under study here are:

- Stock volume

- Pipeline length

- Batch feed. At one extreme the producer can feed one product into the pipeline after each sale, or can add products to the pipeline in a batch.

- Open or closed pipeline. In a conventional fulfilment system the production plan is closed, or invisible, to the sales function.

Customers are modelled as seeking a preferred product specification, but being prepared to tradeoff the specification with the lead time. When a customer enters the system, a search is made through stock and along the pipeline for the 'best' product. The lowest scoring product is allocated to the customer, unless a BTO order would have a lower score, in which case the customer is fulfilled by the BTO mechanism. The score for product $i$ is the sum of the specification difference and its lead time, $l$ :

$\mathrm{s}_{\mathrm{i}}=\left|\mathrm{v}_{\mathrm{i}}-\mathrm{v}_{\text {requested }}\right|+1_{\mathrm{i}}$

The metrics are:

- Compromise received by the customer, measured as the difference between received and requested specification, and expressed as a percentage of the product range.

- Fulfilment mechanism, i.e. proportion of customers fulfilled from stock, pipeline and BTO

- Customer waiting time

- Stock metrics such as volume, age and mix (which is measured as the proportion of variants represented in stock)

\subsection{Pipeline feed methods}

Six methods for feeding the pipeline are tested. These are summarised below with a full description in Brabazon et al (2008):

- Method 1: Random feed. The next variant fed into the pipeline is chosen at random from the target distribution.

- Method 2: Reduce stock-out probability. The next variant to be fed into the pipeline is the one that has the highest probability of stocking out. The cal- 
culation does not lool solely at stock but uses the total number of available products in stock and the pipeline, and therefore the total number of a variant in both the stock and pipeline.

- Method 3: Reduce weighted error from target distribution. This considers the error between the actual number of a particular variant in both stock and pipeline and the expected demand for that variant. The error is weighted according to the demand for the variant. The variant to be fed into the pipeline is the one that has the largest error

- Method 4: Reduce distance (to reduce compromise). This method applies the concept of compromise distance to select a variant. It selects a variant which will minimise the expected compromise suffered by customers. This is a computationally demanding method, with the number of calculations being proportional to the square of the number of variants. Results have not been obtained for all conditions.

- Method 5: Increase forward sales coverage. The expected forward sales cover of a variant is calculated using the binomial approximation. The variant to feed into the pipeline is the one which has the lowest forward sales coverage.

- Method 6: Follow the previous customer's request. In this method the sequence of wholesaled products repeats the sequence of customer orders.

\section{Results}

\subsection{Closed versus Open Pipeline}

In this section the performance of the VBTO system is compared to a conventional fulfilment system which has a closed pipeline.

In neither systems are any customers fulfilled by BTO, therefore in the conventional (closed pipeline) system all customers are fulfilled from stock. It is very clear the customers in the conventional system experience much higher compromise (and variation in compromise) than in the open pipeline system. Once the stock level approaches 500 the two systems converge.

As a result of more customers being fulfilled from stock in the conventional system than in the VBTO system, the age of stock is less in the conventional system. However, the coverage of stock is also less.
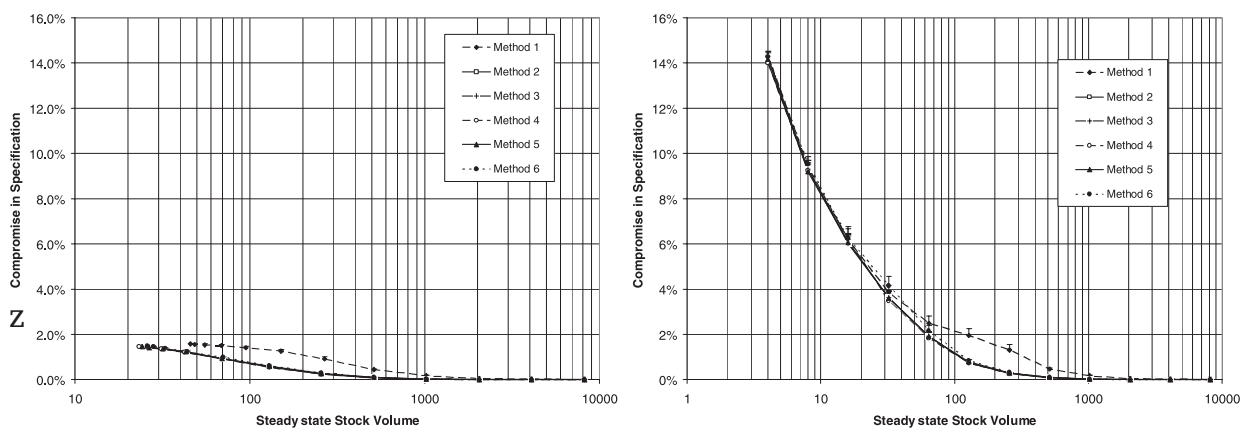

Figure 3.1: Compromise in specification (VBTO left plot, Conventional right plot) 

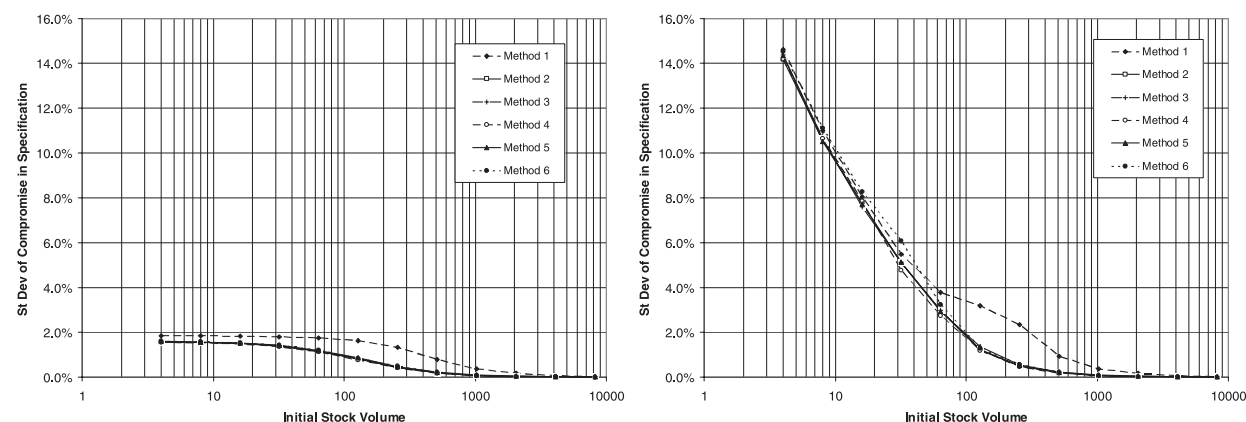

Figure 3.2: Standard deviation of Compromise in specification (VBTO left plot, Conventional right plot)
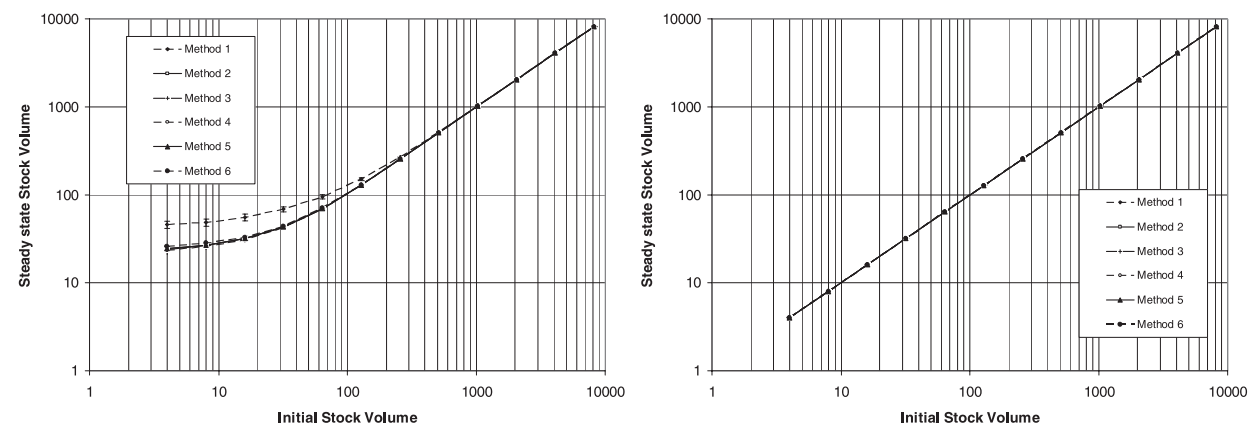

Figure 3.3: Stock volume (VBTO left plot, Conventional right plot)
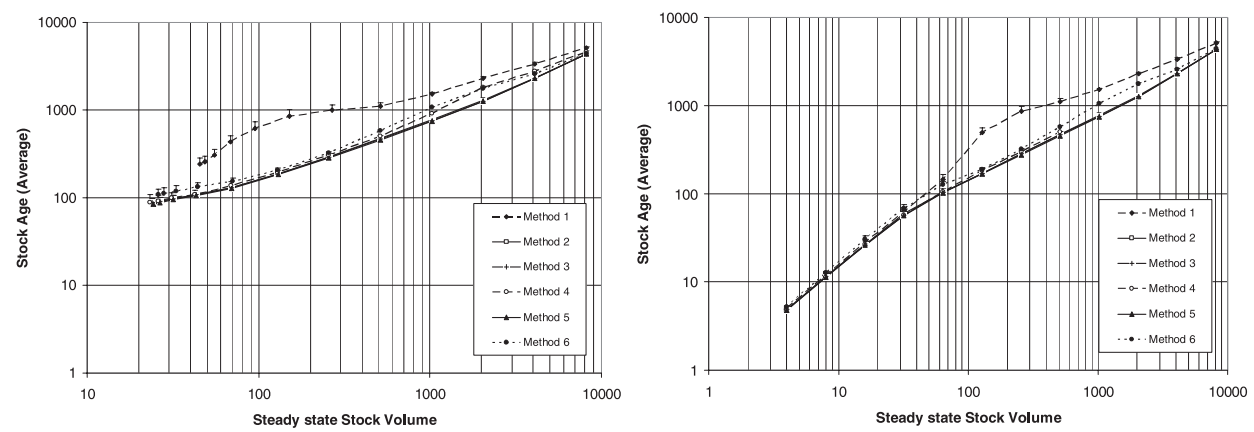

Figure 3.4: Stock age (VBTO left plot, Conventional right plot)
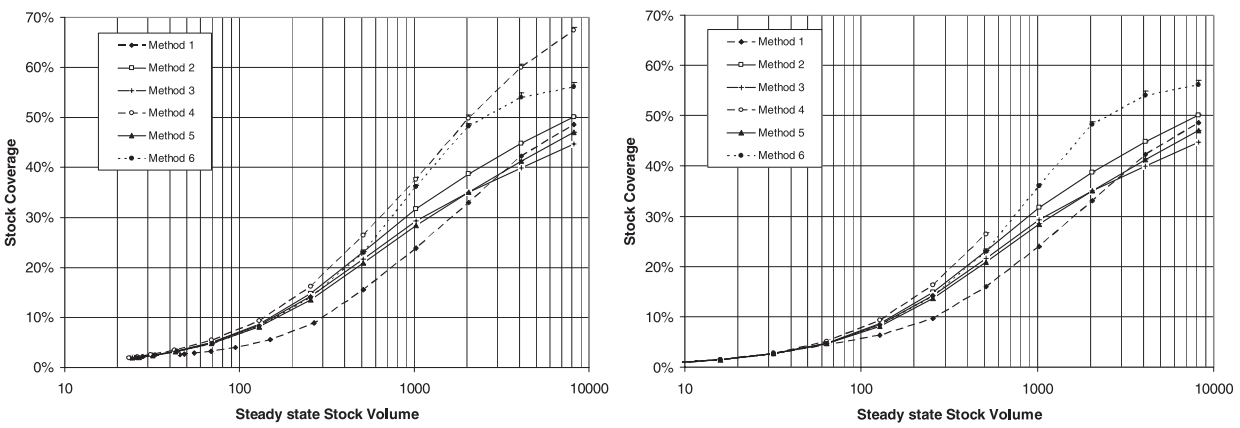

Figure 3.5: Stock coverage (VBTO left plot, Conventional right plot) 


\subsection{Different levels of variety and different pipeline lengths}

Using Method 2 the effect of altering the variety levels is studied, and also the effect of altering the length of pipeline.

It is observed:

- All performance metrics improve as variety is reduced.

- All performance metrics improve as pipeline length is reduced.

- With customers compromising, the consistency in performance for constant $\mathrm{v} / \mathrm{p}$ ratios does not hold as has been when customers require an exact match (Brabazon and MacCarthy, 2006). For example, at a ratio of 4, when variety is 4096 and pipeline is 1024 the proportion fulfilled from stock is $\sim 65 \%$ when the stock level is 100 , whereas it is $\sim 88 \%$ when the variety is 1024 and pipeline is 256 .
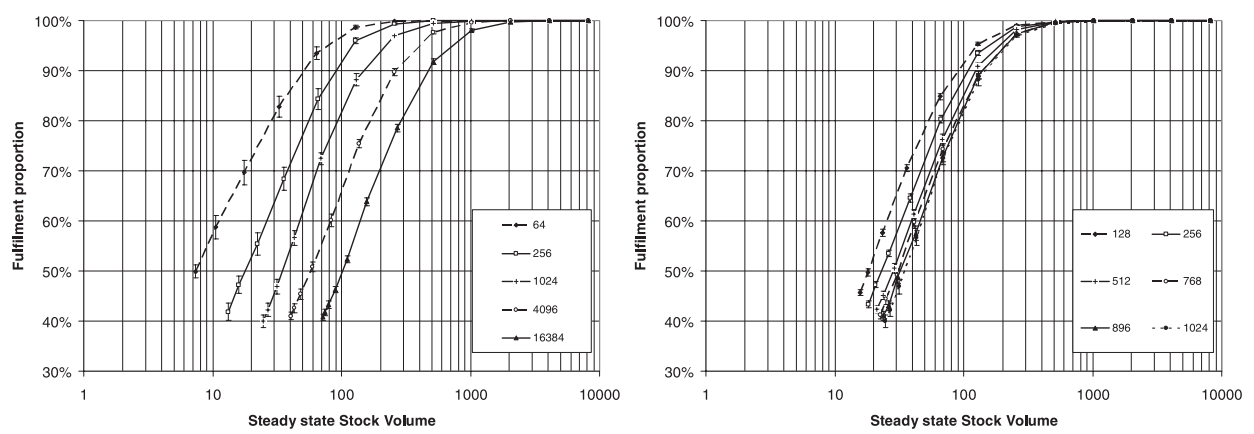

Figure 3.6: Stock fulfilment (multiple varieties left plot, multiple pipe lengths right plot)
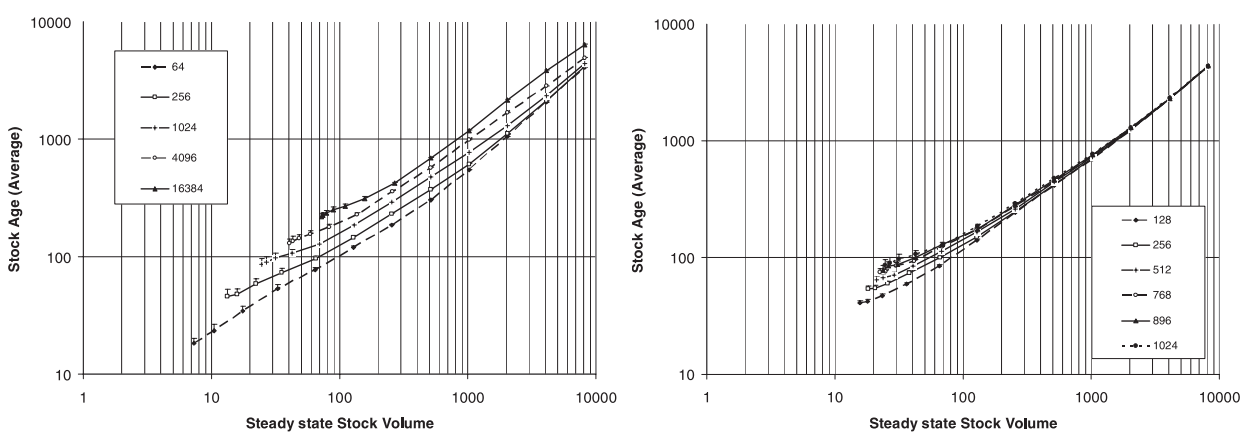

Figure 3.7: Stock age (multiple varieties left plot, multiple pipe lengths right plot) 

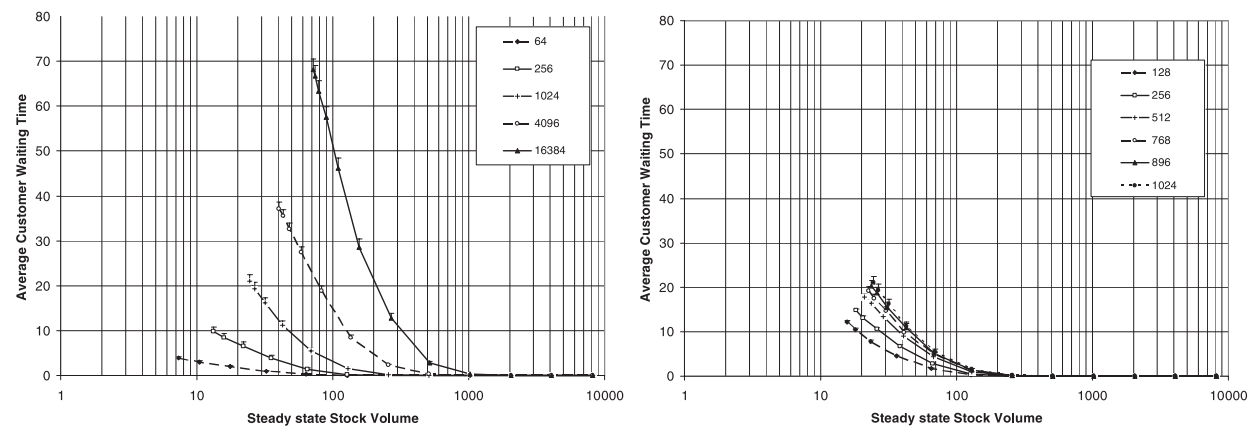

Figure 3.8: Customer Waiting time (multiple varieties left plot, multiple pipe lengths right plot)
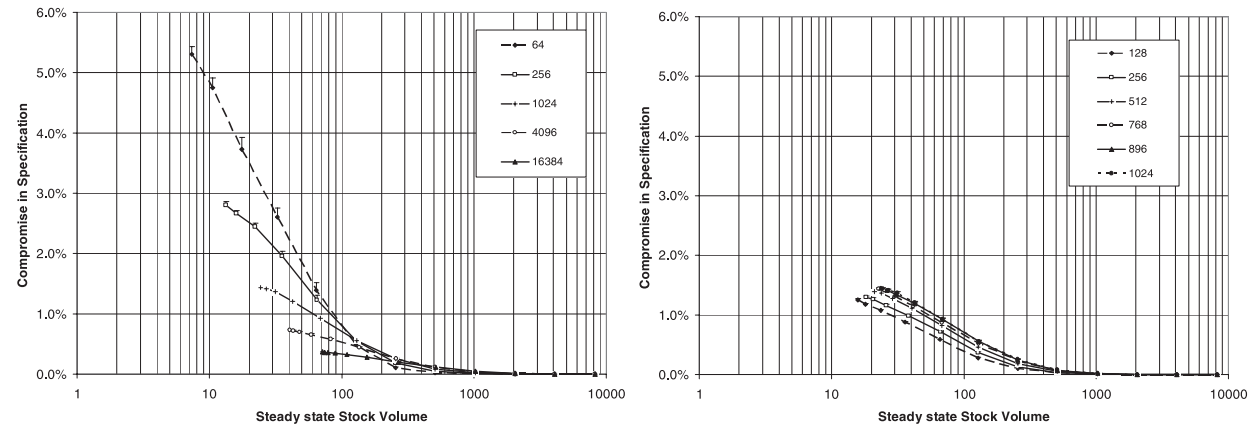

Figure 3.9: Compromise in specification (multiple varieties left plot, multiple pipe lengths right plot)
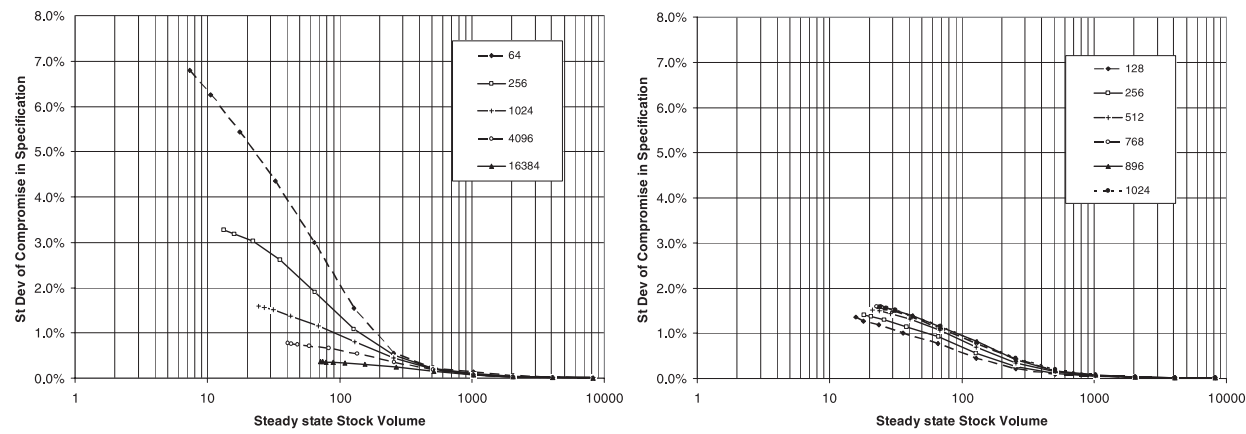

Figure 3.10: Standard deviation in Compromise in specification (multiple varieties left plot, multiple pipe lengths right plot)

\subsection{Impact of feedsize}

In the above experiments the feed into the pipeline has been synchronised with the arrival of customers, i.e. one product is fed into the pipeline, then one customer is processed, then one product is fed in and so on. This section reports on the affect of batch feeding the pipeline. For example, after 32 customers have been processed, during which time no products are fed into the pipeline, a block of 32 products are fed in. Therefore the number of products in the pipeline, if plotted over time, would appear to have a saw tooth pattern, as illustrated in the figure below. 


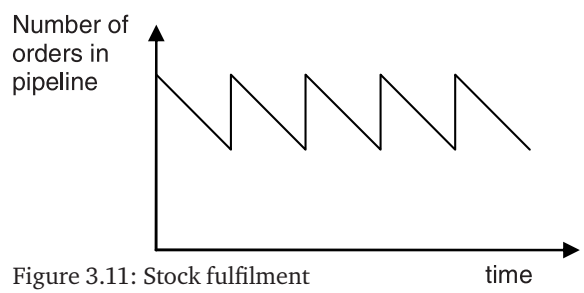

Three batch sizes are analysed - 32, 128 \& 512. If the pipeline, which holds 1024 products, is equivalent to 2 months production plan, then the three batch sizes can be considered to beequivalent to batches of 0.25 week, 1 week and 1 month.

A batch feed does not remove the use of the BTO queue. Any BTO request is stored and given priority when the batch is fed into the pipeline. The lead time for a BTO order, required for scoring the BTO option relative to products in stock and the pipeline, is calculated knowing the time in the cycle and the length of the BTO queue.

From the plots below it is observed batch size changes the metrics little. Only for the largest batch size studied is there perceptible change in some metrics. When the stock level is below 200 there is slight increase in stock fulfilment and consequence reduction in waiting time. Unexpectedly, customer compromise (and variation in compromise) is reduced slightly by the largest batch size.

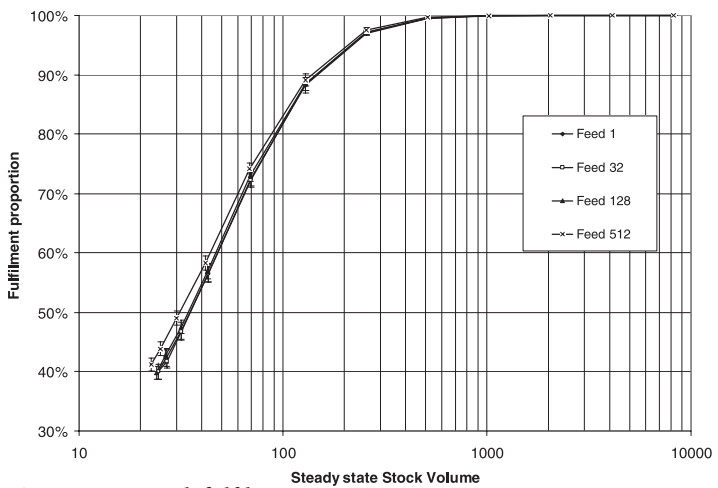

Figure 3.12: Stock fulfilment

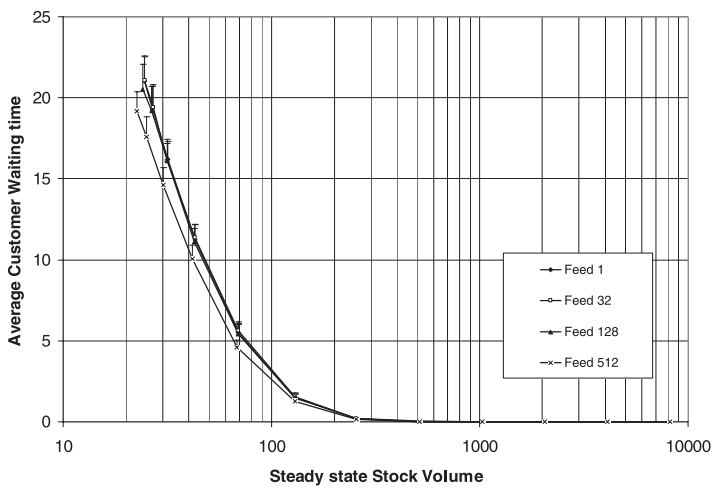

Figure 3.13: Customer Waiting time 


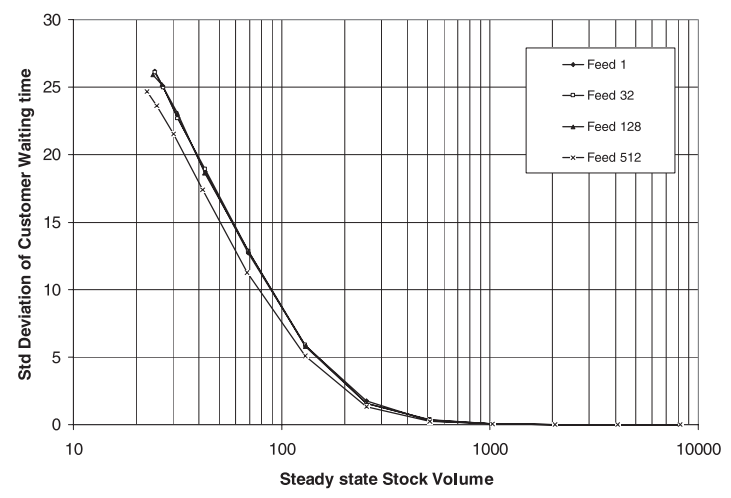

Figure 3.14: Std. Deviation of Customer Waiting time

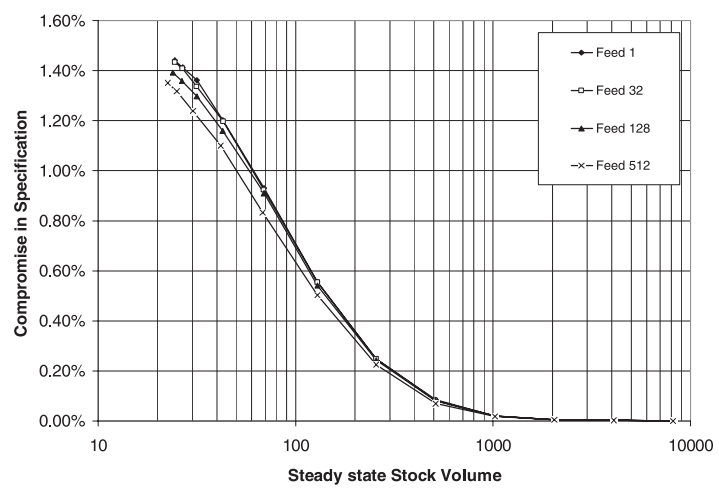

Figure 3.15: Compromise in specification

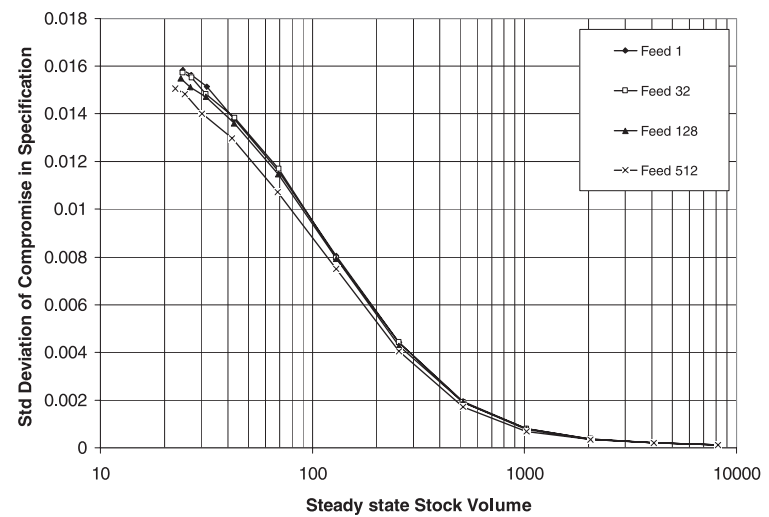

Figure 3.16: Std. Deviation of Compromise in specification

0

0.002

0.004

0.006

0.008 


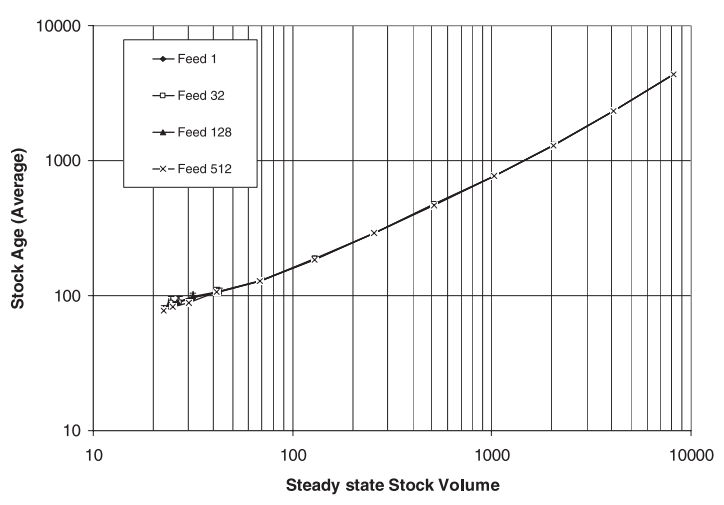

Figure 3.17: Stock age

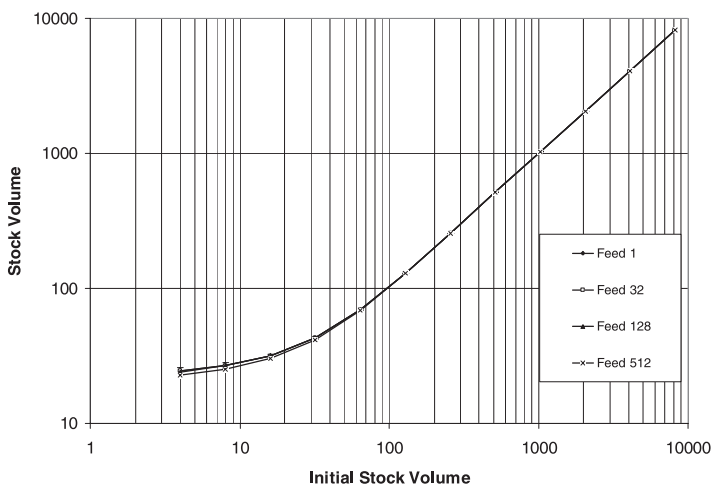

Figure 3.18: Stock volume

\subsection{Mismatch in wholesale and customer demand distributions}

In the above analyses the producer has an accurate model of the customer demand, which follows an 80/20 distribution. The implication of the producer having an inaccurate model of demand is simulated. Two inaccurate distributions are tested:

- 70/30: the producer is aiming to create a mix in the system that matches a $70 / 30$ shape which is a flatter spread than the actual customer demand.

- 90/10: the producer is aiming to create a mix in the system that matches a 90/10 shape which is more peaked than the actual customer demand.

The Random feed method is very sensitive to the change in wholesale distribution. For all metrics it is the method that is most altered. In general, the metrics are more severely affected by the $90 / 10$ skew than 70/30 skew.

The method of following the customer (Method 6) is resilient to the different skews, which is unsurprising since the selection of variant to feed into the pipeline is not dependent on the producer's model of the demand mix distribution.

The performance of the other methods is similar and is summarised in the table below. 
Table 3.1: Interaction of two skew conditions with pipeline feed methods $2,3,4$ \& 5

\begin{tabular}{|c|c|c|c|c|}
\hline \multirow[t]{2}{*}{ Metric } & \multicolumn{2}{|l|}{$70 / 30$} & \multicolumn{2}{|l|}{$90 / 10$} \\
\hline & Change & Comment & Change & Comment \\
\hline Stock fulfilment & $\downarrow$ & $\begin{array}{l}\text { When stock is below } 200 \text { there is } \\
\text { reduction in stock fulfilment }\end{array}$ & $\downarrow \downarrow$ & $\begin{array}{l}\text { The reduction is larger than for } 70 / 30 \\
\text { and is evident for stock below } 2000\end{array}$ \\
\hline Stock volume & $\leftrightarrow$ & No significant change & $\leftrightarrow$ & No significant change \\
\hline Stock age & $\uparrow \uparrow$ & $\begin{array}{l}\text { Across all stock levels the age is } \\
\text { increased. Differences appear between } \\
\text { the methods }\end{array}$ & $\uparrow$ & Small increase in stock age \\
\hline Stock coverage & $\uparrow \uparrow$ & $\begin{array}{l}\text { As expected, a large increase in } \\
\text { coverage, especially at higher stock } \\
\text { levels }\end{array}$ & $\downarrow \downarrow$ & $\begin{array}{l}\text { As expected, a reduction in coverage, } \\
\text { especially at higher stock levels }\end{array}$ \\
\hline Compromise & $\uparrow$ & $\begin{array}{l}\text { Slight increase in compromise when } \\
\text { stock is below } 1000 \text {. Differences } \\
\text { between the methods at low stock } \\
\text { levels }\end{array}$ & $\uparrow \uparrow$ & $\begin{array}{l}\text { Large increase in compromise at all } \\
\text { stock levels. Differences between the } \\
\text { methods except at highest stock level }\end{array}$ \\
\hline $\begin{array}{l}\text { Compromise } \\
\text { (variation) }\end{array}$ & $\uparrow$ & $\begin{array}{l}\text { Slight increase when stock is below } \\
1000\end{array}$ & $\uparrow \uparrow$ & Large increase at all stock levels \\
\hline Waiting time & $\uparrow$ & $\begin{array}{l}\text { Small increase when stock levels } \\
\text { below } 200\end{array}$ & $\uparrow$ & $\begin{array}{l}\text { Small increase when stock levels below } \\
1000\end{array}$ \\
\hline
\end{tabular}

Figure 3.19: Stock fulfilment (upper left Matching feed, upper right 70/30 feed, lower left 90/10 feed)
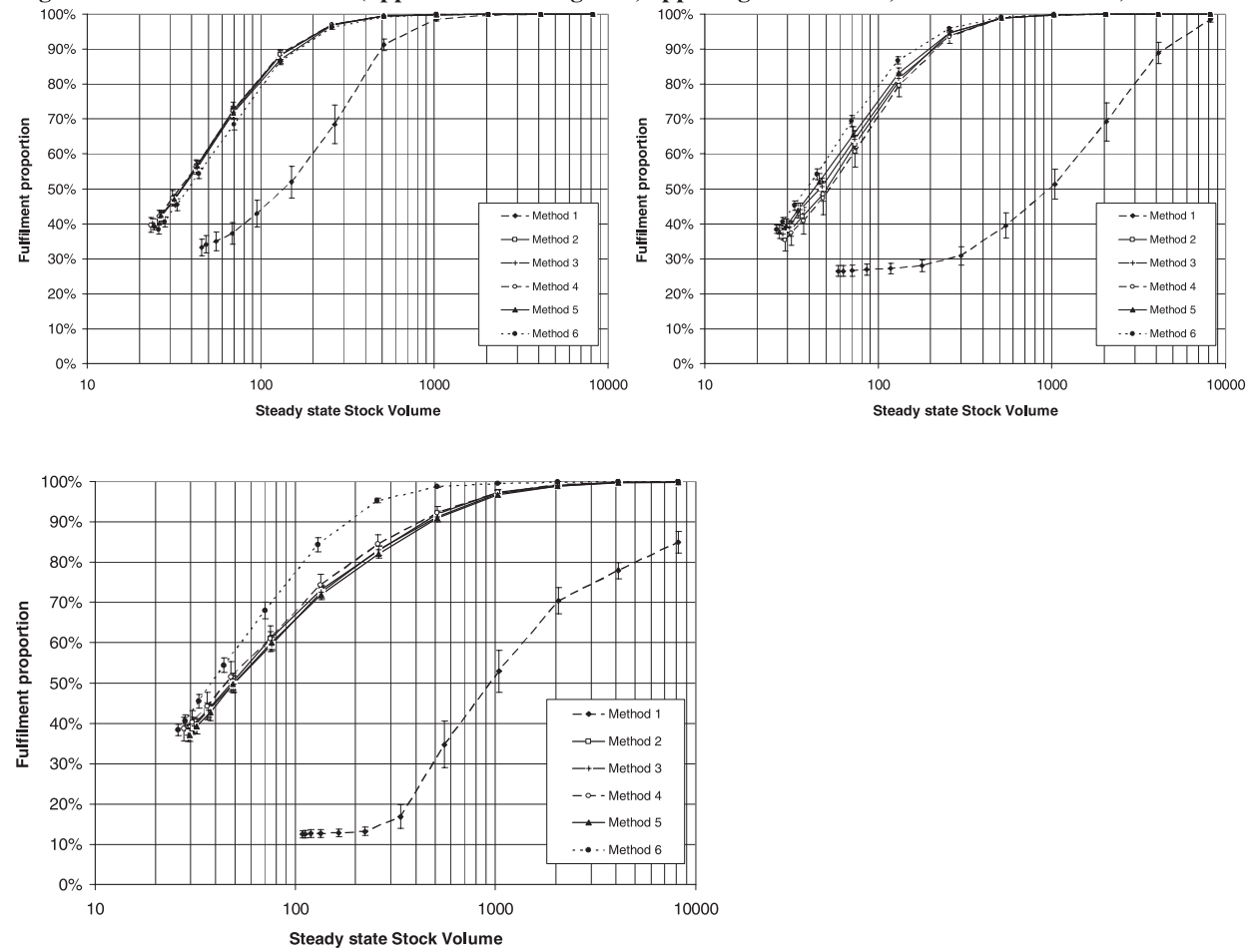

Figure 3.19: Stock fulfilment (upper left Matching feed, upper right 70/30 feed, lower left 90/10 feed) 

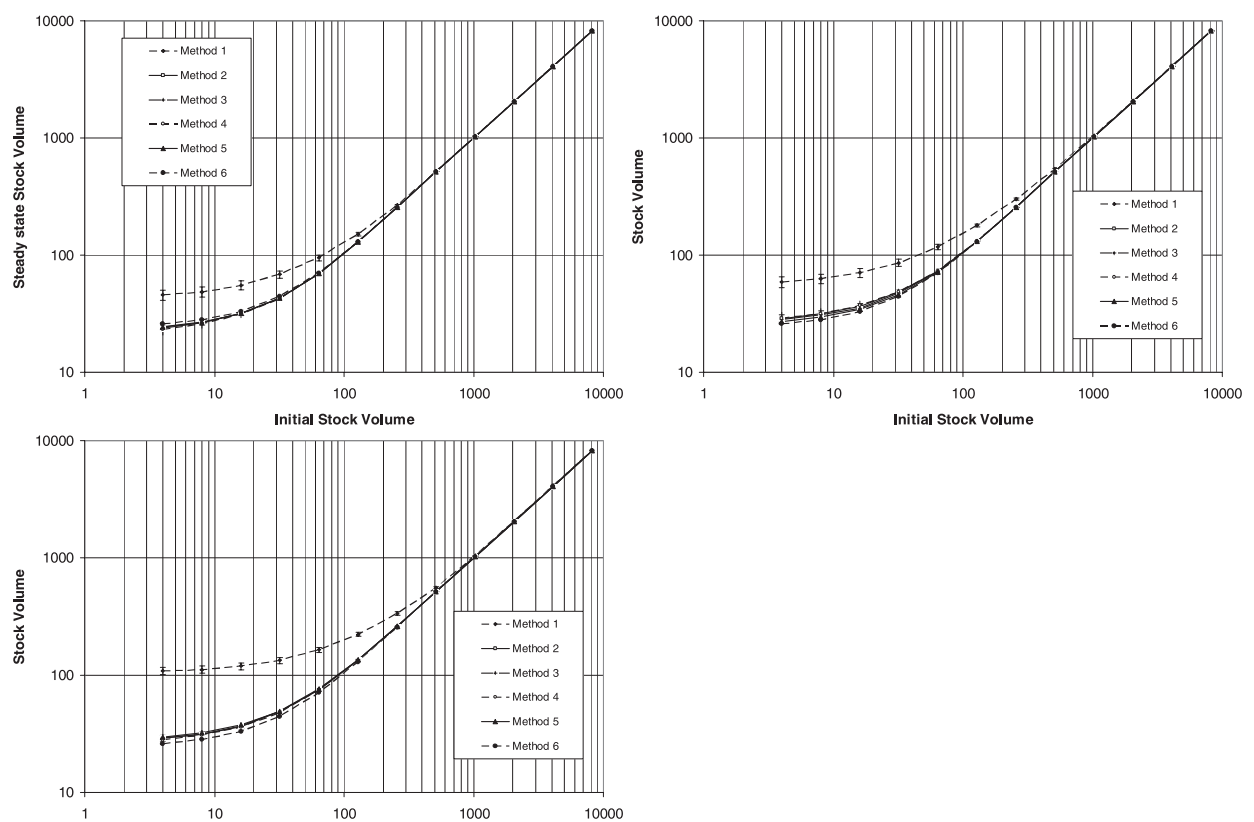

Figure 3.20: Stock Volume (upper left Matching feed, upper right 70/30 feed, lower left 90/10 feed)
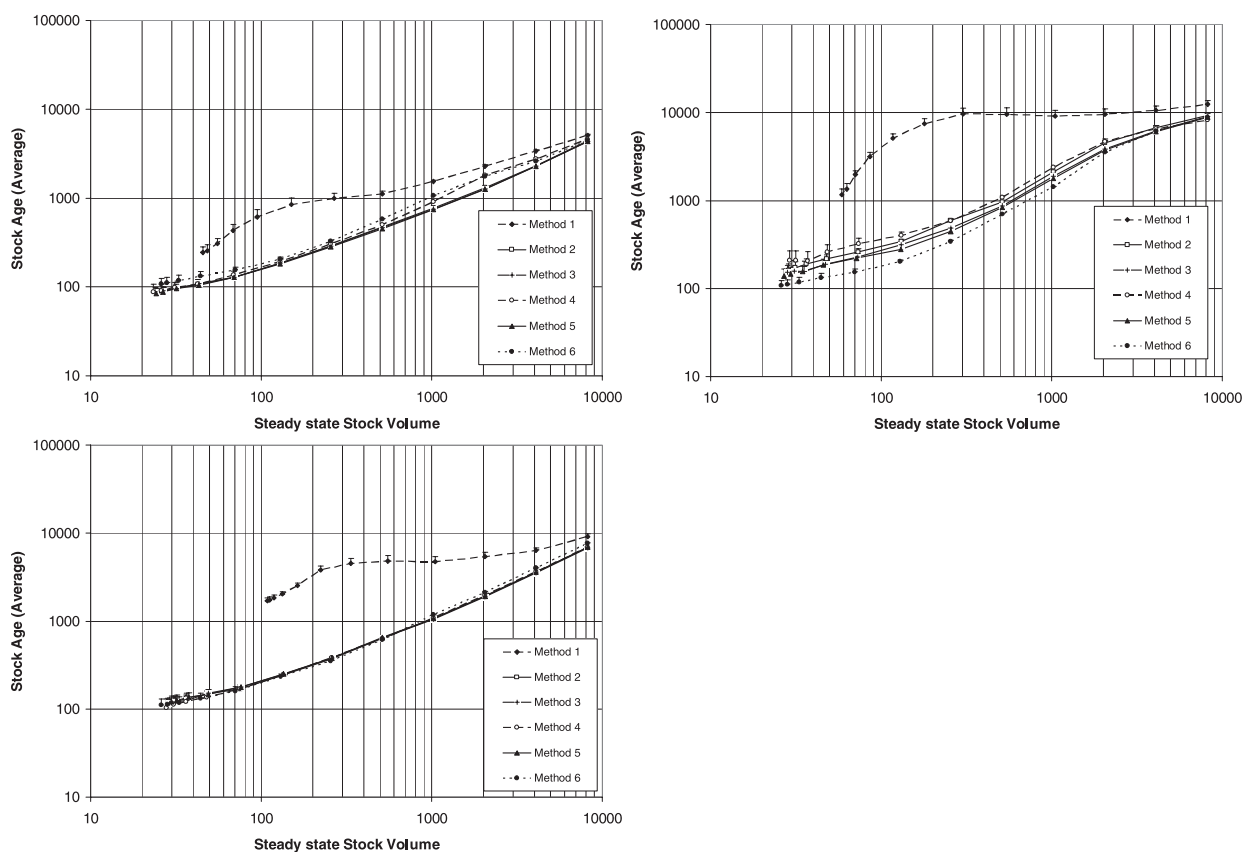

Figure 3.21: Stock age (upper left Matching feed, upper right 70/30 feed, lower left 90/10 feed) 

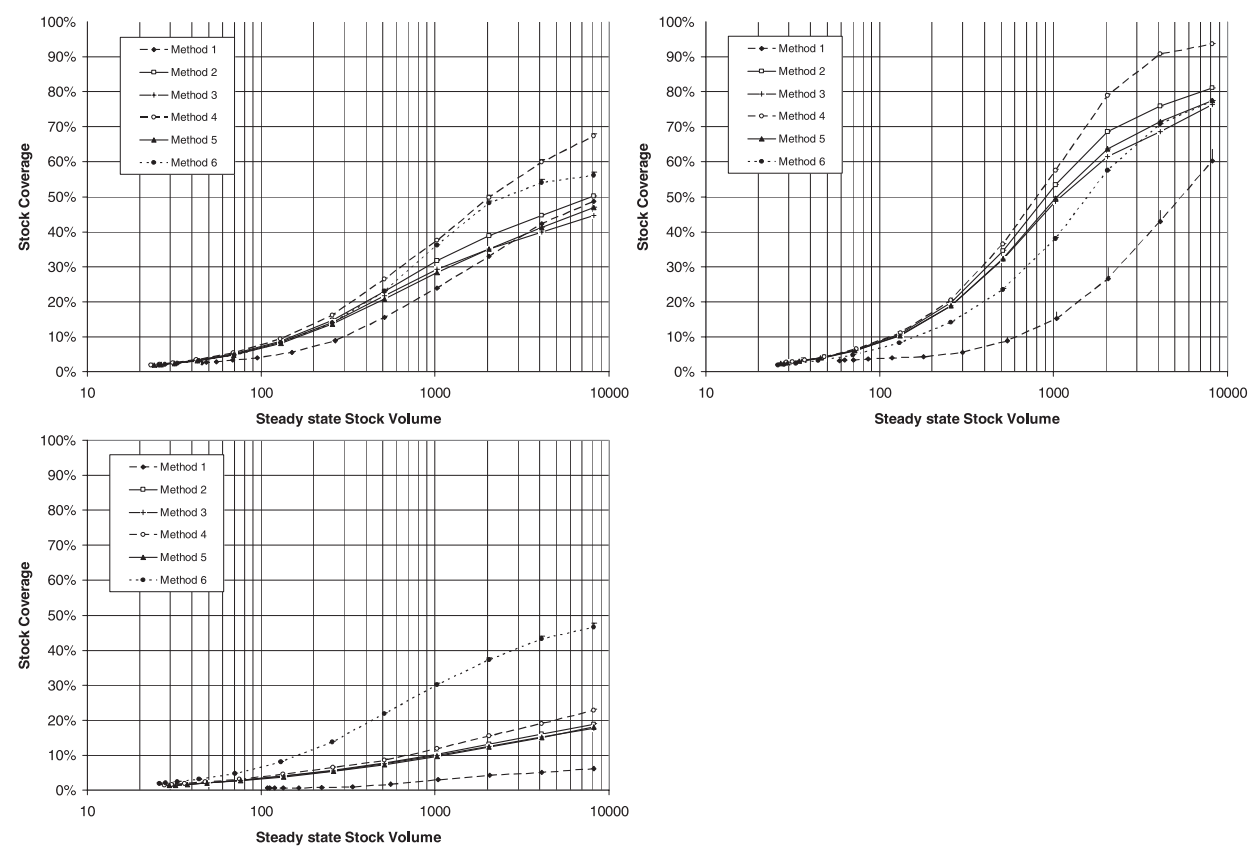

Figure 3.22: Stock coverage (upper left Matching feed, upper right 70/30 feed, lower left 90/10 feed)
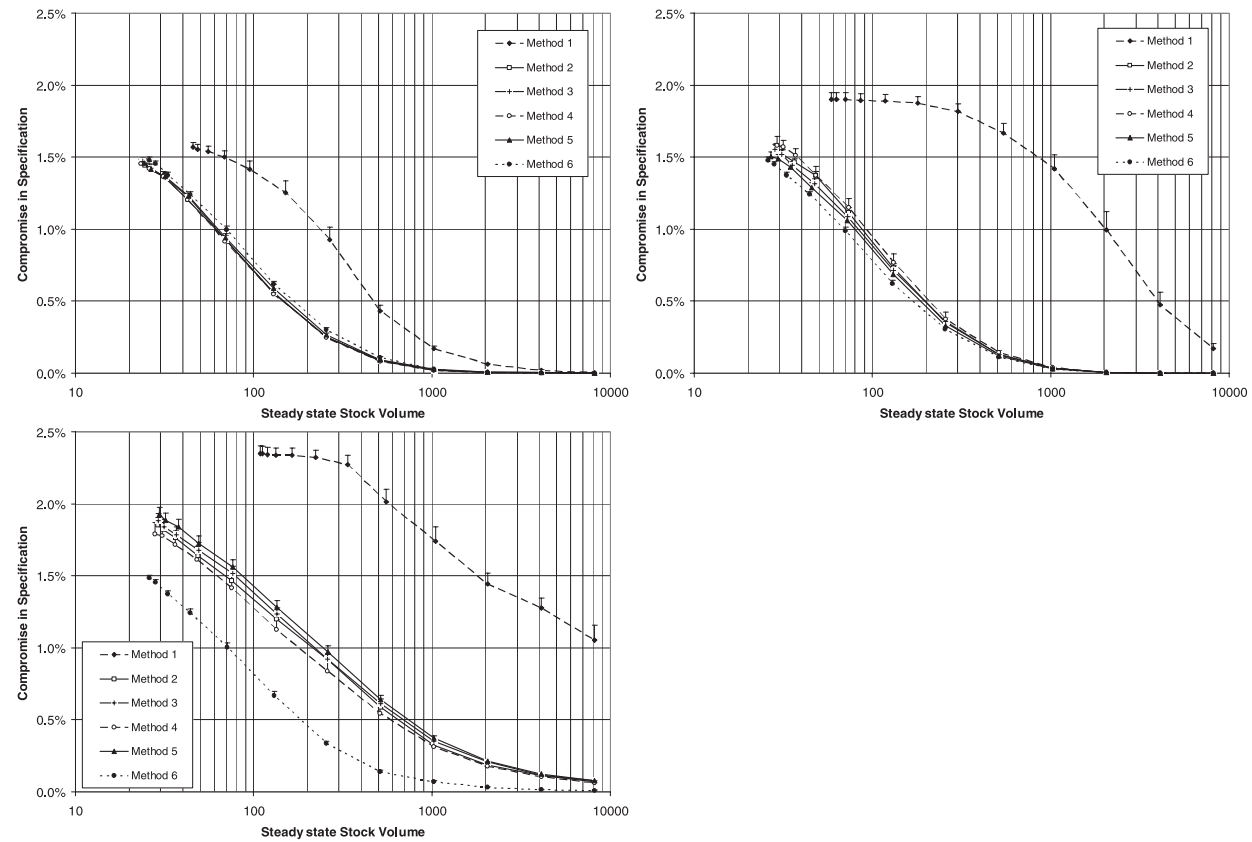

Figure 3.23: Compromise in specification (upper left Matching feed, upper right 70/30 feed, lower left 90/10 feed) 

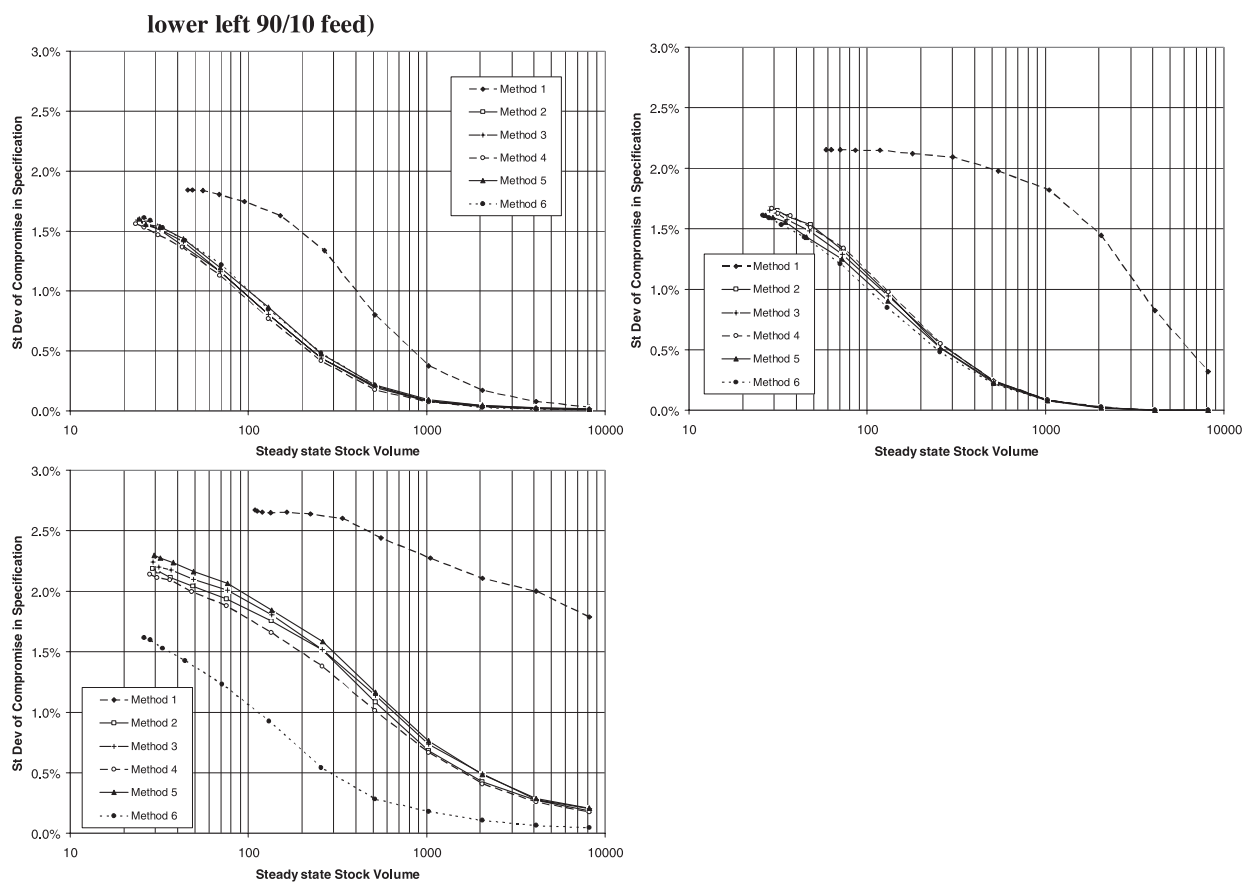

Figure 3.24: Standard deviation of Compromise in specification (upper left Matching feed, upper right 70/30 feed, lower left 90/10 feed)
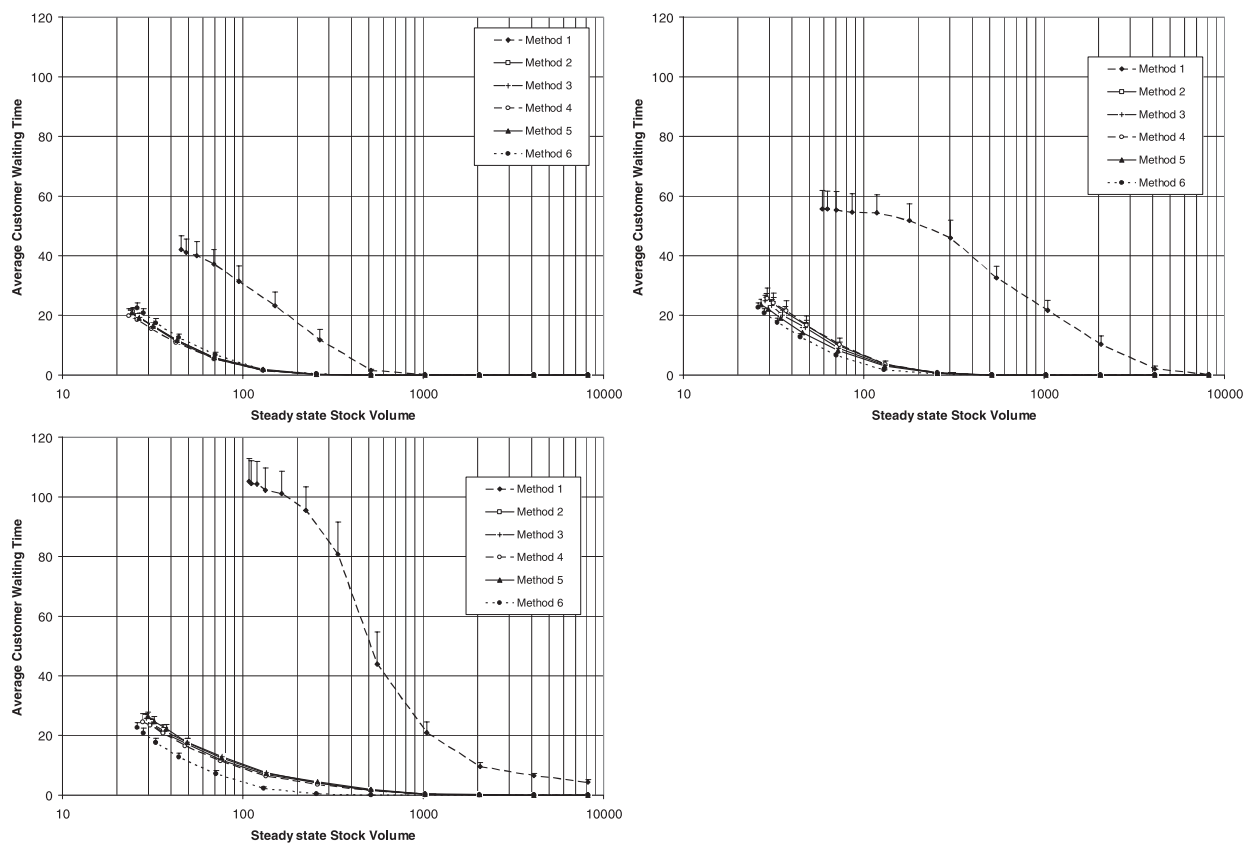

Figure 3.25: Customer Waiting time (upper left Matching feed, upper right 70/30 feed, lower left 90/10 feed) 


\section{Conclusion}

Emerging findings include:

- When the pipeline is closed the customers in the conventional system experience much more compromise (and variation in compromise) than with an open pipeline. The two systems converge as stock levels increase. As a result of more customers being fulfilled from stock when the pipeline is closed, the age of stock is less but the coverage of stock in terms of product variety represented is also less.

- All performance metrics appear to improve as pipeline length is reduced.

- The feed batch size changes the metrics little. Only for the largest batch size studied is there perceptible change in some metrics.

- An error in the producer's perception of the demand function can have a dramatic impact on fulfilment performance. A risk reduction measure is for the producer to follow its customers in regard to what products are fed into the pipeline.

\section{References}

Brabazon, P. G. \& MacCarthy, B. L. (2006). Fundamental behaviour of VirtualBuild-to-Order systems. International Journal of Production Economics, 104(2), 514-524.

Brabazon, P. G., Woodcock, A., MacCarthy, B. L. (2008). Intelligent pipeline control - a simulation study in the automotive sector. Fifteenth International Working Seminar on Production Economics, Congress Innsbruck, Innsbruck, Austria.

Holweg, M. and Pil, F.K. (2004) The second century: reconnecting customer and value chain through Build-to-Order. Cambridge, Mass: MIT Press.v 


\title{
Replanning in an open pipeline fulfilment system - a simulation study in an automotive context
}

\author{
Andrew Woodcock, Philip G. Brabazon, Bart L MacCarthy
}

\begin{abstract}
Automotive producers are adopting multi-modal fulfillment models in which customers can be fulfilled by products from stock, by allocating as yet unmade products that are in the planning pipeline, or by building a product to order. The study reported here considers how fulfillment performance can be improved by replanning the sequence of (virtual) products that are progressing along the pipeline toward manufacture.
\end{abstract}

\section{Keywords}

Order fulfillment, product variety, pipeline, automotive

\section{Introduction}

In the automotive sector it has become common practice for the mass market producers to use several different mechanisms to fulfil customers in their major markets (Holweg and Pil, 2004). A suitable vehicle for a customer can be sought by searching: the stock of finished vehicles, among the vehicles planned for production, or by building a vehicle to order (BTO). The fundamental behaviour of this form of system has been studied by Brabazon \& MacCarthy (2006) and been shown to be different from a conventional system in which the pipeline is closed from view. Methods for sequencing products into the pipeline have been described and compared in Brabazon et al (2008). The study reported here extends the previous research by assessing the impact on system performance of respecifying orders at specific slots in the pipeline.

To study the system a discrete event simulation has been created which models the pipeline as a sequence of products. At each time step of the simulation these products increment one position along the pipeline and one leaving the downstream end. The exiting product goes into stock unless it has been sold already in which case it is removed from the system. Customer arrivals are synchronised with the incrementing pipeline, with one customer served in each time period. Thus, production and customer demand are assumed to be in balance with respect to volume. Every customer is allocated a product, either from stock or the pipeline or by requesting a buildto-order (BTO) product. 


\section{Model description}

A schematic of the order fulfilment system is shown in Figure 1. In this system there are three mechanisms for fulfilling a customer with a product - from finished stock, from allocating a product in the planning pipeline to the customer, or requesting a build-to-order (BTO) product

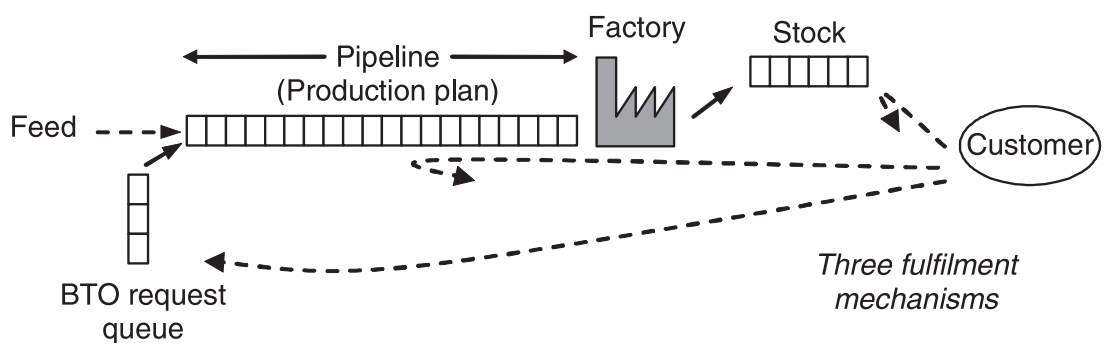

Figure 2.1: Schematic of the order fulfilment model with three fulfilment mechanisms

The challenge for a producer is to configure the system in a way that gives the best fulfilment to customers. The amount of product variety being offered by producers is large, with some passenger vehicles coming in thousands if not millions of feature and option combinations.

In this study, the relative demand for each variant follows an 80/20 distribution, i.e. $20 \%$ of the variants account for $80 \%$ of demand as illustrated in the right plot in Figure 3. This is modelled in the simulation using a Beta distribution with the shape parameters set to 1 and 7.5 .
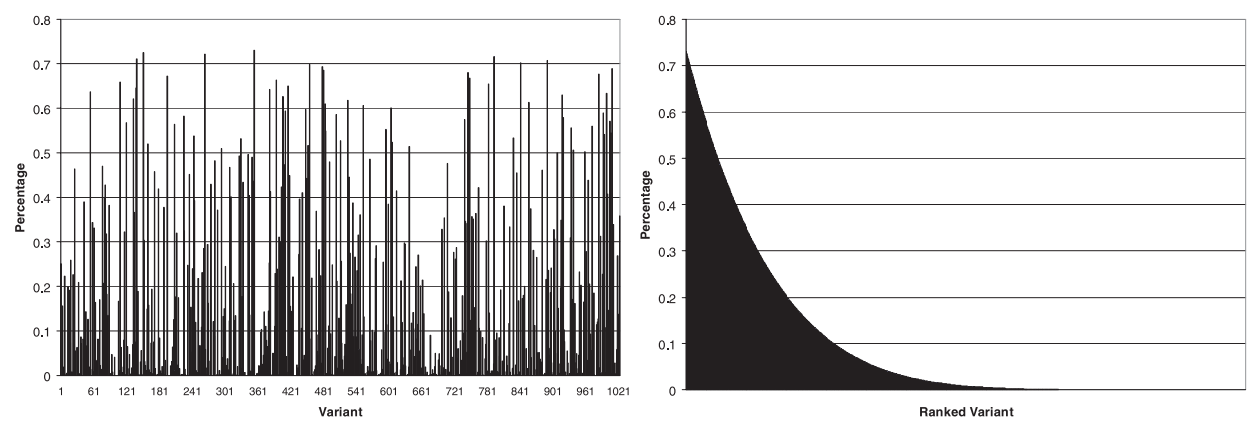

Figure 2.2: Demand for each variant in number order (left plot) and ranked by demand proportion to show the shape of the $80 / 20$ distribution (product range 1024)

Three different levels of variety have been simulated in order to study the effects of replanning at low (128 variants), medium (1024 variants) and high (8192 variants) variety equating to variety/pipeline ratios of $1 / 8,1$ and 8 since the length of the pipeline is fixed at 1024 . Figure 2.2 shows the relative demand for each variant when there are 1024 variants and it is important to note that demand per variant is randomised to avoid a correlation between variant specification and variant 
demand. This is to emulate the real world situation in which the most commonly requested variants from a product range differ greatly.

\subsection{Product selection and replanning strategies}

The producer selects the next product to feed into the pipeline according to one of two policies:

- Feed Method 1: Random feed. The next variant fed into the pipeline is chosen at random from the target distribution.

- Feed Method 2: Reduce stock-out probability. The next variant to be fed into the pipeline is the one that has the highest probability of stocking out. The calculation does not look solely at stock but uses the total number of available products in stock and the pipeline, and therefore the total number of a variant in both the stock and pipeline.

Detailed descriptions of these two feed methods and how they impact on key fulfilment metrics are given in Brabazon et al (2008).

As products fed into the pipeline travel downstream the opportunity to change the specification of an unsold order, based on the current state of the system, is made available at a single pre-determined slot in the pipeline. The decision whether or not to amend an order by changing its specification at this pipeline position is made by considering the stock-out probabilities of each variant downstream of the order and comparing these probabilities with a threshold value. The stock-out probabilities are calculated using the same approach as detailed for feed method 2 with two separate approaches to defining the comparison thresholds as follows:

- Static threshold: A pre-determined stock-out probability threshold value between 0 and 1 is specified at the beginning of each simulation run.

- Dynamic threshold: The mean stock-out probability over all variants downstream of the replanning slot is calculated and used as the threshold value each time respecification of orders is considered.

Replanning is performed by considering the downstream stock-out probability of each variant in the range and then comparing the appropriate stock-out probability values with the threshold value in order to decide whether or not to amend an order. Let the downstream stock-out probability of a variant $i$ be $p_{i}$, then an order at the replanning slot can be respecified from one variant $i$ to another variant $i$ ' if $p_{i}<$ threshold $<p_{i}$ where $i=\arg \max p_{i}$.

\section{Results}

The graphs used to illustrate the effects of replanning on the system have a horizontal axis that represents the position in the pipeline at which orders may be changed. 
A reconfiguration point of 0 should be interpreted as the situation where respecification is not allowed. In all the results that follow customers are fulfilled with an order that exactly matches their requested specification.

\subsection{Using a dynamic threshold}

Introducing pipeline replanning in conjunction with the random feed method 1 results in increasing levels of reconfigured orders as the reconfiguration slot is moved further downstream towards the end of the pipeline as shown in figure 3.1. The level of reconfiguration at a particular slot in the pipeline is higher when variety increases. Contrastingly the level of replanning required reduces with increased variety when the replanning slot is upstream of approximately 660 when using the intelligent feed method 2. Noticeably zero replanning takes place along the entire pipeline at the highest variety level with the intelligent feed method 2.

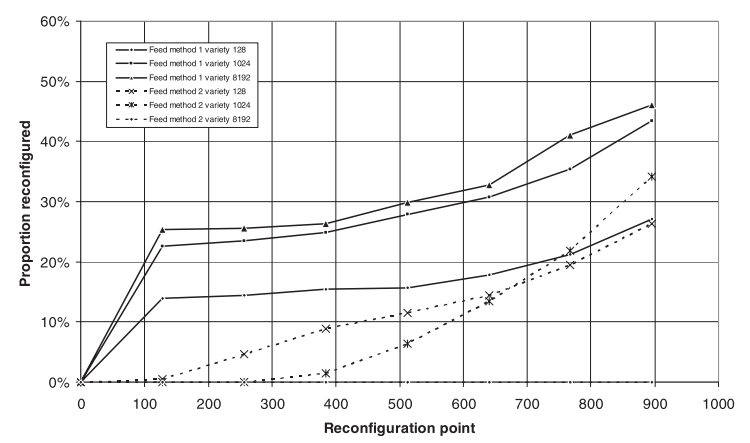

Figure 3.1: Amount of reconfiguration for feed methods 1 and 2 using a dynamic reconfiguration threshold

Figure 3.2 illustrates how increases in stock fulfilment are achievable by implementing reconfiguration with a dynamic threshold. By comparison to the situation where replanning is not allowed increased stock levels occur at all three levels of variety and across the length of the pipeline if random pipeline feed is in operation although increases are greater in the downstream half of the pipeline at low and medium variety. If the intelligent pipeline feed is in operation then clearly there will be no change in stock fulfilment at high variety since replanning does not occur under these circumstances but some increases are experienced at medium and low variety when the reconfiguration point is in the downstream half of the pipeline. Placing the reconfiguration point further towards the downstream end of the pipeline produces higher levels of stock fulfilment at medium and high variety using random feed compared to the intelligent feed method 2 .

Increases in stock fulfilment produced by reconfiguration appear to be largely countered by decreases in pipe fulfilment resulting in little or no change in the BTO fulfilment using feed method 2. The BTO plot in figure 3.2 also shows how the dynamic threshold approach to replanning reduces the level of BTO orders when the 
random feed method is in use but the reductions are not dependant upon where in the pipeline the reconfiguration point is placed except at low variety where there is slight advantage to be gained by having the reconfiguration point towards the upstream end of the pipeline.

The use of a dynamic reconfiguration threshold in conjunction with a single reconfiguration slot in the pipeline has the effect of correcting the system such that it subsequently performs as if the pipeline were being intelligently fed. This effect can be seen from the plots in figure 3.3 which shows how three key performance indicators are affected by the introduction of this replanning approach.
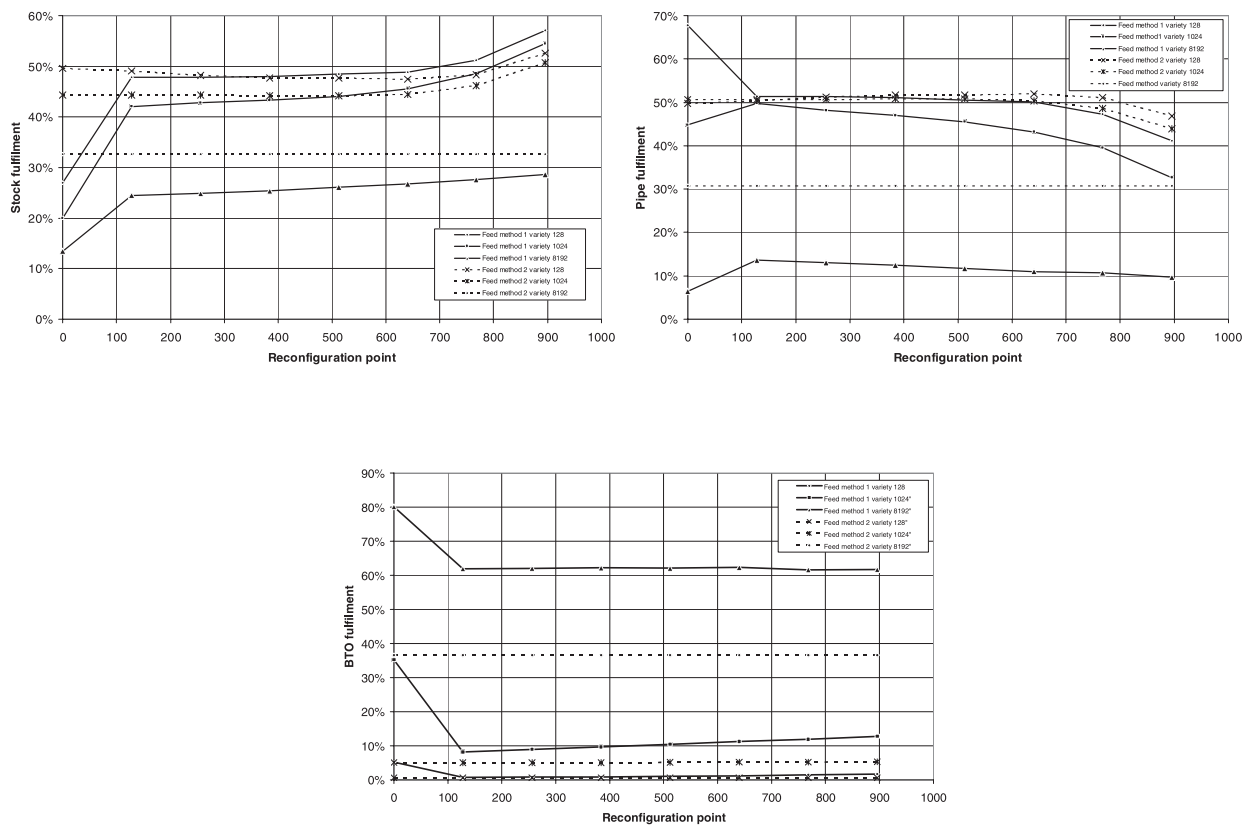

Figure 3.2: Fulfilment proportions for stock (top left), pipe (top right) and BTO (bottom) using the dynamic threshold 

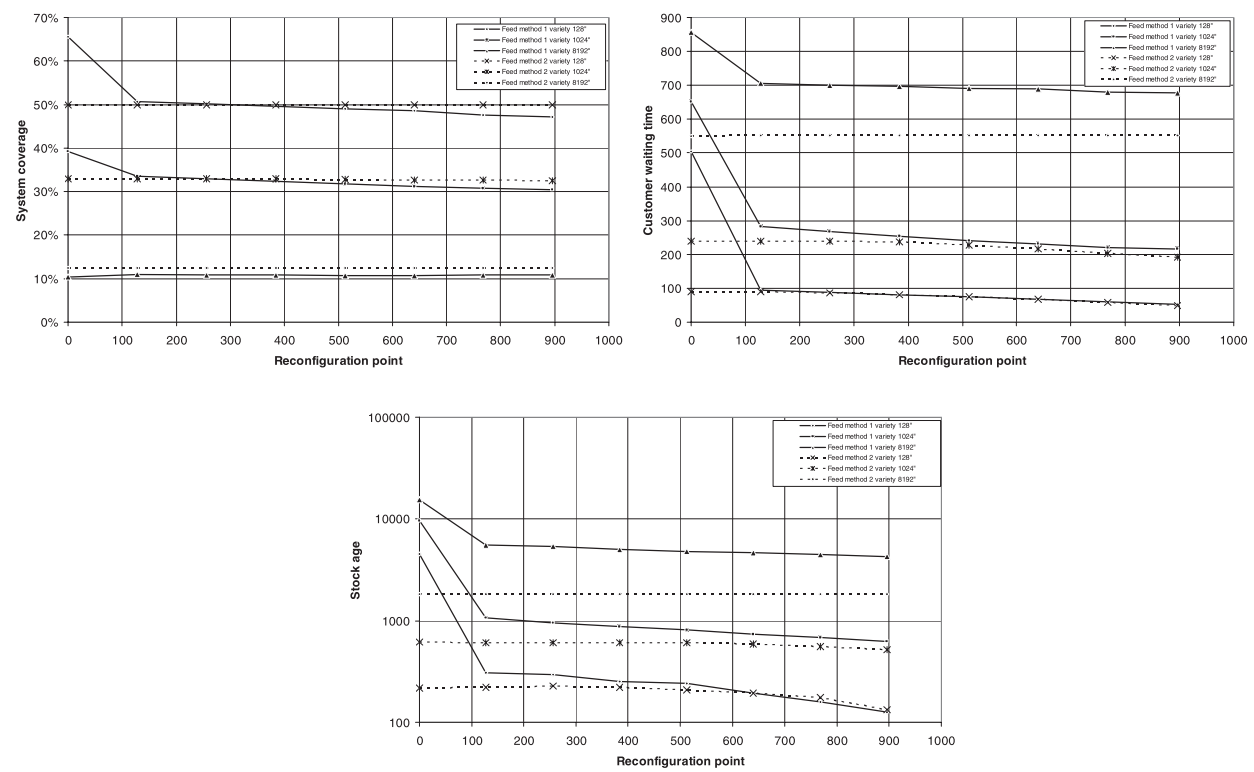

Figure 3.3: Impact on performance when replanning using a dynamic reconfiguration threshold

\subsection{Static reconfiguration threshold approach}

Variations in system responses using the static approach are dependant upon both thereconfiguration threshold set and the position of the reconfiguration slot. Since an order at the reconfiguration slot can only be reconfigured if the variant specified by the order has a stock out probability below the threshold and an alternative variant has a stock out probability above the threshold then setting the threshold to the values 0 or 1 would reduce the system to one where no reconfiguration is allowed. The results that follow have been obtained by setting values of the threshold in the range [0.1,0.9].

\subsubsection{Random pipeline feed}

Figure 3.4 gives an indication of the amount and location in 3-dimensional space of reconfiguration when it is dependant on both position of the reconfiguration point and the reconfiguration threshold when the pipeline is being fed with random variants drawn from the target distribution.. Interpretation of the plot is straightforward when considered as the surface of the 3-dimensional landscape. At low variety a threshold of 0.5 yields the highest proportion of reconfigurations when the reconfiguration point is placed between position 384 and 640 in the pipeline, which would result in between $80 \%$ and $90 \%$ of orders being reconfigured. The proportion of reconfiguration that takes place reduces as the threshold is moved away from 0.5 in either direction.

At medium variety the level of reconfiguration peaks at between $80 \%$ and $90 \%$ if the reconfiguration point is in the upstream half of the pipeline with a threshold 
of 0.5 and drops away as the threshold is moved away from 0.5 at a similar rate in either direction. If the reconfiguration point is placed in the upstream half of the pipeline the landscape changes to one where there is a peak of reconfiguration at a threshold of 0.3 at position 768 in the pipeline where over $90 \%$ of orders are reconfigured. Decreasing the threshold from 0.3 produces greater reductions in the level of reconfiguration than would result from increasing the threshold by the same amount.

At high variety this reconfiguration approach produces no reconfiguration in the downstream half of the pipeline when the threshold is set at 0.5 or above and no reconfiguration takes place at any point along the pipeline if the threshold is set to 0.7 or above. In relation to the 3-dimensional space the reconfiguration is much more concentrated when the level of variety is high, peaking at a threshold of 0.3 in the upstream half of the pipeline between positions 128 and 384 and at a threshold of 0.1 in the downstream half of the pipeline at position 768 .

At low variety increases in stock fulfilment are observable for all threshold values, compared to the no reconfiguration situation, although lower threshold values $(<=0.5)$ produce the largest increases in stock fulfilment. Varying the position of the reconfiguration point, as shown in figure 3.5 , between 128 and 640 causes little or no change in stock fulfilment at any given threshold value. Positioning the reconfiguration point downstream of position 640 produces increased levels of stock fulfilment as the reconfiguration point approaches the end of the pipeline with threshold values $<=0.3$ yielding most stock fulfilment. 

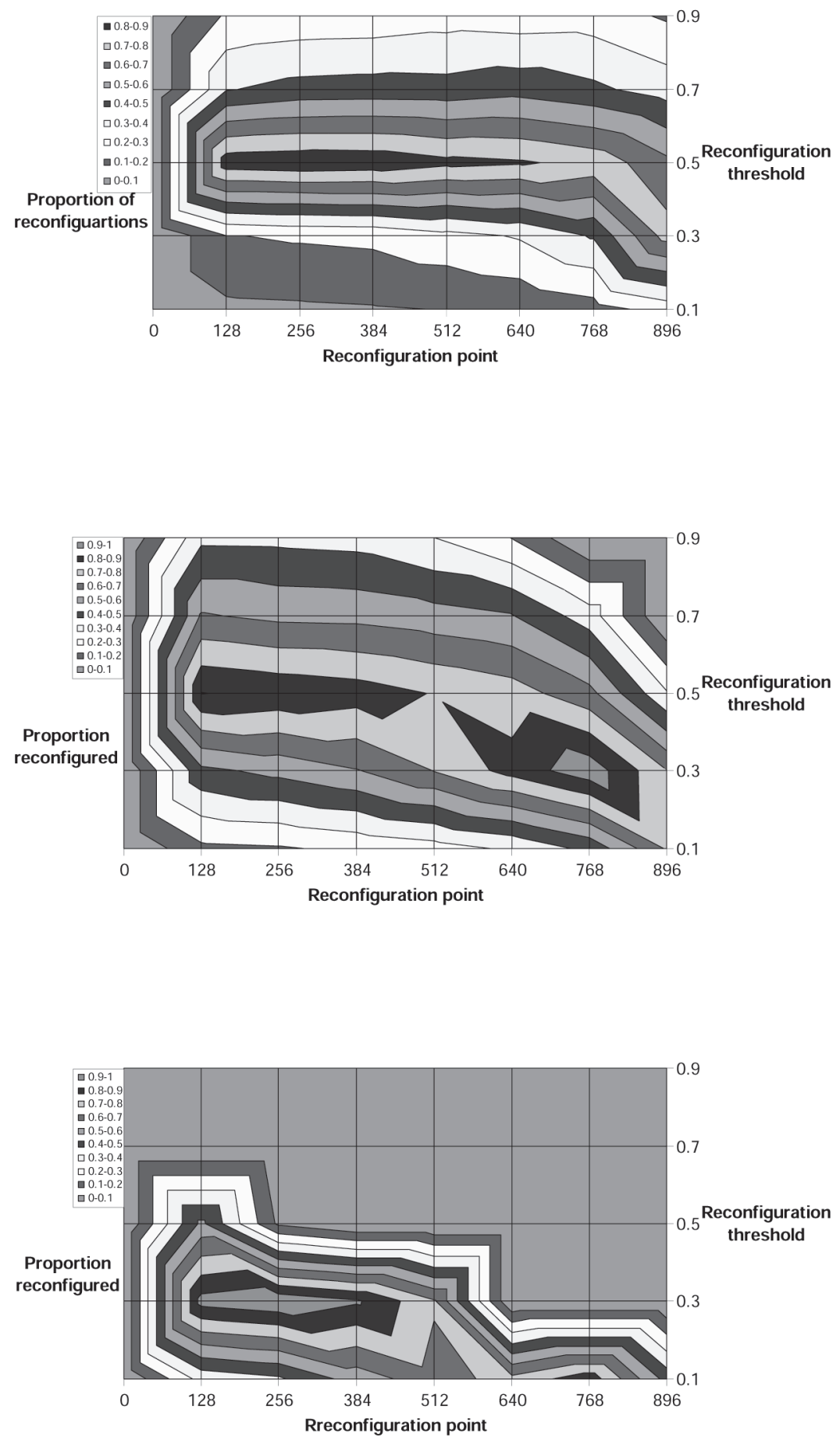

Figure 3.4: Reconfiguration landscapes using static threshold values for variety 128 (top), 1024 (middle) and 8192 (bottom) 

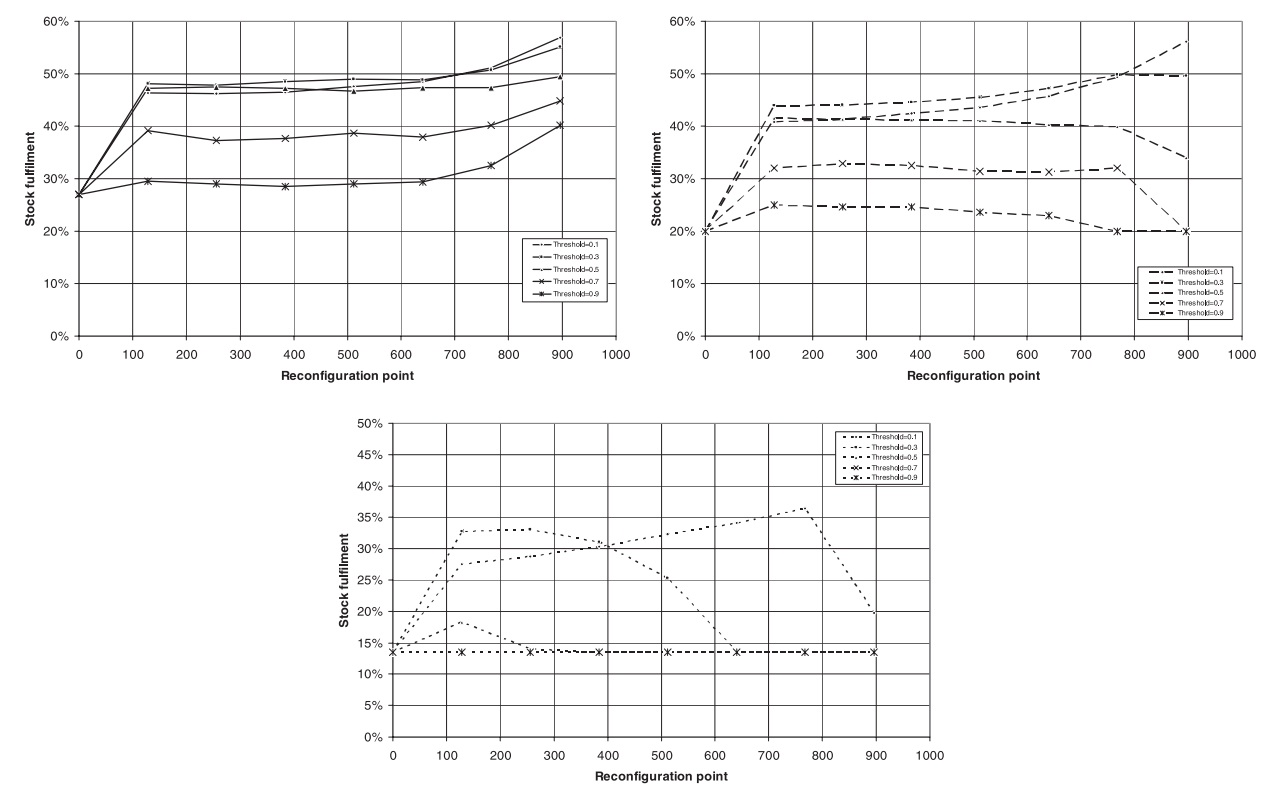

Figure 3.5: Effects of replanning on stock fulfilment at low variety (top left), medium variety (top right) and high variety (bottom)

A noticeable difference is observable in the downstream half of the pipeline at medium variety where, for threshold values $>=0.5$, a reduction in the level of stock fulfilment occurs, compared to the level of stock fulfilment levels in the upstream half of the pipeline, as the reconfiguration slot is moved closer to the end of the pipeline. For threshold values $<=0.3$ increased levels ofstock fulfilment are achieved as the reconfiguration slot is moved along the pipeline from position 128 to 768 . Reducing the threshold to 0.1 beyond position 768 results in further increases.

At high variety noticeable increases in stock fulfilment only occur for threshold values $<=0.3$ in the upstream half of the pipeline and $<=0.1$ in the downstream half of the pipeline.

Figure 3.6 shows how at low variety decreases in the level of pipe fulfilment tend to correspond with increases in stock fulfilment for threshold values $<=0.7$ as the reconfiguration point is moved downstream towards the end of the pipeline. At the 0.9 threshold small increases in pipe fulfilment are evident in the upstream half of the pipeline. At medium and high variety increased levels of pipe fulfilment occur if replanning is allowed in the upstream half of the pipeline at most threshold values with the highest levels of pipeline fulfilment occurring when the reconfiguration point is placed furthest upstream in the pipe.

These changes in fulfilment from both stock and pipeline result in reductions in the level of BTO fulfilment at all three levels of variety. These reductions increase substantially with higher variety as shown in figure 3.7 

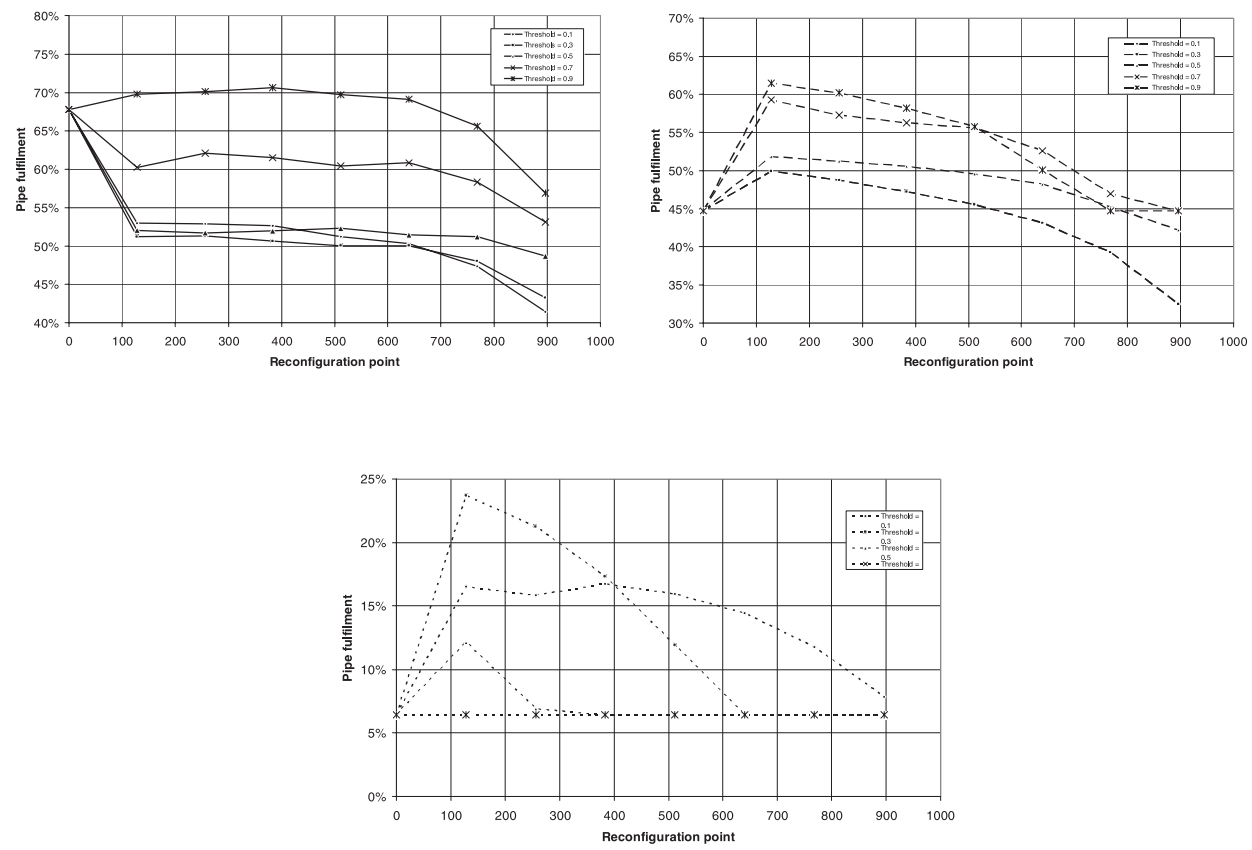

Figure 3.6: Effects of replanning on pipe fulfilment at low variety (top left), medium variety (top right) and high variety (bottom)
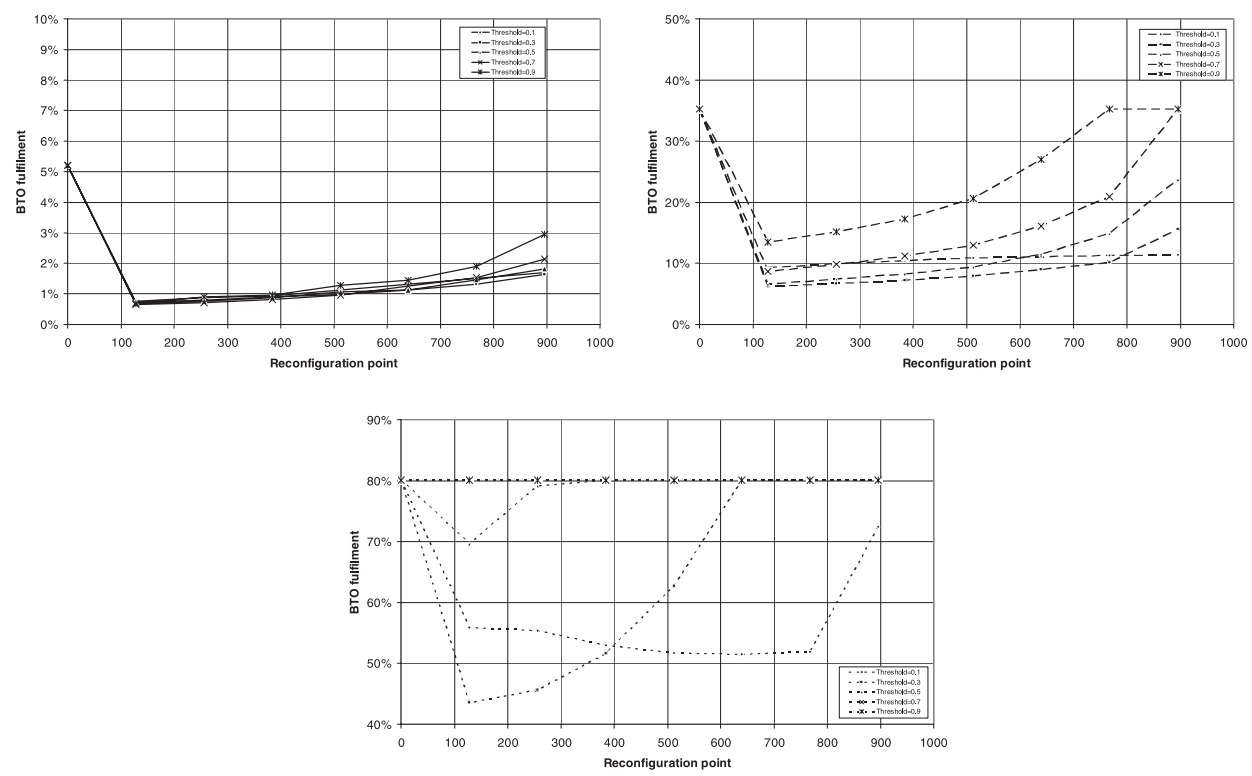

Figure 3.7: Effects of replanning on BTO fulfilment at low variety (top left), medium variety (top right) and high variety (bottom) 
Changes in key metrics such as stock volume and customer waiting time tend to coincide with reductions in BTO at medium and high variety but differ slightly at the lowest level of variety as shown in figure 3.8 where the initial decrease obtained by the introduction of reconfiguration at point 128 is evident but can be further reduced as the reconfiguration point is moved downstream, with the exception of a slight increase towards the end of the pipeline when the threshold is set $>=0.7$.

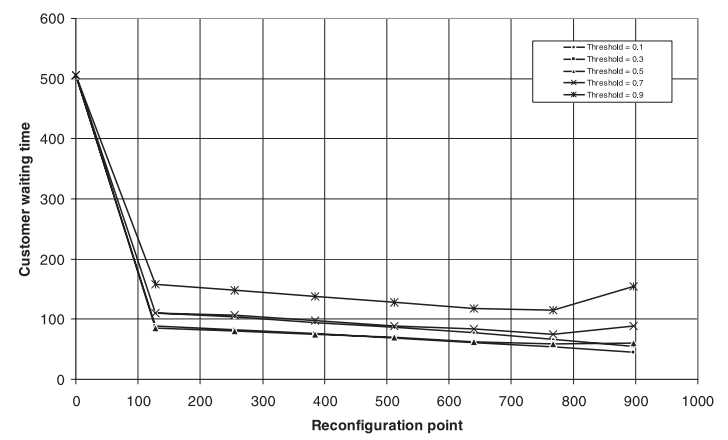

Figure 3.7: Effects of replanning on customer waiting times when using the random feed process

\subsubsection{Intelligent pipeline feed}

The pattern of reconfiguration for feed method 2 is similar to that of feed method 1 at all three levels of variety as seen in figure 3.8 although changes in the proportion of orders reconfigured appear to be slightly more sensitive to changes in the value of the threshold and generally less reconfiguration occurs at the extremities of the threshold range i.e. 0.1 and 0.9 . The peaks in proportions of reconfiguration tend to occur at similar points in terms of threshold value and reconfiguration point and at similar levels. The impact of reconfiguration when using feed method 2, compared to feed method 1, on the fulfilment mechanisms and key metrics is much less however.

At low variety reconfiguration causes reduced stock fulfilment except when the reconfiguration point is placed close to the end of the pipeline and the threshold is set to be less than 0.5. As variety increases little impact on stock fulfilment is experienced when the reconfiguration point is positioned in the upstream half of the pipeline but moving the reconfiguration point towards the end of the pipeline in the downstream half produces noticeable increases in stock fulfilment. At the highest level of variety small increases in stock fulfilment corresponding with increased proportions of reconfiguration are apparent as can be seen in figure 3.9. Decreases in pipe fulfilment again correspond with increases in the level of stock fulfilment although increased levels of BTO fulfilment can be experienced particularly with increased levels of variety as shown in figure 3.10.

Figure 3.11 shows that reconfiguration may be beneficial with regard to key metrics such as average stock age and customer waiting times by moving the reconfiguration point close to the downstream end of the pipeline although at high variety 
small increases in customer waiting times can be experienced by reconfiguring in the middle section of the pipeline if the threshold is set too low. In fact reconfiguration with this feed method has no advantage in terms of reducing customer waiting times.
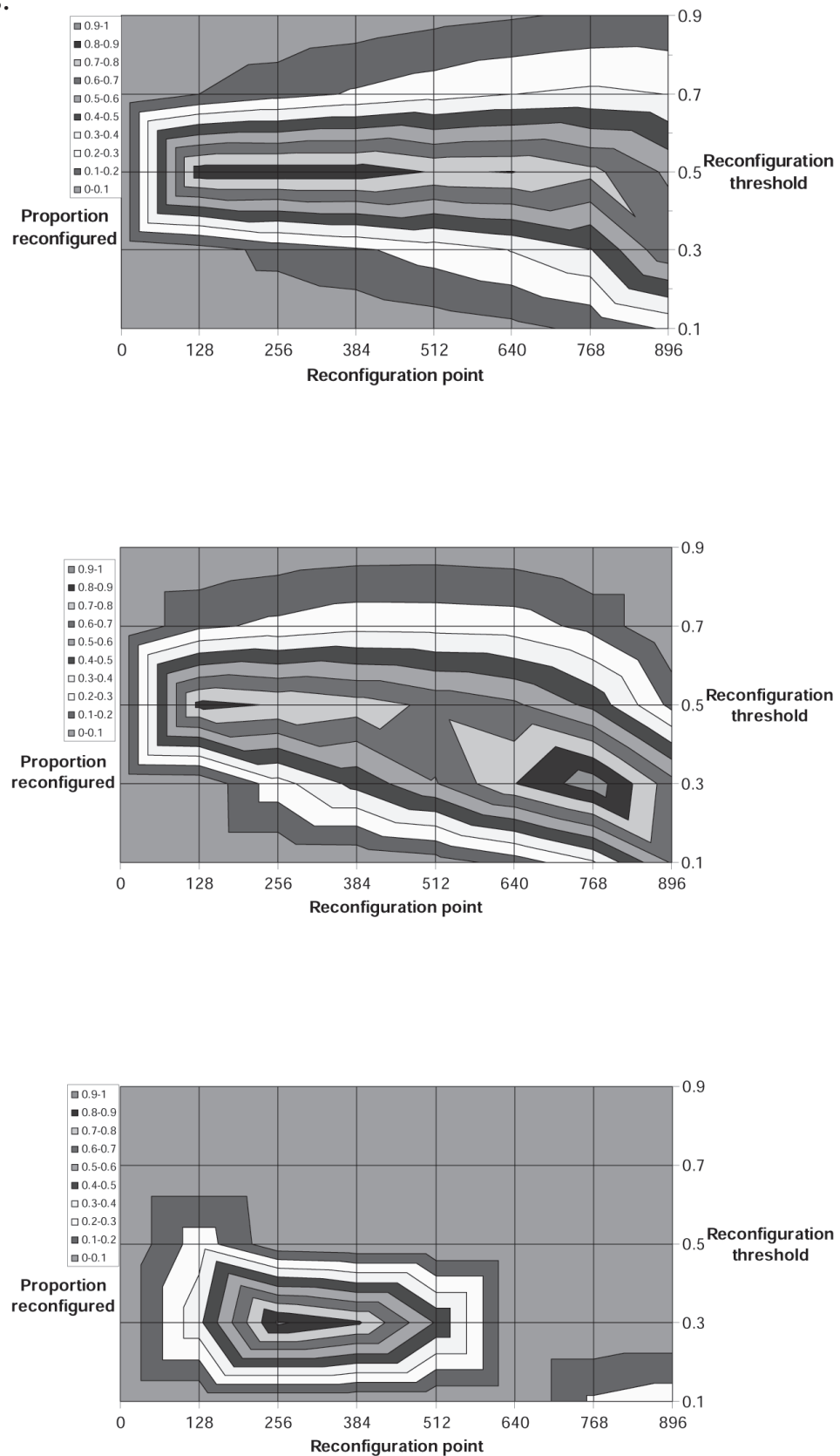

Figure 3.8: Reconfiguration landscapes using static threshold values for variety 128 (top), 1024 (middle) and 8192 (bottom) 

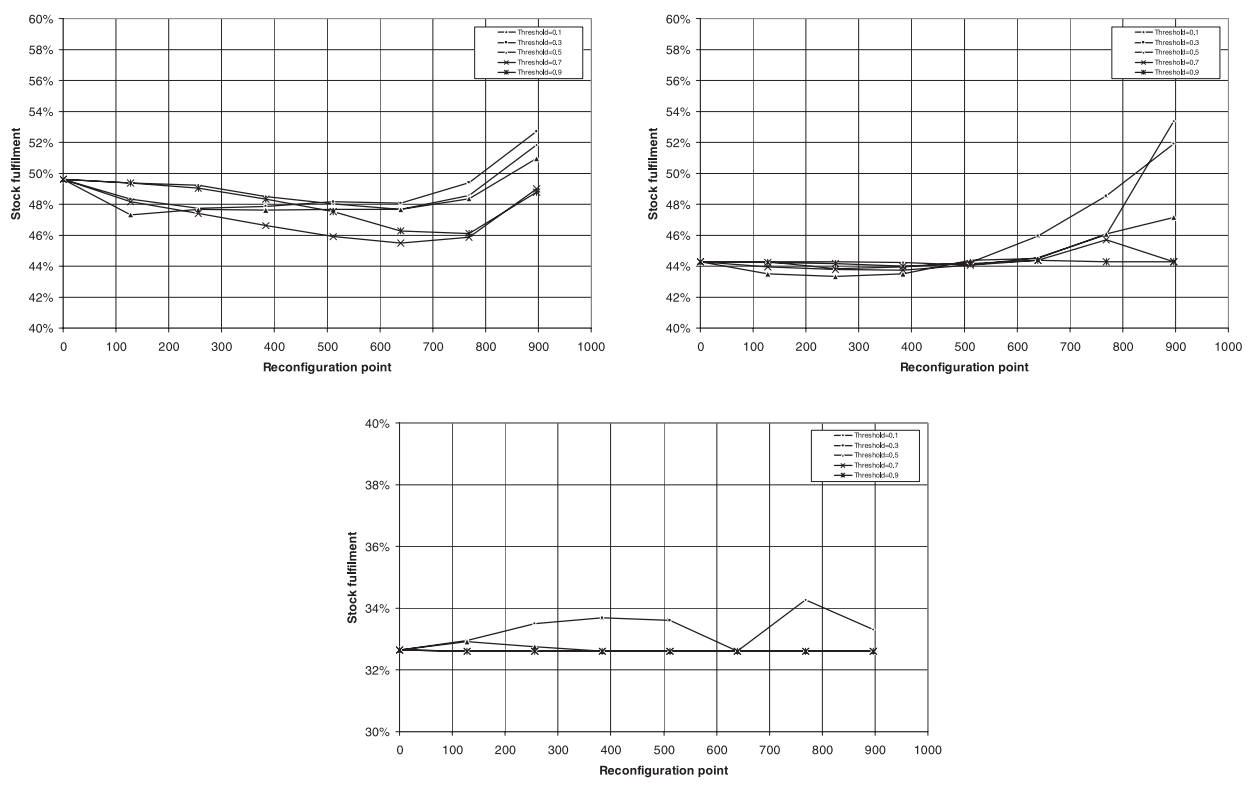

Figure 3.9: Effects of replanning on stock fulfilment at low variety (top left), medium variety (top right) and high variety (bottom)
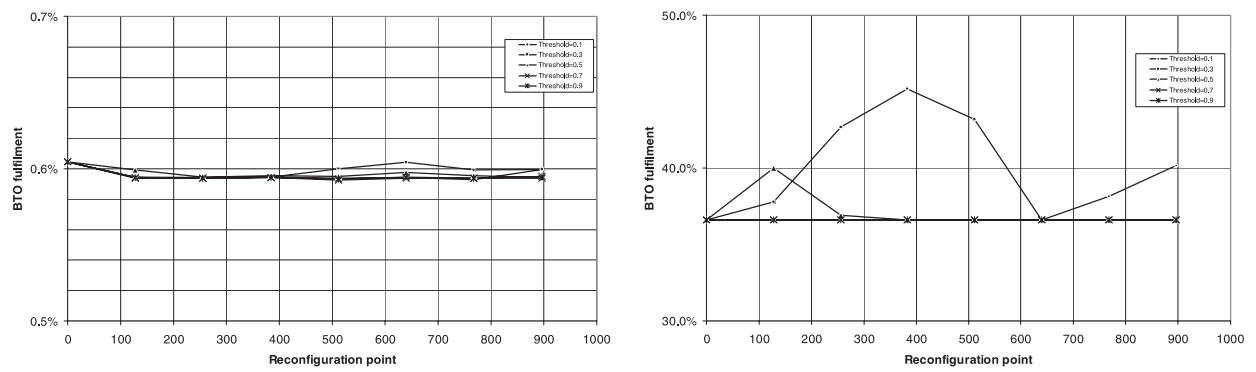

Figure 3.10: Effects of replanning on stock fulfilment at low variety (left) and high variety (right)
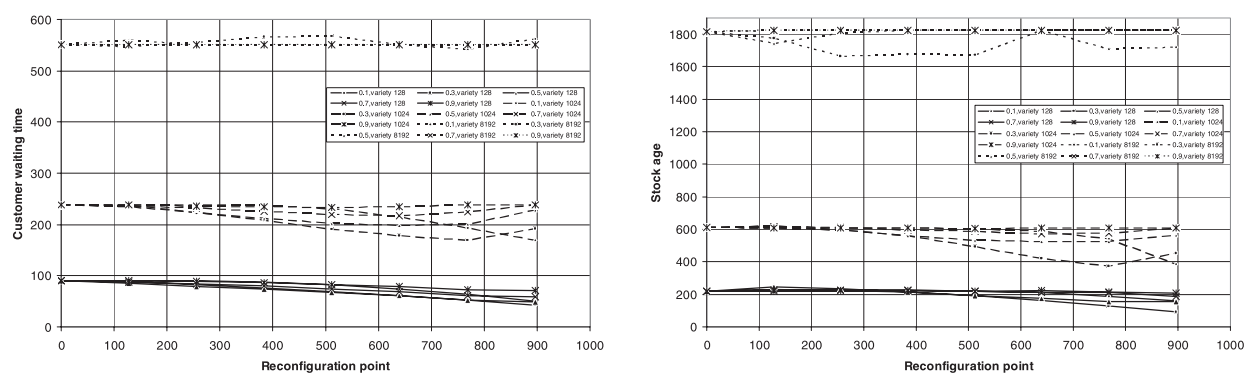

Figure 3.11: Effect on key metrics using the intelligent feed method 


\subsection{Impact on allocation of orders in the pipeline}

The results presented in section 3.2 shows that high levels of respecifying of orders tend to increase stock fulfilment whilst reducing fulfilment from the pipeline. The following results show how the distribution of allocations from the pipeline change as the reconfiguration slot is moved upstream in the pipeline. The following results were obtained with a pipe length of 1024 and a variety/pipeline ratio of 1 from the system at steady state during a processing period of 10000 customers.

Figure 3.12 gives the distribution of pipeline allocations obtained when a random feed method is used without pipeline replanning, allocations of orders in the pipeline occur with greater frequency at the upstream end of the pipe whereas an intelligent feed process can reduce the stripping effect at the upstream end of the pipe with more orders being allocated at the downstream end of the pipeline.
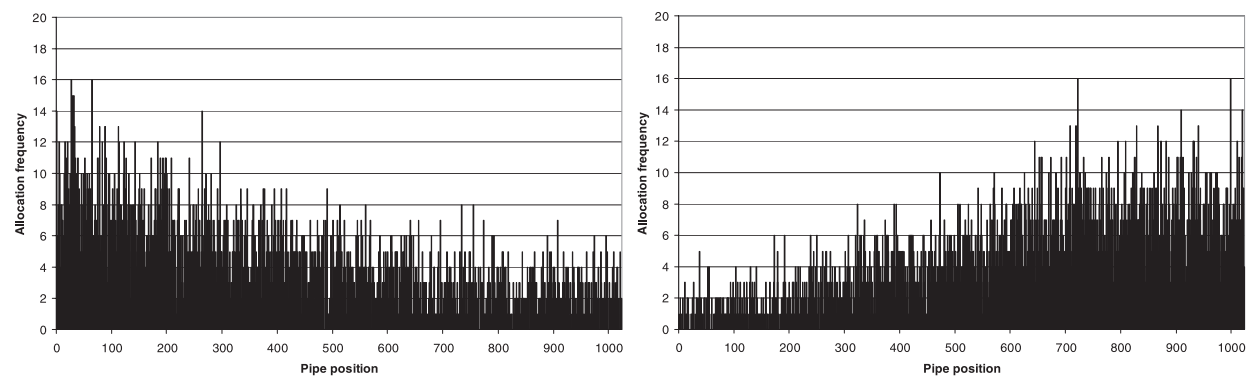

Figure 3.12: Allocation frequencies of pipeline orders using the random feed method (left) and the intelligent feed method(right)

The introduction of a reconfiguration point at the upstream end of the pipeline (position 128) has a noticeable effect on pipeline allocation when the random wholesale process is used by introducing an intelligent aspect to order specification at the upstream end of the pipeline (see figure 3.13).

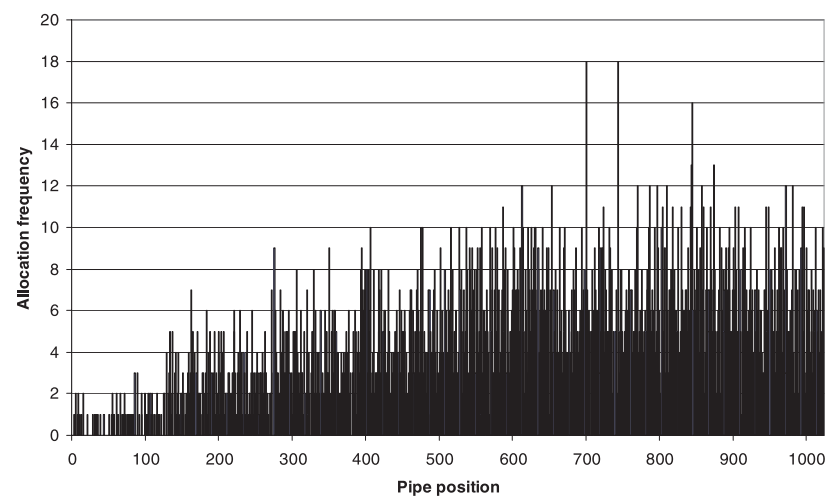

Figure 3.13: Allocation frequencies of pipeline orders using the random feed method with reconfiguration at position 128 
The distributions presented in figure 3.14 show that moving the reconfiguration point further downstream in the pipeline to position 512 shows how most pipeline allocations now occur downstream of the reconfiguration point with much less pipeline allocation taking place upstream of the reconfiguration point. Fewer orders are now allocated upstream of the reconfiguration point when using a random feed process compared to the intelligent feed method.

method.
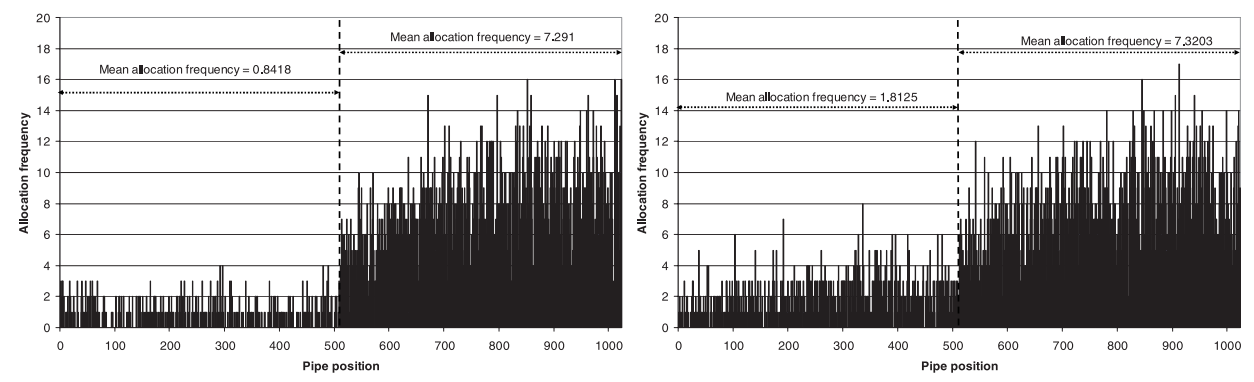

Figure 3.14: Allocation frequencies of pipeline orders using the random feed method (left) and intelligent feed method (right) with reconfiguration at position 512

Figure 3.15 shows how the distribution of allocations changes as the reconfiguration point is moved further downstream towards the end of the pipeline (position 896). The distribution of pipeline allocations becomes even more highly skewed under these circumstances. In this situation 7/8ths of the pipeline has very low frequency of allocations contributing towards the reduction in pipe fulfilment and corresponding increase in pipe availability under these circumstances.
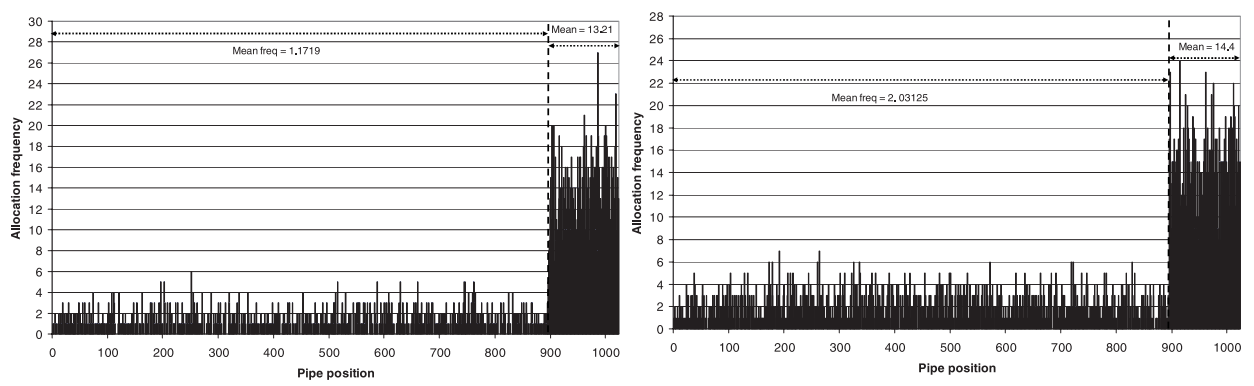

Figure 3.15: Allocation frequencies of pipeline orders using the random feed method (left) and intelligent feed method (right) with reconfiguration at position 896

\section{Conclusions}

In general the introduction of pipeline replanning can be viewed as having a positive impact on the system. When the pipeline feed process randomly selects variants to enter the pipeline according to the target demand distribution then reconfigura- 
tion in the pipeline can have a substantial impact on the fulfilment mechanisms and some key system metrics. The lack of intelligence due to the random feed method is effectively corrected by the intelligence of the replanning process and the performance of the system is more closely aligned with one that has an intelligent feed process. The magnitude of the impact on system performance of replanning is smaller when an intelligent feed method is used with pipeline replanning. This can be seen by comparing the implementation of both feed methods and respecifying orders according to a predetermined threshold. In this situation the amount of reconfiguration taking place at different reconfiguration slots and different thresholds are almost identical for the two methods and yet the impact is greater if the pipeline feed is random. The impact of replanning on system performance is two-dimensional in that it depends both on where in the pipeline reconfiguration occurs and the amount of reconfiguration that is allowed to take place at that reconfiguration point. The amount of reconfiguration that occurs can be controlled by the approach to reconfiguration and in the methods implemented here this is achieved by means of a threshold that informs the decision whether to reconfigure or not.

Interestingly pipeline replanning can have a negative impact when used with the intelligent feed method yielding small increases in the level of BTO fulfilment although this situation does coincide with reductions in metrics such as customer waiting times and stock age particularly as the reconfiguration point is moved downstream towards the end of the pipeline.

Replanning can clearly have a significant impact on pipeline fulfilment and should be considered as a tool for tactically controlling fulfilment from the pipeline.

\section{References}

Brabazon, P. G. \& MacCarthy, B. L. (2006). Fundamental behaviour of VirtualBuild-to-Order systems. International Journal of Production Economics, 104(2), 514-524.

Brabazon, P. G., Woodcock, A., MacCarthy, B. L. (2008). Intelligent pipeline control - a simulation study in the automotive sector. Fifteenth International Working Seminar on Production Economics, Congress Innsbruck, Innsbruck, Austria.

Holweg, M. and Pil, F.K. (2004) The second century: reconnecting customer and value chain through Build-to-Order. Cambridge, Mass: MIT Press. 


\title{
An approach for the Real Time Intelligent Production Control to fulfill Mass Customization requirements
}

\author{
Sarfraz-ul-Haque, Minhas, \\ Ralf, Kretzschmann, \\ Veronica, Vargas, \\ Ulrich, Berger
}

\begin{abstract}
The automotive industry is distinguished by regionalization and mass customization of products. As a consequence, the diversity of products will increase and the lot sizes will decrease. Thus more product types have to be handled along the process chain and common production paradigms will fail. Hence, Rapid Manufacturing (RM) will be used for manufacturing small individual lot sizes. Nevertheless, new solution for joining and assembling these components are needed. The state of the art production control solutions exhibit several disadvantages in response to mass customization. A comprehensive and real time intelligent production control and monitoring system can overcome these limitations which will be the totality of intelligent soft computing algorithms and smart intelligent peripheral devices. The concept is based on three interlinked main modules: a technology data catalogue (TDC) to access, share, process and structure relevant engineering data, an automated scheduling processor (ASP) based on graph theory to get an optimized and adaptable work plan using feature technology and a central programmable automation controller (PAC) for real-time sensor/actor communication. The processes will be controlled through PAC and influenced by humans through scalable human machine interfaces. The PAC and its supported software's widespread functionality for real time applications and compatibility and flexibility with the conventional programming languages will enable customized palette of functions and tools for scalable level of control solutions. It will have a widespread accessibility as it will influence the execution of robot programs after preprocessing the data coming from various peripheral devices and calculating new robot paths by segmentation of detected curves and paths corresponding to the process planning information via one interface. The human machine communication will be based on the ontological approach as a natural language interaction system for filtering and translating into machine command. The entire concept will be demonstrated in a laboratory set-up with distinct machining, assembly and joining processes and will be experimentally validated in European research and development projects.
\end{abstract}




\section{Keywords}

Intelligent Production Control, Knowledge Driven System, Human Machine Interface, Dynamic Navigation, Technology Data Catalogue, Ontology, Mass Customization.

\section{Introduction}

The current situation in the automotive industry is characterized by increasing requirements from the customer side on quality and individualization of products and, at the same time, upcoming pressure on product prices. Car manufacturers create new product segments and enrich existing segments with more possibilities for individualization like regionalization and customization of products. The product diversification is combined with ongoing reduction of product life cycle time and an acceleration of innovation (Kuhn, et al., 2002). Furthermore, the automotive industry is characterized by enabling innovations in light-weight vehicle structure, energy efficient power-train solutions and assistance systems. New manufacturing paradigms for automotive structures and components force the automotive industry to continuously promote the development of cost-efficient and innovative vehicles, with high-added customer value, increased personalization capabilities and environmental sustainability. According to the recent Global Technology Revolution 2020 report, issued by the RAND corporation, there is foreseen a strong trend for on-demand manufacturing of components and small products to individual personal or corporate specifications (Silberglitt, 2006). Although the development and process planning of diversified products is enabled with help of powerful CAD/ CAM and PLM systems however, handling of different products in the production line is still solved unsatisfactorily. Consequently, in a mass customized scenario, the production will be the bottleneck along the life cycle. In this context, Ruohonen, et al., (2006) suggested the need for adopting mass customization along the complete value chain. This means that the whole product lifecycle has to be customized. Besides creating external complexities, mass customization triggers internal complexity inside the company while handling extensive product variety.

\section{State of the art: Mass Customization Manufacturing}

The mass customization manufacturing process covers the manufacturing of diversified components and then assembles them to complicated mass customized products. In this context, most automotive manufacturers are trying to handle mass customization by adapting the standard automated manufacturing cell. This typical cell (see Figure 1 next page) consists of several components. The principal components are the NC machines and the assembly robots.

This whole setup is a combination of on-line and off-line devices. It is a common practice to generate robot programs with an off-line work planning module based on product, process and resources (PPR) information. The robot as well as NC ma- 


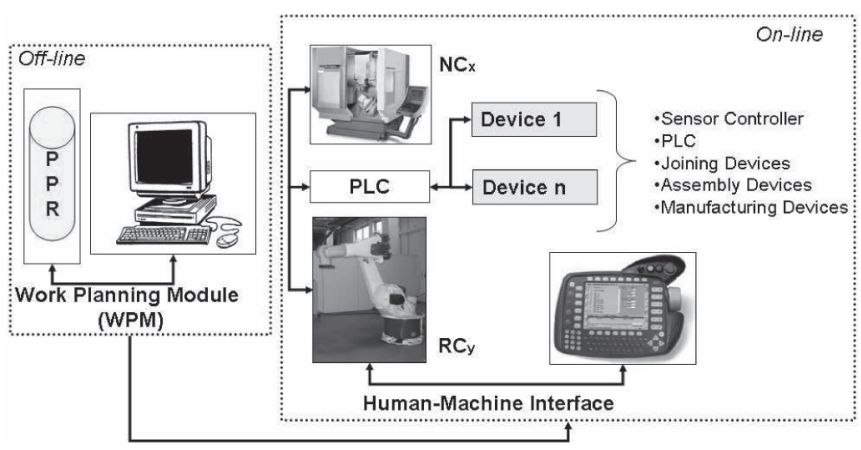

Figure 1: Standard approach of a manufacturing cell

chines are connected to sensor controllers via PLCs and their respective controllers. The manufacturing cell consists of Numerical Controlled Machines (NC), Industrial Robot (IR) with the Robot Controller (RC), the Human Machine Interface (HMI) and the Work Planning Module (WPM) based on Product, Process and Resources (PPR) information as an off-line programming system. This standard approach can work successfully for processing customized applications but a lot of interfaces need to be developed and modification of control parameters is necessary. Consequently, when a manufacturing cell based on this architecture is subjected to mass customization, exhibits certain disadvantages like limited flexibility in programming of industrial robots or machines due to manufacturer dependent programming environment, offline verification of industrial robot programs which in some cases limits the adaptation and consumes lot of time, complicated synchronized simulation of robots and its implementation, large number of interfaces and non compatibility issues and fixed but highly branched program (spaghetti code) execution corresponding to the sensor data inputs. As a result, the automated manufacturing systems have lower capability, high downtimes of the automated machinery and consequently high troubleshooting costs. The non-availability of ubiquitous operational knowledge and the absence of dynamic and explicit knowledge recuperation procedures minimize the achievement of ondemand capabilities. In this context, the Japanese NISTEP report no. 99 from 2005 identifies with high priority the establishment of a technology for converting implicit knowledge on manufacturing and manufacturing technique into explicit knowledge (NISTEP, 2005). In addition to these complexities, the level of precision in adaptation of manufacturing cells is low due to the weak or missing link between the ubiquitous operational knowledge and the dynamic and explicit knowledge. These arguments highlight the incorporation of intelligent systems like knowledge based guidance system in automated manufacturing systems. The above mentioned complexities can be resolved if restructuring of production control is made upon intelligent methodologies and algo- 
rithms to deal mass customization. This paper gives a comprehensive approach for intelligent production control of manufacturing cells in the automotive industry to cope with the mass customized induced internal complexities.

\section{Intelligent Production Control Approach}

The main challenges for coping mass customization in manufacturing are to achieve high production quality in case of high product variety and the mechanism for automatic variant specific product and process configuration. As stated earlier, that existing production control systems have rigid parameter settings and equivalent production rates or throughput times. The control architecture based on Programmable Logic Controllers has limited room for modifications so as to make the system highly responsive to the dynamic demands. In addition to that, the signal processing units have strict working points and thresholds. Therefore production process cannot be effectively controlled in accordance with the requirements of mass customization. As a result, an effective and efficient automation solution is required to face these challenges. It needs a holistic redesign of the manufacturing system exploiting, the state of the art concepts of intelligent and flexible automation, robotic technologies and sensory systems. High flexibility in product development cycle is also needed for reconfiguration and adaptation of manufacturing setups. Modularization of knowledge driven platform for data handling and automatic configuration of products as well as processes is essential for fast customization of these setups. These demands can be catered through fast and responsive intelligent production control system which is triggered by intelligent monitoring. Such system can quickly reconfigure itself to allow flexibility not only in producing new products but also changing the system itself. The intelligent production control can be the totality of intelligent production controller based on knowledge base and other intelligent soft computing algorithms such as artificial neural networks, fuzzy logic, and genetic algorithms and smart and intelligent peripheral devices. Intelligent production automation requires the replacement of deterministic control sequences by a common negotiation schemes and individual decision algorithms. These intelligent algorithms can be considered as a base for optimization, problem diagnosis, multivariant parameters adaptation, multi-criteria decision making, distributed decision making, distributed scheduling, resource allocation, data mining and data fusion as in case of dynamic knowledge navigation or in combining monitoring output data. Such approach helps in increasing the performance and accuracy of control and in predicting failures or disturbances in processes, machines, or devices and to fix deadlocks in overall production processes. A hybrid algorithm can be employed as a self learning base for the system in order to be more efficient in automatic and precise configuration.

The production control approach is devised to achieve high adaptability, extensibility and reusability. The intelligent approach is designed on the following points: 
- Highly knowledge driven platform such as technology data catalogue containing the systematic production parameters and functional correlations and coherencies

- Intelligent modeling methods dealing with multi-variant parameter correlations and production control parameters considering their interdependencies

- Highly flexible and applicable centralized control set-ups taking all technical specifications and functionalities for fast and smooth adaptation of new materials and processes, faster ramp up of new technologies and ensure direct and transparent quality control

\subsection{Customer Requirements and Analysis}

The general structure of mass customized core is divided into three modules (see Figure 2), which will be introduced in the following chapter:

- Planning Module, which determines the parameter settings and the layout of the workbench corresponding to the given customer demands

- Knowledge Driven System, which provides and support the other modules with a efficient and certain product and process data management (Technology Data Catalogue)

- PAC-based Intelligent Production Control, as new intelligent approach for handling the high dynamical manufacturing operations

\subsubsection{Planning Module}

Varying customer demands, which results variable lot sizes and high product variety increases the complexity in production planning and scheduling. Therefore a mass customization supported planning module has to achieve robust production control in order to satisfy customer's needs efficiently. Therefore, the presented planning module is a multi-step approach. At first the manufacturing setup layout will be defined. Afterwards the allocation of manufacturing cells and their reconfiguration will be introduced in the product sequencing phase.

\subsubsection{Layout Definition Phase}

The desired layout has to be highly flexible and reconfigurable in order to handle the different customer demands (CD) in a manufacturing setup. Therefore the probabilities of the CD variants or CD set (CDS) have to be investigated. One well-known method from the field of facilitation technique is the use of a morphological box in combination with the Pareto chart. The customer will select different concrete requirements / demands for its mass customized product from a list of production possibilities or alternatives (A) for each demand (D). Thus, the demands will be ordered in a vertical form whereas alternatives for each demand will be ordered hori- 


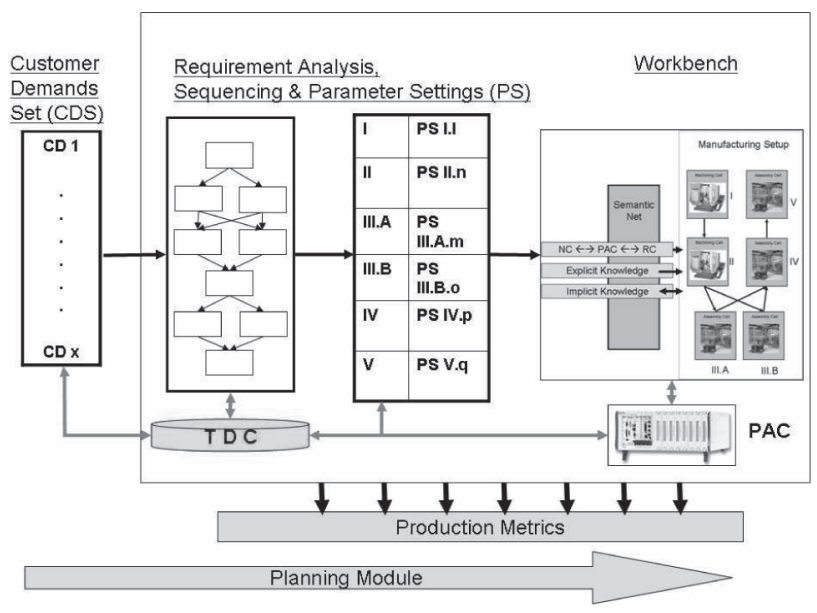

Figure 2: Parameter settings in mass customized core

zontally. Finally, the selection of optimal option for each demand is summarized in a single customer demand set (CDS).

Afterwards the occurrence of the CDS will be analyzed by using Pareto chart. Thus, a classification into A (80\% occurrence), B and C products is enabled. The layout will be defined in accordance with A products so as to handle their manufacturing in efficient way. The evaluation and definition of the most appropriate layout will be done by intelligent, virtual simulation tools like "DELMIA QUEST" or "taraVRbuilder". The evaluation metrics are the overall cycle time, the allocation efficiency of the actual allocation efficiently. The overall objective is to reduce the value-added time by reducing the buffer and transportation time of each part.

\subsubsection{Product Sequencing Phase}

Mass customization supported manufacturing layout definition is followed by utilization of intelligent algorithms for determining appropriate manufacturing cell sequencing and resource allocation. Therefore each CDS, which represent a product, has to be analyzed and the corresponding manufacturing cells in the manufacturing setup layout has to be sequenced, allocated and reconfigured at the right time. This issue was addressed by several different methods and techniques. There are already established methods like MRP I (Material Requirements Planning), MRP II (Manufacturing Resource Planning), KANBAN, Optimized Production Technology (Eversheim 1996) for this purpose. Furthermore, the MES (Manufacturing Execution Systems) were introduced in the last years to handle this optimization issue (Kletti, 2007). In the field of artificial intelligent the following methods are well known in the field of shop-floor job scheduling and manufacturing parameter settings:

- Neural Networks (Duran et al, 2008)

- Heuristical Search with planning rules (Hamelmann, 1996), (Hellmann 1993), (Rudolph, 1993) 
- Genetic Algorithms (Rudolph, 1993)

- Feature Technology and algorithms known from the graph theory (Berger and Kretzschmann 2007)

This presented challenge is comparable with scheduling of NC programs in machine tools. Berger and Kretzschmann (2007) presented an approach for ordering and scheduling machine operations with help of assessed feature-based operations. Furthermore, the possible alternative machining operation sequences are transferred into a directed graph. With the help of algorithm (Floyd-Warshall and Travailing-Salesman-Heuristics) based on graph theory, the processing of this directed graph is enabled to assess feasibility and effort for executing machine operations at alternative machines. This methodology is illustrated in Figure 3. The sequencing system has manifold access to the PPR (process, product, resource) hub to get information about the produced mass customized product and other relevant information such as materials, manufacturing technique applicability and tolerances. The sequencing system classifies the manufacturing layout with their process parameters into the following application categories:

- Capability: This manufacturing cell provides manufacturing operation that has to be executed in order to meet the customer demands.

- Incapability: This manufacturing cell is not executable to meet the customer demands.

- Non-allocatable: This manufacturing cell is not allocatable at the right desired allocation time; therefore this alternative branch will get a high effort.

- Selected: This manufacturing cell with the selected parameter setting is sequenced.

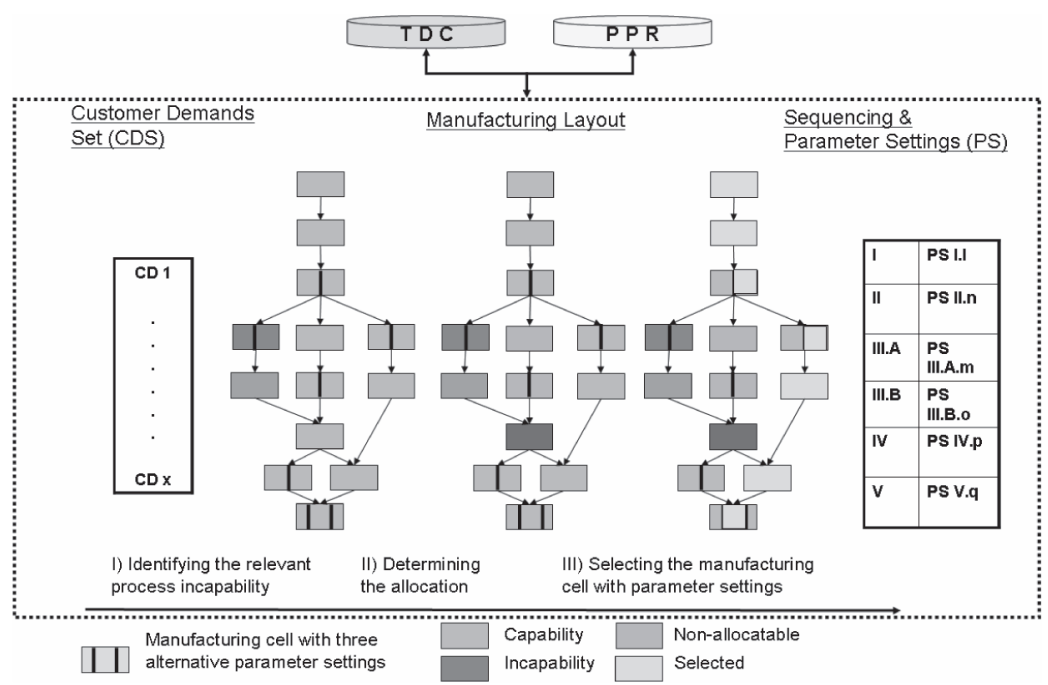

Figure 3: Product sequencing work flow 
At first CDS will be mapped into the processes of the assembly layout (I) with the help of the variant process planning documents which are stored in the PPR hub. At this point, the system can determine the capable assembly cells that can be used to execute joining and assembly tasks in order to influence the work piece for meeting the customer demands. The branches with the incapable assembly cells will be marked as non-sequenced. The capable assembly cells with different parameter settings are then assigned. In the second step (II), the allocatability for each assembly cell will be checked for the desired allocation time. If the cell is not allocated, then the effort of the corresponding branch will be drastically increased. In the final third step, the effort of the remaining assembly cells with concrete parameter settings will be designated (III) with the help of introduced metric. Thus, each parameter setting of each remaining assembly cell is assigned with individual effort. The application of the optimal work plan (using the Floyd-Warshall) algorithm determines in real-time the sequence of assembly cells with parameter setting which has in total the least sum of efforts. The sequenced work plan will used to process the mass customized products in the assembly setup.

\subsection{Knowledge Driven System}

A mass customization manufacturing process can be defined as a cross-functional, intercompany and market oriented process, which requires constant interaction based on the exchange of data to respond to customer needs (Calabrese, 1999). It involves distribution of explicit knowledge within the company through formal communication channels whereas implicit knowledge requires co-operation (Calabrese, 1997). On the other hand, higher levels of knowledge dissemination and information exchange lead to a better understanding of technology capabilities and trends, competitors- and competitive actions, market and customer. This is essential information to design the manufacturing process, determine the product features and specifications (Van der Bij, et al., 2003). It also leads to a considerable decrease of technical uncertainties in the mass customization knowledge driven process. Moreover, greater levels of information exchange within an organization increase the likelihood that the new product is well responsive to the mass customization. Moreover, it has been suggested that information can be exchanged formally within the boundaries of defined mechanisms, such as structured methods and formal processes within the organization (Perks 2000, p. 182; Van der Bij, et al., 2003, p. 164; Calabrese, 1999, p. 440). Efficient knowledge sharing can be carried out by implementing formal procedures for guiding information flows. Moreover, there are mechanisms that can originate such processes for instance: diffusion, information retrieval, information pooling etc. (for more details see Berends, et al., 2006). Nevertheless, sharing knowledge among members of an organization may be a complex activity. As long as the knowledge is not shared, it can not be exploited by the organization (Choo, 1996). 
The literature on organizational memory states that there are sophisticated computerized tools for externalizing personal knowledge such as idea managing tools, expert systems etc. Nevertheless, there is still a danger that tacit knowledge may remain as personal knowledge due to the complexity to share information throughout distributed or big companies (Klammer \& Mathias, 1998). There are many tools, such as CAD systems, that are available to manage the latter type of design information or the final product definition. In contrast, much of earlier type of design information is currently not captured in structured or systematic ways due to its level of abstraction. It is supposed that a high level of abstraction is related to less detailed information. Moreover, the access to information has been solved by largescale computer networks. The processing and interpretation of retrieved information remain a problem due to heterogeneity of the data (Basson, Bonnema and Liu, 2004). There can be distinguished three main heterogeneity problem categories (Stuckenschmidt and van Harmelen, 2005):

1) Syntactical problems, for instance data format heterogeneity

2) Structural problems. These problems are originated because the same objects and facts can be described in different ways using homonyms, synonyms, etc.

3) Problems of semantics. These problems refer to intended meaning of terms in a particular context or application. They are caused due to the inherent context dependency of information that can be understood only in the context of their original source and purpose.

Standards used as interfaces to integrate different information sources can solve the first kind of problem. Further, structural problems and semantic conflicts can be partially solved by one to one structural mappings. If structural mappings do not apply, like in the case of large-scale information sharing, the semantics of the information has to be taken in account in order to decide how different information items relate to each other. Thus, it is proposed to use ontologies as technique to solve the problem of explicating semantic knowledge about information (Stuckenschmidt and van Harmelen, 2005). Ontologies are defined as "a set of formal terms, usually with a hierarchical organization, with associated formal definitions that specify their relationships with the other formal terms, and a set of constraints about their use in the knowledge representation of the domain studied" (Goossenaerts and Pelletier, 2001); or "a term used to refer to the shared understanding of interest" (Uschold and Gruninger, 1996); or an "explicit specification of a conceptualization" (Gruber, 1993a and Gruber, 1993b).

As a result of the previous discussion, it is concluded that a knowledge representation based on semantic net, that develops the intelligent relationship of manufacturing processes and product features as well as other relevant information will be developed. This intelligent knowledge system will include a product and process 
data management which is very essential in mass customization scenarios for making rapid and precise adjustments. Moreover, it will be termed as technology data catalogue (TDC).

\subsubsection{Technology Data Catalogue}

The main aims of the TDC are gathering, retrieving, structuring, processing and sharing relevant engineering data/information in an intelligent way. The TDC will also provide structured information about the "best-practice" settings of the manufacturing system. Data sources have to be defined and a suitable knowledge representation structure has to be created in order to store the relevant implicit and explicit knowledge generated at the different levels of companies.

The TDC will use product data standards STEP to overcome data format heterogeneity of explicit knowledge sources. STEP will provide an unambiguous and computer interpretable representation of product data (Fowler, 1995). The product data will be taken from different sources CAD/CAE/CAM, Product Data Management (PDM) and Enterprise Resource Planning (ERP) systems. The following "STEP-Generic Resources" will be the information resources of explicit knowledge for the Technology Data Catalogue: fundamentals of product description and support, geometric and topological representation, product structure configuration, materials, shape variation, tolerances, and process structure and properties. An ontological system is suggested to workout problems of semantics in knowledge representation. Specifically, this model will contain: (i) A database, (ii) Translators and (iii) Filters.

(i) The database with shared vocabulary (concepts, instances, attributes, etc.) will allow the use of terminology most appropriate to the particular context. It will solve those problems that are generated due to ambiguities among terms. Here the ontologies are built according to the following design criteria: clarity, coherence, extendibility, minimal encoding bias, minimal ontological commitment (for more details see for example Gruber, 1993b).

(ii) Translators will determine the relation among concepts coming from different sources ensuring the coherence of the exchange of data between the systems and the TDC. In the literature, translators have already been proposed and successfully tested, e.g. in Goossenaerts and Pelletier (2001).

(iii) Filters will enable sorting out information that better match the requested technology (Lepratti, 2005; Basson, Bonnema and Liu 2004).

To reflect changes in the production environment, the ontological system needs to be updated consistently. Thus, it is suggested that the ontological system has to be maintained regularly by a knowledge manager, who will obtain constant feedback from other departments (i.e. design and manufacturing). It may also be the responsibility of the knowledge manager to verify any effect on the system before achieving any change. 
Semantic nets are the last components of the TDC. They will facilitate the dynamic navigation through the information, the information visualization and the sharing of information through formal procedures. Moreover, they will support mass customization. A semantic net is defined in the literature as "a graphic notation for representing knowledge in patterns of interconnected nodes and arcs" (Sowa, 1992). Some other advantages of this graphical notation are mentioned (Kendal and Creen, 2007) in the following:

- It is adaptable method of representing knowledge because many different types of objects can be included in the network.

- It is graphical and therefore relatively easy to understand.

- It can be used as a common communication tool between the knowledge engineer and the human expert during the knowledge acquisition.

The external data access of the TDC will be realized through semantic networks. Thus, the fast heterogeneous information access for handling mass customized products requirements is enabled. After defining the structure of the TDC, the content has to be determined. The TDC includes information about manufacturing operations (applicability of parameter settings for manufacturing setups and relevant measuring parameters), process (parameter settings correlated with multi-variant criteria) and products (correlation between independent customer criteria).

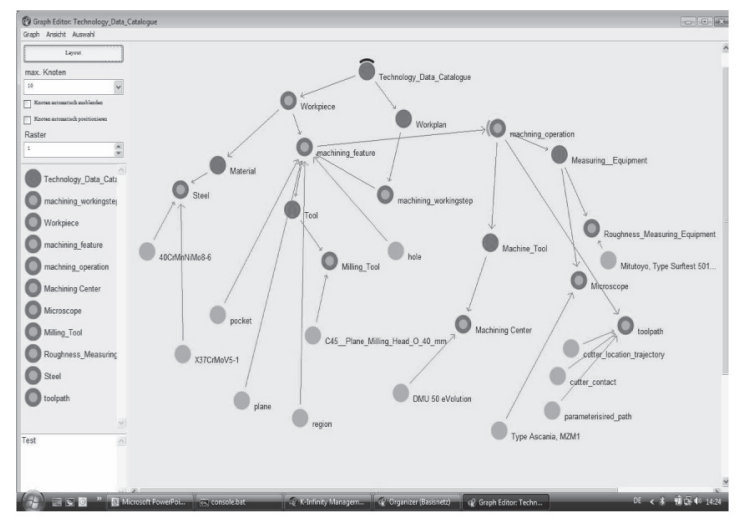

Figure 4: Semantic net developed in K-Infinity

Figure 4 shows a semantic net developed in K-Infinity software. In this graphical representation, the TDC is mainly divided in two nodes: "workpiece" and "workplan". These nodes provide accurate process parameters about materials, tools, ma- 
chines etc. that will be used by the Programmable Automation Controller (PAC) module.

The content of the TDC will consider rapid change of the manufacturing environment and application scenarios. Therefore, the TDC will be enriched regularly with manually acquired data on one side. On the other side, the implementation of data mining and automatic algorithms will be developed to link new applications or cases in the TDC. Finally, the use of the TDC will also fulfill other requirements, highlighted in the literature as general requirements for information systems; some of them are mentioned in (Basson, Bonnema and Liu; 2004)

- Selective extraction of information by avoiding redundancies

- Recording of informal statements at the shop floor level will prevent hiding of personal knowledge

\subsection{Intelligent Production Control}

The state of the art controlling of production processes is carried out through conventional PLCbased architectures (Noe, 2007). These types of architectures are highly non flexible to mass customization as modifications are not only uneconomical but generate lot of interface problems also. Moreover, highly data driven, knowledge intensive system to cope mass customization in an intelligent way cannot be done by traditional control systems like PLCs. Therefore, the Programmable Automation Controller (PAC) and use of intelligent components and modular design promise to bring essential benefits for re-configuration and adaptation of automated production system. This adaptable and reconfigurable control architecture is fast responsive to make quick product changes (adaptability), easy programmable and reprogrammable (flexible) and resilient to manufacturing tolerances (compliant).

This new intelligent approach revolves around the Programmable Automation Controller (PAC) that has several but standardized interfaces and can access all information from Technology Data Catalogue (TDC) and Product Process Resource (PPR) modules (See Figure 5). The PAC accesses the process planning information Automated Scheduling Processor (ASP).It takes the data from multi-sensor monitoring system, pre-processes the data and then calculates new robot paths by segmentation of detected curves and paths (Berger, et. al., 2006) in order to control the robot program corresponding to the process planning information through common interface. The scalable portable and remote human machines interfaces as well as multi user profiles enable the users of all level to influence the process control. One such HMI is in the form of personal digital assistant which is a part of manufacturing demonstrator setup for EU project. Intelligent production control will be achieved by integration of production simulations; intelligent monitoring able to recognize its environment; and novel feedback loops, which link the simulation and the practical validation. This concept is in implementation phase in EU FP6 FUTURA-project. 


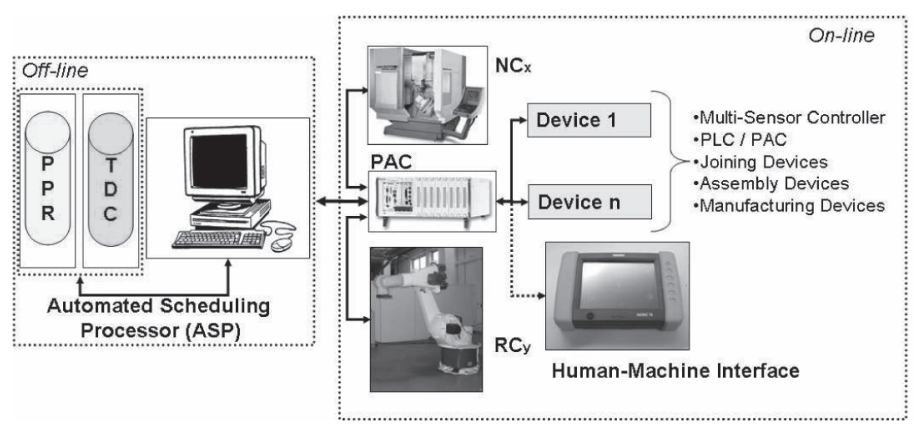

Figure 5: Architecture of Mass Customized Manufacturing Cell

\section{Technical Realization}

At present, the approach for mass customized responsive automated manufacturing cell through intelligent production control is in realization phase. For dealing mass customization in a systematic way, the modularity and scalability of variants is considered so as to handle huge variety of products with fewer modifications. As a first step, the assembly of Body in White (BIW) will be developed with the help of various scalable modules at the shop floor. The intelligent algorithms for sequencing and reconfiguration of manufacturing cells are under practical realization. The reconfiguration in terms of handling is enhanced by means of variant flexible fixtures. The bench marked part i.e. side frame component, is shown in Figure 6. The complete approach is being implemented in a laboratory set-up considering cold joining process. However, this work will be extended to other manufacturing processes in near future.

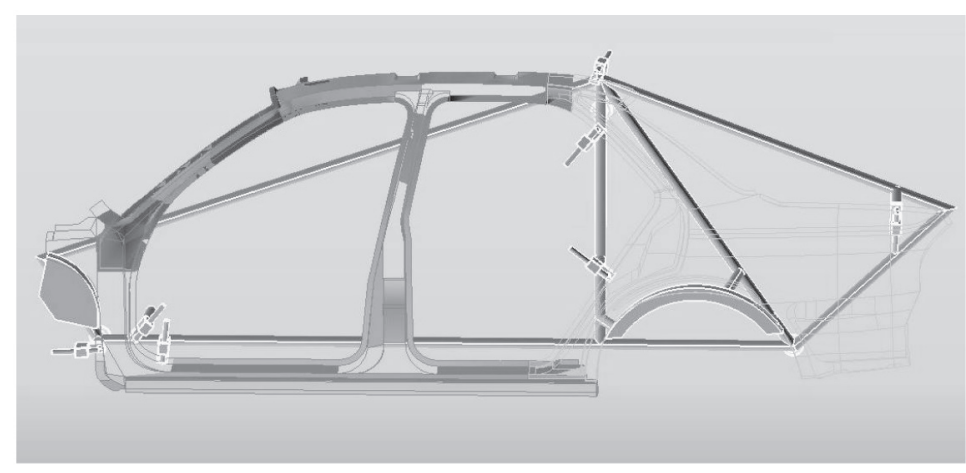

Figure 6: Specimen side-frame

The robot-based experimental setup is focusing on cold joining methods like adhesive bonding with one or two component bonding material. Consequently, the TDC includes technology parameter settings for adhesive joining and the modular and scalable segments of benchmark part. 
The experimental validation is an ongoing process and will be experimentally validated in research and development projects. One of the projects is the EU Sixth Framework Programme "FUTURA - Multi-Functional Materials and related Production Technologies integrated into the Automotive Industry of the Future" (FP62004-NMP-NI-4-026621), which will pursue innovative concepts that will facilitate the integration of multifunctional materials in the automotive industry according to mass customization demands. The objective is the designing and assembly of modular and scalable as well as hybrid body and chassis structures. The scalability will enable an accommodation of size and design requirements for different models, while the modularity concept will facilitate an easy derivation of variants and a flexible assembly and/or joining process.

\section{Conclusions}

In this paper, the comprehensive approach for intelligent control of manufacturing cell has been described. This approach is developed to tackle mass customized induced complexities in automotive industry and to enhance its competency. The intelligent production control system assisted by knowledge base will enable fast modifications to handle highly dynamic volumes and changing variants. Moreover, the knowledge driven system will assist in fast identification of cross correlations of multi-variant parameters as well as automatic setting of thresholds. By this way the conventional way of determination of parameter settings through statistical or experimental means is eliminated. Systematic acquisition and implementation of functional requirements and identification of specifications of peripheral devices at shop floor level through intelligent semantic net methodologies will enhance knowledge level at overall manufacturing cell level. The new materials and object features can be taken for manufacturing processes by easy modifications in manufacturing setups. The standardized data acquisition, filtering and processing of CAD and simulation data through dll and ODBC interfaces will enable a generic solution for handling multi-variants in the same setup, thereby reducing redundancies and rapid adjustments. The intelligent computing algorithms and graphical programming platform will yield wide spread functionality and flexibility conducive for mass customization.

\section{References}

Basson, Anton H. / Bonnema, G. Maarten / Liu, Yang (2007): A flexible ElectroMechanical Design Information System, In: Tools and Methods of Competitive Engineering, Imre Horváth and Paul Xirouc hakis (ed.), v.2, pp. 879- 889, Rotterdam Netherlands: Millpress, 2004.

Berends, Hans / Van der Bij, Hans / Debackere, Koenraad / Weggeman, Mathieu (2006): Knowledge sharing mechanisms in industrial research, In: R\&D Management, Vol. 36, 1, 85-95. 
Berger, Ulrich / Kretzschmann, Ralf (2007): Development of a holistic guidance system for the NC process chain for benchmarking machining operations, In: Proceedings of the 12th IEEE Conference on Emerging Technologies and Factory Automation, Greece, 2007.

Berger, Ulrich / Kretzschmann, Ralf / Vargas, Veronica (2007): Intelligent production monitoring and control for mass customization of automated manufacturing cells in the automotive industry, In: Proceedings of the 12th MCPC 2007 World Conference on Mass Customization and Personalization, USA, October 07-10, 2007.

Calabrese, Giuseppe (1999). Managing information in product development, In: Logistics Information Management, Vol. 12, 6, 439-450.

Calabrese, Giuseppe (1997). Communication and co-operation in product development: a case study of a European car producer, In: R\&D Management, Vol. 27, 3, 239-252.

Choo, C. W. (1996). The knowing organization: How organizations use information to construct meaning, create knowledge and make decisions, In: International Journal of Information Management, Vol. 16, 5, 329-340.

Orlando, Duran / Nibaldo, Rodriguez / Airton, Consalter Luiz (2008): End Milling: A Neural Approach for Defining Cutting Conditions, In: Proceedings of the 4th International Workshop on Artificial Neural Networks and Intelligent Information Processing, ANNIIP 2008, Madeira, Portugal, May 2008.

Eversheim, W. (1996): Organisation in der Produktionstechnik - Band 1 Grundlagen, 3. Auflage, Düsseldorf: VDI-Verlag.

Fowler, Julian (1995): STEP for Data Management, Exchange and Sharing, Great Britain : Technology Appraisals Ltd.

Goossenaerts, J.B.M / Pelletier, C. (2001): Enterprise Ontologies and Knowledge Management, In: K.-D. Thoben, F. Weber and K.S. Pawar (ed.) Proceedings of the 7th International Conference on Concurrent Enterprising: "Engineering the Knowledge Economy through Cooperation”, pp. 281-289, Bremen, Germany.

Gruber, Thomas R. (1993): A Translation Approach to Portable Ontology Specifications, In: Knowledge Acquisition, 5, (2): 199-220.

Gruber, Thomas R. (1993): Toward Principles for the Design of Ontologies Used for Knowledge Sharing, In: International Journal Human-Computer Studies 43, pp. 907-928.

Hamelmann, Stefan (1996): Systementwicklung zur Automatisierung der Arbeitsplanung, In: Fortschriftberichte VDI-Reihe 20 Nr. 195, Düsseldorf: VDI Verlag.

Hellberg, Klaus (1993): Methoden zur automatischen Erzeugung von Arbeitsgangfolgen, Düsseldorf: VDI-Verlag.

Kendal, Simon / Creen, Malcolm (2007): An Introduction to Knowledge Engineering, USA: Springer.

Kletti, Juergen (2007): Konzeption und Einführung von MES-Systemen: Zielorientierte Einführungsstrategie mit Wirtschaftlichkeitsbetrachtungen, Fallbeispielen und Checklisten,Berlin: Springer - Verlag. 
Kuhn / Wiendahl / Eversheim / Wiesinger (2002): Schneller Produktionsanlauf von Serienprodukten, Ergebnisbericht der Untersuchung 'fast ramp-up ', Dortmund: Verlag Praxiswissen.

Lepratti, Raffaello (2005): Ein Beitrag zur fortschrittlichen Mensch-Maschine-Interaktion auf Basis ontologischer Filterung, Berlin: Logos Verlag.

NISTEP (2005): Nistep Report No. 99, Science and Technology Foresight Center National Institute of Science and Technology Policy (NISTEP), Ministry of Education, Culture, Sports, Science and Technology, Japan.

Perks, Helen (2000): Marketing Information Exchange Mechanisms in Collaborative New Product Development, the Influence of Resource Balance and Competitiveness, In: Industrial Marketing Management, Vol. 29, 179-189.

Rudolph, Fritz Nicolai (1993): Konfigurierbare Technische Elemente für Konstruktion und Arbeitsplanung, Düsseldorf: VDI-Verlag, 1993

Ruohonen, M. / Riihimaa, J. / Mäkipää, M. (2006): Knowledge based mass customisation strategies: cases from Finnish metal and electronics industries, In: Int. J. Mass Customisation, Vol. 1, Nos. 2/3, pp.340-359.

Silberglitt (2006): Global Technology Revolution 2020, In-depth Analysis: Bio/Nano/ materials/information, Rand Corp.

Sowa, John F. (1992): Semantic Networks, In: Shapiro Stuart C., Wiley (ed.) Encyclopedia of Artificial Intelligence.

Stuckenschmidt, Heiner / van Harmelen, Frank (2005): Information Sharing on the Semantic Web, Berlin Heidelberg, Germany: Springer-Verlag.

Uschold, Mike / Gruninger, Michael (1996): ONTOLOGIES: Principles, Methods and Applications, In: Knowledge Engineering Review, Vol. 11, 2.

Van der Bij, Hans / Song, Michael X. / Weggeman, Mathieu (2003): An Empirical Investigation into the Antecedents of Knowledge Dissemination at the Strategic Business Unit Level, In: Journal of Product Innovation Management, Vol. 20, 163-179. 


\title{
Driving Mass Customisation in Supply Networks. A Machine Tool Sector Case Study
}

\author{
Eduardo Saiz, Eduardo Castellano, Juan Manuel Besga, \\ Jone Uribetxeberria, Iñaki Zugasti
}

\begin{abstract}
A key issue faced by organisations today is the challenge posed by how to deliver the different products demanded by customers, in different markets, at any given moment in time, and preferably individually customized, as cheaply and as quickly as possible. The research presented in this paper attempts to help globalised organisations to identify alternative multiplant network configurations, and management strategies, to respond, through a mass customisation business strategy, to different demand requirement scenarios, within required cost and time restrictions. In order to facilitate the understanding of the consequences of adding or removing operational units, processes, reallocation of resources, etc, over the global network performance, a computational decision support system approach has been taken.
\end{abstract}

\section{Keywords}

Manufacturing Network, Decision support systems, Hybrid simulation

\section{Introduction}

Market globalisation, world-wide procurement, geographically distributed plants, and more sophisticated customer requirements, are hardening global competition in general, creating a new dynamic environment for supply networks. This new dynamic environment has led to the development of new managerial approaches, due mainly to the transformation of competitive forces, with the appearance of responsiveness to demands, with a greater degree of customisation, as a key factor (Kidd 1994).

Current production systems require the capability to change their performance dimensions in terms of range and response, maintaining in turn high levels of performance in traditional competitive aspects such as time and cost. All of which has led to the need for moving to a multifocus strategy, in which the concept of flexibility takes on special relevance. Mass customization business strategy main idea is that one-of-a-kind type products are manufactured with high levels of quality and fast delivery, with the low costs of mass production (Anderson and Pine II 1997). This strategy achieves, therefore, a compromise between the advantages of product customisation -economies of scope-, guaranteeing fast response times for customized demands, with productivity and low costs associated with economies of scale. 
The set of changes described above affect the way we conceive manufacturing and distribution networks. In order to deepen understanding of the consequences of multi-plant network design, and strategic policies, over the global network performance, a computational decision support system approach has been taken. The simulation approach allow us to conduct, for a multitude of scenarios and conditions, systematic testing of the structure and operation of this type of networks, its behavioural patterns and properties, in order to identify, both alternative flexible multi-plant network structures, as well as those strategies, policies, and rules, that are most adequate for their management, at low cost and risk (Shapiro 2001). And thus, approach the challenge of this research, about how to enable a globalised manufacturing organisation identify, based on demand orders, and following a mass customisation business approach, alternative multi-plant network configurations, and their management strategies, to facilitate respond different customized demand scenarios, in the most efficient, cost and time, possible manner.

The outline of this paper is organised as follows. Section 2 presents some specific demand driven supply networks concepts. In section 3, the research approach is specified. Section 4 very briefly describes the conceptual model building blocks. In Section 5, the Machine Tool Sector Case Study is presented. And in Section 6 the simulations developed as well as its main outcomes are presented. Finally, Section 7 shows the main conclusions of the research.

\section{Demand Driven Networks Concepts}

Depending on the characteristics of product orders, i.e. volume, number of variants, uncertainty in demand, product life cycle length, lead-time accepted, etc, there are different models of supply network (Fisher 1997), different types of manufacturing process (Hayes and Wheelwright 1979), as well as planning and control systems (Berry and Hill 1992), that are more adequate. These three "models", are very significant as background to place in context the development of the conceptual model of this research. We next move on to describe briefly each of them, along with a final note about the decoupling point concept, which allows us to relate the customer orders characteristics, and operations management, to the mass customisation business strategy adopted.

\subsection{Customer Orders Characteristics and Supply Network Structure}

Regarding the relationship between the characteristics of orders and supply network models, Fisher (1997), based on the cases of Campbell Soup and Sport Obermeyer, distinguishes between functional products with foreseeable demand, and innovative products with unpredictable demand. For those belonging to the first group, the author assigns a physically efficient type of supply network, the aim of which is to maximise efficiency at the lowest cost possible, high levels of manufacturing level resources, a strategy of inventory minimisation and a reduction in lead 
times. As regards the second group, innovative products, Fisher recommends market responsive type supply networks. The aim of these ones is to respond quickly to a demand with a high degree of uncertainty for minimising stock-outs and obsolete inventories. For this, excess manufacturing capacity is required, as well as product parts and finished products broad buffers, aggressive investment in lead time reduction, supplier selection based on their speed, quality and flexibility, and a modular type design strategy which facilitates the postponement of customisation of the product as late as possible.

\subsection{Customer Orders Characteristics and Processes}

As regards the relationship between product order characteristics, and manufacturing process type, the product-process matrix (Hayes and Wheelwright 1979) establishes two axes. The first one contains product characteristics, from products with a high number of variations and low volumes of orders (unique and one-of-a-kind products), to products with a low number of variations and high volume of orders (standard products). The second axis in the matrix, which refers to manufacturing processes, assigns, to each one of the previous types, manufacturing process of the type; project manufacturing for unique products, job shop type processes for oneof-a-kind products, flow shop for products with many variants and low volume, line flow for few major products with high volume (i.e. automobile), and finally, continuous manufacturing processes for standard products with high volumes. This model has been empirically validated in different empirical works.

\subsection{Customer Orders Characteristics and Operations Management}

On the management level, manufacturing operations, inventories and transport at each production unit in the supply chain are planned on different hierarchical levels. Depending on the level of aggregation, and planning horizon, can be distinguished: Sales and operations planning; Master scheduling; Materials planning, and; Production activity control. As a function of market order characteristics, different approximations may be adopted at each of these hierarchical management levels (Berry and Hill 1992; Selldin 2005).

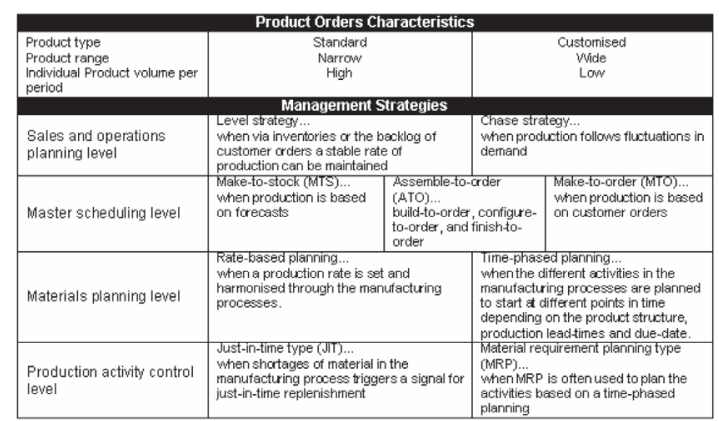

Table 1: Order characteristics and management strategies relationship (Selldin 2005) 


\subsection{Decoupling Points and Flexibility}

The dividing line between orders manufactured based on forecasts (MTS) and those that are assembled, manufactured, or designed, according to customer orders (ATO, MTO, ETO), determines a very important point in manufacturing processes and supply networks, known as the Customer Order Decoupling Point - CODP (Mason-Jones et al. 2000). Upstream the CODP there are few product variants and volumes are high, downstream the CODP each product may be unique and customised for a specific customer. Therefore, the CODP marks the point at which an order becomes attached to a specific customer, conditioning therefore the capacity of a supply network to provide different grades of customised product, at a particular cost and delivery schedule.

In a multi-plant network there may be multiple CODP, i.e. within the same operation unit certain products may be manufactured MTS and others MTO, or even in between plants. For instance, to achieve supply chain mix flexibility, the capacity of the manufacturing system to alter the relative production amount between products in a product mix, both, a multi-product plant strategy, as well as a certain number of single-product plants strategy may be adopted. In this last case, each single-product plant would have a low grade of mix flexibility being able to develop high productivity ratios, and would at the same time form part of a superior structure and strategy leading to mix flexibility on a firm level (Vereecke and Van Dierdonk 1999). The multi-plant option, multi-plant networks, is very interesting for creating flexible production capacity, transferring production volume from overloaded plants to others that at that moment in time have excess capacity. The potential configurations of multi-plant networks can become, therefore, a key aspect in obtaining, via very different means, the levels of flexibility desired on a firm level. Thereby, for each case being analysed one must consider what type of flexibility is desired for each unit of analysis, and define the particular manner in which it shall be developed to obtain the objectives as efficiently as possible for the system as a whole.

\section{Research Approach}

The research methodology adopted can be classified as an engineering approach for performance enhancement of systems (Pritsker 1997). For developing the research we have based on: (1) Previous studies identified in the literature; (2) A case study research of a machine tool sector global manufacturer, denoted in this paper as MACTO-NET, and; (3) A simulation environment.

Case studies are frequently used for exploratory and theory building research (Yin 1994). The selected case study, MACTO-NET, is a machine tool manufacturer with a global multi-plant network and urgent requirements for introducing flexibility in their response to markets demanding products with a high degree of customisation. Machine tools delivered by MACTONET network have a total production lead time bigger than the service time demanded from their markets. Therefore, 
machines manufacturing process has to begin before customer orders arrival, and when orders arrive, a machine that is in process must be assigned to each order. To do this assignment, some relevant information like machines process status, plants load-capacity balance, plants capabilities, subcontracting availability or customers location is used. Moreover, the path that the assigned machine is going to follow across the manufacturing network is generated and the decoupling point where customization will be executed to adapt the machine to the specific order requirements is identified.

The simulation modelling approach is fundamental to this research due to the high levels of interdependencies between constituent elements of these multi-plant networks, their inherent feedback loops, non-linearities, and delays, that make network behaviour in the face of market demand variations a dynamic process which generally produces counterintuitive behaviours over time (Sterman 2000), and therefore makes purely analytical approaches to the problem inadequate (Fowler 1998). The modelling and simulation methodological framework followed in the research is the one of systems dynamics, as its validity has been demonstrated for the design of company simulations and strategic decision-making laboratories (Sterman 2000). Its main steps, in outline, are the following ones (Coyle 1977; Sterman 2000):

- Elicitate MACTO-NET model;

- Implement MACTO-NET model in the simulation environment;

- Calibrate and validate the simulated model based on empirical data;

- Conduct structural and behavioural analysis of the simulated model under different conditions (i.e. product-process structures, policies, operations...), and scenarios (i.e. customer demand scenarios), and;

- Make managerial suggestions based on the knowledge of the model behaviour to improve the real world system performance.

\section{Multi-Plant Network Conceptual Model}

The conceptual model associated with the multi-plant network model for a mass customisation strategy, is structured into five conceptual blocks, as illustrated in Figure 1. For a more detailed description of the model building blocks see Saiz et al (2006).

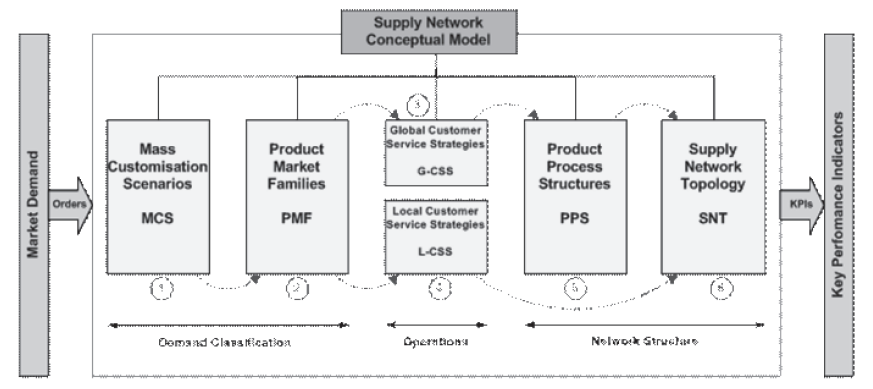

Figure 1: Supply network conceptual model 
As demand orders arrive they classified according to the customization required (MCS), and the product family to which they belong and the market to which they are directed (PMF). As a result of this classification, orders are associated with one or more strategies by which they shall be served to customers (CSS). These strategies can be of two types: (i) Global, whereby it is specified, in product and process structures (PPS), what components are to be manufactured, subcontracted or purchased, and the form of the network topology (SNT) that will supply the product; (ii) Local, whereby the restocking procedure is established for each network node. A set of key performance indicators are obtained for measuring the performance of the system order transmission process: Inventory level; delivery times; resources workload, etc.

\section{MACTO-NET Case Description}

To describe the main characteristics of the case chosen for this paper, the blocks scheme proposed in the multi-plant network model is going to be used.

\subsection{Product Market Families (PMF)}

MACTO is an Spanish company that manufactures and sells machine tools, concretely, milling machines and milling centres (figure 2).

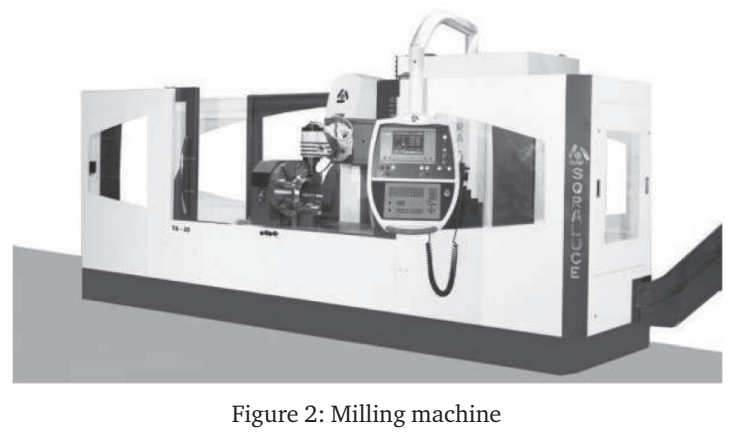

MACTO offers to the market a wide product catalogue that includes several families depending on the machine size, bed type (fixed or mobile) and column type (fixed or mobile). Machines can be standard or incorporate different customization levels. This characteristic allows MACTO to satisfy a wide range of customers belonging to sectors as diverse as aerospace, capital goods, railway, subcontractors or mold \& die manufacturers.

For the case study presented in this paper there has been selected the MC-1 product family basically constituted of small milling machines with fixed bed and fixed column type. This product is principally sold on three markets: Spain, Germany and Turkey. 


\subsection{Mass Customization Scenarios (MCS)}

MC-1 machine orders are done defining the main characteristics of the machine. Every machine has a basic configuration in which basic options such as the $\mathrm{X}$ axis travel length, bed area, or bed weight capacity, have to be chosen. Additionally, it is possible to choose optional equipments as, for example, the incorporation of an automatic tool changer.

One particularity of MC-1 machines is that the delivery time requested by the customer (10 weeks) is often lower than the production lead time (33 weeks). This situation generates the need to do launches to production against forecasts. Launched machines are formed by the most usual options chosen by the customers. When a customer order is received at MACTO, all the launched machines are checked to assign the order to that MC-1 machine already launched which better fits to the technical, delivery time, and cost requirements.

Depending on order attributes and the production stage of the machine that has been assigned to an order, modifications are introduced on the original machine to customize it to the specific customer requirements. Although these modifications can take place in different manufacturing process stages, due to the high level of product standardization, modifications usually take place in advanced stages of the process just before the customer visits MACTO's facilities to proceed to the product reception. Also, although strictly speaking the decoupling point (DP) it's not fixed, the high percentage of cases in which customization takes place in the same point allows to affirm that the DP appears in the same stage of the manufacturing process.

\subsection{Supply Network Topology (SNT)}

MACTO Supply Network (MACTO-NET) has three assembly plants, two of which are located in Spain and the third one in Hungary (figure 3 next page).

For supplying all the commercial components necessary for the machines assembly, MACTO has a Central Warehouse in Spain in which all the purchases are centralized (suppliers S1, S2, ..., Sn). Commercial components are distributed from the Central Warehouse to the assembly plants depending on the needs generated by the assembly planning system.

Assembly operations corresponding to MC-1 machines only take place in the assembly plant 1 of Spain (MACTO AP1-Spain) and in the assembly plant 3 in Hungary (MACTO AP3 Hungary). Initial assembly stages are done in MACTO AP3-Hungary which has its own supplier network in Hungary for the supplying of cast-iron (suppliers SS1 and SS2) and machined (supplier SM1) parts.

Once finished the initial assembly in MACTO AP3-Hungary, machines are transported to MACTO AP1-Spain where customization operations, electrical and mechanical assemblies, careened, tests and painting are done. Also at MACTO AP1Spain takes place the machine inspection and approval for the customer before shipping to the destination where final installation in house will be done. 


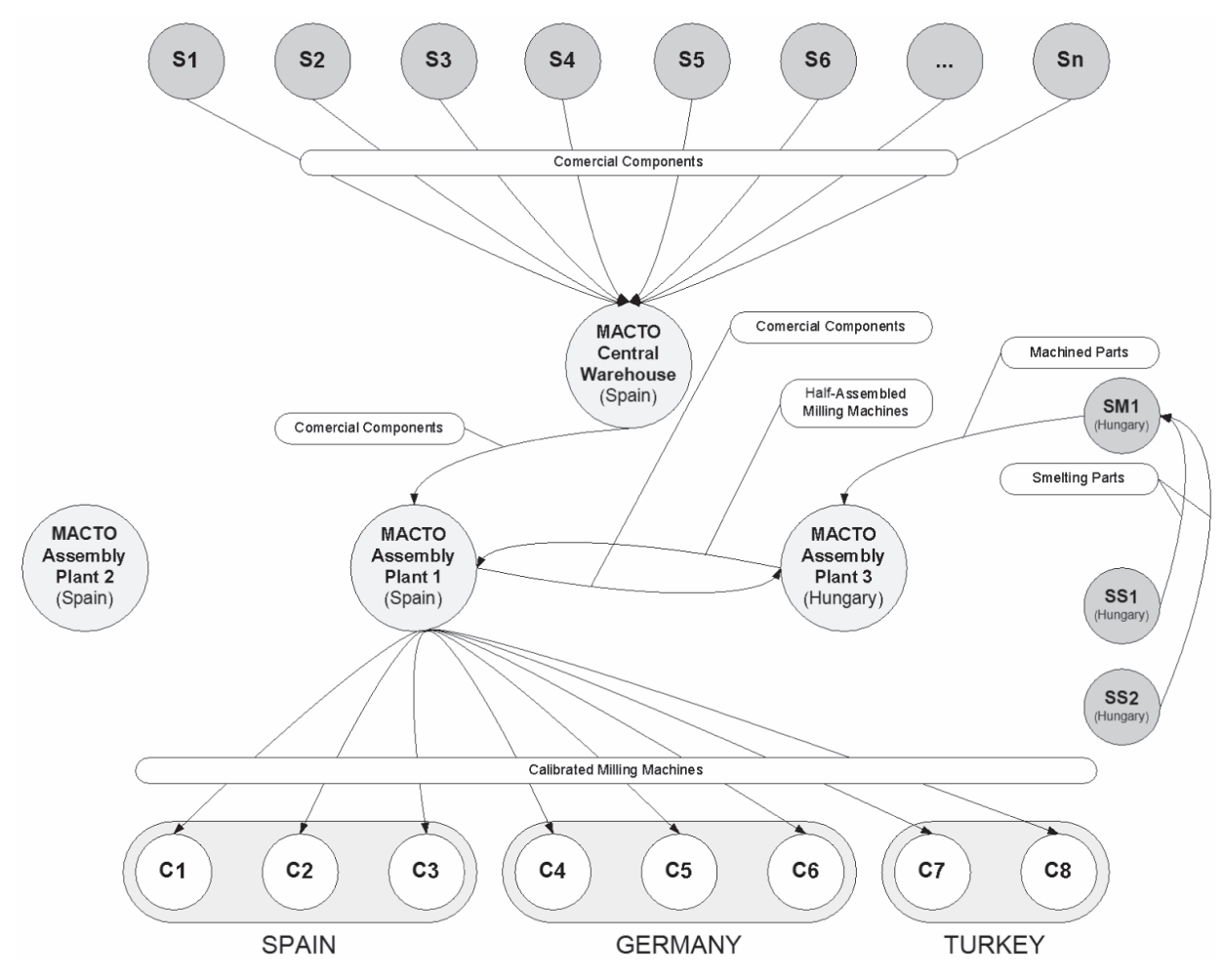

Figure 3: Present MACTO Supply Network Topology

\subsection{Product Process Structures (PPS)}

A simplified scheme of the Product Process Structure (PPS) corresponding to the MC-1 milling machines is showed in figure 4, next page. This structure is a generic representation of the different components that constitute the product family and the operations that are needed for its' manufacture.

The bed component is a purchased part which, in conjunction with other components as the $\mathrm{X}$ linear measurement scale, is assembled to form the subset $\mathrm{X}$ axis travel. In turn, this one is assembled together with the motorization set, Y axis travel and $\mathrm{Z}$ axis travel to constitute the subset half-assembled machine 1 , and so on to obtain the calibrated machine that is sent to the customer.

PPS components are matched from the available options of MACTO's catalogue for MC-1 machines family. Some of them are fixed, e.g. $Z$ axis travel, whereas others are selected by the customer, e.g. the length of the X axis travel, the type of cooling system or the number of swarf conveyors. As it has been mentioned previously, it exists an additional customization level that happens with machines launched to manufacture against forecasts. Later assignment of orders to machines in process require the introduction of modifications to adapt the machines to the specific 


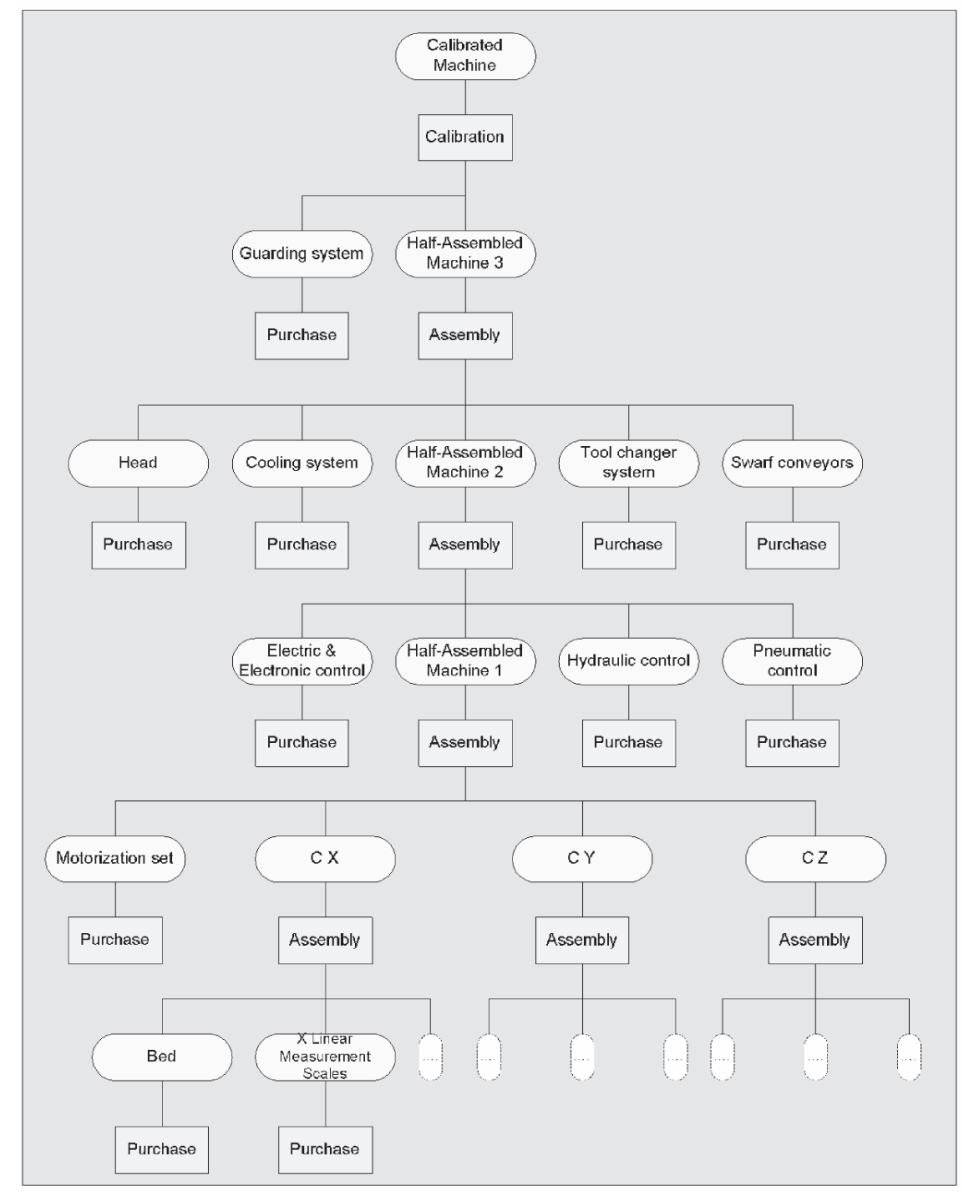

Figure 4: Simplified PPS for MC-1 product family

customer order characteristics. Generally, this adjustment is done in the assembly stages in which the subsets half-assembled machine 2 and half-assembled machine 3 are obtained.

All the machines belonging to the MC- 1 family have the same manufacturing process. Nevertheless, the PPS allows the incorporation of several process alternatives, e.g. the hydraulic control might be assembled in someone of the plants or bought like in the present case. These process alternatives are used in the definition of Customer Services Strategies (CSS) as it is detailed in the following point.

\subsection{Customer Services Strategies (CSS)}

MACTO has established several strategies that determine the form in which its Supply Network (MACTO-NET) is going to provide the product to the market. These strategies can be defined for every product family, customization type and market 
segment (countries, regions, points of sale, etc.). Depending on the impact that they have on the supply network, strategies are named as local or global.

\subsubsection{Local Customer Services Strategies (L-CSS)}

This type of strategies permits MACTO to define the supplying system for the commercial components, cast-iron, machined parts, etc, for each plant that is involved in the manufacturing process of the MC-1 machines. Every purchased component has assigned its own supplying management procedure depending on different characteristics as price, delivery time, consumption pace, etc. Cast-iron and machined parts that have great volume and high cost are provided against forecasts generated from consumption statistics and sales estimations made by the commercial area. On the other hand, commercial components of regular consumption are managed using diverse replenishment systems as order point, min-max, kanban, etc.

\subsubsection{Global Customer Services Strategies (G-CSS)}

This type of strategies allows MACTO to defines the way that the supply network (MACTONET) is going to provide the product demanded to the market. This is done by assigning each machine manufacture stage to one network plant depending on location, production costs, technological competencies and market proximity. Nowadays, MACTO is evaluating/comparing the performance of two different strategies to approach the target market:

\section{CSS0: Present Customer Service Strategy}

This strategy, already mentioned in section 5.3, is the one that MACTO uses nowadays. Half-assembled machine 1 assembly stages are carried out at MACTO AP3Hungary based on the commercial components that are sent from the MACTO Central Warehouse in Spain and the cast-iron and machined parts that are provided by the Hungarian suppliers. Once finished the subset half-assembled machine 1, it is shipped to MACTO AP1-Spain where it proceeds to the customization, assembly ending, acceptance and shipping to the customer.

Transport cost increase associated with this strategy justifies itself for the lower staff cost of MACTO AP3-Hungary and the small technical difficulty of the operations taken place in the machine at this assembly stage. Furthermore, Hungarian suppliers of cast-iron and machined parts provide a quality product with competitive costs.

\section{CSS1: New Customer Service Strategy}

Nowadays MACTO is evaluating the possibility of introducing a new global strategy. It consists on maintaining the same strategy for the Spanish market (G-CSSO), but completely assembly the whole MC-1 machines family at MACTO AP3-Hungary for the German and Turkish markets (figure 5). 


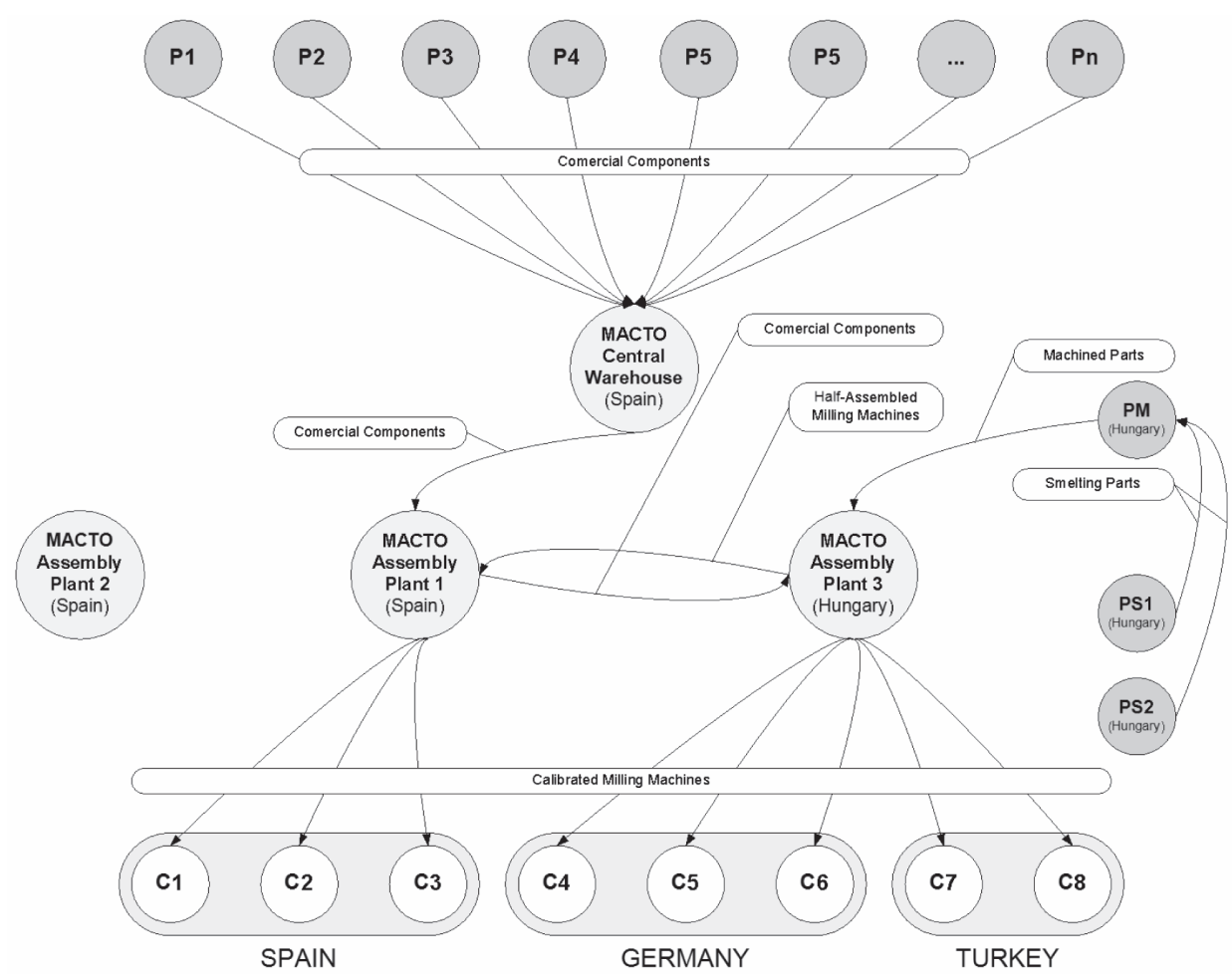

Figure 5: New MACTO-NET Customer Service Strategy

The materialization of this strategy requires a necessary investment in the Hungarian plant facilities and to increase the technological training of its staff. This investment has to be compared with the production cost reduction due mainly to smaller Hungarian staff costs and smaller transport costs for the proximity of the plant to German and Turkish markets.

\section{MACTO-NET Simulations}

MACTO wanted to know the potential benefits of implementing the new customer service strategy CSS1 over its supply network performance metrics. To do so, the simulation tool developed, that implements MACTO-NET conceptual model associated with the multi-plant network model for a mass customisation strategy, was used.

The results obtained from the simulation were used to analyze the viability of CSS1 strategy and therefore its potential real implementation. Following sections describe the type of simulations developed as well as the analysis of the outcomes achieved.

\subsection{Simulation Runs}

Two main simulation runs have been executed that correspond to the strategies that 
MACTO wants to evaluate/compare (see section 5.5.2); Present Customer Service Strategy - CSS0, and New Customer Service Strategy - CSS1.

Both simulations share the same inputs with respect to: (i) Customer orders - 66 milling machines MC-1, obtained from real annual sales sequence, being the configuration of each demanded machine determined from real customer orders statistics; (ii) Local strategies (L-CSS), meaning that replenishment, restocking procedures, were the same for each plant in both simulation runs; (iii) And also neither components purchasing costs nor supply network management costs were modified in both runs.

\subsection{Simulation Interface}

In order to visualize the results of the simulations an interface has been designed (figure 5).

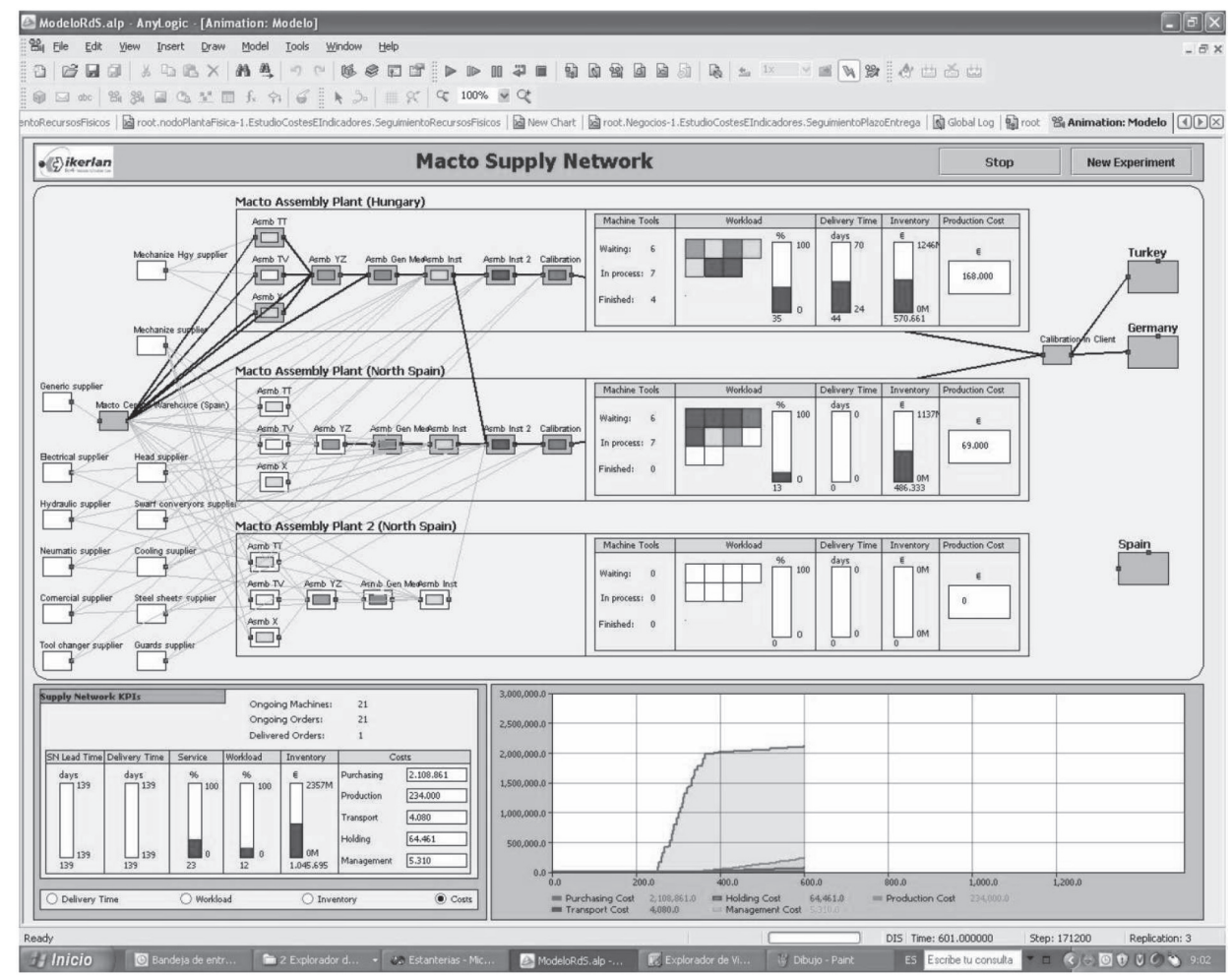

Figure 5: Simulator Interface

The interface shows the topology of the supply network (MACTO-NET); suppliers in the left hand, the three assembly plants in the centre of the image, and customers in the right hand. Each plant is divided into the different MC-1 machines assembly stages. The lines that link plant stages with suppliers and customers show the complexity of MACTO-NET material and information flows that are taking place at each simulation time step. 
Additionally the simulator interface shows different supply chain Key Performance Indicators - KPIs (Lambert 2004) that register some performance metrics of MACTO-NET and each of its plants, under both CSSs, in delivering MC-1 machines to the customers. The KPIs proposed for MACTO-NET case study are the following ones.

- MACTO-NET Lead Time:

The time period that takes the manufacturing/assembly of MC-1 machines along MACTONET also including components suppliers lead times.

- Lead Time:

The time period needed for each plant to manufacturing/assembly MC-1 machines also including components suppliers lead times.

- Delivery Time:

MACTO-NET time period needed to serve customer their orders starting from the moment each order becomes attached to a specific customer in the production process.

- Service: Percentage of customer orders period fulfillment achieved by MACTO-NET.

- Workload:

Ratio between the manufacturing/assembly capacity used and the manufacturing/assembly capacity available at MACTO-NET and at each of the plants that intervene in the manufacturing/assembly of MC-1 machines.

- Inventory:

Economic value of the mean inventory level at MACTO-NET in general and at each of its plants.

- Total Cost:

The total cost of manufacturing the 66 machines. This cost is also presented broken down in the form of Purchasing Costs, Production Costs, Transport Costs, Holding Costs and Management Costs.

- Machine Cost:

Cost of fabrication of each MC-1 machines.

KPIs values are presented both numerically, as well as in a graphical form (min, max and mean value), and Also the KPI can be displayed in the form of a time series.

\subsection{Simulation Outcomes Analysis}

Figure 6 shows the results of MACTO-NET simulation runs for CSSO strategy (Present Customer Service Strategy). 


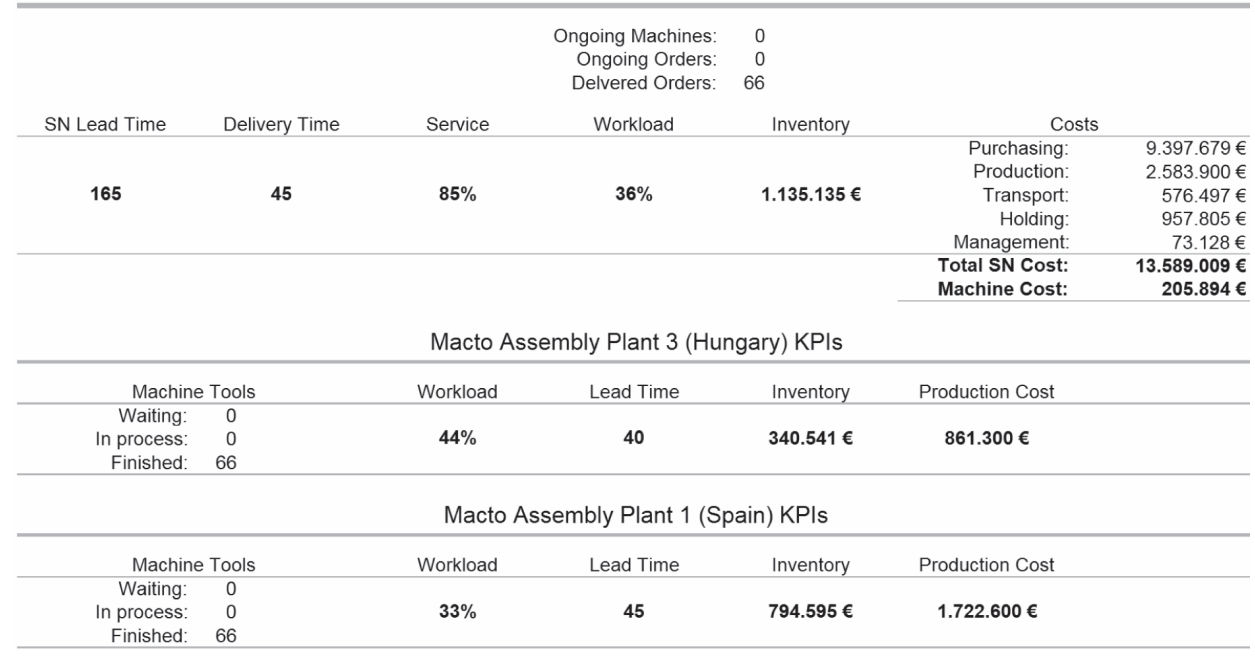

Figure 6: CSS0 - Simulation 1 Outcomes

Figure 7 shows the results of MACTO-NET simulation runs for CSS1 strategy (New Customer Service Strategy under evaluation).

CSS1 - Macto Supply Network KPIs

\begin{tabular}{|c|c|c|c|c|c|c|}
\hline & & & $\begin{array}{l}\text { Ongoing Machines: } \\
\text { Ongoing Orders: } \\
\text { Delvered Orders: }\end{array}$ & $\begin{array}{r}0 \\
0 \\
66\end{array}$ & \multirow[b]{2}{*}{ Cost } & \\
\hline \multirow[t]{3}{*}{ SN Lead Time } & Delivery Time & Service & Workload & Inventory & & \\
\hline & & & & & Purchasing & $9.397 .679 €$ \\
\hline & & & & & Production & $1.866 .150 €$ \\
\hline \multirow[t]{6}{*}{150} & 40 & $92 \%$ & $36 \%$ & $1.031 .941 €$ & Transport & $345.898 €$ \\
\hline & & & & & Holding & $870.732 €$ \\
\hline & & & & & Management & $73.128 €$ \\
\hline & & & & & Total SN Cost: & $12.553 .587 €$ \\
\hline & & & & & Machine Cost: & $190.206 €$ \\
\hline & & \multicolumn{3}{|c|}{ Macto Assembly Plant 3 (Hungary) KPIs } & & \\
\hline \multicolumn{2}{|c|}{ Machine Tools } & Workload & Lead Time & Inventory & Production Cost & \\
\hline Waiting: & 0 & & & & & \\
\hline \multirow{3}{*}{$\begin{array}{l}\text { Finished: } \\
\text { Frocess: }\end{array}$} & 0 & $88 \%$ & 40 & $1.002 .703 €$ & $1.579 .050 €$ & \\
\hline & 66 & & & & & \\
\hline & & Macto & sembly Plant 1 (S & ain) $\mathrm{KPIs}$ & & \\
\hline \multicolumn{2}{|c|}{ Machine Tools } & Workload & Lead Time & Inventory & Production Cost & \\
\hline Waiting: & 0 & & & & & \\
\hline In process: & 0 & $17 \%$ & 40 & $132.432 €$ & $287.100 €$ & \\
\hline Finished: & 66 & & & & & \\
\hline
\end{tabular}

Figure 7: CSS1 - Simulation 2 Outcomes

Comparing CSSO and CSS1 simulation results, an important reduction in the total cost for each MC-1 machine fabricated at MACTO-NET can be highlighted; from 205.894 € with CSS0 strategy to $190.206 €$ with CSS1 strategy. For all the 66 MC1 machines, the improvement goes beyond the 1 million $€$, in fact a 7,62\% cost reduction. Taking into account that purchasing and management costs were kept identical for both simulation runs, the $69 \%$ of the improvement is imputable to 
fabrication costs reduction derived from lower personnel workforce costs, and the $22 \%$ transport costs reduction is due to the proximity of manufacturing/assembly plants to the customers locations. Also, a decrease of inventory level is registered because of the 15 days Lead Time reduction at MACTO-NET, what means a 9\% costs improvement in stock management activities.

\section{Conclusions}

The use of some simulation techniques and tools constitutes a valuable decision support approach in the strategic design of supply networks with mass customisation challenges.

The knowledge of the costs, lead times, and inventory levels are fundamental to take decisions about the potential impact of alternative multi-plant network configurations, the mix of products that should be fabricated at each supply network plant, or for instance the possibility of creating a new warehouse to improve customer service. Risky decisions related to the type of products that are going to be served to each market, the strategy needed for each of them, the level of customisation offered, the location of the decoupling point for each order in the supply chain, etc, require tools that can take a systemic and dynamic perspective and capabilities for processing high amounts of databases.

According to MACTO-NET managers, the conceptual model and simulation decision support tool developed in this research facilitates the definition of demand driven responsive and efficient multi-plant networks based on customer orders, and provides valuable information for complex decision making problems, incorporating the possibility of evaluating alternative supply network customisation strategies and KPIs to measure the performance impact of the different what-if scenarios.

\section{References}

Anderson, D.M., \& Pine II, B.J. (1997). Agile Product Development for Mass Customization, Niche Markets, JIT, Build-to-Order, and Flexible Manufacturing. Burr Ridge, IL, Irwin/McGraw-Hill Professional Publishing.

Berry, W.L., \& Hill, T. (1992). "Linking systems to strategy." International Journal of Operations and Production Management, Vol.12 (10), 3-15.

Coyle, R. (1977). Management Systems Dynamics. Wiley, NY.

Douglas M. Lambert (2004). Supply Chain Management: Processes, Partnerships, Performance. The Supply Chain Management Institute, ISBN-10: 0975994905, ISBN-13: 978-0975994900.

Fisher, M.L. (1997). "What is the right supply chain for your product?." Harvard Business Review, Vol. 75 (2), 105-16.

Fowler, A. (1998). "Operations management and systemic modeling as a framework for BPR." International Journal of Operations and Production Management, Vol.18 (9/10), 1028-1056. 
Hayes, R.H., \& Wheelwright, S.C. (1979). "Link manufacturing processes and product life cycles." Harvard Business Review, Vol. 57 (1), 133-140.

Kidd, P.T. (1994). Agile Manufacturing - Forging New Frontiers. Wokingham: Addison-Wesley.

Mason-Jones, R., Naylor, B., \& Towill, D.R. (2000). "Lean, agile or leagile? Matching yor supply chain to the marketplace." International Journal of Production Research, Vol. 38 (17), 4061-70.

Pritsker, A.A. (1997). "Modeling in performance-enhancing processes." Operations Research, Vol.45 (6), 797-804.

Saiz E., Castellano E., Besga J.M., Zugasti I., \& Eizaguirre F. (2006). Global and Flexible Supply Networks Modelling and Simulation. In Thorsten and Wolfgang Kersten (Eds.) (2006) Operations and Technology Management II: Complexity Management in Supply Chains. Concepts, Tools and Methods. Erich Schmidt Verlag, Berlin.

Selldin, E. (2005). Supply chain design. Conceptual models and empirical analyses, PROFIL 22, Ph.D. dissertation, Linköping Institute of Technology, Linköping, Sweden.

Shapiro, J. (2001). Modeling the Supply Chain. Duxbury Press, North Scituate, MA.

Sterman, J. D. (2000). Business Dynamics: Systems Thinking and Modeling for a Complex World. NY: McGraw-Hill Higher Education.

Vereecke, A., \& Van Dierdonk, R. (1999). Design and Management of international Plant Networks: Research Report. Academia Press, Gent, Belgium.

Yin, R. (1994). Case study research: Design and methods (2nd ed.). Beverly Hills, CA: Sage Publishing. 


\title{
A cost management viewpoint to mass customizable services: a review and considerations
}

\author{
Author: Matti Sievänen
}

\begin{abstract}
Mass customization has been quite a popular topic since the early 90s. However, a deeper understanding of service mass customization is still missing. How should services be mass customized and can methods like modularization and service platforms be used? Furthermore, what kinds of cost management tools should be used? The aim of this paper is to review the literature to find out how services can be mass customized and to give some suggestions as to what cost management accounting tools should be used and how they should be used in the case of mass customized services. Activity-based costing is presented as one way to calculate costs of mass customized services. The cost object can be activity but, similarly, customers would be suitable cost objects.
\end{abstract}

\section{Keywords}

Mass customization, Services, Cost management

\section{Introduction}

Mass customization has been defined as the ability to design and manufacture customized products at mass production efficiency and speed. Customization and mass customization are often justified because different customers can give different values to the same product. Customers require different features and performance and one size does not fit all anymore. There are numerous examples of different mass customized products, in which Dell computers might be the most frequently used. However, most of the studies concentrate on consumer goods, and have typically either a marketing or an operations view, although there are some studies that have focused on management accounting. The results are contradictory; some writers suggest sophisticated management accounting systems, like activity-based costing, and others cannot find them justifiable.

Similarly, services are a popular subject at the moment. The share of services has been increasing and it accounts for over $60 \%$ of gross national product worldwide. In service operations management literature, customization has been recognized. Services can be classified, for example, as professional services, service shops, and mass services based on the degree of customization. It can be seen that, in the 
manufacturing business, resources are used to produce one unit of goods and in the services business, resources are consumed by the client in the fruition of the performance connected with the service. Moreover, service operations management studies point out important factors like contact time, source of value added, etc., which can be used as a drivers in a management accounting system.

The aim of this paper is to review the literature and determine some suggestions for what cost management accounting tools should be used, and how they should be used, in the case of mass customizable services. The paper is structured as follows. First, an introduction to mass customization is given. This is followed with a short literature review of services. Next is a discussion of what mass customization could be, in a service context. Thirdly, a short discussion of management accounting tools, and their connection to mass customization, and customization in general, is given. Finally, some conclusions are made and new directions for further research are suggested. The focus is on how services can be mass customized and what management accounting tools should be used with these mass customized services.

\section{Mass Customization}

The term "mass customization" was first introduced in a book "Future Perfect" by Davis (1987). It was defined as a way to manufacture one-of-a-kind products, based on customer specifications, without sacrificing scale economics. Mass customization became more popular when Pine (1993) published his book "Mass Customization: The New Frontier in Business Competition". Pine defined mass customization as the ability to design and manufacture customized products at mass production efficiency and speed. Furthermore, he defined mass customization as a process by which companies provide variety and customization through flexibility and quick response. The goal was that almost anyone would find exactly what he or she wanted without paying a penalty in price. However, the definition of mass customization remained somewhat unclear. Moreover, the literature is missing good conceptual boundaries for mass customization (Duray et al. 2000). Some scholars include variety management, locate-toorder strategy, customizable (Logman 1997) and self-customizing (Åhlström \& Westbrook 1999) products as mass customization. Personally, I take quite a strict view on mass customization and require customer involvement to take place during the production process. Thus, the view is "full" mass customization - customers are involved in specifying the product configuration and the "mass" in mass customization is obtained primarily by standardized components and product modularity within standardized processes (Åhlström \& Westbrook 1999; Duray et al. 2000). This view is quite similar to Customized Standardization as it is defined by Lampel and Mintzberg (1996): the utilization of product modularity and configurability to assemble customized products with standardized design and standardized components. Piller (2004) has similar ideas of mass customization. Customer co-design and a fixed solution space (stable 
processes and product architectures), in which all operations are performed, are key aspects in mass customization. Fixed solution space ensures that a product or service can be produced at a cost and efficiency close to that of mass production and does not require a switch in an upper market segment.

The path of industrial development started with craft manufacturing, in which every product could be customized. With the industrial revolution, and especially with Ford Model-T production, the era of mass production began. Mass production was followed by continuous improvement, at least in some industries. Mass customization is presented as current paradigm (Pine 1993). However, this path can be questioned many ways. There are still companies in every phase and the shift to mass customization can be from craft manufacturing as well as from mass manufacturing (Duray 2002). Similarly, it is not clear if continuous improvement has ever been the leading paradigm.

The requirements for mass customization manufacturing systems involve product design issues as well as the production and product configuration processes (Blecker \& Friedrich 2007). The often-mentioned prerequisites for product and process design are commonality and modularity (Pine et al. 1993; Feitzinger \& Lee 1997; Anderson \& Pine 1998; Duray et al. 2000; Blecker \& Abdelkafi 2006). With component and process commonality and product and process modularity, the benefits of economies of scale and scope, as well as the goals of reusability and differentiation, can be reached simultaneously (Blecker \& Friedrich 2007). These design requirements may help in reducing internal process variety, which is said to improve flexibility, responsiveness and quality as well as reducing costs (Anderson \& Pine 1998). In line with these requirements, Blecker \& Abdelkafi (2006) define seven sequential strategies of complexity reduction and variety management for mass customization (Figure 1). The authors have identified component families, component commonality, and process commonality as steps below product and process modularity. Implementing the first three stages does not imply the ability to achieve efficient mass customization, but product and process modularity are required to complement the minimum variety management sequence for an efficient and effective implementation of mass customization. After grouping components into families, according to similarity of features or manufacturing processes, in order to decrease the total setup time needed, component commonality can be increased between different products to reduce the number of internal parts needed to create large product variety. Component commonality leads to increased process commonality that signifies the ability to produce product variety with a limited number of processes. 


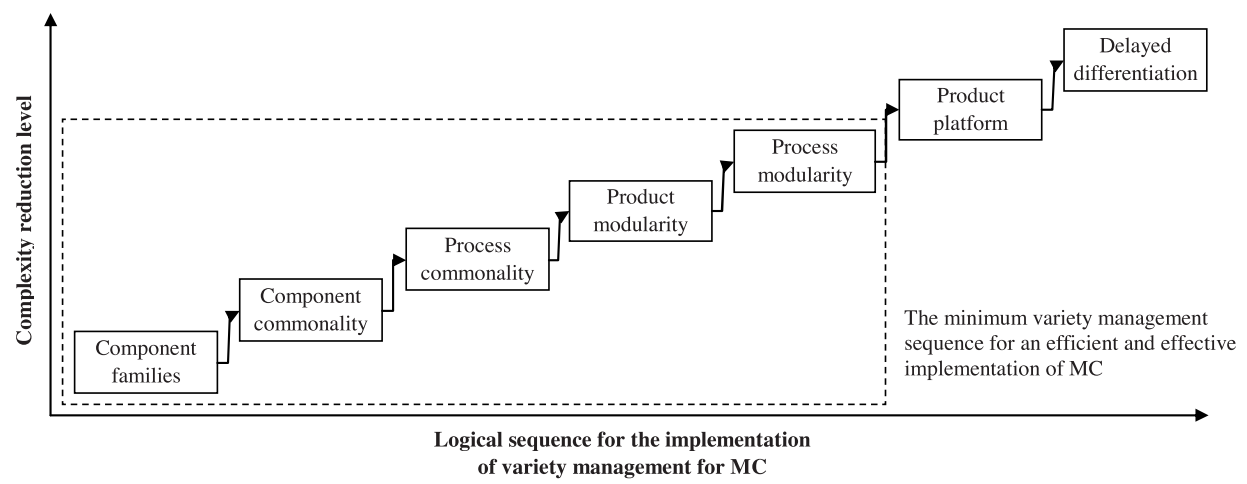

Figure 1: Complexity-based variety management for MC (Blecker \& Abdelkafi 2006).

When products are modular, the modules can be manufactured separately and simultaneously to decrease the production lead times and to localize possible problems more easily (Feitzinger \& Lee 1997). Product modularity makes it possible to mix and match interchangeable modules that have standardized interfaces and, thereby, create product variants efficiently (Blecker \& Abdelkafi 2006). Under ideal conditions, a certain module represents a certain functionality of the product and hence, the effort required to make a functional change to a product can be directed to a single module (Ulrich \& Eppinger 2004).

Product modularity is the key for the achievement of process modularity, product platforms and delayed differentiation (Blecker \& Abdelkafi 2006). Process modularity refers to turning processes into modules and ensuring the rapid and flexible integration between these modules with an adequate architecture (Pine et al. 1993). In other words, regardless of the end product, the process remains generally the same, except that not every part of the process is necessarily in use. According to Blecker \& Abdelkafi (2006), the product and process module standardization should lead to a platform strategy. A product platform can be described as a set of core modules that is implemented in different product variations. With the platform, the life cycle of a product family can also be defined; instead of modifying only one particular module, the redesign of the platform affects the whole product structure and generally results in a new product family being introduced.

The last stage, delayed differentiation, is referred to as an important way to standardize the production process and thereby reduce work-in-process and production lead time and to increase production efficiency. Blecker \& Abdelkafi (2006) regard delayed differentiation as delaying the point where the product variants assume their unique forms in the production process by redesigning products and processes. On the other hand, delayed differentiation can be also seen as delaying some variation of the product until the final stages of manufacturing or until it is sent to the dealer (Selladurai 2004). 
The mass customization production system generally consists of push and pull subsystems separated by the customer order decoupling point (Blecker \& Abdelkafi 2006). Before the customer-driven pull subsystem and the order penetration take place, modules are produced and outsourced components are delivered in an efficient, standardized manner in the push subsystem.

The positioning of the decoupling point is a crucial matter for companies, since it defines the degree of customer integration and the range of customization possibilities and it usually also has a direct effect on delivery times. With delayed differentiation, however, dependence on the decoupling point can be reduced.

\section{Mass Customizable Services}

If mass customization is not well defined, neither are the services. This debate has been ongoing for at least a half century and distinction between services and physical goods has dominated the discussion (Vargo \& Lusch 2004). In most cases, services are defined by using the IHIP (inseparability, heterogeneity, intangibility, and perishability) characteristics to point out their difference from physical goods (Edvardsson et al. 2005). However, any of these characteristics can be questioned. For example inseparability, simultaneous production and consumption are not always realized in a case of financial services. Similarly, many services consist of tangible features; for example, a restaurant service without food or drink would be useless. The most important characteristic regarding mass customization; namely, heterogeneity - the inability to standardize the output of services - will be discussed later.

As can be seen, these commonly used characteristics cannot define services exclusively. However, Rathmel (1966) has made a clear distinction between a good and a service. "....consider a good to be noun and a service a verb... The former is an object, an article, a device, or a material ... where as the latter is a deed, a performance, or an effort. When a good is purchased, the buyer acquires an asset; when a service is purchased, the buyer incurs an expense." There are two main points that can be found in this definition. First, a service is an activity. It can bee seen as doing something for someone. And secondly, it is an expense rather than an asset. Thus, used services are hard to sell. Furthermore, for companies, this means that services are usually expenditures, whereas a good can be treated as a capital investment. Moreover, this definition is not exclusive, but rather, a continuum can be seen between goods and services.

There are interesting aspects of services which connect them to customization. Inseparability can be connected to co-creation/co-design, one of the key elements of mass customization (Piller 2004). In (mass) customization, a customer always takes part in defining processes and therefore inseparability is part of customization. Similarly, as service is a deed or an action, customization can be seen as doing something on behalf of someone. If a standard product is not suitable, it has to be modified either by the user or by someone else. The modification (customization) 
can be done by the manufacturer, a dealer, or an outsider. In any case, it does not matter; delivering a customized product is always a service.

It can be asserted that service is always customized because of the heterogeneity characteristics of services. Quite often services are customized, however standardized services can also be found. Similarly, just as manufacturing has been automated to increase profitability, certain services are automated; for example, in the way that ATMs have replaced the personal service we used to get at banks. Similarly, many services that used to require personal service can be done through the internet, like leaving an insurance claim, or buying an airplane ticket, to mention a few. It is also argued that standardized service delivery increases quality (Zeithaml et al. 1988). However, it is not clear what "better quality" means and we should rather bediscussing a stable quality, which can be met with standardized process. Similarly, standardization and automation can lower our expectations, allowing them to be met.

Sundbo (2002) presents Service Modulization, the idea of combining the advantages of standardization and customization, productivity and customer satisfaction. For service firms, for example, this could mean a standardized production system (back office) and an individualized delivery system (front office). A typical restaurant can be seen as a mass customized service. You can order from a limited menu option of choices, which are manufactured and maybe combined in a kitchen and then delivered by a waiter/waitress who can personalize the service. Silvestro et. al (1992) present a similar idea when they make an analogy between service processes and the production process model in manufacturing operations literature. They present three service processes, which are professional services, service shops, and mass services. Mass customized services are most often produced in service shops, similar to mass customized products in job shops or disconnected lines (Hayes \& Wheelwright 1979; Sievänen 2002).

It is of interest to examine if services develop in the same way as manufacturing, from craft manufacturing towards standardization and mass production (Sundbo 2002) and finally to mass customization. Sundbo (2002) presents evidence that services became less standardized during the period from 1992-97, based on surveys conducted in Denmark. However, personal experience and neoclassical productivity-oriented economics do not support this. More and more services are automated and are referred to as self-services. In any case, traditional manufacturing did not come purely from mass manufacturing to mass customization, and neither do services.

An interesting question is whether the complexity of services can be managed in the same ways as "product manufacturing" (Blecker \& Abdelkafi 2006). What does a component mean in a case of service? In a physical product, a component is quite obvious, but what does it mean in a case of service if service is defined as an activity? Similarly, what is a module? When service is an activity, how activity is defined 
is important. In activity-based costing literature, micro- and macro-activities can be found (Turney \& Stratton 1992) as well as tasks, activities, and processes (Sievänen \& Tornberg 2002). If activity is defined as a set of tasks, then a task is a component and an activity is a module. With the same analogy, service can be seen as a process, which consists of a set of activities. Similarly, these service components and modules have resemblance to components and modules of physical products (Tiihonen et al. 2007). They can all be included, available optionally, or not available. Furthermore, some service elements can be parametric. However, can these service elements be commoditized? At least to some extent, back office elements can be standardized, as well as certain front office elements.

The first four steps of strategies of complexity reduction can be fulfilled. The fifth one, process modularity, becomes problematic. We have defined service product as a process, so we have to now take a slightly different view. In physical goods manufacturing, the modules can be manufactured separately and simultaneously, to decrease the production lead times. However, is this possible with a service? Can some modules be produced separately and used when needed? If we define services using the IHIP characteristics, the answer is no. Inseparability, in particular, makes this impossible. However, as mentioned earlier, in some services there can be features that do not fulfill inseparability characteristics. So, if there are service elements that are not time dependent, process modularity is to a certain extent possible. Anyway, we come back to front and back office definitions, back office processes can be modular but with front office processes, modularity is hard to achieve.

The sixth step, the product platform, is quite complicated with this service definition as well. Are there two different levels, service and service platform, combining tasks and activities? Also, does the platform define interfaces between modules (activities)? This is a bit complicated because it is hard to distinguish product and platform levels. However, although some services can be managed as service platforms, whether these services should be seen as different services, or variations of the same service, is not clear. This question remains unanswered in this study.

The seventh step, delayed differentiation, is even harder to achieve in a service context. It is difficult to conceptualize that first, a standard service is given and then afterwards, it is configured based on a customer requirement. However, the definition of delayed differentiation is not clear, thus nothing exclusive can be said (Mertanen \& Sievänen 2007). Overall, it seems that the presented framework of managing complexity and variety can be used, with certain limitations, in a case of mass customized services.

\section{Mass Customized Services and Cost Management Tools}

If we take the definition of service as an activity as a given, then there is an obvious costing method for services, named activity-based costing ( $A B C)$. In $A B C$, product costs can be determined by assigning the costs of activities to a product that employs 
those activities. The $\mathrm{ABC}$ framework views activity as a task that is undertaken to make or deliver a product or a service. ABC should be especially useful when expenses in indirect and support resources are high, and a diversity exists in products, customers, and processes (Kaplan \& Cooper 1998). In a case of mass customization there is certainly diversity in products and customers. As well, processes differ, even though there is an aim to standardize these. In a case of mass customized services, products are defined as processes so the diversity comes from there.

It is not agreed that investment to $\mathrm{ABC}$ is justified in mass customization manufacturing. It is asserted that if companies use advanced manufacturing technology, the information requirements can be met with less sophisticated costing systems, like one with multiple cost pools and unitlevel cost driver (Abernethy et al. 2001). There are other opinions as well, but the problem lies behind of the definition of $\mathrm{ABC}$. It is not clear what an $\mathrm{ABC}$ system is and what it is not. Rather than speaking of $\mathrm{ABC}$, we should concentrate on features and then think if the costing system fulfills these features (Karjalainen 1997; Mévellec \& Sievänen 2003). If a costing system is used only for product costing purposes, these requirements can be met with less sophisticated costing systems than $\mathrm{ABC}$, like responsibility center costing. However, if cost accounting systems need to be able to offer more than merely giving product cost information, the investment to $A B C$ can be justified. The ABC system has been claimed to contain two dimensions: the cost view and the process view. It is further argued that the emergence of a process view could extend ABC beyond product costing to process improvement (Turney 1991).

Companies have difficulties in knowing the cost of services (Anderson \& Narus 1995). It is said that this might be a result of costs structures in services, with more fixed than variable costs (Mathieu 2001). However, in this kind of environment, with high indirect and support expenses, ABC should be the most suitable choice. Similarly, it has been indicated that the customer, rather than the product or service, would be a suitable cost object (Carú \& Cugini 1999). This also supports the use of $A B C$ because, in $A B C$, there can be multi-level cost drivers.

\section{Conclusions}

In this paper, mass customized services were studied based on literature review. As was shown, services can be mass customized as well as products. However, the problem lies in the definition of services, which is not clear. In this study, service is defined as an activity - a deed, a performance, or an effort. Using this definition, a task or micro activity can be seen as a component of service. Furthermore, the study shows that the same complexity management tools used for products can also be used for services. In this sense, services do not differ that much from products.

When considering cost management tools, it can be said that $\mathrm{ABC}$ can be a useful method for determining the cost of mass customized services. However, how ABC should be applied is not answered. Are there special factors in ex-ante and ex-post 
calculations that should be taken into account? Also, it would be educating to learn how cost management tools are used in practice, knowing that $A B C$ is in fact not widely used even in manufacturing environments (Innes et al. 2000). Mass customizable services are part of a new environment that requires new applications of $\mathrm{ABC}$ and other management accounting tools.

This paper is a short literature review and thus falls short in many ways. However, it gives some ideas about how mass customized services can be developed and managed. To be able to manage services, these have to be defined and service processes have to be understood. Further research is needed, even at the conceptual level, as well as at the practical level.

\section{References}

Abernethy, M. A., A. M. Lillis, et al. (2001). Product diversity and costing system design choice: field study evidence. Management Accounting Research 12(3): 261-279. Anderson, D. M. and B. J. Pine (1998). Agile product development for mass customization: how to develop and deliver products for mass customization, niche markets, JIT, build-to-order and flexible manufacturing. New York, NY, McGraw-Hill.

Anderson, J. and J. Narus (1995). Capturing the Value of Supplementary Services. Harvard Business Review.

Blecker, T. and N. Abdelkafi (2006). Complexity and variety in mass customization systems: analysis and recommendations. Management Decision 44(7): 908-929.

Blecker, T. and G. Friedrich (2007). Guest Editorial: Mass Customization Manufacturing Systems. IEEE Transactions on Engineering Management 54(1): 4-11.

Carú, A. and A. Cugini (1999). Profitability and customer satisfaction in services - an integrated perspective between marketing and cost management analysis. International Journal of Service Industry Management 10(2): 132-156.

Davis, S. (1987). Future Perfect, Addison-Wesley.

Duray, R. (2002). Mass customization origins: mass or custom manufacturing? International Journal of Operations \& Production Management 22(3): 314-328.

Duray, R., P. T. Ward, et al. (2000). Approaches to mass customization: configurations and empirical validation. Journal of Operations Management 18(6): 605-625. Edvardsson, B., A. Gustafsson, et al. (2005). Service portraits in service research: a critical review. International Journal of Service Industry Management 16(1): 107-121.

Feitzinger, E. and H. L. Lee (1997). Mass customization at Hewlett-Packard: The power of postponement. Harvard Business Review 75(1): 116-121.

Hayes, R. H. and S. C. Wheelwright (1979). Link manufacturing process and product life cycles. Harvard Business Review 57(1): 133-140.

Innes, M., F. Mitchell, et al. (2000). Activity-based costing in the U.K.'s largest companies: a comparison of 1994 and 1999 survey results. Management Accounting Research 11: 349- 362 . 
Kaplan, R. S. and R. Cooper (1998). Cost \& Effect - Using Integrated Cost Systems to Drive Profitability and Performance. Boston, Harvard Business School Press.

Karjalainen, J. (1997). Advances in cost accounting of some Finnish companies in the early 1990's : is the slow pace acceptable? Espoo, TKK.

Lampel, J. and H. Mintzberg (1996). Customizing Customization. Sloan Management Review 38(1): 21-30.

Logman, M. (1997). Marketing mix customization and customizability. Business Horizons 40(6): 39-44.

Mathieu, V. (2001). Service strategies within the manufacturing sector: benefits, costs and partnerships. International Journal of Service Industry Management 12(5): 451-475.

Mertanen, M. and M. Sievänen (2007). A practical approach to mass customization - Lessons learned from Finnish machine construction. The 2007 World Conference on Mass Customization and Personalization, MCPC 2007, Boston, USA.

Mévellec, P. and M. Sievänen (2003). ABC, Process-Oriented Systems: a comparative study. 6th International Seminar on Manufacturing Accounting Research, Twente, The Netherlands.

Piller, F. T. (2004). Mass Customization: Reflections of the State of the Concept. International Journal of Flexible Manufacturing Systems 16(4): 313-334.

Pine, B. J. I. (1993). Mass customization: the new frontier in business competition. Boston, Harvard Business School Press.

Pine, B. J. I., B. Victor, et al. (1993). Making mass customization work. Harvard Business Review 71(5): 108-119.

Rathmell, J. M. (1966). What is meant by Services? Journal of Marketing 30(October): 32-36.

Selladurai, R. S. (2004). Mass customization in operations management: oxymoron or reality? Omega 32(4): 295-300.

Sievänen, M. (2002). What is customization? 9th International Conference European Operations Management Association, Copenhagen, Denmark.

Sievänen, M. and K. Tornberg (2002). Process-based costing: The best of activitybased costing. AACE International Transactions: CS151-CS156.

Silvestro, R., L. Fitzgerald, et al. (1992). Towards a Classification of Service Processes. International Journal of Service Industry Management 3(3): 62-75.

Sundbo, J. (2002). The Service Economy: Standardisation or Customisation? Service Industries Journal 22(4): 93-116.

Tiihonen, J., M. Heiskala, et al. (2007). Applying the Configuration Paradigm to Mass-Customize Contract Based Services. The 2007 World conference of mass customization and personalisation, MIT Cambridge/Boston, USA.

Turney, P. B. B. (1991). Common Cents - The ABC Performance Breakthrough. Hillboro, Cost Technology.

Turney, P. B. B. and A. J. Stratton (1992). Using ABC to Support Continuous Improve- 
ment. Management Accounting 74(3): 46-50.

Ulrich, K. and S. D. Eppinger (2004). Product Design and Development. New York, NY, McGraw-Hill.

Vargo, S. L. and R. F. Lusch (2004). The Four Service Marketing Myths: Remnants of a Goods- Based, Manufacturing Model. Journal of Service Research 6(4): 324-335.

Zeithaml, V. A., L. L. Berry, et al. (1988). Communication and Control Processes in the Delivery of Service Quality. Journal of Marketing 52(2): 35.

Åhlström, P. and R. Westbrook (1999). Implications of mass customization for operations management. International Journal of Operations \& Production Management 19(3): 262-274. 


\title{
Products vs. services: The point of view of the customer
}

\author{
Author(s): Jose Angel Lakunza, Juan Carlos Astiazarán
}

\begin{abstract}
Most of the work carried out in the field of Mass Customization has been focused on physical products and there is limited analysis and discussion around the concepts of Mass Customization applied in services. Surprisingly, service is per se a more customizable than physical product; the timing of services is determined usually by the customer, and most of their features are modified on the fly to adapt to the circumstances. Further more, nowadays there are few products unrelated to usual services such distribution, installation, warranty, spare parts ...

This paper tries to build a general frame to extend the benefits and concepts of Mass Customization to services, including both pure services and services as part of extended products. The paper starts identifying services' distinguishing features that differentiate them from physical products, and then studies how to deal with extended products.

As a result of the research carried out, a clear and comprehensive definition of service is proposed and contrasted with previous research work in the literature. Furthermore, a way to translate the concepts and ideas around Mass Customization towards services and, in particular, to extended products is proposed.
\end{abstract}

\section{Keywords}

Customer's point of view, service definition, goods and services, extended product, service

customization.

\section{Introduction}

Traditionally, there have been goods business, and services business. And both academy and industry have accepted this dichotomy; industries positioning themselves as goods business or service business, and academy developing separate scientific disciplines in marketing, management and strategy making decisions.

But nowadays, looking for more value to be added to their product, goods business is investigating different paradigms. Mass Customization is one of them, trying to get the right product for the right customer. A second one is product extension (or enhancement); that can be accomplished adding other goods as complements, and/or mixing goods and services. 
These companies face the unknown: can they manage service as they manage physical goods? If not, which new competences must they learn? Which competences must they keep? How can be mixed goods and services management? It is possible to extend the benefits of Mass Customization to the service side of the extended products? How? Being services more flexible than physical goods, are there undiscovered benefits?

From the academic point of view, these questions can be posed as following: Can pure service concepts and methods be applied to the service side of extended products? Can Mass Customization paradigm and technologies be applied directly to services, or do they need some adaptations?

\section{Objective of the paper}

The goal of the paper is to discuss about the differences between the service side of an extended product and pure services, and its impact on customization.

To deal with, the structure of the paper is as follow:

- A first review of some definitions of service.

- Then, definitions of service result and service execution will be proposed.

- After, goods and services will be defined as a part of the offer, from the customer's point of view.

- Next point will deal with goods based business vs. services based business.

- After comes a discuss on customization in extended products.

- And, finally, conclusions will be extracted.

\section{A survey in service definition}

Literature survey shows a lack of clear and univocal service definition. Existing definitions, although useful over a complex reality, are partial and incomplete, mainly as consequence of a clear difficulty perception of the service definition (Cook et al. 1999, Johns 1999).

Most of the service definitions apply a technical perspective, based on the item to be defined, or a provider point of view. This paper uses a different approach that has not been used before: the customer point of view. It analyzes relationships, and defines a generic relationship between customer and provider, always from the point of view of the customer. Analyzing the different elements and facts involved (actions, materials, tools...), as well as the possible kinds of customization involved, some rules are provided to distinguish between physical products and services, moreover, to be used in mixed relationships.

For every definition found, it's not difficult to find some examples falling out of the given definition. As Cook et al. (1999) remarked, "no single definition of a service is capable of encompassing the full diversity of services and complex attributes 
that accompany them". The title of an often cited article of Johns (1999) is very significant: What is thing called service?

If we analyze the definitions found, most of the authors coincide in saying that service is NOT a physical good:

- ... any act or performance that one party can offer to another that is essentially intangible and does not result in the ownership of anything (Kotler, 1994).

- ...to organise a solution to a problem (a treatment, an operation) which does not principally involve supplying a good (Gadrey et al., 1995).

- ...the delivery of help, utility or care, and experience, information or other intellectual content - and the majority of the value is intangible rather than residing in any physical product (DISR, 1999)

- ...services have some distinguishing characteristics: intangible... (J.P.J. de Jong et al., 2003).

- A service is a time-perishable, intangible experience performed for customer acting in the role of coproducer... A service is intangible and perishable (Fitzsimmons \& Fitzsimmons, 2004).

- In economics and marketing, a service is the non-material equivalent of a good.

Some authors remark the perishability of services:

-...services have some distinguishing characteristics: intangible, simultaneously produced and consumed, and often customized to a customer's needs (J.P.J. de Jong et al., 2003).

- A service is a time-perishable, intangible experience performed for customer acting in the role of coproducer... (Fitzsimmons \& Fitzsimmons, 2004).

- A service is intangible and perishable. It is an occurrence or process that is created and used simultaneously or nearly simultaneously. While the consumer cannot retain the actual service after it is produced, the effect of the service can be retained (Fitzsimmons \& Fitzsimmons, 2004).

From the provider point of view, service is sometimes defined as a process:

-...to organise a solution to a problem (a treatment, an operation) which does not principally involve supplying a good. It is to place a bundle of capabilities and competences (human, technological, organisational) at the disposal of a customer to organise a solution, which may be given to varying degrees of precision (Gadrey et al., 1995).

- ...characterizes services as trying to meet customer needs through actions (Johns, 1999)

- A service is a process consisting of a series of more or less intangible activities... (Christian Grönroos, 2000).

- Services are deeds, processes, and performances... (Fitzsimmons \& Fitzsimmons, 2004). 
- It is claimed to be a process that creates benefits by facilitating either a change in customers, a change in their physical possessions, or a change in their intangible assets... (Wikipedia, 2006).

And some insist in the customer taking action:

- A service is... a series of... activities that normally... take place in interactions between the customer and service employees... (Christian Grönroos, 2000).

- A service is a time-perishable, intangible experience performed for customer acting in the role of coproducer... (Fitzsimmons \& Fitzsimmons, 2004).

After compiling its literature review on Innovation in service firms, J.P.J. de Jong et al. (2003) insist in the delivering phase as the core of a service.

And, to finish, Wikipedia (2006) gives the closest definition to a customer point of view, based in the effects of a service: It is claimed to be a process that creates benefits by facilitating either a change in customers, a change in their physical possessions, or a change in their intangible assets.

\section{Proposed definitions}

As remarked in the previous chapter, found service definitions are elusive, often intuitive and, in any case, no totally comprehensive.

But service can be analysed too from the customer's point of view. That is, the need's point of view. So, service has two closely tied different meanings: service as a result (customer's point of view) and service as a process (provider's point of view).

Based on the most comprehensive point of the previous definitions, this paper will propose two definitions covering both points of view.

A service result (the customer's point of view) is a set of transformations experimented by the customer, his belongings and/or related people, as a consequence of a service execution. In any case, service result can be the absence of a transformation (e.g. security guards).

Service results appear in the literature as outcomes, activity outcomes (Ma et al.), service outcomes (Baida)...

A service (the provider's point of view) is the execution of a process that produces a transformation on the customer, his belongings and/or related people, with his possible presence and/or collaboration.

- Execution... (J.P.J. de Jong et al., Fitzsimmons \& Fitzsimmons).

- Of a process... (Gadrey et al., Johns, Christian Grönroos, Fitzsimmons \& Fitzsimmons, Wikipedia).

- That produces a transformation... (Kotler, Gadrey et al., DISR, de Jong et al., Fitzsimmons \& Fitzsimmons, Wikipedia). 
- On the customer, his/her belongings and/or related people... (Wikipedia).

- With his possible presence and/or collaboration. (Christian Grönroos, Fitzsimmons \& Fitzsimmons).

- Service results can be composed by other more nuclear service results or services, and can be part of a bigger service result. Of course, the same happens with services.

\section{Goods and services as a part of the offer}

As said before, traditionally industries have positioned themselves as goods business or service business. To make this distinction, they have relayed on the main process behind.

But, from the point of view of the customer, the offer of the company is usually a mix of goods and services. In other words, what the business can offer is a goods and services continuum, and at every moment the company chooses what offer (Paloheimo et al., 2004).

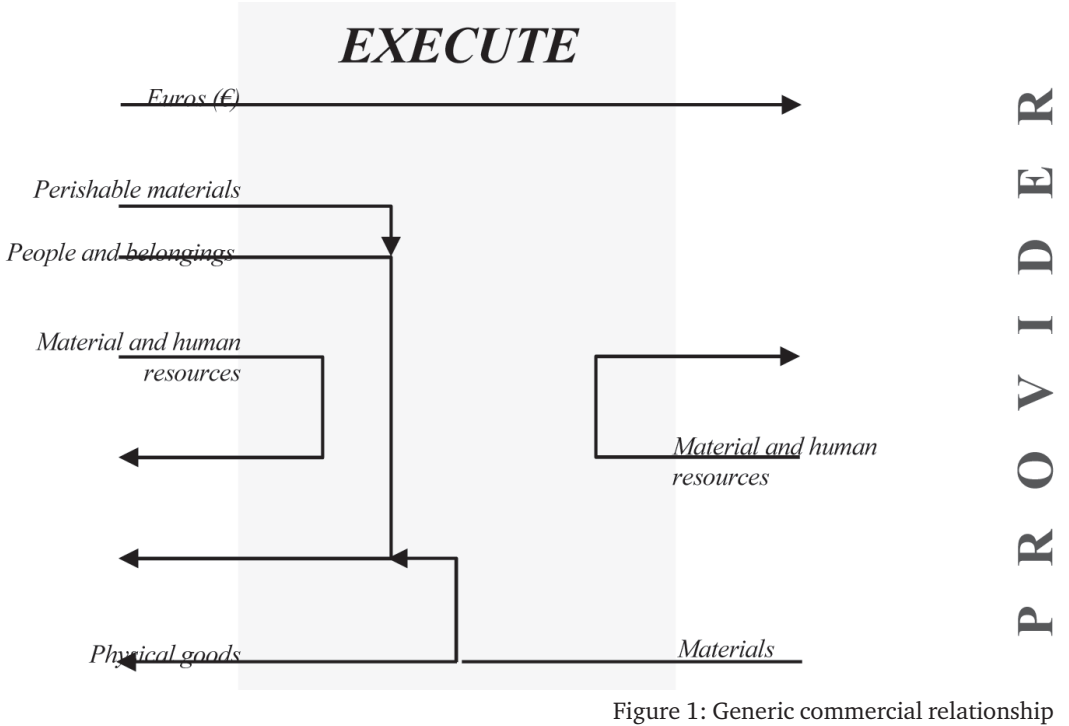

Let's have a look in more detail. As seen in Figure 1, at any commercial relationship the customer gets something (a good and/or a service, customized or not) from a second part (the provider), and rewards this one with a prize (usually money). This relationship can be punctual (buying something and getting it at the moment) or last for a time (assurance, maintenance contract...) We'll analyze the relationship from the customer's and the provider's point of view, the relationship itself and its possibilities of customization. 


\subsection{The customer's point of view}

To perform a commercial relationship, the customer can give:

- Money, to pay the provider.

- Fungibles to be used in the relationship.

- Human and/or material resources to be used in the relationship.

And after the relationship has happened, the customer can perceive some possible changes:

- The customer is the new owner of some goods.

- The customer and/or his environment (related people, belongings) have been transformed in a physical or mental way (welfare, state of mind, information, knowledge...). Or, even has avoided a not desired transformation!

- The customer takes back the human and material resources given form the relationship.

\subsection{The provider's point of view}

To perform a relationship, the provider can give:

- Materials (fungibles) to be used in order to get the goods and/or the physical and mental transformations in the customer, his belongings and/or related people.

- Resources, human and/or material, to be used in the relationship.

As a result of the relationship, the provider can perceive some possible changes:

- The provider is the new owner of some money.

- The provider takes back the resources given for the relationship.

\subsection{The relationship itself, and its customization}

Relationships are carried out by resources. These resources are given by the provider and, in some cases, even by the customer too.

These resources can execute two kinds of actions:

- Building goods from materials.

- Transforming, physically or mentally, the customer, his belongings and/or related people.

In some cases, disposable material given by the customer can be necessary. And, of course, usually some money passes from the customer to the provider.

The relationship can be customized in various ways and moments:

- The goods to be delivered (goods).

- The delivering itself (services).

- Services along the life of the relationship. 


\section{Goods based business vs. services based business}

As stated in the previous definitions, by means of a commercial relationship the customer can obtain some goods, and/or some services' results.

Although it would be possible to find pure goods' relationships, or pure service relationships, the reality shows than, usually, what the customer gets from any relationship is a mix of both.

Most of the so called goods business includes some kind of service in their offer; in a similar way, a lot of service business use to give material goods to their customers. In other words, there is not goods business but goods based business; there is not service business but services based business.

So, from this point of view we could classify business in three groups:

- Goods based business.

- Services based business.

- Mixed business.

\subsection{Goods based business}

The provider, using his material and human resources, produces physical goods and delivers them to the customer. Production can be carried out before or after the selling is made.

Nowadays, there are very few relationships involving only physical goods: usually, the provider aims giving more value to his physical good adding other products and services (see figure 2):

- Add-ons (complementary goods and devices) to with the basic good offered: sauces with the vegetables, tupperware with the fridge...

- Gifts and gadgets: an iPod when purchasing a car.

\section{EXECUTE}

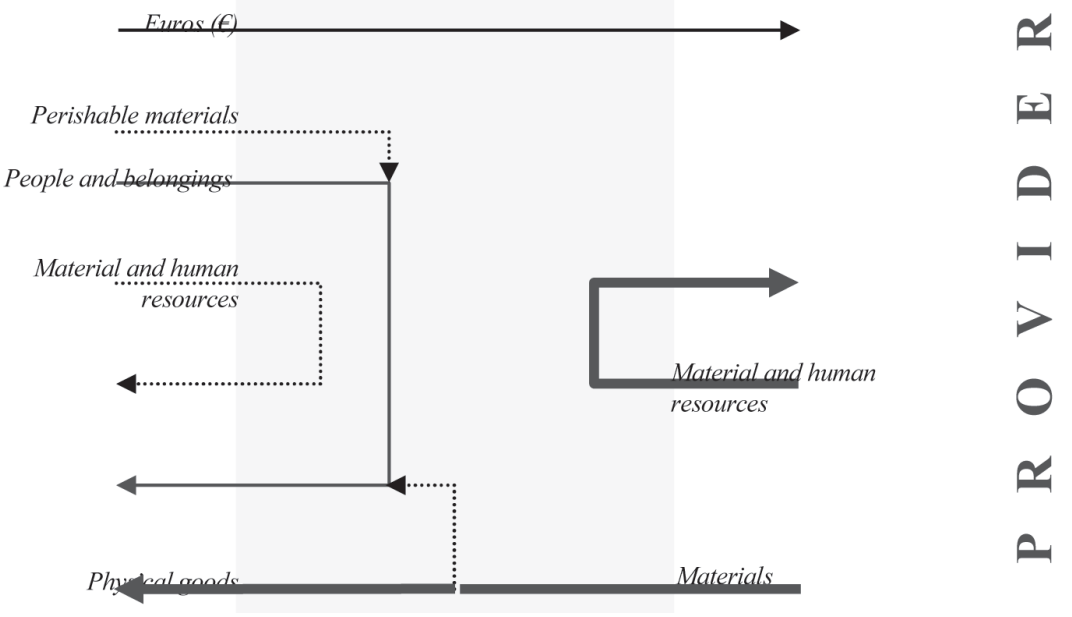

Figure 2: Goods based business 
- Physical or mental transformation of the customer: education and training, user's manuals, entertainment, heating in the sales point, bringing her to the shoreline to have a look on an apartment to sell...

- Physical or mental transformation of people related with the customer: nursery to keep the kids when the customer is buying.

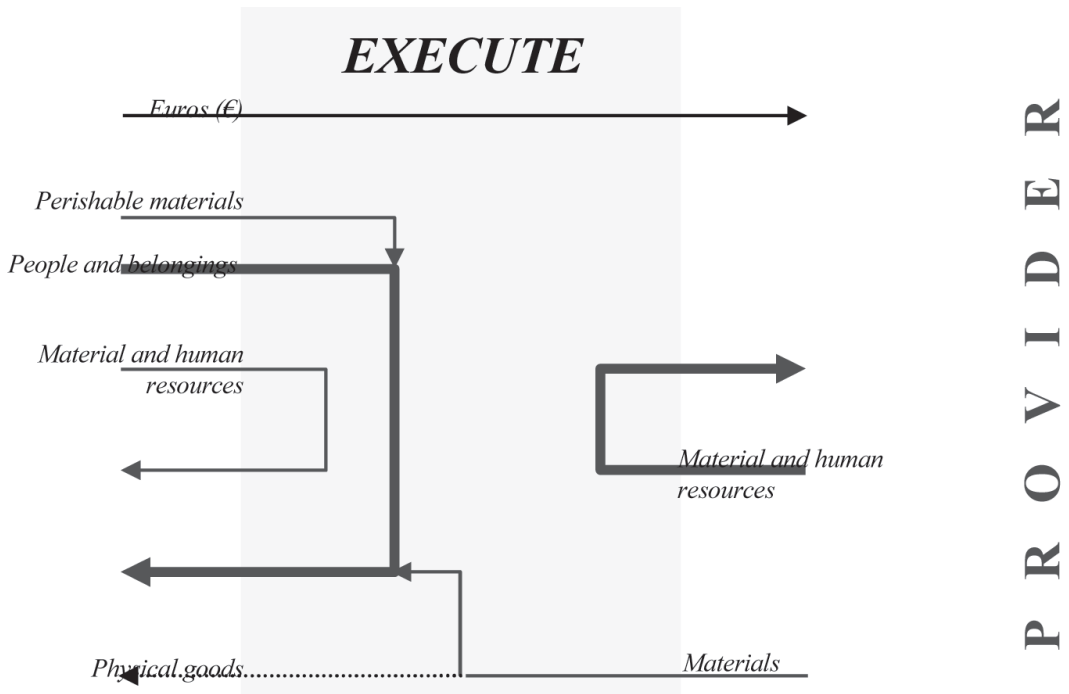

Figure 3: Services based business

\subsection{Services based business}

The provider, using his material and human resources, produces physical or mental transformations on the customer, his belongings or on related people. This transformation is always carried out after the selling is made.

Apart from the resources and materials of the provider, sometimes resources and materials given by the customer can be used (see figure 3).

Being services, by themselves, aggregation items, it's difficult to find a single service offered; usually they are bundled with other services and/or physical goods:

- Physical or mental transformation of the customer: ambient music in the underground, official registers in a wedding...

- Physical or mental transformation of people related with the customer: entertaining kids when the parents are in the spa...

- Add-ons: sticks in a Chinese restaurant...

- Gifts and gadgets: an iPod when purchasing a holiday pack.

\subsection{Mixed business}

These are the most complex business: being both the physical good and the service essentials for the customer, they form part of the core offer of the business. 
Management is mixed too: the physical good could be produced before the selling, and the service execution could be delayed very long time after the selling: house appliances with warranty, an elevator with a 5 years contract of maintenance...

\section{Customization in extended products}

As we have set before, an extended product is usually a mix of goods and services. As a result, customization of extended products mixes both kids of customization.

Customization of goods is carried out by providers, just before its delivering: customer and provider agree in the need customization level (what customize), the provider customized the good and delivers it to the costumer. As an exception, the customer itself can customize the goods by himself in installation or use (build customization).

In the other hand, services are customized by the provider in their execution. Apart from the what, customization can take the when, who, how, where... Depending on the case, more or less choice is left to the consumer. Even more, in some cases customization is made long after the service is sold: e.g. assurances.

So, whit extended products we can have two kinds of customization:

Customization related to the delivering, comprising the goods and its associated services: the delivering itself, installation, training... This kind of service customization can be managed in a similar way than physical goods customization.

Customization related with the life of the product: assistance, maintenance, repair... This kind of customization can be managed like pure services customization.

\section{Conclusions}

After a contrast with previous research work in the literature, a new definition of service is provided, in order to be more comprehensive than the existing ones: A service is the execution of a process that produces a transformation on the customer, his belongings and/or related people, with his possible presence and/or collaboration.

Most business mix goods and services. Depending on the position of their offer in a continuum of physical goods and services, they will be goods based business or services based business.

From this point, it can be inferred that developing the offer towards product extension can be as natural as altering the mix goods-services. A second conclusion can be extracted, that theories, concepts and technologies applied to services are independent of the kind of service's integration in the business offer, pure services or the service side of an extended product.

Last, the way to manage customization in extended products depends on the moment of the relation: around the delivering time point, the goods kind of customization prevails, while the pure service kind of customization is to be applied along the life of the product. 


\section{References}

Cook, D.P., C.H. Goh, C.H. Chung (1999): Service typologies: a state of the art survey, Production and Operations Management 8(3): 318-338.

Kotler, P. (1994): Marketing Management: Analysis, Planning, Implementation and Control, Prentice Hall International, London.

Gadrey, J., F. Gallouj and O. Weinstein (1995): New Modes of Innovation: How services benefit industry, International Journal of Service Industry Management 6(3): 4-16.

Johns, N. (1999): What is this thing called service? European Journal of Marketing, 33(9), 958-974.

DISR (1999): The Australian Service Sector Review 2000, Canberra: Department of Industry, Science and Resources.

Grönroos, C. (2000): Service Management and Marketing: A Customer Relationship Management Approach, 2nd Edition, John Wiley \& Sons, Ltd.

J.P.J. de Jong et al. (2003): Strategic Study B200205, Innovation in service firms explored: what, how and why? Literature review, EIM.

Fitzsimmons, J.A. \& Fitzsimmons, M.J. (2004). Service Management-Operations, Strategy, and Information Technology, ISBN 0-07-121457-7, McGraw-Hill.

Paloheimo, K-S., Miettinen, I. \& Brax, S. (2004). Customer Oriented Industrial Services.

Espoo, Finland: Report Series - Helsinki University of Technology, BIT Research Centre.

Wikipedia (2006): www.en.wikipedia.org 


\title{
Dimensions for classifying service mass customizers
}

\author{
Mikko Heiskala, M.Sc. (Tech), Project Manager
}

Software Business and Engineering Institute,

Department of Computer Science and Engineering,

Helsinki University of Technology

P.O. Box 9210

02015 HUT, Finland

Mikko.Heiskala@tkk.fi

Mikko Heiskala is a project manager and researcher in the Software Business and Engineering Institute (SoberIT) at the Department of Computer Science and Engineering of Helsinki University of Technology (HUT). His main research interests are mass customization and configuration - particularly of services, modelling configurable services for configuration and design, configurators, and business and operations based on these. He has worked in the area of product configuration and modelling since the year 2000. He is currently working towards a Ph.D. at HUT.

\begin{abstract}
We present dimensions for classifying service mass customizers. Our work is based on a conceptual analysis and synthesis of goods mass customization and service classification dimensions found in literature. The dimensions proposed could help service companies striving for mass customization to position their mass customized service offerings related to competitors and to identify management issues they could face.
\end{abstract}

\section{Keywords}

mass customization; services; classification dimensions

\section{Introduction}

\subsection{Background and motivation}

Classifications are used to characterize different companies, their offerings and operations, and strategic positions, amongst other things, and compare these against other companies (Shafti et al., 2007). They are also beneficial in identifying the differences in required competencies, skills, competitive strategies, etc. for a given offering or operations type. This all can be useful for researchers and practitioners alike. Classifications usually employ dimensions, special characteristics or criteria, to categorize or cluster companies, their operations and the like into specific types or classes (Shafti et al., 2007). 
There are several mass customization (MC) classifications in literature (e.g. Pine, 1993; Lampel \& Mintzberg, 1996; Ross, 1996; Gilmore \& Pine, 1997; Duray et al., 2000; Piller, 2002; Piller \& Stotko, 2002; MacCarthy et al., 2003). Many of these are reviewed in (da Silveira et al., 2001; Blecker et al., 2005; Brabazon, 2005; Moser 2007). Classifying MC companies and their products or operations or reviewing such classifications is therefore nothing new. Unfortunately, the extant MC classifications seem to mostly focus on goods and neglect services. An exception is provided by Lampel \& Mintzberg (1996) who do state that their MC strategies classification is geared towards manufacturing and do discuss how to characterize service MC. Pine (1993), Ross (1996), da Silveira et al. (2001), Piller \& Stotko (2002), Piller (2002) all include a similar idea of customizing a standard good or service either by providing customizable, add-on services on top of it or providing a service that customizes the standard part. This does not appear to provide much insight for a company striving for service mass customization. The upshot is there seems to be no classification with a service mass customization focus in MC literature.

The differences between goods and services been discussed in service literature for some time (see e.g. Silvestro et al., 1992; Grönroos, 2000) and is based on the intangibility, heterogeneity, inseparability and perishability (sometimes referred to as IHIP) characteristics attributed to services. These characteristics and the differences between goods and services stemming from them, or at least their exclusiveness to services and their claimed detrimental effects on services when compared to goods, have been questioned recently by Vargo and Lusch (2004) from a marketing and customer perspective. Nevertheless, the literature review by Bowen \& Ford (2002) does conclude that the services operations often involve different management challenges compared to operations related to goods. Therefore, given the different nature of goods and services, at least on some aspects, the extent to which the results from another domain are applicable in the other should be at least questioned (da Silveira et al., 2001; Peters \& Saidin, 2000). This further strengthens the need for a MC classification geared towards services. Moreover, services are an important part of the economy today (Grönroos, 2000; Fitzsimmons \& Fitzsimmons, 2004) and several authors have noted a lack of literature on service mass customization in general (Blodgett Beckett, 1996; Benaroch, 1996; McLaughlin, 1996; Harvey et al., 1997; Duray et al., 2000; Peters \& Saidin, 2000; da Silveira et al., 2001; Papathanassiou, 2004; Paloheimo et al., 2004; Heiskala et al., 2006; Heiskala et al., 2007; Moser, 2007).

The service literature contains numerous classifications of their own (e.g. Schmenner, 1986; 2004; Silvestro et al., 1992; Kellogg \& Nie, 1995; Cook et al., 1999; Buzacott, 2000; Johansson \& Olhager, 2004). Many of these include aspects related to customization and variability, hence being potentially insightful for service mass customization as well. However, the service classifications do not appear to consider service mass customization specifically. 
This paper was written as a part of the Customer-Oriented Systematically Managed Service Offerings (Cosmos) project, which is a three-year project that began in summer 2007 and is coordinated by Software Business and Engineering Institute (SoberIT) of Helsinki University of Technology (HUT). Research partners of Cosmos include Human Capital and Leadership Research Group (HCL) of Laboratory of Work Psychology and Leadership at HUT and Cost Management Center of Tampere University of Technology in Finland and Computer Science and Manufacturing Group at Departments of Informatics of University of Klagenfurt. Cosmos is funded by Tekes (Finnish Funding Agency for Technology). Early ideas for the paper were constructed during the previous SoberIT and HCL co-operation project, Configurable Services on the Web (ConSerWe), which was also funded by Tekes.

\subsection{Research Problem and Objectives}

The research problem of this paper can be expressed as a question:

What dimensions can be used to classify service mass customizers?

The paper aims to answer this question by meeting the following research objectives.

- Gain an understanding of the service and mass customization classification and the dimensions used in them.

- Gain an understanding of management challenges in service mass customization.

- Identify dimensions appropriate to characterize service mass customizers and the management challenges they face from the reviewed literature or by creating new ones by synthesis of service and mass customization dimensions.

\subsection{Overall Research Methodology}

Shafti et al (2007) critique service classifications in literature of being theoretical in nature and consequentially the managerial implications offered in them not to be necessarily rooted in realworld practical phenomena, or at least not to be substantiated in practise. For their own study, they adopt an approach presented in Figure 1. In their approach, they first identify a long list of potentially relevant dimensions from literature. After this, they study management challenges in services, and then study if the identified dimensions indicate similarities or differences between the services related to the management challenges. Finally, they summarize the relationships between the dimensions and management challenges in developed classification models.

In this paper, we adopted a similar approach to Shafti et al.'s, albeit a less rigorous one empirically, see Figure 1. The main research method in this paper was literature study. First, a literature research was conducted on service and mass customization 
A) Traditional approach

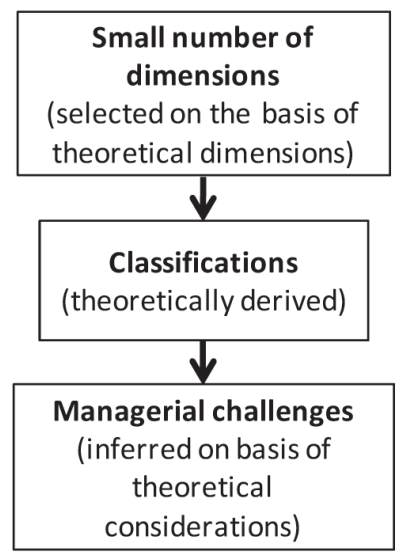

C) The (adapted) approach used in this paper

\begin{tabular}{|c|}
\hline (Long) list of potentially \\
relevant service MC \\
dimensions \\
(derived from service and \\
MC literature) \\
\hline
\end{tabular}

B) The (empirical) approach proposed

by Shafti et al. in (2007)

Managerial challenges

(studied by collecting empirical data on a long

\begin{tabular}{|c|}
\hline Long list of \\
potentially relevant \\
dimensions \\
(derived from \\
literature)
\end{tabular}
list of managerial themes)

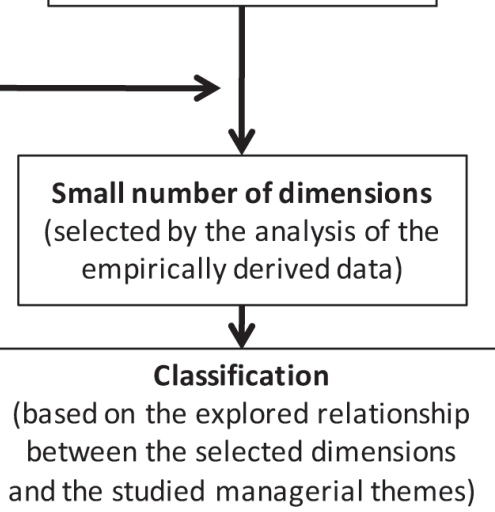

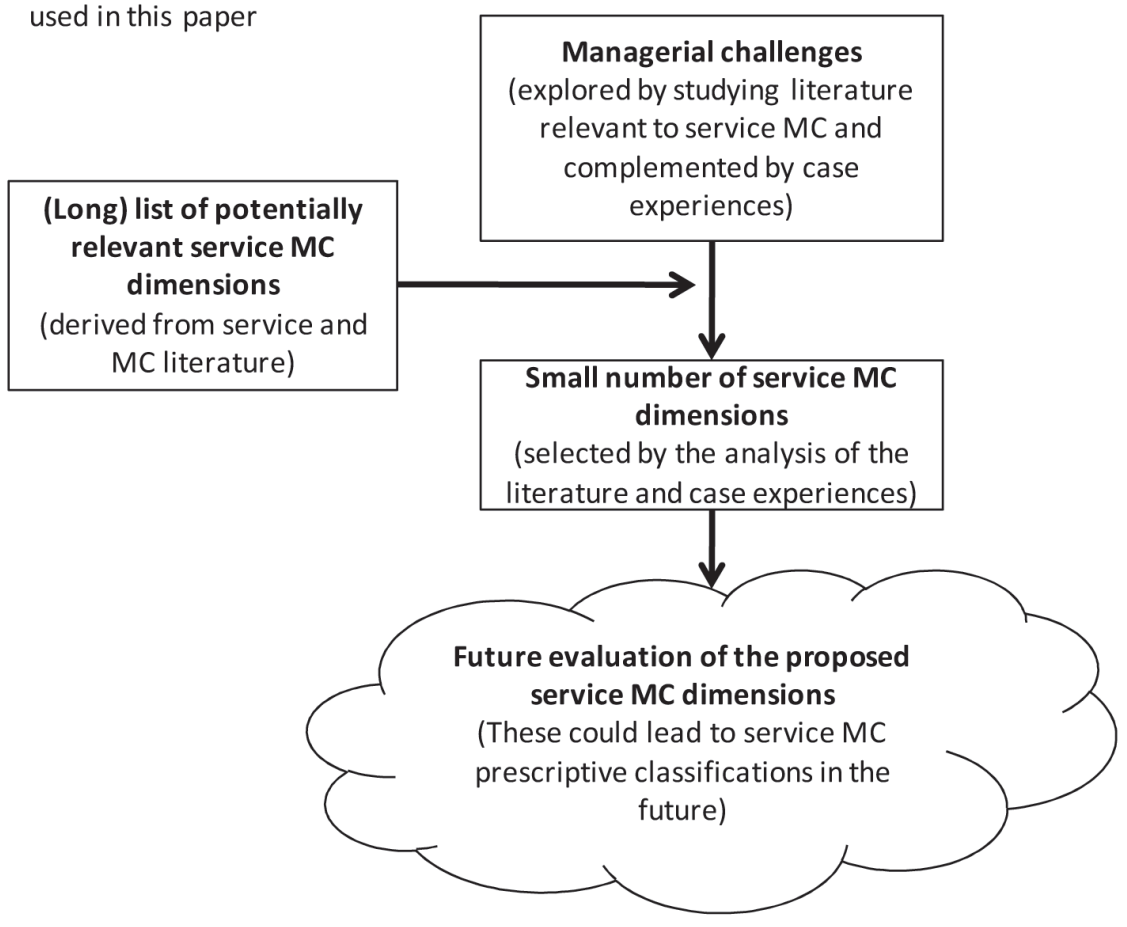

Figure 1 A) Traditional approach used in service classifications (adapted from Shafti et al., 2007), B) The empirical approach used by Sfafti et al. (adapted from Shafti et al., 2007), and C) The approach used in this paper. 
classifications. The crux of this research was to identify a long list of potentially relevant dimensions for service mass customization. Next, literature was studied to gain an understanding of service mass customization management challenges. This included service literature having a variation or customization viewpoint, mass customization literature on goods, and some limited number of papers focusing on service mass customization problems. Subsequently, dimensions deemed potentially useful for characterizing service mass customization, in relation to the management challenges, were identified, both ones from literature and new ones.

\subsection{Scope of the Research}

We have focused our attention in this paper to management challenged relating to product structures, operations, and marketing rather than strategic level issues.

\subsection{Structure of the Paper}

Next, chapter 2 reviews mass customization and services literature from the viewpoint of trying to identify management challenges relevant for service mass customization. Chapter 3 reviews literature on service and mass customization classifications. Chapter 4 presents the main thrust of this paper, the proposed dimensions to classify service mass customizers. Subsequently, chapter 5 includes the discussion, comparison to previous work, the limitations of the study and directions for future work. Conclusions end the paper in chapter 6 .

\section{Services, Mass Customization, and Service Mass Customization}

This chapter reviews literature on services, mass customization and service mass customization with the viewpoint of trying to identify management challenges in service mass customization.

\subsection{Services}

Researchers interested in services have to live with the challenge that no single and consistently used definition of what services are and are not exists (Shafti et al., 2007). Cook et al. (1999) argue that this is due to the diversity in services and also why service classifications, trying to address the complex nature of services in more structural way, are abundant in literature. This challenge is probably why Grönroos "reluctantly" proposes in (2000, pp. 46) the following definition for services. "A service is a process consisting of a series of more or less intangible activities that normally, but not necessarily always, take place in interactions between the customer and service employees and/or physical resources or goods and/or systems of the service provider, which are provided as solutions to customer problems." Another definition is given in (Fitzsimmons \& Fitzsimmons 2004, p. 4): "A service is a time-perishable intangible experience performed for customer acting in the role of co-producer". As touched upon in Introduction, services are often characterized 
with the following IHIP attributes (Grönroos 2000, p. 47; Fitzsimmons \& Fitzsimmons 2004, p. 21; Sampson \& Froehle, 2006).

In services, production and consumption are simultaneous and inseparable, significant parts of the production cannot begin or proceed until some customer inputs are provided or the customer is present. Services therefore often fail in the presence of the customer whereas in goods you might be able to spot an error at the factory, before customers become aware of it. In services, customers might be the only ones noticing the failure of services to meet their expectations.

Perishability refers to the time-sensitivity of service provider's capacity to produce a service (maintenance engineer's time or empty dentist's chair), not the service product itself (the work and its effects the engineer does on a machine or the dental work) (Sampson \& Froehle, 2006).

Because of the simultaneity characteristic, also the customer's time "perishes" during the production and consumption. Contrary to the relatively common misconception, services processes can hold inventory according to Sampson and Froehle (2006). If customer inputs arrive in the service process in excess of the available production capacity, some of those inputs can be held in "inventory", e.g. in queues or waiting lines. This inventory is more time-sensitive than in goods as customers can withdraw their inputs at any time. Due to the perishability of production capacity and time sensitivity of "customer input inventory" managing capacity and demand can be challenging for service companies.

Heterogeneity refers to the variability and consistency in the worker or customer performance in the service process (assuming human involvement) and in the customer expectations of the service (Sampson \& Froehle, 2006). As customers differ and do not necessarily know what to expect from the service their participation creates unpredictability and variation to the service process. The more complex the customer involvement is the more challenging it is for the service company to accommodate for the customer-induced variability in the service process (Bowen \& Ford, 2002).

Intangibility is often attributed to services, and can be divided to physical intangibility, i.e. incapable of being perceived by senses or mental intangibility, i.e. difficult to be grasped mentally (Lovelock \& Gummesson, 2004). Because of intangibility, customers have difficulties in evaluating a service (Grönroos, 2000, pp. 49). However, many acts in the service process are tangible, and they often produce tangible outcomes.

Sampson and Froehle (2006) argue that customer participation, ranging from providing information, tangible belongings or self-inputs to the process, is what distinguishes service processes from other processes and that it is exactly these customer-inputs that are the cause of the unique challenges in service management. They also argue that the management challenges differ based on the customer-inputs. Moreover, Chowdhury and Miles (2006) argue that it is exactly the customer-induced uncertainty that creates the management challenges that characterize 
especially services. Decoupling the process to front-office (customer physically or consciously present) and back-office (low-customer contact, easier to standardize and often more efficient) processes is a common notion in service literature. In Sampson \& Froehle's (2006) view, customer self-inputs require a front-office process whereas customer-provided information and tangible belongings can be handled with a back-office process.

Service is often understood along two main streams: as activities, process or as outputs of a system (Grönroos 2000, pp. 51, 63). When understood as activities a service is performed for others, usually the customers. As an output a service is "a deed", "a performance", "an offering", or "a benefit". From the service provider's view, much of the service management deals with the process and outcome consumption. Grönroos (2000, pp. 63-4) argues that the service quality seen by customers has two dimensions: a technical (what received, outcome) and functional (how received, interactions, process) quality. In goods, the consumption is usually dominantly outcome consumption, i.e. using the manufactured good which is produced separately from the customer. Services can also be divided to continuously rendered services, where the provider and customer may form even very long-lasting relationships (like insurance and industrial maintenance service), and discrete transactions (e.g. hair dressers) (Grönroos, 2000, pp. 50). Grönroos (2000, p. 244) sees that services involve the firm giving a promise and then fulfilling the promise. The customer actions and expectations can be managed with the promise, specifying beforehand what the customer can expect to receive and needs to do in order to receive the service.

\subsection{Mass Customization}

Mass customization (MC) can be defined as a strategy of providing even individually customized goods or services at production costs and lead-times of, or close to, large-scale mass production (Da Silveira et al. 2001). Hart (1995) gives a more broad, idealistic definition for MC: "the ability to provide your customers with anything they want profitably, any time they want it, anywhere they want it, any way they want it". In reality, the profitability requirement of the above definition compromises the other goals or at least restricts them to a "pre-determined envelope of variety" (Hart 1995).

Zipkin (2001) states that to successfully implement MC the firm should have three key capabilities in place: elicitation, process flexibility, and logistics capabilities, discussed in the following. Elicitation is a mechanism of interacting with the customer to learn about customer specific information and their needs. Learning about needs is difficult. Often customers don't know what they really want, or are unsure about it, and often cannot communicate their needs accurately. Customer information is significant for MC; you can't give customers what they want without learning what that is first. 
A high-volume but flexible production process is needed in $\mathrm{MC}$ to translate the customer specific information into the actual product. How difficult it is to achieve sufficient process flexibility and keep costs comparable to mass scale processes, depends on the products fabricated.

The logistics steps into play after (and partly during) the product is fabricated. To be able to deliver the goods to the right customer at least information about the customer's identity must move along with the goods. Da Silveira et al. (2001) argue that efficient information transfer among the operations of a MC company determines largely the success of the MC program: customer needs must accurately elicited, translated into production instructions, and then ensuring the production process functions accordingly.

\subsection{Management Challenges Service Mass Customization}

Service mass customization is not a very studied subject (da Silveira et al., 2001; Heiskala et al., 2007). Case examples are few and far between (one is Peters \& Saidin, 2000). Heiskala and coworkers have reviewed literature for management challenges in mass customization (Heiskala et al., 2007) and have also conceptually analyzed their relevance in service settings in (Heiskala et al., 2006). In both their reviews, the difficulties in elicitation or specification are mentioned as important management challenges. In (2006) Heiskala et al argue that intangibility of services may even highlight elicitation problems. Erroneous specifications are often realized during the consumption and by the customer because of simultaneity. For continuously rendered services, the elicitation will be done repeatedly as customer needs change.

Achieving production process flexibility and balancing the offered customization with the company capabilities are probably both relevant challenges in service MC as well (Heiskala et al., 2006). The heterogeneity in customer inputs probably hampers achieving the process flexibility. Peters and Saidin (2000) argue managing resources capacity is challenging in service MC.

Management of information transfer from elicitation phase to production and during production is an important challenge in MC (Heiskala et al., 2007). Maybe even more so in services, at least in those services where the information has to flow more frequently between humans than in goods manufacturing (Heiskala et al., 2006). Intangibility of services can also imply the existence of tacit knowledge which is more difficult transfer. In services, it may be necessary to relay information to the customer about how to perform in different process phases. In continuously rendered services the customer relationships may be long. This might imply that information transfer between process tasks is more difficult than in goods manufacturing where time elapsed between tasks might be shorter, and even more so if customers are involved in front-office tasks. Consider for example two machinery maintenance visits taking place two months apart and the assembly of a PC. 


\section{Review of Classification Dimensions}

\subsection{Service Classification Dimensions}

There are several service classifications, typologies or taxonomies that use different dimensions to characterize services. Service classifications have been reviewed in articles by Cook et al. (1999) and Silvestro et al. (1992). Johansson and Olhager (2004) and Shafti et al. (2007) also discuss many of the classifications. Instead of reviewing service classifications in similar depth as in these papers, we focus on dimensions that might be relevant for the service mass customization challenges discussed in the previous chapter.

Cook et al. (1999) categorize service dimensions broadly according to organizational ownership, product and marketing-oriented, process and operations-oriented, and the interaction and integration of the marketing- and operations-oriented dimensions. Johansson and Olhager (2004) also divide the dimensions they reviewed along market and service offering aspects and service production aspects. Similar division to product/offering and production/process dimensions is present in classifications of Kellogg and Nie (1995) and Buzacott (2000).

Cook et al. (1999) list several marketing-oriented dimensions. The already discussed tangibility is one. Differentiation of offered services from competitors (which Cook et al argue not to be particularly useful discriminating between different groups of services) and commitment of customer to provider are other ones. They also identify object of transformation, i.e. object of the service process like persons or their possessions. This should probably include customerprovided information, following Sampson \& Froehle (2006). Johansson and Olhager (2004) have identified dimensions used to denote the level of customization range in the offering. Similar dimension is present in both Kellogg and Nie's (1995) and Buzacott's (2000) classifications. Johansson and Olhager (2004) also include another dimension, the level of diagnosis offered, ranging from complex to none, which seems similar to the elicitation activities in MC.

Of the operations-oriented process dimensions Cook et al. (1999) identify customer contact describing the time spent in them, the level of intimacy or confiding required, and the information richness in them. Similar dimension is customer involvement characterizing how much the customer can actually influence the service process and interacts with the service system, present also in Kellogg and Nie (1995). Silvestro et al. (1992), Cook et al. (1999), Buzacott (2000), and Johansson and Olhager (2004) identify the provider discretion i.e. how much provider can exercise own judgment in creation and delivery of the service, offering or process-wise, without referring to superiors as one service dimension. Dimensions describing the customization range or capability to handle variability in the production process are present in (Silvestro et al., 1992; Buzacott, 2000; Johansson \& Olhager, 2004). 
Many of the classification reviewed by Cook et al. (1999) and Johansson \& Olhager (2004) include dimensions describing the resources needed in service process, the skills workers need in diagnostic or production phase, whether the service is produced by humans, machinery or other systems, and the like. Another interesting dimension in (Silvesto et al., 1992) is how the value added activities are divided between front- and back-office.

\subsection{Mass Customization Classification Dimensions}

Many of the MC classifications reviewed in (da Silveira et al., 2001; Blecker et al., 2005; Brabazon, 2005; Moser, 2007) categorize MC efforts according to how deeply the provider's activities are done in response to individual customer needs or in other words the place in value chain where customization begins (Brabazon, 2005, pp. 7). Often this implies manufacturing modules or sub-assemblies to stock and the combining and assembling these in response to customer orders. As services are perishable similar production of modules to stock to wait for customer orders is not possible. Another viewpoint is the method of how the product is customized, its architecture or structure (Duray et al., 2000). Duray et al categorize MC along what kind of modularity is used in the product structure. Their idea of sometimes just combining existing modules in response to customer needs, sometimes altering modules for customer, sharing modules across different product architectures for scale economies could be useful in services as well. Modularity is mentioned in (Pine 1993) as one way to achieve MC. MacCarthy et al. (2003) discuss the use of resources as one classifying dimension for MC. Companies can serve their customers with fixed resources, i.e. the provider does not acquire resources outside its current capabilities in response customer needs and therefore implicitly commits to limiting the customer requests to the predetermined envelope of variety, or with mixed resources i.e. possibly obtaining extra or external resources to fulfill a customer order.

Services are not present in most of the MC classifications. Pine (1993) claims that one way to achieve MC is to develop and offer customization services on top of their standard products or services. Da Silveira et al. (2001) include a similar idea in their classification. However, Lampel and Minzberg (1996) concede that their classification is geared towards the operating processes of a manufacturing company and thus is not suitable to e.g. services for which (according to them) there is no design, fabrication or assembly. They argue that for services (and similar industries) the classification has to be extended to include products (ranging from commodities to unique) and transactions (ranging from generic to fully personalized) by which buyers come to agreements with sellers. Transactions seem similar to the elicitation capability of MC. 


\section{Proposed Dimensions for Classifying Service Mass Customizers}

\subsection{General}

This chapter describes the proposed dimensions for classifying services mass customizers. The dimensions were divided to three categories: to offering dimensions and to two process oriented categories i.e. elicitation and production process categories.

MC is a customer-oriented strategy. In services, customers see that both the process and its outcomes affect the service quality they experience. Process flexibility is a key capability in MC. Many of the service classifications use the extent of customization in the service process and the service offering as dimensions. Therefore it is arguable that the service process and service offering (equivalent of product in MC classification) should be incorporated in the service MC dimensions. Customers often participate in the service process. This causes variation in the process and the depth of customer involvement may affect it. Customer involvement has some similarities to the customer order decoupling in manufacturing value chains. It has been suggested that the service company can manage the customer participation by communicating in advance how the customer should act. The more customized the process, the more difficult it may be for customers to participate as the company wishes and for the company to communicate its wishes. Division of the production process to front- and back-office tasks and the type of customer inputs (self, information, possessions) could also be useful dimensions in service MC.

An issue intertwined with processes is the resources. Some service classification dimensions characterize the resources at the company's disposal, e.g. workers. MacCarthy et al. (2003) use resources, either fixed or modifiable, to classify MC. Resources could be useful for service MC classification as well. A key capability in MC is the elicitation. It is similar to the transaction dimension Lampel \& Mintzberg (1996) put forward. Further, some service classifications use the diagnosis of the customer needs as a dimension. Based on these, elicitation should probably be included in the service MC dimensions. As customers affect the process, there may be information required of them that is needed in order for the company to be able to deliver the service successfully.

Another MC capability is logistics. It is required to deliver the customer specific product to the right customer. Similarly, a customized service has to be delivered according to the customer specific service agreement, as promised. Information about the agreement must be available in the process for the service workers to be able to deliver accordingly. According to Da Silveira et al. (2001) efficient information transfer is a key success factor in MC.

\subsection{Offering Dimensions}

Two dimensions are suggested for service offering. Here the service offering is un- 
derstood as the pre-delivery agreement options, the terms and conditions of insurance, McDonald's menu, etc. for example.

- Range of offering customization: It describes potential built-in to the service offering to meet different customer needs. Or in other words, the extent to which the pre-determined service options allow customization for individual customer needs. It describes the predetermined envelope of variety (Hart 1995) in the service offering.

- Degree of offering discretion: It depicts the extent of additional customization allowed to be made to the service offering outside the pre-determined, predesigned service options.

\subsection{Elicitation Dimensions}

Elicitation is the phase when the buyer and the service company come into agreement of the service to be delivered. Its end result is a specification of the service to be delivered for a particular customer. As elicitation is a process, it has many similar dimensions as the service production process has. In McDonald's order-taking is equivalent to elicitation.

- Range of elicitation customization: It describes the potential for customization in the predesigned elicitation process. Possible determinants are the number of different paths through the process and the number of distinct ways to perform a process task. For example, in Starbucks or in a similar coffee house, the elicitation includes a certain set of questions asked from the customer, like the size of a cup, the coffee beans used, milk, cream, sugar, etc. The questions are asked in a certain order but can result in different paths depending on customer choices. The questions are not necessarily the same for cappuccino or latte.

- Degree of elicitation discretion: It depicts the extent of additional customization allowed to be made in the elicitation outside the pre-determined, pre-designed elicitation process. This can happen by adding new tasks and paths and customization in how a task is carried out. If you compare the elicitation at Starbucks or McDonald's to investment counselling the differences are clear. Investment counselling gives much more freedom for workers in the elicitation, even if some of the end results it should produce may be pre-designed.

- Elicitation resource flexibility: It describes whether the resources the company uses in elicitation are fixed or modifiable. For example, house managers may use outside help in eliciting the needs for a heating system repairs because of insufficient competencies in the house managing agency or consultancies may acquire extra resources from partners.

- Elicitation task resource interchangeability: The dimension describes how interchangeable the company's resources are for different tasks in the elicitation 
process. For example, in a financial services company offering insurance, investment counselling and pension saving, the complexity of the different offerings may necessitate the use of different sales clerks for insurance, investment counselling, and pension saving, respectively.

- Customer-specific information intensity: This dimension describes the amount and complexity of information that needs to be elicited from the customer for the company to be able to deliver the service successfully. For example, the characteristics of maintained machinery may be required in advance so that the maintenance engineer knows which spare parts to bring with him. In insurance, customer information is necessary for risk-oriented pricing. The dimension does not include the customer choices between offering options.

\subsection{Production process}

The service process is the actual process with which the service is delivered to the customer. In McDonald's it is the preparation of the food and serving the food.

- Range of process customization: It describes the potential for customization in the predesigned process. Possible determiners are the number of different paths through the process and the number of distinct ways to perform a process task (see Figure 1).

- Degree of process discretion: It depicts the extent of additional customization allowed to be made in the service process outside the pre-determined, predesigned service process. This can happen by adding new tasks and paths and customization in how a task is carried out.

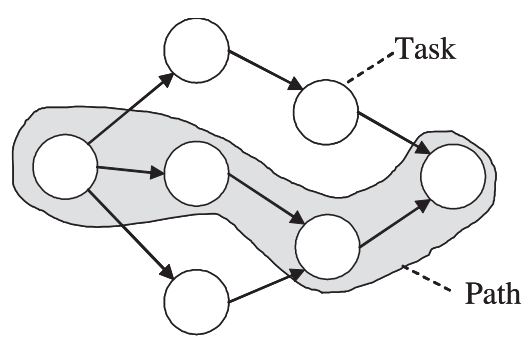

Figure 2 Illustration of process paths and tasks

- Production resource flexibility: Following MacCarthy et al. (2003) it describes whether the resources the company uses are fixed or modifiable. Fixed resources means that the service is delivered with the resources already available to the company when the order is placed. In case of modifiable resources, the company acquires new (external) resources for the delivery of a particular service.

- Task resource interchangeability: The dimension describes how interchange- 
able the company's resources are for different tasks in the process. For example, at McDonald's all the employees are trained to take orders, prepare food, serve food, and clean the facilities, thus making them easily interchangeable.

- Customer-specific information flow intensity: The dimension describes the amount of information the internal and external resources of the company need to know in the service process about the customer-specific aspects of the service agreement. The intensity is affected by the amount of information itself, in how many tasks customer-specific information is required, and how many different resources require the information.

- Information flow intensity to customer: This dimension describes the amount of information the customer (or different actors in the customer organization) needs to know in the service process in order for the service to be delivered successfully. This dimension characterizes the in-advance management of customer participation in the production process. For example, in some machinery maintenance services the customer organization is supposed to manage on-site spare parts stock, do some simple preventive maintenance themselves, etc. In transaction type services, the time elapsed between the customer receiving the information and acting accordingly can be even considerably shorter than for continuously rendered services with long-term relationships and life-cycles.

- Depth of customer involvement: This dimension describes how deeply the customer participates actively in the production process as self-inputs (of Sampson \& Froehle, 2006). It is closely related to the back- or front-office dichotomy.

- Type of customer-inputs processed: Following Sampson \& Froehle (2006), the production process deals with different customer inputs. The options are customer-self, customerprovided information, and/or customer possessions.

\section{Discussion}

\subsection{General}

We set out to identify what kind of dimensions could be useful in classifying service mass customizers. On the basis of mostly literature study of both classifications and management challenges in services and mass customization we identified 15 dimensions divided to three categories of service offering, elicitation, and production process. Some of the dimensions were applied more or less directly from literature and whereas some are synthesis of literature or entirely new ones. Currently, the dimensions are more descriptive than prescriptive.

\subsection{Comparison with Previous Work}

Arguably, the proposed dimensions take service-oriented issues into account more than the previous mass customization classifications studied. However, admittedly 
focus in most of the previous MC classifications has been squarely on goods manufacturing. The division to the offering, elicitation and production process oriented issues is present e.g. in Lampel and Mintzberg (1996). However, they use their equivalents (product, process, transactions) as dimensions and are only seemingly interested in the level of customization in them. Resource flexibility dimension is the same as MacCarthy et al. (2003) use.

Many of the proposed dimensions are present in the studied service classifications, with slightly different names. However, the three different information intensity dimensions are not present in the service classifications and thus can be considered an extension to previous work. They reflect the importance of information transfer in MC operations and the role that customer participation has in the service process. Moreover, the dimensions specifically focused on the elicitation phase have been put forth as successful elicitation is critical for MC.

The proposed classification dimensions synthesize general services and MC literature and service and MC classifications extending them by adding aspects not present earlier. The work presented in this paper particularly extends the MC literature that has neglected services.

\subsection{Limitations of the study}

The literature research on mass customization, services, and service mass customization cannot be considered exhaustive, especially for services classification which are abundant judging from (Cook et al., 1999; Johansson \& Olhager, 2004). Some relevant dimensions may have gone unnoticed. The proposed dimensions are still mostly based on conceptual analysis and need to be evaluated empirically.

\subsection{Future work}

The most pressing need is evaluation of the dimensions. Service mass customization cases reported in the literature are however very few and far between so the evaluation can only proceed with empirical case analysis. Analyzing service MC cases and later conducting such an extensive empirical evaluation as was done in (Shafti et al., 2007) could validate the dimensions and unearth insights and prescriptive recommendations for managers.

\section{Conclusions}

This study has presented dimensions that can be used to describe service mass customization companies. The dimensions were based on a literature research of service and mass customization classifications. The proposed dimensions both synthesize and extend the previous work. However, the dimensions need to be evaluated in future work. Moreover, the literature research the proposed dimensions are based on is not exhaustive, particularly for the service classifications. 


\section{References}

Benaroch, M. (1996). Knowledge Reuse in Mass Customization of Knowledge-Intensive Services. In: Ein-Dor, P. (Ed.) Artificial Intelligence in Economics and Management, Kluwer Academic Publishing, Boston 1996, 107-127.

Blecker, T., Friedrich, G., Kaluza, B., Abdelkafi, N. \& Kreutler, G. (2005). Information and Management Systems for Product Customization. New York, USA: Springer.

Blodgett Beckett, S. (1996). Achieving competitive advantage through customer value: exploring mass customization as a strategy for service organizations. Ph.D. Thesis, The University of Texas at Arlington, 1996. ISBN 0-591-15025-5.

Bowen, J. \& Ford, R.C. (2002). Managing Service Organizations: Does Having a "Thing" Make a Difference?. Journal of Management, 28(3), 447-469.

Brabazon, P.G. (2005). Mass Customization: fundamental modes of operation and a study of an order fulfilment model. Ph.D. Thesis, The University of Nottingham.

Buzacott, J.A. (2000), Service system structure. International Journal of Production Economics, 68(1), 15-27.

Cook, D.P., Goh, C.-H., Chung, C.H. (1999). Service typologies: a state of the art survey. Production and Operations Management, 8(3), 318-338.

Chowdhury, S. and Miles, G. (2006). Customer-induced uncertainty in predicting organizational design: Empirical evidence challenging the service versus manufacturing dichotomy. Journal of Business Research, 59(1), 121-129.

Da Silveira, G., Borenstein, D., Fogliatto, F.S. (2001), Mass customization: Literature review and research directions. International Journal of Production Economics, 72(1), 1-13.

Duray, R., Ward, P.T., Milligan,G.W., Berry, W.L. (2000). Approaches to mass customization: configurations and empirical validation. Journal of Operations Management 18, 605-625.

Fitzsimmons, J.A. \& Fitzsimmons, M.J. (2004), Service Management - Operations, Strategy, and Information Technology, Fourth Edition, International Edition, McGraw-Hill.

Gilmore, J.H. \& Pine II, B.J. (1997). The Four Faces of Customization. Harvard Business Review, 75(1), 91-101.

Grönroos, C. (2000), Service Management and Marketing: A Customer Relationship Management Approach, 2nd Edition, John Wiley \& Sons, Ltd.

Harvey, J., Lefebvre, L.A. \& Lefebvre, E. (1997). Flexibility and technology in services: a conceptual model. International. Journal of Operations \& Production Management, 17(1), 29-45

Hart, C.W.L. (1995). Mass customization: conceptual underpinnings, opportunities and limits. International Journal of Service Industry Management, 6(2), 36-45.

Heiskala, M. Paloheimo, K-S., Tiihonen, J. (2006), Mass Customization of Services: Benefits and Challenges of Configurable Services, Frontiers of e-Business Research (FeBR 2005). Conference proceedings of eBRF, 26.-28.9.2005, Tampere, Finland 
Heiskala, M., Tiihonen, J., Paloheimo, K-S., Soininen, T. (2007), Mass Customization with Configurable Products and Configurators: A Review of Benefits and Challenges. Blecker, T. \& Friedrich, G. (Eds.), Mass Customization Information Systems in Business, Idea Group Publishing, ISBN 978-1-59904-039-4, 1-32

Johansson, P. \& Olhager, J. (2004). Industrial service profiling: Matching service offerings and processes. International Journal of Production Economics, 89(3), 309-320.

Kellogg, D.L. \& Nie, W. (1995), A framework for strategic service management. Journal of Operations Management, 13(4), 323-337.

Lampel, J. \& Mintzberg, H. (1996). Customizing Customization, Sloan Management Review, 38(1), 21-30.

Lovelock, C. \& Gummesson, E. (2004). Whither Service Marketing? In Search a New Paradigm and Fresh Perspective. Journal of Service Research, 7(1), 20-41.

MacCarthy, B., Brabazon, P.G., Bramham, J. (2003), Fundamental modes of operation for mass customization, International Journal of Production Economics, 85(3), 289-304.

McLaughlin, C.P. (1996), Why variation reduction is not everything: a new paradigm for service operations. International Journal of Service Industry Management, 7(3), 17-30.

Moser, K. (2007). Mass Customization Strategies, PhD Dissertation, Lulu Enterprises Inc. USA, ISBN 978-1-4303-0932-1, 2007

Paloheimo, K-S., Miettinen, I., Brax, S. (2004), Customer Oriented Industrial Services, Helsinki University of Technology, BIT Research Centre, ISBN 951-22-6867$1,2004$.

Papathanassiou, E.A. (2004). Mass customization: management approaches and internet opportunities in the financial sector in the UK. International Journal of Information Management, 24(5), 387-399

Peters, L. \& Saidin, H. (2000), IT and the mass customization of services: the challenge of implementation. International Journal of Information Management, 20(2), 103-119.

Piller, F.T. (2002). Customer interaction and digitizability - a structural approach. In: Claus Rautenstrauch et al. (Eds.): Moving towards mass customization, Berlin/ New York: Springer 2002, pp. 119-138.

Piller, F.T. \& Stotko, C. (2002). Four approaches to deliver customized products and services with mass production efficiency. In: Durrani, T. S. (Ed.): Proceedings of the IEEE International Engineering Management Conference. Managing Technology for the New Economy, Cambridge University, UK, 18-20 August 2002, 773-778.

Pine, B.J. II (1993). Mass Customizing Products and Services. Strategy \& Leadership, 21(4), 6- 13, 55.

Ross, A. (1996). Selling uniqueness. IEE Manufacturing Engineer, 75(6), 260-263.

Sampson, S.E. \& Froehle, C.M. (2006). Foundations and Implications of a Pro- 
posed Unified Services Theory, Production and Operations Management, 15(2), 329-343.

Schmenner, R.W. (1986), How Can Service Business Survive and Prosper?, Sloan Management Review, 27(3), 21-32.

Schmenner, R.W. (2004), Service Businesses and Productivity. Decision Sciences, 35(3), 2004, 333-347.

Shafti, F., Van Der Meer, R. and Williams, T. (2007). An Empirical Approach to Service Classification for Productivity Management Studies. The Service Industries Journal, 27(6), 709-730.

Silvestro, R., Fitzgerald, L., Johnston, R. (1992). Towards a Classification of Service Processes. International Journal of Service Industry Management, 3(3), 62-75.

Vargo, S.L. \& Lusch, R.F. (2004). The Four Service Marketing Myths. Remnants of Goods-Based, Manufacturing Model. Journal of Service Research, 6(4), 324-335. 


\title{
Cognition of retrieved product information in configuration projects
}

\author{
Anders Haug
}

\begin{abstract}
Product configurators are software-based expert systems that support the specification of customer specific products. When creating configurators for the configuration of complex industrial products, knowledge acquisition is often one of the most challenging tasks of such a project. In spite of this fact, the knowledge acquisition process of product configuration projects is something that has been almost neglected in configuration literature, besides propositions of different modelling techniques. Therefore, this paper deals with improving the basic understanding of this aspect of product configuration.

Based on two earlier papers that deal with tacit knowledge and the types of information that a domain expert possesses/delivers in a knowledge acquisition context, this paper moves on to focus on how knowledge engineers in different ways cognize the information that they retrieve from domain experts. The increased understanding of the knowledge acquisition process of configuration projects, which this paper provides, can be seen as an improvement of the basis for future configuration research.
\end{abstract}

\section{Keywords}

Product Configuration, Knowledge Engineering, Knowledge Acquisition, Knowledge Elicitation, Knowledge Representation

\section{Authors and addresses}

Anders Haug:

Department of Entrepreneurship and Relationship Management, University of Southern Denmark, Engstien 1, 6000 Kolding.

Email:adg@sam.sdu.dk.

\section{Biographical notes}

Anders Haug is assistant professor at the Department of Entrepreneurship and Relationship Management at the University of Southern Denmark. His main research areas are knowledge management, knowledge representation, knowledge engineering and knowledge sharing - from an individual, organizational and company network perspective. Anders has produced a number of international publications that deals with the development of product configurators, representation of complex industrial knowledge, sharing of product information and more. 


\section{Introduction}

A product configurator is a software-based expert system that supports the specification of customer specific products by restricting how different elements and their properties may be combined. For many manufacturers of products engineered for the individual customer, the use of product configurators has produced a range of benefits, such as: shorter lead times, improved quality of product specifications, preservation of knowledge, use of fewer resources for specifying products, optimized products, less routine work, improved certainty of delivery, and less time needed for training new employees (e.g. Ardissono et al., 2003; Hvam, 2004; Hvam et al., 2008; Forza and Salvador, 2002; Forza and Salvador, 2007). In such cases the knowledge base of a configurator often consists of thousands of classes, attributes/ properties, rules/constants and methods. However, at the beginning of the configuration projects, most often much of this information does not exist in a well-structured and unambiguous form. These factors imply that the representation of the information that should be implemented in a configurator often is one of the most challenging parts of a configuration project (Sabin and Weigel, 1998; Hansen et al., 2003; Hvam et al., 2008). In spite of this fact, the knowledge acquisition process of configuration projects has not received much attention so far, in particular compared to the more technical aspects (Hvam et al., 2006; Haug, 2007).

Based on two earlier papers (Haug and Hvam, 2007a; 2007b) that deal with the types of knowledge/information that domain experts (e.g. product and process experts) posses and deliver in a knowledge acquisition context, this paper moves on to discuss how knowledge engineers in different ways cognize the information that they retrieve and are to make representations of in configuration projects. For instance, the retrieved information may be incorrect, which implies that the knowledge engineer may obtain a wrong understanding of the problem domain. Also, just because a knowledge engineer retrieves correct and relevant information, this does not mean that he/she necessarily interprets such information correctly. This and other situations can lead to the development of a configurator that does not deliver the required outputs. To create an understanding of how knowledge engineers in different ways cognize the information that they retrieve from domain experts, this paper proposes a classification that describes this aspect. In practice, the classification can be applied as a list of situations for a knowledge engineer to be aware of during the process of retrieving domain expert information and creating representations of this information.

The remainder of the paper is structured as follows: In section 2, relevant concepts from the field of knowledge engineering are described. Next, in section 3, tacit knowledge is discussed in relation to the knowledge acquisition process in configuration literature. Section 4 proposes a classification of how knowledge engineers in different ways cognize the information that they retrieve from domain experts. Section 5 discusses the validity of the proposed classification and outlines how the 
future investigation of the classification is planned to be carried out. The paper ends with a conclusion in chapter 6 .

\section{Knowledge engineering}

Knowledge engineering is a concept that has evolved from the late 1970s' building of knowledge systems (in this context used to describe expert systems, knowledgebased systems, and knowledge-intensive information systems). Knowledge systems are the most important industrial and commercial offspring from the field of artificial intelligence (Schreiber at al., 1999).

In order to create a knowledge system, the knowledge that this should include needs to be described in an explicit fashion. Knowledge elicitation is the process where the information needed for the creation of the first knowledge representations is obtained. The ones to provide the information that forms the basis for the creation of a configurator are the relevant domain experts of a company. The knowledge engineer is the one who is responsible for retrieving and formalizing domain expert information into a conceptual model (also called analysis model). Typically, knowledge engineers are persons who do not posses a deep knowledge of the domain that they are describing, but they are equipped with methods and techniques for eliciting and representing such information. The information which knowledge engineers receive can be delivered verbally, in body-language and in documents of physical or electronic form. The delivered information can be in the form of diagrams, tables (spreadsheets), formulas, informal rules, sketches etc., and be of declarative character (entities, relationships and constraints) and/or procedural character (how declarative knowledge is manipulated).

Conceptual models are abstractions of domain knowledge that only include selected aspects and leave others out. By focusing only on certain aspects, conceptual models provide a clearer and more unambiguous basis for discussions of relevant domain knowledge. Two commonly applied modelling languages for the creation of conceptual models in configuration projects are product variant masters (PVMs) and class diagrams (Haug and Hvam, 2007c; Hvam et al, 2008; Felfernig et al., 2000). Through the years, different PVM definitions have been proposed by Mortensen et al. (2000), Harlou (2006) and Haug and Hvam (2006). Figure 1 shows a principal example of the PVM notation, based on the latter definition, which represents the most extensive and formalized of these definitions. In a PVM model, the left column describes whole-part structure (aggregation), while the right column describes specializations of classes (generalization structure).

A class diagram describes object classes and their relations. Class diagrams are included in the Unified Modelling Language (UML) and is the most commonly applied diagram type of UML (Fowler, 2005). Oppose to PVMs, class diagrams have widespread use in general software development. In figure 2 a principal example of a class diagram in a configuration context is shown. 


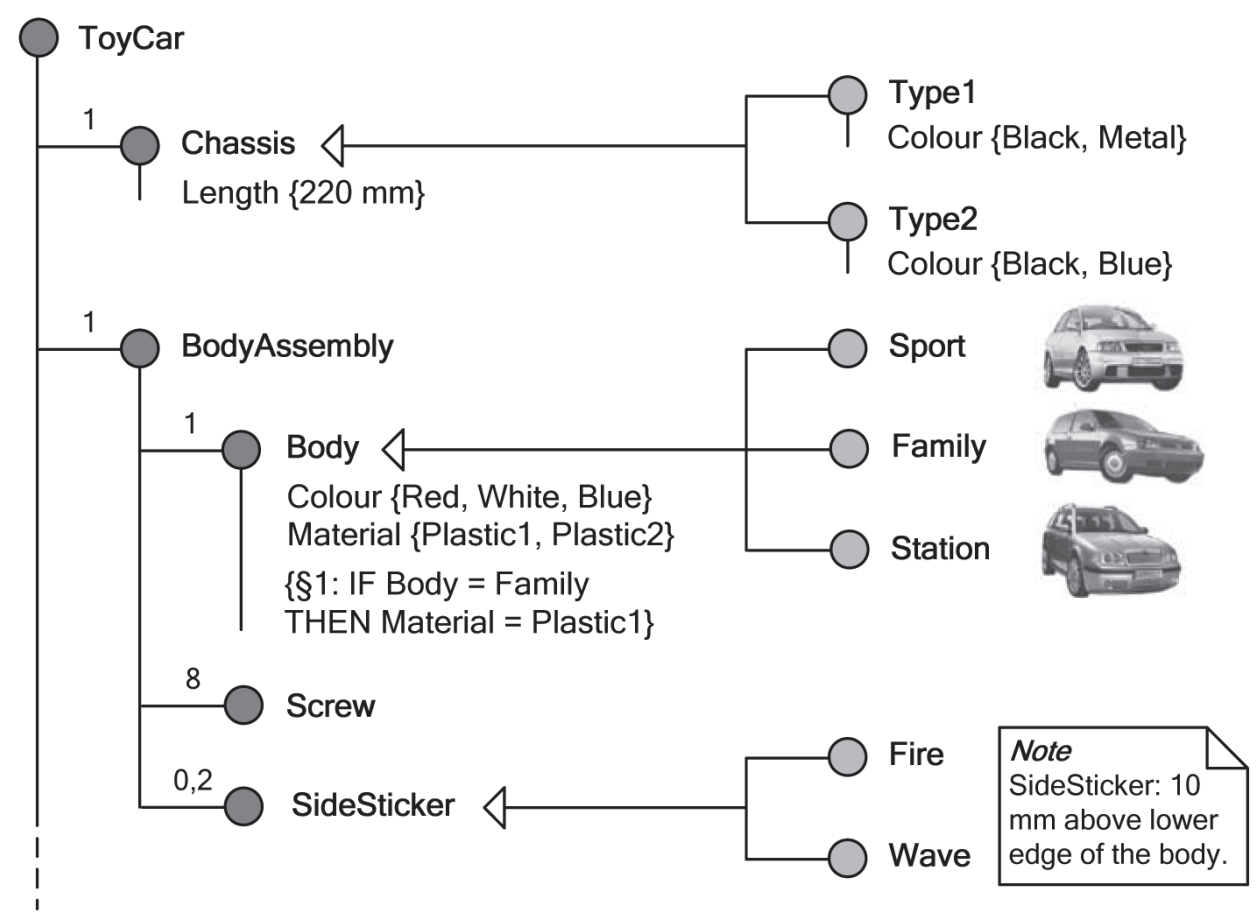

Figure 1: Example of the PVM technique

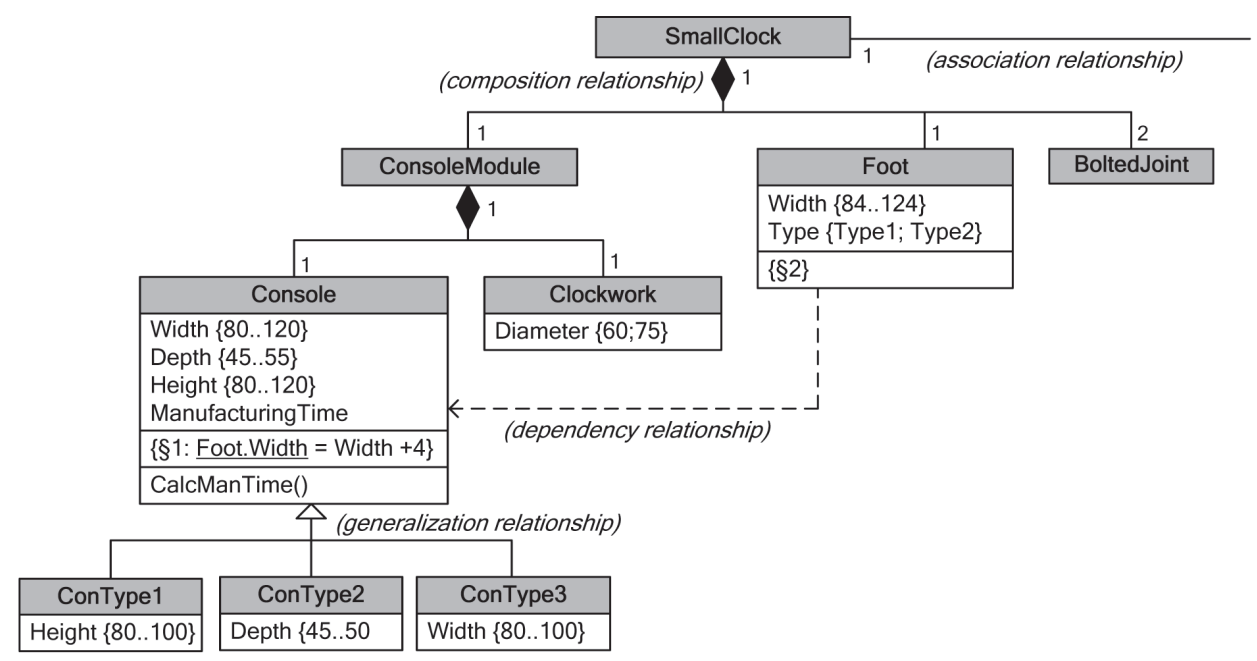

Figure 2: Example of class diagram

In the early 1980s, the development of software-based expert systems (and other knowledge-based systems) was seen as a transfer process. The principal perspective was that the required knowledge already existed in an organization, and that this 
knowledge was to be collected and implemented. However, today there is an overall consensus that the process of building an expert system is better perceived as a modelling activity, in the sense that the purpose is not to create an identical copy of domain expert knowledge, but to create a model that simulates the domain expert knowledge (Clancey, 1989; Studer et al., 1998; Speel et al., 2001; Schreiber at al., 1999). Therefore, the modelling process should rather be seen as a 'knowledge construction process' than a 'knowledge transfer process'.

Different ways exist for eliciting the information that forms the basis for the creation of conceptual models. Schreiber at al. (1999) mention five types of techniques for knowledge elicitation:

1) Interviews (Which can be unstructured, semi-structured or structured.)

2) Protocol analysis (The knowledge engineer takes record of what a domain expert is doing while solving a given task. The domain expert may be instructed to think out loud.)

3) Laddering (The knowledge engineer and the domain(s) expert construct a graphical representation together, which later may be refined by the knowledge engineer.)

4) Concept sorting (This can e.g. be done by asking the domain expert to organize cards, which represent relevant concepts, in piles. Based on this, relevant characteristics and rules of the represented concepts can be deduced.)

5) Repertory grids (This involves that the domain expert(s), based on a set of relevant elements, describes the attributes (constructs) of the elements.)

Although, the described knowledge representation and elicitation techniques provide means for carrying out knowledge acquisition in configuration projects, more deep understandings of the process also seem beneficial in order to ensure efficient and successful acquisition processes. Some of the questions that need to be answered are: which kind of knowledge do domain experts possess, which kind of information do they deliver, and how is this information cognized by the knowledge engineer, as a basis for the creation of knowledge representations? These topics are the focus of the next sections in this paper.

\section{Tacit and explicit knowledge in configuration literature}

In the configuration literature, the knowledge of domain experts has often been described in terms of tacit and explicit knowledge, in the sense that it is the tacit knowledge of the domain experts that causes the problems when creating knowledge representations, while explicit knowledge is unproblematic (Haug and Hvam, 2007a). However, such explanations of the knowledge acquisition process have been criticized by Haug and Hvam (2007a) as being unclear and misleading. The first problem is the self-contradiction that emerges from focusing on tacit knowledge as the factor that makes the knowledge acquisition difficult, while reducing 
the meaning of tacit knowledge to something as 'knowledge that is unknown to an observer'. If tacit knowledge includes all the knowledge that is unknown to an observer, some of this tacit knowledge can easily be articulated and represented, for which reason it is not much more difficult to deal with than explicit knowledge. On the other hand, just because some information is explicit, this does not mean that it is unproblematic. If the knowledge engineer that receives the information does not understand this or the knowledge engineer retrieves information that contradicts other information, such explicit information can cause great problems.

The second problem is that the use of the term 'tacit knowledge' in such a broad meaning reduces the clarity of the term and is not in line with its philosophical roots. In management studies the concept of 'tacit knowledge' has become a popular term, to a large extent because of Nonaka and Takeuchi's "The Knowledge-Creating Company" (Nonaka and Takeuchi, 1995) (Tsoukas, 2003). They cite the main founder of the term, Michael Polanyi, but as pointed out by some researchers they apply the term in another meaning than defined by Polanyi (1966; 1969). One of the critics is Tsoukas (2003), who argues that Nonaka and Takeuchi more or less interpret tacit knowledge as 'knowledge not yet articulated'. This kind of use implies that tacit knowledge is reduced to something that awaits translation or conversion into explicit knowledge. Tsoukas argues that although such interpretations have been widely adopted in management studies, they are erroneous, because they ignore the essential ineffability of tacit knowledge and reduces it to what can be articulated. Instead Tsoukas advocates that tacit and explicit knowledge should be seen as two sides of the same coin, and not two ends of a continuum, since even the most explicit kind of knowledge is underlain by tacit knowledge. From this perspective pure explicit knowledge is not 'knowledge', but merely information. Another critique of the way in which the term 'tacit knowledge' is often used in management literature comes from Gourlay (2004). He argues that although Polanyi was responsible for the term 'tacit knowledge', his work shows that he was concerned with a process, namely tacit knowing and not a static form of knowledge. In order to avoid the ambiguous and potentially misleading use of the term tacit knowledge, Gourlay (2006) recommends that the term 'tacit knowledge' is only to be used in cases in which there is evidence of action/behaviour of which the actors could not give account (excluding situations where inhibitions of communication are the reason). He further suggests that the issue of whether or not tacit knowledge can be made explicit is reframed in terms of "whether functionally equivalent descriptions of behaviours can be made". Haug and Hvam (2007a) advocate that a similar perception of tacit knowledge should be applied within product configuration research in order to: (1) ensure that a common language is used, so that misunderstandings of the literature can be avoided, and (2) to avoid the dilution of the term, which reduces its meaning to something that holds too broad a meaning to describe phenomena in a unambiguous manner. 
Even when using a more strict definition of the term 'tacit knowledge', applying the term to explain difficulties of the representation of domain knowledge in configuration projects is still problematic. First, if some required knowledge is tacit, but it is easy to create some new knowledge or information that can simulate the use of this tacit knowledge, such tacit knowledge does not represent any problems. Second, empirical investigations of configuration projects indicate that the focus on tacit knowledge is misleading. Haug and Hvam (2007b) investigated four configuration projects and found that in these cases, inarticulable information/knowledge played an insignificant if any direct role.

In order to provide a better basis for understanding configuration projects, Haug and Hvam (2007b) propose a classification that includes eight types of information that domain experts do or do not provide to a knowledge engineer in a knowledge elicitation situation. The defined types of information are:

- Information that does not leave the domain expert:

1) Concealed information

2) Unrecognized information

3) Non-possessed information

- Information that is not usable:

4) Incorrect information

5) Irrelevant information

- Information that requires analysis:

6) Inarticulable information (knowledge)

7) Contradicting information

- Directly usable information:

8) Relevant explicit information

The focuses of the classification above and the one proposed in the current paper are shown in figure 3.

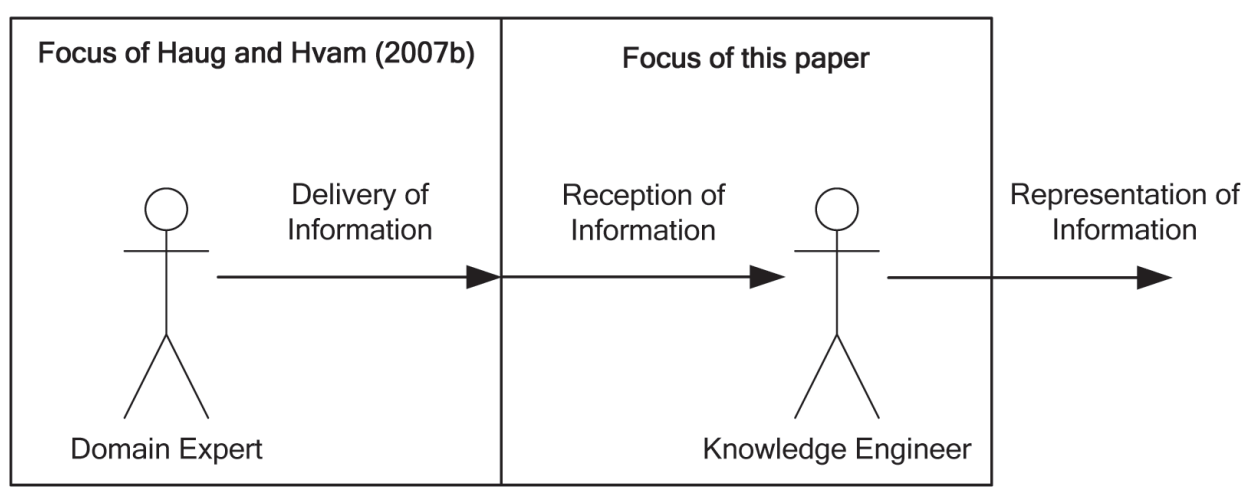

Figure 3: Focus of this paper 


\section{Proposition of a new classification}

Figure 4 shows a classification that describes how knowledge engineers cognize information retrieved from domain experts, as a basis for the creation of knowledge representations in configuration projects. The classes of the classification are further explained subsequently. The classification has been developed based on the experience from the configuration projects in which the author has been involved and discussions with knowledge engineers in the industry and other researchers.

\begin{tabular}{|c|c|c|c|}
\hline \multicolumn{4}{|c|}{ A classification of how knowledge engineers cognize information retrieved from domain experts } \\
\hline Category & No. & Types of information & Further explanation \\
\hline \multirow{3}{*}{$\begin{array}{l}\text { Information that } \\
\text { is not } \\
\text { represented }\end{array}$} & 1 & Non-cognized information & $\begin{array}{l}\text { Information that is not registered by the receiver } \\
\text { because it is ignored or not heard. }\end{array}$ \\
\hline & 2 & $\begin{array}{l}\text { Cognized, but unrecognized } \\
\text { information }\end{array}$ & $\begin{array}{l}\text { Information that is gained (unconsciously) without the } \\
\text { receiver being aware of its relevance. }\end{array}$ \\
\hline & 3 & $\begin{array}{l}\text { Cognized non-representation } \\
\text { related information }\end{array}$ & $\begin{array}{l}\text { Relevant information, but not to be part of the } \\
\text { knowledge representations. }\end{array}$ \\
\hline \multirow{2}{*}{$\begin{array}{l}\text { Information that } \\
\text { may lead to } \\
\text { incorrect } \\
\text { representations }\end{array}$} & 4 & Cognized incorrect information & $\begin{array}{l}\text { Incorrect information that is retrieved without the } \\
\text { receiver knowing that it is incorrect. }\end{array}$ \\
\hline & 5 & $\begin{array}{l}\text { Cognized misunderstood } \\
\text { information }\end{array}$ & Information that is misunderstood by the receiver. \\
\hline $\begin{array}{l}\text { Superfluous } \\
\text { information }\end{array}$ & 6 & Cognized irrelevant information & $\begin{array}{l}\text { Information that is irrelevant, without the receiver } \\
\text { being aware of this. }\end{array}$ \\
\hline \multirow{4}{*}{$\begin{array}{l}\text { Information that } \\
\text { can be difficult } \\
\text { to represent }\end{array}$} & 7 & $\begin{array}{l}\text { Cognized information to be } \\
\text { translated }\end{array}$ & $\begin{array}{l}\text { Information that needs translation to be represented, } \\
\text { e.g. due to limitations in notation formalism. }\end{array}$ \\
\hline & 8 & $\begin{array}{l}\text { Cognized non-articulable } \\
\text { information }\end{array}$ & $\begin{array}{l}\text { Recognized non-articulable information gained by } \\
\text { observing or doing. This can be tacit knowledge. }\end{array}$ \\
\hline & 9 & $\begin{array}{l}\text { Cognized contradicting } \\
\text { information }\end{array}$ & $\begin{array}{l}\text { Domain experts have different perceptions of which } \\
\text { information is correct. }\end{array}$ \\
\hline & 10 & $\begin{array}{l}\text { Cognized, but not understood } \\
\text { information }\end{array}$ & $\begin{array}{l}\text { Information that is cognized, but which the receiver } \\
\text { does not understand. }\end{array}$ \\
\hline $\begin{array}{l}\text { Directly } \\
\text { representable } \\
\text { information }\end{array}$ & 11 & $\begin{array}{l}\text { Cognized, understood and } \\
\text { representable information }\end{array}$ & Information that can be represented instantly. \\
\hline
\end{tabular}

Figure 4: A classification of how knowledge engineers cognize information retrieved from domain experts

1) Non-cognized information occurs in situations when a knowledge engineer fails to notice some information delivered by a domain expert. The knowledge engineer may not hear it or may ignore it because it is perceived not to be relevant. However, if such information is essential, this would imply incomplete models of the domain knowledge, if not later recognized.

2) Cognized, but unrecognized information occurs in situations when a knowledge engineer gains some information without recognizing the relevance of this. This 
may be in a situation when a knowledge engineer cognizes some information, but does not include it in the knowledge representation because the knowledge engineer believes that the information is irrelevant. Not being aware of the importance of this information, the knowledge engineer would create an incomplete model unless the relevant aspects are pointed out or the knowledge engineer at some point recognizes the importance of this information.

3) Cognized non-representation related information occurs when a domain expert cannot provide the required information and informs the knowledge engineer: that the required information does not exist, who possesses it, or that he/she does not know. Although such information is not to be represented in the created models, this sort of information provides a basis for further investigations and is, therefore, relevant.

4) Cognized incorrect information occurs when a domain expert intentionally or unintentionally provides incorrect information. If not discovered, such information would lead to the development of a software system that does not deliver the desired output.

5) Cognized misunderstood information occurs when a knowledge engineer does not understand what the domain expert is telling him and cognizes what has been told in an incorrect manner. Unless discovered, this would lead to incorrect representations.

6) Cognized irrelevant information occurs when a domain expert provides some information that is not needed in order to create the desired knowledge representations. Such information may be included in early models and later be excluded when it is discovered that it is not relevant.

7) Cognized information to be translated occurs in situations when some retrieved information cannot be represented in the delivered form, but needs translation or restructuring. Reasons for this may be: expressional limitations of the modelling languages applied for the creation of the conceptual models, limitations of the software used to draw models, or lack of skills of the knowledge engineer who creates the models. The distinction between 'information to be translated' and 'understood and representable information' can be a bit fluid. However, the focus of 'information to be translated' is the situations in which converting the retrieved information into a conceptual model is not straightforward. This may for instance be the case when the selected modelling software does not allow the expression of a certain type of relation between two classes, for which reason this relation needs to be formulated in another way, e.g. in a constraint or rule. 
8) Cognized non-articulable information is information that cannot be articulated, but apparently makes a domain expert capable of doing certain actions, i.e. tacit knowledge, according to the definitions by Haug and Hvam (2007a). To deal with such knowledge, the creation of new information that can simulate the domain expert is a way of getting around this obstacle.

9) Cognized contradicting information occurs when a knowledge engineer retrieves information that contradicts other information already retrieved. The challenge in such cases is to determine which information should be included in the final model, while ensuring that the relevant domain experts accept the model. If a domain expert disagrees with the information implemented in a configurator, the chances of his later use of the system decrease.

10) Cognized, but not understood information occurs when a knowledge engineer manages to cognize the information that a domain expert delivers, but does not understand what it means. This lack of understanding can be very problematic in situations where the information cannot be represented directly as delivered, since this may lead to incorrect representations.

11) Cognized, understood and representable information occurs in situations when the retrieved information is cognized and understood by the knowledge engineer, who is capable of representing this.

During the elaboration of the classification, another type of information was considered, namely 'non-representable information'. This type of information was intended to cover situations in which the selected notation formalism or software did not allow such information to be expressed. However, this type was excluded because of the argument that delivered information per definition is represented, at least by words or gestures. Therefore, if the chosen notation formalism or software does not support the retrieved information, such information belongs to the class 'information to be translated'.

\section{Investigations of the validity of the proposed classification}

As mentioned, the proposed classification was elaborated based on the experience from several configuration projects of the author and on discussions with knowledge engineers. Therefore, the proposed classification is to a large extent related to empirical experience and has some build-in validation. However, additional investigations are needed to strengthen this aspect. Two aspects of the classification are to be investigated: (1) is the classification adequately extensive or do other classes exist? and (2) are all the included classes in the classification relevant, i.e. do they occur in configuration projects? 
To investigate these questions an approach similar to the one applied by Haug and Hvam (2007b) is planned. This means that the knowledge engineers from different configuration projects are to be interviewed with the purpose of comparing their experience to the proposed classification. This includes verification (obviously under the recognition of the subjectivity of their judgement and memory) that all classes are relevant, which can be obtained by asking the knowledge engineers to provide examples of situations in which each of the classes of the classification have occurred. Secondly, the knowledge engineers are to attempt to falsify the assumption that the classification is adequately extensive by providing examples in which other classes than the ones included in the classification have occurred. If they are not capable of doing this, the assumption that the classification is adequately extensive, for the moment can be considered to have some validity.

\section{Conclusions}

This paper took a basis in two earlier papers by the author in which the author criticizes the use of the term 'tacit knowledge' to explain why knowledge elicitation can be difficult in configuration projects and proposed a more nuanced classification of the information that domain experts posses and deliver in configuration projects. With this basis this paper moved on to propose a classification of the different ways in which knowledge engineers cognize the information that they retrieve from domain experts in configuration projects. The classification includes eleven classes divided into five groups: Information that is not represented: 1) Non-cognized information, 2) Cognized, but unrecognized information, 3) Cognized non-representation related information; Information that may lead to incorrect representations: 4) Cognized incorrect information, 5) Cognized misunderstood information; Superfluous information: 6) Cognized irrelevant information; Information that can be difficult to represent: 7) Cognized information to be translated, 8) Cognized nonarticulable information, 9) Cognized contradicting information, 10) Cognized, but not understood information; Directly representable information: 11) Cognized, understood and representable information.

While much configuration literature almost solely applies the term 'tacit knowledge' to describe knowledge that represents difficulties in configuration projects, this paper presented ten types of problematic situations in which a knowledge engineer cognizes retrieved information. This classification therefore provides a more nuanced way of perceiving the cognition process of knowledge engineers in configuration projects. Besides being useful in a product configuration context, the classification may also be applicable in other lines of research that deals with situations where information is elicited from domain experts. 


\section{References}

Ardissono, L., Felfernig, A., Friedrich, G., Goy, A., Jannach, D., Petrone, G., Schäfer, R. and Zanker, M. (2003): A Framework for the Development of Personalized, Distributed Web-Based Configuration Systems. AI Magazine, 24(3): 93-108.

Clancey, W.J. (1993): The Knowledge Level Reinterpreted: Modelling Socio-Technical Systems. International Journal of Intelligent Systems, 8: 33-49.

Felfernig, A., Friedrich, G. and Jannach, D. (2000): UML as domain specific language for the construction of knowledge based configurations systems. International Journal on Software Engineering and Knowledge Engineering, 10(4): 449-470.

Forza, C. and Salvador, F. (2002): Managing for variety in the order acquisition and fulfilment process: The contribution of product configuration systems. International Journal of Production Economics, 76(1): 87-98.

Forza, C. and Salvador, F. (2007): Product Information Management for Mass Customization. New York: Palgrave MacMillan.

Fowler, M. (2005): UML Distilled (3rd edition). Boston, MA: Addison-Wesley.

Gourlay, S.N. (2004): Knowing as semiosis: steps towards a reconceptualization of 'tacit knowledge'. Organizations as Knowledge Systems (Ed. H. Tsoukas and N. Mylonopoulos), pp. 86-105. London: Palgrave Macmillan.

Gourlay, S. (2006): Towards conceptual clarity for 'tacit knowledge': a review of empirical studies. Knowledge Management Research \& Practice, 4(1): 60-69.

Hansen, B.L. (2003): Development of Industrial Variant Specification Systems. PhD thesis. Lyngby, Denmark: Department of Manufacturing Engineering and Management, Technical University of Denmark.

Harlou, U. (2006): Developing product families based on architectures - Contribution to a theory of product families. PhD thesis. Lyngby, Denmark: Department of Mechanical Engineering, Technical University of Denmark.

Haug, A. and Hvam, L. (2006): Merging models with different perspectives on product configuration knowledge. Research in Interactive Design, Volume 2 (Proceedings of Virtual Concept, Cancun, Mexico, Nov. 26 - Dec. 1, 2006). France: SpringerVerlag.

Haug, A. and Hvam, L. (2007a): Tacit knowledge in configuration projects. Innovative Processes and Products for Mass Customization (Proceedings of the Joint Conference IMCM'07 \& PETO'07). Berlin: GITO-Verlag.

Haug, A. and Hvam, L. (2007b): A classification of the information that domain experts do and do not provide in configuration projects. Proceedings of the 12th annual international conference on Industrial Engineering Theory, Applications \& Practice. Cancun, Mexico, Nov. 4-7, 2007.

Haug, A. and Hvam, L. (2007c): A comparative study of two graphical notations for the development of product configuration systems. International Journal of Industrial Engineering, 14(2): 107-116.

Haug, A. (2007): Representation of Industrial knowledge - as a basis for developing 
and maintaining product configurators. PhD thesis. Lyngby, Denmark: Department of Industrial Management and Engineering; Technical University of Denmark.

Hvam, L., Pape, S. and Nielsen, M.K. (2006): Improving the quotation process with product configuration. Computers in Industry, 57: 607-621.

Hvam, L., Mortensen, N.H. and Riis, J. (2008): Product Customization. Berlin and Heidelberg: Springer-Verlag.

Hvam, L. (2004): A multi-perspective approach for the design of Product Configuration Systems - an evaluation of industry applications. Proceedings of the International Conference of Economic, Technical and Organizational aspects of Product Configuration Systems (PETO), Lyngby, Denmark, June 28-29, pp. 13-25.

Mortensen, N.H., Yu, B., Skovgaard, H. and Harlou, U. (2000): Conceptual modeling of product families in configuration projects. Workshop at the 14th European Conference on Artificial Intelligence. Berlin, Germany, Aug. 20-25.

Nonaka, I. and Takeuchi, H. (1995): The Knowledge-Creating Company. New York: Oxford University Press.

Polanyi, M. (1966): The Tacit Dimension (Reprint 1983). Gloucester, MA: Peter Smith Publishers Inc.

Polanyi, M. (1969): Knowing and Being. Essays by Michael Polanyi (Ed. M. Grene). Chicago: University Of Chicago Press.

Sabin, D. and Weigel, R. (1998). Product Configuration Frameworks - A survey. IEEE Intelligent Systems \& Their Applications, 13(4): 42-49.

Schreiber, G., Akkermans, H., Anjewierden A., de Hoog, R., Shadbolt, N., Velde, W.V. and Wielinga, B. (1999): Knowledge Engineering and Management: The CommonKADS Methodology. Cambridge, MA: MIT Press.

Speel, P.H., Schreiber, A.T., Joolingen, W., Van Heijst, G. and Beijer, G.J. (2001). Conceptual Models for Knowledge-Based Systems. Encyclopedia of Computer Science and Technology. New York: Marcel Dekker Inc.

Studer, R., Benjamins, V.R. and Fensel, D. (1998). Knowledge Engineering: Principles and Methods. Data \& Knowledge Engineering, 25(1-2): 161-197.

Tsoukas, H. (2003). Do we really understand tacit knowledge? The Blackwell Handbook of Organizational Learning and Knowledge Management (Ed. M. EasterbySmith, M.A. Lyles), pp. 410-427. Oxford: Blackwell Publishing Ltd.v 


\title{
Time Postponement: Modified Models to Improve Delivery Lead Time
}

\author{
Soroosh Saghiri \\ University of East Anglia \& University of Greenwich Business School \\ E-mail:s.saghiri@uea.ac.uk
}

\begin{abstract}
To achieve higher flexibility in managing sporadic market demand, a number of value adding activities can be delayed to a later time or stage in supply chain. These delays which can happen on time, form or place are recognised as postponement strategies. The aim of this study is to improve the performance of time postponement in finishing and shipment stages of the supply chain. The main shortfall of time postponement is prolonging delivery lead time. To improve the performance of production and shipment postponement strategies, this paper introduces two modified postponement models. Models are developed to postpone forecasting, finishing and shipment operations. Provided modifications on postponement strategies improve the performance of the system. Buyer waiting time and the conformity between shipment quantity and real demand quantity are defined as the performance measures for the postponement model.

The developed models have been tested empirically. Modified postponement models indicate considerable improvement in performance, and provide important contribution to the application of time postponement strategy.
\end{abstract}

\section{Keywords}

Time Postponement, Packaging postponement, Labelling Postponement, Shipment Postponement.

\section{Introduction}

Postponement is known as a supply chain strategy which delays product differentiation until more accurate information about demand is obtained (Naylor et al., 1999). By delaying a number of value-adding activities (e.g. manufacturing, assembly, packaging, or shipment), postponement strategy tries to reduce inventory and increase service level (Nair, 2005). However, application of postponement depends on a series of requirements in product, process and supply chain relationships, which may make achievements of postponement debatable (Christopher, 1998; Van Hoek, 2001). In effect, despite its simple definition, developments in postponement theory and applications are not very straight forward. Identification of activities to delay, postponement time and form, inventory planning in the delayed activities, and postponement requirements and enablers are challenges of post- 
ponement strategy (Pagh and Cooper, 1998; Van Hoek, 2001; Salvador and Forza, 2004; Saghiri and Lowson, 2005; Boone et al., 2007).

Towards application of different types of postponement strategy, Lee and Tang (1997) analyses cost and benefits of delayed production. They examine three key practices of Standardisation, Modularisation, and Process Restructuring, and discuss how they support postponement. Skipworth and Harrison (2004) compare the form postponement strategy with make-to-stock and make-to-order strategies. They discuss the impact of delivery reliability and demand behaviour on postponement. Gupta and Benjaafar (2004) explore the role of capacity on pursuing postponement strategy. They compare and contrast delayed differentiation and maketo-order approaches. Suet al. (2005) also analyse the relationship between product and process types and success of a postponement strategy.

The literature review has indicated that it is widely required to study techniques which maintain or enhance application of postponement strategies. It has also been highlighted by the literature that despite many studies on the benefits of postponement, little is still known about its implementation (Boone et al., 2007; Yang and Burns, 2003; Skipworth and Harrison, 2004). Hence, it seems that the research on postponement is at the stage of investigating and understanding how the concept can be operationalised.

This study develops the postponement problem while it focuses on the postponed packaging, labelling and shipment operations. Two performance criteria for the applied postponement strategy are considered: buyer waiting time and the mismatch between the real demand and the amount shipped to the buyer. Clearly, by postponing some operations, there is a higher chance to provide customer with what exactly it demands. However, the more delay in those operations, the more lead time for delivery (delivery time is called buyer's waiting time in this study).

By distancing from pure postponement, this study tries to develop modified postponement models. Models include postponed forecasting and a mixture of postponed forecasting and postponed finishing (packaging and labelling) and shipment operations. Developing the modified postponement models (postponed forecasting and combination of postponed forecasting and postponed finishing and shipment) is the main contribution of this paper.

\section{Methodology}

Case study has been recognised as an appropriate research strategy to answer "how" questions in research (Saunders et al., 2003). In a case study, the researcher finds the opportunity of in depth investigation collection through review of documents, observation of activities, and direct interviews. Accordingly, the current research focuses on a case study where it gather data through interviews and study of working documents. Data collection and testing the models are based on a case study in clothing industry, in a men's underwear and sock production corporation. 
The men's underwear and sock firm which is called company Alpha later on, makes its products based upon limited predetermined designs and production processes. Changes in design or material happen rarely, even the colour mix is fixed. Products in this firm are distributed through wholesalers (which are called 'buyers' later in this paper) which update information of the consumer market frequently. Wholesalers send their orders to the firm almost regularly. Changes in demand usually happen to the packaging, labelling and quantity of the orders. As the main alteration in orders occurs to the last stages of the production process and quantity of shipment, the firm considers the application of packaging and labelling and shipment postponement. In next section, this study starts with modelling no-postponement and fullpostponement situation. Then it tries to modify the initial models to improve the performance of the systems in terms of buyer waiting time and mismatch with the buyer order.

\section{Problem description and the models}

This study focuses on last stages of the production process of the company Alpha and the shipment of the product to the buyer's site. The last stage of the production process includes packaging and labelling (called "finishing stage" later on) which can be customised based upon the buyer order. The customisation means finishing can vary for different orders. The operations and their time for finishing operations are almost similar. Time postponement of the finishing operations and shipment is focused by the models introduced in this study. The main incentive to pursue time postponement strategy is matching supply and demand in a fluctuating demand situation.

The time postponement problem is analysed by two basic models and four modified models. Two basic models include "advanced finishing" (no postponement) and "postponed finishing" (full postponement). The two basic models reflect extreme situations. In advanced finishing, postponement does not happen. Products are packaged and labelled according to the forecast much prior to receiving customer order. Quite the opposite, in full postponement, product is not prepared and shipped before receiving customer order. Focusing on the time postponement approach, this study tries to overcome the main shortfall of time postponement which is long delivery lead time. To do this, four models (called "modified models") are developed to improve the full postponement model. Before explaining each model individually, assumptions which are considered in the models are explained. The assumptions largely reflect the real-life situation of the case study described earlier. Simplifying assumptions are applied if necessary.

\subsection{General assumptions}

To consider the finishing operations time in the models, parameters $\theta$ and $\tau$ are defined as follows: 
$\theta$ : Finishing time for each unit of product (hour/unit).

$\tau$ :Time available in each period for finishing operations (e.g. 8 hours if a period is assumed as one working day).

Thus, total number of products which can be produced (finished) in each period is $\theta / \tau$.

Products are delivered to the buyer in one shipment. Shipment time is shown by $L r$ and is defined as:

Lr :Transport lead-time - duration of shipping products to the buyer's site (hour).

Finishing operations and shipment are considered in a planning horizon $T$. Each period is indicated by index $t$. Hence, $t \in T$. In the current case study, each period $t$ refers to "one day".

Buyer demand is received by direct order, and also is forecasted by the company Alpha. They are notated as:

$\operatorname{Dr}(t)$ : Real demand (buyer order) in period $t$

$\operatorname{Dr}(t)$ :Forecasted demand for period $t$

When buyer places its order in period $t$, the target is meeting buyer's demand as quick as possible. This is mainly because of the volatile final consumer market and its impact on the company Alpha's buyers. Thus, buyers have no accurate plan when exactly they need the order they place in period $t$. Roughly, they just need it for near future. In that respect, the company Alpha has two main options. Finishing and shipping products based on the demand prediction, or delaying those operations by the time it receives buyer order in each period.

In the first approach, delivery is done in advance. Although the buyer receives the product on time, as shipment is done based on the prediction (ship-to-stock), it may be not exactly what they want in terms of quantity they need and in terms of minor customisation in packaging and labelling. In the second approach, the buyer will exactly receive what it orders but with a time lag - which is usually lengthy.

Two variables of the models which show customer waiting time and difference between the delivery quantity and the real demand are defined as:

$B W T$ - Buyer's waiting time (hours): the time between customer order and product delivery (see ).

$\varepsilon(t)$ - Error: the difference between real demand (buyer order) in period $t$ and product units prepared to meet that demand (of period $t$ ) based on the forecast for period $t$.

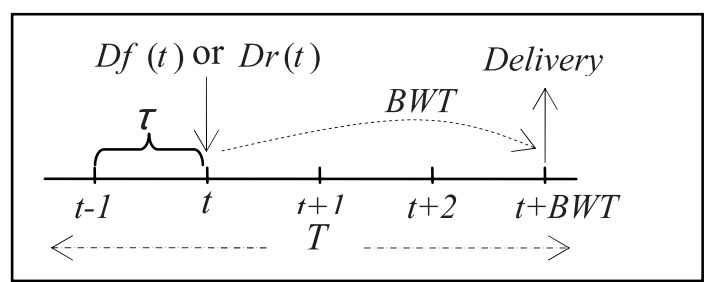

Figure 1. Time elements of the postponement models. 


\subsection{Advanced shipment model}

In advanced shipment model, decision about the quantity which should be sent to the buyer's site is made based on the forecast. Forecast data is received from master planning department which generates and updates demand forecast information. Finishing operations and shipment are done in advance, regardless to the real order of the buyer - which may be received in few periods. Number of products delivered to the buyer in this model is indicated by $X s(t)$.

$X s(t)$ : Advanced shipment based on the forecast for demand of period $t$ (units of product) when finishing and shipment operations are done much earlier than period $t$.

As explained above, $X s(t)$ follows the forecasted demand for period $t$. Hence, $X s(t)=D f(t)$. Products are finished and shipped to the customer prior to the customer order, thus, $B W T=0$. Error, is also defined as $\varepsilon s(t)=\operatorname{Dr}(t)-X s(t)$. Figure 2 illustrates the time structure of the advanced shipment model.

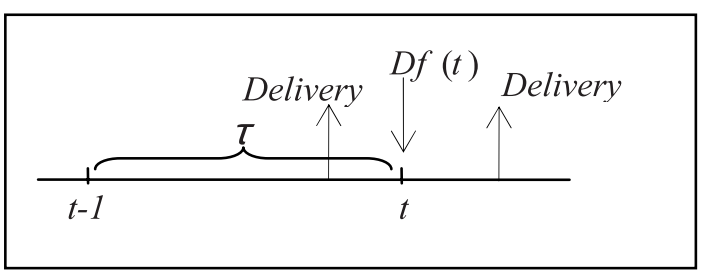

Figure 2. Advanced shipment postponement model.

\subsection{Full postponement model}

In this model, finishing operation starts once the company Alpha receives the buyer order. Output of the finishing stage $(X p(t))$ is equal to the buyer order for period $t$ $(\operatorname{Dr}(t))$. The same amount is shipped to the buyer. As defined earlier, the finishing operations take $\operatorname{Dr}(t) * \theta$ and shipment takes Lr hours. Hence, $B W T=(\operatorname{Dr}(t) * \theta+L r$. As the amount of the products delivered to the buyer is exactly same as what the buyer ordered, the error is zero $(\varepsilon \mathrm{p}(t)=0)$ ). Figure 3 illustrates the time structure of the full postponement model.

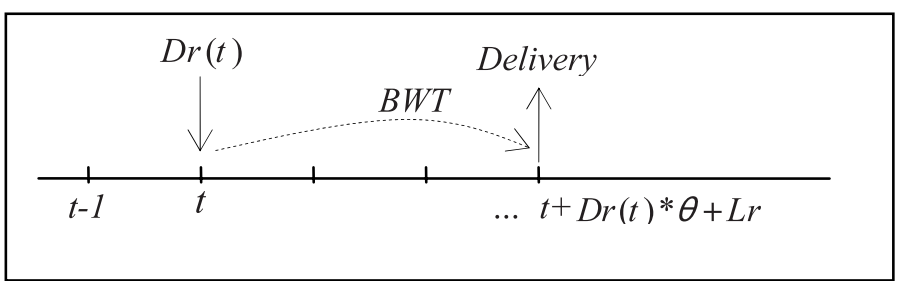

Figure 3. Full postponement model.

\subsection{Modified postponement models}

To improve the long buyer waiting time (BWT) in the full postponement model and high error $(\varepsilon s(t))$ in advanced shipment approach, in this section four modi- 
fied model are developed. All of them follow the time postponement strategy, with some modifications. In modified models, finishing and shipment of the demand of period $t$ are postponed, but not to the period $t$. Instead, modified models, try to generate more accurate forecasts of the demand in a nearer time to period $t$, or to mix the forecasting data and buyer order. Details of each model are explained in the following sub-sections.

\subsubsection{Modified postponement model I and II- postponed forecasting:}

Modified postponement models $I$ and $I I$ are explained in one sub-section as the rational of both is same, but they just use different criteria in their forecasting.

In both models finishing operations (and consequently, shipment) of the period $t$ demand, are started $k$ period earlier. $k$ is much closer to period $t$, than the time of general forecast generated by the master planning department of the company Alpha. Once the forecasting and planning are done (for period $t$ in period $t-k$ ), the company follows it, regardless to the buyer's order (real demand) for period $t$ which may arrive later.

In the modified postponement model $I$, the demand of period $t$ is forecasted in period $t k$, and finishing and/or shipment operations start in the same period $(t-k)$. Number of products which are planned to start operations of finishing in period $t-k$ is indicated by $X m I(t, k)$, and is defined as:

$X m I(t, k)$ : Modified postponed finishing (Model $I$ ) for the demand of period $t$ when finishing operations are started in period $t-k$ (units of product).

$\mathrm{XmI}(t, k)$ is identified by the demand forecast generated in period $t-k$. That forecast is based on the general demand forecast for period $t$ and the buyer order (real demand) in period $t-k$. Thus, it is calculated as:

$X m I(t, k)=(D f(t)+D r(t-k)) / 2$

As the emphasis of all modified models is on the time postponement strategy, they try to work as much as possible with real data. In view of that, they avoid to follow complex forecasting systems which are mostly inefficient in prediction of highly volatile demand.

Error and BWT in the modified postponement model I are calculated as: $\varepsilon m I(t, k)=\operatorname{Dr}(t)-X m I(t, t)$ $B W T=M A X\{0,(X m I(t, k) * \theta+L r)-k * \tau\}$

In the modified postponement model II, the demand of period $t$ is also forecasted in period $t, k$, and finishing and shipment operations start in the same period $(t, k)$. Number of products which are planned to start operations of finishing in period $t, k$ is indicated by $X m I I(t, k)$, and is defined as: 
$X m I I(t, k)$ : Modified postponed finishing (Model II) for the demand of period $t$ when finishing operations are started in period $t-k$ (units of product).

$\mathrm{XmII}(t, k)$ is identified by the demand forecast generated in period $t-k$ which is based on the buyer order (real demand) in period $t-k$. It is calculated as XmII ( $t, k$ )$=D r(t-k)$ Error and BWT in the modified postponement model $I I$ are:

$\varepsilon m I I(t, k)=\operatorname{Dr}(t)-X m I I(t, k)=\operatorname{Dr}(t)-\operatorname{Dr}(t-k)$

$B W T=M A X\{0,(X m I I(t, k) * \theta+L r)-k * \tau\}$

Figure 4 shows the modified models $I$ and II. If XmI (or XmII) are finished and delivered before period $t, B W T$ will be zero. Otherwise $B W T=X m I(t, k) * \theta+L r)-k$ ${ }^{*} \tau$ (or $\left.\left.B W T=X m I I(t, k) * \theta+L r\right)-k * \tau\right)$.

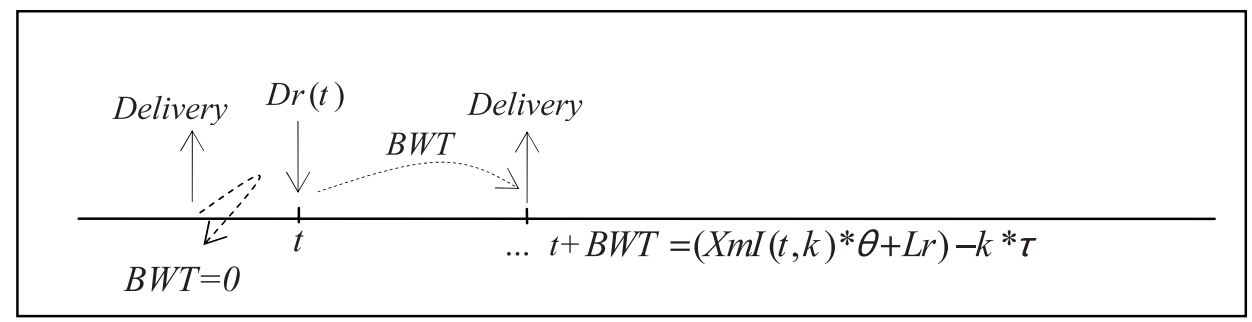

Figure 4. Modified postponement model.

\subsubsection{Modified postponement model III and IV-postponed forecasting and}

finishing: Modified postponement models III and IV both follow same rational, but they use different criteria in their forecasting.

In both models finishing operations (and consequently, shipment) of the period $t$ demand, start $k$ period earlier. The forecasted amount in this model is equal to the forecast of the first modified model $(X m I(t, k))$. Once the forecasting and planning are done (in period $t-k$ for period $t$ ), the company starts the finishing (and consequently, shipment). The finishing operations carry on by period $t$ or by the time $\mathrm{XmI}(t, k)$ units are prepared. If all XmI $(t, k)$ are prepared before period $t$, the shipment is also done. However, if total amount finishes by period $t$ is less than predicted demand for period $t(X m I(t, k))$, the real demand of period $t(\operatorname{Dr}(t))$ is considered. If total amount finished by period $t$ is more than $\operatorname{Dr}(t)$, then the finishing is stopped and finished items by period $t$ are shipped to the buyer's site. If total amount finished by period $t$ is less than $\operatorname{Dr}(t)$, then finishing is carried on by the time totally $\operatorname{Dr}(t)$ items are prepared for the shipment. This modified model actually follows a mixture of forecasting and real data, where both forecasting and finishing are partially postponed. Variables of the Modified postponement model III are defined as follows. 
XmIII $(t, k)$ : Modified postponed finishing (Model III) for the demand of period $t$ (units of product) when finishing operation is postponed to period $t-k$.

$$
X m I I I(t, k)=\left\{\begin{array}{llll}
X m I(t, k) & \text { if } & Y m I I I(t, k)=X m I(t, k) \\
D r(t) & \text { if } & Y m I I I(t, k)=k * \frac{\tau}{\theta}
\end{array} \quad \text { AND } \quad Y m I I I(t, k)<\operatorname{Dr}(t)\right.
$$

$Y m I I I(t, k)=$ Total production from period $t-k$ to period $t$ (in the Model III)

$Y m I I I(t, k)=\operatorname{MIN}\left\{X m I(t, k), k * \frac{\tau}{\theta}\right\}$

$\varepsilon m I I I(t, k)=|\operatorname{Dr}(t)-X m I I I(t, k)|$

$$
B W T=\left\{\begin{array}{lll}
\operatorname{MAX}\{0, X m I I I(t, k) * \theta+L r-k * \tau\} & \text { if } \quad Y m I I I(t, k)=X m I(t, k) \\
X m I I I(t, k) * \theta+L r-k * \tau & \text { if } \quad Y m I I I(t, k)=k * \frac{\tau}{\theta} \operatorname{AND\quad YmIII}(t, k)<\operatorname{Dr}(t) \\
L r & \text { if } \quad \operatorname{YmIII}(t, k)=k * \frac{\tau}{\theta} \operatorname{AND} \quad \operatorname{YmIII}(t, k) \geq \operatorname{Dr}(t)
\end{array}\right.
$$

Similar to the model III, the modified postponement model $I V$ uses a mixture of forecasting and real data. The only difference is that it applies the forecasting method of model II. Accordingly, variables of the Modified postponement model IV are defined as follows.

$X m I V(t, k)$ Modified postponed finishing (Model IV) for the demand of period $t$ (units of product) when finishing operation is postponed to period $t-k$.

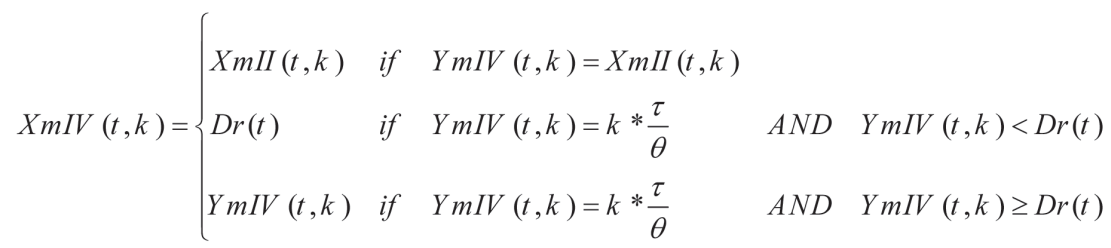

$\operatorname{YmIV}(t, k)=$ Total production from period $t-k$ to period $t$ (in the Model $I V)$

$Y m I V(t, k)=\operatorname{MIN}\left\{X m I I(t, k), k * \frac{\tau}{\theta}\right\}$

$\varepsilon m I V(t, k)=|\operatorname{Dr}(t)-X m I V(t, k)|$

$$
B W T=\left\{\begin{array}{lll}
M A X\{0, X m I V(t, k) * \theta+L r-k * \tau\} & \text { if } \quad Y m I V(t, k)=X m I I(t, k) \\
X m I V(t, k) * \theta+L r-k * \tau & \text { if } \quad Y m I V(t, k)=k * \frac{\tau}{\theta} \operatorname{AND} \quad \operatorname{YmIV}(t, k)<\operatorname{Dr}(t) \\
L r & \text { if } \quad Y m I V(t, k)=k * \frac{\tau}{\theta} \operatorname{AND} \quad \operatorname{YmIV}(t, k) \geq \operatorname{Dr}(t)
\end{array}\right.
$$




\subsection{Computational Results and Managerial Implications}

In this section, models developed earlier are applied in the case study for fifty periods. The real data and forecasting data applied for "full postponement model", "advanced shipment model" and the four modified models. The modified postponement models are run for all $k s(k \in\{1, . ., 5\})$.

Figure 5 shows the mean absolute error (MAE) for all six models. MAE indicated the average of errors in fifty periods for which the models are run:

$$
\mathrm{MAE}=\sum_{t=1}^{50} \varepsilon_{t} / n ; n=50 .
$$

As illustrated by Figure 5, error is highest in advanced shipment model, while it is zero in full postponement model. By applying modified models, although MAE is still more than fully postponement situation, but considerable improvement is achieved comparing to the advance shipment model. The analysis indicates that the modified models $I$ and $I I$ are not highly sensitive to $k$. It means that while just forecasting is postponed, the system still confronts high level of error, and relying more on the forecast (i.e. increasing $k$ does not reduce the error). On the other hand, models III and $I V$, which postpone both forecasting and finishing operations are highly successful in reducing the error. Those models are sensitive to $k$. This implies that by relying more on forecasting data (i.e. increasing $k$ ) the error of the system is increasing as well.

The other factor which should be considered is buyer waiting time (BWT). As it is clear from the definition of advance shipment the BWT is zero in that model. It is also expected that BWT is in its maximum in fully Postponement situation (see Figure 6). By modifying the fully postponement models and increasing $k$, all four modified models show improvement in BWT.

\section{Conclusion}

Advance shipment and fully postponement approach has serious shortfall in meeting customer needs. Advance shipment does not send the right quantity to the buyer (high error) and fully postponement sends the buyer order with significant delay (high $B W T$ ). Modified models have tried to mitigate those shortfalls. Finding a k, which can satisfy both error and BWT is crucial. Right $k$ is different in different systems and different situations. It also depends on the importance of error and BWT factors for the buyer and operations management system as well.

In future studies, applying more efficient forecasting methods can be studied. Moreover, other performance criteria can be considered. 


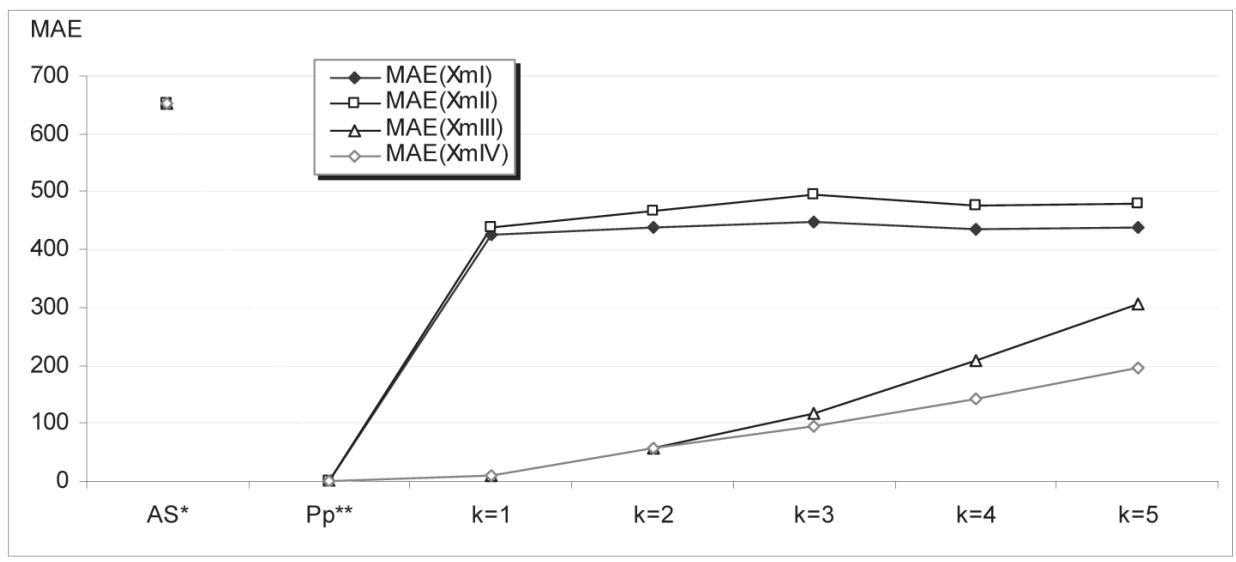

Figure 5. Mean Absolute Error in different postponement strategies. * Advance Shipment, ** Fully Postponement

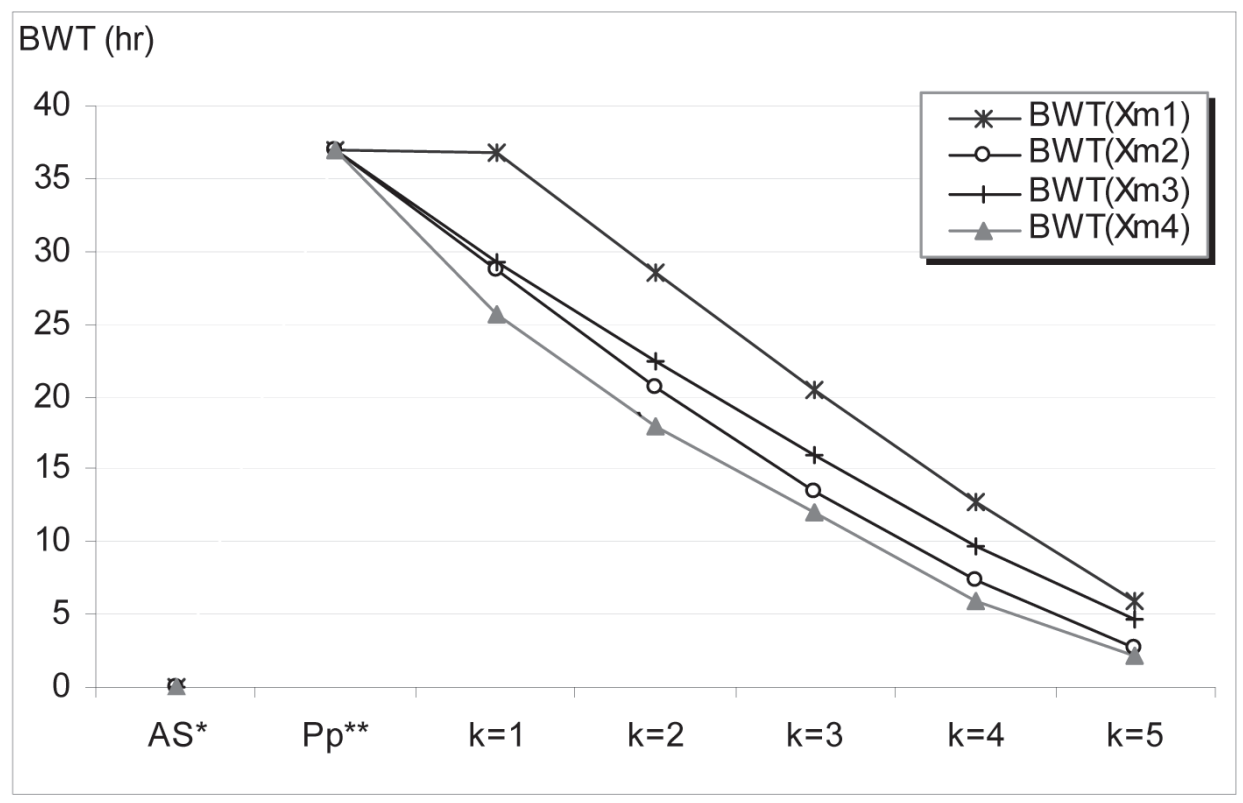

Figure 6. Buyer waiting time (BWT) in different postponement strategies. * Advance Shipment, ** Fully Postponement 


\section{References}

Boone, C.A., Craighead, C.W. and Hanna, J.B. (2007). Postponement: an evolving supply chain concept. International Journal of Physical Distribution \& Logistics Management 37(8): 594-611.

Christopher, M. (1998). Logistics and supply chain management: strategies for reducing cost and improving service. 2nd edition. London, Financial Times Pitman.

Gupta, D. and Benjaafar, S. (2004). Make-to-order, make-to-stock, or delay product differentiation? A common framework for modeling and analysis. IIE Transactions 36(6): 529-546.

Lee, H.L. and Tang, C.S. (1997). Modelling the Costs and Benefits of Delayed Product Differentiation. Management Science 43(1): 40-53.

Nair, A. (2005). Linking manufacturing postponement, centralized distribution and value chain flexibility with performance. International Journal of Production Research 43(3): 447-463.

Naylor, J.B., Naim, M.M. and Berry, D. (1999). Leagility: Integrating the lean and agile manufacturing paradigms in the total supply chain. International Journal of Production Economics 62(1-2): 107-118.

Pagh, J. and Cooper, M.C. (1998). Supply chain postponement and speculation strategies: how to choose the right strategy. Journal of Business Logistics 19(2): 13-33.

Saghiri, S. and Lowson, R.H. (2005). Agile Supply Chain Management Operations Tactics: An Architectural Viewpoint. in 10th Annual Conference of the Logistics Research Network: International Logistics and Supply Chain Management, Plymout, UK.

Salvador, F. and Forza, C. (2004). Configuring products to address the customizationresponsiveness squeeze: A survey of management issues and opportunities. International Journal of Production Economics 91: 273-291.

Saunders, M., Lewis, P. and Thornhill, A. (2003). Research Methods for Business Students. 3. London, FT Prentice Hall.

Skipworth, H. and Harrison, A. (2004). Implications of form postponement to manufacturing: a case study. International Journal of Production Research 42(10): 2063-2081.

Su, J.C.P., Chang, Y.L. and Ferguson, M. (2005). Evaluation of postponement structures to accommodate mass customization. Journal of Operations Management 23: 305-318.

Van Hoek, R.I. (2001). The rediscovery of postponement: a literature review and directions for research. Journal of Operations Management 19(2): 161-184.

Yang, B. and Burns, N. (2003). Implications of postponement for the supply chain. International Journal of Production Research 41(9): 2075-2090. 


\title{
Web-based VR for Pre-Sales Service Customization
}

\author{
Boris Tudjarov \\ Angel Bachvarov \\ Ilia Boyadjiev
}

\begin{abstract}
The new products are introduced to the market traditionally trough the "DesignMake-Sell"- cycle, typical for manufacturing industries. Manufacturers capture and aggregate customer needs to product specifications, pass the information to the designers to design the product, produce it and display it for sale. This long cycle time is not enough responsive to the specific needs of the contemporary dynamic and competitive markets. Customers' knowledge and direct involvement in the design process can leverage product and product related information, facilitate marketing research and bring different customers' attitudes towards buying decision making. As a consequence, the traditional cycle of "Design - Make-Sell" shall be replaced by the new one of "Design - Sell-Make". Customers design the product they need, buy it, and send the order to be manufactured.

The main goal of our work is to present and discuss an approach to provide a direct customers' participation in the design process in form of a pre-sale service, based on the use of the Web Virtual Reality. Core element of implementation such a service is the 3D product model web configurator. An experimental 3D Web Configurator is realized, mainly by the use of eXtensible 3D language (X3D). It aims to realize an eXtensible Markup Language (XML) environment for 3D Modeling and XML based Integration of the Customer preferences, Engineering calculations and Model of Assembly. This 3D configurator provides as feedback in the web-browser of the customer not only the appropriate graphical representation of the newly developed product (system), but the information about the product installation and operational environment, animation of the product functions and dynamical change of the assembly model and its parts parameters such as dimensions of form, dimensions of dispositions and etc. In order to design, analyze and document products (not only for change of the surfaces colors, viewpoints and etc.), we use the enriched depth of the knowledge that XML based documents can provide for direct modeling.

The presented pre-sales service supports and maintains the development and marketing of a "virtual family" of modular positioning and handling systems called DriveSets.
\end{abstract}

Presented work was partially funded by the Research Contract BY-TH-110 with the Bulgarian Ministry of Education and Science. 


\section{Keywords}

Product Customization and Individualization, VR and VE Methods, 3D Web Configurator, Virtual Product Family, Product Design.

\section{Introduction}

The ability to create, transfer, assemble, integrate, protect and exploit knowledge assets is a main factor for the existence and successful work of the industrial companies. The knowledge base of the firm includes its technological competences, its knowledge of customer needs and supplier capabilities. The approach for the direct customers' participation in the design process by expressing their preferences allows customers to be actively involved in the product definition process rather than passively receive the end product designed by the producer. Presently the proposed approach is applied successfully to continue and maintain development of a "virtual product family" of modular positioning and handling systems called DriveSets [Boyadjiev, 2005].

In recent years, technological developments have made it possible to build interactive 3D models of objects and 3D Virtual Environments (VE) that can be experienced through the Web, using common, low-cost personal computers. As a result, $3 \mathrm{D}$ content is increasingly employed in different Web application areas, such as education and training, e-commerce, virtual communities etc. Application of such 3D Web Sites is still not so popular for solving real world engineering and des problems [Chittaro, 2007].

The main goal of presented work is to propose use of a configurator to build a 3D product models and a correspondent virtual environment for the needs of Designby-the-Customer process of modular handling and assembly systems called DriveSets representing an extension of early developed XML CAx knowledge structuring environment. Constructing such a virtual product configurator shall enable the accurate assessments of the customer acceptance, better informed development of "tailored products", and a sort of "binding" the customer to the designed product (system).

\section{Customer Product Co-Design Facilitated by Internet}

The new products are introduced to the market traditionally trough the "DesignMake-Sell"- cycle, typical for manufacturing industries. Manufacturers capture and aggregate customer needs to product specifications, pass the information to the designers to design the product, produce it and display it for sale. The long cycle time is not enough responsive to the specific needs of today's dynamic and competitive markets concentrating on small number final products increases the risk of misjudging the real customer needs [Simpson, 2003].

Customers' knowledge and direct participation in the design process leverages product and product related information, facilitates marketing research and brings different customers' attitudes towards buying decision making. As a consequence, 
the traditional cycle of "design - make - sell" will be replaced by the new one of "design - sell - make". Consumers design the product, buy it, and send the order to be manufactured; this will create a new product design and development process, which benefits both customers and manufacturers. In this evolvement manufacturers are able to gain advantages, reducing amount of iterations caused by unsatisfactory products, and building up a knowledge base and providing a more effective product knowledge transfer from the product development process to the sales-delivery process.

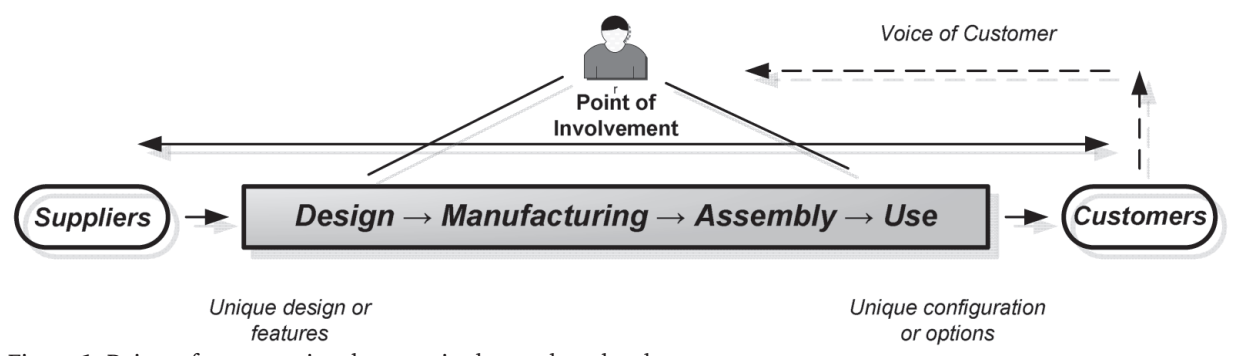

Figure 1: Points of customer involvement in the product development

Customer Co-Design, which term we adopted for the well known "Design-by-theCustomer" approach of development of new products, refers to a process in which customers are allowed to express their product requirements and carry out the mapping process to the physical domain of the product. If implemented as a presale service it will bring a lot of benefits for both the producer and the customer which could be summarized extending the presented by Piller in [Piller, 2005] as (i) providing a better starting product configuration through direct use of customer needs, (ii) supporting the product design through fostering joint creativity problem solving capability of the stakeholders (iii) reducing cost and efforts, (iv) changing the customer attitude toward new developed product through trust building trust relation and reducing the risk perception.

The Customer Co-Design comprises following two stages: (i) Customers' Needs Identification and Structuring and Product Design [Kurniwan, 2004]. Within Customers' Needs Identification and Structuring stage following steps can be identified (Fig. 2):

- Elicitation of tacit, implicit or hidden customers' needs

- Translation identified customer needs in engineering oriented customers' requirements

- Enhancing the clarity of customers' needs

In the Product Design stage customers are enabled to alter and improve products interactively and so to participate in the product definition process. Customers are presented a product configurator, in which a set of product attributes and their possible values are given for selection and modification. Here the configuration is 

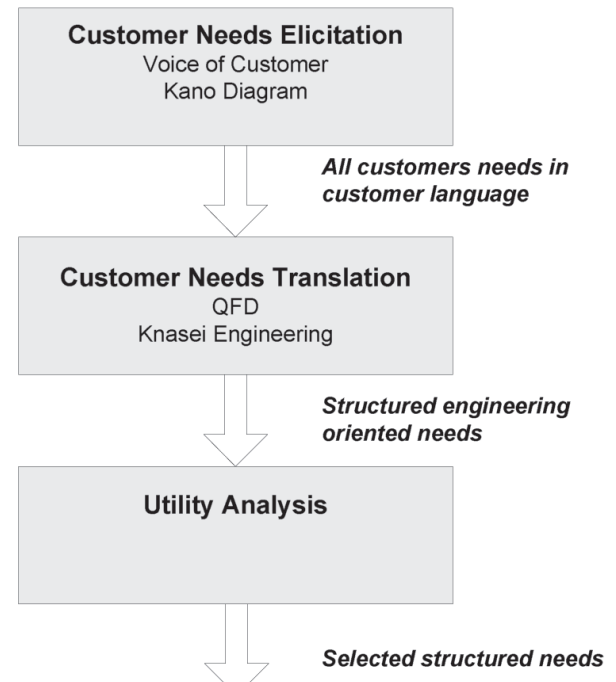

Figure 2: Framework for structuring customer needs for Design by Customer [Kurniwan, 2004]

referred to a process of choosing different attributes and attribute values and combining them into a final product. The detailed graphical representation of the Customer Product Co-Design process could be obtained on Fig. 3.

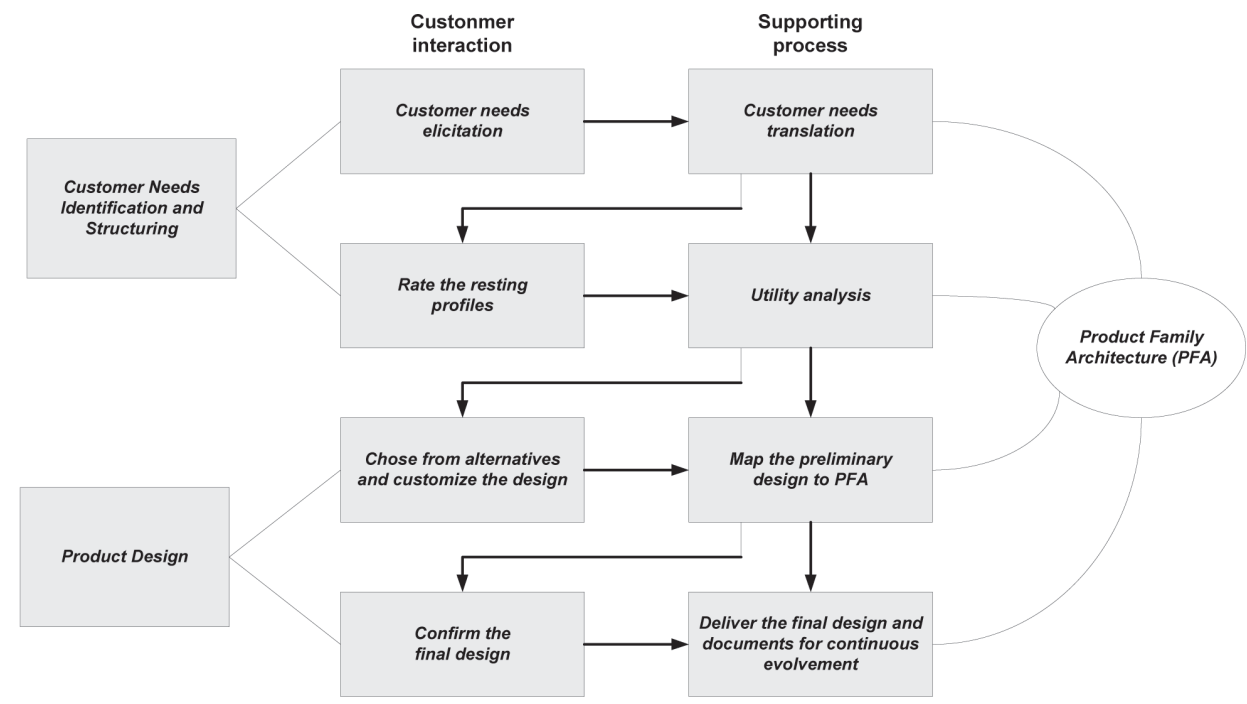

Figure 3 Customer Product Co-Design according to [Kurniwan, 2004]

According to [Choi , 1999] the nowadays computer-mediated global economy will accelerate the process of customization through its technologies. The Internet provides new channels for promoting products and making sales in B2B market segment and so becomes an extremely important factor for the business in a wide range of industrial branches. Considerable research in using the Internet for supporting 
the design and production process is already done [Simpson, 2003]. The Internet enables customer to improve his involvement in the early stages of product development through use of interactive web-based platform customization as an extensions of product family design.

One of the most promising approaches to facilitate design and customization and to improve customer involvement within product development is use of so called Virtual Manufacturing (VM) environments that goes far beyond the scope of traditional modeling and simulation in CAD/CIM [Lacroix, 2003]. Different scenarios for the use of Web-based VR in the small and medium-scale companies are reasonable, such as Component catalogues, Digital procurements, Electronic bids, Partner selection, Customization etc [Banerjee, 2002]. Recently a trend for developing of so-called Virtual Products is established. Here the concept of "the virtual product" concerns not only the conventional computer aided planning and simulation of the developed products. According to the adopted definition "the virtual product" is a technical system, which allows instant and direct access and management from every $\mathrm{PC}$, everywhere and on every stage and level of its life-cycle in order to increase the competitiveness and to guarantee the market share of the vendor through high diversity of the covered technical parameter ranges and possibility for promptly development and "materialization" of pre-engineered "custom-tailored" solutions according to the individual clients needs. Here "access" is referred to a full duplex data flow performed by appropriate transfer protocol (usually TCP/IP) which can be configured and optimized on-line and independently of the place and time.

One of the possible ways for direct involvement of the Customer within virtual product development process is the use of 3D Web Sites. A common idea behind this is that the $3 \mathrm{D}$ model of the product over Internet could enhance communication and collaboration between customers and the product developer, located in geographically dispersed places and enabling full product customization. The customers could select some of the product features and their correspondent parameters using the product VR-model and standard web controls.

In general, a VR model contains data that are used to render a 3D digital world in which the customer could navigate in real-time and interact with its objects. The user could examine the digital product in 3D space from different angles and with different resolutions. The user could also interact with the digital product to trigger animations showing the dynamic behavior of the product such as the assembly or disassembly sequence, operational movement, and so on.

VR-model is good suited for customer-company interaction purpose for following reasons. VRmodel has strong support in multi-media integration and interactive behavior specification, which are two important functions for developing product catalog and showing the dynamic behavior of the product. Further VR model could be dynamically generated on the fly to let the customer immediately see a proposed product customization in 3D space. 


\section{Notes on VR-modeling and 3D Web Sites Implementation}

The composing elements of a VR model are organized into three types of data structures: nodes, scene graph, and animation circuits (Fig.4). Nodes are the building blocks of a VR model. They contain data and methods to define the composing elements of a virtual environment. Nodes composing a virtual environment could be further classified into eight categories: (i) shape, (ii) appearance, (iii) grouping, (iv) environment, (v) viewing, (vi) animation, (vii) interaction, and (viii) miscellaneous [Tien, 2005]. In the VR model of a product, the shape or geometry nodes describe the 3D shape information of the product. The polyhedral approximations rather than geometric models are stored in the shape nodes. The appearance nodes provide detailed control over the color, texture, and transparency of the product. The grouping nodes simply serve as the parent node in the scene graph to manage a list of children nodes. The environment nodes define the show room of the product. The viewing nodes control the viewing camera. The animation nodes are used to show the dynamic behavior of the product, such as assembly or disassembly sequence or the operations. The interaction nodes are used to sense the users and then trigger the animation. At last, the miscellaneous nodes contain extra data that could not be classified by previous categories such as scripts. Nodes in a VR model are organized into a tree-like structure called a scene graph. The third type of data structure used to organize data in a VR model is a process diagram called animation circuit, which specifies the execution sequence and logics of the animation and interaction nodes in the scene graph. The animation circuit is composed of nodes wired together. Each node has inputs and outputs. Messages are passed among the nodes to change the status of the nodes and correspondingly cause changes in the virtual environment. The VR model of a product contains all data necessary to generate the 3D representation of the product.

The web sites containing 3D data can be divided into two basic groups: (i) sites that display interactive 3D models of objects embedded into Web pages, and (ii) sites that are mainly based on a 3D virtual environment which is displayed inside the Web browser.
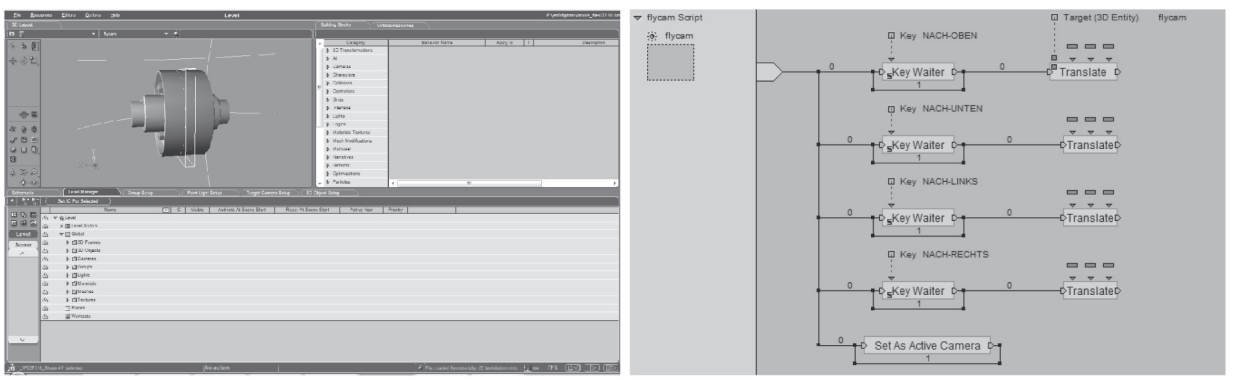

Figure 4: Product VR model implementation 
In the first case, the primary information structure and user's interaction methods are still based on the hypermedia model, with the additional possibility of inspecting 3D objects. In the second case, the primary information structure is a 3D space, within which users move and perform various actions.

Technologies for implementation of 3D Web sites are based on the common used technical and architectural solutions typical for the "conventional" web. The content, represented in a proper format, is stored on a server, requested by a client, through HTTP, and displayed by a browser, or, by a plug-in for a Web browser. 3D content can be integrated with other kinds of Web content such as images, sounds, videos inside a 3D VE accessible through the Web.

Beside the possibility for immersive 3D experience of the content in case of use of special hardware, 3D Web content normally is experienced with the common I/O - devices (CRT or LCD monitors, keyboard and mouse) allowing more realistic representations in comparison to the classical web content and enabling customers to inspect, manipulate and customize products before purchasing, as they are accustomed to do in the real world [Brusilovsky, 2004].

For implementation of 3D Web following two main open ISO standards are used VRML and X3D. VRML (Virtual Reality Modeling Language) is most know and used technology for building and delivering 3D Web content. VRML documents are text files that describe 3D objects and 3D VEs using a hierarchical scene graph. VRML defines different node types, including geometry primitives, appearance properties, sound and video, and nodes for animation and interactivity [X3D, 2004]. Recently a new ISO standard, called eXtensible 3D Graphics (X3D), has been proposed as a successor of VRML. X3D inherits most of the features of VRML improving upon VRML mainly in adding new nodes and capabilities, mostly to support advances in 3D graphics techniques and hardware, such as shaders etc.

Besides open ISO standards, there are many other (non-standardized) technologies for 3D on the Web. The best known examples are probably Java3D <http:// java.sun.com/products/javamedia/3D/ $>$, an extension of the Java language for building 3D applications and applets and Shockwave 3D < http://www.adobe. com/products/shockwaveplayer/> from Macromedia.

Access to VRML/X3D Web content is possible through one of the available Web browser plugins, such as Octaga Player <http://www.octaga.com/joomla/index. php $>$, Parallelgraphics Cortona < http://www.parallelgraphics.com/products/ cortona $>$, and Mediamachines Flux player < http://www.mediamachines.com/ developer.php $>$.

\section{Systematic Approach for Customer Product Co-Design}

The systematic design provides an effective way to rationalize the development and production processes. Such design method is prerequisite for continuous computer support of the design by using stored data. Systematic processing makes possible 
consideration of cost and quality of the designed products on early stage, which enables better market chances. In our work we have combined the main principles of the systematic design [Pahl, 2000] with the 3D web technologies in order to provide the direct participation of the customers at very early stage of the modular product development in form of a pre-sale service for SMEs.

Our approach compromises four stages and includes following basic elements:

- a 3D Web configurator and an appropriate virtual environment assisting the identification, structuring and mapping the customer requirements and realtime graphical representation of the developed product enabling direct interac tion with the customers;

- a product frame describing the significant parameters and features of the product (or product family member) derived on the ground of preliminary analysis of the customer needs and requirements;

- a functional structure and UML-model of the product (product family);

- a classification of the functional units performing the single sub-functions of the product (product family members) stored in a data base;

- a morphological matrix to compose the functional unit set of the single sub functions in overall product functional structure;

- an algorithm generating the possible variants of the product structures;

- a procedure for selection the optimal product variant for specific set of objectives; • a validation procedure;

- an algorithm for automatic generation of the technical documentation and webpresentation of the newly created product variant.

The concise and simplified linear organization of the above mentioned elements within particular stages of the proposed approach for customer co-design approach within product development process can be described as follows (see Fig. 5):

(i) Within the first stage the "Voice of Customers" (VoC) is captured and stored by the 3D web in (XML1); (ii) The captured data are processed, analyzed, "translated" and structured in form of engineering specification (XML2); (iii) On the ground of the obtained results (XML2), the particular predetermined product modules (XML3) are selected, the values of their parameters are identified and an assembly model is generated (XML4) - third stage; (iv) Within fourth stage a selection of the optimal handling or assembly system for specific requirements and constraints is made. Then some simulation tests can be run to determine whether the intended functionality and purposes have been satisfied by the identified product configuration. After the validation a 3D graphic model (XML5-X3D) describing the proposed structure is built and the proposed product configuration is stored in a database for further use and modification. The technical documentation and HTML-code for web-representation of the new developed product are generated at the end of the working cycle. 


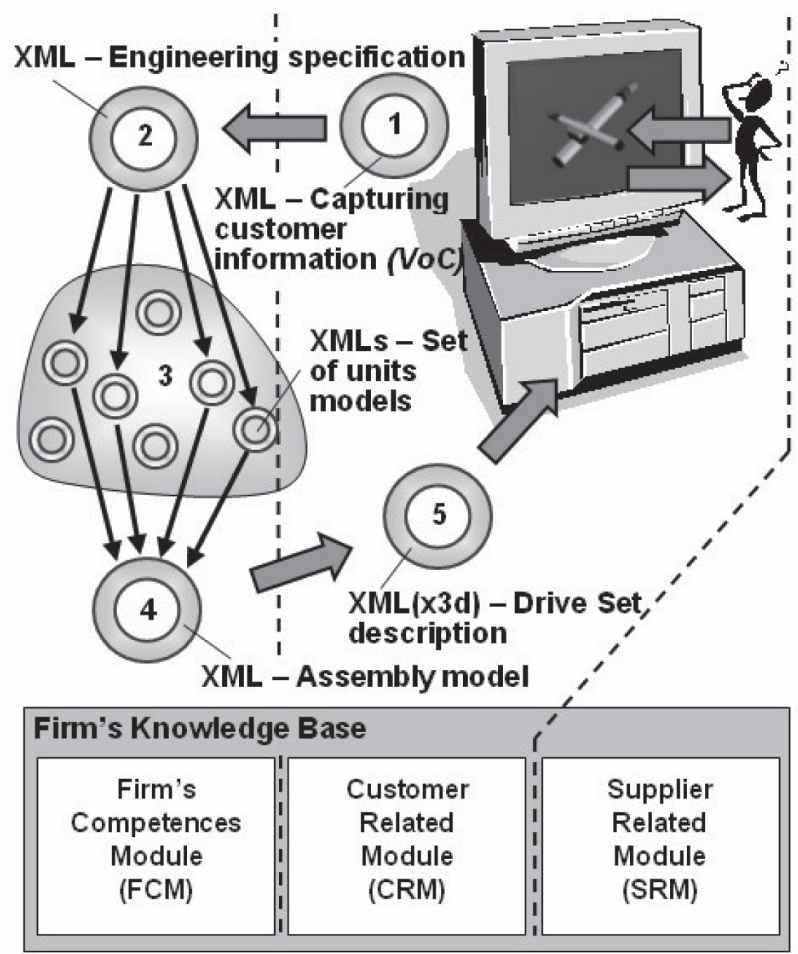

Figure 5: Systematic processing of customer input through Customer Co-Design approach using 3D Web configurator

\section{3D Web Product Configurator for Customer Product Co-Design}

The core element of the proposed systematic approach for Customer Product CoDesign is the 3D Web Configurator. On higher level it represents one of the components of so called "Customer Related Module" (CRM), which stores and manages the information about customer's needs, requests and advices of the producer. The 3D configurator is responsible for the 3D graphical representation within the configuration and development of the modular products, enabling the direct participation of the customers within system designing. Customers' needs identification and structuring for the Customer Co-Design within early Product Design stages of the products allow interactively altering and improvement the products and direct participation within product definition. Customers are presented a wizard, in which a set of product attributes and their possible values are presented for selection and modification. The frame of the parametric range was built by systematic variation and full combination of some significant technical and structure product parameters. Beside that come the common for VR operations like turning, aligning, flying etc. enables customer to change some parameters of the designed system.

The 3D Web Configurator provides as feedback in the web-browser of the customer not only the appropriate graphical representation of the newly developed 
system, but the model of the systems installation and operational area, animation of the systems action and dynamical change of the model parameters such as dimensions of form, dimensions of dispositions etc.

For our experimental implementation of the 3D Web configurator we have used virtual product model of the DriveSets-family brought to the market by Systec E $+S$ $\mathrm{GmbH}$, Germany < http://www.drivesets.de>. DriveSets have been designed as a scalable frame based parametric range of modular linear positioning and handling systems with application in the industrial and laboratory automation, evolved through the integration of OEM-components (see Fig. 6).

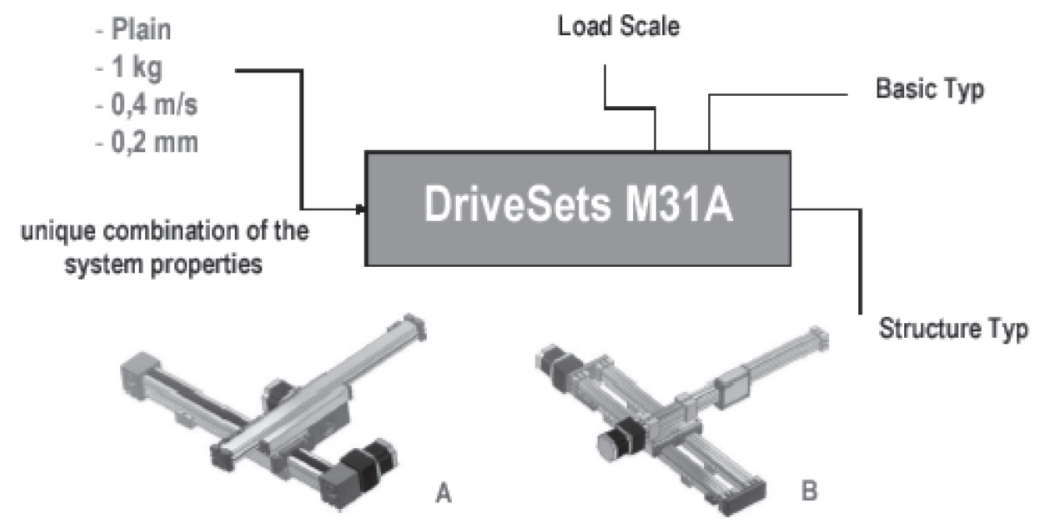

Figure 6: An example for a DriveSet positioning and handling system: significant system parameter set and its correspondent product family

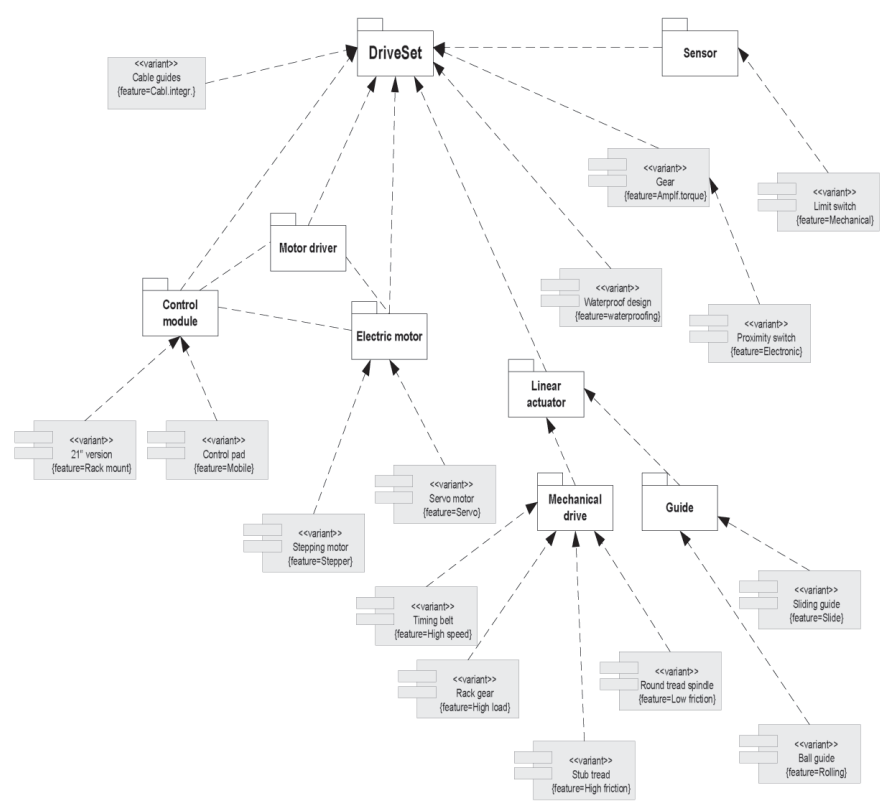

Figure 7: A Simplified UML-representation of the DriveSets product platform 
The particular DriveSets form a "virtual product family" in accordance with the predefined sets of systems properties (such as paying load, maximum speed, repeatability, operating area, design type, stroke) described in a form of a product platform (Fig. 7). The specific value of the system parameters for each family member is determined at the design phase on the ground of the mapped customer requirement and constraints providing a sort of tailored customization. Every unique property value combination has a particular number identifying the correspondent representative of the family. In such a manner some 144 basic structures have been specified. The customer could select among these "virtual existing" pre-engineered basic structures and find the system most appropriate for his specific needs using a simple selection procedure in ndimensional system feature space (online or supported by hard copy catalogue). Further he can choose the stroke for every single axis of the system. 10 standard stroke lengths are available for every system axis. Various additional properties of the system like as cable chains, control unit type, material etc. are optional for the customer and enable him to perform a sort of standardized customization.

In our work we have focused on the development of a prototype of an experimental 3D Web configurator providing "deeper" involvement of the customer within process of configuration new or existing DriveSets-structures using XML-technologies to ensure exchangeability and extensibility of data and models. The easy to understand XML description of the virtual product model could be obtained from Fig. 8. On the grounds of the XML description of the product a X3D file is generated, which can be visualized directly in the web browser of the customer. The flexibility and support of dynamical changes are provided trough the 3d Ajax technology. The experiments are carried out through the Document Object Model (DOM) - Scene Access Interface (SAI) connection. This connection is used to change dynamically the X3D scene.

For the needs of the X3D modeling of the DriveSets, they have been decomposed in single subassemblies (single axes), which have been denoted as unit1, unit2 and unit3 (see Fig. 9).

For the purposes of the animation the elements of the X3D model have been "nested" as it is shown on the Fig. 10. This is because every unit (it has its own movement) derives the movement from the preceding one. The parts of each unit are divided into two groups: fixed and dynamically altered (animated). The elements of the "offspring" unit must be included within the animated group of parts of its "parent".

There are two kinds of connection between units "slide - slide" or "slide - linear guide" and these relations are very important for the determination of which part within which group (fixed or animated) have to be included. 


\section{Contents of driveset_base.xml}

$\left\langle\times \mathrm{ml}\right.$ version $=" 1.0^{\prime}$ encoding $="$ UTF -8 " ?>

- edriveset_base>

- sgenerals

- cuariants <type>001001</type> \&/variant>

- Lanimations stime />

$</$ animation $>$

$\&$ generals

- cunitss

- <unit>

stype $>1</$ type $>$

$<d i r e c t i o n>0</$ direction $>$

- <parts> Direction means - Axial Sub

- <engines Structure (unit) direction-from sdimention_1 /> the side of the engine! sdimention_2 /> <dimention_3/> \&lengines

$+\langle$ transmission $\rangle$

+ <connector $>$

$+\langle$ end $1>$

+ <guide_beams

$+<e n d 2>$

$+<$ carriage $>$

$</$ parts $>$

<unit>

+ sunit>

+ sunit>

$<$ units >

ddriveset_base>

Number 001004 1 row 4 column (numbered)
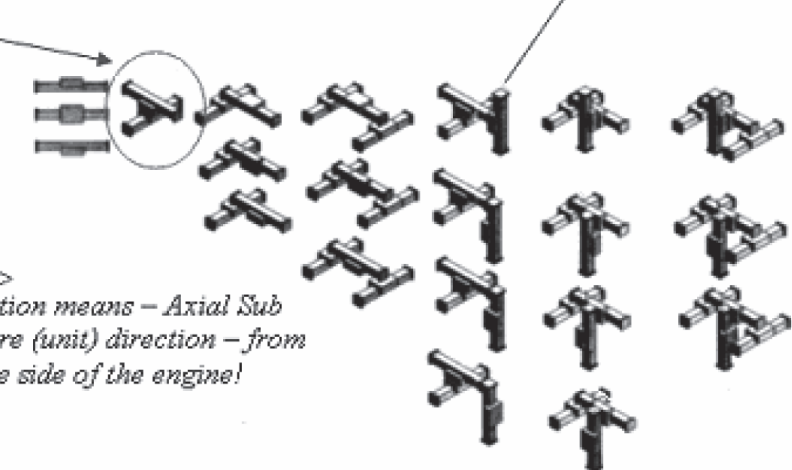

Figure 8: XML-description of the DriveSets product family
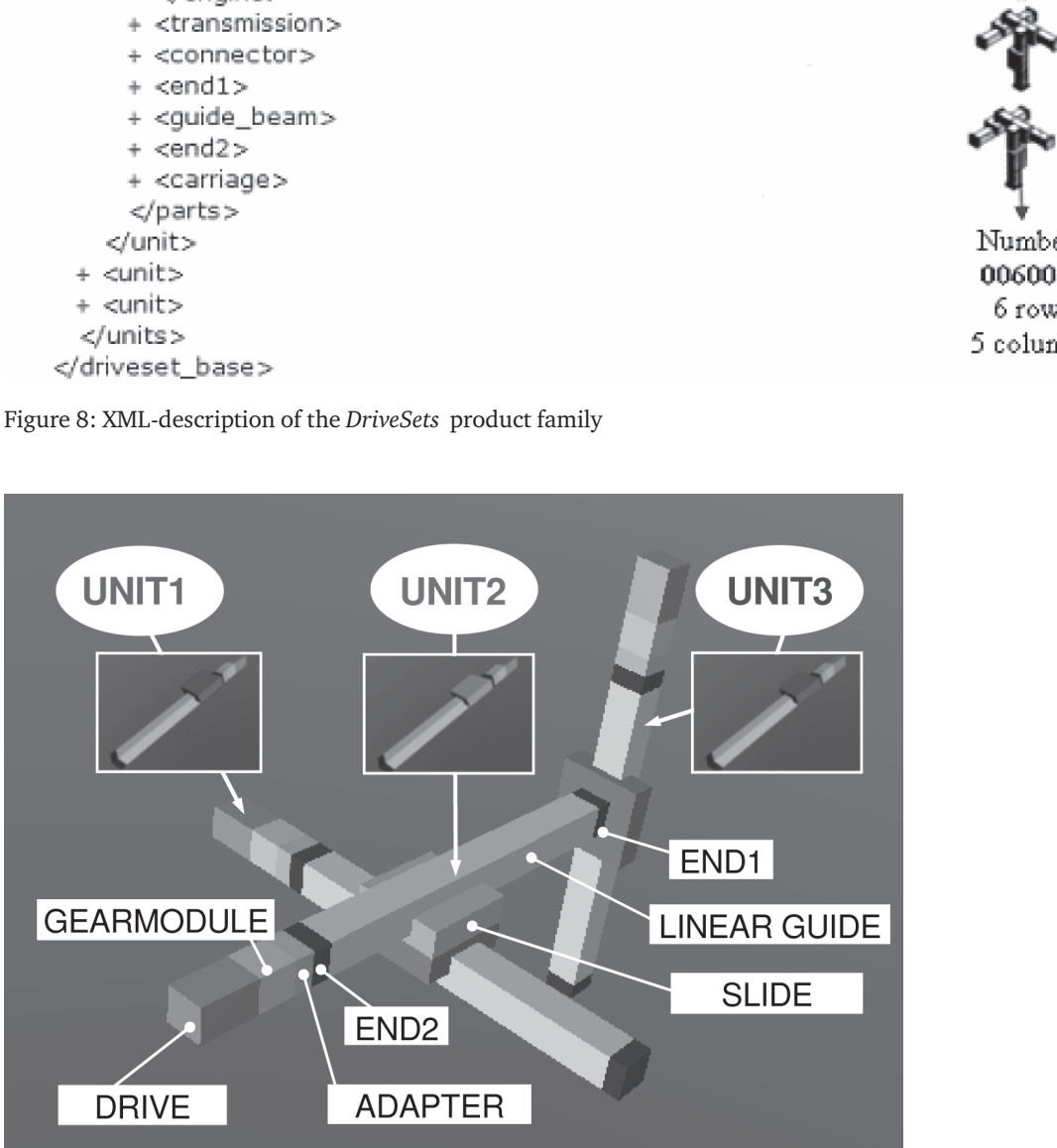

Number 006005

6 row

5 column

Figure 9: Decomposition of DriveSets for X3D-modelling 


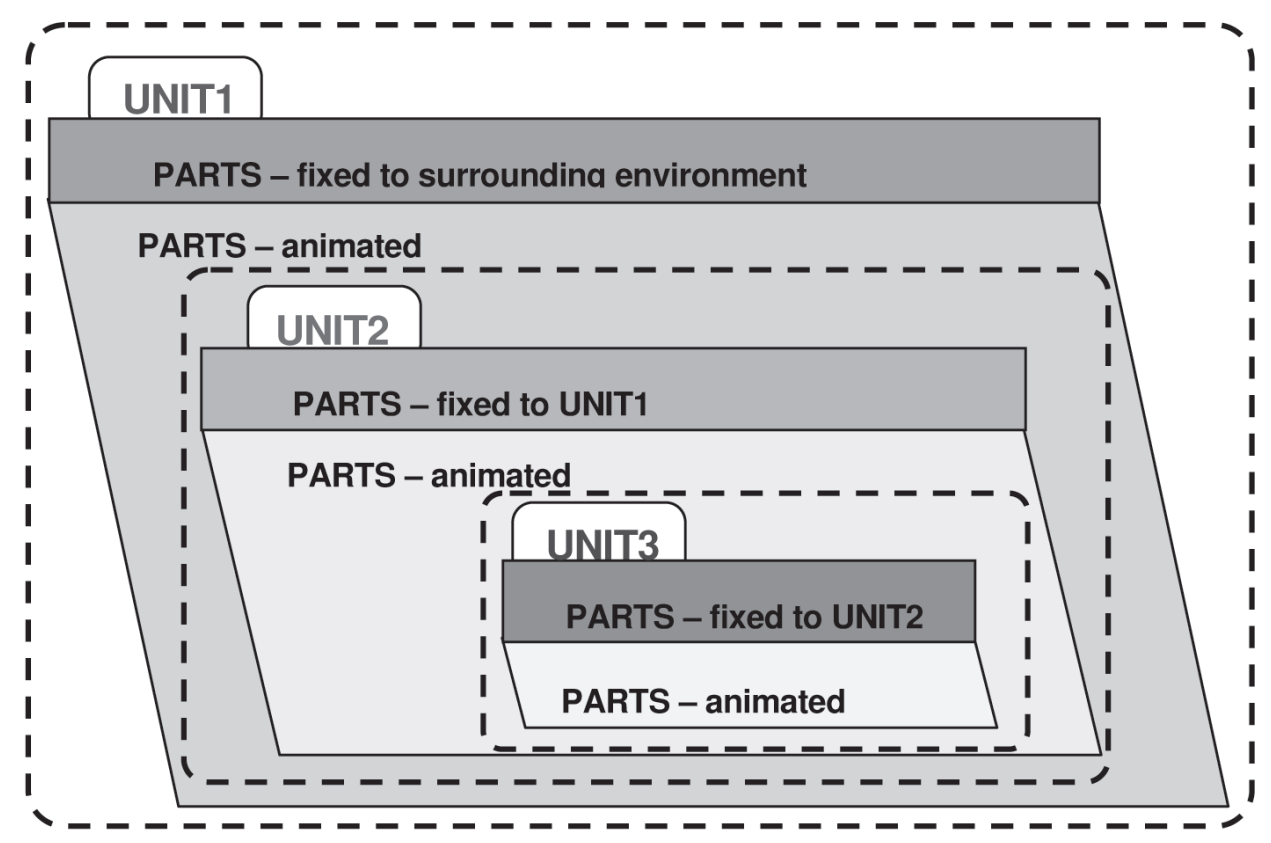

Figure 10: Structure of X3D-representation of the DriveSets.

The experimental implementation of the 3D Web Configurator aims at providing an environment for 3D modeling and XML integration of the customer preferences, engineering calculations and reasoning and generating the product assembly model. It can work on two ways: (i) by the use the information from the other algorithms for engineering calculations working in background (the particular data are stored in the ASM.X3D file) and (ii) a) by the direct participation of the customer within design process or b) by conventional capturing the customers' requirements as already described (Fig. 11).

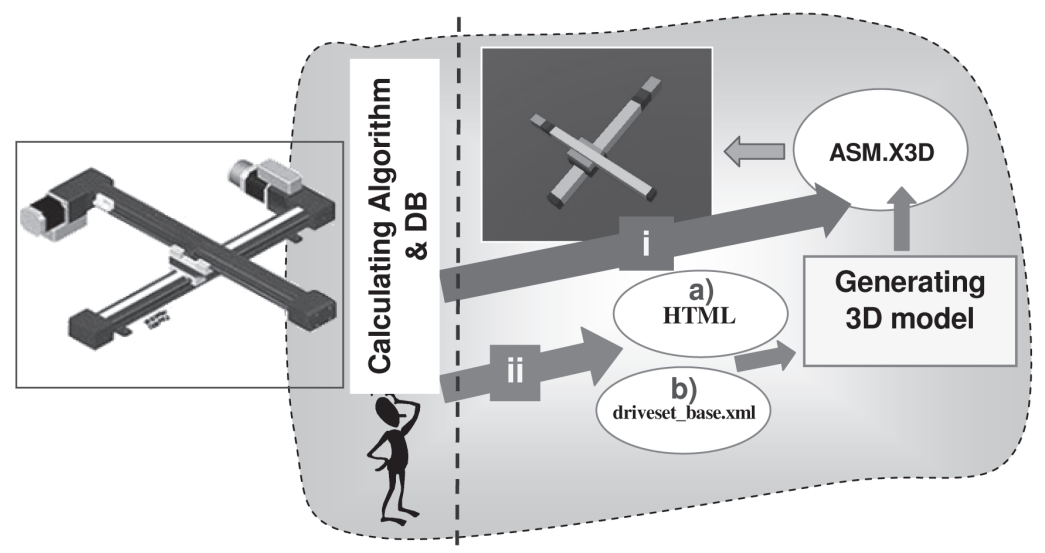

Figure 11: 2-ways operation of the experimental software 
The system architecture of the framework for Customer Product Co-Design is presented on Fig. 12. The place of the 3D Web configurator is in the client's web browser. The calculation algorithm is implemented as a $\mathrm{C}++$ application, which communicates from one site with the database and from other with a PHP-application using TCP/IP socket technology. The PHPapplication generates HTML-pages based on predefined templates. The HTML-rages are served by the web browser to the single customers browsers.

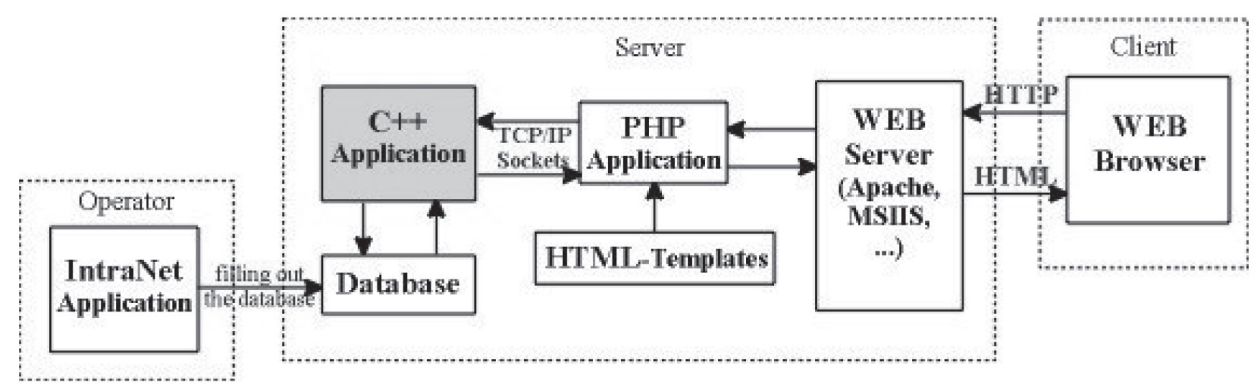

Figure 12: System architecture of the web based Customer Product Co-Design framework.

The following snapshot (Fig. 13 next page) shows the graphical user interface of the experimental implementation and the way of 3D model configuration of each of the single axis and their combination in a complex spatial structure. As could be seen in the prototype the quality of the visualized 3D model is still rough (with low Level of Detail) and will be further enhanced. In particularly the proposed 3D configurator allows dimension modification and structure alteration (position of the elements within the system) for every single axis or the complete system. The montage and operational areas of the designed system are visualized as well (Fig. 14 next page). Further it improves data transfer between geometrical model and other calculation modules and database running in background on the server.

X3D provides unsurpassed interoperability for 3D data and significant flexibility in manipulating and displaying scenes interactively. By the represented experimental X3D Product Configurator the challenge of creating real-time 3D applications using a standard XML language for the Web has been realized.

\section{Conclusions}

It is obvious that a direct customer participation in the design process saves time and money, reduces the engineering efforts in respect to the solving of the design problem, improves the quality, changes the attitude of the customers towards the product and this way facilitates the product market realization. This involvement can be particularly "amplified" and leveraged using a 3D Web Configurator as a pre-sales service based on the X3D within XML CAx knowledge structuring environment. Here we conclude by pointing following obtained experimental results and outlook: 


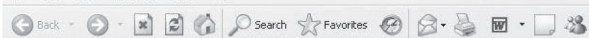

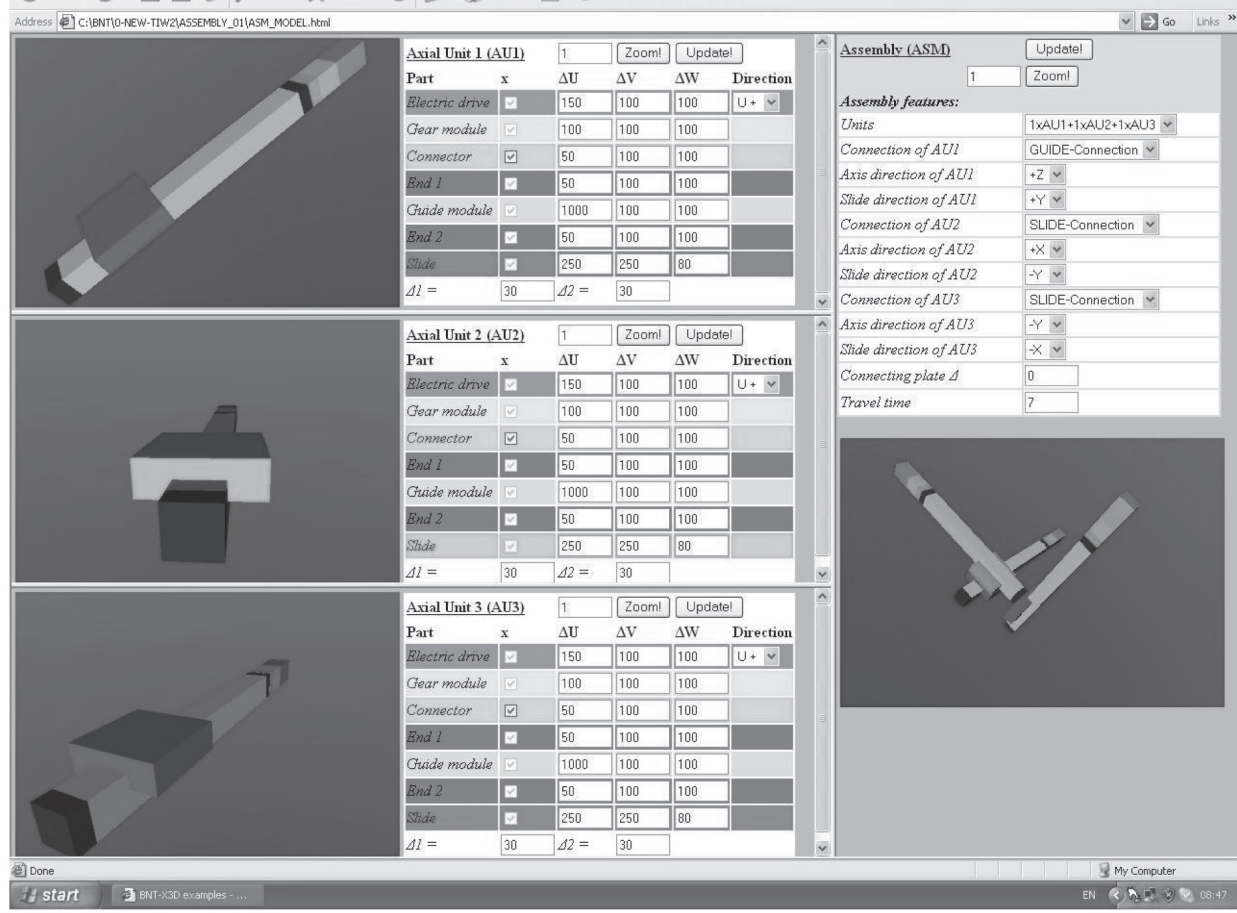

Figure 13: Experimental 3D Web Product Configurator - graphical user interface
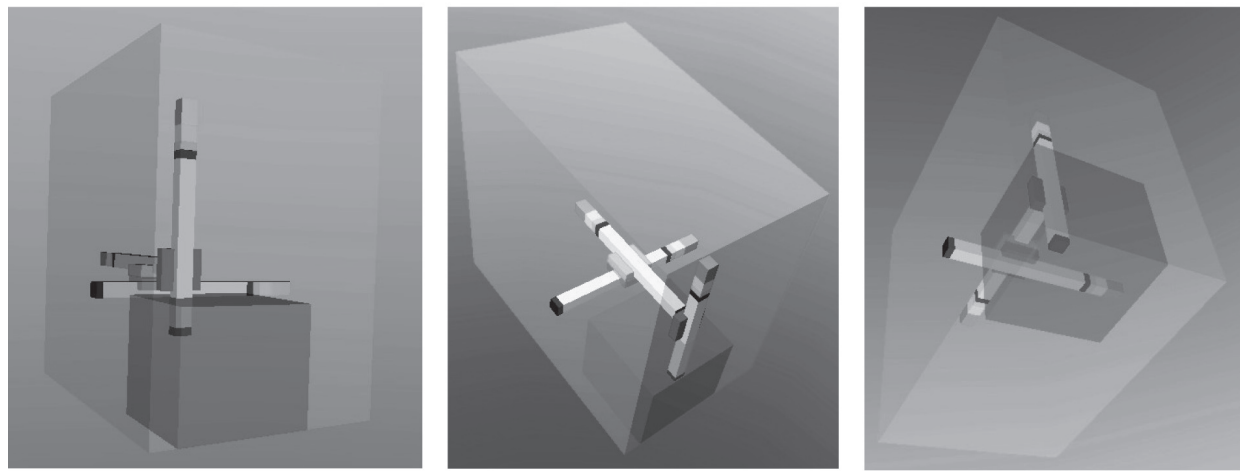

Figure 14: Visualization of the montage and operational areas by transparent boxes - different view points 
(1) The proposed approach ensures overcoming the negative effects of the communicational fragmentation: it is based on XML sets of applications;

(2) The customers' knowledge and direct participation within the design process facilitates marketing and brings different customers' attitudes towards buying decision making;

(3) As a consequence, the traditional cycle of "design - make - sell" is replaced by the new one: "design - sell - make";

(4) Use of the X3D language enables the attractive, real-time, interactive and high quality graphic representation of the results obtained, as well as video and 3D data and allows components to be added to extend its functionality;

(5) By additional attachment of functional information it is expected a X3D based model of the mechanical assembly to be developed for the needs of the more sophisticated VR immersive representation techniques;

(6) The formalized description of DriveSets developed for the needs of this work can be observed as a basis for the development of neutral 3D Web Configurator for design of various products.

\section{References}

Banerjee, P./ Zetu, D. (2002): Virtual Manufacturing. John Wiley \& Sons Inc. NewYork, 2002.

Boyadjiev, Ilia/ Bachvarov Angel/ Bojkov Dimitar (2005): Connecting Customer to the Design - Approach on the Example of the Development of Modular Handling Systems, 50. Internationales Wissenschaftliches Kolloquium, Technische Universität, Ilmenau, Germany, 2005.

Brusilovsky, Peter (2004): Adaptive Navigation Support:From Adaptive Hypermedia to the Adaptive Web and Beyond. PsychNology Journal, 2004 Volume 2, Number 1, 7 - 23, 2004.

Chittaro L./ Ranon R. (2007): Adaptive 3D Web Sites, Published in: Brusilovsky, P., Kobsa, A., Nejdl, W. (eds.), The Adaptive Web - Methods and Strategies of Web Personalization, Lecture Notes in Computer Science Vol. 4321, Springer-Verlag, Berlin, 2007.

Choi, S.-Y. / Whinston, A. B. (1999): The future of the digital economy. Published in Handbook on Electronic Commerce (Shaw, M., Blanning, R., Strader, T. and Whinston, A., eds.), pp. 25-52, New York: Springer, 1999.

Kurniwan, S.H./ Zhang, M./ Tseng, M.M. (2004): Connecting Customers in Axiomatic Design. Proceedings of ICAD2004, Seoul, 2004.

Lacroix, E. / St-Denis, R. (2003): Web technologies in support of virtual manufacturing environments. Emerging Technologies and Factory Automation. Proceedings. ETFA '03. IEEE Conference, 2003.

Pahl, G./ Beitz, W. (2000): Konstruktionslehre. Springer-Verlag. Berlin Heidelberg, 2000. 
Piller, F./ Schubert, P./ Koch, M./ Möslein, K. (2005): Overcoming mass confusion: Collaborative customer co-design in online communities. Journal of Computer-Mediated Communication, 10(4), 2005.

Simpson, T.W. / Karthikeyan Umapathy (2003): Development of a Framework for Web-Based Product Platform Customization. Journal of Computing and Information Science in Engineering -- June 2003 -- Volume 3, Issue 2, pp. 119-129 , 2003.

Tien-Lung Sun/ Tsung-Han Chiang, (2003): Supporting Customer-Company Interaction in Product Customization Using a Web-Based Collaborative VR Environment. Journal of the Chinese Institute of Industrial Engineers, Vol. 22, No. 3, pp.235-242, 2003.

X3D International Standard, (2004): X3D framework \& SAI. ISO/IEC FDIS (Final Draft International Standard) 19775:200x. Available at www.web3d.org/x3d/specifications (last access on April 2007), 2004.

Dr. Boris Tudjarov is an Associate Professor at the Faculty of Mechanical Engineering, Department of Design Fundamentals. He is a lecturer on: "Design Fundamentals and CAD systems" and "XML Based CAD/CAM/CAx Integration". His research interests comprise Fundamentals and Automation of Design; Computer Modeling; Web-based Information Systems; XML (eXtensible Markup Language); Ubiquitous Collaboration; Scheduling for the Contemporary Manufacturing Systems.

Angel Bachvarov is an Assistant Professor for Product Design and Virtual Engineering at the Faculty for German Engineering and Management Education of the Technical University of Sofia. His research interests comprise use of VR for solving real world engineering problems, advanced product design techniques and modelling, product customization and individualization, multiobjective evolutionary algorithms, complexity and chaos theory.

Dr. Ilia Boyadjiev is a Professor for Product Design at the Faculty for Mechanical Engineering and Dean of the Faculty for German Engineering and Management Education of the Technical University of Sofia. His research interests comprise theory of the engineering design, assembly and discrete processes automaton, optimization methods and VR engineering application. 


\title{
A software tool for design and documentation of configurator knowledge bases
}

\author{
Anders Haug, Torben Lisbjerg and Lars Hvam
}

\begin{abstract}
A seven-phase procedure for the development and maintenance of product configurators from Centre for Product Modelling (CPM) at the Technical University of Denmark has been applied in several Danish configurator development projects. The representation techniques to be applied during analysis, design and maintenance of configurator knowledge bases prescribed by the CPM-procedure are the product variant master technique (PVM), CRC-cards, and in some cases class diagrams. In the cases studied at CPM, PVMs are most often created in programs such as MS Visio, while CRC-cards are created by using MS Word. However, the use of MS Visio and Word can be time-consuming, since such software has significant modelling limitations and does not provide integration between PVMs and CRC-cards. Also, a few companies apply Lotus Notes for documenting their configurator. This software, however, does not support the PVM technique, for which reason other software is also used during product analysis processes, implying manual transfers of information between software systems.

For several years configuration literature has dealt with the topic of creating better software to support the analysis, design and maintenance tasks of configurator projects. However, only recently such software has emerged on the market. This software is named Product Model Manager (PMM) and was released by Incore Systems. This paper describes the PMM software and compares it to the existing possibilities of IT-supported documentation in configuration projects.
\end{abstract}

\section{Keywords}

Product Configuration, Documentation of Configurator Knowledge Bases, Knowledge Representation, Product Model Manager

\section{Introduction}

This paper describes a new type of software, which has recently emerged on the market and is aimed at supporting the tasks of knowledge elicitation, knowledge base design and maintenance of product configurators.

A product configurator is a software-based expert system that supports the users in the specification of customized products by restricting how different elements and their properties may be combined. In configurator projects, one of the most difficult tasks is the representation of the domain knowledge that should be im- 
plemented in a configurator (Sabin and Weigel, 1998; Hansen, 2003; Hvam et al., 2008). In industrial companies, which apply configurator technology, the configurator knowledge base often consists of thousands of classes, attributes, rules/constraints and methods. When the product assortment changes the configurator has to be updated in order to support the company processes. Such updates in many cases imply a need for product experts to evaluate the existing implemented knowledge in order to change or extend this. However, the modelling environment of configurators seldom provides overviews of the implemented product information that are adequately comprehensible for the domain experts. Thus, for domain experts to understand the implemented knowledge, external representations would be needed. Documentation of configurator knowledge bases, however, is a timeconsuming task that seems often to be neglected.

A study of 12 Danish industrial companies, that apply configurator technology, showed that the maintenance of configurators is often a task creating great difficulties if the implemented knowledge is not documented in an external system (Edwards et al., 2005). However, in the cases studied by Edwards et al., the documentation task was often the first activity to be postponed or cut away from a configuration project. In the short run, such a strategy may be economically sound, but in the long run it may have big negative consequences, as several of the investigated companies were not able to maintain or further develop their configurators. Also, for some of the companies, the poor documentation had led to problems in the daily communication between persons involved in product development and those involved in configurator development. Based on the many configuration projects studied at the Centre for Product Modelling (CPM) at the Technical University of Denmark, the impression of Hvam et al. (2005) is that the manual documentation of configurator knowledge bases requires much effort as a model grows bigger and more complex, for which reason many companies settle for less comprehensive and more inadequate documentation. This lack of configurator documentation software implies that different kinds of software must be used at the same time, which causes a need for manual transfers of information between models. Such tasks are timeconsuming activities, which to some extent may explain that configurator documentation has been given a lower priority. Hvam et al. (2005) therefore emphasize the need for an IT-based documentation system that provides easily understandable overviews of what shall be or has been implemented in a configurator, and thereby can increase the quality of the documentation and relieve companies of the timeconsuming tasks of ensuring consistent product models. Unfortunately, software, which in a satisfactory way supports the modelling techniques recommended by CPM (Hvam et al., 2008) in an integrated manner, has not until recently been available. Compared to existing documentation approaches in configurator projects, the use of this new emerging documentation system is associated with a number of advantages when it comes to knowledge acquisition, configurator knowledge base de- 
sign and documentation of configurator knowledge bases. This paper aims to clarify the advantages and possible drawbacks of using the new documentation software.

The remainder of the paper is structured as follows: First, section 2 describes relevant modelling techniques for the development and maintenance of configurator knowledge bases. Next, section 3 discusses existing ways of documenting configurator knowledge. Section 4 describes a recently emerged software-based configurator documentation system, and compares the use of this system to existing approaches. The paper ends with a conclusion in section 5 .

\section{Representation techniques}

In 1994 Hvam proposed a seven phase procedure for the development and maintenance of product configurators (Hvam, 1994). At CPM this procedure has since been further developed, as experience with its use has been obtained, into a form as that described by Hvam et al. (2008). In the CPM-procedure the prescribed three representation techniques to support the tasks of knowledge elicitation, design of the configurator knowledge base and documentation of the implemented knowledge are the product variant master technique (PVM), class diagrams and CRCcards. Having successfully been applied (or partly applied) in many Danish configuration projects, the CPM-procedure offers a solid basis for defining which modelling techniques a configurator documentation system should be capable of supporting.

\subsection{PVMs}

The PVM technique offers formal notation for the representation of information about a product assortment (Mortensen et al., 2000; Hvam et al., 2008). A PVM includes two generic sections for describing part-of (aggregation) structure and kindof (generalization) structure respectively. Furthermore, in the recent CPM definitions (Hvam et al., 2008), PVMs include attributes, attribute values, constraints (often only the most important ones, while the rest are described elsewhere), class description and cardinality. Figure 1 shows the most recent definition of the PVM notation formalism as prescribed by CPM.
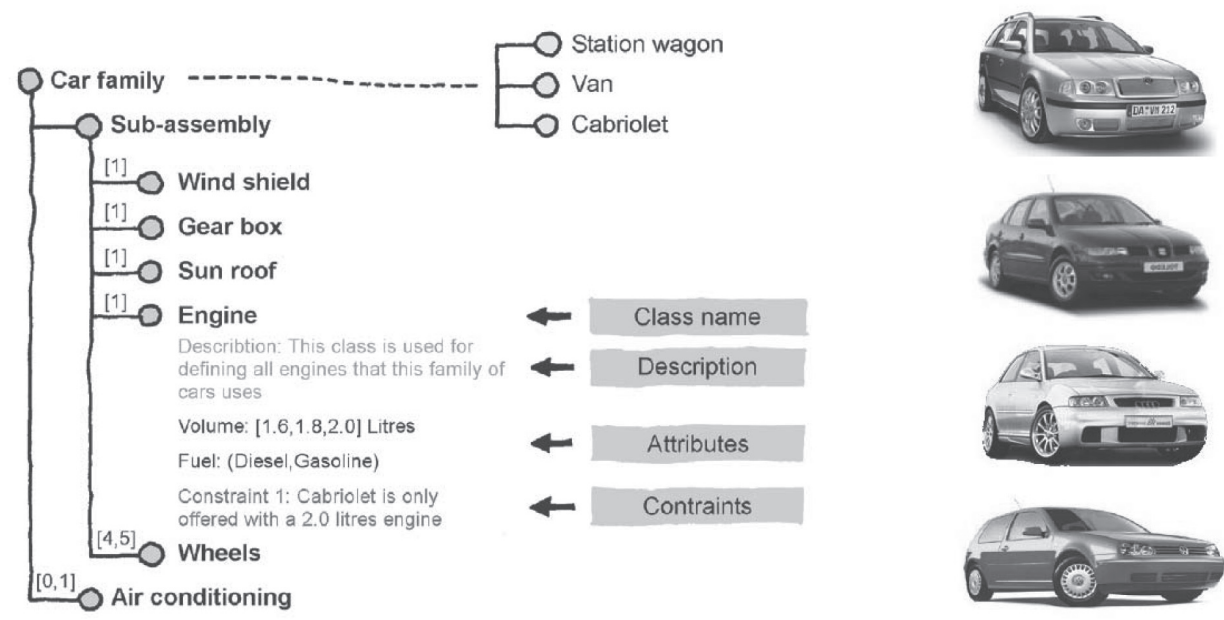

Figure 1: PVM formalism (Hvam et al., 2008 from Harlou, 2006) 


\subsection{Class diagrams}

A class diagram describes object classes and their relations and is the most commonly applied diagram of the Unified Modelling Language (UML) (Fowler, 2005). An example of the use of the class diagram notation, including some of the most common relationship types, is shown in figure 2 (navigability arrows can be used to show the direction of association and composition relationships).

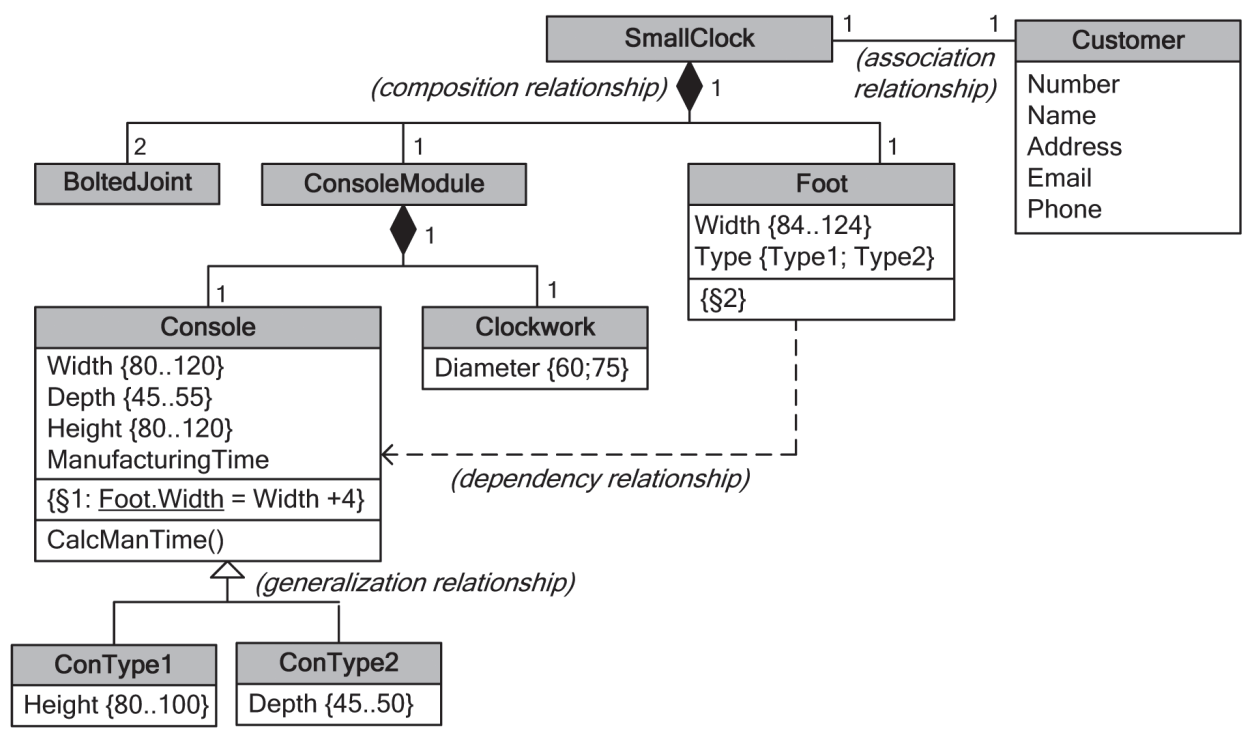

Figure 2: Example of class diagram

In a configuration context, the expression of constraints regarding possible combinations of elements is essential. For the expression of constraints, UML includes a language called OCL (Object Constraint Language). However, the use of OCL is not required to conform to normative UML, but any formalism can be used as long as placed within braces $(\{\})$ (Fowler, 2005). Stereotypes are another important concept of UML, that are used for representing variations of existing types of model elements, such as classes or relations. Stereotypes, therefore, make the use of domain specific terminology or notation possible. All UML model elements can be extended by stereotypes (OMG, 2005), which is shown by placing the name of the stereotype in guillemets $(<<\ldots>>)$.

\subsection{CRC-cards}

The CRC-card technique was invented by Ward Cunningham in the late 1980s (Fowler, 2005) and originally presented in a paper by Beck and Cunningham (1989), described as a way of teaching the object-oriented way of thought. These CRC-cards consist of the class name together with two columns for 'responsibilities' and 'collaborators'. In brief, responsibilities are summaries of the things that an 
object from the class should do, while collaborators are the other classes that the class works together with (Fowler, 2005). In the CPM-procedure the purpose of CRC-cards is to organize detailed information about classes represented in PVMs and class diagram models. Therefore, the CRC-cards of CPM consist of additional fields compared to the original definition. Figure 3 shows the most recent CRC-card definition from CPM. In these cards the 'methods fields' are for describing information about methods and what in other contexts is referred to as constraints or rules. 'Product methods' concern products and their life-phase properties, while 'system methods' concern software aspects of a configurator. 'Internal methods' refer to the internal structure, functions and properties of a class, while 'external methods' refer to interfaces to others classes.

\begin{tabular}{|l|l|l|}
\hline \multicolumn{1}{|c|}{ Class name: } & \multicolumn{1}{|c|}{ Date: } & \multicolumn{1}{|c|}{ Author/version: } \\
\hline Responsibilities: & Generalisation \\
\hline Aggregation & Superclasses: \\
\hline Superparts: & Subclasses: \\
\hline Subparts: & \\
\hline Sketch: & \\
\hline Attributes: & Collaborators: \\
\hline System methods: & \\
\hline & \\
\hline Product methods: & \\
\hline Internal methods: & \\
\hline
\end{tabular}

Figure 3: The latest CRC-card definition by CPM (Hvam et al. 2008)

\subsection{The use of PVMs, class diagrams and CRC-cards}

Hvam et al. (2008) prescribe the use of PVMs together with CRC-cards for product analysis and class diagrams together with CRC-cards for the design of the know- 
ledge base of a configurator. Basically PVMs and class diagrams describe the same thing, for which reason the use of both techniques in the same project implies a transfer of information from one diagram to the other. The argument for recommending the use of both structural diagrams is that PVMs are considered to be more easily understood and overviewed, for which reason this diagram type is well-suited in the analysis phase where product experts without significant diagrammatic modelling prerequisites are often involved. This assumption has to a large extent been confirmed by the studies of Haug and Hvam (2007). On the other hand, class diagrams provide a richer, more flexible and more formalised notation. Therefore, class diagrams would often be better suited for making adequately extensive and exact definitions of what to implement in a configurator knowledge base (see 'Haug and Hvam, 2007' for further description). According to Hvam et al. (2008), configurator knowledge is best documented in either PVMs combined with CRC-cards or class diagrams combined with CRC-cards, but not in both PVMs and class diagrams at the same time, since documenting configurator knowledge in both diagram types would imply much redundant documentation work and make the documentation task extra time-consuming.

\section{Documentation of configurator knowledge bases}

Since software, which supports PVMs and the CPM defined CRC-cards in an integrated manner, has not existed until recently, in the cases studied at CPM, PVMs are most often created in a program such as MS Visio (sometimes Excel or CAD), while CRC-cards are created by using MS Word. Class diagrams can be drawn in MS Visio or in one of the many UML drawing and CASE tools (see www.omg.org for a list of such software). The companies GEA Niro and American Power Conversion (APC), which are involved in two of the most extensive configuration projects, apply Lotus Notes for the documentation of their configurators (Hvam, 2004). However, all these kinds of software use have significant drawbacks when applying the CPMprocedure to carry out configuration projects.

\subsection{MS Visio, Word and UML tools}

The use of MS Visio (or similar drawing tools) in configurator projects can be timeconsuming. Such software does not know the PVM notation and therefore does not include PVM models elements. Therefore, if using MS Visio, model elements may be drawn and placed in stencils, from where defined elements can be placed repeatedly in a model by using drag-and-drop. However, these elements do not know where in a PVM model they can be placed (i.e. connection points) and will be placed exactly where dropped instead of being connected to the nearest element. Placing such model elements therefore requires some precision work. But the creation of PVM models in MS Visio gets even more tedious when making changes to a model. Insertion of classes between already drawn classes means that all the elements of 
a model below the insertion point need to be marked and be moved down manually. Another great shortcoming of MS Visio, when used to create PVM models, is that when rules/constraints are formulated, the names of already defined classes and attributes have to be retyped instead of being selected from a list of attribute names. Based on these limitations of MS Visio, one may think that other software could provide better support for the creation of PVM models. This, however, has not been the case.

For the creation of the CPM defined CRC-cards, MS Word or similar software is normally applied. This means that elements shown in both PVM model and CRCcards (i.e. class names, attribute names, attribute values, rules/constraints, pictures, comments etc.) need to be retyped in the CRC-cards. Besides this in itself being a time-consuming task, great chances of errors are associated with this manual synchronization of information. For creating class diagram models, as mentioned, a long list of dedicated software exists. Even MS Visio includes a template for the creation of class diagrams, which means that some of the mentioned problems of creating PVMs do not exist when creating class diagrams. In spite of this fact, the use of PVMs in the configuration projects studied at CPM is far more extensive than class diagrams (Hvam, 2004; Haug and Hvam, 2007; Hvam et al., 2008), seemingly because PVMs are found to be easier to use. Also, the class diagram modelling tools do not solve the problem of the integration to the CPM defined CRC-cards, but imply carrying out a significant amount of retyping of class names, attribute names, attribute values, rules, comments etc. in the class diagram model or the CRC-cards.

In the maintenance phase of configuration projects, other problems with the mentioned types of software emerge. In this phase the need for administrative functionalities increases, such as being able to administer change requests, share models, handle different user-access, use version control etc. Such tasks are not supported by the mentioned kinds of software, for which reason some companies have taken up the use of Lotus Notes for documentation of configurator knowledge bases.

\subsection{The Lotus Notes alternative}

Hvam and Malis (2001) describe how to apply Lotus Notes for the documentation of configurator knowledge and an actual implementation of Lotus Notes at GEA Niro. This form of documentation system is based on the use of standard Lotus Notes templates as CRC-cards. As mentioned, Lotus Notes is also applied by APC, although with a different CRC-card layout. The layout of GEA Niro is shown in figure 4 (next page).

According to Hvam (2004) the experience from the use of Lotus Notes as a configurator documentation system at GEA Niro and APC shows that to some extent 


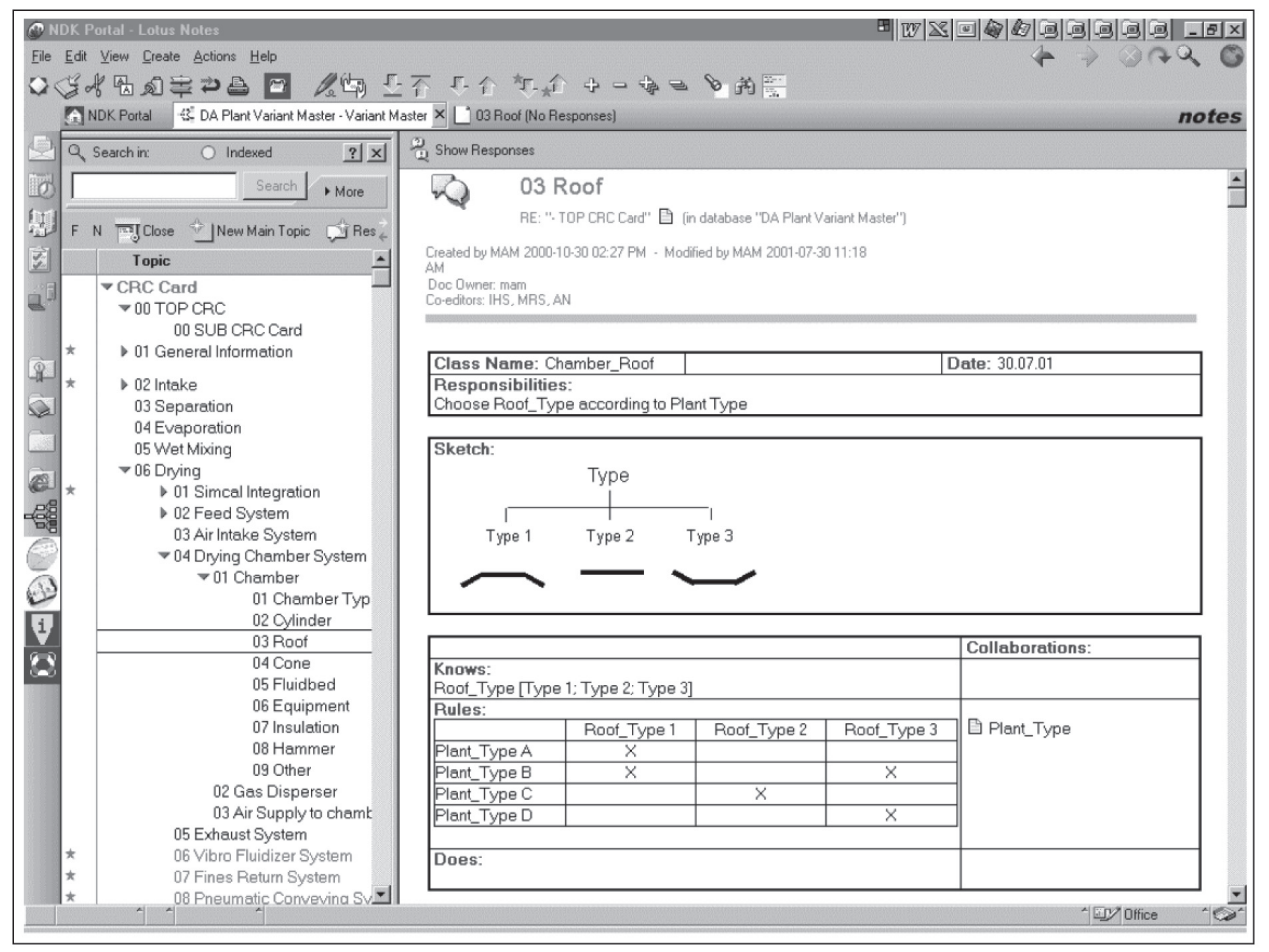

Figure 4: Lotus Notes at GEA Niro (Hvam and Malis, 2001)

this system supports the communication concerning relevant product information and the maintaining/further development of product configurators. However, also many shortcomings of this approach exist. Firstly, Lotus Notes includes no real functionality for administering change requests. For this reason GEA Niro applies different colour of texts in the CRC-cards to differentiate between elements of different states (Haug and Hvam, 2006a), which obviously can be hard to administer with the many users. Secondly, in connection with the CPM-procedure, the Lotus Notes-based documentation system does not support the elaboration of PVMs and class diagrams. For this reason, when product information is acquired at GEA Niro and APC this is described by using other software. Therefore, some data have to be transferred from the analysis/design models to the documentation system manually. Thirdly, there is no integration between the documentation system and the configurator. The update of information in both the configurator and the documentation system is time-consuming and places strict demands for discipline to avoid that these systems become inconsistent. Therefore, in some cases, the use of Lotus notes within the mentioned two companies implies that some model information has to be typed three times, i.e. in analysis models (MS Visio), in the documentation system (Lotus Notes) and in the configurator (Oracle). Furthermore, Lotus Notes has a long list of other limitations, such as not supporting: print of all CRC-cards 
at once, search for particular elements of the CRC-cards, insertion of defined attributes into rules and more. These limitations are further described later in this paper. Based on the drawbacks of the use of Lotus Notes in configuration projects, literature has pointed out the need for a more efficient system for the documentation of configurator knowledge bases (e.g. Riis, 2003; Hvam et al., 2005; Haug and Hvam, 2007).

\subsection{Towards a documentation system}

To ensure more efficient and user-friendly PVM and CRC-card modelling environments, including the automation of the synchronization of PVM and CRC-card information (in some cases also class diagrams), configuration literature has for several years dealt with the creation of a documentation system that supports the CPM procedure.

Hvam and Malis (2001) study five standard configurators and conclude that these do not provide adequate documentation-related functionality. Therefore, based on the experience gained from several Danish configuration projects, Hvam and Malis define a list of requirements for a documentation system. Later, based on these requirement definitions and interviews with four Danish manufacturing companies who apply configuration systems, Hvam et al. (2005) propose an extended list of requirements for a documentation system. Hvam et al. furthermore provide a high level description of a possible architecture of such a documentation system. However, in spite of these definitions, a documentation system was not created. A major problem may have been that although Hvam and Malis (2001) and Hvam et al. (2005) specify which modelling techniques should be included in a documentation system, this literature does not in a detailed manner deal with topics like: user interface design, detailed definitions of the included modelling techniques, the mutual mapping between modelling techniques etc. In this context it should also be considered that different companies apply different variants of the modelling techniques defined by the CPM-procedure in their configuration projects, for which generic definitions would be needed in order to support specific company needs.

Some of the required definitions of adequately flexible and software-prepared notation formalisms were provided by Haug and Hvam (2006b). This definition of a documentation system includes three main views, namely a CRC-card view, a PVM view, and a class diagram view. The paper primarily focuses on defining the PVM and class diagram formalisms, and how these formalisms can be mapped to each other, to the CRC-cards and to the CRC-card navigator (i.e. class tree). Another step towards the creation of a configurator documentation system was taken by Haug and Hvam (2006a). Based on the existing definitions of CPM (preliminary edition of: Hvam et al., 2008) and an analysis of two of the companies that to the greatest extent apply CRC-cards for the documentation of their configurators, they propose a highly extended definition of the CPM CRC-cards. Being a generic layout concept, 
this definition takes into account that different companies have different requirements for information to document.

Based on the definitions by Haug and Hvam (2006a; 2006b), the first prototype to support the modelling techniques of the CPM-procedure in an integrated manner was created in 2006 (Haug et al., 2007). Screen shots from the prototype are shown in figure 5 and 6.

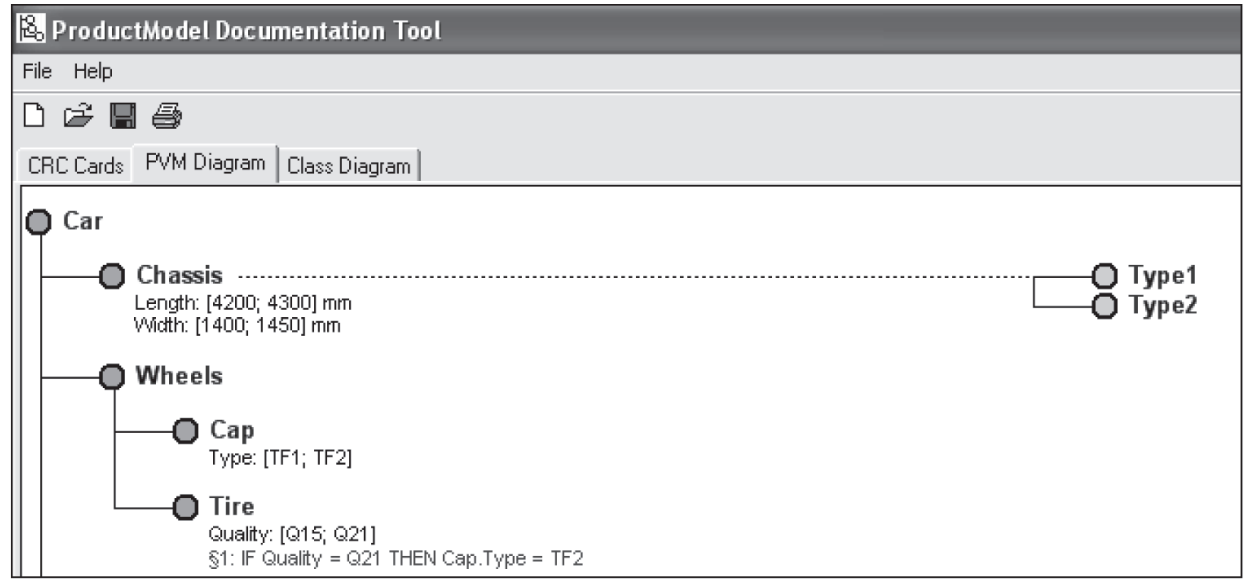

Figure 5: Prototype PVM view

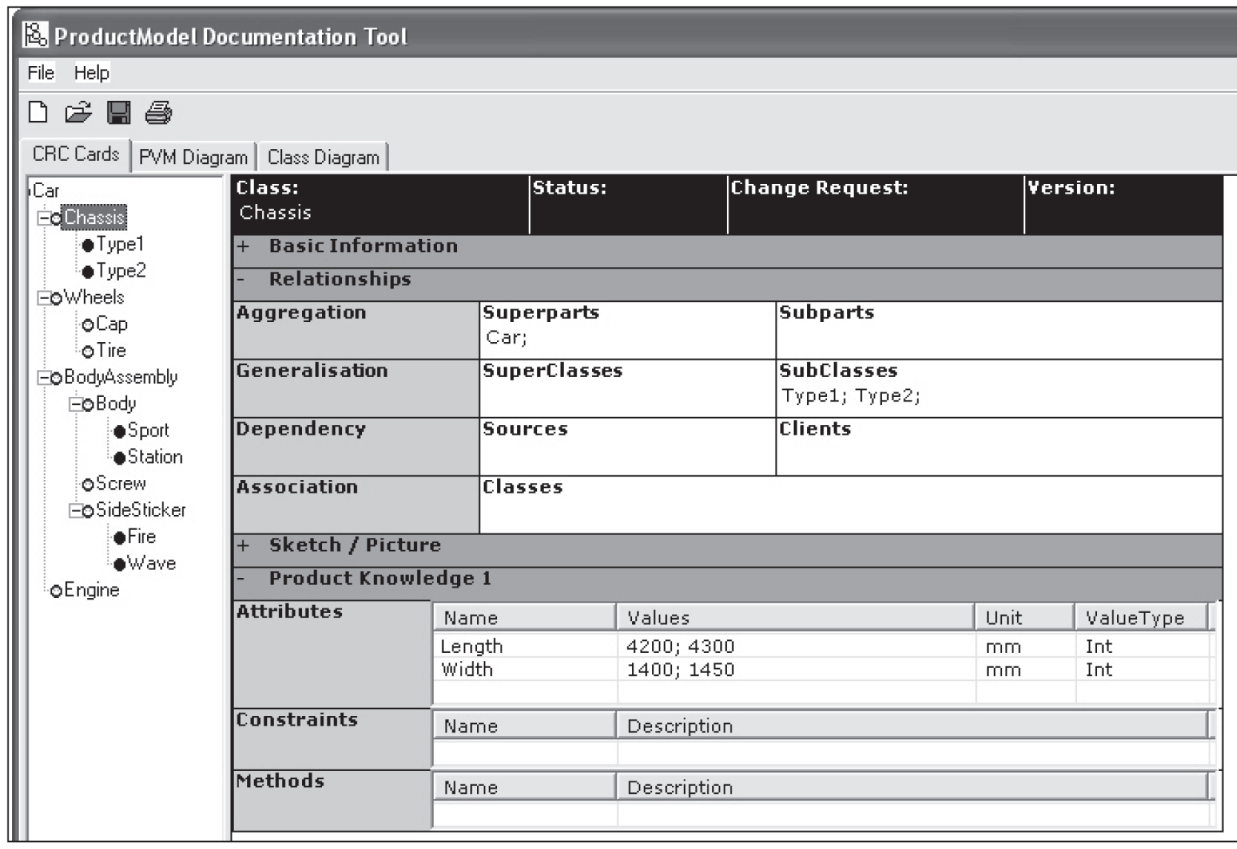

Figure 6: Prototype CRC-card view 
The purpose of the described prototype was to test the definitions of Haug and Hvam (2006a; 2006b). To some extent the tests showed that the defined principles were consistent and provided a solid basis for the creation of such a system. However, because of this focus and time-constraints, the prototype was not developed into a state where it was really applicable in practice. Some of the most important limitations of the prototype are e.g.: it is too inflexible concerning the layout and contents of PVM and class diagram models; it does not support the generic CRCcard principle defined by Haug and Hvam (2006a); it is not adequately stable or complete to be suitable for use in practice; and almost no administrative functionality is included.

\section{Introducing Product Model Manager}

\subsection{Background}

Although the prototype, described in the previous section (Haug et al., 2007), to some extent was able to show that the definitions by Haug and Hvam (2006a; 2006b) were consistent and convertible to software, more definitions were needed to ensure an adequately user-friendly, comfortable and flexible modelling environment. Based on the definitions of Haug and Hvam (2006a; 2006b) additional specifications were made during 2007 by the first two authors of this paper. Based on these specifications several new prototypes were created, and in the end a full-scale system. This software was named Product Model Manager (PMM) and the first version of this software was released in December 2007 by Incore Systems. The name (i.e. Product Model Manager) serves to illustrate, that while PMM bears some resemblance with PDM (Product Data Management) systems, it is the documentation of structured product models rather than product data that PMM is aimed at. Based on the first customer feedbacks version 1.1 of PMM was released in March 2008. Compared to the 1.0 version, this version includes new modelling features, better means to support the handling of multiple users and simple version control.

\subsection{Main features}

A major difference between PMM and the prototype described by Haug et al. (2007) is that the class diagram environment has been left out of PMM. This decision was taken based on the argument that since PVMs and class diagrams basically illustrate the same thing, but class diagrams are richer, by extending the PVM notation to have a similar richness and flexibility as that of class diagrams, the class diagram environment could be left out without loosing the benefits of having this environment. The topic of how to enrich the PVM notation is to some degree described in the papers by Haug and Hvam (2006b) and Haug and Hvam (2007), which formed part of the basis for this work.

In the current PVM notation, as defined by CPM (Hvam et al., 2008), classes include: attributes, description (notes), sketches/pictures and constraints (rules). 
Beside this, the classes of the PVM notation in PMM also include the entities: comments; links to external document/file; and sub-projects. The use of sub-projects means that models created can be applied in multiple models, while being maintained centrally. For this reason the use of generalisation structure is possible, not just within a model, but across several models. Another important feature of PMM is the Product Explorer (PE) view. Using the PE view together with the PVM view means that a simple overview of the model can be utilized while elaborating the more detailed PVM model. Furthermore, navigation and modelling in the PVM and PE views can be done simultaneously, i.e. clicking on an element in one view highlights the same element in the other view. The PE view in combination with the PVM view is shown in figure 7. All elements (i.e. classes, attributes, rules etc.) of a PVM can be dragged-and-dropped in a desired position while the connection lines of the element are automatically created by PMM.

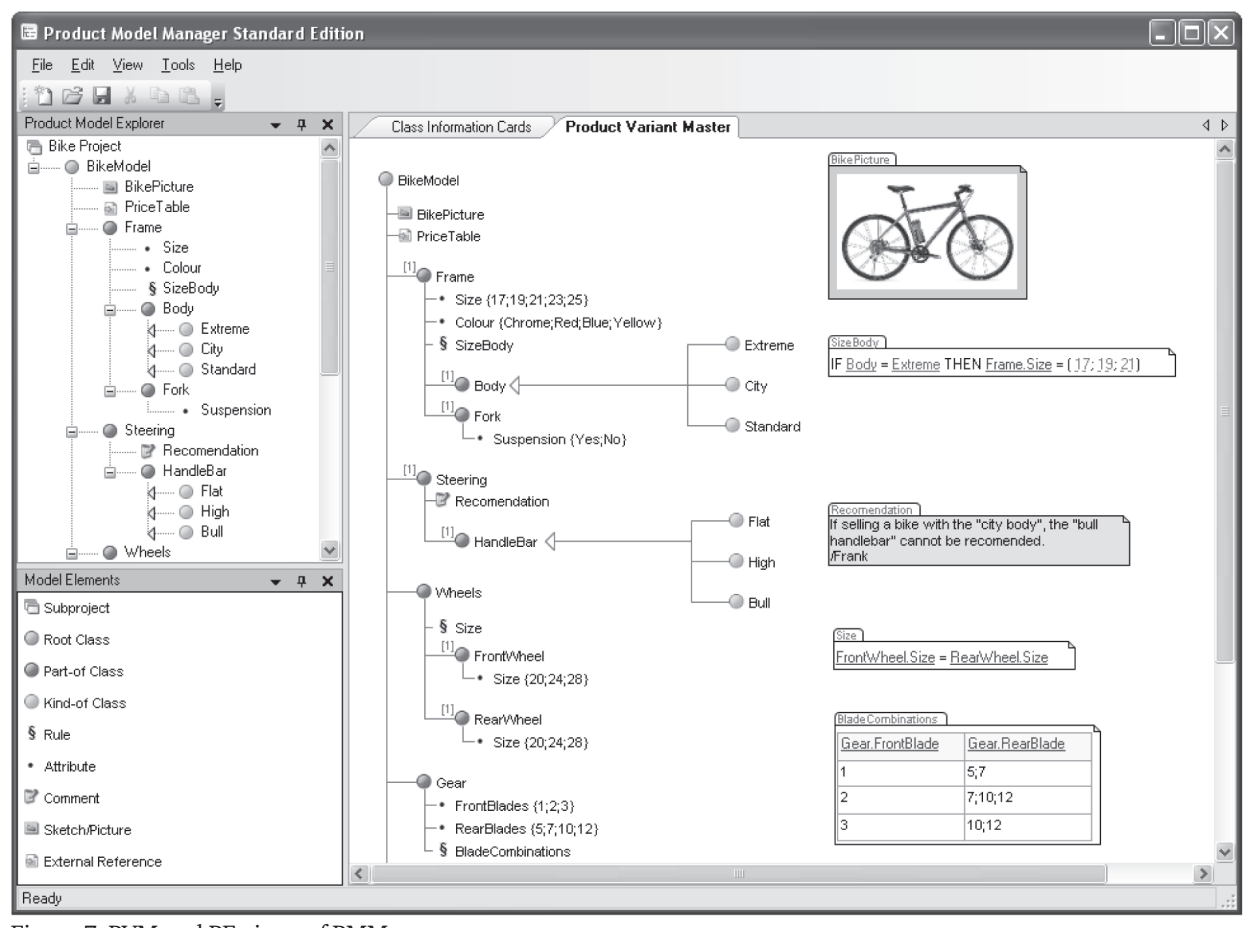

Figure 7: PVM and PE views of PMM

The third modelling environment of PMM is the Class Information Card (CIC) view. The name 'Class Information Cards' has been chosen instead of, as in CPM literature, 'CRC-cards' in order to avoid associations with the original kind of CRC-cards (Beck and Cunningham, 1989), which are mainly applied for a rather different purpose and have a much more simple layout. 
In figure 8 the CIC view of PMM is shown. It should be noticed that the image is only an example of a possible configuration of the layout, which in a XML file can be defined to consist of the fields exactly desired by a specific company, group or user. Additionally, PMM contains a report engine that will transform the model into other applications native formats. For instance, a style-sheet is included that enables a user to preview all Class Information Cards in a web-browser.

As seen in figure 8, the folders of the CICs of PMM can be expanded ('Basic Information' and 'Product Knowledge') and collapsed ('Relationships'), depending on which information of a CIC the user is interested in. To navigate in and between CICs, besides using the PE, such navigation is also possible by using the hyperlinks in the CICs.

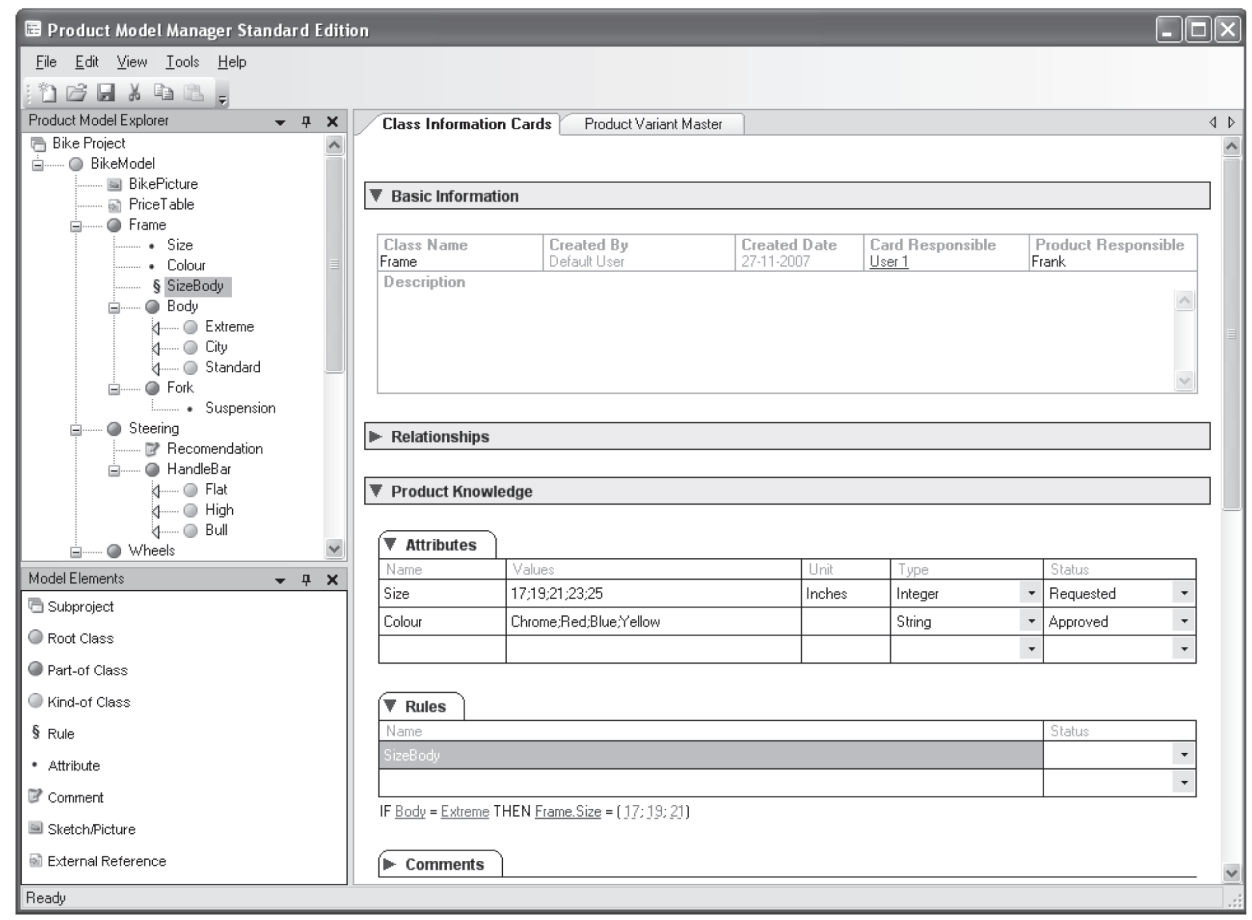

Figure 8: The Class Information Card view of PMM

To evaluate the value of PMM as an alternative to existing documentation systems in configuration projects, PMM is compared to the MS Visio/Word and Lotus Notes alternatives in the following two sections.

\subsection{Comparison to the use of MS Visio and Word}

In table 1, the advantages of the use of PMM compared to MS Visio and Word in configuration projects are described. The primary focus in this context is on the use in the analysis and design phase, i.e. before the implementation of the created models into the configurator knowledge base. 


\begin{tabular}{|c|c|}
\hline Task & Advantages of using PMM instead of MS Visio/Word \\
\hline \multirow[t]{8}{*}{$\begin{array}{l}\text { (1) Creation of PVMs } \\
\text { (PMM vs. Visio) }\end{array}$} & $\begin{array}{l}\text { In PMM, the model elements of a PVM model do not need to be placed at } \\
\text { their exact position, but can be placed at areas near possible insertion points, } \\
\text { based on which PMM automatically repositions elements and creates } \\
\text { connection lines. }\end{array}$ \\
\hline & $\begin{array}{l}\text { In PMM, already defined entities that are to be included in a rule (i.e. classes, } \\
\text { attributes and values) can be chosen from a list. }\end{array}$ \\
\hline & $\begin{array}{l}\text { In PMM, when inserting classes in positions between existing classes, the } \\
\text { part of a model below the insertion point automatically repositions. }\end{array}$ \\
\hline & In PMM, PVM elements can be inserted from context menus. \\
\hline & $\begin{array}{l}\text { In PMM, parts of models can be maintained separately/globally by the use of } \\
\text { sub-models. }\end{array}$ \\
\hline & $\begin{array}{l}\text { In PMM, when clicking on an element of a rule statement, its position in the } \\
\text { model is shown in both the PE and PVM view. }\end{array}$ \\
\hline & $\begin{array}{l}\text { In PMM, due to the built-in model validation (e.g. a class cannot be a part of } \\
\text { an attribute), a model is always consistent with the PVM notation. }\end{array}$ \\
\hline & $\begin{array}{l}\text { In PMM, files can be dragged from the file system and be attached to the } \\
\text { model as sub-models, sketches or external references. }\end{array}$ \\
\hline \multirow[t]{4}{*}{$\begin{array}{l}\text { (2) Creation of CRC- } \\
\text { cards/CICs (PMM vs. } \\
\text { Word) }\end{array}$} & $\begin{array}{l}\text { In PMM, links to referenced elements (e.g. a class mentioned in a rule) are } \\
\text { automatically created, so that navigation is possible by clicking on elements } \\
\text { belonging to other CICs. }\end{array}$ \\
\hline & $\begin{array}{l}\text { In PMM, an overview of CICs and a means for navigation between the CICs } \\
\text { is provided by the Product Explorer view. }\end{array}$ \\
\hline & $\begin{array}{l}\text { In PMM, reports that include only specific parts of a CIC can be created by } \\
\text { using XSLT and the report engine. }\end{array}$ \\
\hline & In PMM, different user rights can be set up in the PMM configuration file. \\
\hline \multirow[t]{2}{*}{ (3) Synchronization } & $\begin{array}{l}\text { In PMM, when creating or changing an element in the PE, PVM or CIC view, } \\
\text { this is automatically reflected in the other views. }\end{array}$ \\
\hline & $\begin{array}{l}\text { In PMM, when the entities (class names, attribute names, attribute values), } \\
\text { which are included in a rule, are changed, this change will automatically be } \\
\text { effected in all rules where the given entity is included. }\end{array}$ \\
\hline \multirow[t]{4}{*}{ (4) General features } & $\begin{array}{l}\text { PMM provides an XML interface that allows the import and export of models } \\
\text { to other software (obviously the XML formats have to match). }\end{array}$ \\
\hline & $\begin{array}{l}\text { In PMM, new export formats can be generated through the use of XSLT and } \\
\text { the report engine. }\end{array}$ \\
\hline & $\begin{array}{l}\text { In PMM, when inserting and downsizing pictures, their file sizes are } \\
\text { automatically reduced, while it being possible to reload the original. }\end{array}$ \\
\hline & $\begin{array}{l}\text { PMM can be extended by plug-ins, thus facilitating a seamless integration } \\
\text { with other systems like product configurators or ERP-systems. }\end{array}$ \\
\hline
\end{tabular}

Table1: Comparison of PMM to MS Visio and Word

One may imagine that the use of PMM instead of MS Visio for the creation of PVM models implies some drawbacks related to the freedom of placement of model elements. However, model elements in the PVM view of PMM can be placed anywhere desired by dragging the element (or using the arrow keys) to the desired position, while connections automatically adapt to this new placement. Also, like MS Visio/ Word, PMM supports operations such as: use of hyperlinks to external files; insertion of pictures; creation of tables; copy/paste of tables from/to Word and Excel; search for texts in models; turn of display of certain model elements (in PMM by checkboxes, in MS Visio by the use of layers); and print functionality. On the other 
hand, there may be different views on whether it is an advantage allowing the creation of new graphical symbols outside the defined notation formalism, as in MS Visio, or ensuring that only normative notation is used, as in PMM. Furthermore, it may seem that MS Word or Visio are more flexible for the creation of CRC-cards. However, like Word/Visio, PMM supports the creation of CIC templates according to particular requirements, i.e. folders and fields can be created, named and placed as desired. Also printing of CICs and copy-paste of texts from/to MS Word/Excel is possible.

\subsection{Comparison to the use of Lotus Notes}

The information about Lotus Notes being used as a configurator documentation system was retrieved by using data from earlier interviews (Haug and Hvam, 2006a) and by subsequent clarification through the conducting of a semi-structured interview with persons from the configuration team of GEA Niro. Based on this information, compared to Lotus Notes, PMM can be said to hold the advantages, described in table 2 (next page).

Two of the main reasons for the many advantages from the use of PMM compared to Lotus Notes for configurator documentation are: (1) the inclusion of the PVM environment, and (2) the way in which PMM stores information compared to Lotus Notes. The inclusion of the PVM notation in PMM gives significant advantages compared to the use of Lotus Notes. In the collection of information from products experts, the use of Lotus Notes is associated with numerous limitations because of the limited possibilities of the CRC-card navigator. Therefore, as mentioned, both GEA Niro and APC use other programs to build PVM models when retrieving information from product experts (MS Visio and Excel). This, however, means that the information of these PVM models has to be retyped in Lotus Notes later, which is time-consuming and some times implies that information is lost because of the expressional limitations of Lotus Notes. The second advantage of PMM is that in Lotus Notes, the elements of classes (i.e. attribute name, attribute values, unit, status etc.) are not stored as separate fields in the Lotus Notes database, but in a way corresponding to a text in a Word document. This means that the elements of the CRC-cards of Lotus Notes are not linked, e.g. a rule name is not linked to a rule expression. For this reason searching across models and setup of data exchange is not supported by Lotus Notes, whereas PMM does. The XML-interface of PMM means that it is possible to create a data exchange application for exchange of data between PMM and a configurator. Using such interface applications with PMM implies that most of the retyping of information in either the configurator or the documentation system can be avoided.

On the other hand, it may at first glance seem that Lotus Notes holds an advantage over PMM, because users can edit different CRC-cards of the same model si- 


\begin{tabular}{|c|c|}
\hline Task & Advantages of using PMM instead of Lotus Notes \\
\hline (1) Use of PVM models & $\begin{array}{l}\text { To make PVM models, when using Lotus Notes, these must be created in } \\
\text { another program and the information manually transferred to the CRC- } \\
\text { cards of Lotus Notes. PMM supports the creation of PVMs and class } \\
\text { information cards (CRC-cards) in an integrated fashion. }\end{array}$ \\
\hline \multirow[t]{5}{*}{$\begin{array}{l}(2) \mathrm{CRC}-\mathrm{card} / \mathrm{CIC} \\
\text { navigator }\end{array}$} & $\begin{array}{l}\text { The CRC-card navigator of Lotus Notes (simple class hierarchy) only } \\
\text { displays class names, while the Product Explorer window of PMM also } \\
\text { provides information about attributes, rules/constraints, pictures/sketches } \\
\text { etc. }\end{array}$ \\
\hline & $\begin{array}{l}\text { The CRC-card navigator of Lotus Notes cannot differentiate between part- } \\
\text { of and kind-of relationships - the Product Explorer of PMM can. }\end{array}$ \\
\hline & $\begin{array}{l}\text { The CRC-card navigator of Lotus Notes does not support insertion of } \\
\text { classes (and other kinds of entities) by using drag-and-drop, whereas the } \\
\text { Product Explorer of PMM does. }\end{array}$ \\
\hline & $\begin{array}{l}\text { The class hierarchy of the CRC-card navigator of Lotus Notes cannot be } \\
\text { printed directly, but only as screen-shots. This implies that for a big model } \\
\text { there will be several screen shots that must be printed individually. In PMM } \\
\text { PVM models can be print-previewed and printed. }\end{array}$ \\
\hline & $\begin{array}{l}\text { In Lotus Notes classes cannot be reorganized by using drag-and-drop } \\
\text { functionality, whereas PMM allows this. }\end{array}$ \\
\hline \multirow[t]{5}{*}{ (3) CRC-cards/CICs } & $\begin{array}{l}\text { In Lotus Notes a text or a field cannot be given a status (e.g. 'request', } \\
\text { implemented' etc.). PMM allows the setup of the required fields and fixed } \\
\text { values through a XML configuration file. }\end{array}$ \\
\hline & $\begin{array}{l}\text { When creating rules/constraints in the CICs of PMM, classes and attributes } \\
\text { and values can be chosen from a list of already defined entities. In Lotus } \\
\text { Notes such entities have to be retyped. }\end{array}$ \\
\hline & $\begin{array}{l}\text { The ClCs of PMM allow the setup of possible values for custom fields. This } \\
\text { is not possible in standard Lotus Notes. }\end{array}$ \\
\hline & $\begin{array}{l}\text { In Lotus Notes one cannot print out all CRC-cards at once, but only one at } \\
\text { the time. In PMM all CICs can be printed directly (or exported as HTML). }\end{array}$ \\
\hline & $\begin{array}{l}\text { Lotus Notes does not support the reuse of classes or models, but such } \\
\text { classes have to be copied and maintained individually. PMM supports the } \\
\text { use of sub-models that can be inserted several times and maintained from } \\
\text { a central position, so that all instances are automatically updated. }\end{array}$ \\
\hline \multirow[t]{7}{*}{ (4) General features } & $\begin{array}{l}\text { You cannot export a model in Lotus Notes to XML or another exchange } \\
\text { format. PMM provides an XML interface that allows the import and export of } \\
\text { models to other software (obviously the XML formats have to match). }\end{array}$ \\
\hline & $\begin{array}{l}\text { Lotus Notes does not allow data exchange with configurators. PMM allows } \\
\text { definition of an export that only includes selected parts of a model. This is } \\
\text { done by defining a transformation file and attaching it to the report engine. }\end{array}$ \\
\hline & $\begin{array}{l}\text { When inserting and downsizing pictures in PMM, the file sizes are } \\
\text { automatically reduced, while it is still possible to reload the original. Lotus } \\
\text { Notes does not include such functionality. }\end{array}$ \\
\hline & $\begin{array}{l}\text { Lotus Notes does not have version control of the models created. In PMM, } \\
\text { using the web plug-in, a new version of the model is defined at each } \\
\text { subsequent upload. Hence in its basic and non-customized form simple } \\
\text { version control is available. }\end{array}$ \\
\hline & $\begin{array}{l}\text { Lotus Notes does not support rollback of a model to an earlier state. In } \\
\text { PMM, the web-plug-in can be used to retrieve a specific version. }\end{array}$ \\
\hline & $\begin{array}{l}\text { In Lotus Notes, it is not possible to search for entities (classes, rules, } \\
\text { attributes etc.) in an entire model. PMM includes a search-function that } \\
\text { allows searching through models, and it is even possible to search in the } \\
\text { web report of the CICs. }\end{array}$ \\
\hline & $\begin{array}{l}\text { In Lotus Notes one can only give the users 'read-only' or 'unlimited access' } \\
\text { to the CRC-cards. In PMM you can define specific classes or fields in the } \\
\text { XML configuration file that only specific users have access to. Furthermore, } \\
\text { you can define users that are not allowed to change the model structure but } \\
\text { only the model content. }\end{array}$ \\
\hline
\end{tabular}

Table 2: PMM compared to Lotus Notes 
multaneously, i.e. if more users open the same CRC-card, only the first will be allowed to edit it, while the rest would open a read-only file. In PMM it is in fact possible to do exactly what Lotus Notes does, namely making references to external documents (e.g. Word documents), which obviously can be edited in parallel. This can be done by using the PMM entity 'external reference' for pointing at these documents. Furthermore, PMM supports multiple user access by dividing a model into sub-models, that can be edited independently of the main model and other submodels. Also the possibility of using a version control system for merging different versions of models exists when using PMM. Therefore, it also seems hard to identify any real advantages of the use of Lotus Notes instead of PMM in a proper setup.

\section{Conclusion}

For several years the need for a documentation system to support the development and maintenance of product configurators has been described in literature. However, although several definitions of such a system have been produced, such software did not exist on the market until recently. Even greater is the amount of papers and books that describe the use of the PVM notation and the CPM-defined CRC-cards, whereas no IT-systems have been capable of supporting these notations in an efficient and integrated manner. The emergence of PMM therefore represents a significant event because it is the first commercial application that is capable of supporting PVMs and extended CRC-cards in an integrated manner.

As mentioned, PMM version 1.1 was released in March 2008, and although some companies have used PMM version 1.0 since January 2008, the current version includes many new features, e.g. for better handling of multiple users and model versions. Because of this, in order to make a fair evaluation of the use of PMM in practice, more time has to pass. Instead this paper evaluated the features of PMM compared to the existing software alternatives, which are applied by the companies involved in configuration projects. For the application in the analysis and design phase of configuration projects, PMM was compared to MS Visio/Word. This comparison showed that PMM holds a long list of advantages, while the mentioned alternative does not hold significant advantages. For the application as a configurator knowledge base documentation system, PMM was compared to the use of Lotus Notes, which may represent the most successful application of a software system for such. Also here it seems that PMM includes a long list of advantages compared to Lotus Notes, while no significant drawbacks. Therefore, based on this study, it seems that companies who apply PVMs and/or CRC-cards in their configuration projects could achieve significant advantages from using PMM.

Much investigation concerning the use of PMM remains to be carried out. However, based on the analysis carried out in this paper, it seems that PMM has a great potential for efficiently improving the way in which the analysis and design phases are carried out in configuration projects by enhancing the possibilities of model- 
ling relevant information in a faster and more consistent way than what has been possible so far. But the impact of the use of PMM may be even greater on the maintenance phase of configuration projects, where PMM provides a basis for achieving great reductions in the resources required for this task.

\section{References}

Beck, K. and Cunningham, W.A. (1989): A laboratory for teaching object-orien-ted thinking. Proceedings of OOPSLA '89 and Special issue of SIGPLAN Notices, 24(10): 1-6. Edwards, K., Hvam, L., Pedersen, J.L., Møldrup, M. and Møller, N. (2005): Udvikling og implementering af konfigureringssystemer: Økonomi, Teknologi og Organisation. Final report from PETO research project. Lyngby, Denmark: Department of Manufacturing Engineering and Management, Technical University of Denmark.

Fowler, M. (2005): UML distilled: A brief guide to the standard object modeling language (3rd edition). Boston, MA: Addison-Wesley.

Hansen, B.L. (2003): Development of Industrial Variant Specification Systems. PhD thesis. Lyngby, Denmark: Department of Manufacturing Engineering and Management, Technical University of Denmark.

Harlou, U. (2006): Developing product families based on architectures - Contribution to a theory of product families. PhD dissertation. Lyngby, Denmark: Department of Mechanical Engineering, Technical University of Denmark.

Haug, A. and Hvam, L. (2006a): CRC-cards for the development and maintenance of product configuration systems. Customer Interaction and Customer Integration (Proceedings of the Joint Conference IMCM'06 \& PETO'06, Hamburg, Germany, June 22-23, 2006). Berlin: GITO-Verlag.

Haug, A. and Hvam, L. (2006b): The modelling techniques of a documentation system that supports the development and maintenance of product configuration systems. Customer Interaction and Customer Integration (Proceedings of the Joint Conference IMCM'06 \& PETO'06, Hamburg, Germany, June 22-23, 2006). Berlin: GITO-Verlag.

Haug, A. and Hvam, L. (2007): A comparative study of two graphical notations for the development of product configuration systems. International Journal of Industrial Engineering, 14(2): 107-116.

Haug, A., Degn, A., Poulsen, B. and Hvam, L. (2007): A prototype of a documentation system that supports the development and maintenance of product configuration systems. WSEAS Transactions on Information science and Applications, 4(5): 1048-1055.

Hvam, L. and Malis, M. (2001): A knowledge based documentation tool for configuration projects. Proceedings of World Congress on Mass Customization and Personalization, Hong Kong, Oct. 1-2, 2001.

Hvam, L., Pape S., Jensen K.L. and Riis, J. (2005): Development and maintenance of product configuration systems: Requirements for a documentation tool. Inter- 
national Journal of Industrial Engineering - Theory Applications and Practice, 12(1): 79-88.

Hvam, L. (2004): A multi-perspective approach for the design of Product Configuration Systems - an evaluation of industry applications. Proceedings of the International Conference of Economic, Technical and Organizational aspects of Product Configuration Systems (PETO), Lyngby, Denmark, June 28-29, pp. 13-25.

Hvam, L. (1994): Application of product modelling - seen from a work preparation viewpoint (Trans). PhD thesis. Lyngby, Denmark: Department of Industrial Management and Engineering, Technical University of Denmark.

Hvam, L., Mortensen, N.H. and Riis, J. (2008): Product Customization. Berlin and Heidelberg: Springer-Verlag.

Mortensen, N.H., Yu, B., Skovgaard, H. and Harlou, U. (2000): Conceptual modeling of product families in configuration projects. Workshop at the 14th European Conference on Artificial Intelligence, Berlin, Germany, Aug. 20-25, 2000, pp. 68-73. OMG (2005): Unified Modeling Language: Superstructure: Version 2.0 (formal/0507-04). Nedham, MA.

Riis, J. (2003): Fremgangsmåde for opbygning, implementering og vedligeholdelse af produktmodeller. PhD dissertation. Lyngby, Denmark: Department of Manufacturing Engineering and Management, Technical University of Denmark.

Sabin, D. and Weigel, R. (1998): Product configuration frameworks - A survey. IEEE Intelligent Systems and Their Applications, 13(4): 42-49.

\section{Authors and addresses}

\section{Anders Haug:}

Department of Entrepreneurship and Relationship Management, University of Southern Denmark, Engstien 1, 6000 Kolding.

Email: adg@sam.sdu.dk.

\section{Torben Lisbjerg:}

Incore Systems ApS,

Diplomvej 381,

2800 Kgs. Lyngby.

Email: torben@incoresystems.dk.

\section{Lars Hvam:}

Department of Manufacturing Engineering and Management, Technical University of Denmark,

Building 425,

2800 Kgs. Lyngby, Denmark.

Email: 1hv@ipl.dtu.dk. 


\section{Biographical notes}

Anders Haug is assistant professor at the Department of Entrepreneurship and Relationship Management at the University of Southern Denmark. His main research areas are knowledge management, knowledge representation, knowledge engineering and knowledge sharing - from an individual, organizational and company network perspective. Anders has produced a number of international publications that deals with the development of product configurators, representation of complex industrial knowledge, sharing of product information and more.

Torben Lisbjerg is managing director of Incore Systems ApS. Torben holds a M.Sc. in Engineering, and have several years of experience from management of software development projects particularly in the automotive industry.

Lars Hvam is associate professor at the Technical University of Denmark and head of the Centre for Product Modelling (www.productmodels.org). The main research area of Lars Hvam is the development of methods for analysing the tasks of engineers/technicians for specifying products, and in addition to this, to define the contents of the IT-systems (product models) which are to support the specifying tasks of engineers and technicians. Lars Hvam has been the supervisor of several $\mathrm{PhD}$ projects regarding the construction and application of configuration systems as well as the project leader of four major research projects on product configuration. 


\title{
CONFIGURATION OF PRODUCT-CENTRIC SERVICES
}

\author{
Philipp Ackermann
}

\begin{abstract}
Buyers of investment goods are increasingly considering the full cost of a product, i.e., not just purchase costs but also operational costs. Manufacturers are therefore forced to provide industrial product service systems where life cycle cost data reflect operating expenses, service costs, and energy efficiency impacts. The necessity of enhancing product data with service elements exists in product design as well as in application engineering. Manufacturers are therefore asking for configuration technology that manages their integrated product-service mix.
\end{abstract}

\section{Keywords}

Product-centric Services, Integrated Product and Service Lifecycle Management, Service Visualization, Configuration of a modular Product-Service Portfolio

\section{Front-loading Custom-specific Design and Engineering}

In today's highly competitive global marketplace, producers of equipment, machines, plants, and installations are forced to provide basic engineering tasks for free during the sales and acquisition phase. Due to the increasing levels of pressure in the market there is a fundamental need to reduce the associated technical and financial risks, which means great engineering efforts are required during the offer process. Manufacturers are therefore shifting from unprofitable designto-order models to individually configurable product systems based on reusable modules and assemble-to-order fulfilment.

\section{Cross-functional Standardization and Modularization}

In order to increase the reuse of existing product elements, manufacturers are establishing modular product systems. They typically start with standardising norm parts and then modularise their core product, e.g., the mechanical parts of a machine. It is often the case that an offer includes more than one machine, so that transport and handling systems are standardised and brought into a platform strategy as well.

\subsection{Mechatronical Portfolio}

After achieving benefits from standardizing and modularising the mechanical parts, the electric, electronics, hydraulic and pneumatic elements will be incorporated in the modular solution portfolio by the classification of established corporate 
standards. As both piping and cabling need conduits for optimum performance, architectural building elements become part of the modular system. The automation software needed to control machines and plants has to adapt to the standardised components, and is encoded as modular building blocks in order to reflect the flexibility gained with the physical modules.

\subsection{From Product to Solution Provider}

More and more manufacturers in the discrete industries are enhancing their product offerings with added services in order to become solution providers. This pro-cess helps manufacturers to provide a fully-fledged product-service mix for their customers, while activities such as transportation, installation, commissioning, testing, maintenance, or financing move into the focus of standardisation and modularisation. Ultimately the whole portfolio - including mechanics, electronics, software and services - will be transformed into modular systematics so as to improve competitive flexibility and business efficiency. In the sales process, the product information that has been separated from all the diverse engineering disciplines needs to be aggregated into a unified view for the customer. Providing a cross-functional, integrated planning and configuration environment is therefore a crucial element in effectively supporting sales engineering.

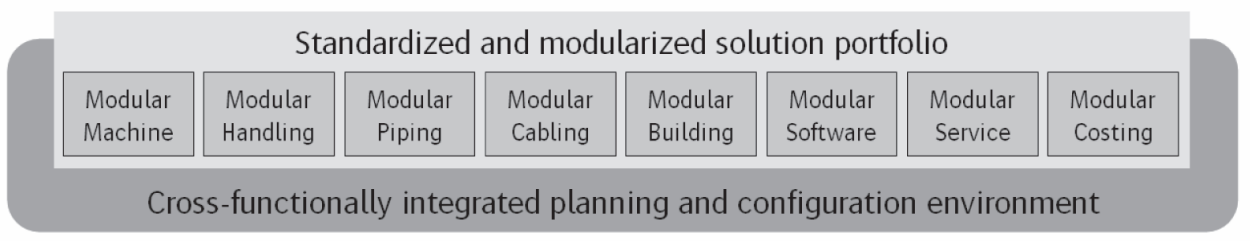

Fig. 1: Cross-functionally integrated configuration

\section{Solution Lifecycle Management}

\subsection{PLM-integrated Sales and Service}

Detailed technical and cost accounting clarifications need to be carried out in order to create competitive offers. In many industries the traditionally separate departments of sales and engineering will therefore increasingly converge. To support an integrated work process, technical, logistical and commercial aspects need to be combined into an explicit knowledge base. While the product development process is well-supported by $3 \mathrm{D}$ CAD tools and the production process profits from wellestablished ERP and PPS systems, machine and plant manufacturers often suffer from the missing digital link inbetween, where product management, sales and custom-specific engineering take place. 
Effective marketing and sales support for investment goods needs to combine technical and logistical aspects, while also integrating CAD data created during the development process with ERP data used for order fulfilment. Companies are therefore likely to move from developmentoriented Product Data Management (PDM) towards enterprise-wide and collaborative Product Lifecycle Management (PLM) [Stark, 2005]. The most demanding integration challenge is the use of unified product knowledge at the touch points of customer interaction in sales and service processes. Within complex products each assembly section is developed in specialised expert tools, such as mechanical CAD for geometric forms, electro CAD for cabling and wiring, P\&ID for hydraulics and pneumatics, software tools, and other applications for service and project planning. Modern sales and service processes are extremely demanding, which means that the information that has been generated by all the diverse expert tools needs to be aggregated into a unified view focussed on the customer.

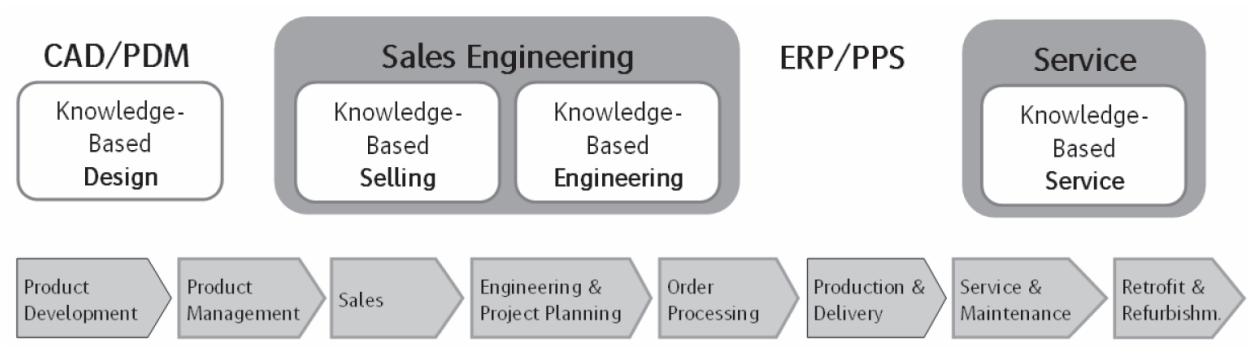

Fig. 2: Knowledge-based Sales and Services

\subsection{From PLM to Solution Lifecycle Management (SLM)}

To manage customization of a product-service mix in an efficient way, services should systematically be structured and modularized similar to approaches in product development. In recent years, service engineering [Bullinger/Scheer, 2003] has therefore gained general interest. To streamline the service engineering process between design teams, sales people, project engineers, planning partners, customers, and operators, the relevant portfolio information has to be handled in an integrated and collaborative way that bridges CAD, PDM, ERP and CRM systems. Within IT strategies, companies shift focus from supporting technology-oriented product innovation to manage the whole lifecycle of integrated product-service portfolios. To become full-fledged "Solution Lifecylce Management" systems, current IT tools for Product Lifecycle Management (PLM) have therefore to be enhanced with Service Lifecycle Management functionality. 


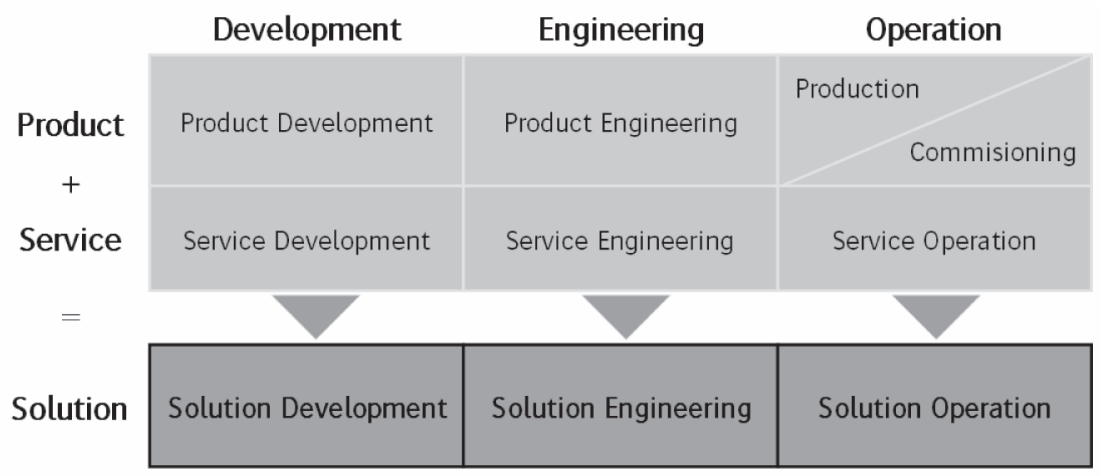

Fig. 3: Solution Lifecycle Management

In order to enhance PLM systems towards service support, the following topics along the lifecycle are considered of specific research interest:

1.) Service Development

- Service classification

- Integrated models of a product-service mix

- Service composition / service sets / service bundles

- Tools for an integrated design of modular product-service portfolios

- Spatial anchoring of product-centric services

- Information visualization of services in the spatial context of machines/ plants/buildings

2.) Service Engineering

- Customer-individual configuration of modular product-service solutions

- Interaction design for service configuration

- Knowledge-driven automation for layouting customer-individual products and services

- Enhancing price calculations towards life-cycle costing (LCC)

3.) Service Operation

- Extending installed base data with service and LCC information

- Supporting service fulfilment with graphical service visualization

- Data handover from PLM engineering back-end to mobile users and operators/owners

- Managing repair/overhaul/retrofit as re-configuration using a unified knowledge base

\section{Product, Service and Project Configuration}

\subsection{Solution Configuration}

It is difficult for customers to specify configurable products and services, particu- 
larly when preferences and priorities are unclear and change continually during the process of explorative solution finding. Therefore, manufacturers have to communicate their modular product portfolio in an intuitive way and should provide consultative help to their customers.

Configuration knowledge will be necessary in every phase of the lifecycle. It is not sufficient that elaborated parametric CAD models in the development department record the design intent on flexibility and modularity. If that product knowledge does not reach the sales man, project engineer, customer or service engineer, a large part of its potential benefit is not used. To take into account a set of well-defined restrictions on how the customer, sales or service engineer can combine product and service components, product configurators [Riitahuhta/Pulkkinen, 2001] are using modelling techniques such as declarative product descriptions, compatibility matrices, constraint solving, and rule-based systems. Machine and plant configuration in the context of custom-specific solution finding compromises four steps often run through iteratively: requirements configuration, product configuration (of physical parts), service configuration, and project configuration. It might be sufficient to clarify only some configuration steps together with the customer and subsequent steps are done by inhouse specialists. But customers are more and more demanding full-fledged envolvement in the solution specification with detailed decision-makings.

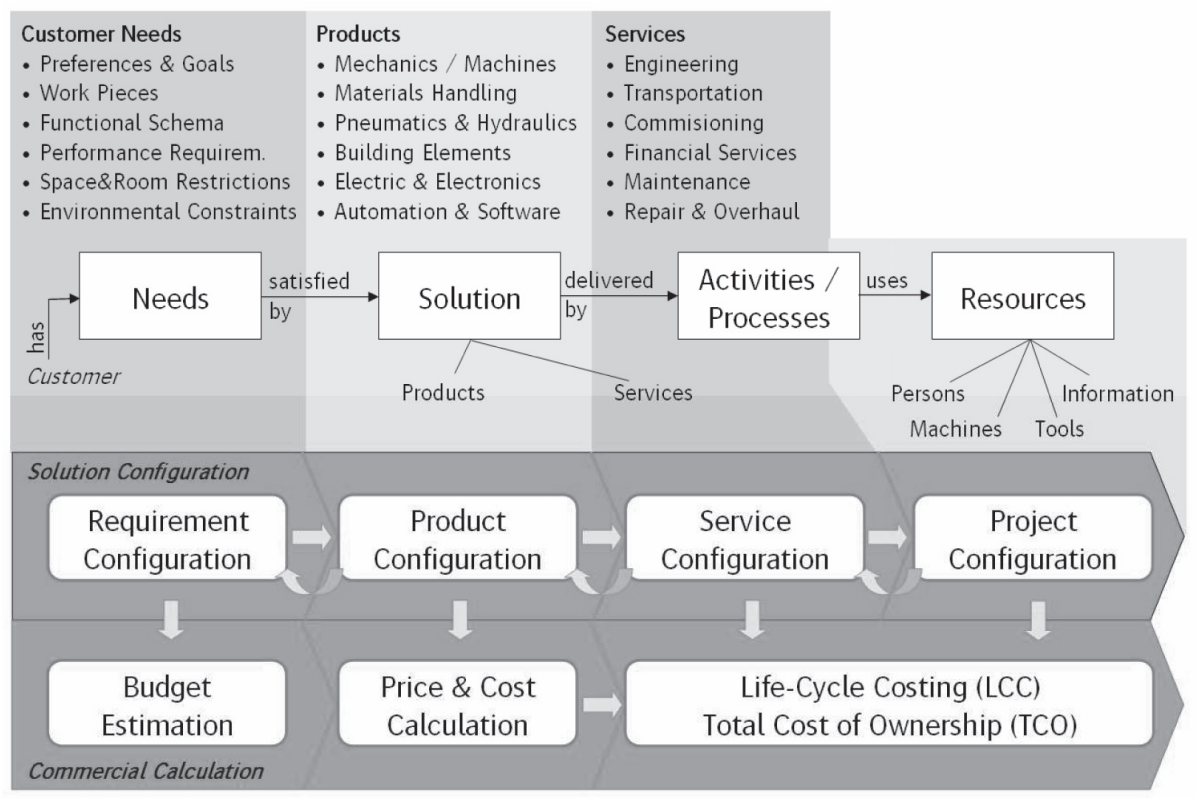

Fig. 4: Concept for Solution Configuration

Beside technical features and functions, the economical dimension is relevant in the layouting of customer-specific solutions. Customers demand decision support 
not only reflecting procurement and construction costs of goods, but considering operational costs (energy efficiency, service charges, labor costs of operators, maintenance \& repair costs, down-time costs, ...). The solution configuration has therefore to go along with a concurrent calculation of commercial effects on the base of simulation data to predict Total Cost of Ownership (TCO) reflecting the whole lifecycle.

\subsection{Product-centric After-sales Services}

While pre-sales and sales activities are typically not explicitly charged, after-sales services for the operating phase are subject to negotiate between tenderer and buyer. Therefore, operational services have to be planned carefully in order to calculate accurate total cost of ownership and to reach a common understanding on Service Level Agreements (SLA). Common types of productcentric after-sales services are:

- Transport

- Commissioning

- Supply of operating resources

- Monitoring

- Calibration

- Cleaning

- Disposal (of waste)

- Inspection

- Repair

- Replacement

- Retrofit

After-sales services are heavily dependent on physical product attributes (size, weight, position, material, ...) and service requirements are already defined in the early design phase of the product. It is desirable to model service needs as selfcontained elements into the product structure during the product definition. Service requirements may then become part of reusable product modules and "Design for Service" aspects may be considered straightforward in application engineering processes.

\section{3 "Pluggable" Services}

An important aspect in supporting the definition of services within the product structure, is the separation of "service needs" and "service fulfilments". Service needs can be defined by the product developer in the design phase, the fulfilment of service needs will be determined by application engineers and by customers according to a chosen service strategy (quality level, collaboration and outsourcing scenario, on-site/online compliance, ...). 
The characteristics of Service Need classes are:

1.) Temporal attributes

- Occurrence: periodic (planned), case-based (unplanned)

- Interval: planned period, mean time between failure (MTBF)/mean time to repair (MTTR)

\section{2.) Spatial attributes}

- Dimension: service spot, service route, service area, service volume

- Position: absolute, relative to floor, relative to housing, ...

- Access: unrestricted, after shutdown, after disassembling, outside, inside, ...

By incorporating Service Needs into the product structure, parametric propagation of part attributes may align services to product options. Examples of variant-dependent services influenced by spatial-geometrical product features are a) inspection depending on route length of a cable, b) cleaning depending on the affected area, and c) disposal of waste depending on tank volume. Reusable modules that are managed in the configurator's catalogue include physical parts together with their service needs in an integrated product structure (Fig. 5 on the left).

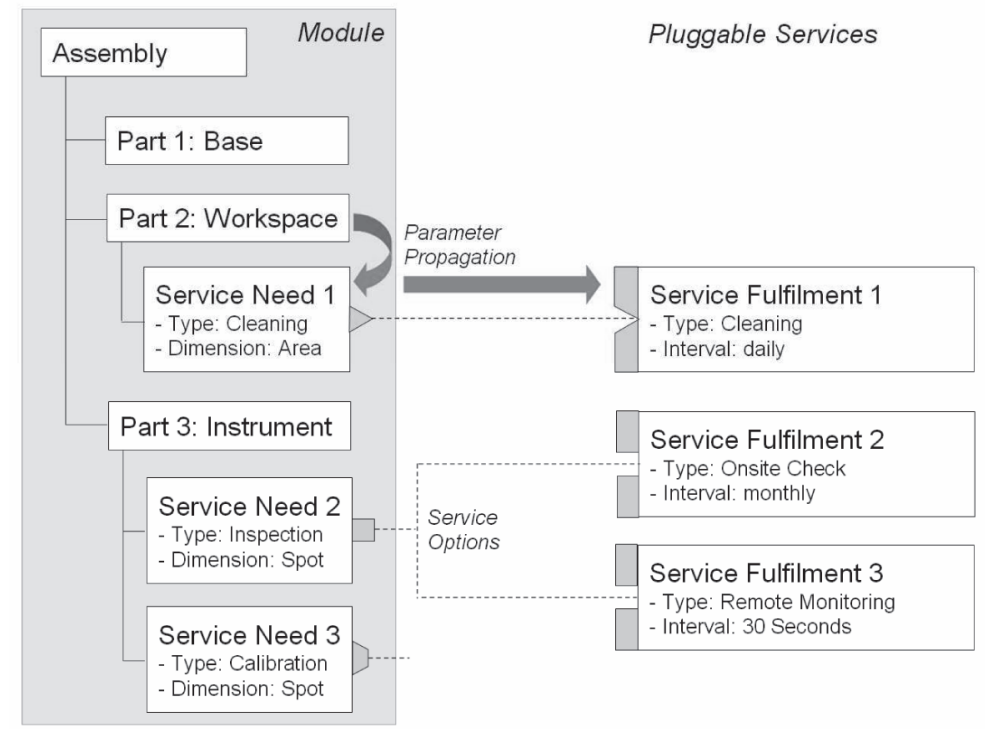

Fig. 5: Configuration as composition of Service Fulfilments to predefined Service Needs

The configuration of services is handled as a composition of service fulfilments to the predefined service needs of all product modules that are layouted in a customspecific project. The Service Needs will propagate parameters to the Service Fulfilment elements, where required resources (man hours, material, tools) and the corresponding costs are calculated. 


\subsection{Knowledge-driven Automation for Service Configuration}

By using rule-based configuration and calculation logic, the following tasks can be automated:

1.) Automatic generation of services

- Generate and compose service fulfilments to service needs

- Choose service options according to a service strategy / to customer preferences

- Calculate services on the base of module compositions (e.g., installation services considering needed boltings and weldings for on-site commissioning)

2.) Automatic aggregation of service bundles

- Per service type/category: all cleaning services, all maintenance services, ...

- Per spatial arrangement: per machine, per room, per access (e.g. outside building), ...

- Per tempori: weekly periodic services, unplanned services, ...

- Per responsibility: manufacturer, operator, user, contractor

3.) Validate services

- Find missing but needed services

- Find inconsistencies, e.g. an overlapping mix of on-site and online services

- Find optimization potentials, e.g. quality improvements or cost savings due to remote services

\section{4.) Automatic Cost Calculation}

- Calculate resources and corresponding costs for service fulfilment

- Simulate unplanned services (MTTR) and their cost impacts

- Map costs to life-cycle phases

The configuration results include the product elements listed as Bill of Material (BOM), defined services documented in a Service Level Agreement (SLA), and an illustration of the Life-Cyle Costing (LCC).

\subsection{Service Visualization}

Thanks to the integration of services into the product structure, service elements have a spatial reference to the physical parts. If the product parts provide $3 \mathrm{D}$ representations, the service visualization can be displayed in the context of the virtual 3D model as well. Services may be displayed as graphical enhancements (colors, outlines) of the affected service dimension (spot, route, area, volume) and as 3D symbols depicting the service type (Fig. 6). This provides userfriendly communication of complex product-service models by an intuitive visual access via a 3D graphical user interface (Fig. 6). 


\section{Conclusions and Outlook}

The described concepts of service configuration were developed in projects covering industries such as machine construction, plant engineering, electrical engineering, and furniture equipment. The experiences and best outcomes were elaborated into the P'X5 software platform

[Perspectix].

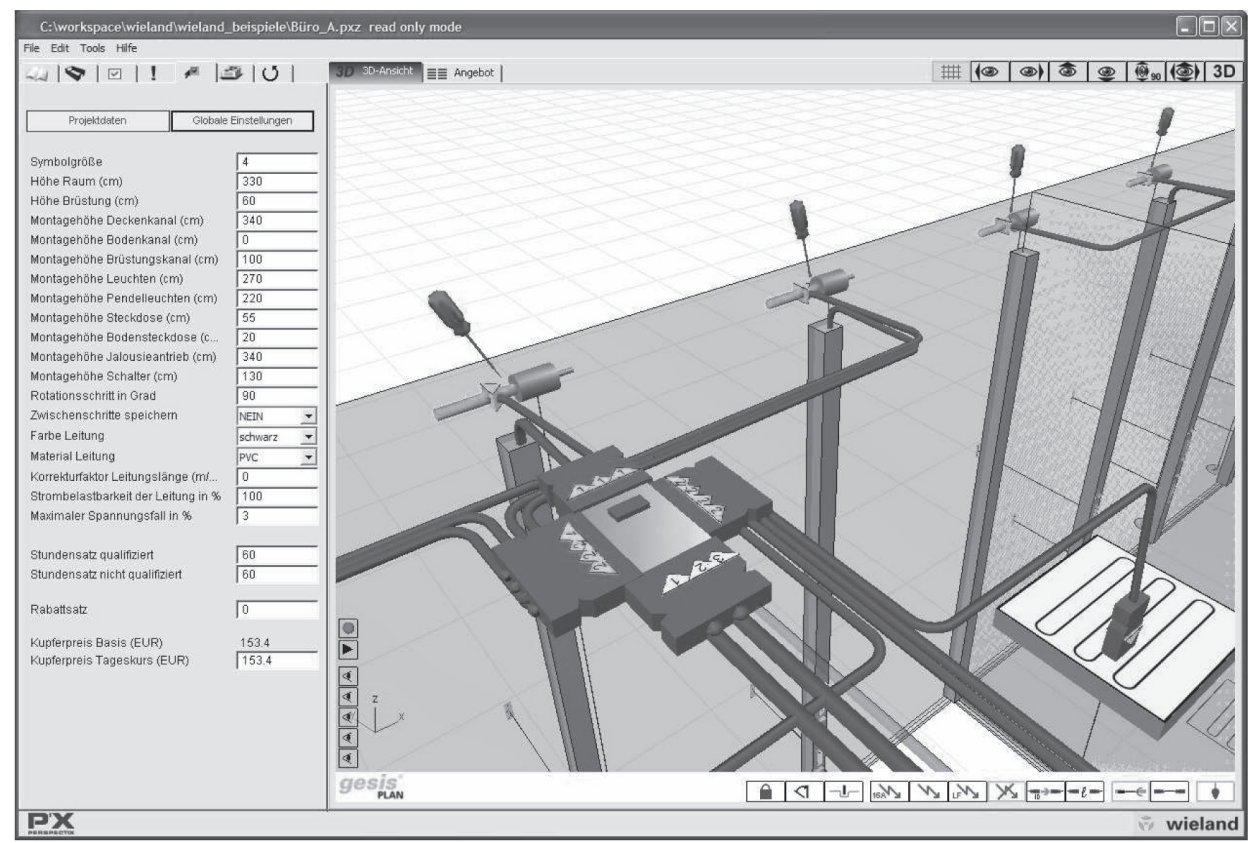

Fig. 6: Visualization of installation services as symbols in 3D

The P'X5 configurator uses a declarative product knowledge base that consists of object-oriented classifications of product characteristics, components, geometric interfaces and assemblies [Ackermann/Eichelberg, 2004]. These classified product entities are enriched by knowledgedriven automatisms based on technical and commercial calculations with constraint solving and rule-based conditions. The modelling of service aspects into the product knowledge base has demonstrated that an integrated configuration of goods and services will foster a more optimal solution finding between different stake holders and between different design dimensions such as functional fitness, technical feasibility, economical as well as ecological efficiency. Currently the ideas are more detailed and applied to the building industry within the EU research project "Industrialised, Integrated, Intelligent Construction" [I3CON]. 


\section{References}

Stark, J.: Product Lifecycle Management -21st Century Paradigm for Product Realisation, London, Springer Verlag, 2005.

Bullinger, H.-J. / Scheer, A.-W., eds.: Service Engineering. Entwicklung und Gestaltung innovativer Dienstleistungen, Berlin, Heidelberg, New York: Springer Verlag, 2003.

Riitahuhta A. / Pulkkinen A., eds.: Design for Configuration, Berlin, Springer Verlag, 2001.

Ackermann, P. / Eichelberg, D.: Product Knowledge Management, in: International Conference on Economic, Technical and Organisational Aspects on Product Configuration Systems, Copenhagen: Technical University of Denmark, 2004.

Perspectix: www.perspectix.com

I3CON: www.i3con.org

\section{Philipp Ackermann}

Philipp Ackermann is co-founder and Managing Director of Perspectix AG, a software company which specialises in product configuration and sales tools for technical industries. He studied Information Technology, Business Administration and Social Ethics at the University of Zurich, from where he also received his doctoral degree.

Perspectix AG, Hardturmstrasse 253, CH-8005 Zurich, Switzerland

email: ackermann@perspectix.com 


\title{
Coupling interactively Product and Project Configuration: a Proposal using Constraints Programming
}

\author{
Authors: \\ Elise VAREILLES, Michel ALDANONDO, \\ Meriem DJEFEL, Paul GABORIT
Ecole des Mines d'Albi-Carmaux - Industrial Engineering laboratory
Campus Jarlard
81013 ALBI CT Cedex 09, France.
\{vareille, aldanond, mdjefel, gaborit\}@enstimac.fr

\begin{abstract}
This communication is a prospective study which looks at the possibility of coupling together interactive aiding design tools relevant to product and project design, in order to propagate decisions made from one to the other. This project involves ten academic laboratories and industrialists. Its purpose is the development of an open source software able to help industrialists to simultaneously design a product and its associated project. Indeed, an analysis of the current practices in the aeronautic, space and transport sectors highlights the need to bring closer together the two processes of product design and project organization, on a methodological level but also by means of dedicated tools, in order to obtain a higher consistency in the decisions and hence better efficiency.

As we are at the initial state of the project, this communication limits product design to product configuration (extreme routine design) and project design to production activities configuration (manufacturing activities). An example, which illustrates our proposition, considers the configuration of a sailing boat respecting the international one-meter-class rule (http://www.iomclass.org/class-rules) and of its project of realization.
\end{abstract}

\section{Keywords}

Configuration, Constraint satisfaction problem, Filtering technique, Project planning

\section{Introduction}

The aim of this communication is to present a prospective study about the development of a configuration system that will simultaneously allow interactive configuration of product and project organization. This problem originates from a French project called ATLAS whose purpose is the development of an open source software 
able to help industrialists to design a product and its associated project simultaneously, by passing decisions made from one to the other.

\subsection{The Origin of our Study}

A large number of big companies in the aeronautic, space and transport sectors are located in the South-West region of France. The products provided by these companies are highly complex and must be reliable. In the actual context of market competitivity, the control and optimization of design processes are necessary in order to improve the diversity, performance and quality of such products and in the same time to shorten their development time and decrease their cost. This phenomenon involves a constant development and renewal of methodologies and procedures, in order to obtain a better-organized contribution of all the available information and the optimal satisfaction of the requirements defined in terms of delays, economy, safety and quality constraints [1].

The instrumentation of the activity of design was, in a first phase, focused on the product by integrating collaborative processes, for example in the paradigm of concurrent engineering. A certain maturity in this domain having been reached, we think that it is the interaction between product design and project organization, which must now be studied and optimized. Indeed, product and project are often treated either in an independent way (which causes inconsistencies) or in a sequential way (which causes additional delays). An analysis of the current practices in these domains highlights the need to bring closer together the two processes of product design and project organization, on a methodological level but also by means of dedicated tools, in order to obtain more coherence in the decisions and hence better efficiency.

\subsection{Solution Investigation}

According to [2] and [3], product design can be characterized with respect to a degree of recurrence in: creative, innovative and routine design. In the case of extreme routine design called configuration, all design possibilities can be investigated completely and all the tasks can be identified. Many authors such as [4], [5] have shown that product configuration could be efficiently modelled and aided when considered as a CSP (Constraints Satisfaction Problem). In the same way, many authors such as [6], [7] have shown that project planning could be modelled and aided when considered as a temporal CSP. Some studies have already started considering the coupling together of these aspects with constraints [8].

A CSP is a triplet $\{\mathrm{X}, \mathrm{D}, \mathrm{C}\}$ where $\mathrm{X}$ is a set of variables, $\mathrm{D}$ is a set of finite domains (one for each variable) and $\mathrm{C}$ a set of constraints linking the variables [9]. The variables can be either discrete or continuous. The constraints can either be of compatibility, when defining the possible or forbidden combinations of values for a set of variables (lists of compatible values, mathematical expressions, temporal 
relations), or of activity, when allowing the activation of a subset of variables and constraints [10].

As we are at the initial state of the project, the example, which illustrates our proposition, is the configuration of a sailing boat respecting the international onemeter-class rule (http://www.iomclass.org/class-rules) and of its project of realization. This real example is used in a preliminary validation of our approach. The resulting configuration model is composed of two interconnected parts: the first corresponds to the design of the sailing boat and the second to the design of its realization project.

\subsection{Organization or the Paper}

The second section presents the configuration model of a sailing boat. This model is described as a CSP and outlines the kind of product configuration variables and constraints necessary to make it. The model assembles different constraint approaches: discrete CSP, continuous CSP and dynamic or conditional CSP, in order to represent the permissible or forbidden combinations of parameter values and to modulate the existence of some configuration parameters.

The third section presents the configuration model of the realization project seen as a CCSP and the kind of constraints needed to make it. The same set of constraint approaches is used to represent tasks. Each task is described by a set of parameters such as possible starting times, finish times and durations defined with intervals. The tasks are linked with anteriority constraints and logical connectors, such as $X O R$ and $A N D$ in order to express the different paths and choose among them the one to follow. This set of CSP can be extended with TemporalCSP and, Conditional Composite and Temporal CSP.

The last section presents the coupling of the previous models and some ideas on the filtering engine, which is able to propagate choices made from one model to the other. Being based on constraints, which do not have propagating directions, the proposed configuration system can therefore be used in two operating modes. The first, named Product Configuration consists in interactively inputting some restrictions on the product variables in order to configure a product and to propagate the consequences on the tasks necessary to realize it and relevant parameter values. This mode allows the behaviour: "What is going to become my realization planning if I select this configuration option ?". The second one, named Product Configuration consists in inputting restrictions only on non-negotiable product variables and thresholds on the parameters of the tasks (possible starting times, finish times, resource selection, duration, etc) in order to propagate the consequences on negotiable product variables. The behaviour would be: "What will be my product options if I want my product in 3 weeks without subcontracting ?". These two operating modes can, of course, be mixed in order to pass on the decisions made independently in product and project design and are illustrated by the previous examples. 


\section{Product Model}

The aim of this section is to present the product configuration part of the sailing boat model. The model is described as a CSP and outlines the kind of variables and constraints necessary to make it.

\subsection{Sailing Boat Configuration}

Our example is a model of sailing boat respecting the international one-meter-class rule (http://www.iomclass.org/ class-rules) as shown in Fig. 1. Around 50 parameters have been identified by experts and characterize the hull, the sails (the mainsail and the headsail), the hull appendages (the keel composed of a fin and a bulb, and the rudder) and the mast.

Let us focus on a subset of parameters of the hull, the fin and the weight of the complete sailboat in order to illustrate our propositions. The hull is characterized by a geometry (u-shape, v-shape, wide u-shape, wide v-shape), a weight (in grams, notated g), a material (wood or fibreglass) and a finish (varnished, painted, coated and painted). The fin is characterized by a geometry (narrow or large) and a weight (in grams). Two kinds of constraints exist and reduce the configuration possibilities:

- the first are due to the design of the sailing boat itself. For instance, it is obvious that, for hydrodynamic reasons, a v-shaped or a wide v-shaped hull would not be compatible with a large fin and that the weight of the hull is different according to its shape.

- the second are due to the respect of the international one meter- class rule. For instance, in order to take part in competitions, the weight of the keel must be between $2200 \mathrm{~g}$ and $2500 \mathrm{~g}$ and the global weight of the sailing boat must be superior or equal to $4000 \mathrm{~g}$. If the global weight is under this limit, the sailboat must be weighted in order to reach it.

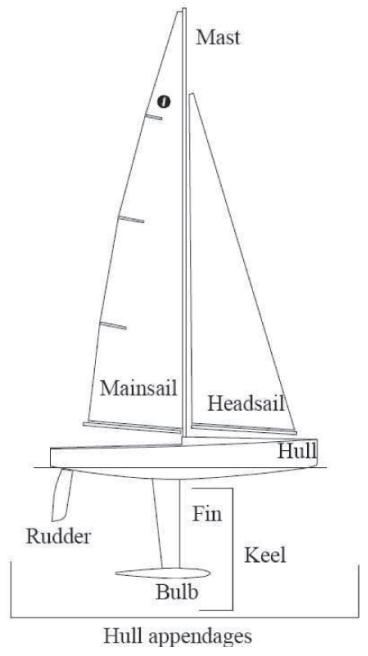

Figure 1: Sailing boat example 


\subsection{Sailing Boat Configuration as a CSP}

Translating this configuration model into a CSP means that each product parameter is associated with a configuration variable and each parameter value corresponds to one value of the variable. For instance, in our example, 8 variables must be defined:

- a variable, called Geo-H, corresponding to the geometry of the hull, whose domain is discrete and corresponds to \{u-shape, $v$-shape, wide $u$-shape, wide $v$ shape\},

- a variable, called Mat- $H$, corresponding to the material of the hull, whose domain is discrete and corresponds to \{wood, fiberglass, FaS \}, with FaS for From a Supplier,

- a variable, called Finish- $H$, corresponding to the finish of the hull, whose domain is discrete and corresponds to $\{V, P, C$ and $P\}$, with $V$ for varnished, $P$ for painted and $C$ and $P$, for coated and painted,

- a variable, called Weight- $H$, corresponding to the hull weight, whose domain is continuous and greater than $0 \mathrm{~g}$,

- a variable, called Geo-F, corresponding to the geometry of the fin, whose domain is discrete and corresponds to \{narrow, large\},

- a variable, called Weight-F, corresponding to the fin weight, whose domain is continuous and greater than $0 \mathrm{~g}$,

- a variable, called Weight-Bo, corresponding to the global weight of the boat, whose domain is continuous and greater than $0 \mathrm{~g}$,

- a variable, called Weight-Ba, corresponding to the ballast weight, whose domain is continuous and greater than $0 \mathrm{~g}$.

In order to structure our model, we can group the variables in sets, relevant to the different parts of the sailing boat. The model has four main sets of variables corresponding to: (i) the hull G-Hull, (ii) the rigs G-Rigs composed itself of two sub-sets corresponding firstly to the sails G-Sails and secondly to the mast G-Mast and (iii) the hull appendages $G$-Hull-Appendages composed itself of two sub-sets corresponding to the rudder G-Rudder and to the keel G-Keel, the keel itself being composed of two sub-sets corresponding to the fin G-fin and the bulb G-Bulb. The last is the sailing boat itself $G$-sailing Boat encompassing all the other groups. For reasons of clarity, only a part of the following groups are shown in Fig. 2: G-Sailing Boat, GHull, G-Hull-Appendages, G-Keel and G-Fin (next page).

Compatibility constraints represent the permissible (solid lines in Fig. 2) or forbidden (dot lines in Fig. 2) combinations of parameter values. These kinds of constraints can be described as tables of permissible values or as mathematical expressions. For instance, the compatibility constraint between the geometries of the hull and the fin is described in table 1. 


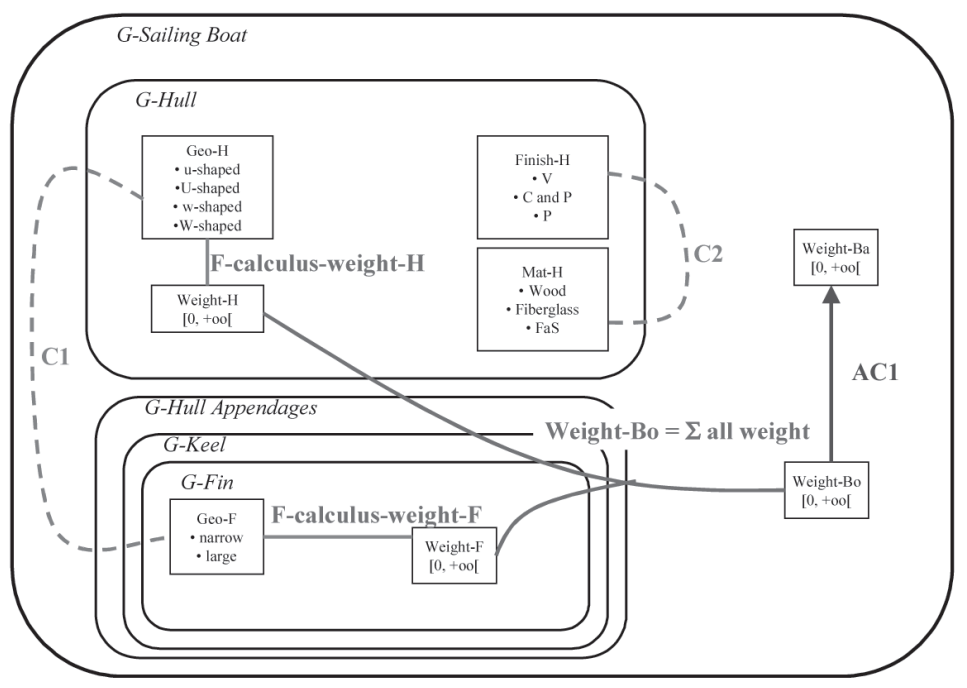

Figure 2: Sailing boat CSP Model

\begin{tabular}{|c|c|}
\hline Geo-H & Geo-F \\
\hline v-shaped & $\neq$ large \\
\hline wide v-shape & $\neq$ large \\
\hline u-shape, wide u-shape & large, narrow \\
\hline
\end{tabular}

Table 1: Example of Design Compatibility Constraint

The weights of the hull and the fin depend on their geometries and are computed by the functions F-calculus-weight-H and F-calculus-weight-F respectively. The global weight of the boat can be defined as follows:

$$
\text { Weight }- \text { Bo }=\text { Weight }-\mathrm{H}+\text { Weight }-\mathrm{F}+\sum \text { all other Weights }
$$

In order to modulate the existence of some parameters, it is necessary to be able to express that some variables have their existence conditioned. These kinds of constraints, called activity constraints, have been introduced by [10] in Dynamic CSP. These constraints allow the control of the existence of variables in the following way: if a variable $X$ has a particular value ' $\mathrm{X}$ ' then another variable $Y$ has to be taken into account in the configuration solution: $X={ }^{\prime} X ' \rightarrow$ Y exists (arrow in Fig. 2). Then, two disjunctive sets of variables are defined in the model: the initial set of active variables (variables that exist always in any solution) and the set of inactive variables (variables that do not exist in all the solutions). For instance, an activity 
constraint, named AC1, controls the existence of the ballast (additional weight), which is inactive at the beginning of the configuration:

$$
\text { AC1 : Weight }- \text { Bo }<4000 \rightarrow \text { Weight }- \text { Ba exists }
$$

\section{Project Model}

The aim of this section is to present the project configuration part of the sailboat model. The model is described as a CSP and outlines the kind of variables and constraints that are necessary to build it.

\subsection{Project Configuration}

Around 120 tasks have been identified in order to realize a sailing boat respecting the one-meter-class rule and correspond to the realization of the hull, the sails (the mainsail and the headsail), the hull appendages (the keel composed of the fin and the bulb, and the rudder) and the mast.

Each task is characterized by three intervals: the first corresponds to the possible starting times (earliest and latest start times), the second to the possible finish times (earliest and latest finish times), the last to the possible length of time (optimistic and pessimistic lengths of time). In our example, the resources will not be considered.

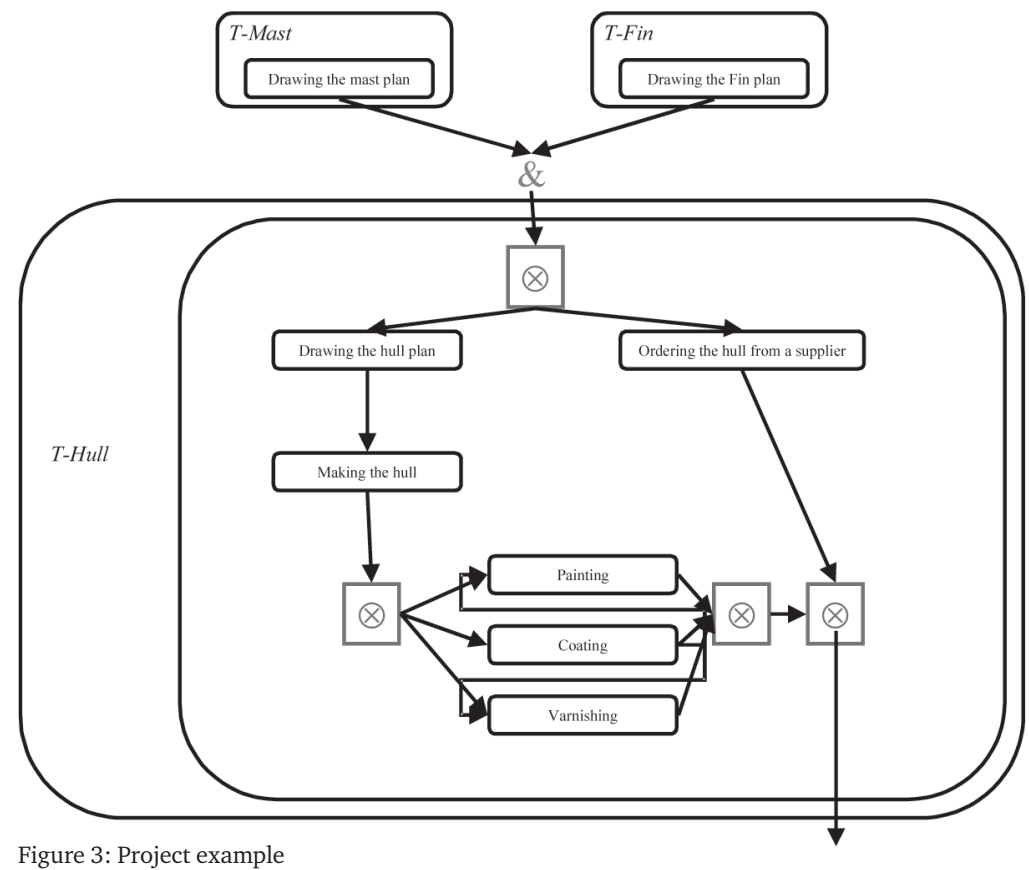


Let us focus on a subset of tasks corresponding to the hull, as shown in Fig. 3. The hull can either be ordered from a supplier or homemade. In order to make a hull, we have first to draw its plan, then to make the hull itself in a material and to make its finish (varnished, coated and/or painted).

Two kinds of constraints exist and reduce the configuration possibilities:

- the first are due to the making of the sailing boat itself. For instance, the drawings of the plans of the fin and the mast must have been made before drawing the plan of the hull because of the interface between these components.

- the second are due to the choices made by the user. For instance, if the hull is homemade, different tasks must be carried out, such as drawing the plan and making the hull, and some tasks can be carried out, such as varnishing, coating and painting.

\subsection{Project Configuration as a CSP}

Translating this project configuration model into a CSP means that each task could be associated with a set of three continuous variables representing the possible start times called pst, finish times called $p f t$ and duration called pdt. For each task, these three variables could be linked by a numerical constraint, called Calcul-dates, which tells us that the possible finish time equals the possible start time plus the possible length of time: $p f t=p s t+p d t$. In the rest of the paper, we have noted the access to one of these variables pst, pft and pdt of the tasks T: T-pst, T-pft and T-pdt.

For instance, in our example, 8 tasks must be defined:

- a task corresponding to the design of the mast, called Drawing the mast plan, with pst $=[0,5]$, pet $=[1,7]$ and $p d t=[1,2]$ (notated $([0,5],[1,7],[1,2])$ in the rest of the paper).

- a task corresponding to the design of the fin, called Drawing the fin plan, with $([0,10],[2,13],[2,3])$,

- a task corresponding to the design of the hull, called Drawing the hull plan, with $([2,7],[4,15],\{0,[2,8]\})$,

- a task corresponding to the supplying of the hull, called Ordering the hull from a supplier, with ([2, 7], [17, 32], \{0, [15, 25]\}),

- a task called Making the hull, with ([4, 15], [6, 19], \{0, [2, 4]\}),

- a task called Varnishing, with ([6, 22], [7, 25], \{0, [1, 3]\}),

-a task called Coating, with ([6, 19], [8, 22], \{0, [2, 3]\}),

-a task called Painting, with ([6, 22], [7, 25], \{0, [1, 3]\}),

In order to structure our model, we can group the tasks in sets relevant to the different tasks of the project. The model has four main sets of tasks corresponding to the groups already identified in the sailboat configuration model (sub section 2.2): 
(i) the hull T-Hull, (ii) the rigs T-Rigs composed itself of two sub-sets corresponding firstly to the sails T-Sails and secondly to the mast T-Mast and (iii) the hull appendages T-Hull-appendages composed itself of two sub-sets corresponding to the rudder T-rudder and to the keel T-Keel, the keel itself being composed of two sub-sets corresponding to the fin T-Fin and the bulb T-Bulb. The last corresponds to the sailboat TSailing Boat and encompasses all the other sets. For reasons of clarity, only T-Mast, T-Fin and T-Hull are shown in Fig. 4.

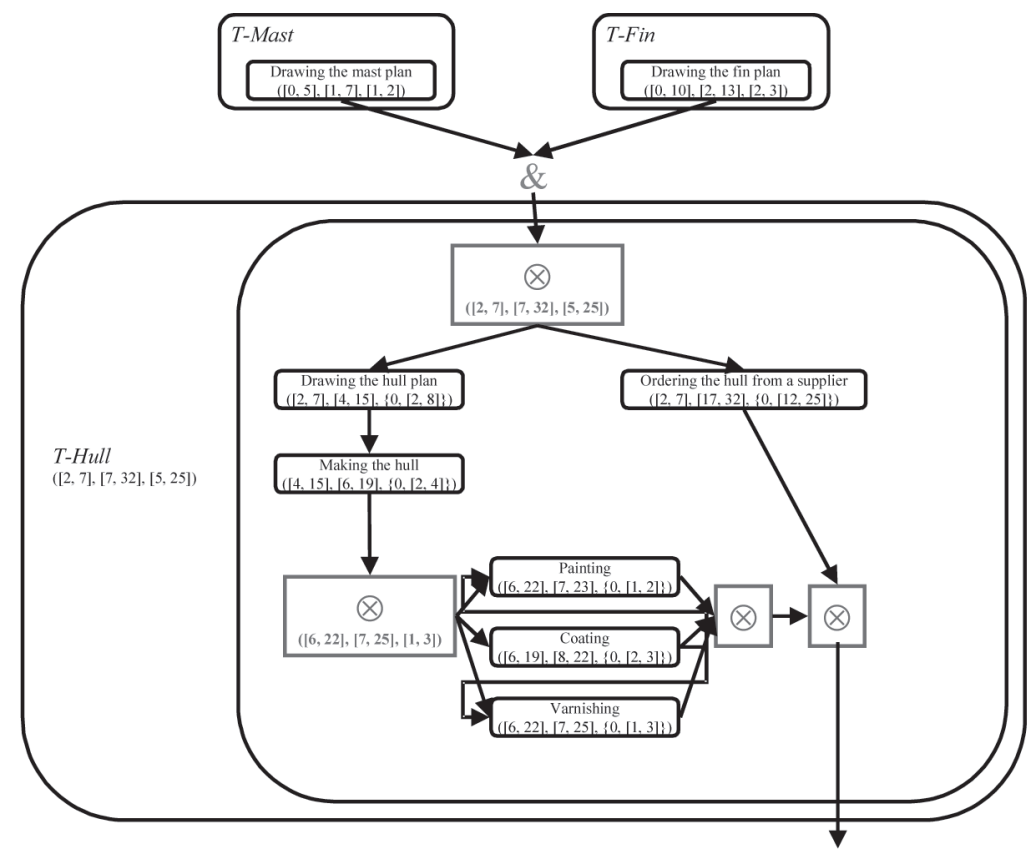

Figure 4: Project CSP Model

Each of these sets of tasks $S$ has a possible start time S-pst, a possible finish time S-psf and a possible length of time S-pdt. Each set is linked with its sub-tasks by numerical constraints.

Compatibility constraints represent temporal relations between tasks expressed as continuous constraints on their pst, pft and pdt. These kinds of constraints have certain similarities to the Allen's primitives [11] (before, after, starts, finishes) but they can be applied to intervals of start and finish times. For instance, the fact that we need the plans of the fin and of the mast before designing the hull can be described by this pair of numerical constraints:

Drawing the hull plan-pst $\geq$ Drawing the mast plan-pft

Drawing the hull plan-pst $\geq$ Drawing the fin plan-pft. 


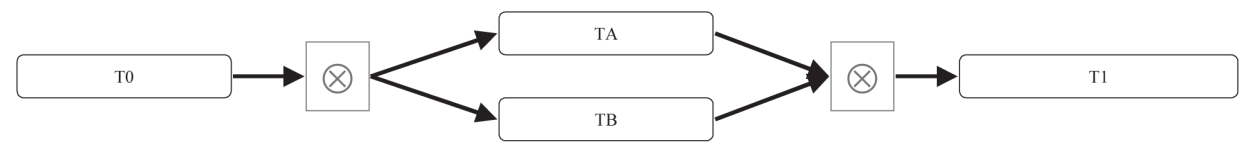

Figure 5: XOR example

In our example, each arrow in Fig. 4 corresponds to a temporal relation expressed as continuous constraint. In order to modulate the existence of some tasks or sets of tasks, it is necessary to be able to express that some of them have their existence conditioned. Some studies have already proposed solutions called Conditional and Composite Temporal Constraint Satisfaction Problems or CCTCSP [12], which are based on the Dynamic CSP, proposed by [10]. But, CCTCSP does not allow the tasks (represented by events) to have an interval for their length of time. We have therefore defined a meta-task entity XOR that assembles two tasks that are linked with a XOR node. Assume that we have a process TO then TA or TB then T1 (as in Fig. 5).

In order to modulate the existence of $T A$ and $T B$ we add:

- the single numerical value $O$ in the definition domain of the possible duration of $T A$ and $T B$, meaning that the task has not to be taken into account in the solution,

- a constraint that excludes two null values or two non-null values for the duration of $T A$ and $T B$, meaning that a path has to be selected. The possible combinations are therefore expressed in table 2 :

\begin{tabular}{|c|c|}
\hline$T A-p d t$ & $T B-p d t$ \\
\hline$=0$ & $\neq 0$ \\
\hline$\neq 0$ & $=0$ \\
\hline
\end{tabular}

Table 2 : Constraint expressing the selection of the task TA or TB

The possible starting and finishing times of the $X O R \_A B$ meta task assembling $T A$ and TB, XOR_AB.pst and XOR_AB.pft, are constrained by:

- XOR_AB.pst > TO.pft meta task starts after TO

-XOR_AB.pst $\leq$ TA.pst meta task starts before TA

-XOR_AB.pst $\leq$ TB.pst meta task starts before TB

- TA.pft $\leq X O R \_A B . p f t$ meta task finishes after TA

- TB.pft $\leq X O R \_A B . p f t$ meta task finishes after TB

- T1.pst > XOR_AB.pft meta task finishes before T1

The possible duration of the $X O R \_A B$ meta task is constrained by:

- XOR_AB.pdt $=$ TA.pdt U TB.pdt, expressing that the possible duration of the 
meta task equals the union of the possible duration of tasks $A$ and $B$

- XOR_AB.pdt $>0$, expressing that the possible duration of the meta task is always strictly positive (a path has to be selected either TA or TB).

That's why a $O$ was added to the $p d t$ domain of the tasks Drawing the hull plan, Ordering the hull from a supplier, Painting, Coating and Varnishing.

\section{Coupling the Models Together}

The aim of this section is to present the connection between the sailing boat design model and its project of realization model. The way these two aspects can be coupled together with constraints, is described in the first subsection. In the second, the different ways in which such a configuration tool can be used, are presented in order to pass decisions made from one to the other.

\subsection{Coupling with Constraints}

Around thirty constraints between the two previous models have already been identified. Two kinds of constraints exist and reduce the configuration possibilities:

- the first are due to the feasibility and technical aspects. For instance, a relationship exists between the material selected for the hull and the length of time needed to make it. The making of a hull in fiberglass takes much longer than in wood. It takes between 3 or 4 units of time instead of 2 or 3, depending on who is making it.

- the second are due to the choices made to design the boat or the project. For instance, if a user

wants his hull to be coated and painted, the tasks corresponding to these choices Coating and Painting must be activated and the task T-Hull must be updated.

Let us translate these two constraints into our model:

- the compatibility constraint between the material of the hull Mat-H described in section 2.2 and the time needed to make the hull, pdt of the task Making the hull described in section 3.2, is described in table 3.

\begin{tabular}{|c|c|}
\hline Mat-H & Making the hull-pdt \\
\hline Wood & {$[2,3]$} \\
\hline Fiberglass & ] $3,4]$ \\
\hline
\end{tabular}

Table 3: Example of Coupling Compatible Constraint

- the activation of the tasks Coating and Painting by the variable Finish- $H$ described in section 2.2 could be described as follows: 
Finish- $H=$ coating and painting $\rightarrow$ Coating-pdt $\neq 0$, Painting-pdt $\neq 0$ and Varnishing-pdt $=0$

\subsection{Running Modes}

The interactive aiding design consists in giving a value to or limiting the domain of a variable v belonging either to the product or project model. The modification of the domain of $\mathrm{v}$ is propagated through the constraints network to the other variables by retrieving all the values from their domain, which are now inconsistent with the reduced domain of $\mathrm{v}$. This mechanism, repeated several times, progressively restricts the solution space in order to reach a solution in product and project designs simultaneously. Aiding design being interactive, we could use the filtering techniques of constraints programming:

- compatibility tables, such as 1 and 3, are filtered by using arc-consistency [13] and [14]

- mathematical formulae are filtered by 2B-consistency [15], based on interval arithmetic [16]. Interval arithmetic extends real arithmetic to intervals by applying the operators of a formula to the endpoints of the intervals of its arguments. For example, if we consider the mathematical formula $\mathrm{f}:(\mathrm{x}, \mathrm{y}) \rightarrow \mathrm{x}+\mathrm{y}$ that adds the variables $\mathrm{x}$ with $\mathrm{Dx}=[\mathrm{x}, \mathrm{x}]$ and $\mathrm{y}$ with $\mathrm{Dy}=[\mathrm{y}, \mathrm{y}]$, the result of $\mathrm{f}$ yields in: $\mathrm{Dx}+\mathrm{y}=\mathrm{Dx} \oplus \mathrm{Dy}=[\mathrm{x}+\mathrm{y}, \mathrm{x}+\mathrm{y}]$

- If we consider the constraint $\mathrm{f}:(\mathrm{x}, \mathrm{y}) 7 \rightarrow \mathrm{x}-\mathrm{y}$ that subtracts the variables $\mathrm{y}$ with $\mathrm{Dy}=[\mathrm{y}, \mathrm{y}]$ to $\mathrm{x}$ with $\mathrm{Dx}=[\mathrm{x}, \mathrm{x}]$, the result of $\mathrm{f}$ yields in: $\mathrm{Dx}-\mathrm{y}=\mathrm{Dx} \Theta \mathrm{Dy}$ $=[\mathrm{x}-\mathrm{y}, \mathrm{x}-\mathrm{y}]$

Being based on constraints, which do not have propagating directions, the general configuration model can therefore be used in two operating modes. The first, named Product configuration consists of interactively inputting some restrictions on the design variables in order to configure a product and to determine the tasks necessary to realize it. The second mode, named Project configuration consists of inputting restrictions on non-negotiable design variables and a threshold on either the $p f t$, pst and/or $p d t$ of the tasks in order to deduce the value of negotiable design variables. These two operating modes are illustrated by the previous examples.

Product Configuration: In the first operating mode, the user restricts, step by step, the domain of the design variables to find a configuration and its project of realization. Let us consider the example presented in subsections 2.2 and 3.2, and focus on the variables corresponding to the hull.

At the beginning, as no choice has been made in the sailboat configuration part, nor in the project configuration part: the time needed to make the hull equals $[5,25]$.

If the user reduces the variable $M a t-H$ to $F a S$, this reduction has a direct impact through the compatibility constraint c2 in Fig. 2 on the finish of the hull Finish- $H$ which is reduced to $C$ and $P$. 
The reduction of the variable Mat- $H$ to FaS has an impact through an activity constraint on the project: the task Ordering the hull from a supplier is selected $\rightarrow$ its duration must be non-null while each of the durations of the tasks corresponding to the homemade hull is null.

The selection of the task Ordering the hull from a supplier has an impact through the constraints Calcul-dates on the task T-Hull. The pst of T-hull stays equal to [2, 7] because the two tasks have the same $p s t$.

The $p d t$ of $T$-hull is reduced to [15, 25] $=$ T-hull-pdt $\cap$ Ordering the hull from a supplier-pdt $=[5,25] \cap[15,25]$, and the $p f t$ of T-hull is reduced to $[17,32]=T$ hull-pdt + T-hull-pft $=[15,25] \cap[2,7]$.

Then, with a hull ordered from a supplier, the user has no influence on the finish of his hull and he will receive it between the 15th and the 25th units of time.

Project Configuration: In the second operating mode, the user can input restrictions on non-negotiable design variables and a threshold on either the pft, pst and/or pdt of the tasks in order to see the impact on the values of design variables.

For instance, let us consider that the user wants his hull finished before or on the 8th unit of time. He reduces the possible finish times of the variable T-Hull to $\leq 8$. The $p f t$ of this task is then reduced to $[7,32] \cap[0,8]=[7,8]$.

This reduction has a direct impact on the task Ordering from a supplier whose $\mathrm{pft}$ is greater than 8: this task cannot be selected and the other path can $\rightarrow$ its duration is valuated to null while each of the durations of the tasks corresponding to the homemade hull is non-null.

The reduction of the T-Hull-pft to [7, 8] is reflected through the other tasks Painting, Coating and Varnishing. The pst, pft and $p d t$ are reduced:

- for the Painting task to:

- pft: $[7,8]=$ Painting-pft $\cap$ T-Hull-pft $=[7,23] \cap[0,8]$.

-pst: $[6,7]=$ (Painting-pft $\cap$ Painting-pdt $) \cap$ Painting -pst $=([7,8] \cap$ $[1,2]) \cap[6,22]=[5,7] \cap[6,22]$.

- pdt: $[2,3]=($ Painting-pft $\cap$ Painting-pst $) \cap$ Painting-pdt $=([7,8] \cap$ $[6,7]) \cap[1,2]=[0,2] \cap[1,2]$.

- for the Coating task to:

- pft: $[8,8]=$ Coating-pft $\cap$ T-Hull-pft $=[8,22] \cap[0,8]$.

- pst: $[6,6]=($ Coating-pft $\cap$ Coating-pdt $) \cap$ Coating-pst $=([8,8] \cap$

$[2,3]) \cap[6,19]=[5,6] \cap[6,19]$.

- pdt: $[2,2]=($ Coating-pft $\cap$ Coating-pst $) \cap$ Coating-pdt $=([8,8] \cap$ $[6,6]) \cap[2,3]=[2,2] \cap[2,3]$.

- and for the Varnishing task to:

-pft: $[7,8]=$ Varnishing-pft $\cap$ T-Hull $\cap$ pft $=[7,25] \cap[0,8]$.

- pst: $[6,7]=($ Varnishing-pft $\cap$ Varnishing-pdt $) \cap$ Varnishing-pst $=$ $([7,8] \Theta[1,3]) \cap[6,22]=[4,7] \cap[6,22]$. 
- pdt: $[1,2]=($ Varnishing-pft $\cap$ Varnishing-pst $) \cap$ Varnishing-pdt $=$ $([7,8] \Theta[6,7]) \cap[1,2]=[0,2] \cap[1,2]$.

In the same way, the reduction of the pst of the three previous tasks has an impact on the task Making the hull whose pft is reduced to [6,7], whose pst is reduced to $[4,5]$ and whose $p d t$ is reduced to $[2,3]$.

In the same way, the reduction of the pst of the task Making the hull has an impact on the task Drawing the the hull plan whose pft is reduced to [4, 5], whose pst is reduced to $[2,3]$ and whose $p d t$ is reduced to $[2,3]$.

The reduction of the $p d t$ of the variable Making the hull has an impact through the constraint presented by table 3 on the variable $M a t-H$ which is reduced to wood.

The reduction of the variable Mat- $H$ to wood has a direct impact through the compatibility constraint c2 in Fig. 2 on the finish of the hull Finish- $H$ which is reduced to $C, V, C$ and $P$.

The fact of wanting the hull ready for the 8th unit of time implies that the hull is homemade, made in wood and either coated, varnished or coated and painted.

\section{Conclusion}

The aim of this communication has been to present a prospective study about the development of a configuration system, based on constraints, that allows interactive configuration of product and project organization simultaneously. Firstly, we have presented our problem and one of the solution investigations. Then, two configuration models, seen as CSP, have been presented: one for the configuration of a sailing boat respecting the one-meter-class rule and the other for the configuration of its project organization. The last section has presented the coupling of the two models thanks to some coupling constraints, in order to pass decisions made from one to the other. As constraints have no propagating directions, two main operating modes have been identified:

The first operating mode, called Product configuration consists in interactively inputting some restrictions onto the design variables in order to configure a product and to determine the tasks necessary to realize it. The second operating mode, called Project configuration consists in inputting a threshold on either the pft, pst and/or pdt of the tasks in order to deduce the set of tasks necessary and the value of the design variables. This operating mode can help the user to see the impact of a choice made in the project part. Of course, these two operating modes can be mixed in order to pass on the decisions made independently in product and project design. This kind of approach will be used in a real-size problem to help users designing their products and their projects simultaneously. A model corresponding to the needs of the industries involved in the national project ATLAS will be available in one and half year. 


\section{Acknowledgement}

The authors would like to thank their partners: Anyware Technologie (Toulouse, France), Pulsar Innovations (Toulouse, France), Sigma Plus (Toulouse, France) LAASCNRS (Toulouse, France), LGP-ENIT (Tarbes, France), IMSLAPS (Bordeaux, France), the French National Research Agency and the 7th Strategic Activity Domain Architecture and Integration of Aerospace Valley for their involvement in this project.

\section{References}

[1] C. Baron, S. Rochet, C. Gutierrez, Proposition of a Methodology for the Management of Innovative Design Projects, International conference on system Engineering (INCOSE 2005), Rochester, NY USA, INCOE Publishers, 2005.

[2] D.C. Brown, B. Chandrasekaran, Expert systems for a class of mechanical design activity, Knowledge Engineering in Computer-Aided Design, North-Holland, pp. 259-282, 1985.

[3] R.D. Coyne, M.A. Rosenman, A.D. Radford, M. Balachandran, KnowledgeBased Design Systems, Addison-Wesley, 1990.

[4] E. Tsang, Foundations of constraints satisfaction, Academic Press, London, 1993.

[5] D. Sabin, F. Freuder, Configuration as Composite Constraint Satisfaction Technical report FS-96-03, Workshop on configuration, AAAI Press, pp.28-36, 1996.

[6] R. Dechter, I. Meiri, J. Pearl, Temporal Constraint Satisfaction Problems, Artificial Intelligence, 49, pp. 61-95, 1991.

[7] F. Focacci, P. Laborie, X. Nuijten, Solving scheduling problems with setup times and alternative resources, 5th International Conference on Artificial Intelligence Panning and Scheduling, pp. 92-101, 2000.

[8] M. Aldanondo, E. Vareilles, C. Baron, Y. Lahmar, Product Development and Project Management: towards a Constraint based Approach International Conference on Industrial Engineering and Systems Management, IESM 07, Beijing, China, Tsinghua University Press, ISBN: 978-7-89486-439-0, 2007.

[9] H. Montanari, Networks of constraints: Fundamental Properties and Application to Picture Processing, Information Sciences, vol. 7, pp. 95-132, 1974.

[10] S. Mittal, B. Falkenheiner, Dynamic Constraint Satisfaction Problems, Proceedings of the 8th National Conference on Artificial Intelligence, AAAI Press, Boston, MA, pp. 25-32, 1990.

[11] J. Allen, Maintening knowledge about temporal intervals, Communication of the CACM, tome 26(11), pp. 832-843, 1983.

[12] M. Mouhoub and A. Sukpan, A New Temporal CSP Framework Handling Composite Variables and Activity Constraints, the 17th IEEE

International Conference on Tools with Artificial Intelligence (ICTAI'05), pp. 143149, Hong-Kong, November 14-16, 2005.

[13] A.K. Mackworth, Consistency in Networks of Relations, Artificial Intelligence, pp. 99-118, 1977. 
[14] B. Faltings, Arc Consistency for Continuous Variables, Artificial Intelligence, pp. 363-376, 1994.

[15] O. Lhomme, Consistency techniques for numeric CSP, International Joint Conference on Artificial Intelligence, pp. 232-238, 1993.

[16] R.E. Moore, Interval Analysis, Prentice-Hall: Englewood Cliffs, NJ, 1966. 


\title{
Issues in the implementation process of tailored logistics services for mass customization
}

\author{
Frank Straube, Stefan Alexander Doch and Kathrin Klaar
}

\begin{abstract}
Tailored logistics services have the potential to greaten customer satisfaction by the provision of customer individual delivery times, reconfiguration options and after-sales-service reaction times. Based on an exploration process, the concept elements for the implementation of tailored logistics services were systematized in the following five subsystems: the Customer Relationship System, the Logistics Service tailoring System, the internal Coordination System, the Logistics Operations System and the Information System. The implementation of logistics systems contains all activities, which support the transformation process to the desirable state as well as the enforcement for sustaining the transformed logistics system. Possible identified issues for tailoring logistics services had been classified in a grid, which contains technological, organizational and social psychological components. This effective procedure allows inducting activities according to the situation and progress of the implementation.
\end{abstract}

\section{Keywords}

Tailored logistics services, tailoring process, implementation process, implementation issues.

\section{Introduction}

One of the mayor questions in mass customization is how to tailor products and services to customer needs in an efficient and effective way. While well known concepts like product modularization and production postponement support an efficient process of product individualization, there is a lack of research as well as practical experience in the customer interface and service individualization aspect of mass customization.

Also in a mass customization environment the differentiation based on products gets more and more complicated. Therefore differentiation strategies based on services build up the new focus area (Woratschek, 2001). Especially tailored logistics services have the potential to greaten customer satisfaction by the provision of customer individual delivery times, reconfiguration options and after-sales-service reaction times. Tailoring logistics services in a Supply Chain Management context can be described as the design of Supply Chains based on customer relations. These 
Supply Chains aim to deliver process efficient logistics services that are focused on specific customer requirements.

Tailored logistics services can have a positive influence on two of the major objectives of mass customization strategies: Offering individualized products and services as well as doing that to competitive costs. Several research projects have shown that suppliers often provide customers with more services than they want or need, at prices that often reflect neither the value of those services to customers nor the cost of providing them (Anderson/Narus, 1995). Companies often do not know what services are core elements of a product and which of them can be offered as customer tailored add-ons, because some of the customers would pay a premium for these services. Result of these misinterpretations is a missing surcharge willingness for additional services, lost-sales due to missing demand flexibility and rising logistics costs that cannot be amortized with a higher customer satisfaction level (Payne/Peters, 2004). All these observations refer to a design of Supply Chains that is based on average customer expectations (Fuller et al., 1993). To overcome this so called Averaging-Effect the tailoring of logistics services on customer preferences is the central concept of interest in this research paper.

The research approach is oriented on a qualitative and applied research process: Based on a brief literature review and personal interviews the state of tailored logistics services was identified and the main conceptual parts of the tailoring process were analyzed and the implementation issues were described. The objective of this research is the development of a design and implementation model for the tailoring of logistics. Therefore major implementation management concepts were adapted to the logistics context. This made it possible to map known implementation management concepts (i.e. workshops, customized training courses, team building activities, agreement on objectives, implementation-controlling) to the identified implementation issues.

In Summary, the research of this paper is oriented on the following research question: How can logistics systems that are oriented on differentiated customer requirements successfully be established to ensure the provision of customized logistics services in simultaneously efficient and effective supply chains?

Individual customer services and specific value-added services enable logistics management to gain sustainable competitive advantages based on a comprehensive differentiation strategy (Janowski, 2004). The concept of service differentiation is based on well known strategic management ideas, especially in the areas of marketing, business strategy, but also in production management or in the product development process. Also for the area of supply chain management, the basic strategic options to tailor logistics processes to customer expectations are already established. However, there is no design concept, neither in research or practice, that integrates the conceptual parts of the tailoring process and the implementation issues in an overall model (Mentzer et al., 2001). 


\section{Conceptual parts of the tailoring process}

The concept of tailored logistics services requires focusing logistics operations on specific goals and customer requirements (Janowski, 2004). Based on an overview over the recent developments in research on focused management approaches in different disciplines, the basic principles of customer focused supply chains for the development of differentiated logistics services can be worked out. The research on tailored logistics services is mainly based on the work of Skinner in the field of production management (Skinner, 1974). He describes the objectives of focused production systems with the terms "[...] simplicity, repetition, experience, and homogeneity of tasks breed competence [...]" (Skinner, 1974). For the concept implementation "[...] each key functional area in manufacturing must have the same objective, derived from corporate strategy. (Skinner, 1974)" The scope of this approach was expanded by Fuller (Fuller et. al., 1993) to the functional area of logistics and by Fisher (Fisher, 1997) to the design of the entire spectrum of supply chain management. Focusing concepts are the basic strategy for the implementation of a tailoring process for logistics services. By that focus of the service operations process it is possible to meet customer requirements and to overcome the already described Average-Effect (Childerhouse et. al., 2002). The main challenge in the implementation of logistical services differentiation concepts is the choice of an optimal level of focus. The location of this point can be influenced by different factors such as the heterogeneity of product characteristics, environmental conditions or customer demand. Figure 1 shows an exemplary focusing cost curve and the trade-off between the degree of focus and the associated focusing costs.

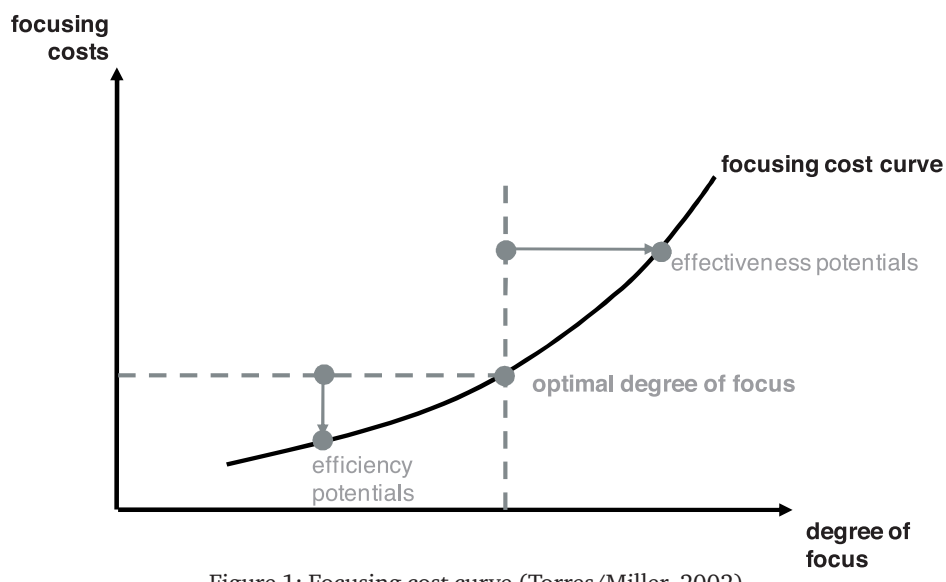

Figure 1: Focusing cost curve (Torres/Miller, 2002)

A high degree of focus allows the efficient fulfillment of specific customer requirements. This objective is analogous to the well-known marketing concept of "segments of one" where in very small customer segments the highly individual customer requirements are met. In contrast, a low degree of focus results in decreasing 
logistics service costs, because specific customer requirements are not taken into consideration. This situation is analogous to the marketing concept of "one-sizefits-all" where the marketing strategy is only oriented on average customers' needs. For Supply Chain Management it is therefore a tension between efficient logistics operations on the one hand and effectively satisfying the customer's needs on the other hand. The effectiveness and efficiency principle is the basic design principle for logistics processes.

A literature review indicated that for building up tailored logistics services a high degree of market knowledge and customer orientation is needed (Bowersox et al., 2000). It is also mentioned that further implementation issues are the lack of metrics for the measurement of the costs and benefits of tailored logistics systems (Pfohl et al., 2006),. Additional barriers in the implementation process refer to internal collaboration problems (i.e. with the sales and marketing department) (Mentzer/Williams, 2001), the higher complexity of logistics processes and missing IT capabilities to adapt information systems to the changing requirements (Kaipia/ Holmström, 2007).

Based on this exploration process, the conceptual elements for the implementation of tailored logistics services were systematized in the following five subsystems (Figure 2): the Customer Relationship System, the Logistics Service tailoring System, the internal Coordination System, the Logistics Operations System and the Information System.

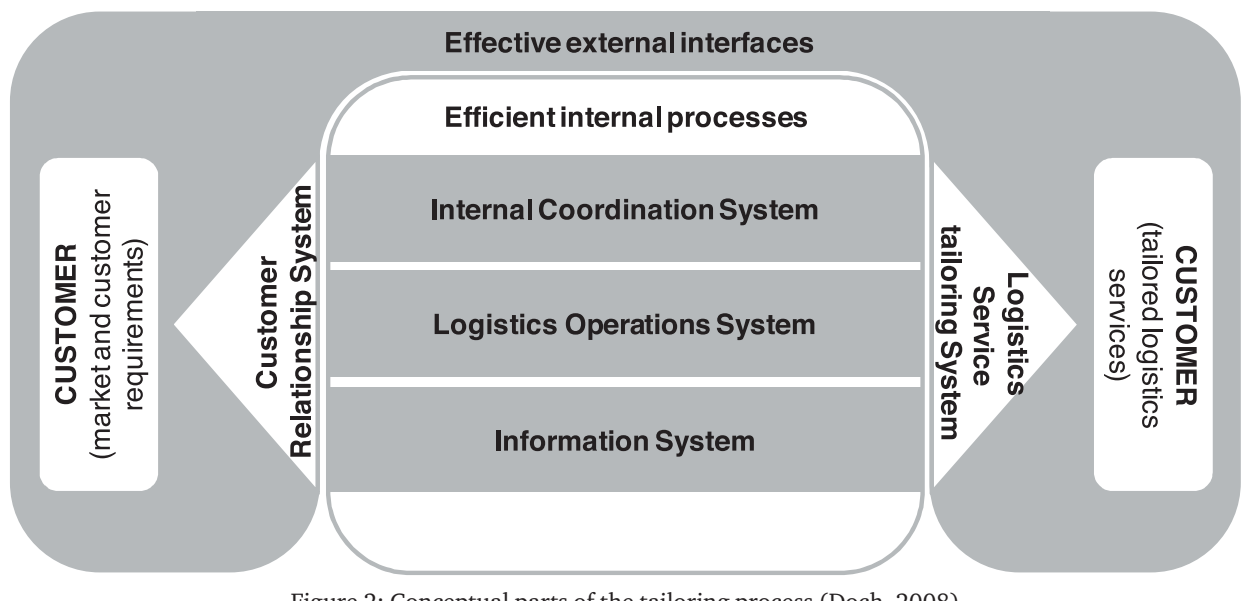

Figure 2: Conceptual parts of the tailoring process (Doch, 2008)

In the design field of the customer relationship system, it should be noted that an integrated customer order process is an essential requirement to realize tailored logistics services. Due to the fact that the customer contact at manufacturing com- 
panies is often constrained by retail structures and the logistics department is often not involved into the customer relationship process, the organizational integration of logistics and sales functions within the company plays a crucial role. This internal coordination requires clearly defined processes and harmonized performance objectives. Furthermore the interrelations between logistics, sales and marketing service components and their impact on the overall customer satisfaction have to be worked out. This allows forming logistical customer segments and selecting the right logistics service criteria to tailor. For the adjustment of the logistics structures and processes to the needs of tailored logistics services, a combination of various logistics concepts and methods is required. Postponement strategies, the modularization of logistical processes or the adaption of the Revenue Management concept to the logistics context are examples for this wide range of concepts that have to be implemented in the logistics operations system. To support these operations systems a higher service orientation and extended planning scale of various information systems such as Enterprise Resource Planning and Advanced Planning Systems are feasible. The service oriented design of the information system landscape allows it, to reduce the complexity of the information system that is needed to serve the different requirements of eachsingle logistics service configuration. An extension of the planning scale to the consideration of corporate restrictions and the integration of customer and supplier restrictions enables companies to benefit from the flexibility and efficiency potentials tailored logistics services have.

Based on this systematization 27 practitioners were asked to evaluate the overall relevance of the of the conceptual parts of the tailoring process (Figure 3 ). On a 1 to 5 scale the managers agreed on the concept (average 3,83). Furthermore, the practical relevance of each of the conceptual parts was discussed. Practitioners should therefore rate the relevance of each part, based on their company specific problem situation. This rating is an indicator for the importance of each part and gives an idea were the focus of the concept development should lie on. Interestingly, for managers has the design of the information system (average of 4,41), the customer relationship system (average of 4,12 ) and the internal coordination system (average of 3,88 ) the highest priority. Managers argued, that these conceptual parts are mainly based on personal experiences and concept specific management decisions, whereas management of the logistics service tailoring system (average of 3,47 ) and the logistics operations system (average of 3,46 ) is predictable.

In summary, the identified conceptual parts of the tailoring process fit to the generic requirements of industrial companies. The outcome of this research step is a conceptual toolkit that maps known management concepts to the different subsystems. Furthermore, the interdependencies of the single conceptual parts were made transparent. The tailoring of logistics services to individual customer expectations consists of concepts and strategies to greaten customer orientation (effec- 


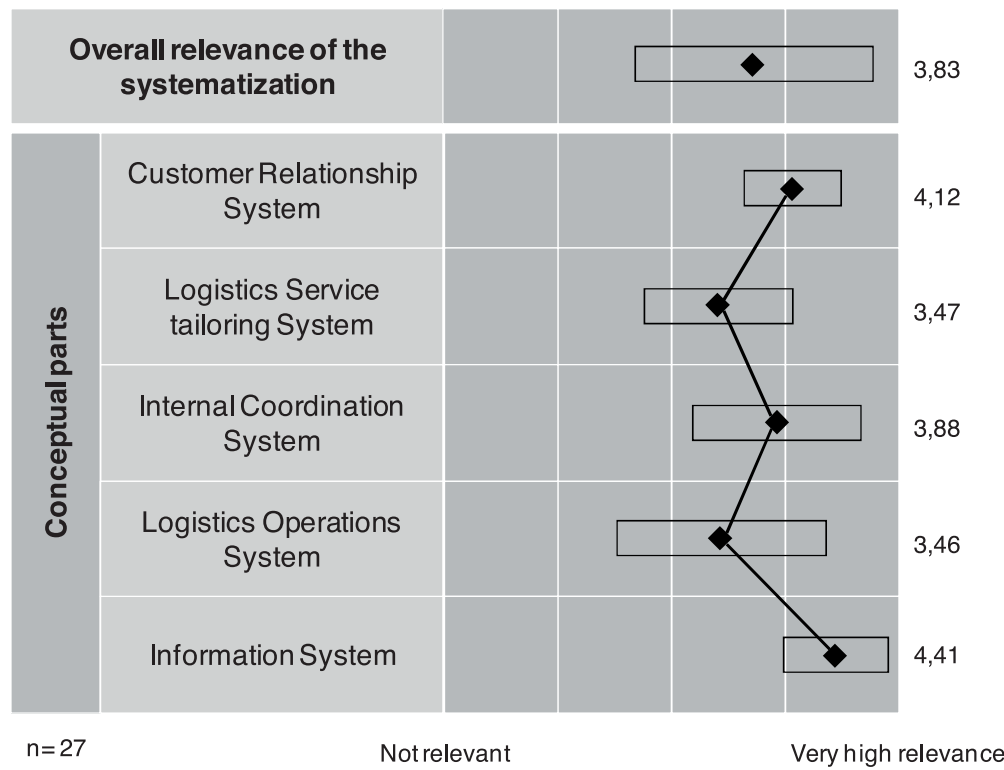

Figure 3: Relevance of the systematization

tiveness) and support logistics operations efficiency. These two interrelated objectives are integrated in the presented concept.

\section{Phases of the implementation process}

A general definition for the implementation of logistics systems contains all activities, which support the transformation process to the desirable state as well as the enforcement for sustaining the transformed logistics system (Pladeck, 2004). This effort has to be executed throughout all process phases of the implementation. Even though these phases do not reflect a common standard, it is possible to deduct five generic phases for the implementation process of logistics systems by comparing different implementation projects and the mentioned phases in literature (i.e. Bowersox et. al., 2007, Slack et. al., 2001, Gudehus, 2005, Waller, 2002). The implementation process therefore includes the phases of analysis, conceptual design, planning, realization and adjustment as shown in figure 4.

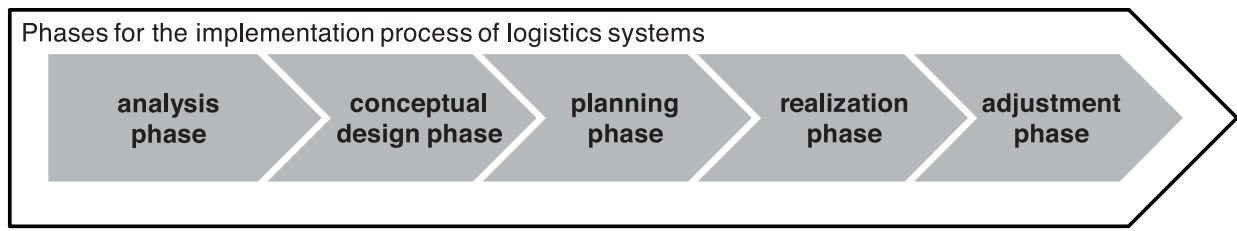

Figure 4: Phases for the implementation process of logistics systems (Klaar, 2007) 
The analysis phase intends to establish precise implementation needs to define the specific target for the whole implementation process. The result of this phase conduces to the following tasks as an essential fundament. A theoretical and detailed elaboration of the full prospective logistics design takes place in the conceptual design phase. Hereafter the specific configuration scheme of what has to be implemented should be developed in total. During the planning phase it is necessary to make decisions on the sequence of realization, the responsibilities for the realization tasks and the use of supporting instruments. The quality of all these preparations will be tested in the realization phase, where the detailed plan will put into action. Every mistake or not considered influencing factor that appears during realization will become an issue for the adjustment phase. Additionally the performance of the complete logistics system, including the interfaces to adjoining processes, will be tested under full load in this phase. If this ends up successfully, the change over from the old to the new implemented system can be initiated.

This framework is an approximated procedure with smooth transitions between the phases. It is also possible that some iterative loops between phases will deliver better results for further decisions (e.g. doing more analytical steps in the conceptual design phase). Nevertheless the framework offers the possibility to describe the steps to undertake for complex implementation processes in a structured way.

The application of this implementation process shows the different importance of the conceptual parts over the phases. The critical point in the analysis phase is to examine the general conditions for the implementation of a tailoring process. Managers stated that the reputation of the logistics department in comparison to the other departments and the already realized coordination efforts between company departments are major organizational characteristics that need to be identified in the analysis phase. Having transparency about these aspects enables managers to anticipate major implementation issues. The focus of the conceptual design phase is to set a common vision for the company with clearly pointing out the benefits of a tailoring process for logistics services. Therefore the requirements on logistics operations and IT systems as well as the internal organization have to be defined. This includes the definition of the additional competences and qualifications of the logistics management and planning staff which is needed for the management of customer relationships and the tailoring process itself. The basic decisions in the planning phase consist of the adaption of all operations processes to the requirements of tailored logistics services. The critical point in this transformation process is, to clearly define the responsibilities for the implementation, especially between sales and logistics department. The most important point managers stated to this phase was the decision how to integrate logistics segments in the already existing sales segmentation. Getting the tailoring process into actionrequires a high degree of process transparency and well designed performance figures. Furthermore customers should be strongly integrated in the implementation process to get a direct 
feedback from the market. Therefore it is possible to evaluate the costs and benefits of tailored logistics services and to adjust the processes in the adjustment phase efficiently. The special challenge in the adjustment phase is to establish a high degree of flexibility in the logistics service tailoring and the logistics operations system to realize an efficient adaption process regarding changing customer expectations. The focus of each implementation phase concerning the different conceptual parts is shown in figure 5 in summary.

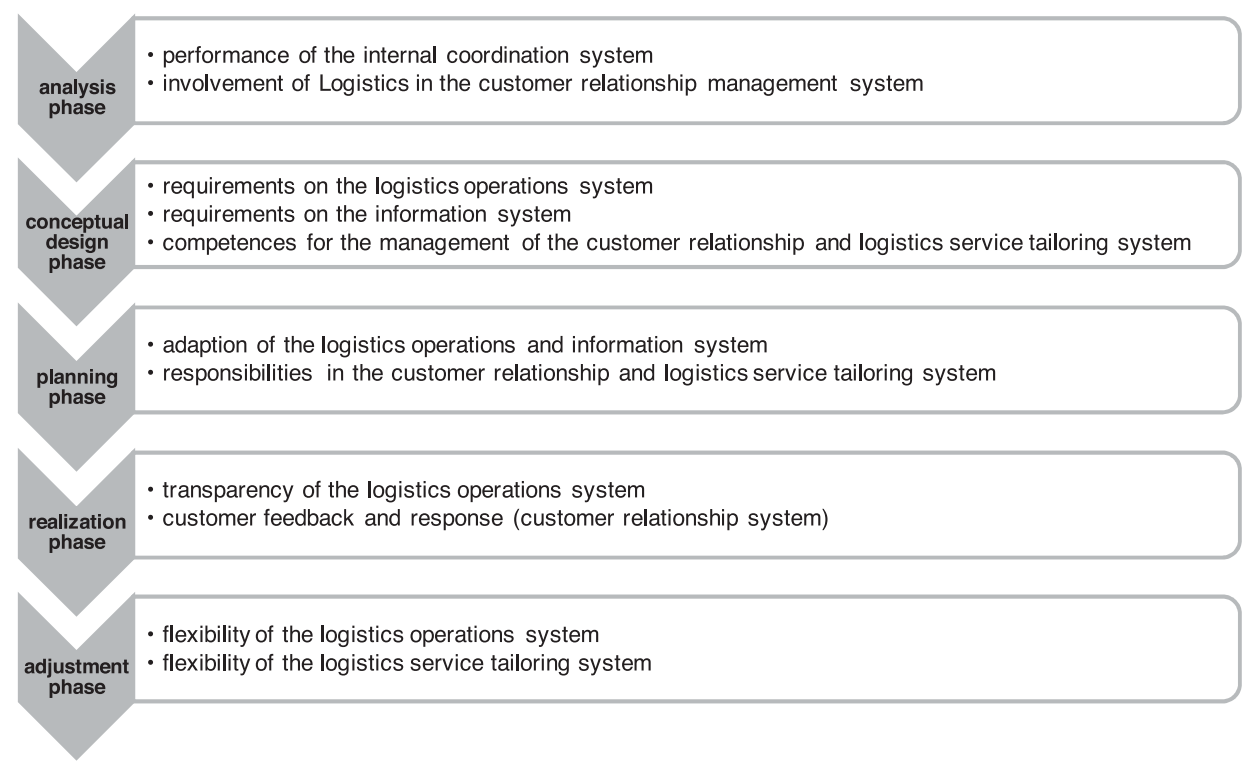

Figure 5: Focus of each implementation phase

\section{Issues in the implementation process}

The challenge for the implementation process is to foresee and prevent critical barriers and in case they are coming up, to overcome them. In practice there is a plurality of issues that could avoid a successful implementation of the logistics system. Solving these issues, especially that one that is the root cause, seems to be a complex task. Therefore a generic classification for the potential issues that may appear during the implementation process of logistics systems was developed (Klaar/Doch, 2007). It contains technological, organizational and social psychological components that will be distinguished briefly below (Figure 6).

The technological class contains all issues that are directly related to the subjectmatter of the logistics systems. It concerns the technical perspective (e.g. material handling technology) and the IT-infrastructure. Especially logistics focuses on linking technical resources through appropriate processes. Hence, logistics strategies for planning and operating the implementation subject are subsumed in this category of technological issues. To specify the technological issues, it can be further 
categorize into issues belonging to robustness that is technical oriented, performance, where the processes affiliate and compatibility, which is mainly addressed to ITspecific issues.

The organizational implementation issues are related to the specific context of the logistics project itself. This includes all tasks and activities belonging to the preparation and realization of the implementation. It contains providing all required resources, monitoring the methodological approach of the whole project as well as the planning and initiation of attendant or supporting activities during the implementation process.

The socio-economic system, i.e. the individual attitudes and action of the company's members, value-adding partners and logistics service providers in the supply chain as well as their corporate culture, are subjects of the social psychological implementation issues. They concern communication and leadership matters as well as all types of resistance in the change process that could endanger the implementation success.

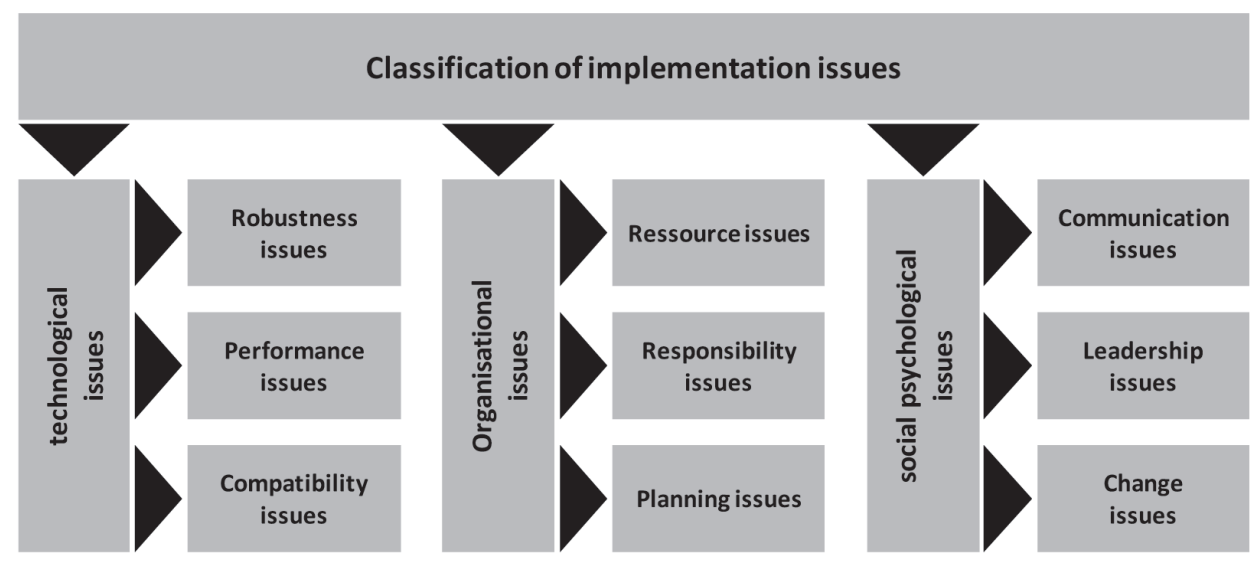

Figure 6: Classification of issues in the implementation process of logistics systems

For tailoring logistics services the main implementation issues had been elicited by literature analyses and been classified in a grid (Figure 7). This grid contains the described technological, organizational and social psychological components as one systematization aspect and the conceptual parts of the implementation process as the other systematization aspect. Additionally to the discussion of the conceptual parts of the tailoring process, the practitioners were asked what aspects they foresee as relevant issues in the implementation process. Highly relevant implementation issues ( 3 or more points on a 1 to 5 scale) were arranged into the grid.

The main technological issues in the customer relationship system refer to missing conceptual and managerial competencies to realize tailored logistics services. In detail, a lack of knowledge in the logistics function about methods for the analy- 
sis of customer expectations and the missing involvement of the logistics function in the customer interaction process were the major implementation issues in this area. The same reasons where mentioned as organizational issues in the customer relationship system. Missing resources and responsibilities of the logistics function describe the main issues in this design area. Communication issues, cross-functional as well as cross-organizational and an suspected loss of importance of the sales function were the main social psychological barriers in the implementation of the required customer relationship system.

The main technological issues in the logistics service tailoring system refer to conceptual difficulties to distinguish logistics, sales and marketing components of the overall customer service and to match logistics service segments into the existing customer segmentation. Missing resources and responsibilities of the logistics function describe the main organizational issues in this design area in analogy to the customer relationship system. The willingness of the sales function to adapt the existing customer segmentation to the requirements of tailored logistics services describes the main social psychological issue in the implementation of the required logistics service tailoring system.

Implementation issues for the internal coordination system are mainly affected by social psychological barriers. In detail, company organization structures with a strong functional focus, missing top management support for cross-functional coordination efforts and power-political change barriers are the major implementation issues.

In contrast to the focus of the implementation issues of the internal coordination system on social psychological barriers, the logistics operations system is mainly affected by technological issues. The implementation of a tailoring process fails due to negative influences on the stability of logistics and production processes through the higher process complexity. Furthermore, concepts and strategies to boost the flexibility and transparency of the logistics processes are not implemented accurately. These failures in the design of the logistics operations system appears in a low overall system performance of the company. Due to the fact that the compatibility of the adapted logistics processes with other company's and supplier's processes is missing the performance gap is bred to the entire supply chain.

The same performance outcomes can also be affected by issues in the implementation of the information system. A low IT-performance and missing planning capabilities can have a major influence on the overall system performance as well. The main reason for these technological problems refers to organizational issues in the implementation of the information system. Missing knowledge of the system requirements for tailored logistics services in the early stages of the implementation process causes the barriers in this design area.

An overview of the identified issues in the implementation process of tailored logistics services is presented in figure 7. 


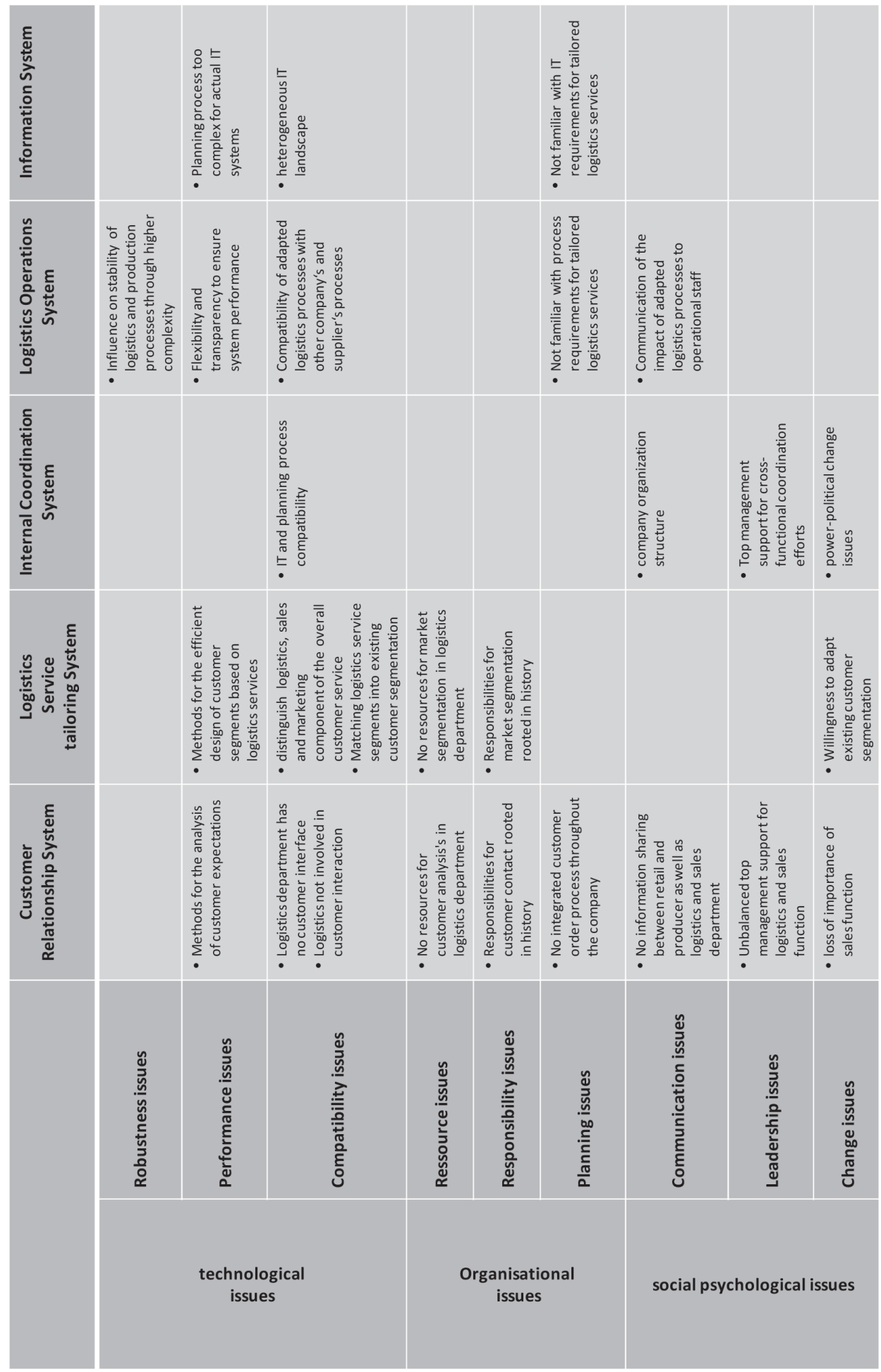

Figure 7: Issues in the implementation process of tailored logistics services 
To face the anticipated issues in the implementation process of tailored logistics services a range of context specific instruments should be deployed. Especially instruments for the mentioned technological issues should focus on professional training and development to understand the required changes of the logistics process and learn how to deal with this new amount of complexity between the participating organizational functions. The management and the staff that will be confronted with a modified scope of duties need to be trained for different tasks,responsibilities, IT-systems and a new communication process. This can be executed with seminars, learning on the job with supervising coaches, methodological workshops as well as special help desks or online advisory tools.

However, in the end it is almost more important that all involved managers and staff learns and understands the advantage of the whole context that is changing and the part they will contribute to make the implementation work. Because of this, the development of a vision and strategic objectives for the context of the implementation of tailoring logistics services are instruments with a suitable impact to start with. It is necessary to break these objectives down to the operational level. Value-Mapping is therefore an adequate method in order to enforce defined KPI's at the same time. Due to the new allocation of responsibilities, tasks and resources another powerful instrument concerning organizational issues is to define or reassign strictly new roles and functions at the management level.

Closely linked to the organizational issues are those of the social economic perspective. The implementation of logistics systems which contains a high proportion of changes to be taken requires the largest effort for the management level. They have to connive at possible changes concerning their power and status in the hierarchy and have to initiate activities to communicate convincingly the benefits of the upcoming implementation process. To create and support a persistent motivation and appropriate incentive system for all of the concerned and involved persons is a major challenge. An increasing effort in direct and indirect individual communication, management by objectives, marketing activities tailored to the staff, teambuilding activities etc. are of avail to prevent and face individual or group resistance.

\section{Conclusions}

The result of this research is a model for the implementation of tailored logistics services that integrates the conceptual parts of the tailoring process and the implementation phases to realize these customer differentiated logistics services. Based on this systematization it is possible to structure the implementation process regarding two different dimensions: the conceptual and the implementation process dimension. The definition of the conceptual parts of the tailoring process helps to systematize the mutual requirements, methods and tools to tailor logistics services to individual customer expectations. Due to the fact that the tailoring process influences multiple functional departments and management areas within 
the company, the shown systematization reduces planning complexity and allows focusing on critical elements of the tailoring process. Researchers can find it helpful to develop further strategies for the tailoring process based on this systematization approach. The application of a standardized implementation process on the topic of tailored logistics services helps to understand the typical realization problems. It was argued, that additional research has to be done in the implementation part of the tailoring process. This paper has shown the link between the mutual design areas and the different implementation phases. The developed grid therefore has identified the basic issues in the different phases and the mutual concept areas and mapped them in a systematized way. Feedback from the involved practitioners has shown that the application of this grid helps companies to realize tailored logistics services more successfully.

\section{References}

Anderson, J./ Narus, J. (1995): Capturing the Value of Supplementary Services, in Harvard Business Review, issue 73, no. 1, 1995, p. 75-83.

Bowersox, D. J./ Closs, D. J./ Cooper. M. B. (2007): Supply Chain Logistics Management, 2nd edition, New York: McGraw-Hill, 2007.

Bowersox, D. J./ Closs, D. J./ Stank, T. P. (2000): Ten Mega-Trends That Will Revolutionize Supply Chain Logistics, in: Journal of Business Logistics, issue 21, no. 2, 2000, p. 1-16.

Childerhouse, P./ Aitken, J./ Towill, D. R. (2002): Analysis and design of focused demand chains, in: Journal of Operations Management, issue 20, no. 1, 2002, p. 675-689.

Doch, S. A. (2008): Logistische Leistungsdifferenzierung im Supply Chain Management, Working Paper, Berlin Institute of Technology, 2008.

Fuller, J. B./ O'Conor, J./ Rawlinson, R. (1993): Tailored logistics: the next advantage, in: Harvard Business Review, issue 71, no. 3, 1993, p. 87-98.

Gudehus, T. (2005): Logistik: Grundlagen - Strategien - Anwendungen, Berlin Heidelberg New York: Springer, 2005.

Janowski, J. (2004): Kundenindividuelle Logistik als strategischer Wettbewerbsvorteil?, in: DBW, issue 64, no. 6, 2004, p. 739-752.

Kaipia, R./ Holmström, J. (2007): Selecting the right planning approach for a product, in: Supply Chain Management: An International Journal, issue 12, no. 1, 2007, p. 3-13.

Klaar, K. (2007): Change Management in der Logistik, Working Paper, Berlin Institute of Technology, 2007.

Klaar, K./ Doch, S. A. (2008): Implementierung im Supply Chain Management, Working Paper, Berlin Institute of Technology, 2008.

Mentzer, J. T./ Flint, D. J./ Hult, G. T. M. (2001): Logistics Service Quality as a Seg- 
ment- Customized Process, in: Journal of Marketing, issue 65, no. 4, 2001, p. 82-104. Mentzer, J. T. / Williams, L. R. (2001): The Role of Logistics Leverage in Marketing Strategy, in: Journal of Marketing Channels, issue 8, no. 3/4, 2001, p. 29-47.

Payne, T./ Peters, M. J. (2004): What Is the Right Supply Chain For Your Product?, in: The International Journal of Logistics Management, issue 15, no. 2, 2004, p. 77-92.

Pfohl, H.-C./ Elbert, R./ Gomm, M. (2006): Supply Chain Finance: Antwort auf die Forderung nach einer wertorientierten Logistik, in: Wolf-Kluthausen, H. (Ed.): Jahrbuch der Logistik, Korschenbroich: free Beratung, 2006, p. 18-29.

Skinner, W. (1974): The focused factory, in: Harvard Business Review, issue 52, no. 3, 1974, p. 113-121.

Slack, N./ Chambers, S./ Johnston, R. (2001): Operations Management, 3rd, Harlow: Pearson Education, 2001.

Torres, L./ Miller, J. (2002): Aligned Logistics Operations: Tailoring Logistics to the needs of Customers, in: Gattorna, J. (Ed.): Strategic Supply Chain Alignment, Hampshire: Gower Publishing, 2002, p. 42-59.

Waller, D. L. (2002): Operations Management. A supply chain approach, London: Thomson Education, 2002.

Woratschek, H. (2001): Zum Stand einer Theorie des Dienstleistungsmarketing, in: Die Unternehmung, issue 55, no. 4/5, 2001, p. 261-278. 


\title{
Configuration and Product Modularization for the flexible Network: A Contribution to Mass Customization
}

\author{
Wendelin Gross, Sven Kuhn
}

\begin{abstract}
Manufacturers of complex investment goods face the challenge of increasing product variety due to individual customer requirements, short product life cycles and new technologies. Subsequently, process complexity and thereby costs and delivery times rise.

Product modularization supports the achievement of Mass Customization benefits. Established modularization methods consider customer requirements and product functions. Additionally, the presented approach covers product specific, cost and time-consuming manufacturing processes as well as flexibility of network partners concerning manufacturing, product development as well as maintenance and repair. As product modularization requires measures of network partners' flexibility, the relevant components of flexibility have to be estimated systematically.

The developed concept of integrated configuration incorporates the modular product as well as the flexible network. It enables manufacturers and service providers to determine the most adequate product and network set-up in order to meet customer demand on the basis of product modularization and network flexibility estimation.
\end{abstract}

\section{Keywords}

Modularization, Product Design, Supply Network Flexibility, Configuration

\section{Introduction}

Manufacturers of complex investment goods face the challenge of increasing product variety due to individual customer requirements, short product life cycles and new technologies. In addition to functionality, quality and price, the delivery time of the products and product-related services plays a decisive role for the customer. High product variety causes turbulences in the processes of the OEM (Original Equipment Manufacturer) as well as in the entire product development and production network. The consequences are long lead times and high costs in direct, e.g. assembly and manufacturing, and indirect, e.g. purchasing, processes.

There are different approaches to reduce product variety or to minimize its impact on production processes and network. Measures like product modularization, 
modular sourcing or postponement in logistics are frequently used, but as we will show, they are not yet adequate forthe manufacturers in consideration here. Enhancements are necessary to cover the particularities of complex investments goods, which are produced to a specific order. In this regard, product complexity and the dependence on suppliers with unique product and manufacturing knowledge already working at their capacity limits require an analysis of the entire network.

Network particularities have to be considered in product modularization. Only if the needs of the entire network are taken into account, the potential of improved flexibility in enterprise specific and cross enterprise processes can be exploited. Therefore, the suppliers' capability in research and development, in manufacturing and pre-assembly, in product documentation and in maintenance and repair has to be regarded within modularization.

Any bottleneck on the critical path within the development and production of a consumer-specific module leads to a delay in delivery of the final product. The product designer should be aware that a multitude of suppliers with frequent delivery problems, which represent additional bottlenecks for the different components of one module, are adverse to short lead times, for example. Delays sum up and cannot be counterbalanced.

Depending on the customer needs, the network planner has to adapt the network to new situations. He must be able to change the network configuration based on predefined scenarios. An immediate modification of the network for a newly developed customer-specific module is hardly possible, since the expected delivery times are mostly shorter than the time necessary to choose and qualify a new supplier. In case of frequent and extensive changes of the product or in case of exceptional combinations of modules, alternative suppliers can be chosen to improve efficiency. Therefore, suppliers with different performance attributes have to be available to the OEM.

\section{Flexibility of Suppliers and Supply Network}

Supply network flexibility is an issue of Supply Chain Management that has not yet been exhaustively investigated (Tachizawa / Gimenez Thomson 2007). It is, however, a capability required for economic success, because of competition among supply chains and the increasing importance of reduced delivery time for customer satisfaction (Kumar et al. 2006). Work on flexibility of the supply chain originates in survey and description of manufacturing flexibility, which reflects the ability of a system to properly and rapidly adjust production of goods according to internal or external changes (Garavelli 2003). In the course of reducing the real net output ratio, i.e. the fraction of production processes performed by the OEM himself compared to the total value added, it becomes crucial to equally highlight the flexibility of supply relations and the entire supply network (Kumar et al. 2006) (Duclos et al. 2003). Furthermore, network flexibility concerns product development as well as maintenance and repair processes of the suppliers in addition to manufacturing. 
According to the literature review of Duclos et al. including strategic and manufacturing flexibility, six components of supply chain flexibility can be defined (Duclos et al. 2003): operation system flexibility, logistics flexibility, supply flexibility, organizational flexibility, new product flexibility and information systems flexibility. The supply network can be considered as consolidation of several linear supply chains (Pfohl / Buse 2000). This extends the understanding of flexibility by the possibility of shifting production to different sites of the supply network. From the viewpoint of a single company that aims at estimating the flexibility of a single supplier, only the supplier's ability to shift production is of interest. Thus, we will refer to production shift flexibility as the suppliers' ability to choose another site for production in the following. Hence, as the supply network consists of several supply chains in our approach, the flexibility components of the above mentioned authors are extended by the production shift aspect.

In order to deal with the challenge of increasing product variety and ensuing process complexity, it is vital to arrange the supply network in face of flexibility. Thus, supply network flexibility has to be considered within network design and planning. Alternative network set-ups are required in order to benefit from a flexible network. These alternative set-ups will be employed in the course of network configuration according to the customer's requirements (see chapter 4).

\subsection{Flexibility Components with Relevance for Product Modularization}

According to the findings on flexibility stipulated above, we consider the following flexibility components to be relevant for product modularization:

- Operation system flexibility,

- Logistics flexibility,

- Organizational flexibility,

- New product flexibility,

- Information systems flexibility,

- Production shift capability

Operation system flexibility refers to manufacturing flexibility as it comprises product mix and product volume changing ability and capacity at network nodes. Obviously, it is one of the most important components of flexibility and subsequently a determinant of product modularization. Product modules have to be easily replaceable with others in the manufacturing and repair processes and have to be usable in a wide range of product variants in order to perfectly meet the flexibility requirement.

Logistics flexibility implies both supply and distribution flexibility. In the context of product modularization, logistics flexibility is an input parameter that comprises the capability of network partners to switch from one procurement process to another, e.g. direct delivery to milk runs. 
Organizational flexibility refers to workforce assignment to processes, organizational structures and business practices that are appropriate to guarantee responsiveness at the different network sites (Duclos et al. 2003). Flexibility means that alternative business processes are defined, which can be easily applied, or that well educated and qualified staff is available that can react flexibly to new situations.

New product flexibility describes the capability to integrate newly developed products or components into the order processing as well as product design and manufacturing processes. New product flexibility makes demands on product architecture in terms of manufacturing techniques required and comprehensive documentation provided. Only if the modules can be designed and manufactured with common methods and tools, it is possible to switch to concurrent suppliers.

Information systems flexibility comprises information sharing within the network and data interfaces between the network partners. The ability of the network to react and to adjust its structure depends on the ability to integrate network partners case-specifically without lengthy adaptation. The integration relies on data processing and exchange that is conducted with support of information systems. Unlike the former components, information system flexibility is not strictly linked to product modularization endeavors. More precisely, information systems will not be of major concern in the process of product modularization, because in this case flexibility of the network is not directly correlated with the respective product.

Production shift flexibility as described above poses demands on product architecture. Furthermore, the capabilities of production sites and relevant suppliers have to be kept in mind accurately in the course of product modularization.

For the modularization approach presented, it is vital to identify network-related determinants for product modules as well as functional and process related ones. As these network-related determinants for modularization include flexibility, a further detailed understanding of flexibility is required to operationalize the approach. Also, it is indispensible to develop a rough estimation of network partners' flexibility as a base for the definition of viable alternative network structures, which can be applied to meet customer demand.

In practice, planning and administration effort have to be considered in any attempt to boost flexibility within the network. Only if there is demand for flexibility, attention should be paid to its enhancement. Suppliers of replaceable low-cost components do not need to be very flexible for example. So, in a preliminary step of flexibility estimation, the network's elements relevant for flexibility consideration have to be identified.

In the following chapter we will describe a framework for the evaluation of network flexibility.

\subsection{A Framework for the Evaluation of Flexibility}

Similar to network flexibility, scientific work on the measurement of flexibility is 
scarce. As Stevenson et al. state, "literature that attempts to measure supply chain flexibility remains in its infancy" (Stevenson / Spring 2007). Regarding flexibility and its integration in processes of decision-making, the theoretic concepts of network flexibility have to come down to relations between supplier and customer. For practical implementation, the measurement or the estimation of flexibility must refer to a certain supplier and a defined part or product component. However, the definition of figures on a numeric scale seems inadequate and not feasible because of the dynamic behavior of relations (Giannakis 2007) and the interdependencies of relations within the network. Flexibility of a certain network partner, e.g. supplier, thus is dependent on the respective network partner within a bilateral supply relation, e.g. customer. Consequently, flexibility is an attribute of a one-to-one relation between one supplier and one customer. However, as each supplier maintains relations to several customers, flexibility and capacity have to be subdivided.

Nonetheless, it is indispensable to measure or at least to estimate the flexibility of suppliers in order to find the appropriate network out of a range of applicable alternative supply structures. Therefore we propose an interval scale of flexibility estimation as introduced by Garavelli. For the sake of simplicity and manageability Garavelli differentiates the flexibility categories 'no flexibility', 'limited flexibility'and 'total flexibility' (Garavelli 2003). As these three categories are a very rough approximation and thus insufficient to represent the potentials of product modularization, we map the above mentioned flexibility components on five flexibility categories for the requirements of manufacturing (see table 1). These categories allow evaluating flexibility more detailed because interdependencies between influencing factors are considered within each category. In our approach we assume that network f lexibility is one parameter of product modularization. One subsequent implication is the pretension that successful modularization permits the leap to an upgrade level, i.e. category, of flexibility.

The five generic flexibility categories we assess are 'no flexibility', 'partial flexibility', 'limited flexibility', 'extensive flexibility' and 'total flexibility'. Table 1 (next page) displays the characteristics of the flexibility categories related to flexibility components.

The flexibility framework cannot be consolidated to a single index or figure, but must be evaluated per product module and network partner respectively. A supplier, for instance, who is able to raise output volume of a certain product, but cannot adapt the supply process, is not comparable by figures to another supplier, who can deliberately process a wide range of new products, but is not able to standardize the new production process, due to the lack of organizational flexibility.

Measuring flexibility in five clusters is a simple approach to estimate the extent to which suppliers can adapt their operational system, logistics, organization, new product integration, information systems and production site. However, the approach seems appropriate for our practical intentions. The flexibility estimation of 


\begin{tabular}{|c|c|c|c|c|c|c|}
\hline $\begin{array}{r}\begin{array}{r}\text { Flexibility } \\
\text { Components }\end{array} \\
\text { Flexibility } \\
\text { Categories }\end{array}$ & $\begin{array}{c}\text { Operation System } \\
\text { Flexibility }\end{array}$ & $\begin{array}{l}\text { Logistics } \\
\text { Flexibility }\end{array}$ & $\begin{array}{l}\text { Organizational } \\
\text { Flexibility }\end{array}$ & $\begin{array}{l}\text { New Product } \\
\text { Flexibility }\end{array}$ & $\begin{array}{c}\text { Information } \\
\text { System Flexibility }\end{array}$ & $\begin{array}{c}\text { External } \\
\text { Production } \\
\text { Shift }\end{array}$ \\
\hline $\begin{array}{l}\text { No } \\
\text { Flexibility }\end{array}$ & $\begin{array}{l}\text { No adaption of } \\
\text { product volume } \\
\text { and mix possible }\end{array}$ & $\begin{array}{l}\text { Fixed supply } \\
\text { process (direct } \\
\text { relation) }\end{array}$ & $\begin{array}{l}\text { No adaption of } \\
\text { workforce } \\
\text { assignment and } \\
\text { business } \\
\text { processes }\end{array}$ & $\begin{array}{c}\text { No processing } \\
\text { of new } \\
\text { products }\end{array}$ & $\begin{array}{c}\text { No data interfaces } \\
\text { and information } \\
\text { sharing }\end{array}$ & $\begin{array}{l}\text { No shift of } \\
\text { production to } \\
\text { alternative sites }\end{array}$ \\
\hline $\begin{array}{l}\text { Partial } \\
\text { Flexibility }\end{array}$ & $\begin{array}{l}\text { Limited adaption } \\
\text { of product volume } \\
\text { OR mix }\end{array}$ & $\begin{array}{l}\text { Supply processe } \\
\text { including service } \\
\text { provider ( } 3 \mathrm{rd} \\
\text { party) }\end{array}$ & $\begin{array}{l}\text { Limited adaption } \\
\text { of workforce } \\
\text { assignment OR } \\
\text { business } \\
\text { processes }\end{array}$ & $\begin{array}{l}\text { Processing of } \\
\text { products with } \\
\text { scaled } \\
\text { geometry }\end{array}$ & $\begin{array}{c}\text { Specific uni- } \\
\text { directional data } \\
\text { interfaces and } \\
\text { limited information } \\
\text { sharing }\end{array}$ & $\begin{array}{l}\text { Production shift } \\
\text { to one } \\
\text { alternative site } \\
\text { with high } \\
\text { investment }\end{array}$ \\
\hline $\begin{array}{l}\text { Limited } \\
\text { Flexibility }\end{array}$ & $\begin{array}{l}\text { Total adaption of } \\
\text { product volume } \\
\text { OR mix }\end{array}$ & $\begin{array}{c}\text { Supply } \\
\text { processes } \\
\text { affecting other } \\
\text { partners } \\
\text { (milkrun) }\end{array}$ & $\begin{array}{l}\text { Total adaption of } \\
\text { workforce } \\
\text { assignment OR } \\
\text { business } \\
\text { processes }\end{array}$ & $\begin{array}{l}\text { Processing of } \\
\text { products with } \\
\text { new manu- } \\
\text { facturing } \\
\text { techniques }\end{array}$ & $\begin{array}{l}\text { Standard uni- } \\
\text { directional OR } \\
\text { specific bi- } \\
\text { directional data } \\
\text { interfaces and } \\
\text { limited information } \\
\text { sharing }\end{array}$ & $\begin{array}{l}\text { Production shift } \\
\text { to one } \\
\text { alternative site } \\
\text { with mean } \\
\text { investment }\end{array}$ \\
\hline $\begin{array}{l}\text { Extensive } \\
\text { Flexibility }\end{array}$ & $\begin{array}{l}\text { Total adaption of } \\
\text { product volume } \\
\text { OR mix and ltd. } \\
\text { adaption of volume } \\
\text { OR mix resp. }\end{array}$ & $\begin{array}{l}\text { Supply } \\
\text { processes with } \\
\text { advanced time } \\
\text { sensitivity (JiT) }\end{array}$ & $\begin{array}{l}\text { Limited adaption } \\
\text { of workforce } \\
\text { assignment AND } \\
\text { business } \\
\text { processes }\end{array}$ & $\begin{array}{l}\text { Processing of } \\
\text { products with } \\
\text { new design } \\
\text { effort }\end{array}$ & $\begin{array}{c}\text { Standard bi- } \\
\text { directional data } \\
\text { interfaces and } \\
\text { limited information } \\
\text { sharing }\end{array}$ & $\begin{array}{l}\text { Production shift } \\
\text { to one } \\
\text { alternative site } \\
\text { without } \\
\text { investment }\end{array}$ \\
\hline $\begin{array}{l}\text { Total } \\
\text { Flexibility }\end{array}$ & $\begin{array}{l}\text { Total adaption of } \\
\text { product volume } \\
\text { AND mix }\end{array}$ & $\begin{array}{c}\text { Supply } \\
\text { Processes with } \\
\text { additional } \\
\text { services } \\
\text { (sequencing etc.) }\end{array}$ & $\begin{array}{l}\text { Total adaption of } \\
\text { workforce } \\
\text { assignment AND } \\
\text { business } \\
\text { processes }\end{array}$ & $\begin{array}{l}\text { Processing of } \\
\text { any new } \\
\text { product } \\
\text { including } \\
\text { prototyping }\end{array}$ & $\begin{array}{l}\text { Standard bi- } \\
\text { directional data } \\
\text { interfaces and full } \\
\text { information sharing }\end{array}$ & $\begin{array}{l}\text { Production shift } \\
\text { to multiple } \\
\text { alternative sites } \\
\text { without } \\
\text { investment }\end{array}$ \\
\hline
\end{tabular}

Table 1: A Framework for the Evaluation of Flexibility

single suppliers and specific parts or modules consolidates in the network to a product-specific flexibility map. Using this map, the flexibility demand, the flexibility offer and the resulting gap can be determined. If necessary, measures can be taken to reduce the gap, e.g. supplier qualification.

\section{Product Modularization for the Flexible Network}

Product design determines the greatest part of costs and times required for the production processes of customer-specific products within the network (Pahl et al. 2004). Applying modularization, it is expected that a wide range of products can be produced without variant-specific efforts. Modularization means to build a product from mostly standardized components, so that a high number of final products can be achieved by combining different components, i.e. modules. The aim is to divide the product into modules while minimizing interconnections between modules and maximizing dependencies between different parts of one module (Mikkola 2001).

Since modularization is an important prerequisite for Mass Customization, an optimized product structure for complex investment goods must be identified. Especially for long product life cycles and products covering a considerable market share and market growth respectively, modularization seems to be efficient. Else wise costs are too high to compensate the efforts of modularization (Doran et al. 2007).

As a product s structure and therewith its modules cannot be changed easily and quickly, a multitude of influencing factors has to be taken into account within mo- 
dularization in order to design a product structure that lasts over the product lifecycle. As mentioned above, these factors are functionality and process as well as network and flexibility related aspects.

Furthermore, modularization has to represent future requirements of customers, processes and network partners. Thus, it is necessary to forecast customer demand in order to deduce product variants and quantities. Product modules have to be classified according to their variety distinguishing invariant modules from those with foreseeable frequent and extensive changes.

Scenarios have to be developed representing probable future requirements. These scenarios must contain for instance information about possible changes of customer requirements, new technical design demands, new production technologies and new suppliers in the network. Using methods like the Quality Function Deployment as a basis (see figure 1), product modules, which undergo extensive changes, can be deduced.

We have developed the simple index „level of change' in order to identify products and modules, which undergo extensive changes. The index is a multiplication of the expected number of changes over the life cycle and the expected costs per change at involved partners. This level of change is sufficient for an efficient evaluation of possible changes. It is of importance for the integration of flexibility into modularization.

\subsection{Network-related Requirements}

The modules' demand for flexibility is characterized by the highest demand for flexibility of any module component. A module that consists of many simple components like screws, clamps and levers and one complex component, e.g. engine, demands the flexibility of the most challenging component, i.e. the engine, for example. Thus, parts of minor relevance for flexibility are not regarded in the applied method in order to limit modularization efforts.

In the following, the above mentioned flexibility components (see table 1) will be regarded in order to identify flexibility and network requirements for modularization.

Following the definition of operational system flexibility, parts with high flexibility requirements should be pooled together to one module in order to reduce flexibility requirements within one module. In case of modular sourcing only one supplier is affected then.

The module structure should allow the production of different module variants with virtually the same technologies. Thus, the variants of a module should have similar technology, quality, measuring and testing equipment and capacity requirements. These requirements have to be regarded, if specialized suppliers are necessary to repair the module. Furthermore, the automation level of module variants should be similar to the variant with the highest production volume in order to level flexibility demand within a module. 
Since technical capabilities of suppliers normally do not change fundamentally within a considerable timeframe, the module components should require only a limited number of technologies. Obviously, this applies especially to modules that probably will be changed during product life cycle.

Within product modularization, logistics flexibility refers to logistic parameters, e.g. dimensions and weight, of module variants as well as their loading device. Parameters and loading device should be standardized in order to allow switching between alternative suppliers and module variants respectively.

The organizational flexibility as defined above is only of importance when specific knowledge or competences are necessary for the production of different module variants. The production of a module should therefore demand similar skills and level of experience in order to enhance flexibility.

These modularization requirements have to be weighted for each supplier regarded and taken into account by the applied modularization method.

\subsection{Established Modularization Methods}

Several modularization techniques are available in scientific literature and practice. It is the aim to use and enhance existing techniques wherever possible within this work. Therefore it is necessary to examine, which method is applicable according to their characteristics.

One of the best known modularization techniques is the Modular Function Deployment (MFD) method (Ericsson / Erixon 1999). The user defines modules by using predefined module drivers. A module driver is an experience-based criterion for modularization which represents similar requirements that a module has to fulfill. Module drivers result predominantly from one functional area of the enterprise, e.g. assembly (Erixon 1996).The comprehensible structure and the multitude of influencing factors considered are the advantages of this method. However, influencing factors resulting from flexibility and network considerations are not included.

Another technique for modularization is the Design Structure Matrix (Pimmler / Eppinger 1994). An existing product is decomposed into modules by considering only interfaces of complex components. Only customer related, physical and a few strategic requirements are regarded.

The Modular Product Development method (Pahl / Beitz 1996) is based on the functional decomposition of the product and on functional trees, while criteria for designing different modules are not given explicitly. Network aspects and flexibility requirements are not considered.

The Function Structure Heuristic method uses functional decomposition block diagrams of product functions as well as material, energy and information flows between the components (Stone et al. 2000). Stone et al. separate modules from a single product's function structure by finding the 'dominant flow', 'branching flows', or 'conversion-transmission' pairs. Since these flows between modules can hardly 
express flexibility and network requirements, this method is not integrated in the presented approach.

Modular structures can also be defined using Dendrograms (Hölttä et al. 2003). Since this is another possibility of representing and defining the modular structure that covers functional aspects only, the method will not be pursued further.

Dynamic Modularization (Lehtonen et al. 2003) is another method to modularize a product using cause and effect diagrams for individual modularization causes. This method is considered as being too complex to integrate flexibility and network aspects because of interdependencies between the regarded causes.

The method House of Modular Enhancement also takes life cycle costs into account (Sand et al. 2002). The shortfalls of this method are the difficulties to calculate life cycle costs for complex industrial goods and the lacking possibility to weight the influencing factors of modularization within the method.

Since the existing modularization methods consider neither network nor flexibility requirements, a new approach has to be developed or the existing methods have to be enhanced. The MFD method is the most advantageous of the methods presented, because it corresponds to different requirements, it allows the weighting of module drivers and it is proven and commonly accepted. Subsequently, it will be used as a basis for our approach.

\subsection{The Advanced Product Modularization Method}

As mentioned above, variants and future product requirements have to be determined as input parameters for modularization (see figure 1). Product classes can be defined, if high efforts are necessary to determine the impact of future customer preferences, technical developments and network parameters. Each product class represents different products with similar modularization requirements.

Then, demand forecast has to be surveyed in order to define the importance of functionalities, i.e. product modules, for the customer. The aim is to prioritize modularization endeavors according to forecast volume.

The next step within modularization contains the determination of suppliers and product components that pose important requirements. These requirements have to be integrated into the modularization technique. In the case of a newly developed product, which is not yet in series production, the supplier's capability to produce the number of variants within the required cost and time frame has to be estimated.

Within modularization, the flexibility and network requirements of any part must be compared with the requirements stated by customer, functionality and processes. Using an ordinal scaled measure, which is based on the results of standardized questions to the modularization team, this comparison can take place. As figure 1 shows, requirements for modularization can be summarized in the Modular Indication Matrix of MFD. The aim is to develop a modular structure that is adequate 
for all product variants since otherwise this structure has to be optimized for many single product variants.

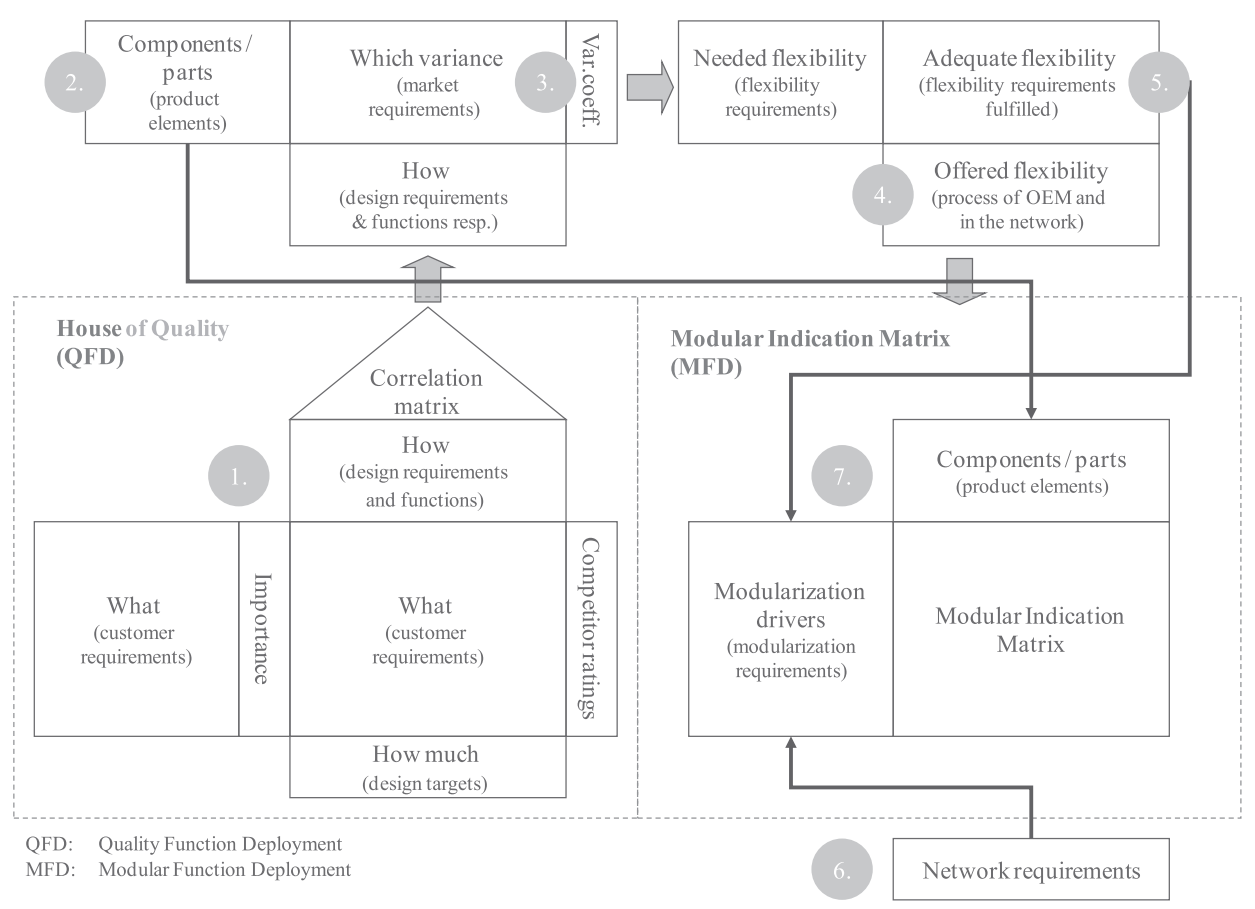

Figure 1: Integrating Network and Flexibility Aspects into Modularization

Given the modular structure of the product family, which fulfills the most important requirements including flexibility and network requirements, the resulting product variants have to be managed efficiently. As we will show in the next chapter, the configuration of the product and the network is a viable approach to cope with product variety.

\section{Configuration of Modular Product and Flexible Network}

The use of software-based product configurators is widely spread in businesses that follow the Mass Customization approach. The configuration of the supply network and the consideration of network flexibility in contrast have not yet been described in detail (Gross et al. 2007). In this work we aim at combining product and network configuration within an integrated concept while taking network flexibility and the result of product modularization as described above into account. 
When configuring the product, the foremost goal is to obtain a Bill of Material (BOM) and a working plan that is suitable to generate a product according to the customer demand (Helo 2006). A secondary goal is to establish configuration software as a data base for expert knowledge (Büttner 1997). There are various approaches how to technically implement this aim ranging from case-based to constraint-based methods (see Blecker et al. 2004 for an overview).

Within network configuration two distinctive use cases have to be distinguished: First, the concealed, automated configuration in the background while the customer customizes a product. Second, the obvious configuration when the network designer or supply chain manager, who has access to the available information, configures the most adequate network, e.g. for a set of customer orders, for a newly developed product or even for repair orders. While the first case originates in an immediate customer requirement, the latter's trigger is a planning task. The differences in the cases concern the different goals of operation, the diverse knowledge of the user and the scope of data, which is available or visible to the user. The purpose of configuration as regarded in this work meets the second of these two cases. Practically, the configuration will be performed in order to analyze the impact of new or changed products, modules or specific features or to define the most adequate network to deal with a bulk order.

When configuring, a data base of the subject has to be available covering its components and their combinability. The result of the configuration process is a set of components, which meets preliminary stipulated functional requirements, general rules and specific directives, e.g. minimize costs or maximize product performance (similar: Ladeby et al. 2007). The resulting set of components needs to be described in a modality that is usable within further business processes, i.e. a Bill of Material and preferably working plans in case of product configuration. This is especially of importance in the course of integrating the configuration concept into existing or newly developed software tools. Consistent business processes then allow coping with high product variety.

Our approach of integrating modularization and configuration emphasizes the correlation of both aspects by including the supply network and flexibility within modularization and within configuration. Based on the modular product and flexible supply network structures we aim at configuring both aspects in a single working environment and with a common data base.

As displayed in figure 2 (next page), the configuration process originates in a conceptional planning task. Within this step, the user has to define the requirements, which the product has to fulfill, as well as his preferences regarding costs, time of delivery and flexibility that are required for network configuration.

The result of integrated configuration is a specific product and network set-up. In the following, the product configuration, network configuration and data base according to figure 2 are described. 


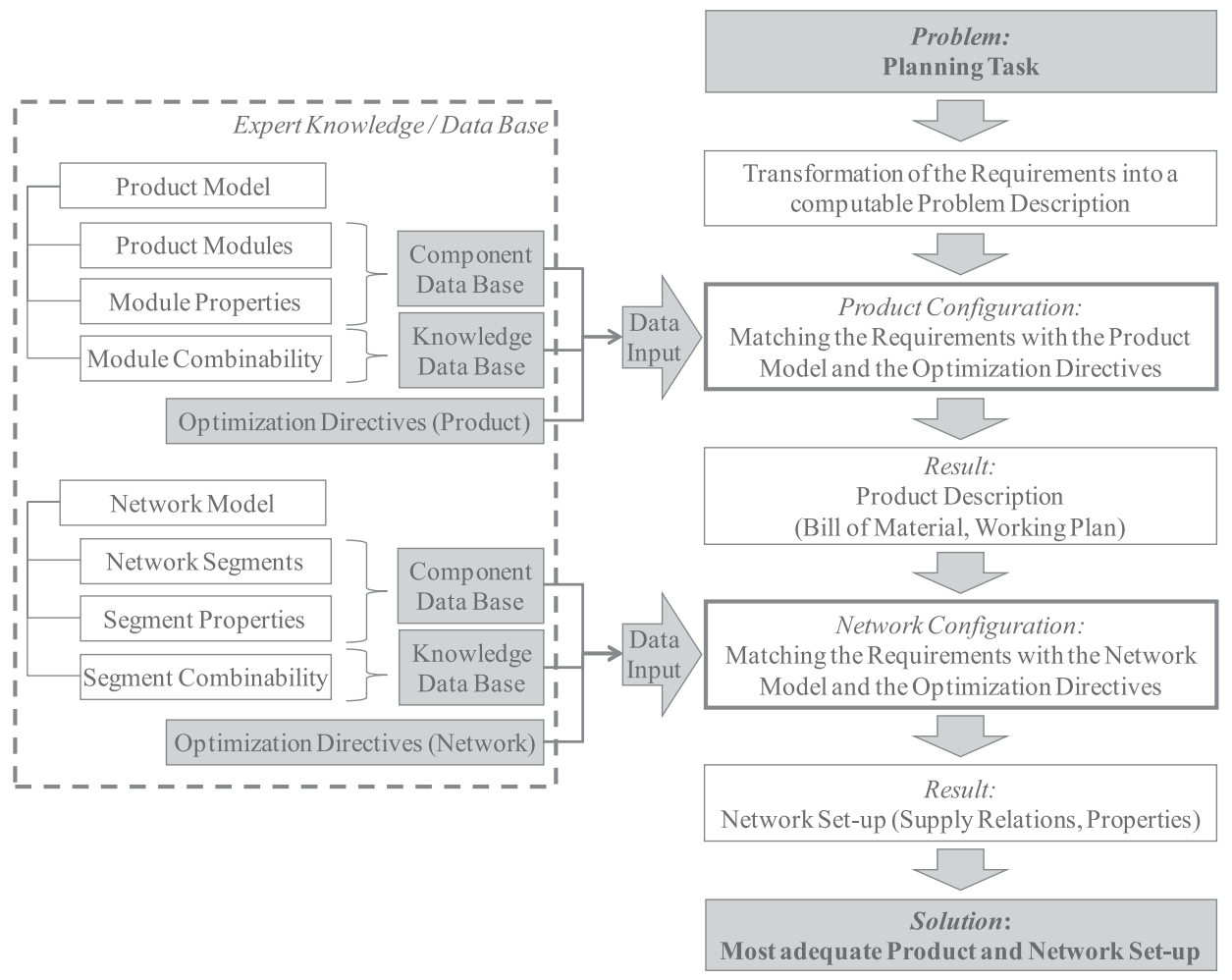

Figure 2: Data Base and Process of Integrated Configuration

\subsection{Product Configuration}

Product modularization is a prerequisite for configuration. The product configuration in the sense of composing a product set-up is only possible on the basis of a product model, which includes the compatibility of product components.

Based on the product modules that have been defined within the modularization process as described above, on the rules and constraints, which constitute the modules' combinability, and on optional directives, product configuration is performed. According to Ladeby et al. the following data-sets are required for product configuration (Ladeby et al. 2007):

- A fixed, pre-defined set of components, where a component is described by a set of properties

- Ports / interfaces for connecting it to other components

- Constraints at each port that describe the components that can be connected to this port

- Other structural components 
In other words, these data sets comprise the product modules, their combinability and properties. Additionally, the configuration process uses directives, which are used to evaluate the quality of a solution (see figure 2). Core functionality of configuration is to match the initially posed requirements with the module properties. In this process the modules' combinability defines the constraints that have to be fulfilled. Finally, the best out of a set of possible solutions is identified by application of the optimization directives. The most obvious optimization directive in product modularization is minimizing the costs of the configured product.

Result of the product configuration step is a comprehensive description of the configured product, i.e. a Bill of Material with the selected modules. Furthermore, it is advisable to provide working plans for manufacturing and assembly of the product and the modules respectively. This however requires working plans stored in the data base in addition to the above mentioned elements.

Within the consecutive step, the network configuration, the resulting product setup is used as input for computation.

\subsection{Network Configuration}

Similar to the product configuration step, we search a network set-up that optimally meets previously stated requirements, rules and directives within network configuration. Requirements refer to the previously defined product and to customers' preferences, rules state the interdependencies between network segments and directives define how to detect the best out of a set of possible solutions. Obviously, the result of the previous step, i.e. the configured product is incorporated into the network configuration process. Thus, the solution space of permitted network segments is limited to those that are applicable to the previously configured product and its modules.

While product configuration relies on a product model, network configuration requires a network model containing combinable segments (see figure 2). The combinability of the segments has to be described in the network model to ensure that the solution is both complete and feasible. Equally to the product model, the network model contains interfaces between the segments and constraints describing combinability, i.e. which segments can be connected to the respective interface.

Network segments include the supply relation, i.e. supplier and customer, the assigned product module and properties, like transportation and stock policy. Flexibility according to the framework of components and categories (see table 1) is a property of network segments within network configuration as well. The flexibility property is used as an input parameter in the course of network configuration: Depending on the user preferences, some network segments are more or less adequate for network set-up.

The optimization directives in network configuration cover logistic costs, the resulting time of delivery of the product and required network flexibility. The user 
preferences concerning these dimensions are incorporated into the configuration process.

The result of the network configuration step is a complete network that matches requirements, rules and directives and that allows the adequate productions of the previously configured product.

\subsection{Data Base and Software Tool Requirement}

As figure 2 displays, the configuration of product and network can be performed without support of information technology (IT) on basis of expert knowledge. Actually, this is the daily task of product designer and supply chain manager. However, in order to manage product variants and alternative networks, a digital data base and the support of computer software is advisable.

As shown in the product and network configuration sections, integrated configuration requires a model of product and network respectively that consists of components, i.e. modules and segments, their combinability and properties. In conclusion, we propose a data base that contains the elements 'component data base', 'knowledge data base' and 'optimization directives'. The component data base co-vers product modules and network segments respectively and their specific properties. The knowledge database comprises information about component combinability and optimization directives describe how to find the best out of a set of possible configurations.

The component data base of the product represents a list of all available modules and their properties, which are relevant for configuration. These properties include the interfaces available to other modules and their parameters as well as information on costs.

In the knowledge data base of the product, each interface, which has been listed in the component data base, is opposed to any other interface in a matrix. The cells of the matrix contain the bandwidth within the parameters may vary until the interfaces do not fit to each other anymore.

The component data base of the network contains available network segments. As described above, these segments consist of a supply relation, the assigned product module and properties. In detail, these properties are:

- Transportation: Means of transportation, capacity and loading device are defined here.

- Stock policy: Defines stock level and safety level at origin and destination.

- Flexibility: Defines the flexibility of the supply relation according to the framework in table 1.

- Cost and time information: Costs and lead time of the supply relation.

The knowledge data base of the network contains interfaces between network segments. These interfaces constrain combinability of network segments according to their origin (supplier), destination (customer) and properties, e.g. loading device. 
Optimization directives for product and network are mapped within a dataset that contains a brief description of the directive and the stipulated constraints. As mentioned above, these optimization directives concern costs, time of delivery and network flexibility.

Further directives like mandatory service level or local content, i.e. the procurement of parts from local suppliers, can extend the proposed trias of directives. However, this requires further research endeavor on the influencing factors and the expected result.

The data base is an element of integrated configuration that allows a software tool to support product and network configuration. The configuration process of matching requirements and available components, i.e. product modules and network segments, can only be performed by an automated software routine, if a consistent data base is available.

As a result of the integrated configuration process described, a software tool, which can perform the integrated configuration, has to provide the following core elements:

- A data base, which consists of component data base and knowledge data base for both product and network.

- Search algorithms, which allow identifying possible solutions by matching requirements and combinable components.

- Analyzing algorithms, which allow identifying the most adequate out of possible solutions according to stated optimization directives.

Since the presented approach of integrated configuration is complex and innovative, these rough requirements to software are a first estimation that has to be refined in consecutive work.

\section{Conclusions}

Customer expectations regarding costs and time of delivery and high product variety affect production processes as well as the value creation network. Mass Customization is a promising approach to cope with these challenges, because a modular product structure and customized products enhance order processing. Thereby, costs and time of delivery can be reduced.

As manufacturers of complex investment goods depend on specialized suppliers for development, production and repair of product components, a flexible network is crucial in order to satisfy individual customer demands. Thus, network and flexibility requirements have to be considered in modularization endeavors. A method was developed approving the integration of network and flexibility aspects into product modularization.

As networks change frequently, network flexibility has to be considered continuously. The concept of integrated configuration of product and network incorporates 
network flexibility as input parameter into the configuration process. It has been shown, that this concept enables companies to determine the most adequate product and network set-up in order to meet customer demand.

Computer software for the integrated configuration has to map product modules and network segments as well as their combinability and properties in order to implement the concept.

Further developments and advancements of the modularization and configuration concepts presented are advisable. The modularization method must be refined with the aim to measure and integrate all important flexibility components accurately. The modularization technique then has to be supported by software products. The integrated configuration concept has to be enhanced and detailed in order to develop a software tool that supports the automated configuration.

\section{Acknowledgement}

This work has been partly funded by the German Federal Ministry of Education and Research within the national research project "BAU-MO 2008".

\section{References}

Blecker, T. / Abdelkafi, N. / Kreuter, G. / Friedrich, G. (2004): Product Configuration Systems: State-of-the-Art, Conceptualization and Extensions, Proceedings of the Eighth Maghrebian Conference on Software Engineering and Artificial Intelligence, Sousse: May 9.-12., 2004.

Büttner, K. (1997): Rechnerunterstutztes Konfigurieren von Baukastenprodukten, Düsseldorf: VDI-Verlag, 1997.

Doran, D. / Hill, A. / Hwang, K. / Jacob, G. (2007): Supply chain modularization: Cases from the French automobile industry, International Journal of Production Economics, Vol. 106, No. 1, March 2007, pp. 2-11.

Duclos, L. K. / Vokurka, R. J. / Lummus, R. R. (2003): A conceptual model of supply chain flexibility, Industrial Management \& Data Systems, Vol. 103, No. 6, 2003, pp. 446-456.

Ericsson, A. / Erixon, G. (1999): Controlling design variants: Modular product platforms, New York, NY: ASME Press, 1999.

Erixon, G. (1996): Modular Function Deployment (MFD), supportfor good productstructure creation, 2nd WDK Workshop on Product Structuring, Delft: June 3.-4. 1996.

Garavelli, A. C. (2003): Flexibility configurations for the supply chain management, International Journal of Production Economics, Vol. 85, No. 2, 2003, pp. 141-153.

Giannakis, M. (2007): Performance measurement of supplier relationships, Supply chain Management: An International Journal, Vol. 12, No. 7, 2007, pp. 400-411.

Gross, W. / Heimel, J. / Schwab, C. / Kuhn, S. (2007): Towards case-based Product and Network Configuration for Complex Construction Machinery, in: Blecker, T. / Edwards, K. / Friedrich, G. / Hvam, L. / Salvador, F. (eds.): Innovative processes and 
products for mass customization, Berlin: GITO-Verlag, 2007, pp. 121-135.

Helo, P. T. (2006): Product configuration analysis with design structure matrix, Industrial Management \& Data Systems, Vol. 106, No. 7, 2006, pp. 997-1011.

Hölttä, K. / Tang, V. / Seering, W. P. (2003): Modularizing Product Architectures using Dendrograms, Proceedings of the International Conference on Engineering Design 03, Stockholm: August 19.-21., 2003.

Kumar, V. / Fantazy, K. A. / Kumar, U. / Boyle, T. A. (2006): Implementation and management framework for supply chain flexibility, Journal of Enterprise Information Management, Vol. 19, No. 3, 2006, pp. 303-319.

Ladeby, K. / Edwards, K. / Haug, A. (2007): Typology of Product Configuration Systems, in: Blecker, T. / Edwards, K. / Friedrich, G. / Hvam, L. / Salvador, F. (eds.): Innovative processes and products for mass customization, Berlin: GITO-Verlag, 2007.

Lehtonen, T. / Juuti, T. / Pulkkinen, A. / Riitahuhta, A. (2003): Dynamic Modularisation - A Challenge for Design Process and Product Architecture, Proceedings of the International Conference on Engineering Design 03, Stockholm: August 19.-21., 2003.

Mikkola, J. (2001): Modularity and Interface Management of Product Architectures, International Conference on Management of Engineering and Technology PICMET '01, Portland: 2001.

Pahl, G. / Beitz, W. (1996): Engineering Design - A Systematic Approach, Berlin, Heidelberg, New York, London, Paris, Tokyo: Springer, 1996.

Pahl, G. / Beitz, W. / Feldhusen, J. / Grote, K.-H. (2004): Konstruktionslehre - Grundlagen erfolgreicher Produktentwicklung, Berlin, Heidelberg, New York, London, Paris, Tokyo: Springer, 2004.

Pimmler, T. U. / Eppinger, S. D. (1994): Integration Analysis of Product Decompositions, ASME Design Theory and Methodology Conference, Minneapolis, MN, 1994.

Pfohl, H. C. / Buse, H. P. (2000): Inter-organizational logistics systems in flexible production networks: An organizational capabilities perspective, International Journal of Physical Distribution \& Logistics Management, Vol. 30, No. 5, 2000, pp. 388-408.

Sand, J. C. / Gu, P. / Watson, G. (2002): HOME: House Of Modular Enhancement - a Tool for Modular Product Redesign, Concurrent Engineering, Vol. 10, No. 2, 2002, pp. 153-164.

Stevenson, M. / Spring, M. (2007): Flexibility from a supply chain perspective: definition and review, International Journal of Operations \& Production Management, Vol. 27, No. 7, 2007, pp. 685-713.

Stone, R. B. / Wood, K. L. / Crawford, R. H. (2000): A heuristic method for identifying modules for product architecture, Design studies, Vol. 21, No. 1, 2000, pp. 5-31.

Tachizawa, E.M. / Giménez Thomson, C. (2007): Drivers and sources of supply flexibility: An exploratory study, International Journal of Operations \& Production Management, Vol. 27, No. 10, 2007, pp. 1115-1136. 


\title{
Development of Product Configurators Levels of Customisation
}

\author{
Author: Kaj A. Jørgensen
}

\begin{abstract}
Product configuration is an approach for implementing Mass Customisation (MC). With product configuration, each individual product is specified by configuration and the customer then makes decisions about the solution based on a set of available options. Typically, such options are presented by a configurator, which can support the customer in the configuration process. Hence, with such a configurator it is possible to configure multiple individual solutions - perhaps a large set of products.

When a configurator is designed, a large number of design parameters must be considered and balanced decisions must be made. In order to support this decision making, a model for customisation has been developed. This model arranges customisation in four different levels of customisation, ranging from the structure level at the bottom, through the performance level and the value level to the learning level at to top. The model has a dual view on customers at one side and products at the other side and it is developed so that designers must decide how far up in levels the configurator should reach. A specific configurable building, the Pregnant House, is used as the case product.
\end{abstract}

\section{Keywords}

Product configuration, product family model, product configurator, customisation levels.

\section{Address}

Aalborg University, Department of Production

Fibigerstræde 16, DK-9220 Aalborg Øst, Denmark.

Phone: +45 9940 8945. Fax: +459815 3030.

E-mail: kaj@production.aau.dk.

Web: http://www.iprod.aau.dk/ kaj.

\section{Biographical Notes for Kaj A. Jørgensen}

Associate professor at Aalborg University, Department of Production, Denmark. His primary research activities are about product modelling, building modelling, 
product configuration and product family modelling. These research activities are based on previous research on information modelling, information systems and databases.

His teaching activities are related to industrial manufacturing and management and include primarily database systems, development of information systems, product configuration, product family modelling and building modelling.

\section{Introduction}

Mass Customisation (MC) was initiated more than one decade ago as a research topic with Davis' publication "From Future Perfect: Mass Customisation" (Davis, 1989), presenting how products and services could be realised as a one-of-a-kind manufacture on a large scale. Davis also presented the idea that the customisation could be done at various points in the supply chain. In 1993, Pine published a major contribution to the mass customisation literature: "Mass Customization: The new Frontier in Business Competition" (Pine, 1993), (Pine et al., 1993), which was an extensive study of how American enterprises during the seventies and eighties had been overrun by the efficient Japanese manufacturers, which could produce at lower costs and higher quality. Since its introduction, MC has called for a change of paradigm in manufacturing and several companies have recognised the need for mass customisation. Much effort has been put into identifying, which success factors are critical for an MC implementation and how different types of companies may benefit from it (Lampel and Mintzberg, 1996), (Gilmore and Pine, 1997), (Sabin, 1998), (Silveira et al., 2001), (Berman, 2002).

For obvious reasons, there are different strategies on how to implement MC most appropriately and it varies naturally also between different companies, markets and products. Because there is not a single generic strategy, it is important to look at the issue from different viewpoints. The fact that products must be easily customisable in order to achieve MC has been described comprehensively in the literature. (Berman, 2002) and (Pine, 1993) proposed that the use of modular product design combined with postponement of product differentiation would be an enabler to a successful MC implementation. This issue of course also relates to the question of readiness of the value chain.

\section{Mass Customisation and Product Configuration}

An often used approach for implementation of MC is product configuration, in which a series of products is defined by one single model - a product family model (see figure 1) (Jørgensen, 2003). Hence, a product family can be viewed as the set end products, which can be formed by combining a predefined set of modules (Faltings, 1998), (Jørgensen, 2003). In the product family model, it is described, which modules are included in the product family model and how they can be combined. The result of each configuration will be a model of the configured pro- 
duct, configured product model. From this model, the physical product can be produced (see figure 1).

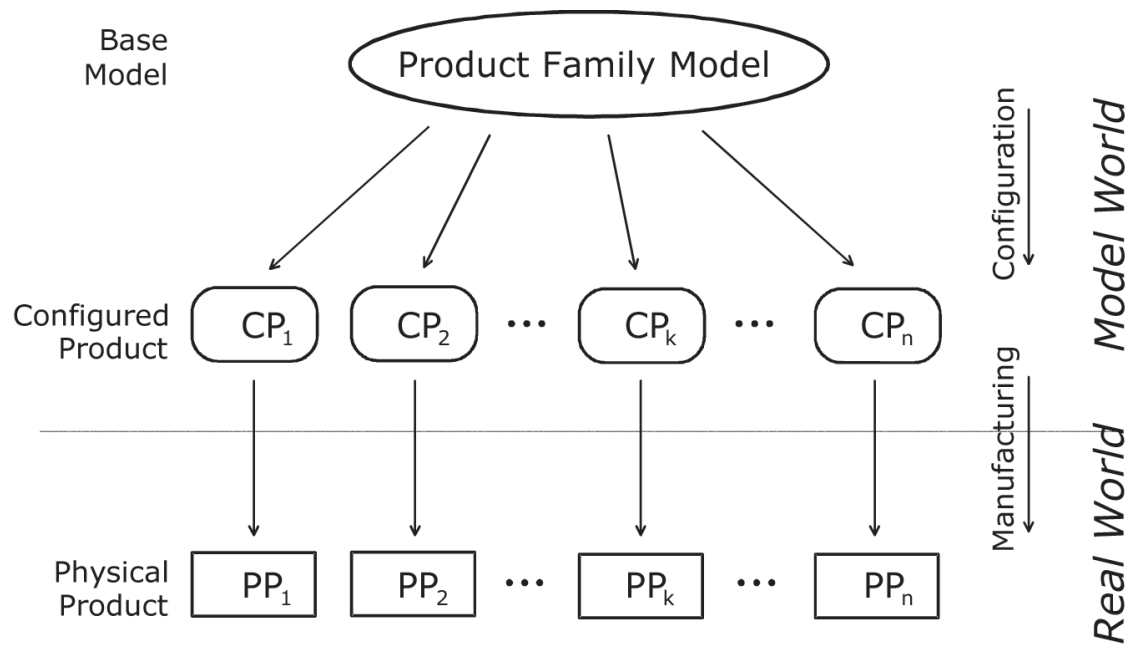

Figure 1 - Product family model as basis for configuration

From time to time, several different methods for defining product family models and product configurators have been proposed, each with their own advantages. A "Procedure for building product models" is described in (Hvam, 1999) based on (Hvam, 1994). It is a rather practical approach with a seven step procedure, describing how to build a configuration system from process analysis and product analysis onto implementation and maintenance. For the product modelling purpose it uses the Product variant master method followed by object-oriented modelling to describe both classification and composition in a product family. The objectoriented approach is also applied by (Felfernig et al., 2001), who uses the Unified Modelling Language (UML) to describe a product family. This is done by using a UML meta model architecture, which can be automatically translated into an executable logical architecture. In contrast to (Hvam, 1999) this method focuses more on formulating the object-oriented product structure, rules and constraints most efficiently. The method also focuses on how the customers' functional requirements can be translated into a selection of specific modules in the product family.

A product family model is often the basis for development of a product configurator. A product configurator can be defined as a tool, computer software, which can support users in the configuration process (Faltings, 1998), for instance by selecting modules to compose products. Hence, with a product configurator, it is possible to configure multiple individual solutions - perhaps a large set of products. 


\section{Application of Product Configuration}

Mass Customisation and product configuration is relevant for many enterprises and great benefits are normally found, where customisation is common and where the idea is introduced gradually. In general, however, the benefits depend very much on the product and the market. In the relationship between the manufacturer and the market or more precisely the product and the customer, the product configurator plays a major role.

A major distinction regarding markets/customers is between business-to-business (B2B) and business-to-consumers (B2C) and an important dimension here is the degree of personalisation. Personalisation is most relevant in relationship with $\mathrm{B} 2 \mathrm{C}$ and a high degree of personalisation towards individual customers or small groups of customers generates special requirements to product configurators but, on the other hand, this also raises new opportunities for increased volume.

Development of computer based configurators provides a range of opportunities for adding new dimensions to the subject and configuration may also add more value to customers. Therefore, when a configurator is designed, a large number of design parameters must be considered and balanced decisions must be made. Many of the parameters are related to development of software systems, e.g. usability, reliability, flexibility and security. Some of these parameters will not be considered in detail in this paper. The primary focus will be on relationship between market/customer and the product and a new model with identification of customisation levels is developed and presented in the following.

\section{Customisation Levels}

Most of the methods, which exist for product family modelling, focus on modelling of the solution space of a configuration process. This means that they describe the possible attributes of the products and the product structure. Hence they do typically not focus on additional information which goes beyond, what must be used to perform the configuration itself. This kind of information, which could include e.g. customer, market, logistics and manufacturing information, is according to (Reichwald et al., 2000) similarly important, since a successful implementation of MC must integrate all information flows in the so called "Information Cycle of Mass Customisation".

In order to support the decision making regarding customisation of products, the following model for customisation has been developed (see figure 2). This model arranges customisation in four different levels of customisation, ranging from the structure level at the bottom, through the performance level and the value level to the learning level at to top. The model has a dual view on customers at one side and products at the other side and it is developed so that configurator designers must decide how far up in levels the configurator should reach. 
Customer

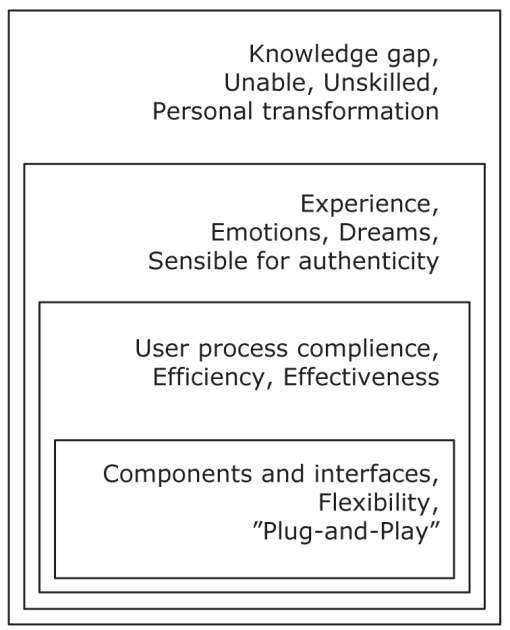

Figure 2-Customisation on four different levels

\section{Product}

Complexity reduction,

Knowledge transfer,

Product $=$ configurator + product

Imaginary attributes,

Communication, Presentation,

Story telling, Service

Functionalities, Attributes,

Integration, Optimisation

Architecture,

Modularity,

Platforms

\subsection{Customisation: Structure Level}

Configuration on the structure level is a rather common view of configuration and is characterised as a matter of acquiring components, which can be used as building blocks - the well known LEGO bricks. Important issues are modularity, interfaces of modules and product platforms. Modules are defined as assemblies of components and end products are composed of modules (see figure 3). Very often, modularity is recommended as a precondition for implementation of product configuration and modules are most preferably identified with clear separation of functionalities, i.e. modularity is in contrast to integration. Further, different architectures of modularity are worth considering.

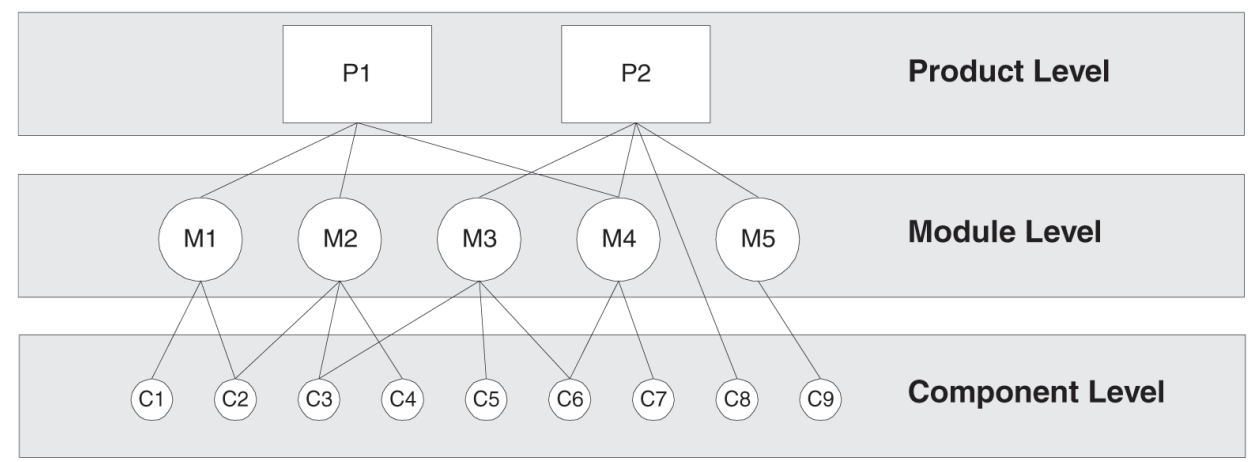

Figure 3 - Model of the structure with the three levels. 


\subsection{Customisation: Performance Level}

On the next level, the performance of products is essential. When products are installed in their user environment, they perform their functions - hopefully in the expected way. Therefore, considerations about the ability to perform the functions, which are required by the customer, are very important and should be a significant subject of configuration. Hence, the focus of product configuration is shifted to identification and definition of product attributes instead of modules and components. This is particularly important in companies, where order horizons are long and where many changes often have to be managed.

Figure 4 gives an overview of how underlying modules/components of an endproduct in a product family can be determined on the basis of decisions regarding attributes.

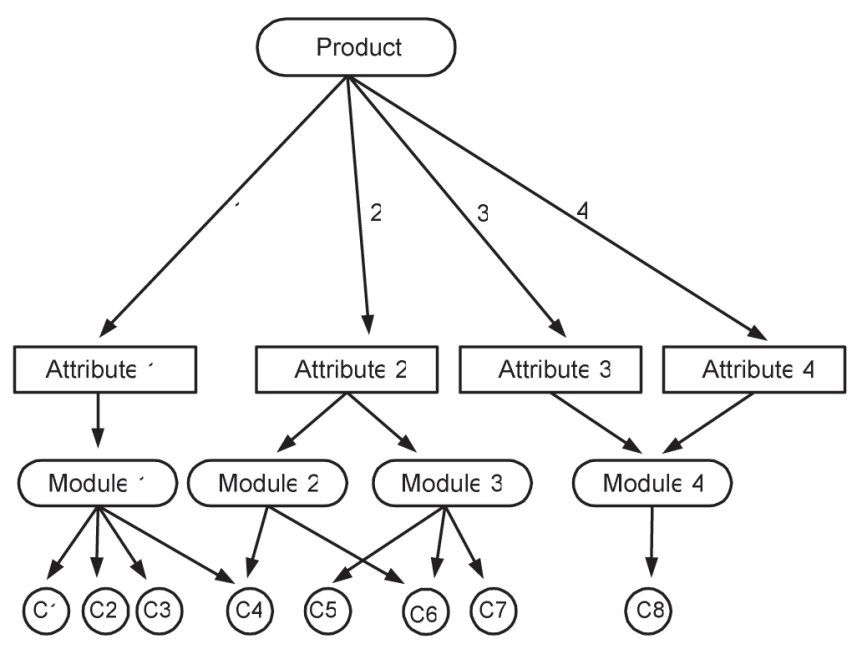

Figure 4-Specification of modules directly or indirectly through functionalities.

Attribute 1 corresponds to one module whereas attribute 2 determines two modules. Further, the figure shows that module 4 is determined by two attributes.

In special situations, products are units, which are not composed of multiple modules, e.g. parametric products, services and software products. In such cases, the end-product is defined by adjusting a set of parameters.

Mapping of functional requirements to specific modules is considered in (Jiao et al., 1998), (Du et al., 2000) and (Männistö, 2001). Jiao proposes to use a triple-view representation scheme. The three views are the functional, the technical and structural view. The functional view is used to describe, typically the customer's functional requirements and the technical view is used to describe the design parameters in the physical domain. The structural view, which corresponds to the structural level described above, includes the mapping between the functional and technical view as well as the rules of how a product may be configured. The descrip- 
tion of this modelling approach is however rather conceptual, and does not easily implement in common configuration tools.

The two lower levels of customisation, the structure level and the performance level, are rather common and widely used with many products and on all types of markets. Further levels of customisation will primarily relate to customers and products with higher degree of personalisation.

\subsection{Customisation: Value Level}

The next level, the value level, focuses on special attributes of products and also on imaginary attributes, which are related to customer emotions and dreams. Involvement in a configuration process will for many customers result in a higher degree of satisfaction and the customer will likely feel a stronger attachment to the solution (Pine and Gilmore, 1999). The value level of customisation is therefore strongly related to personalisation. Hence, customers are primarily individual persons or relatively small groups. Many fashion and service products, for instance, are highly personalised and aim at giving the customer specific experiences. Examples are entertainment, personal care, wellness and travel. Many examples show that configurators for these types of products aim at special values of the products for the customers. But for many customers, ordinary products may be looked at with extra dimensions of personal valuation. Customer's concern for the environment may for instance give more preference for ecologic products.

Because the value level focuses primarily on attributes, Figure 4 can also be used as an illustration of this case. In order to create good support for the value level, it is important that the available options are matched properly with the customer needs and it is important to analyse, what effect different attributes have on customers, whether they are real or imaginary attributes. Many products are presented with images of apparently happy people and admirable locations. An important aspect of this customisation level is authenticity (Gilmore and Pine, 2007). There is a tendency that customers are becoming more sensitive and expect higher and higher quality of goods and services. Practically all consumers desire authenticity. Every person is unique and intimately aware of and valuing his own uniqueness. The consumer sensibility for authenticity evidences itself whenever informed individuals independently purchase any item with which they are intensely involved. According to this theory, many companies fail if they act differently than they announce that they do. In such cases, there may be a great risk that configuration will give a negative effect. If a company claims to be very conscientious, it may very fast loose great respect, if it is disclosed that some products for instance are produced by children and perhaps under poor circumstances.

Means for good configurator support on the value customisation level are to present the perhaps unseen values of products and to provide good and reliable guidance to the user, to display consequences of choices. If the options are limited, 
it is important to be selective regarding customer segments. However, some customers may be intimidated by getting a wrong message. In many cases it is like balancing on a knife edge; if you fall, you may cut yourself.

\subsection{Customisation: Learning Level}

At the top level of customisation, the learning level, the configurator offers services that may result in further impact on the involved customer. At this level, the transformation of the customer is the primary aim. A primary product is available but special aspects of the product lead to a learning process with the customer. Consequently, a further amount of services are added and such services may include a range of subjects that represent a gap between the customer's knowledge and what the product can offer. The lower customisation levels may be identified, i.e. a modular or otherwise configurable product is offered and appealing attributes can be presented, but addition of the learning level can create further attraction from the customer towards the underlying product.

The customer's knowledge gap may be related to different areas. The product may be complex and difficult to understand and assess or it may be difficult for the customer to estimate, how the product can fulfil the requirements. Perhaps the product must fit into complex processes at the customer's site. Maybe the customer is a first time buyer so many issues are new for the customer. Therefore, it should be possible for the customer to find answers to questions about issues, which the customer finds complex. If customers are unable or unskilled to make decisions about such issues, the configurator must include trustworthy guidance. In this way, the configurator is integrated with the product or it can be seen as a part of the product. Like for the previous presented customisation levels, adding such additional features also requires a good segmentation of customers in order not to give a negative effect. Well skilled customers may find this kind of support as a barrier, so it is important that the configurator is able to adjust itself to different customers.

\section{Product Case: Pregnant House}

In this paper, product configuration is primarily aimed at configuration of buildings, i.e. buildings are in this situation regarded as the products. A specific configurable apartment building, the Pregnant House, is used as the case product. The Pregnant House building is extensible and the name pregnant refers to the occasion that, if the family gets pregnant, the house can also get "pregnant".

Customers will be able to select a variety of configurations and also to some degree participate in design of certain solutions. The customers of apartment buildings are typically individuals or families.

Application of configuration to building construction is well known, but development of computer based configurators provides a range of opportunities for adding new dimensions to the subject and configuration may also add more value to customers. 
The Pregnant House has many qualities and possibilities and embraces the best of different types of apartments. It has a unique architecture but, on the other hand, it also supports the demand for individual solutions.

Pregnant House is configurable in multiple ways. The primary architecture includes a ground floor with the possibility to add a tower, which can be one or two stories high (see figure 5). In addition, the width and position of the windows can be changed within some limitations. By this flexibility, Pregnant House offers special possibilities to bring light from the exterior into the rooms.

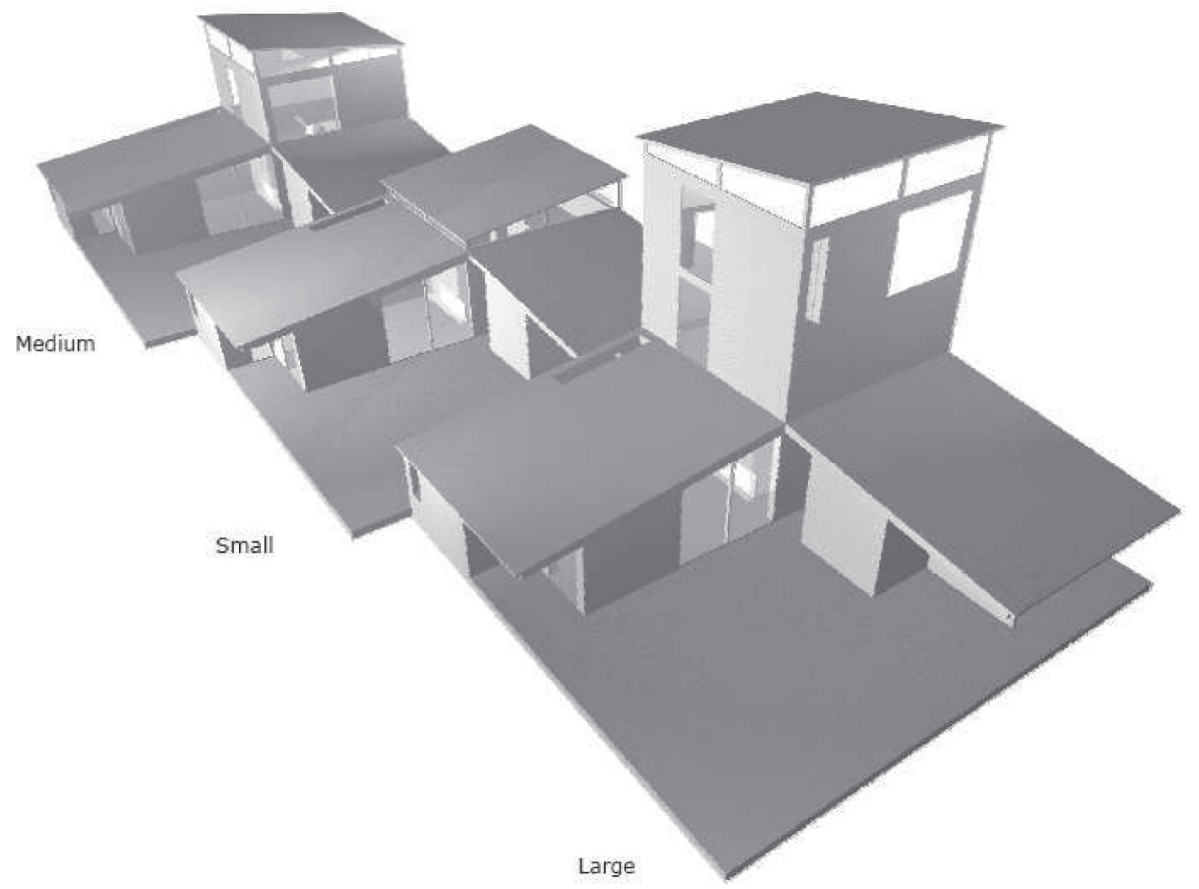

Figure 5 - Three different configurations of Pregnant House

At the facades, it is the intention to offer a range of different materials which, thereby, creates a variety in architecture even though the configurations are standardised. Internally, it is possible to make a number of arrangements of rooms and equipment of rooms (see figure 6). The number of rooms can be selected and rooms can have different size. More over, it is the intention also to add some limited possibilities for customers to design certain details.

With these configuration possibilities, it is the aim with Pregnant House to offer attractive apartment buildings, which can satisfy a larger number of individual needs compared to traditional building types. Especially, the flexibility adds great value to Pregnant House because possible extensions of the building are planed in advance. A consequence of this is that it is possible to have such extensions prepared and ready for economic manufacturing. 

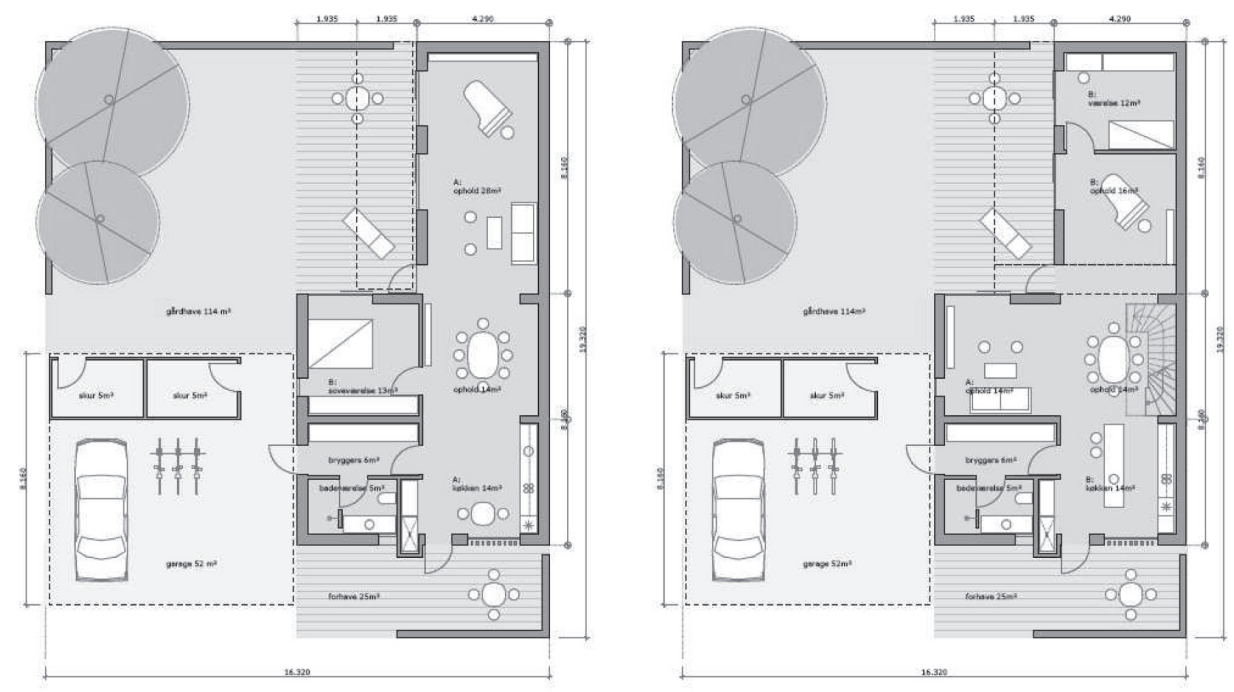

Figure 6 - Different arrangement of rooms on the ground floor of Pregnant House

\section{Pregnant House Configuration}

The Pregnant House case is ideal because there are a reasonable large number of configuration options and it is possible to illustrate all levels of customisation. Apartment houses relate to the $\mathrm{B} 2 \mathrm{C}$ market and, as mentioned, the typical customers are individuals or small groups like families. Hence, a considerable degree of personalisation is also present.

The structure level of customisation relates to the organisation of construction components and, in fact, it is the intention to prepare the Pregnant House for industrial manufacturing. Many design issues has been related to the division of the building in order to get the most appropriate sections for manufacturing, transportation, assembly, etc. In addition, each section may be configured differently prior to the manufacturing. These configurations cover a number of areas like room arrangement, selection of interior, selection of coverings, selection of colours and selection of various building services. Segmentation of the building includes special development of assembly components and methods.

At the performance level, a number of attributes can be identified in response to the customer needs. These attributes include the size of the living space, the selection of types of rooms, the size of the rooms, the orientation of the building on a given site, the arrangement of windows and how it is drawing light from outside into the rooms. If customers, in accordance with the model for customisation (figure 2), focus on these attributes, it will be possible to generate automatically the selection of modules of the building. The customers do not need to know about the segments and will expect that the underlying structure is unified and optimised. 
At the value level, a number of issues are also relevant. First of all, the thoroughly prepared architecture should make strong appeal to many customers and should support the creation of good bonds between the customer and the product. Secondly, the well planed options for extending the building should draw significant attention. In addition, it should be valuable for many customers that it is possible to specify a large number of different configurations without substantial increase of cost. Further attributes includes quality, usability, maintainability, serviceability, etc. Normally, the location of the building is a very important attribute, but this is considered out of scope of this project.

At the learning level, the Pregnant House project has also a number of excellent examples. A building is in many ways a complex product, which can be difficult to manage in an optimal way. Especially for customers, who are buying or renting for the first time, a number of issues may be complicated to understand and to decide about. A number of authorities have requirements and regulations about buildings, so what are the constraints and what rights does the owner or the tenant have? Operation and maintenance of buildings must be performed adequately, but there are many alternative methods and it may be difficult to choose an appropriate solution. Financing may also be complicated and in order to develop budgets it may be difficult to calculate economic estimates regarding e.g. heating expenses, maintenance expenses, financial payments, tax payments, etc. Support for customisation on this level must include the possibility to be guided sufficiently about such issues and many potential customers may learn from it. However, it is important that it is presented as extra features, which may be used on demand. No doubt such features may attract and appeal to certain customers but may be irrelevant for others.

Based on the model for customisation and the identified levels in relationship with Pregnant House, a development of a configurator has been initiated. As part of the development approach, a product family model (see figure 1) is structured by following the ideas presented in (Jørgensen, 2006). The model will be independent on any development tool and differs some from the approach described in (Hvam, 1999). The primary content is the identified set of model component types and the identification of these types is performed by classification and related to each other in a hierarchical structure - the taxonomy. Relations about the structure are modelled afterwards. The model component types include the different types of rooms and the types of building components but also component types available for description of the customers. When this model is completed and the user interface is designed, the detailed development of the configurator can be performed.

\section{Conclusion}

Implementation of Mass Customisation (MC) by using product configuration provides the possibility that each individual product is specified by a configuration process, where customers make decisions about the solution based on a set of avai- 
lable options. It is thereby possible to configure multiple individual solutions. Product configuration can be applied to many products and many markets or customers and the development of configurators will depend on these application areas. A major distinction regarding markets/customers is between business-to-business (B2B) and business-to-consumers (B2C). An important dimension here is the degree of personalisation because a high degree of personalisation towards individual customers or small groups of customers generates special requirements to product configurators. Implementation of such requirements, however, may also raise new opportunities for increased volume.

Development of configurators, which can offer a good support for configuration processes and produce well specified individual product models, is a great challenge and, when a configurator is designed, a large number of design parameters must be considered and balanced decisions must be made. In order to support this decision making, a model for customisation has been developed and presented above. The model arranges customisation in four different levels of customisation, ranging from the structure level at the bottom, through the performance level and the value level to the learning level at to top. The model includes a dual view on customers at one side and products at the other side. Designers must decide how far up in levels the configurator should reach.

In this paper, product configuration is primarily aimed at configuration of buildings, where the customers typically are individuals or families. A specific configurable building, the Pregnant House, is used as the case product. Customers will be able to select a variety of configurations and thereby to some degree participate in design. Application of configuration to building construction is well known, but development of computer based configurators provides a range of opportunities for adding new dimensions to the subject. With specific address to the Pregnant House case, it is argued that the presented model for customisation on different levels can add more value to a product and make it more attractive for customers to select.

\section{References}

Berman, B. (2002). Should your firm adopt a mass customization strategy? Business Horizons, 45(4):51-60.

Davis, S. (1989). From future perfect: Mass customizing. Planning Review.

Du, X., Jiao, J., and Tseng, M. M. (2000). Architecture of product family for mass customization. In Proceedings of the 2000 IEEE International Conference on Management of Innovation and Technology.

Faltings, Boi and Freuder, Eugene C. (Ed.): Configuration - Getting it right. Special issue of IEEE Intelligent Systems. Vol.13, No. 4, July/August 1998.

Gilmore, J. and Pine, J. (1997). The four faces of mass customization. Harvard Business Review 75 (1).

Gilmore, J. and Pine, J. (2007). Authenticity. Harvard Business School Press. 
Hvam, L. (1999). A procedure for building product models. Robotics and Computer-Integrated Manufacturing, 15:77-87.

Jiao, J., Tseng, M. M., Duffy, V. G., and Lin, F. (1998). Product family modeling for mass customization. Computers \& Industrial Engineering, 35:495-198.

Jørgensen, Kaj A. (2003): Information Models Representing Product Families. Proceedings of 6th Workshop on Product Structuring, 23rd and 24th January 2003, Technical University of Denmark, Dept. of Mechanical Engineering.

Jørgensen, Kaj A. (2006): Product Modelling on Multiple Abstraction Levels. In: Mass Customization, Challenges and Solutions, pp. 63-84, Springer Verlag, 2006.

Lampel, J. and Mintzberg, H. (1996). Customizing customization. Sloan Management Review, 38:21-30.

T. Männistö, H. Peltonen, T. Soininen and R. Sulonen (2001): Multiple Abstraction Levels in Modelling Product Structures. Data and Knowledge Engineering no.36, pp.55-78, 2001.

Pine, B. Joseph (1993): Mass Customization - The New Frontier in Business Competition. Harvard Business School Press, Boston Massachusetts, 1993.

Pine, J., Victor, B., and Boyton, A. (1993). Making mass customization work. Harvard Business Review 71 (5), 71(5):108-119.

Pine, J. and Gilmore, J. (1999). The Experience Econmy : Work Is Theater \& Every Business a Stage.

Reichwald, R., Piller, F. T., and Möslein, K. (2000). Information as a critical succes factor or: Why even a customized shoe not always fits. In Proceedings Administrative Sciences Association of Canada, International Federation of Scholarly Associations of Management 2000 Conference.

Sabin, D. and Weigel, R. (1998): Product Configuration Frameworks - A survey. In IEEE intelligent systems \& their appplications, 13(4):42-49, 1998.

Silveira, G. D., Borenstein, D., and Fogliatto, F. S. (2001). Mass customization: Literature review and research directions. Int. Journal of Production Economics, 72:1-13. 


\title{
Developing appropriate metrics to measure supplier load in Mass Customization and high variety environments
}

\author{
Lorena Scarpulla, Bart MacCarthy, Philip Brabazon
}

\begin{abstract}
A business strategy focused on Mass Customization implies that the demand for different product variants is less predictable than in a conventional operational environment where production is based largely on forecasts. A Mass Customization strategy can also imply a potentially greater number of distinct product variants than would occur in a conventional forecast-driven production system. In this paper we consider how to measure the load placed on the supply base in Mass Customization and high variety environments. We examine metrics that include the level of variety, the stability and degree of change in the plan and the amount of time given to the supplier to respond. A number of metrics are proposed incorporating these aspects. The metrics are tested in a simulation environment. The implications for supplier flexibility are discussed.
\end{abstract}

\section{Keywords}

Mass Customization, Product Variety, VBTO, Flexibility, Uncertainty measure 1 Introduction Mass Customization requires process configurations and operational strategies that enable flexibility in meeting variable demand (MacCarthy et al, 2003). In particular, the flexibility of the supply base to respond to variable demand must be addressed. A business that customizes products on a mass scale needs to ensure that its supply base can respond flexibly and quickly. Despite the importance of supply base responsiveness as an enabler for Mass Customization, measures to evaluate the load placed on the supply base have not been reported in the literature. More generally, product variety is increasing across sectors (Bils and Klenow, 2001) and thus the burden placed on suppliers to respond flexibly is increasing.

Here we propose measures to evaluate supplier load and test them in a simulation environment. The behaviour of the supplier load metrics under different experimental conditions is analysed. Desirable features for effective supplier load metrics are considered with respect to different types of supply base flexibilities (D'Souza and Williams; 2000). The significance of the work for Mass Customization and high variety environments is discussed and recommendations are made for further work in different producer-supplier contexts.

The paper is organized as follows. In the next section relevant literature is reviewed. Then the concept of supply load is introduced, the main elements impact- 
ing on it are highlighted and measures to evaluate it are proposed. In section 4, the simulation environment is described and adaptations of the generic metrics for the simulation are given. Illustrative experimental results are shown in section 5 . The implications for supplier flexibility and the limitations of the present work are discussed in section 6, while conclusions and indications for further work are presented in the last section.

\section{Literature review}

Managers understand how critical variety is to add value to their product offerings (Zipkin, 2001). A careful approach to product variety management is an essential condition to improve long term profitability (Ramdas; 2003). Offering a wider range of options to customers and ensuring that all are available 'off the shelf' may be very costly and infeasible in some situations. Furthermore, a strategy to increase variety should be pursued only after obtaining a better understanding of target customer needs i.e. the marketing and production strategies need to be aligned.

As noted by (Pine et al; 1995), customers often do not want more variety but want more precision in satisfying their specific requirements. Mass customization has thus emerged as a competitive strategy relevant to a number of industries, as different as computer assemblers and bicycle manufacturers. It potentially allows the customization of products for individual customers in high volumes at low producer cost (Gilmore and Pine, 1997; MacCarthy et al., 2003). Different classifications have been proposed for Mass Customization and its implementation with respect to the stage in the value chain where customization happens and the level of product customization offered (MacCarthy et al, 2003).

Modular product design, postponement (Feitzinger et Lee; 1997), part commonality (Fisher et al; 1999), product family-based design (Tseng et al; 1996), have all been suggested as enablers of Mass Customization. More generally, greater demand variability, shorter life cycle of products and technologies, shorter delivery times, together with increased variety and customization characterize today's competitive scenarios. All of these factors require consideration of operational flexibility (De Tony and Tonchia, 2001).

Mix and volume flexibilities have been suggested as a means to achieve Mass Customization. Mix flexibility allows the customers to be offered products that are closer to their desired specifications, while volume flexibility keeps the efficiency level, and thus costs, near to Mass Production settings (Zhang and al; 2003). Although widely recognized as a competitive priority, it is still difficult to find a fully recognised framework for flexibility definitions, types, measures and enablers.

Agreement exists that flexibility is a multi-dimensional concept and that it can be realized in different ways depending on environmental and strategic factors. Suarez et al., $(1991,1995,1996)$ distinguish "first order" flexibilities - directly visible to customers, and thus affecting a firm's competitive position, namely mix, volume, 
new product and delivery time flexibilities - and lower order flexibilities such as routing flexibility, that are enablers of the former, albeit not directly visible to the customer. First order flexibilities are also important to define the competitive position and the relationship of intermediate firms that sell goods to other firms. In this case it is suggested (Suarez et al., 1991) that end-product characteristics also influence intermediate firms' flexibility requirements - the type and level should be chosen after evaluating the product and operating policy of the downstream node.

Much of the focus on the flexibility and responsiveness has related to the manufacturing firm (Lummus et al; 2003), while less attention has been devoted to the effort required in the supply chain to enable a firm to satisfy its customers. Although mentioned in previous work (Suarez et al., 1991), the role of suppliers as flexibility enablers has been mainly neglected in the literature. More recently, increasing attention on a firm's external environment has forced consideration of flexibility issues in a wider way, in terms of which level of flexibility the supply chain is able to provide in its current settings, in terms of network robustness (Ferdows, 1997) and in the ability to reshape the whole supply chain to better match customers' requirements (Stevenson et al, 2007). This is particularly important if a manufacturer is pursuing a Mass Customization strategy, since "mass customization often means higher volatility in the demand for different types of components" (Westbrook et al; 1993) and thus requires an increased effort for suppliers to cope reliably with incoming orders. In the work reported here we investigate ways of measuring the actual requirements that the manufacturing approach imposes on the supply base in order to highlight the most appropriate types and levels of flexibility required by suppliers.

\section{Supply load measures - proposition}

When analysing the impact of a manufacturer's requirements on a supplier's flexibility, we are interested in the supplier's external flexibility i.e. the flexibility perceived by its customer- the manufacturer itself. The main elements of such flexibility have been suggested to be volume, mix and delivery flexibility. Uncertainty and variations have been identified as the main factors requiring flexibility (Correa and Slack; 1996). Here we consider the variety of products required and the uncertainty in the requested orders as the main elements to be taken into account in evaluating the load on supplier. Furthermore, flexibility has not only a range dimension, but also a response dimension defined as 'the ease (in terms of cost, time, or both) with which changes can be made within the capability envelope' (Slack; 1987). We include in our measure the time element to take into account this response dimension (De Toni and Tonchia; 1998).

We propose that the load imposed by a manufacturer on its supply base can be evaluated as a function of (1) the variety of products required, (2) the uncertainty and changes in the requirements and (3) the time available to the supplier to respond to requirements. 
To evaluate the level of variety we can count the number of different products that may be required. This approach is simple and relevant where products show low complexity level (Da Silveira; 1998). In this study we will define variety as the number of options that the manufacturer can produce.

The order fulfilment time is the time span from order receipt by the supplier to the delivery at the manufacturing plant. The order fulfilment time should be smaller than the manufacturer's planning horizon, otherwise the supplier would not be able to deliver the requested components on time. The time available to the supplier is more critical when the supplier's order fulfilment time approaches it. Thus, we use the ratio of the order fulfilment time and the manufacturing planning time to take into account the time factor.

The most challenging element to capture and evaluate is the uncertainty and degree of change in the stream of orders received by the supplier from the manufacturer. The instability of the planned orders should be carefully evaluated, since 'instability is usually passed and amplified in the next tier of supplier' (Inman et al; 1997). The definition of this uncertainty measure is described in the following section.

The supply load is expected to increase as variety increases and as change increases, and to diminish as the time available to fulfil the order increases.

Thus a relationship between the principal variables may be stated as follows:

$$
\text { SupplyLoad }=v * c^{\alpha} *\left(\frac{f}{t}\right)^{\beta}
$$

where $v$ is the variety requested from the manufacturer, $c$ a measure of uncertainty and change, $f$ the order fulfilment time of the supplier and $t$ the time given to the supplier to respond (from order receipt to delivery date). $\alpha$ and $\beta$ are user-defined parameters explained below.

Since our aim is to understand the impact on flexibility requirements of different manufacturing policies and approaches, we can consider the previous expression with respect to the extreme conditions that different policies may generate. Let $V$ be the maximum level of variety that may be required and $C$ the maximum theoretical level of change that could occur, we can calculate Supply Load as

$$
\text { SupplyLoad }=\frac{v}{V} *\left(\frac{c}{C}\right)^{\alpha} *\left(\frac{f}{t}\right)^{\beta}
$$

In theory, this expression provides a measure between 0 (lowest load) and 1 (highest load). 
Choosing different values for the parameters $\alpha$ and $\beta$ allows the impact of 'uncertainty and change' and 'time' to be weighted according to the specific features of the environment and the supplier production and order management system. Modifying the value of $f$ allows different classes of supplier subject to different time constraints to be taken into account. An empirical investigation of the conditions that are considered by managers as important in terms of the load imposed on suppliers could be a way to calibrate these coefficients in different contexts.

\subsection{Uncertainty evaluation}

The uncertainty evaluation is particularly challenging because we are interested in a measure taking into account both the average level of uncertainty and the most extreme conditions that may be experienced. We can approach the problem in terms of deviation from what the supplier is expecting to provide according to a forecast or in terms of stability of the orders placed in different instants of time and evaluate uncertainty as some combination of the two.

Looking at a sequence of orders for which a supplier may have to respond, an intuitive way to evaluate the uncertainty is to consider the most extreme conditions the supplier has to face, that is the maximum difference of the quantity ordered for each variant.

We can see it graphically in Figure 1, showing the quantities ordered for nine different variants in ten orders, where the upper black line represents the highest quantity ordered for each variant across the ten orders, while the lower dashed lines represents the lowest quantity required for each variant across all the orders.

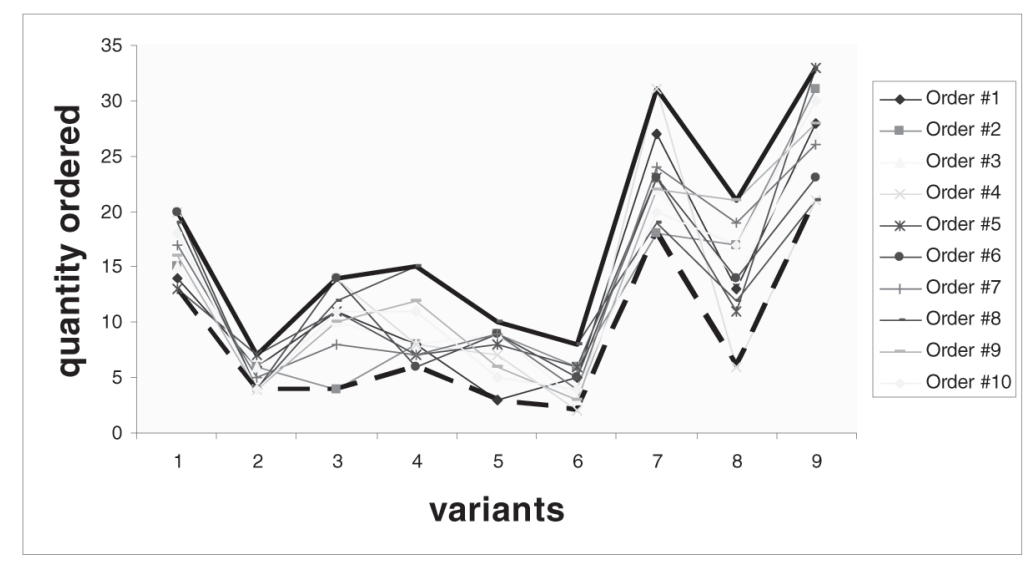

Figure 1: Example of composition of a sequence of orders

Let:

$O$ be the total number of orders placed by the manufacturer in the relevant time horizon 
$o=1, \ldots, O$ be the index of the order placed

$v$ be the variety level currently offered by the manufacturer (assumes a fixed upper limit)

$i=1, \ldots, v$ be the index of the different variants ordered

$n_{i}{ }^{\circ}$ be the number of items of variant $i$ requested at order $o$

$d_{i}$ be the forecast of the number of items of variant $i$ required at each order (we assume this value is constant for all orders in the time horizon)

$Q$ be the total number of items ordered in each order; we assume this value is constant across the orders and equals to the sum of the forecasts for all the variants offered:

$$
Q=\sum_{i=1}^{v} d_{i}
$$

The difference

$$
A_{i}=\max _{o}\left\{n_{i}^{o}\right\}-\min _{o}\left\{n_{i}^{o}\right\}
$$

is the maximum difference experienced by the supplier among all orders for variant $i$. Summing for all the variants we get the total maximum difference

$$
A=\sum_{i=1}^{v} A_{i}
$$

which is the difference between the highest and the lowest curve in Figure 1. Dividing for the overall volume required, gives a measure not depending on the volume required and interpretable as the percentage of variant volume variation that the supplier should be able to cope with.

$$
C_{1}=\frac{A}{Q}
$$

The interpretation of this measure is straightforward: the smaller the value of $\mathrm{A}$, the smaller is the deviation along all the orders placed to the supplier, meaning that the ability of the supplier to potentially provide extremely high or low volumes for each variant can be smaller. The theoretical maximum values of this measure is $\mathrm{v}^{1}$.

In considering only the extreme conditions, this measure fails to take into account some aspects of instability in the orders requested i.e. the average change in variant quantities during the time considered (Kadipasaoglu et al; 1995). A different way to address the issue is to compare each variant quantity ordered with the expected (forecasted) quantity, $d_{i}$. The average deviation for variant $i$ is: 


$$
D_{i}=\frac{\sum_{o=1}^{o}\left|n_{i}^{o}-d_{i}\right|}{O}
$$

Summing for all variants gives the average total deviation per order,

$$
D=\sum_{i=1}^{v} D_{i}
$$

and dividing it by the volume ordered gives a percentage of average deviation.

$$
C_{2}=\frac{D}{Q}
$$

The maximum value 2 of $\mathrm{C} 2$ is

$$
\frac{2\left(Q-\min _{i} d_{i}\right)}{Q} \approx 2
$$

Another important element that could be taken into account is the number of setup changes that the supplier may incur at each order (Jensen; 1993). If we suppose that a setup is incurred each time a variant is manufactured at order o when it was not manufactured at order $o$ - 1 , the total number of setup changes in one order can be computed, summed for all orders and divided for the numbers of orders, giving the average number of setups per order. The ratio between the actual number of setups and the maximum number of setups changes may then be used as a measure of instability. We have not included the setup metrics here because of space limitations.

\section{Simulation Environment}

The proposed supply load measure has been tested using a simulation model. The simulation environment considers a producer with a dynamic planning pipeline that is fed with a distribution of product variants, which then feeds a stock of finished products. Customer demand is satisfied in one of three ways - fulfilment from stock, fulfilment from the planning pipeline and fulfilment by feeding a required variant into the pipeline plan (a Build-to-Order or BTO product). Stock

\footnotetext{
${ }^{1}$ This value is experienced in the theoretical situation where for each variant $\mathrm{i}^{*}$ there is at least an order $\mathrm{o}^{*}$ where $\mathrm{n}_{\mathrm{i}}^{* \mathrm{o}^{*}}=\mathrm{Q}$ and for all the other $\mathrm{i} \mathrm{n}_{\mathrm{i}}^{\mathrm{o}^{*}}=0$ and an order $\mathrm{o}^{* *}$ where $\mathrm{n}_{\mathrm{i}}{ }^{\mathrm{o} \mathrm{o}^{* *}}=0$

2 This value would be experienced if, for all the orders, the only variant ordered were the one showing the smallest forecasted value.
} 
fulfilment is the preferred method, followed by pipeline fulfilment and then by BTO. At each instant one new item is fed into the pipeline and one leaves to be assembled for a particular customer or to add to the stock of unsold products. This makes the pipeline volume constant and equal to the pipeline length. We test the supply load measures in a Virtual-Build-To-Order system (Brabazon and MacCarthy; 2006). This system can allow pipeline searching and reconfiguration as additional ways to satisfy customers' orders (Figure 2).

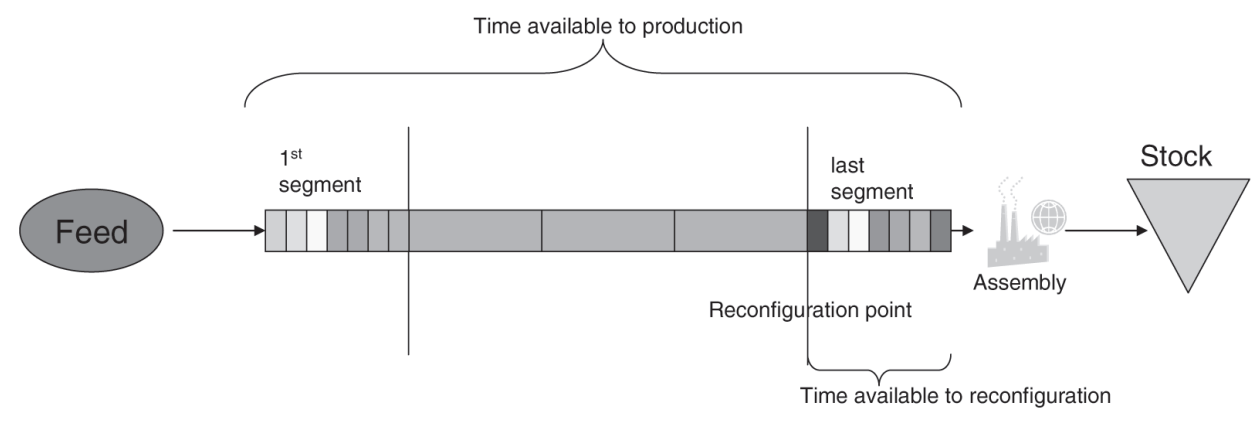

Figure 2: The VBTO system

The system model is thus the manufacturing planning process starting with the decision about which item should be fed into the pipeline and ending in the stock of products. We consider the pipeline split into a number of segments each of the same length with a reconfiguration point set at the beginning of the last segment. When a product arrives that is unallocated at the reconfiguration point, its specifications may be reconfigured to a product matching or closer to an incoming customer's needs. We assume that an order is sent to the supplier for all the items in the first segment of the pipeline at the beginning of the planning process. Thus the order volume is equal to the segment length. In the rest of the paper we will refer to the first segment composition as the order composition.

In this system we can evaluate a 'long term supplier load' due to the variability of the orders launched at the beginning of the pipeline and a 'short term supplier load' due to reconfiguration at the reconfiguration point. We assume that the delivery of an order is due at the end of the pipeline at the assembly facility (i.e. a short time is required to realize assembly and is negligible compared to the pipeline length) thus providing the supplier with the whole pipeline length as time available for 'ordinary delivery' and the last segment length as time available for 'reconfiguration delivery'. We want to evaluate the load imposed on the supplier as a consequence of the variability of orders launched in the long run and the modification of the order due to reconfiguration in the short run.

The simulation environment allows the different supplier load metrics to be investigated under a range of experimental conditions and parameter settings includ- 
ing: different levels of variety; whether or not fulfilment from the pipeline is allowed; whether or not variant reconfiguration is allowed in the pipeline; different pipeline control policies; different demand scenarios. Illustrative results are presented in section 5 .

The time available to the supplier is the pipeline length in the long run and the segment length in the short run; both of these are parameters that can be set in the model and thus are inputs to the simulation. The variety level is also a parameter of the simulation. The effect of changing the level of variety and/or the time available to the supplier can be evaluated using the simulation model. To evaluate the uncertainty in the long run we use the metrics discussed in the previous paragraph. We use as forecasts value di the mix of items computed, drawing the probability from the beta distribution used to feed the pipeline.

Defining $p$ as the pipeline length, $s$ as the segment length (equal to the order volume), $v$ as the variety level in a simulation run, and $\mathrm{V}$ as the maximum variety level, then the long term supply load for the simulation model is:

$$
\text { SupplyLoad }=\frac{v}{V} *\left(\frac{c}{C}\right)^{\alpha} *\left(\frac{1}{p}\right)^{\beta}
$$

To evaluate the uncertainty in the short run, some further considerations are required to adapt the generic measures proposed in section 3 . Since the reconfiguration is the modification of a single order, the most onerous condition for the supplier is the maximum amount of reconfiguration experienced in a single order.

For each order the number of reconfigurations is calculated comparing the distribution of variants before and after the reconfiguration point; the absolute value of such differences are summed for each variant in order to obtain the total amount of reconfiguration. As defined above, no $i$ is the number of items of variant $i$ fed into the first segment at order o. Defining ni o,aft as the number of items of variant $i$ present in the last segment corresponding to order $\mathrm{o}$, then the maximum amount of reconfiguration is:

$$
\text { reconamount }=\max _{o}\left\{\sum_{i=1}^{v}\left|n^{o}{ }_{i}-n_{i}^{o, a f t}\right|\right\}
$$

The maximum theoretical level of reconfiguration calculated as stated is equal to twice the volume processed in the last segment, $s$.

A measure of uncertainty due to reconfiguration is the reconfiguration ratio $r r$ - the ratio of reconamounut and twice the volume produced in the segment, a value is between 0 and 1 . 


$$
r r=\frac{\max _{o}\left\{\sum_{i=1}^{v}\left|n_{i}^{o}-n_{i}^{o, a f t}\right|\right\}}{2 s}
$$

The supply load expression in the presence of reconfiguration is thus:

$$
\text { SupplyLoad }=\frac{v}{V} * r r^{\alpha} *\left(\frac{1}{s}\right)^{\beta}
$$

where $s$ is the time between the reconfiguration point and order delivery, which is the time available to the supplier to satisfy the reconfiguration request.

Different values may be used for the variety parameter in the previous formula: the total variety as an input of the simulation; the average/maximum variety expressed in a single order (because the supply load measure is local, referring to what happens in a single order); the variety experienced in the order requiring the highest level of reconfiguration; the number of variants present in the last segment and not present before (for which a set up is thus required).

For the reconfiguration load it is useful to evaluate the average level of reconfiguration to understand the types and levels of flexibility that may be required. The calculation is straightforward:

$$
\text { averagerecon }=\frac{1}{O} \sum_{o=1}^{o} \frac{\sum_{i=1}^{v}\left|n_{i}^{o}-n_{i}^{o, a f t}\right|}{2 s}
$$

\subsection{Experimental settings}

The simulation has been run using a pipeline split in four segments with two values for pipeline length, 128 and 256, giving segments of length 32 and 64 respectively. The reconfiguration point is set at point 96 and 192 respectively. The parameters settings are influenced by typical scenarios in the automotive sector, which have motivated this study. Long simulation runs have been conducted to get reliable estimates for the load measures.

The experimentation investigates both a closed pipeline and an open pipeline. Two different methods of pipeline feeding are tested (random feeding and selection of the products with the largest probability of stockout), and two levels of variety are considered, namely 8 and 16. For each pair of pipelength and variety, 8 scenarios are investigated: 


\begin{tabular}{|c|c|c|c|}
\hline \multicolumn{4}{|c|}{ Pipelength_Variety } \\
\hline & Feed & closed pipe & open pipe \\
\hline No rec & random & 1 & 2 \\
\hline & stockout & 3 & 4 \\
\hline Rec & random & 5 & 6 \\
\hline & stockout & 7 & 8 \\
\hline
\end{tabular}

Table 1: Experimental settings

Three different pairs of values are used for the coefficients a and b, to highlight situations where variety, uncertainty and time respectively are the most onerous elements to cope with.

\begin{tabular}{|c|c|c|}
\hline High weight to & $\alpha$ & $\beta$ \\
\hline Variety & 0.15 & 0.04 \\
\hline Change & 6.73 & 0.28 \\
\hline Time & 1 & 6.09 \\
\hline
\end{tabular}

Table 2: Coefficients values

\section{Results}

Because of space limitations, only a small selection of results can be presented here. To give a comprehensive overview of the metrics behaviour we will focus only on long term supply load.

Figure 3 shows the percentage of volume variation, $C_{1}$ for all the simulated settings. Each curve represents a specific combination of pipelength and variety level, while each $\mathrm{x}$ axis setting represents one of the eight experimental conditions given in Table 1

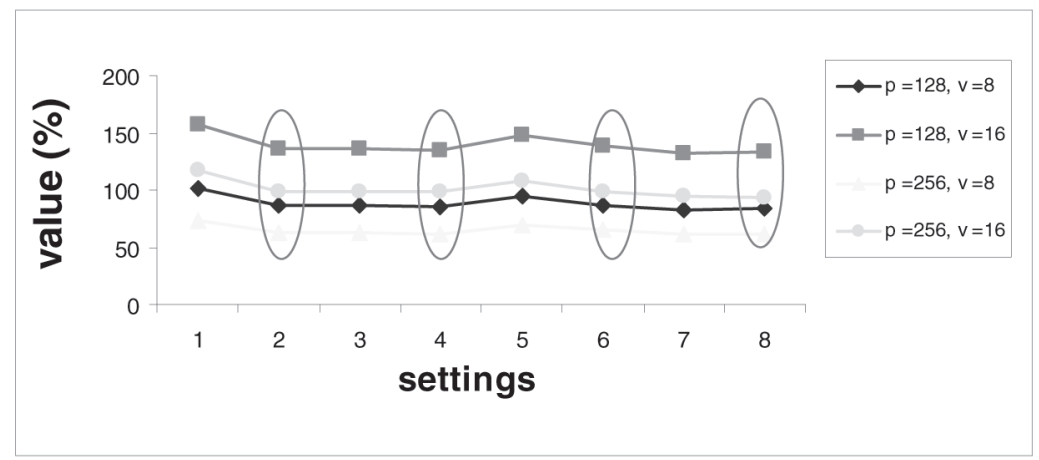

Figure 3: Percentage of volume variation 
The two upper curves are obtained with variety 16 and the lower two with variety 8 . In both cases, the shorter pipeline length gives a higher value of the measure. The curves do not overlap, showing that primarily variety and then pipeline length are the main elements impacting on this measure of change, while only minor effects are due to the feed and allocation policies followed by manufacturer. Of the three manufacturing options allowed in the simulation, namely allowing the reconfiguration, using an intelligent feeding and opening the pipeline, the one with the biggest impact is the latter. The settings where allocation from the pipeline is allowed, circled in Figure 3, usually perform no worse and usually slightly better than their counterparts.

To understand how the measure performs, let us compare two settings where the measure shows respectively a low and a high value. For example with a closed pipeline, reconfiguration allowed and random feeding (setting 5), Figure 4a and Figure $4 \mathrm{~b}$ show the results in the extreme situations of a pipeline length of 256 and variety 8 and a pipeline length of 128 and variety 16, respectively.

a

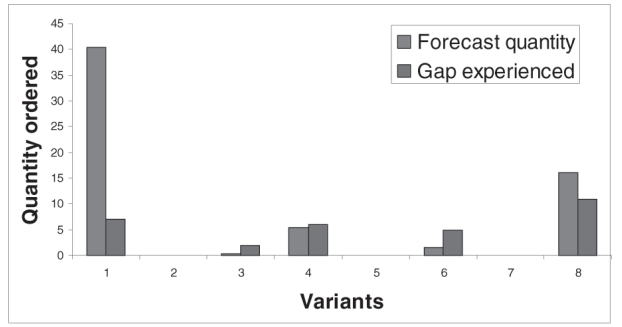

b

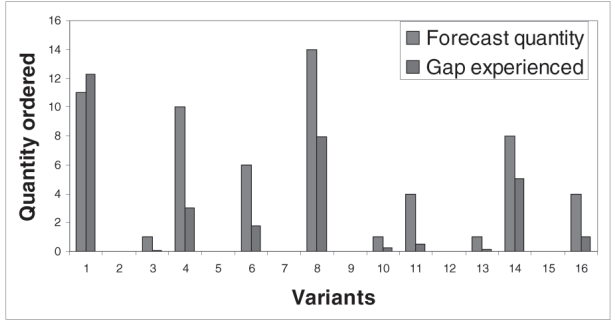

Figure 4: Order deviation as variety increases

Both graphs show in red the forecast volume, constant along all the orders, and in blue the maximum gap experienced in a series of 16 orders for each variant, what we have previously defined Ai. The first graph shows a low deviation compared to the forecast volume resulting in a low level of $\mathrm{C}_{1}$ measure, while the second graph shows a higher relative gap, revealed by the higher value of the measure.

Using percentage of volume variation as a measure of change, Figure $5 \mathrm{a}$ and Figure $5 \mathrm{~b}$ show the value of Supply Load obtained with two different pairs of coefficients $a$ and $b$, as suggested in Table 2 . Figure 5 a shows the situation where variety is the most highly weighted element, while for Figure $5 \mathrm{~b}$ greater is weight is given to time, and thus pipelength. 

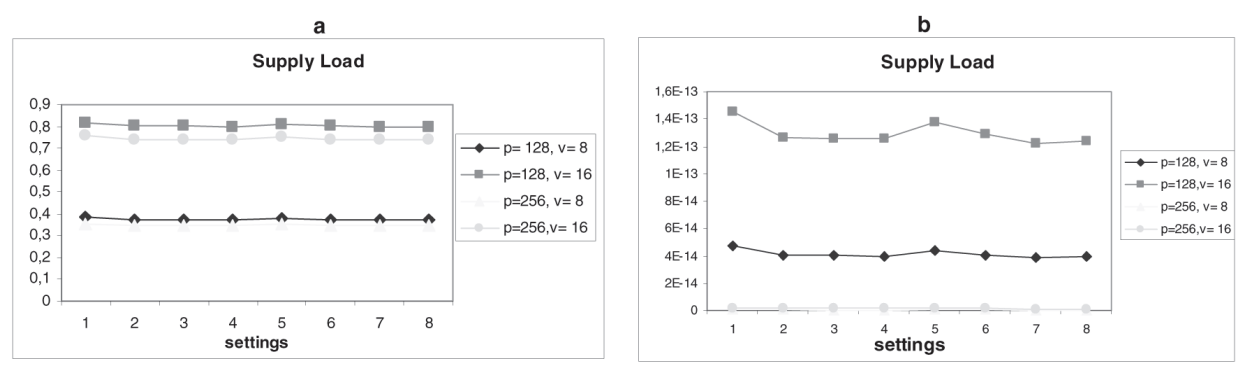

Figure 5: Supply Load comparison using different weights

Figure 5a shows that the load measure, when high weight is given to variety, correctly gives a worse value to higher variety settings, making all the other elements of small impact. The ranking between the curves is modified when the time factor is more important as in Figure 5b: the settings with a longer pipeline outperform their counterparts so much as to be almost out of scale.

A similar evaluation of the measure has been performed for the second dimension of change highlighted in section 3 , and the reconfiguration issue discussed in section 4 , showing the ability of the proposed metrics to capture the instability in a sequence of orders and the impact of the reconfiguration level experienced.

\section{Discussion/Interpretation}

Evaluation of the load placed on the supplier should allow supplier flexibility requirements to be considered. We have so far considered two different measures for uncertainty, one taking into account deviation and the other stability. Measures $\mathrm{C}_{1}$ and $\mathrm{C}_{2}$ provide different insights and have different managerial implications. A useful way to relate the manufacturer requirements to the supplier system is by computing both of them and classifying the stream of orders placed by the manufacturer both in terms of deviation and stability, as shown in Figure 6.

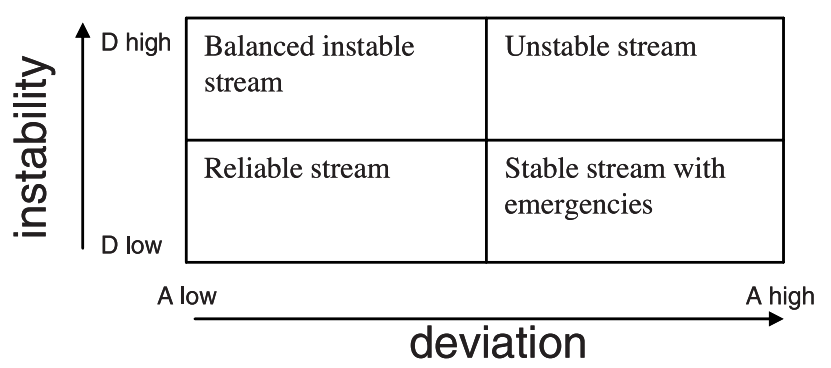

Figure 6: Classification based on level of deviation and level of instability

In the case of high deviation and low instability, the high value of the measure $A$ is due to a few orders showing a peak of demand for some components. In this case 
the capacity of the supplier to cope with the unusual orders is the main issue. In the case of high deviation and high instability the peaks of demand are less wide but more frequent, requiring the system to continuously modify its settings. In the case of low deviation and high instability in orders different quantities of many variants are present; in this case the cost or time of set up is the main issue, and calculating the setup-related instability measure would be useful. The last case of low deviation and high stability is of course the one to be preferred, allowing for the smoothest stream of orders.

A similar approach may be adopted in the presence of reconfiguration by computing both the average and maximum level of reconfiguration, Figure 7:

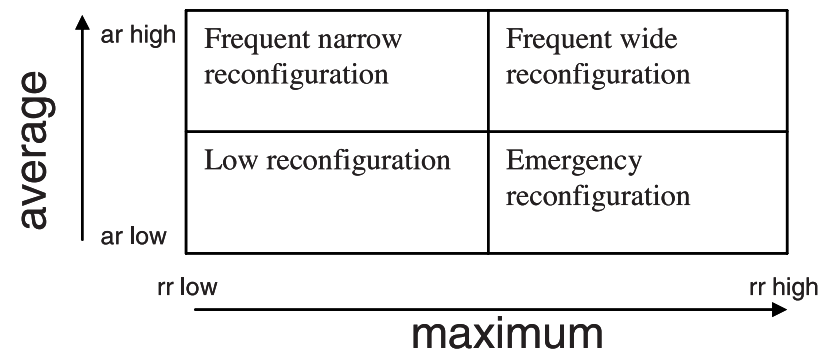

Figure 7: Classification based on maximum and average level of reconfiguration

This approach can be developed to provide insights on the types and levels of supplier flexibility needed, as illustrated in Figure 8.

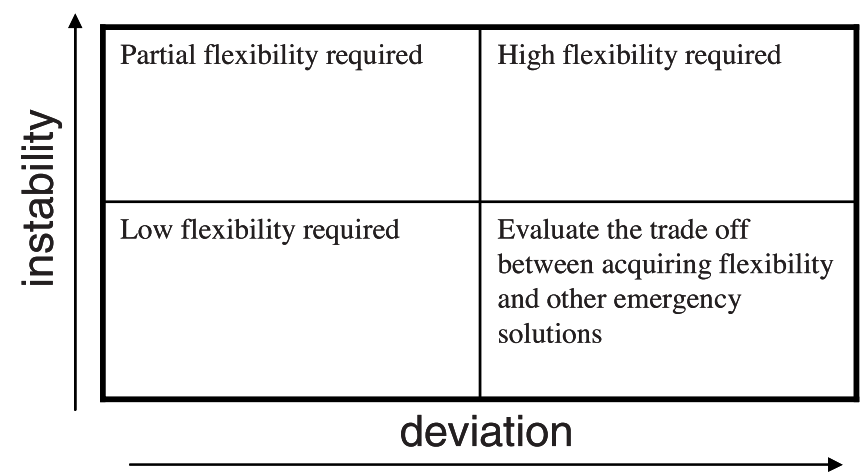

Figure 8: Relating the types and levels of flexibility to deviation and instability

To evaluate the level of variety we have counted the number of different products required. This approach is simple and is relevant where products show low complexity level (da Silveira; 1998). Gupta (1993) advocates a more comprehensive measure, describing 'the number of products in the set, the degree of component commonality and the degree of processing commonality'. Upton (1997) finds this measure unrepresentative of the range flexibility. Considering only the number of 
different products fails to take into account the degree of synergy between them (Ramdas; 2003) and thus the range-heterogeneity dimension of flexibility they require. Using product variety as a proxy for the requested component level variety may not allow the impact of component level commonality to be investigated (Chandra et al; 2005). In this exploratory work our primary aim is to propose useful measures for supplier load in Mass Customisation and high variety environments. Product architecture and component commonality are highlighted as potential areas for further research.

\section{Conclusion}

The paper has proposed an approach to measuring the load placed on the supply base in Mass Customization and high variety environments. Specific metrics have been developed based on the conceptual ideas proposed. A simulation environment has been used to illustrate how the metrics behave under different scenarios. The approach provides a basis for future work in estimating supplier load and in informing the types and levels of flexibility needed in specific Mass Customization and high variety environment.

\section{References}

Bils, M., and Klenow, P. J., (2001). The Acceleration in Variety Growth. The American Economic Review, 91(2), 274-280.

Brabazon, P. G., MacCarthy, B. L. (2006). Fundamental behaviour of virtual-buildto-order systems. International Journal of Production Economics, 104, 514-524.

Chandra, C., Everson, M., Grabis, J. (2005). Evaluation of enterprise-level benefits of manufacturing flexibility. Omega, 33, 17-31.

Correa, H. L., Slack, N. (1996). Framework to analyse flexibility and unplanned change in manufacturing systems. Computer Integrated Manufacturing Systems, 9(1), 57-64.

Da Silveira, G. (1998). A framework for the management of product variety. International Journal of Operations and Production Management, 18(3), 271-285.

De Toni, A., Tonchia, S. (1998). Manufacturing flexibility: a literature review. International Journal of Production Research, 36(6), 1587-1617.

D'Souza, D. E. and Williams, F. P. (2000). Toward a taxonomy of manufacturing flexibility dimensions. Journal of Operations Management, 18(5), 577-593.

Er, M., and MacCarthy, B.L. (2006). Managing Product Variety in Multinational Corporation Supply Chains: A Simulation Study. Journal of Manufacturing Technology Management, 17(8), 1117-1138.

Feitzinger, E., Lee, H. L. (1997). Mass Customization at Hewlett-Packard: The Power of Postponement. Harvard Business Review, 75(1), 116-121.

Ferdows, K. (2003). Making the most of your foreign factories. Harvard Business Review, 75(2), 73-88. 
Fisher, M.L., Ramdas, K. and Ulrich, K. (1999). Component Sharing in the Management of Product Variety: A Study of Automotive Braking Systems. Management Science, 45,(3), 297-315.

Gilmore, J. H., Pine, B. J. (1997). The four faces of mass customization. Harvard Business Review, 75(1), 91-101.

Gupta, D. (1993). On measurement and valuation of manufacturing flexibility. International Journal of Production Research, 31(12), 2947-2958.

Inman, R. R., Gonsalvez, D. J. A. (1997). Measuring and analysing supply chain schedule stability: a case study in the automotive industry. Production Planning and Control, 8(2), 194- 204.

Jensen, T. (1993). Measuring and improving planning stability of reorder-point lotsizing policies. International Journal of Production Economics, 30-31, 167-178.

Kadipasaoglu, S. N., Sridharan, V. (1995). Alternative approaches for reducing schedule instability in multistage manufacturing under demand uncertainty. Journal of Operations Management, 13(3), 193-211.

Lummus, R. R., Duclos, L. K., Vokurka, R. J. (2003). Supply Chain Flexibility: Building a New Model. Global Journal of Flexible Systems Management, 4(4), 1-13.

MacCarthy, B.L., Brabazon, P.G., Bramham, J. (2003). Fundamental modes of operation for mass customization. International Journal of Production Economics, 85, 289-304.

Pine, B. J., Peppers, D., Rogers, M. (1995). Do you want to keep your customers forever? Harvard Business Review, 73(2), 103-114.

Ramdas, K., (2003). Managing Product Variety: An Integrative Review and Research Directions. Production and Operations Management, 12(1), 79-101.

Slack, N. (1987). The flexibility of manufacturing systems. International Journal of Operations and Production Management, 7(4), 35-45.

Stevenson, M., Spring, M. (2007). Flexibility from a supply chain perspective: definition and review. International Journal of Operations and Production Management, 27(7), 685-713.

Suarez, F. F., Cusumano, M. A., Fine, C. H. (1991). Flexibility and performance: a literature critique and strategic framework. Working Paper No. 50-91, Sloan School of Management, MIT.

Suarez, F. F., Cusumano, M. A., Fine, C. H. (1995). An empirical study of flexibility in manufacturing. Sloan Management Review, 37(1), 25-32.

Suarez, F. F., Cusumano, M. A., Fine, C. H. (1996). An empirical study of manufacturing flexibility in printed circuit board assembly. Operations Research, 44(1), 223-240.

Tseng, M. M., Jiao, J., Merchant, M. E. (1996). CIRP Annals-Manufacturing Technology, 45(1), 153-156.

Upton, D. M. (1997). Process range in manufacturing: an empirical study of flexibility. Management Science, 43(8), 1079-1092. 
Westbrook, R., Williamson, P. (1993). Mass customization: Japan's new frontier. European Management Journal, 11(1), 38-45.

Zhang, Q., Vonderembse, M. A., Lim, J. S. (2003). Manufacturing flexibility: defining and analyzing relationships among competence, capability, and customer satisfaction. Journal of Operations Management, 21(2), 173-191.

Zipkin, P. (2001). The Limits of Mass Customization. Sloan Management Review, 42(3), 81-87. 


\title{
Fuzzy Cognitive Map based Health ServiceCustomisation
}

\author{
Dimitrios K. Kardaras, Bill Karakostas, Eleutherios A. Papathanassiou, \\ Stavroula G. Barbounaki, Aikaterini P. Kikilia
}

\begin{abstract}
In today's intensive competitive environments the design and delivery of innovative, flexible, and effective services is of paramount importance for business success. Increasing competition and informed customer put companies under pressure to offer better and more competitive services. The customisation of services implies that service providers can understand customers' priorities and assumes that they can design and develop services that meet consumers' requirements. This paper proposes a service modelling and customisation approach that draws on the fuzzy cognitive maps theory and illustrates it with an example in the health services domain. This research suggests that cognitive mapping enables customers and service providers to express their views on service design and quality. Additionally fuzzy logic supports the modelling of qualitative terms that pertain to service quality and allows the manipulation of the subjectivity and contradiction that is embedded to service customisation process.
\end{abstract}

\section{Keywords}

Service, Customisation, Fuzzy Cognitive Maps, Business Process

\section{Introduction to E-health quality}

Quality is a very important strategic competence for every organization and a key element that no institution can ignore. E-Health, which implies the use of information and communication technologies (ICT) in health care, is one of the most rapidly growing areas in health today. The practice of healthcare delivery using interactive audio, video, or data communications to improve the quality of care offered to patients by the ability to access to a higher level of medical expertise while decreasing their overall operational costs.

E-Health, services and health service related information, is supported by information

technology applications that span across the following systems:

- Electronic Health Records (eHR)

- Patient Information Systems (PIS)

- Hospital information Systems (HIS) 
- General Practitioner Information Systems (GPIS)

- National electronic registries

- National drug registries

- Directories of healthcare professionals and institutions

- Decision Support Systems (DSS)

- Tele-health

There are five dimensions of health service quality, and these are: tangibility, reliability responsiveness, assurance and finally empathy and their purpose is to measure patient's satisfaction (Scholefield, 2007).

\section{Tangibles.}

- Excellent health services hospital would have up-to-date facilities. Examples are: buildings, equipment, X-ray department, laboratories etc.

- The physical facilities at excellent health services hospitals would be visually attractive. E.g. reception area, corridors, wards, out-patient department, signs, carpark.

- Hospital staff would be neat in appearance. E.g. smart and clean uniform, wellgroomed personnel.

- Materials associated with the hospital's service. E.g. appointment card, brochures, directions, documentation.

\section{Reliability.}

- Excellent hospitals would provide their services at the time they promise to do so. E.g. time of the operation, investigation, medicine, food.

- When a patient has a problem, excellent hospitals would show a sincere interest in solving it.

- Excellent hospitals would carry out services right the first time.

- Excellent hospitals would provide error-free documentation. E.g. keeping records correctly.

- Hospital staff in excellent hospitals would tell patients exactly when services will be performed. E.g. date of the operation, progress laboratory results.

Responsiveness.

- Hospital staff in excellent hospitals would give prompt service to patients. E.g. making appointments quickly, returning phone call quickly, and resolving problems quickly.

- Hospital staff in excellent hospitals would always be willing to help patients. E.g. willing to answer questions, provide advice.

- Hospital staff in excellent hospitals would never be too busy to respond to patients requests. E.g. responsiveness to complaints, patient information. 
- The attitude of hospital staff in excellent hospitals would instil confidence in patients. E.g. honest, trustworthy, hospital name/image, reputation.

\section{Assurance.}

- Patients would feel secure in receiving medical cars at excellent hospitals.

- Hospital staff in excellent hospital would always feel courteous with customers. E.g. good telephone manners, showing consideration, pleasant and efficient service.

- Hospital staff in excellent hospitals would have the knowledge to answer patients' question. E.g. knowledge and skill of staff regarding medical and health information.

- Excellent hospitals would be approachable. E.g. ease access to management, telephone access, ease of contact.

\section{Empathy}

- Excellent hospital would give patients individual attention. E.g. learning a patient's specific medical history, flexibility to accommodate individual patient's requirements, preferences, dislikes.

- Excellent hospitals would listen to patients and keep patients informed. E.g. listening to patients' ideas, new operations, and general enquiries.

- Excellent hospitals would have 24-hour availability. E.g. evening appointments, 24-hour emergency availability.

- Excellent hospitals would have the patient's best interest at heart. E.g. building long-term relationships.

- Hospital staff would understand the specific needs of patients.

E.g. recognizing the importance of the patient, what the patient wants.

Patients' rank reliability as the most important of the five dimensions. Reliability from the stand point of the patient depends on the following (Scolefield, 2007):

- Ease of access.

- Guarantee for treatment quality.

- Free access to second opinion within the hospital.

- Prevention of diagnosis errors.

It is necessary for all the hospitals to investigate, the quality level of services they provide. Will they continue to offer such an inadequate service to their patients? It is important to provide the better service quality and also to have continuous quality improvement.

\subsection{Mobile Outpatient Service System (Moss)}

The MOSS is a system that services outpatients, physicians and administrators. 
This system provides messages through mobile cellular phones, through e-mails and also provides voice messages for all those people who are not acquainted with mobile cellular phones (Wen-Yuan-Jen, 2006). MOSS focuses on illness treatment, illness prevention, and patient relation management for outpatient services users (Wen-Yuan-Jen et al, 2006). Below 18 functions of MOSS follow:

1. Medication safety notification.

2. Pre-surgical operation notification.

3. Inpatient wards waiting notification.

4. Pre-physical check-up and precaution procedure notification.

5. Pregnant women routine check-up notification.

6. Current treatment notification.

7. Pediatric disease vaccine service notification.

8. Pre-appointment patient reminder.

9. Pap smear notification.

10. Patient appointment injury.

11. Past due patient notification service.

12. Hospital adult check-up service notification.

13. Missed appointment notification.

14. Abnormal test result from check-up notification.

15. Hospital community activities notification.

16. Lectures notification.

17. Physician and patient relationship management.

18. Birthday greeting notification.

\section{Introduction to Fuzzy Cognitive Maps}

Typically, a fuzzy cognitive map is a non-hierarchical graph (Irani et al. 2002), where a change in a concept may cause a change in the whole network. These changes result from a series of causal increases and reductions. Generally, these variations are in the form of a canonical reasoned meter, form - 1 to +1 . A FCM constitutes a Cognitive Map (CM) with a fuzziness of the relationships among the CM's concepts. Thus, a fuzzy cognitive map is comprised of nodes, which represent the cognitive concepts related to the environment under study, and of arrows, that indicate the different causal relationships, positive or negative, between those factors. A fuzzy cognitive map can also be presented with the use of a connection matrix " $\mathrm{M}$ " with dimensions NxN, where $\mathrm{N}$ the number of nodes - concepts of the graph. The strength and direction of causality among different concepts is represented by values in the cells of the table, while the causality value $\mathrm{m}_{\mathrm{ij}}$ takes on values from $[-1$, +1 ] interval. Depending on the rate (Schneider et al. 1998):

$\mathrm{m}_{\mathrm{ij}}>0 \rightarrow$ causal increase or positive relationship between nodes $\mathrm{i}$ to $\mathrm{j}$.

$\mathrm{m}_{\mathrm{ij}}=0 \rightarrow$ there in no causality between nodes $\mathrm{i}$ and $\mathrm{j}$.

$\mathrm{m}_{\mathrm{ij}}<0 \rightarrow$ causal relationship of negative causality between $\mathrm{i}$ and $\mathrm{j}$ nodes. 
The values on relationships can be represented by linguistic terms such as low, medium, high, which are the result of a defuzzification process (Ross 2004).

The change of a causal relationship can be presented with the help of vector $\mathrm{V}_{1}$, which, multiplied with the connection table (M) of the FCM, gives the opportunity to examine the influence of this change (Banini \& Bearman 1998). The system, with repeated multiplications of the new vector $\left(\mathrm{V}_{\mathrm{i}}\right)$ with the primary table $(\mathrm{M})$ until a dynamic equilibrium is reached $\left(\mathrm{MxV}_{1}=\mathrm{D}_{2}, \mathrm{MxV}_{2}=\mathrm{V}_{3}\right.$ and in general $\mathrm{MxV}_{\mathrm{i}}=$ $\mathrm{V}_{\mathrm{i}}+_{1}$ ), gives the final vector $\mathrm{V}_{\mathrm{i}}+_{1}$, which is the final result of the influence $\mathrm{V}_{1}$. In addition, all results point the course towards the final result and, as a consequence, the points of the process's improvement.

\section{Overview of the Approach}

The proposed approach for service customisation engages the following steps:

\section{Step 1: Modelling Service Quality Design Domain}

In order to be able to design and customise a service the providers of the service need to capture the dimensions of its quality. This study suggests that factors that affect the quality of a service can be modelled as a FCM. The interrelationships of the factors represent the strength of the impact that may exert one factor to others. Service quality factors are considered to be part of the strategic domain within which the services development takes place. Thus, the simulation of the FCM can lead to conclusions regarding to the service quality and its the strategic implications. Let us consider the following subset of the health service quality factors' set.

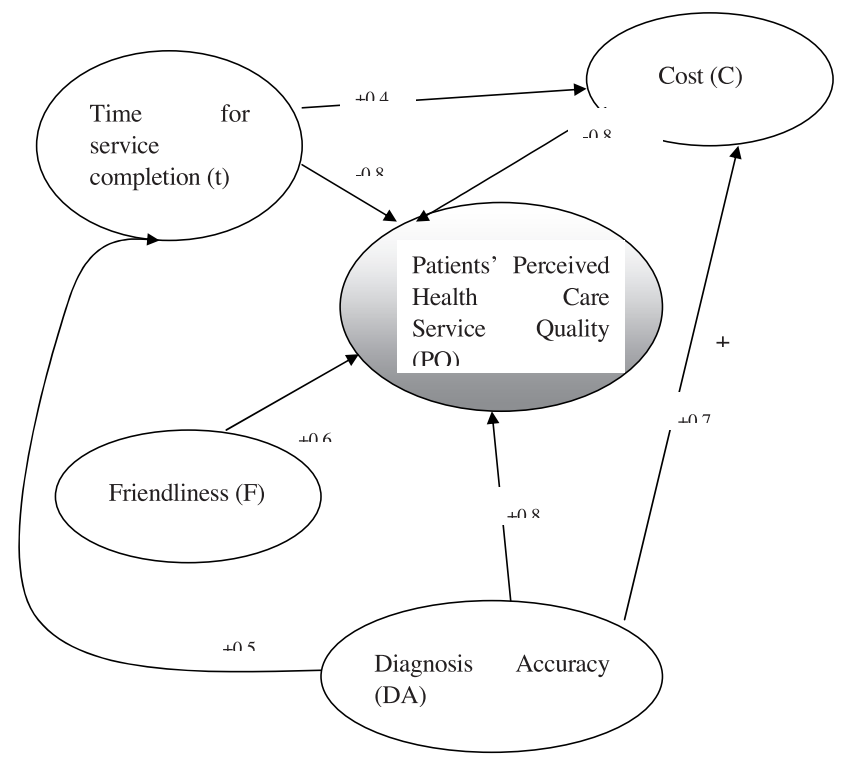

Figure 1: The Health Service Quality Factors FCM (HS-FCM) 
The FCM in the previous figure can be represented as an ( $\mathrm{nXn}$ ) matrix, where (n) is the number of factors in the FCM. Thus, the above FCM can be modelled as an 5X5 matrix. The numbers on Patients' Perceived the arrows indicate how much and to what direction, one factor affects another. For example, the time for service completion reduces the perceived quality by (0.8), i.e. strongly. The following figure shows the HS-FCM matrix.

\begin{tabular}{|l|l|l|l|l|l|}
\hline & PQ & $\mathbf{C}$ & $\mathbf{t}$ & $\mathbf{D A}$ & $\mathbf{F}$ \\
\hline $\mathbf{P Q}$ & 0 & 0 & 0 & 0 & 0 \\
\hline $\mathbf{C}$ & $\mathbf{- 0 . 8}$ & 0 & 0 & 0 & 0 \\
\hline $\mathbf{t}$ & $\mathbf{- 0 . 8}$ & $\mathbf{+ 0 . 6}$ & 0 & 0 & 0 \\
\hline DA & $\mathbf{+ 0 . 9}$ & $\mathbf{+ 0 . 4}$ & 0 & 0 & 0 \\
\hline F & $\mathbf{+ 0 . 7}$ & 0 & 0 & 0 & 0 \\
\hline
\end{tabular}

Figure 2: The HS-FCM matrix

The values in the cells represent the weights of the relationships in the HS-FCM.

\section{Step 2: Modelling Services}

A service can be modelled as a vector, the Service Vector, which is similar to a row of the HSFCM. It is not identical to a row of the HS-FCM for the factors perceived quality is included only once in order to accumulate the impact from all other quality factors. It is an end node that only receives incoming arrows. The following figure shows the service vector.

\begin{tabular}{|l|l|l|l|l|l|l|l|l|l|l|l|l|}
\hline PQ & C & t & NEx & DA & F & SE & A-C & A-t & A-NEx & A-DA & A-F & A-SE \\
\hline 0 & 0 & 0 & 0 & 0 & 0 & 0 & 0.3 & 0.7 & 0.4 & 0.4 & 0.8 & 0.2 \\
\hline
\end{tabular}

Figure 3: The Service Vector

The values of the vector are taken form the interval $[-1,+1]$. The values indicate how well a service that the vector represents performs in terms of the quality factors that are taken into consideration. In this study the factors that are taken into account are the factors shown in figure 1 . The closer to +1 value the highest the performance of that factor. For example, if cost $=+0.9$, it implies that the service's cost is high. 


\section{Step 3: Process Modelling}

A service is being delivered by one process or by a combination of processes. The proposed approach suggests that object oriented models can be used to model the process(es) that support the development and delivery of a service.

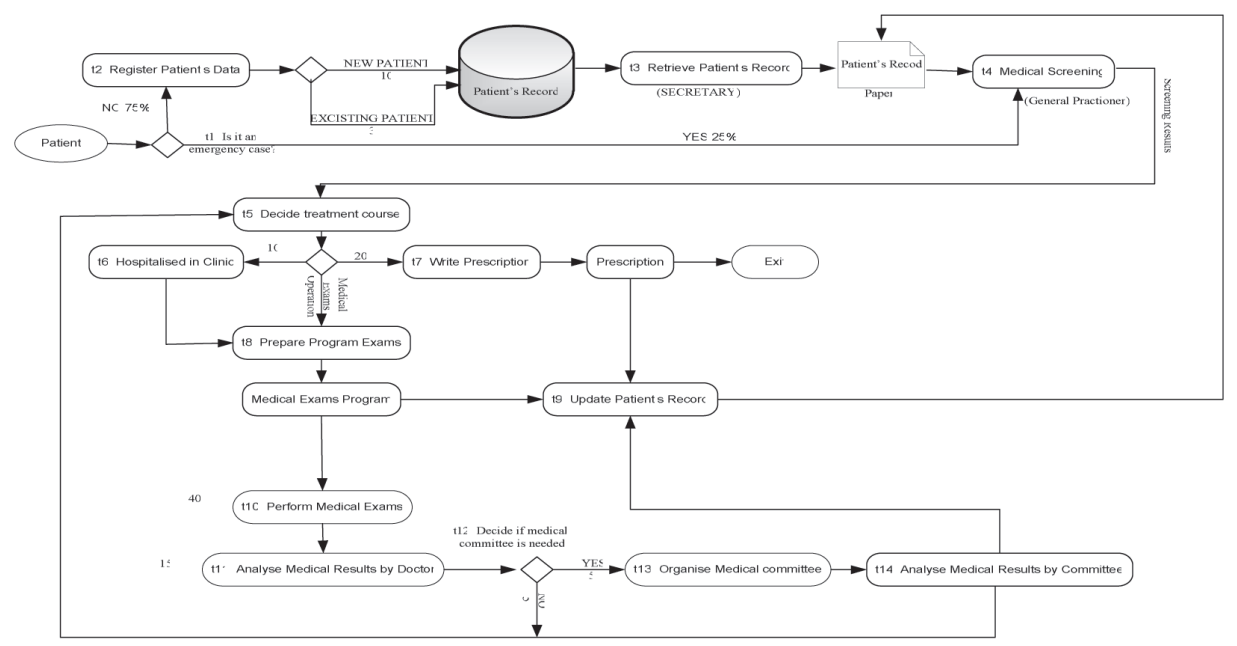

Figure 4: The Admittance to Hospital Process

The above process model represents the tasks that develop and deliver a service that is offered to patients. That service is called Admittance to Hospital (A) for the purpose of this study. The process consists of tasks, which performances affect the quality of service (A). Therefore, the proposed approach to service customisation suggests that processes and corresponding tasks should be assessed in the same terms that services are evaluated. These terms are a subset of the HS-FCM model shown in figure 1.

The process and its tasks are then modelled by the Task Matrix. It is a kXk matrix, where $\mathrm{k}$ is the number of tasks, taken into consideration that each task is modelled in terms of the service quality factors considered in the HS-FCM. Figure 2 shows that the process of this case consists of 14 tasks. Each task is then modelled in terms of the factors shown in figure 1 (but not the end factors perceived quality). Therefore, the process matrix will have 14X4 rows and 14X4 columns.

\section{Step 4: Integrating Strategies, Services and Processes}

The integration of services and tasks means that service providers would be able to investigate the impact of a new service proposal at the strategic as well as at the process level. Such a conceptual approach to integration can be modelled by an FCM that amalgamates all concepts that appear in the HS-FCM, the services and 
the tasks models. It can then represented by a matrix that integrates the HS-FCM matrix, the service vector and the task matrix. The columns will represent all factors of the HS-FCM and the evaluation of each service in terms of the HSFCM factors as well as it includes all the process tasks that are delivering a service ( $\mathrm{j}$ ) as modelled in terms of the HS-FCM. The Service-Task matrix is shown in the following figure. Foe clarity reasons only two tasks ( $\mathrm{t} 1$ and $\mathrm{t} 2$ ) of the process shown in figure 4 are included in the following matrix.

\begin{tabular}{|l|l|l|l|l|l|l|l|l|l|l|l|l|l|}
\hline & PQ & C & $\mathrm{t}$ & DA & F & A-C & A-t & A-DA & A-F & $\begin{array}{l}\text { T1- } \\
\text { C... }\end{array}$ & T1-F & $\begin{array}{l}\text { T2- } \\
\text { C... }\end{array}$ & F \\
\hline PQ & 0 & 0 & 0 & 0 & 0 & 0 & 0 & 0 & 0 & 0 & 0 & & \\
\hline C & $\mathbf{- 0 . 8}$ & 0 & 0 & 0 & 0 & 0 & 0 & 0 & 0 & 0 & 0 & & \\
\hline $\mathrm{t}$ & $\mathbf{- 0 . 8}$ & $\mathbf{+ 0 . 6}$ & 0 & 0 & 0 & 0 & 0 & 0 & 0 & 0 & 0 & & \\
\hline NEx & $\mathbf{+ 0 . 7}$ & $\mathbf{+ 0 . 5}$ & $\mathbf{+ 0 . 6}$ & $\mathbf{+ 0 . 8}$ & 0 & 0 & 0 & 0 & 0 & 0 & 0 & & \\
\hline DA & $\mathbf{+ 0 . 9}$ & $\mathbf{+ 0 . 4}$ & 0 & 0 & 0 & 0 & 0 & 0 & 0 & 0 & 0 & & \\
\hline F & $\mathbf{+ 0 . 7}$ & 0 & 0 & 0 & 0 & 0 & 0 & 0 & 0 & 0 & 0 & & \\
\hline SE & $\mathbf{+ 0 . 8}$ & $\mathbf{+ 0 . 7}$ & 0 & 0 & 0 & 0 & 0 & 0 & 0 & 0 & 0 & & \\
\hline A-C & 0 & $\mathbf{+ 0 . 7}$ & 0 & 0 & 0 & 0 & 0 & 0 & 0 & 0 & 0 & & \\
\hline A-t & 0 & 0 & $\mathbf{+ 0 . 8}$ & 0 & 0 & $\mathbf{+ 0 . 7}$ & 0 & 0 & 0 & 0 & 0 & & \\
\hline A-DA & 0 & 0 & 0 & $\mathbf{+ 0 . 3}$ & 0 & $\mathbf{+ 0 . 4}$ & 0 & 0 & 0 & 0 & 0 & & \\
\hline
\end{tabular}

\begin{tabular}{|l|l|l|l|l|l|l|l|l|l|l|l|l|l|}
\hline A-F & 0 & 0 & 0 & 0 & $\mathbf{+ 0 . 9}$ & 0 & 0 & 0 & 0 & 0 & 0 & & \\
\hline T1-C & & & & & & & & & & & & & \\
\hline$\ldots$ \\
\hline T1-F
\end{tabular}

Figure 5: The Service-Task Matrix that integrates strategies, services and tasks

Te values in the cells show how each factor affects another and range within the $[-1,+1]$ interval. Thus, the service-task matrix for example shows how a task affects some (not necessarily all) of the HS-FCM factors. If a task does not affect an HS-FCM factor then the value of the corresponding cell equals to (0). 


\section{Step 5: Modelling Customer Expectations}

Customer requirements are also modelled as a vector, which is identical to the service vector. The values of the customer vector also range within the interval [-1, +1 ]. The closer the value of an attribute to +1 the higher the expectation for the corresponding attribute to be part of a service design.

\section{Step 6: Developing Customisation Scenarios}

The service vector expresses the current level of services and their current level of quality in terms of the factors in the HS-FCM. The service vector represents the service provider's perspective. On the other hand the customer expectations vector represents the customer requirements. The closer the service vector to the customer vector the better the quality of the services. Their distance indicates what the provider needs to offer in order to meet the customer requirements.

Therefore, this study suggests that service proposal can be formed by calculating the distance between the service vector and the customer vector. The distance is calculated according to the following rule (Schneider et al, 1998):

Consider the two vectors (V1) and (V2) that are monotonically increasing (direct relation), then the distance (di) between two elements (vi) of the vectors is defined as:

$$
\mathrm{d} \quad \mathrm{i}=|\chi 1(\mathrm{vi})-\chi 2(\mathrm{vi})|,
$$

where $\chi 1$ and $\chi 2$ is the degree of membership for the (i) element of the vectors (V1) and (V2) respectively.

In the case of monotonically decreasing vectors (reverse relation) the distance of the two elements (vi) is defined as:

$$
\mathrm{d} \quad \mathrm{i}=|\chi 1(\mathrm{vi})-(1-\chi 2(\mathrm{vi}))| .
$$

For a company to be competitive it needs to minimise the distance between actual service quality, as defined by the actual contribution of each service attribute, and customer expectations. The differences indicate the service attributes that designers should consider changing in order to meet customer needs. The larger the differences are the more urgent the changes but also the more important the potential benefits from customisation.

A service customisation scenario is represented as a vector that is identical to the service vector. The values of the customisation vector are either calculated or defined by the distance of the service vector and the customer vector or they indicate what the service provider wants to offer. This is the top-down route to customisation, for it considers the strategic level and translates the needs to tasks and services 
design. The values range within the interval $[-1,+1]$. The closer to +1 a value is the more of that factor the service provider plans to offer or the more urgent a requirement is from the customer's perspective. For example if friendliness $=+0.7$, it implies that the provider plans a service that would offer a high level of friendliness to its customers. The bottom-up route to customisation allows the service provider consider organisational changes at the task level and then investigates their impact on the service quality, i.e. at the strategic level and on the end variable(s).

The MS excel system can be used to implement the HS-FCM matrix and all the necessary vectors of the proposed approach. The multiplication of the customisation vector and the HSFCM matrix, according to the FCM theory indicates the impact of the new service proposal to all of the factors that are included in the HS-FCM matrix. The result of the multiplication is a vector identical to a row of the HS-FCM matrix. Therefore, the multiplication can assist the service provider estimate the impact on the strategic level values (figure 1), the performance of the service and the tasks of the process that is responsible for delivering the service.

The multiplications, in the bottom-up route, continue until the results vector returns (0) to all cells but the one that represents the end factors, i.e. the perceive quality. In the top-down route the multiplications continue until the impact reaches the tasks level attributes. The current study considers four levels of abstraction in the service customisation.

- The task level.

- The service level.

- The strategic level and

- The Perceived Quality level.

Each multiplication between the customisation vector and the HS-FCM vector estimates the impact of one level of abstraction to the next. Therefore in the case of the bottom-up route, the first multiplication indicates the impact of the tasks on the service attributes. The second indicates the impact of the services on the strategic level and the third shows the impact of the strategic factors on the end factor. The fourth multiplication indicated the overall cumulative impact of all factors to the end variable. The last result vector would have all cells equal to (0), except the cell that corresponds to the perceive quality variable. If a scenario sets a target for a required level of the perceived quality (top-down service and task evaluation) then the final multiplication will give values to cells that correspond to the lowest level of abstraction, i.e. that of the tasks. The one before the last multiplication will give values to the cells that correspond to the service(s) attributes. The values would indicate what the provider needs to offer in a service and what changes should be made at the tasks level. 
For example let us consider the following scenario. The service provider needs to evaluate a proposal of a new service (A) that should be far better in terms of the perceived quality (PQ) compared to other competitors' achievements. So the provider considers the following vector, which take the +0.9 value in the cell (PQ). It means that the perceived quality of service (A) should be (or become) high.

\begin{tabular}{|l|l|l|l|l|l|l|l|l|l|l|l|l|l|}
\hline & PQ & C & t & DA & F & A-C & A-t & A-DA & A-F & $\begin{array}{l}\text { T1- } \\
\text { C... }\end{array}$ & T1-F & $\begin{array}{l}\text { T2- } \\
\text { C... }\end{array}$ & $\begin{array}{l}\text { T2- } \\
\text { F }\end{array}$ \\
\hline PQ & 0.9 & 0 & 0 & 0 & 0 & 0 & 0 & 0 & 0 & 0 & 0 & & \\
\hline
\end{tabular}

Figure 6: New Service proposal modelling

The multiplication of the vector in figure 6 with the HS-FCM matrix would return a vector, which in turn is multiplied with the HS-FCM matrix. Each result vector is multiplied with the HS-FCM vector. Let us suppose that after four multiplications the final results vector in figure 6, with the HS-FCM matrix would return the following vector.

\begin{tabular}{|l|l|l|l|l|l|l|l|l|l|l|l|l|l|}
\hline & PQ & C & t & DA & F & A-C & A-t & A-DA & A-F & $\begin{array}{l}\text { T1- } \\
\text { C... }\end{array}$ & T1-F & $\begin{array}{l}\text { T2- } \\
\text { C... }\end{array}$ & $\begin{array}{l}\text { T2- } \\
\text { F }\end{array}$ \\
\hline PQ & 0 & 0 & 0 & 0 & 0 & -0.8 & 0 & 0.7 & 0.4 & -0.4 & 0 & -.09 & \\
\hline
\end{tabular}

Figure 7: Results Vector example

The vector in the above figure indicates that if the service provider want to increase the perceived quality of service (A), in this case only one service is assumed, then the provider needs to reduce (-0.8) strongly the cost of service (A), increase substantially the diagnosis accuracy and achieve a medium increase in the service's friendliness. The qualitative terms used in these expressions may represent fuzzy sets that are used depending on the modelling.

In order to achieve the above levels of performance the provider needs to focus on task (T1) and task (t2) and in particular to achieve a medium reduction in task's t1 cost, and a strong reduction in task's $\mathrm{t} 2$ cost. Similar implications can be estimates in other factors that are modelled in the service-task matrix, which are not shown in this study for keeping complexity in low level.

\section{Conclusions}

This research suggests that FCM can be used in order to support modelling of service customisation at strategic, service and process level. Fuzzy logic allows for contradicting and subjective perceptions to be modelled and to investigate their impact on key service quality issues. The analysis of FCMs can integrate customer and ser- 
vice providers' views and support customisation. FCM simulation can suggest new service proposals, evaluate existing services and propose process design that could improve service quality.

\section{References}

Banini, G. A. \& Bearman, R. A. (1998). Application of fuzzy cognitive maps to factors affecting slurry rheology, International Journal of Mineral Processing, 52, 233 -244 .

Irani, Z., Sharif, A., Love, P.E.D. \& Kahraman, C. (2002). Applying concepts of fuzzy cognitive mapping to model: The IT/IS investment evaluation process. International Journal of Production Economics 75, 199 - 211.

Ross. T.J. (2004). Fuzzy Logic with Engineering Applications. Publisher John Wiley. Schneider, M., Shnaider, E., Kandel, A. \& Chew, G. (1998) Automatic construction of FCMs. Fuzzy Sets and Systems 93, 161 - 172.

Scholefield, H. (2007). Embedding quality improvement and patient safety at Liverpool women's NHS foundation trust. Best Practice and Research Clinical and Gynecology. Vol. 21, No 4, 93-607.

Wen-Yuan-Jen, Chia-Chen Chao, Ming Chien Hung, Yu-Chuan Li, \& Chi Y.P. (2006). Mobile information and communication in the hospital outpatient service. International Journal of Medical Informatics. 76, 565-574.

\section{Dimitrios K. Kardaras}

Assistant Professor in Information Management

Business Informatics Lab, Dept. of Business Administration, Athens University of Economics and Business,

Patission 76 str. 104 34, Athens, Greece

e-mail: kardaras@aueb.gr

\section{Bill Karakostas}

Senior Lecture in Software Engineering

Centre for HCI Design, School of Informatics,

City University,

Northampton Sq. London EC1V 0HB, London, UK.

e-mail: billk@soi.city.ac.uk

\section{Eleutherios A. Papathanassiou,}

Professor in Business Informatics

Business Informatics Lab, Dept. of Business Administration,

Athens University of Economics and Business,

Patission 76 str. 104 34, Athens, Greece

e-mail: eap@aueb.gr 


\section{Stavroula G. Barbounaki}

Lecturer in Knowledge Management

Tei Piraues,

Petrou Ralli, Athens, Greece

e-mail: sbarb@teipir.gr

\section{Aikaterini P. Kikilia}

Lecturer in Knowledge Management

Tei Piraues,

Petrou Ralli, Athens, Greece

\section{Authors Bio Data}

Dr Dimitris K. Kardaras is an assistant professor in information systems management in the Department of Business Administration, at the Athens University of Economics and Business (AUEB), Athens, Greece. He holds a BSc (Hons) in informatics and a BSc (Hons) in management both from the Athens University of Economics and Business, an MSc in information systems engineering and a $\mathrm{PhD}$ in information systems from the Department of Computation at the University of Manchester Institute of Science and Technology (UMIST), England. Dr Kardaras has participated to many research projects in IS/IT since 1990 and he has been teaching in IS courses for over 12 years. He has published journal and conference papers in the areas of IS planning, fuzzy cognitive maps, IS modelling and e-commerce.

Dr Bill Karakostas is a senior lecturer at the School of Informatics at the City University, London. Dr Karakostas holds a BSc (Hons) in computer engineering from the University of Patras, Greece, an MPhil and a PhD from the Department of Computation at the University of Manchester Institute of Science and Technology (UMIST), England. He has been leading research projects in IS/IT for over 15 years and he has published extensively in the areas of software engineering and IS modelling and e-commerce technologies. He is scientific advisor in IT to English and Greek companies.

Dr Eleutherios A. Papathanassiou is a professor in MIS and director of the Business Informatics Lab at the Department of Business Administration, at the Athens University of Economics and Business (AUEB), Athens, Greece. He holds a BSc (Hons) in mathematics from the University of Athens and a $\mathrm{PhD}$ in computer science from the University of St. Andrews, Scotland. He is scientific advisor to Greek companies and head of the center for distance learning (TeleEducation Centre) of the AUEB. He has published in the areas of IS modelling, e-commerce and computer science.

Dr Stavroula G. Barbounaki is a lecturer in knowledge management with Technological Institute of Education at Piraeus, Greece. She holds diploma in Electri- 
cal and Computer Engineering from the National Technical University of Athens an Master's and a PhD bio-medical technology from the Université de Paris 05 France. She has published journal and conference papers in the areas of mathematical modeling and intelligent systems applications in biomedical technology.

Dr. Aikaterini P. Kikilia is a lecturer with the Technological Institute of Education at Piraeus, Greece. Her research interests focus on learning customisation. 


\title{
Developing a Frame of Reference for understanding configuration systems
}

\author{
By Klaes Ladeby Jensen and Kasper Edwards
}

DTU Management, Technical University of Denmark, Building 423, 2800 Lyngby, Denmark. (Corresponding author: Edwards@ipl.dtu.dk)

\begin{abstract}
This paper uses the theory of technical systems to develop a frame of reference of product configuration systems. Following a definition of the configuration task, product model and product configuration system the theory of technical systems are presented.

Configuration systems are then related to the theory of technical systems and it is shown that it is possible to understand configuration systems in this context. By doing so the configuration system is expanded to include human system. Three operators of the technical system are identified and analyzed.

In this perspective it is realized that users, organization and the configuration system influence each other and the configuration process. Consequently design of such a system must be approached holistically.
\end{abstract}

\section{Keywords}

Product configuration system, Technical system

\section{Introduction}

The purpose of this paper is to place product configuration systems within the larger frame of reference of technical systems (Hubka \& Eder 1988). The reason for this endeavour is to leverage the explanatory power of technical systems to help our understanding of the problems of implementing product configuration systems.

The move towards mass customization has two forms: 1) from mass production to mass customization and 2) from engineering to mass customization. The two forms are distinctly different as the move from mass production entails a larger scope or greater product variation. This is contrasted by the move from engineering where product variation is greatly reduced. Employees exposed to either of these moves will find this a significant change in their work life. While we do not intend to explore the organizational change in great depth it must be appreciated that such changes must induce some degree of organizational inertia if not outright resistance. 
Fact of the matter remain that many attempts to implement mass customization and product configuration systems fail or at least reach a level of maturity far below the expected benefits. Organizational resistance has been the predominant explanation for failure within the move from engineering (Edwards \& Riis 2004), (Edwards \& Møldrup 2004). Still, these results have been based on empirical evidence and have lacked a broader theoretical foundation.

Placing product configuration systems within the technical system frame of reference is an attempt to provide such a theoretical foundation.

It is our claim that this will allow us to explain the impact of product configuration systems as well as why some procedures from implementing product configuration systems are out right wrong.

This paper begins with a brief literature review of the configuration task, product model and configuration system. We then introduce and elaborate on the theory of technical systems. Armed whit this knowledge we then place product configuration systems within the technical system and discuss the implications hereof.

\subsection{The Configuration Task}

There is a fine line between product configuration and design, and many refer to configuration as being a subclass of the design activity. Traditionally, configuration is often considered to be a part of the design science discussion, and even a special case of the general field of design activities. Stumptner ( 1997) and Sabin and Weigel (1998), assumes that in configuration the design goals and requirements are fully specified, and subcomponents and functions are already known.

Many authors have offered their definition of configuration beginning with Mittal and Frayman (1989), where the core of the configuration task is selecting and arranging combinations of parts that satisfy given specifications. No new component types can be created nor can the interface of the existing components be modified. Several other definitions of configuration have been put forwards among other by Felfernig, Friedrich, \& Jannach ( 2000), Soininen et al. ( 1998), (Salvador \& Forza (2004), and Haug (2007). Needles to say these definitions differ somewhat. While it is not our intention to discuss the differences in detail we return to Mittal and Frayman (1989).

As Brown ( 1998) points out, there are problems with the definition of configuration by Frayman and Mittal. The use of connect throughout the definition indicates that the components in the configuration actually physically connects. Brown (1998) indicates that this might always not be thru, and that a logically explanation of their use of 'connect' is that they have a background related to the configuration of computer equipment. Configurations are more often about relationships between components, where touch and connect are examples of relationships.

In this paper a configuration is the output of the configuration task - i.e. a description of a product that satisfies given requirements. Hence, the definition of the configuration task is: 
Configuration task: To combine predefined entities (physical or non-physical) and define their variable properties, while obeying constraints and legal interface combinations, in a way that satisfies given requirements.

\subsection{Product model}

The term product model, commonly used in the literature and related to product configuration systems, has also been defined by many authors including (Andreasen 1994), (Hvam 1999), (Schwarze 1996), (Shaw, Bloor, \& de Pennington 1989) Hvam, Mortensen, and Riis (2008).

The product model in a configuration system should constitute generic model cable of generating a vide variety of configurations, to support the business model of the implementing company. The definition by is particularly well suited for our purpose:

"A product model is an abstract representation or description, describing (a) the structure of $P$ and (b) facts, object, concepts and properties that are relevant in any life cycle phase of $P$. $P$ can be a single product or a family of products. A product is a thing, a substance or a service produced by a natural or artificial process." (Schwarze 1996)

\subsection{Configurators or Configuration Systems}

Configuration systems are simply tools to generate a particular instance of a product. A configuration system can be implemented in a vide variety of ways ranging from simple schematics of which components make up a given product to advanced computer systems. For our purpose a configuration system is a software tool. Going through the literature, three terms are often used to define these types of ITsystems: 1) 'configurator', 2) 'product configuration systems', and 3) 'configuration system'. As (Haug 2007) notes, this would not represent a problem if there was consensus on using the three terms interchangeably. For instance, Forza and Salvador (2007) include the human resources in their definition.

In this paper, the three terms are used interchangeably but only referring to the software system. The system which Forza and Salvador refers to will be referred to as 'total configuration system' (TCS). We further narrow our scope to only configuration systems that are expert systems or knowledge-based systems. Although it is argued that expert systems are a subset of the more general knowledge-based systems (Hopgood 2001; Jackson 1999). Expert systems are typically defined as computer programs that represents and reasons with knowledge of specialist matter with the purpose of solving problems or giving advice (Jackson 1999). Knowledge-based system is broader defined as a computer system that is programmed to imitate human problem-solving by means of artificial intelligence and reference to a database of knowledge on a particular subject. In conclusion a configuration system can be both an expert system and a knowledge-based system. It dependents on the configuration task it needs to perform. However, the basis of any product configuration system is knowledge. 
The essential components are a knowledge base, that contains the knowledge about the domain, and which the inference engine uses to draw conclusions. The results are presented to the outside world by an interface that handles connections to humans, hardware, data, and/or other software. Expert systems often have extra frills in terms of a knowledge acquisition module, or an explanation module to explore the knowledge bases, see Figure 1-1.

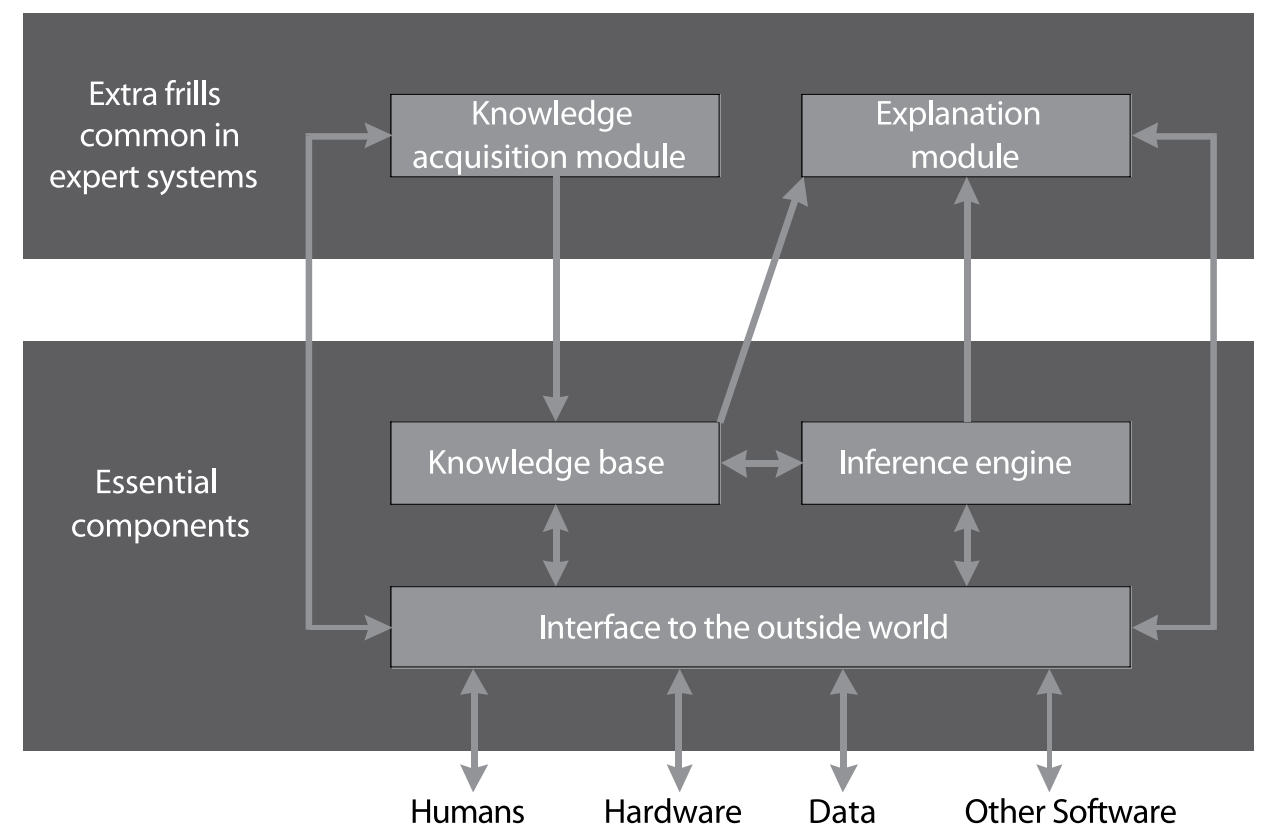

Figure 1-1: The main components of a knowledge-based system (Hopgood 2001)

The goal of a configuration system is thus to build a specification of which a selection of components satisfies the needs of the configurer, and how the components are connected or detect inconsistencies in the requirements giving by the user.

\section{The Technical System (the product)}

Technical systems (TS) are objects, products, things, machines, implements, technical objects, etc., which is made by mankind. In other words, TS refer to all types of human artefacts. The primary aim of theory of technical Systems (TTS) was to: "...classify and categorise the knowledge about technical systems into a ordered set of statements about their nature, regularities of conformation, origination, development, and various empirical TS-related observations."(Hubka \& Eder 1988) 
Technical systems can be used to fulfill human needs and if the means to fulfill a need exist at the time, or are capable of being realized, then a process of designing and manufacturing a product (a TS) can supply the means to fulfil that need. Hubka and Eder ( 1988) calls processes in which TS are applied to fulfill a need for technical processes. A technical process transforms an operand from an existing state to a desired state by use of operators. Hubka and Eder (1988) describes three kind of operators that has an effect in relation to the transformation process, and these are: (i) Human systems, (ii) technical systems, and (iii) active environments. A system of operators that through a technical process transforms an operand from an existing state to a desired state is called a transformation system. The effect posed on the transformation system can be described as material, energy or information, or any combination of those.

Each transformation system has a well-defined purpose that is to perform the intended transformation on the appropriate operands. Hubka and Eder ( 1988) divides the major elements of the total transformation system into a process (an operand that is being transformed), and the operators that drive and guide the process.

The total transformation system is described by four subsystems, (Figure 2-1):

(i) A technical system (TS - the product),

(ii) A human system (HuS - human operator),

(iii) The active environment (AEnv - influence from the environment), and

(iv) A technical process (where an operand is transformed by effects from the above three subsystems).

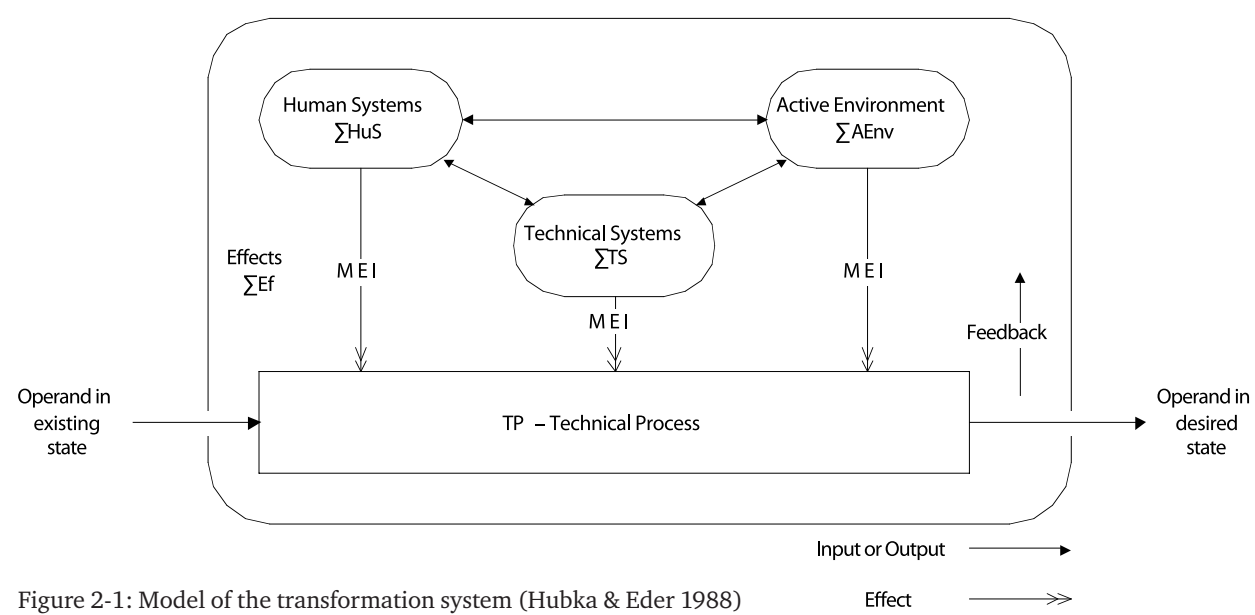

There can be distinguished among four kinds of operands: (i) Biological objects, (ii) Materials, (iii) Energy, and (iv) Information. These four kinds of operands are not exclusive, most often an operand consist of a combination of the classes. The tech- 
nical process transforms an operand from an existing state to a desired state (the attributes of the operand changes). Like in mathematics an operand are modified by operators. When TS are used by humans as artificial tools to carry out the transformation, the transformation process is referred to as the technical process.

The technical process can be described using a variety of tools. In management science and operations management often referred methodology to describe processes is Business Process Modelling Notation (BPMN) (White 2006). Hubka and Eder ( 1988) mentions six main types of representation, and I will not go deeper into the issue of representation for now. The technical process is often realized by various structures of processes and consequently of different technologies, TS, HuS, and AEnv.

Let us explore the operators of the total transformation system. HuS is the subset of humans who exert any effect on the operand in a particular technical process. Likewise the TS as operator is that subset of technical systems that directly exert an effect on the particular operand. These two are main elements of the execution system that drives the technical process. The AEnv as operator compromises all sources of effects exerted by the surroundings (most of which are not explicitly stated) (Hubka \& Eder 1988).

Every operation in a technical system is the consequence of one or more causes, and is simultaneously the cause of one or more other consequences. Consequently, TS can be described as being fully deterministic (Hubka \& Eder 1988). However, in many cases the causes are so complex and with a multitude of interactions that is difficult to assign a cause to each consequence (this is especially true when humans or an active environment is involved). On the other hand each TS has an element of natural variation inherent in form of the variance through production, assembly etc. This should be easily accommodated for by statistics. TS wield planned and goal-oriented effects on the operands of the technical transformation process (see Figure 2-1).

Here it is necessary to distinguish purpose from actual abilities. What we define as the effect of a TS serves to satisfy the need of the human (the purpose of the TS). So the purpose of the TS is thus represented by the system of its output effects. The actual abilities of the TS are referred to as functions. Functions represent an introvert view on how the effects of the TS are derived.

The constituents of TS are fitted together so that a given input will lead to a given output in form of effects to operand. In order to obtain a certain result (i.e. an output effect), various phenomena are linked together in an action chain (the TS-internal processes). The TS-internal processes describes how the input is converted to an output, and this can be described by (i) describing the function structure, or (ii) describing the organ structure (Hubka \& Eder 1988).

Two questions now arise: (i) How and with what means can one accomplish the planned output effects on the operand, and (ii) how can interior of the TS be described at various levels of abstraction? 
The highest level of abstraction is the design specification. The design specification states only the requirements, needs and constraints, independent of process or hardware (Hubka \& Eder 1988).

The next level of abstraction is to perceive a given TS as a 'black box' that based on inputs from the active environments, human systems, and other technical systems yields outputs in form of an effect to a given operand. This does not per see describes the technical system in detail but recognises the effects that the technical system exerts onto the operand in the technical process (the purpose of the TS). So the 'black box'-view of a given TS shoes the functional connections, at the TS output between the TS and the technical process, and the input to various operators (Hubka \& Eder 1988).

According to (Hubka \& Eder 1988) the TS can be described as a functional structure, a organ structure, and a component structure. The three TS structures (function, organ, and component) are different views or representations of the same TS at different levels of abstractions (Hubka \& Eder 1988). The terminology is related as follows:

- "the effects of the TS (as aims) are achieved by certain function structures (as means);

- these function structures (as aims) can be realized by various organ structures (as mean);

- the organ structures (as aims) can only be realized from various component structures (as mean)." (Hubka \& Eder 1988)

The three levels of abstraction will be described in the following three sections.

\subsection{Function structure of the TS}

The TS as an object is defined for the purpose of this structure in term of its functions. The functions of TS can be understood as a unique coupling of independent input measures to dependent output measures. Each function can be described more or less concrete. This influences the number of possible or available organs that can be used as a mean to realize the function. Hubka and Eder ( 1988)

There exist two principle ways to work out a function structure. One is analytical and starts by abstracting from the component or organ structure. The alternative is to concretising from the higher abstraction level, which implies identification of functions and their relationship as the means to achieve the aim in form of the desired output effect that will drive the technical process.

\subsection{Organ structure of the TS}

The organ structure is an abstract model of TS that permits analysis and representation of the operational state of TS. Organs are carriers of functions and are coupled in the action chain to yield desired effects. These couplings are the main relationships between organs, as the output from one organ is the input to the next organ. 
As for the function structure, the organs can be defined at various levels of abstraction. The organ structure can be established by either concretising the function structure or abstracting from the component structure of TS.

\subsection{Component structure of the TS}

The component structure is the most tangible way to represent a TS, and at the same time the most concrete stage in the design process of a technical system, as you work with the constructional elements of the TS, and the relationship between them. (Hubka \& Eder 1988)

The component structure contains all necessary properties to describe how the TS fulfils the user requirements, and how the TS fulfils them. When the component structure is compared with the more abstract structures described above it is clear that the number of criteria that is used to evaluate the TS has increased by a large amount. The more abstract structures is mainly assessed by a functional viewpoint whereas the component structure is a detailed description of every constructional element down to the last bolt and washer (Hubka \& Eder 1988). A component structure is typically established by concretising from a functional or organ structure, and this task is often complicated (Hubka \& Eder 1988).

A technical system (product) can be described on four levels of abstraction, from purpose with few attributes to component structure with most attributes, see Figure $2-2$.

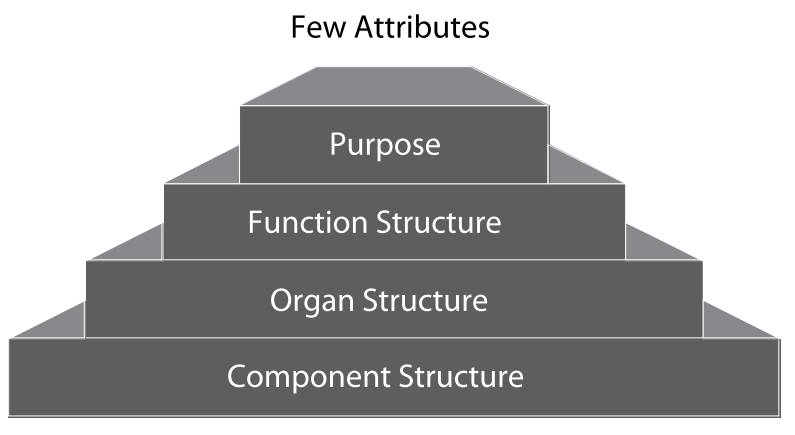

Several Attributes

Figure 2-2: Description of TS on different levels of abstraction

\section{The Total Configuration System as a Technical System}

While Forza and Salvador (2007) describes two subsystems (the human system, and the computing system), the theory of technical systems describes three important elements in a total transformation system: (i) A process, (ii) an operand that is being transformed, and (iii) the operators that drive and guide the process (Hubka \& Eder 1988). Applying the same logic to a configuration system, the total configuration system also consists of an operand, operators, and a process, and this is illustrated in Figure 3-1. 


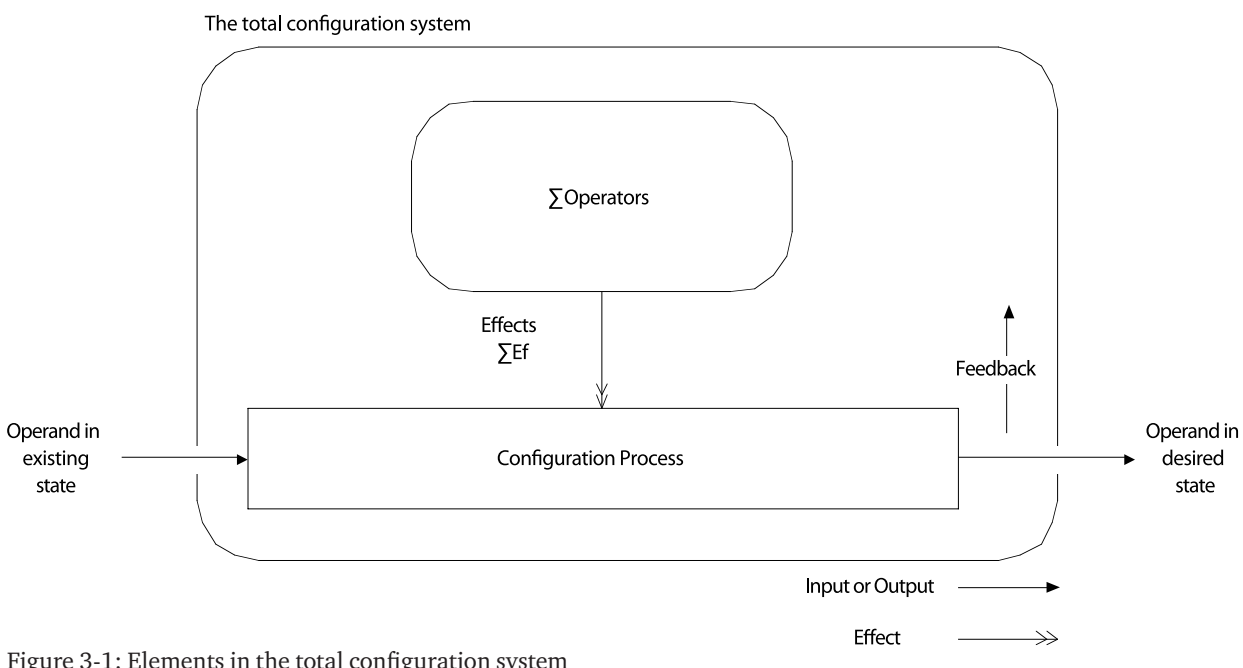

Figure 3-1: Elements in the total configuration system

\subsection{The operand of the TCS}

A very central part of understanding the product configuration systems as technical system is defining the operand. The operand is the description of a product (in it self a technical system). In existing state it is a description of a high abstraction level, but yet it describes the needs of a customer. The operand in desired state is a configuration of a product. Therefore, we define the total configuration systems as the system that transforms the need of a customer to a specification of the products component structure. This is a transformation of a description of the customers' requirements for the product on a high abstraction level, to a description of product on a very concrete.

The Customers knowledge of the product (technical system) he wishes to configure/buy is not complete. The customer is rarely able to describe the product completely on any one of the four different abstraction levels of a technical system. The customer perhaps has complete knowledge about the purpose that the product has to fulfill, some knowledge about the function structure, none knowledge about the organ structure, and a perhaps a bit knowledge about the components structure of the product.

The goal of the configuration process is to concretize the users understanding of the product. This is done by combining predefined entities (physical or non-physical) and defining their variable properties, while obeying constraints and legal interface combinations, in a way that satisfies given requirements.

The configuration process in the total configuration system changes the description of the product, and thus, the operand is defined as the description of the product. Following this logic, the operand in the existing state is the description of the product before it is configured, and the operand in the desired state is the description after the product has been configured. 


\subsection{The Operators of the TCS}

Operators guide and drive the configuration process. We identify the following classes of operators in the total configuration system: 1) Users (human system), 2) Product configuration system (technical system), and 3) Organization (active environment).

In configuration literature all three classes of operators have traditionally been perceived as rational operating systems that act in a rational or at least predictable way. Often this has been justified by referring to general system theories such as (Bertalanffy 1972;Bertalanffy 1950). As it might not be that sufficient to understand all three systems as being rational systems, they will be described in the following three sections.

\subsubsection{The User as Operator}

The knowledge needed to solve the configuration task depends on the abstraction level of the user. If the user has an enormous knowledge of the product, he might wish to configure the product on a fully structural level, selecting and configuring components. A user with less product knowledge might wish to configure the product on a more functional level, ensuring that the product gets the desired functionality and paying no attention to the components used, as long the desired functionality is delivered.

\subsubsection{The Product Configuration System as Operator}

The basis of any product configuration system is knowledge; knowledge about the product being configured, and knowledge about the process the configuration system supports. As previously mentioned, we define the purpose of any configuration system as to support parts of the configuration process.

The product knowledge needed to solve the configuration task depends on the abstraction level of the user and the abstraction level at which the customer presents his needs as input to the configuration process.

If the user has a high degree of knowledge of the product, he might wish to configure the product by picking and configuring components. A user with less product knowledge might wish to configure the product on a more functional level, ensuring that the product gets the desired functionality and paying no attention to the components used, as long the desired functionality is delivered.

The foundation of the product configuration system is an abstract model of the product (a product model) that can transform user requirements to a more or less concrete component structure of the product. In relation to the effect delivered to the configuration process I can identify three different effects that a product configuration system exerts onto the configuration process:

- Concretizing knowledge about the product

- Abstracting knowledge about the product

- Validating knowledge about the product 
These three effects are not mutually exclusive. A configuration system can be designed to concretize from i.e. function structure to component structure, and then validate the component structure as components are interchanged by the user, and finally presenting the results of the configuration process by abstracting to a higher abstraction level. The three effects of product configuration systems are illustrated in Figure 3-2.

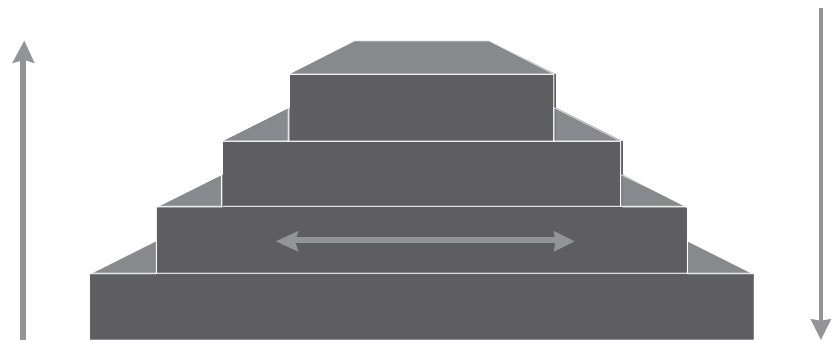

Figure 3-2: The three possible effects of a PCS, abstracting, concretising and validating product knowledge

\subsubsection{Understanding the Organization as Operator}

The active environment of the configuration process is comprised of actors, technical systems, and structures. The active environment is the part of the environment which has a direct relationship to the product configuration system. The active environment is of course dependent on how the total configuration system is defined. In this model for the application of product configuration systems we focus on the organization as being the active environment.

\subsection{Understanding the Configuration Process of the TCS}

By definition the configuration task combine predefined entities and define their variable properties, while obeying constraints. As a technical system the configuration process is driven by the three operators of the total configuration system. In this perspective we can no longer understand the configuration process as a simple technical process where software translates customer requirements into product specifications.

An important lesson learned from the theory of technical systems is that, the technical process can not be designed. The only thing, which a designer can be totally in control of and design, is the technical system (the product). Likewise the configuration process can not be designed either. (This is less true when the users of a configuration system are employed by the company and more true when the users are customers not employed by the company).

The only thing you can be in control of, and which behave in a deterministic way is the product configuration system. And it is possible to organize the user interface of the product configuration system and the design the system by using user-centred development techniques such as contextual design as presented by (Beyer \& 
Holtzblatt 1998), and in that way motivate the user to perform a sequence of task in a given order.

\section{Discussion and summary}

By using the theory of technical systems it is possible to understand product configuration systems from a new perspective emphasizing the system as a whole. Most literature on product configuration systems merely discusses the technical problems of product configuration or a procedure for their implementation.

The theory of technical systems provides a holistic approach to the use and implementation of product configuration systems. The product configuration system depends on the organization and uses, and these three influence and drive the configuration process. As such, each change in the configuration process must be accompanied by changes in the three operators.

This understanding is helpful when designing a new or changing an existing configuration system. Depending on what is to be configured this can have significant implications. In engineering firms product knowledge is complex and cannot be transformed into rules in a configuration system. The engineers retain a lot of knowledge and in particular knowledge needed to develop the products. A configuration system, on the other hand, can only solve the configuration problems thought to exist at the time of the latest revision. Engineering products are dynamic in nature and the organization has the knowledge to redesign the engineering products. Using the theory of technical systems we can deduce that a product configuration system must be general and allow the organization who has a much broader knowledge base to be part of the configuration process. Again this will allow us to understand the users who must have sufficient knowledge to interface the organization, product configuration system and the customer.

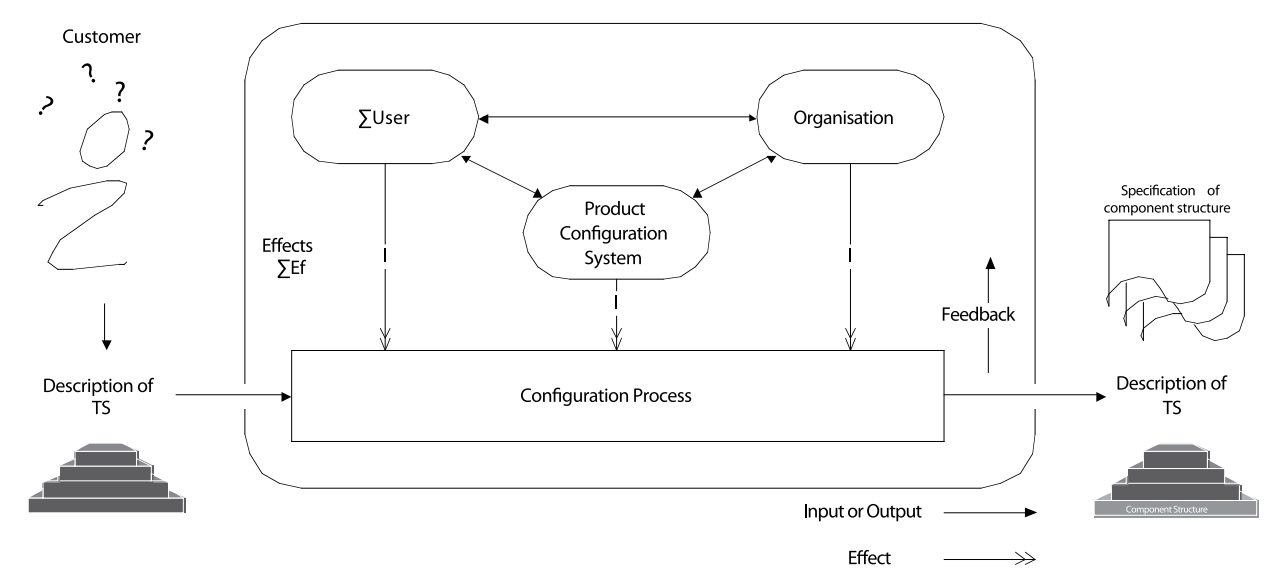




\section{Refrences}

Andreasen, M. M. 1994, "Modelling - The Language of the Designer", Jornal of Engineering Design, vol. 5, no. 2, pp. 103-115.

Bertalanffy, L. V. 1950, "An Outline of General System Theory”, British Journal for the Philosophy of Science, vol. 1, no. 2, pp. 134-165.

Bertalanffy, L. V. 1972, "The History and Status of General Systems Theory", Academy of Management Journal, vol. 15, no. 4, pp. 407-426.

Beyer, H. \& Holtzblatt, K. 1998, Contextual Design - Defining Customer-Centered Systems Morgan Kaufmann Publishers.

Brown, D. C. 1998, "Defining configuring", (AI EDAM) Artificial Intelligence for Engineering Design, Analysis and Manufacturing, vol. 12, no. 4, pp. 301-305.

Edwards, K. \& Møldrup, M. "Unexpected emergence of a Community of Practice when implementing Product Configuration Systems”, in HAANAHA '04, Dept. of Industrial Engineering National University of Ireland, Galway Ireland, pp. 191-205.

Edwards, K. \& Riis, J. 2004, "Expected and Realized Costs and Benefits when Implementing Product Configuration Systems", in Design2004.

Felfernig, A., Friedrich, G. E., \& Jannach, D. 2000, "UML as domain specific language for the construction of knowledge-based configuration systems", International Journal of Software Engineering and Knowledge Engineering, vol. 10, no. 4, pp. 449-469.

Forza, C. \& Salvador, F. 2007, Product Information Management for Mass Customization Palgrave Macmillan, New York.

Haug, A. 2007, Representation of Industrial Knowledge - as a Basis for Developing and Maintaining Product Configurators.

Hopgood, A. A. 2001, Intelligent systems for engineers and scientists, 2nd ed. edn, CRC Press LLC, Boca Raton, Florida.

Hubka, V. \& Eder, W. E. 1988, Theory of Technical Systems - A Total Concept Theory for Engineering Design, 2nd edition edn, Springer-Verlag, Berlin.

Hvam, L. 1999, "A procedure for building product models", Robotics and ComputerIntegrated Manufacturing, vol. 15, no. 1, pp. 77-87.

Hvam, L., Mortensen, N. H., \& Riis, J. 2008, Product Customization Springer-Verlag, Berlin Heidelberg.

Jackson, P. 1999, Introduction to expert systems, 3rd edn, Addison Wesley, Essex.

Mittal, S. \& Frayman, F. 1989, "Towards a generic model of configuration tasks", IJCAI-89 Proceedings of the Eleventh International Joint Conference on Artificial Intelligence pp. 1395-1401.

Sabin, D. \& Weigel, R. 1998, "Product configuration frameworks-a survey", IEEE Intelligent Systems, vol. 13, no. 4, pp. 42-49.

Salvador, F. \& Forza, C. 2004, "Configuring products to address the customization-responsiveness squeeze: A survey of management issues and opportunities", International Journal of Production Economics, vol. 91, no. 3, pp. 273-291. 
Schwarze, S. 1996, Configuration of Multiple-Variant Products - Application Orientation and Vagueness in Customer Requirements, Doctor of Technical Sciences, BWI Betriebswissinschaftliches Institut ETH Zürich.

Shaw, N. K., Bloor, M. S., \& de Pennington, A. 1989, "Product data models", Research in Engineering Design, vol. 1, no. 1, pp. 43-50.

Soininen, T., Tiihonen, J., Mannisto, T., \& Sulonen, R. 1998, “Towards a general ontology of configuration", Artificial Intelligence for Engineering Design, Analysis and Manufacturing: AIEDAM, vol. 12, no. 4, pp. 357-372.

Stumptner, M. 1997, "An overview of knowledge-based configuration", Ai Communications, vol. 10, no. 2, pp. 111-125.

White, S. A. 2006, Introduction to BPMN. 